\title{
Clinic-Based Family Planning and Reproductive Health Services in Africa: Findings from Situation Analysis Studies
}

E D I T O R S

Kate Miller - Robert Miller - Ian Askew Marjorie C. Horn - Lewis Ndhlovu 


\section{Clinic-Based Family Planning and Reproductive Health Services in Africa: Findings from Situation Analysis Studies}

\section{EDITORS}

Kate Miller • Robert Miller • Ian Askew

Marjorie C. Horn • Lewis Ndhlovu

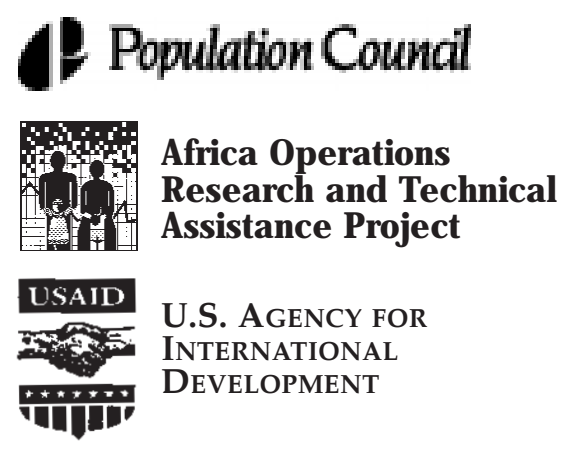


The Population Council seeks to improve the wellbeing and reproductive health of current and future generations around the world and to help achieve a humane, equitable, and sustainable balance between people and resources. The Council, a nonprofit, nongovernmental research organization established in 1952, has a multinational board of trustees; its New York headquarters supports a global network of regional and country offices.

Population Council

One Dag Hammarskjold Plaza

New York, New York 10017 USA

tel: (212) 339-0500

fax: (212) 755-6052

e-mail: pubinfo@popcouncil.org

www.popcouncil.org

(C) 1998 by The Population Council, Inc.

Any part of this document may be reproduced without permission so long as it is not sold for profit.

\section{Population Council Cataloging-in-Publication Data}

Clinic-based family planning and reproductive health services in Africa : findings from situation analysis studies / by Kate Miller, Robert Miller, Ian Askew, Marjorie C. Horn and Lewis Ndhlovu. ; forewords by Elizabeth Maguire and Ayo Ajayi. — New York : The Population Council, 1998.

p. cm.

ISBN 0-87834-094-7

1. Birth control programs - Africa. 2. Women - Health and Hygiene Africa. I. Miller, Kate. II. Miller, Robert. III. Askew, Ian. IV. Horn, Marjorie C. V. Ndhlovu, Lewis. VI. Population Council. Africa Operations Research and Technical Assistance Project. VII. United States Agency for International Development.

HQ 766.5 .A35 C439 1998

This publication was supported by the Population Council's Africa Operations Research and Technical Assistance Project II. The Africa OR/TA Project II is funded by the U.S. Agency for International Development (USAID), Office of Population, Contract No. CCP-3030-C-00-3008-00, Strategies for Improving Family Planning Service Delivery.

The observations, conclusions, and recommendations set forth in this publication are those of the authors and do not necessarily represent the views of USAID. 


\section{Contents}

Abbreviations

iv

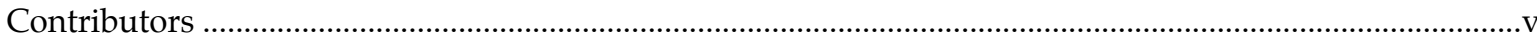

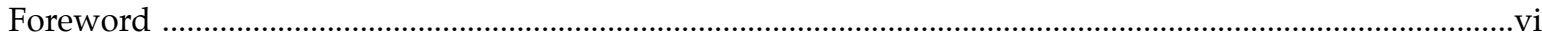

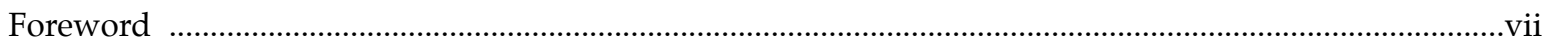

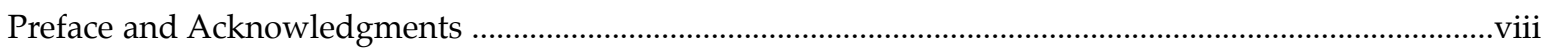

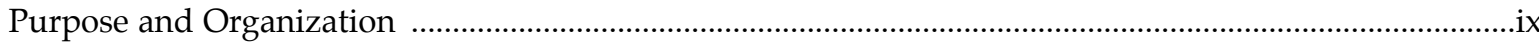

\section{OVERVIEW}

1. Introduction .3

2. Descriptions of the Family Planning Programs Studied

\section{BASIC STUDY FINDINGS \& THEIR UTILIZATION}

3. Indicators of Readiness and Quality: Basic Findings

4. Using Situation Analysis to Improve Reproductive Health Programs .87

\section{FACTORS AFFECTING QUALITY}

5. Determinants of Quality of Family Planning Services:

A Case Study of Kenya 107

6. Unrealized Quality and Missed Opportunities in Family Planning Services .125

7. Urban and Rural Family Planning Services: Does Service Quality Really Differ?

\section{STANDARDS AND GUIDELINES FOR SERVICES}

8. How Providers Restrict Access to Family Planning Methods: Results from Five African Countries

9. Tests and Procedures

Required of Clients in Three

Countries of West Africa 181

\section{CURRENT AND FUTURE PROGRAM DIRECTIONS}

10. Integrating STI and HIV / AIDS Services at $\mathrm{MCH} /$ Family

Planning Clinics

11. Changes in Quality of Services Over Time

VI. SUMMARY, CONCLUSIONS, FUTURE DIRECTIONS, AND RECOMMENDATIONS

12. Clinic-Based Family Planning and Reproductive Health Programs in Sub-Saharan Africa 


\section{Abbreviations}

\begin{tabular}{|c|c|c|c|}
\hline AIDS & $\begin{array}{l}\text { acquired immuno-deficiency } \\
\text { syndrome }\end{array}$ & $\begin{array}{l}\text { MSH } \\
\text { ML/GA }\end{array}$ & $\begin{array}{l}\text { Management Services for Health } \\
\text { mini-laparotomy/general anesthetic }\end{array}$ \\
\hline ANOVA & analysis of variation & $\mathrm{ML} / \mathrm{LA}$ & mini-laparotomy/local anesthetic \\
\hline AVSC & AVSC International & $\mathrm{MOH}$ & Ministry of Health \\
\hline BOTSPA & $\begin{array}{l}\text { (not an abbreviation) } \\
\text { Botswana Population Assistance }\end{array}$ & MOH\&CW & $\begin{array}{l}\text { Ministry of Health and } \\
\text { Child Welfare }\end{array}$ \\
\hline & Project & NCC & Nairobi City Commission (Kenya) \\
\hline $\begin{array}{l}\mathrm{BP} \\
\mathrm{CBD}\end{array}$ & $\begin{array}{l}\text { blood pressure } \\
\text { community-based distribution }\end{array}$ & NCPD & $\begin{array}{l}\text { National Council for Population and } \\
\text { Development }\end{array}$ \\
\hline $\mathrm{COC}$ & combined oral contraceptives & NFP & natural family planning \\
\hline COPE & client oriented and provider efficient & NGO & nongovernmental organization \\
\hline CPR & contraceptive prevalence rate (modern & OC & oral contraceptive \\
\hline & contraceptives only) & OR & Operations Research \\
\hline CYP & couple-years of protection & ORS & oral rehydration salts \\
\hline DfID & $\begin{array}{l}\text { Department for International } \\
\text { Development }\end{array}$ & PID & pelvic inflammatory disease \\
\hline DHS & Demographic and Health Survey & PNPF & $\begin{array}{l}\text { Programme National pour la } \\
\text { Panification Familiale }\end{array}$ \\
\hline FHI & Family Health International & POP & progestin-only pill \\
\hline $\mathrm{FP}$ & family planning & PPAG & Planned Parenthood Association \\
\hline GFPHP & $\begin{array}{l}\text { Ghana Family Planning and } \\
\text { Health Program }\end{array}$ & PPEN & $\begin{array}{l}\text { of Ghana } \\
\text { Planned Parenthood Federation of }\end{array}$ \\
\hline GHANAPA & $\begin{array}{l}\text { Ghana Population and } \\
\text { AIDS Project }\end{array}$ & & Nigeria \\
\hline GRMA & $\begin{array}{l}\text { Ghana Registered Midwives } \\
\text { Association }\end{array}$ & $\mathrm{P}$ & $\begin{array}{l}\text { Primary Health Care Operations } \\
\text { Research }\end{array}$ \\
\hline GTZ & $\begin{array}{l}\text { Gesellschaft für Technische } \\
\text { Zussammenarbeit }\end{array}$ & $\begin{array}{l}\text { RH } \\
\text { RTI }\end{array}$ & $\begin{array}{l}\text { reproductive health } \\
\text { reproductive tract infection }\end{array}$ \\
\hline HIV & human immuno-deficiency virus & SA & Situation Analysis \\
\hline HSD & Honestly Significance Difference & SDP & service delivery point \\
\hline ICPD & $\begin{array}{l}\text { International Conference on Population } \\
\text { and Development, Cairo, } 1994\end{array}$ & SEATS & $\begin{array}{l}\text { Family Planning Service Expansion } \\
\text { and Technical Support Project, } \\
\text { John Snow Inc. }\end{array}$ \\
\hline IEC & $\begin{array}{l}\text { information, education, and } \\
\text { communication }\end{array}$ & STD & sexually transmitted disease \\
\hline INTRAH & $\begin{array}{l}\text { Program for International } \\
\text { Training in Health }\end{array}$ & $\begin{array}{l}\text { STI } \\
\text { TA }\end{array}$ & $\begin{array}{l}\text { sexually transmitted infection } \\
\text { technical assistance }\end{array}$ \\
\hline IPPF & $\begin{array}{l}\text { International Planned } \\
\text { Parenthood Federation }\end{array}$ & $\begin{array}{l}\text { TFR } \\
\text { TL }\end{array}$ & $\begin{array}{l}\text { total fertility rate } \\
\text { tubal ligation }\end{array}$ \\
\hline $\begin{array}{l}\text { IUD } \\
\text { IHPIFCO }\end{array}$ & intrauterine device & UNAIDS & $\begin{array}{l}\text { Joint United Nations Programme on } \\
\text { HIV / AIDS }\end{array}$ \\
\hline JHPIEGO & $\begin{array}{l}\text { Johns Hopkins Program for } \\
\text { International Education in } \\
\text { Reproductive Health }\end{array}$ & UNDP & $\begin{array}{l}\text { United Nations Development } \\
\text { Programme }\end{array}$ \\
\hline $\mathrm{JHU} / \mathrm{PCS}$ & Johns Hopkins University / Population & UNFPA & United Nations Population Fund \\
\hline & Communication Services & USAID & United States Agency for International \\
\hline JSI & John Snow Incorporated & & Development \\
\hline LAM & lactational amenorrhea method & VSC & voluntary surgical contraception \\
\hline LMP & last menstrual period & WHO & World Health Organization \\
\hline MAQ & $\begin{array}{l}\text { Maximize Access and Quality (USAID } \\
\text { initiative) }\end{array}$ & ZNFPC & $\begin{array}{l}\text { Zimbabwe National Family Planning } \\
\text { Council }\end{array}$ \\
\hline $\mathrm{CH}$ & maternal and child health & & \\
\hline
\end{tabular}

iv 


\section{Contributors}

\section{EDITORS}

Kate Miller, M.P.H.

Formerly Staff Program

Associate, Population Council,

New York; currently doctoral

student, Department of

Demography, University of

Pennsylvania, Philadelphia

Robert Miller, Dr.P.H.

Senior Program Associate,

Population Council, New York

Ian Askew, Ph.D.

Senior Associate and Project

Director, Africa Operations

Research and Technical

Assistance Project II, Population

Council, Nairobi, Kenya

Marjorie C. Horn, Ph.D.

Deputy Chief, Research

Division, Office of Population,

U.S. Agency for International

Development, Washington, D.C.

Lewis Ndhlovu, M.Sc.

Associate, Population Council,

Nairobi, Kenya

\section{AUTHORS}

Lisanne F. Brown, Ph.D.

Research Assistant Professor,

Department of International

Health and Development, Tulane

School of Public Health and

Tropical Medicine, New Orleans

Judith Bruce, B.A.

Senior Associate and Program

Director, Gender, Family, and

Development Program,

Population Council, New York

\section{Goli Fassihian, M.P.H.}

Data Analyst, Population

Council, New York

Andrew Fisher, Sc.D.

Senior Associate and Program

Director, Horizons Project,

Population Council,

Washington, D.C.

\section{Martin Gorosh, Dr.P.H.}

Clinical Professor of Public

Health, Center for Population and Family Health, Joseph L.

Mailman School of Public

Health, Columbia University,

New York; and Consultant,

SEATS Project, John Snow Inc.,

Arlington, Virginia

Nicole Haberland, M.P.H.

Program Associate, Population

Council, New York
Heidi Jones, B.A.

Data Analyst, Population

Council, New York

Ndugga Maggwa, M.D., M.Sc.

Associate, Population Council,

Nairobi, Kenya

Gwendolyn T. Morgan, M.P.H.

Doctoral student, Department of

International Health and

Development, Tulane School of

Public Health and Tropical

Medicine, New Orleans

Melinda Ojermark, M.P.H.

Formerly Regional Director for

Africa, SEATS Project, John

Snow Inc., Arlington, Virginia;

currently Chief Advisor to the

Vietnam-Sweden Health

Cooperation, Ministry of Health, InDevelop, Hanoi, Vietnam

Elizabeth Pearlman, B.A.

Program Assistant, Population

Council, New York

Brian Pence, B.A.

Program Assistant, Population

Council, New York

Carolyn Gibb Vogel, M.P.H.

Formerly Technical Officer,

SEATS Project, John Snow Inc.,

Arlington, Virginia; currently

Research Associate, Population

Action International,

Washington, D.C. 


\section{Foreword}

The United States Agency for International Development (USAID) is proud to have supported publication of Clinic-Based Family Planning and Reproductive Health Services in Africa: Findings from Situation Analysis Studies. This volume well reflects USAID's strong commitment to improving the quality of reproductive health care and expanding access for underserved groups. Nowhere are these efforts more important than in Africa, where use of family planning and other measures of reproductive health status are lowest among the world's regions. Helping to provide high-quality health services that meet couples' reproductive needs is a socially just and humane goal in itself. Moreover, higherquality services can reasonably be expected to result in better outcomes with regard to measures of client satisfaction, continuation of use of contraception, and reproductive health, which in turn have positive implications for both the individual client and the population at large.

As we strive to improve quality of care, it is important to be able to define and measure it. Building on the pioneering work of Bruce and Jain in this area, staff of the Africa Operations Research and Technical Assistance Project operationalized the definition of quality at the field level with the Situation Analysis methodology, transforming the definition into measurable variables. Managers and donor agencies welcomed these studies because they provided the tools necessary to diagnose and treat critical service-delivery problems. The project has demonstrated that when program managers are involved in all phases of the research, its results will be utilized. USAID Missions, program managers, and other donors have been using Situation Analysis findings for the last decade in Africa and other regions to better identify and understand the extent and nature of problems of access and quality, and to help focus our assistance and programmatic support on overcoming these problems.

While much progress has been made in providing access to quality services in sub-Saharan Africa, the Situation Analysis data from the studies reported here highlight major challenges for improving reproductive health service delivery. Inadequate client counseling is a pervasive pattern in the region, along with insufficient attention to infection prevention. Further, the integration of sexually transmitted infection (STI)/HIV/AIDS prevention with family planning services is shown to exist in only rudimentary form in many programs, and even the relatively straightforward promotion of condoms as a way of preventing both pregnancy and the spread of STIs/HIV/AIDS is often found to be weak.

The findings reported in this volume, along with the entire body of material from which they were taken, will require discussion, critique, and debate. Utilization of these findings is key for making important policy and program changes to improve service delivery. Ultimately, many of the solutions to the programmatic problems described in this volume will need to be further developed, tested, evaluated, expanded to the national level, and diffused throughout the region and beyond.

Operations Research will address many of these tasks through the new FRONTIERS in Reproductive Health Program, funded by USAID. In addition, USAID will continue to provide support for additional studies of program operations at the field level through the MEASURE program, which began this year. Through these and other research activities, we plan to expand the use of qualitative research on issues such as client satisfaction, which are not captured well by facility- or clinic-based studies.

Responding to the critical issues raised in this volume will require the support and cooperation of program managers, policy makers, and donors. Our challenge and responsibility now is to undertake the sustained efforts necessary to use these findings to vastly improve the reproductive health of women and men around the world.

Elizabeth Maguire Director

Office of Population United States Agency for International Development 


\section{Foreword}

Organized family planning services have been offered in one form or another in sub-Saharan Africa for the past three decades. During most of this period, contraceptive services have been offered within the context of broader maternal and child health $(\mathrm{MCH})$ services, which should make integration of services much easier. Yet the studies documented in this volume show that while millions of women and men have been able to obtain contraceptive methods of their choice at these clinics, the degree of integration of family planning services with other reproductive health services is extremely poor. The paucity of integrated services for diagnosis and treatment of preexisting conditions such as reproductive tract infections (RTIs) and the lack of counseling of clients on their risk of sexually transmitted infections (STIs) raise not just concerns about inefficient utilization of resources, but also serious ethical issues in an environment in which levels of AIDS-related mortality and morbidity are the highest in the world. Services should focus not only on enabling individuals to avoid unwanted childbearing, but also on helping them prevent disease. The paradoxical situation of unintegrated services within an $\mathrm{MCH}$ context is a product of the history and evolution of family planning services in the region, whereby family planning services were initiated and established with donor funds.

The Situation Analysis methodology provided the first tool for a systematic assessment of the state of readiness of service delivery points (SDPs) to offer family planning services. Although later adapted and revised to assess family planning services within the context of a broader reproductive health approach, most of the studies included in this volume were conducted prior to the 1994 International Conference on Population and Development. The poor performance of the clinics studied on a wide range of variables is a clear indication of the amount of work that needs to be done to fully operationalize the reproductive health approach at the level of the SDP. This volume provides the most comprehensive review to date of clinic-based services in Africa and represents the state of the art in measuring, ensuring, and improving the quality of family planning services. The results presented herein form a common knowledge base and serve as a framework that should guide current and future efforts to improve the quality of family planning services and ensure that the limited and declining resources available for health care are utilized in the most effective and efficient way.

The 12 country assessments included in this volume highlight two important points about the central role of the service provider in improving the quality of services provided to clients. First, through their attitudes, knowledge, skill, and enthusiasm, service providers serve as the main link between the entire service system and its clients. Equally important, however, is the content of the information that is exchanged between the provider and the client. In addition to information relating to specific contraceptive methods, this information should include the role of sexual partners in the risk of infection, the key symptoms of the most serious RTIs, and the degree of protection from RTIs and STIs offered by various contraceptives. It is obvious, therefore, that the proper selection, training, and supervision of service providers offer perhaps the most direct and costeffective approach for improving the quality of family planning and reproductive health services received by clients.

The Situation Analysis approach, which was pioneered in Africa and of which Africans are justifiably proud, has made significant contributions to the family planning field. The continent now has an opportunity to lead efforts to expand the approach to include broader reproductive health services. If such efforts enable us to discover how best to give clients the information they need to increase their knowledge and change their behavior to prevent both disease and unwanted childbearing, we will have bridged the gap between what is and what should be.

Ayo Ajayi

Regional Director, East and Southern Africa

Population Council, Nairobi 


\section{Preface and Acknowledgments}

As was noted in the Preface and Acknowledgments to The Situation Analysis Approach to Assessing Family Planning and Reproductive Health Services: A Handbook, published in 1997, the Situation Analysis study methodology was developed and first used in Africa. As the study methodology diffused through much of Africa and the world, the studies could not have been implemented without the cooperation and support of national family planning program managers and ministry of health officials who were committed to seeing how their programs were functioning at the field level, "warts and all."

The thousands of field visits, interviews, and observations documented in this volume attest to the dedication and hard work of many hundreds of field researchers and the patience and openness of both service providers and the women attending service delivery points. We remain deeply indebted to the thousands of family planning and reproductive health staff in all 11 countries who welcomed our research teams at their facilities, often found places for them to sleep, opened their cupboards and records for inspection, allowed their clients to be interviewed and observed, and patiently answered our numerous questions. We thank the thousands of women who allowed us to observe them receiving services and who then proceeded to answer dozens of sometimes intimate questions. We received so much assistance from so many Population Council staff in the implementation of the studies that we are hesitant to attempt to name them all. Yet we would definitely be remiss if we did not recognize our heavy debts to Nafissatou Diop, Joanne Gleason, Inoussa Kabore, Barbara Mensch, Naomi Rutenberg, Diouratie Sanogo, Kathleen Siachitema, John Skibiak, Julie Solo, Placide Tapsoba, and Mounir Toure.

This volume presents a summary of many findings and an analysis of several program issues. We are grateful that so many reproductive health researchers and practitioners in a variety of institutions have been interested in using the Situation Analysis data to explore these issues. We do not doubt that many additional issues deserve similar treatment, and we hope our database can continue to be used productively by other researchers. Despite the fact that this volume represents the culmination of 10 years of effort in conducting Situation Analysis studies in Africa, we hope that the data will remain useful long into the future as a source of additional insights for program managers, as a source of data for researchers interested in a variety of program issues, and as a baseline for assessing future program progress.

Throughout the process of implementing the many studies included in this volume, we received considerable financial and technical support from USAID Washington and the many USAID Mission staff who provided approvals for all of the studies and were frequently contributors to the research process. We remain greatly indebted to USAID for this support and encouragement. The high level of encouragement, frequent utilization of study findings, and numerous technical suggestions for improving the study methodology are gratefully acknowledged.

We are greatly indebted to Rona Briere, who painstakingly edited the entire volume and provided numerous creative suggestions for its formatting. We are indebted as well to the staff of the Population Council's Office of Publications for designing the cover and text, and offering additional creative formatting suggestions. Alisa Decatur assisted our editor by typing the manuscript and facilitating quick transmission. Brian Pence provided research assistance, proofread all of the chapters, and coordinated communications throughout the entire editing and production process. We would also like to thank Nicholas Gouede and Peggy Knoll for developing an initial distribution plan for this volume.

We benefited greatly from the review, comments, and suggestions of many of our colleagues and friends, including Michael Commons, Ralph Frerichs, Steve Green, Anrudh Jain, Young-Mi Kim, Gitanjali Pande, James Shelton, and Eugene Weiss. The production of this volume has been an intensive, collaborative process among the editors and authors. We would appreciate receiving comments and suggestions from readers, and reports from those who carry out situation analysis studies.

The Editors 


\section{Purpose and Organization}

This volume presents results from 12 Situation Analysis studies conducted in sub-Saharan Africa between 1989 and 1996. It summarizes the study findings on about 100 variables; analyzes significant regional patterns and trends, including the integration of family planning and HIV/AIDS activities; identifies major problems with the quality of ser- vices; and attempts to measure changes in the quality of services over time. By synthesizing these findings from nearly a decade of research, this volume is intended to contribute to the understanding and improvement of family planning and reproductive health programs in sub-Saharan Africa and around the world. This volume is organized in six parts:

\section{Overview}

presents the background and context for the 12 studies and describes the Situation Analysis approach.

\section{Basic Study Findings \& Their Utilization}

reviews the overall study findings on indicators of readiness and quality of family planning and reproductive health services and examines the various purposes for which these findings have been used.

\section{Factors Affecting Quality}

provides a detailed look at the factors found to affect the quality of family planning services.

IV. Standards and Guidelines for Services describes the restrictions and requirements imposed by providers on those seeking family planning services.

V. Current and Future Program Directions
examines the trend toward integrated services and documents pro-
gram changes over time.





\section{OVERVIEW}

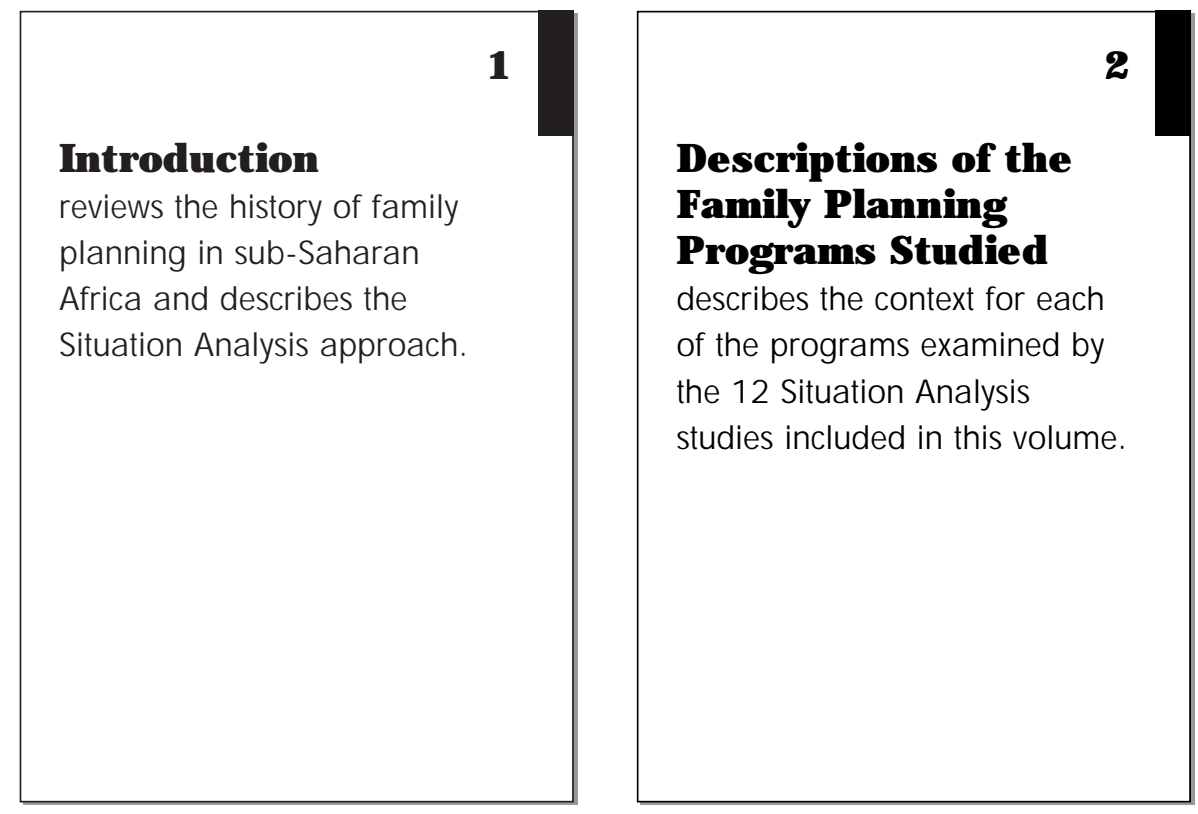





\section{Introduction}

\section{Robert Miller, Andrew Fisher, and Ian Askew}

Access to high-quality family planning and reproductive health services, including the control of sexually transmitted infections (STIs), is a central and growing concern in sub-Saharan Africa today for many reasons. First, sub-Saharan Africa has the highest population growth rates of any region, averaging almost 3 percent per year, and governments are increasingly concerned about the adverse effects of such rapid population growth on development efforts. Women in Africa have children early and in large numbers, with completed family size averaging around 6 children. Second, an estimated 22 million women in the region have an unmet need for family planning services, meaning that they are not currently using family planning, but want to delay or avoid future pregnancies (Rosen and Conly, 1998). Third, 40 percent of the world's 215,000 annual deaths among women in childbirth occur in the region (Rosen and Conly, 1998). Fourth, both health problems stemming from illegal abortion and the increasing sexual activity of adolescents fuel a growing interest in and response to family planning and broader reproductive health programs (Alan Guttmacher Institute, 1998). Finally, and perhaps most important, the world's HIV/AIDS pandemic is hitting Africa-especially East and Southern Africaharder than any other region; sub-Saharan Africa is home to over two-thirds of all people in the world living with HIV and the site of 83 percent of global AIDS deaths (UNAIDS and World Health Organization, 1998). Thus the need for more comprehensive high-quality health services is apparent from many different perspectives.

Yet despite these compelling reasons for family planning and reproductive health services, and despite the unmet need in the region, those services that do exist often are underutilized (Fisher and Miller, 1996). One hypothesis explaining this conflict between need and practice is the poor quality of the services that are offered. (Other explanations relate to high demand for children, low levels of motivation for avoiding pregnancy, and women's lack of empowerment to implement their goals.) Situation Analysis is a tool for examining the quality of family planning and reproductive health services, with the ultimate objective of helping program managers identify and solve problems that compromise the quality of their programs.

This volume reports the results and implications of 12 Situation Analysis studies undertaken in 11 countries since 1989 under the Population Council's Africa Operations Research and Technical Assistance (OR/TA) Projects, funded by the United States Agency for International Development (USAID). The purpose of these studies was to determine the quality of family planning and reproductive health services in sub-Saharan Africa. The context for the studies includes an international advocacy movement that culminated in the Cairo International Conference on Population and Development (ICPD) of 1994, which ratified a worldwide commitment to the provision of comprehensive reproductive health services, including family planning and the control of STIs, and to a broad focus on the special problems of women and girls.

\section{HISTORY OF FAMILY PLANNING IN AFRICA}

In the 1970s, access to modern contraception was extremely limited in Africa, except for pilot program activities (National Research Council, 1993) 
and the early efforts of International Planned Parenthood affiliates and other nongovernmental organizations (NGOs), which operated mainly in urban areas. African policy makers did not experience the absolute numbers and the high population density that characterized the Asian context. Consequently, they expressed little support for population control, which was the stimulus for the first family planning programs in India in the 1950s and in much of the rest of Asia and Latin America in the 1960s. Further, policy makers tended to shy away from family planning, which was controversial in the sociocultural setting in much of Africa. This was especially true in Francophone West Africa, which was strongly influenced by conservative French laws. At the Bucharest World Population Conference in 1974, African leaders joined others from the developing world in voicing support for socioeconomic development and "a new world order," rather than a more demographically oriented approach to Third World problems (National Research Council, 1993; Miller and Rosenfield, 1996).

During the 1980s, considerable change occurred in the African policy climate. The climate became increasingly favorable for population policies and family planning programs as governments documented and grew more concerned about high population growth rates. In 1984, African leaders endorsed the Kilimanjaro Programme of Action for African Population and Self-Reliant Development, formulated in Tanzania, which called for the provision of family planning services and their integration into maternal and child health $(\mathrm{MCH})$ programs (National Research Council, 1993).

Thus in contrast with the Asian context, where family planning services were often developed independently from health services in special vertical programs supported by economic and demographic rationales, African policy makers opted for a health rationale, an emphasis on spacing (rather than limiting) of births, and the delivery of family planning and reproductive health services within integrated health programs. In Africa, the health approach was considered both culturally and politically more appropriate than a demographic orientation for dealing with the interconnected problems associated with reproductive health, rapid population growth, and economic development. At the ICPD, the world endorsed integrated reproductive health programs more in line with the ideal (but infrequently realized) African models than with the earlier vertical Asian models.

\section{GOVERNMENTAL PROGRAMS IN CLINIC SETTINGS}

In most African countries in the 1990s, the vast majority of women ${ }^{1}$ receive modern family planning methods from governmental sources, rather than from nongovernmental agencies, pharmacies, or private practitioners. Among users of modern methods, the proportions receiving them from governmental sources range from 95\% in Botswana and $71 \%$ in Kenya (two of the most successful programs) to a low of $43 \%$ in Ghana (Ross et al., 1993). These governmental sources are most frequently health facilities rather than community-based distribution (CBD) systems, which have been implemented on a much smaller scale in Africa than was the case in Asia in the 1970s and 1980s (Phillips and Greene, 1993). A wide range of health facilitieshospitals, clinics, and health posts-are still the major source of supply of modern methods in Africa. The rationale behind clinical programs in health settings is succinctly described by Bertrand (1991:21-22):

Clinic-based programs can offer a wider range of contraceptive methods than any of the other service delivery mechanisms because they provide methods that can be administered only by clinical personnel (male and female sterilization, IUDs, implants, and injectables), as well as the so-called non clinical methods (the pill, condoms, and spermicides).... What clinic-based facilities have in common is that the personnel serving the public have received clinical training as physicians, nurses, and in some cases midwives; that they are capable of doing a clinical examination in the course of prescribing contraceptives (if they so choose); that they generally have basic gynecological equipment; and that in urban areas, they usually have access to laboratory facilities (either on the premises or nearby). 
The clinic-based service-delivery system in Africa has been a major focus of African policy makers and the donor community. However, recent developments-ICPD and the worsening of the HIV/AIDS pandemic in the region-have resulted in still greater emphasis on the clinicbased system. In response, Ministries of Health (MOHs) and the donor community are increasing efforts to test potential strategies for some of the most important and relevant ICPD components. Particular emphasis is being placed on the integration of family planning with the prevention and treatment of STIs, including HIV / AIDS (Maggwa and Askew, 1997). (Detail on the program context for each of the 12 Situation Analysis study sites is provided in Chapter 2.)

Views on the effectiveness of the clinic-based approach to family planning programs in Africa have changed substantially in the last decade. Caldwell and Caldwell referred in 1988 (p. 21) to "...the complete failure of African family planning programs to reduce fertility...." However, the decade since that comment was made has seen dramatic declines in fertility in several African countries with active family planning programs (such as Kenya, Zimbabwe, and Botswana), along with significant changes in education, family economics, urbanization, and other factors. Kirk and Pillet (1998:17) conclude that "an assessment of fertility trends has uncovered evidence of initial fertility decline in two-thirds of the countries of subSaharan Africa that had conducted a DHS [Demographic and Health Survey] before mid1995," but "within a group of countries in East and Southern Africa...the fertility transition is now well established and progressing at a rapid pace." Moreover, Kirk and Pillet indicate that "contraceptive use is by far the most important factor accounting for across country differences (in fertility)."

\section{OPERATIONS RESEARCH AND THE SUPPLY-DEMAND CONTROVERSY}

As family planning programs in Asia and Latin America expanded rapidly during the 1970s and 1980s and became more comprehensive in coverage and services, they also became more complex and expensive. New approaches were needed to make them more efficient, more effective, and less costly. In this context, the five-stage problem-solving process of operations research $(\mathrm{OR})^{2}$ (Fisher et al., 1991) was well suited to helping programs focus on supply-side problems and test new service-delivery approaches. USAID provided substantial funding for such studies in every region. Throughout Asia and Latin America, the experimental findings from numerous OR studies were instrumental in helping family planning programs identify new approaches and fine-tune existing service-delivery mechanisms. Family planning programs introduced numerous new program techniques and ways of expanding service delivery-initially tested through a process of OR experimental and quasi-experimental studies. These new approaches included CBD; competency-based, experiential training programs; traditional and modern forms of information, education, and communication (IEC); social marketing; and integrated reproductive health services (Shane and Chalkley, 1998).

In Africa, on the other hand, few family planning programs even existed in the 1970s, and those that did (or were subsequently initiated in the 1980s) generally suffered from very serious weaknesses or hardly functioned at all. Program effort scores were universally weak in 1982 (Ross et al., 1993), and contraceptive prevalence rates were universally low. However, whether low contraceptive prevalence rates were due to poorly functioning programs, lack of demand for family planning, or both was controversial (van de Walle and Foster, 1990; Pritchett, 1994).

Commenting on the increasing change in experience and perspectives that pervaded Africa in the mid-1990s, Fisher (1993:20) notes that until recently, conventional wisdom suggested that "regardless of how effective African family planning programs are in making services available and accessible, the use of family planning services in Africa will remain low because the demand for these services is very low." However, he points to three new sources of data that challenge this "weak demand" hypothesis. DHS surveys conducted throughout Africa indicate that demand for family planningespecially for purposes of spacing births-exists, 
often at levels far higher than expected. Further, the Situation Analysis studies that form the basis for this volume reveal that in every country where these studies have been conducted, significant weaknesses in the supply of services affect the ability of programs to satisfy demand. And OR studies completed throughout the continent demonstrate that "when supply side weaknesses are corrected, when services are made more available, easily accessible, and of higher quality, the use of family planning increases substantially and rapidly" (Fisher, 1993:20). In short, there is demand for family planning services, there are severe servicedelivery weaknesses, and numerous OR studies clearly demonstrate the potential to satisfy the demand when those weaknesses are corrected.

\section{THE SITUATION ANALYSIS APPROACH ${ }^{3}$}

If demand is less of a factor restricting family planning use than was thought to be the case just a few years ago, it would seem likely that the supply of services may be more of a barrier to use than was expected. Indeed, in each of the 11 countries in which a Situation Analysis study has been conducted, major weaknesses have been observed in the availability, functioning, and quality of family planning services, and substantial opportunities for strengthening the quality of care have been identified.

Correcting the weaknesses of African family planning and reproductive health programs is no easy task. In addition to the usual complicationsscarce resources, lack of trained personnel, poor communications, nonavailability of proven, appropriate models-management information systems are generally nonexistent or nonfunctioning. Anecdotal accounts and the opinions of "experts" abounded until 1989, when the first Situation Analysis study was implemented in Kenya. Prior to this study, there was little or no information based on field-level assessments about how programs were functioning. There were no baseline measures by which to evaluate the impact of innovations. Beginning in 1989, representative studies of national service-delivery systems, which included actual observations of the quality of care being received by clients, began to provide systematic information on program strengths and weaknesses that could be used to evaluate and improve programs.

Situation Analysis is a comprehensive and standardized approach for systematically assessing both the readiness of family planning/reproductive health programs to deliver services and the quality of care received by clients. The Situation Analysis approach grew out of a perceived need on the part of program managers to know the actual state of their programs at the field level. It evolved from a simple request by the Division of Family Health within the Kenyan $\mathrm{MOH}$ for assistance in determining their equipment needs.

In developing a response to this request, Africa OR/TA Project staff were influenced by the systems thinking of the Primary Health Care Operations Research (PRICOR) Project (Center for Human Services, 1988), the Rapid Survey Methodology (Frerichs, 1989a; Frerichs and Tar Tar, $1989 \mathrm{~b})$, and the quality-of-care framework outlined by Bruce and Jain (Bruce, 1990). The staff recommended a data collection procedure that would provide a more comprehensive picture of program operations than that represented by the original request, including the functioning of each of the program's subsystems, as well as the quality of care being delivered to clients. The $\mathrm{MOH}$ accepted this proposal.

The Situation Analysis approach is defined as follows:

A description and evaluation of: 1) current family planning policies and service delivery standards, and the availability and functioning of family planning subsystems at a representative sample of service delivery points (SDPs) or all SDPs in a geographic area; 2) the readiness of these subsystems to deliver quality of care to clients; 3) the actual quality of care received by clients at these SDPs; and 4) the impact quality of care has on the fertility behavior of clients (Miller et al., 1997:5). As Mensch et al. (1994:19) note:

Although Situation Analysis borrows from other methodologies, it is considered innovative because it integrates a number of approaches to family planning program 


\section{Figure 1-1. Model underlying Situation Andysis studies}

Program Readiness

+ Controceptives and
supplies
+ Facilities and
equipment
+ Staff training and
attitudes
+ IEC materiak
+ Superyision and
manogement

Qualit' of Care

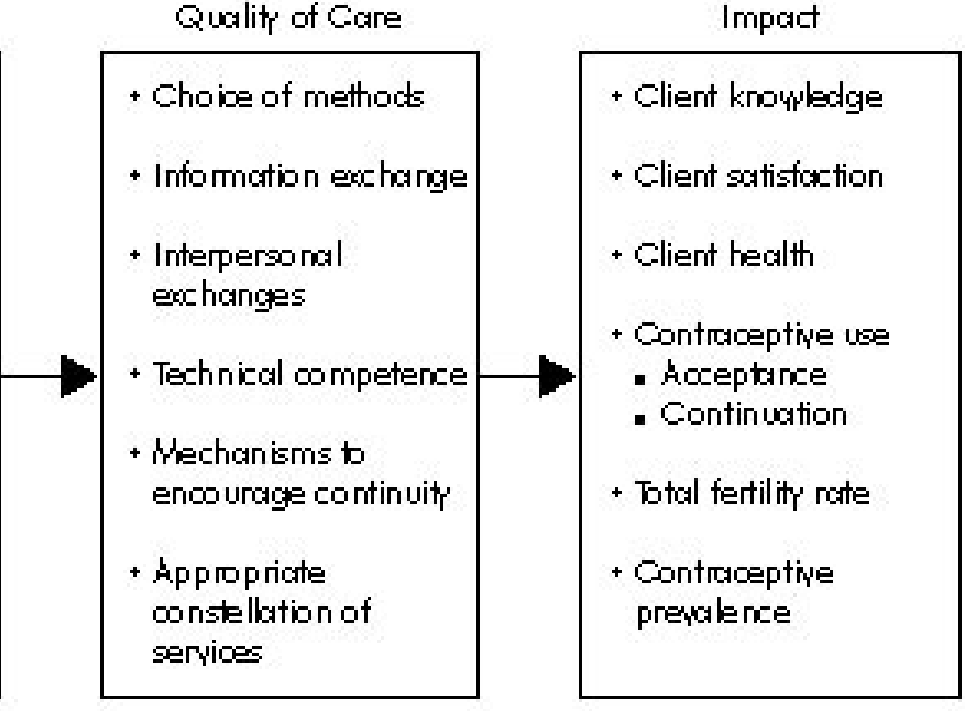

evaluation. These include (1) a systems perspective for identifying crucial subsystem components of program operation; (2) visits to a large sample of SDPs rather than visits to only a few SDPs or reliance on expert opinion; (3) a client-oriented focus on quality of care; (4) structured interviews with managers, providers, and clients rather than with community informants as is the case with the DHS availability module; (5) recording of clinic facilities, equipment and commodities available on the day of the team visit; and (6) nonparticipant direct observation of all family planning clientprovider interactions on the day of the research team's visit.

The core set of Situation Analysis data collection procedures includes the following:

- A representative sample of $\mathrm{SDPs}^{4}$ or all SDPs within a geographic area of interest (country, city, district, province) are visited for a minimum of a full day by a team of three or more people, including at least one with clinical training (a physician, nurse, or nurse/midwife) and at least one with a social science background and field interview experience.

- A complete inventory is taken of equipment and supplies.
- Service statistics (if available) are recorded for the past 12 months.

- All family planning service providers are interviewed regarding family planning and other reproductive health issues.

- Observations are made of the interaction between service providers and all new and continuing family planning clients on the day of the visit.

- All clients observed are subsequently interviewed as they leave the SDP. A selection of $\mathrm{MCH}$ clients are interviewed as well.

Some Situation Analysis studies also include interviews with program managers, observations of non-family planning services, and specialized questionnaires for CBD agents and pharmacies. Examination of the quality of services received follows the Bruce-Jain quality-of-care framework (Bruce, 1990), which has the following components:

- Choice of methods refers to the number and intrinsic variability of methods actually offered.

- Information given to clients relates to the range of information provided to clients during counseling that allows them to choose and employ contraception effectively. It includes information on advantages and disadvantages of various methods; possible side effects and their management; relationship of the methods 
to STIs, including HIV / AIDS; and the fact that the client can switch to another method if she is not satisfied with her initial choice.

- Technical competence involves the clinical techniques of the providers, including proper attention to cleanliness and asepsis during clinical procedures.

- Interpersonal relations relate to the personal component of provider-client interactions.

- Mechanisms to encourage continuity refer to supporting well-informed users in managing continuity on their own, and follow-up mechanisms such as revisit appointments and home visits for checkups and support.

- Appropriate constellation of services refers to situating services so they are convenient and acceptable to clients and respond to clients' related health needs.

The basic underlying model for Situation Analysis studies (see Figure 1-1) holds that the functioning of subsystems-such as IEC, equipment and supplies, logistics, supervision, and records and reporting-represents a degree of readiness to provide a certain level of quality of care, and that this readiness influences the actual quality of care delivered by providers and received by clients.

Situation Analysis emphasizes the collection of data on quality-especially the important components of the counseling process-by trained observers. In separate studies, researchers have determined that such observation data are relatively reliable (Huntington et al., 1996), and while it may be somewhat positively biased, it is probably of greater validity than client reports (Ndhlovu, 1998). Five basic minimum data collection instruments were used in all of the studies documented in this report:

- Inventory for Facilities Available and Services Provided at the Service Delivery Point
- Observation Guide for Interaction Between Family Planning Clients and Service Providers

- Exit Interview for Family Planning Clients

- Interview for Staff Providing Family Planning/Reproductive Health Services at the Service Delivery Point

- Interview for $\mathrm{MCH}$ Clients Attending the Service Delivery Point

The units of analysis for a Situation Analysis study are SDPs, providers, and clients. The sample sizes and other background information on each of the 12 studies included here are provided in Annex 1.1 .

The usability of the Situation Analysis approach was demonstrated in the Kenyan context with the results of the first national study (Miller et al., 1992) and the Nairobi City Commission study (Mensch et al., 1994). Managers quickly developed a variety of uses for the data as a basis for administrative decision making. These included conducting problem-solving discussions among various levels of program managers; ordering/redistributing needed equipment; redesigning and reorienting training programs; redesigning staff deployment plans to better reflect actual case loads; redesigning technical assistance programs; and documenting and representing program needs, such as missing equipment, to donor agencies. Additionally, the findings were used in OR training programs as a basis for selecting important problems to be addressed through the design and implementation of OR subprojects (see chapter 4 and Miller and Frerichs, 1992-1993).

Although the Situation Analysis approach was originally designed for the African context, it diffused rapidly around the world (Miller et al., 1997). USAID recently funded an initiative (DHS+) that will develop 25 facility-based surveys between 1998 and 2002. 


\section{Annex 1-1: Sampling and Weighting}

Annex Table 1-1 gives the sample sizes ( $\mathrm{n}$ 's) for four of the data collection instruments in all 12 study sites. ${ }^{5}$ The data in the inventory are weighted, but those collected by the other three instruments are not. The inventory data are fairly easy to weight because their unit of analysis, the SDP, is also the sampling unit, so the sampling plans yield the weights quite clearly. Nonetheless, the weighted results in the inventory are quite close to the unweighted results, so the effect of weighting is not extreme. The one exception is IUD-related items in Zimbabwe, which are greatly affected by weighting (see endnote 5 in Chapter 3).

Ideally, all the data would have been weighted, but this was not possible for the staff interviews, client-provider observations, and exit interviews. To properly weight the staff interviews, one would need information on the universe of staff at each SDP on the day of the visit. This information would then need to be combined with the SDP weight to yield a final weight. However, the universe of staff is not collected in all studies, and where it is, the data are not particularly reliable. One test of weighting the staff interviews in Senegal, where the universe of staff was collected, showed that doing so resulted in extremely small differences in results.

To weight the client-provider observations, one would need information on the universe of clients who visited the SDP on the day of the study visit.

\section{Annex Table 1-1. Sample sizes, by module}

\begin{tabular}{lcccc} 
Study & Inventories & $\begin{array}{c}\text { Staff } \\
\text { Interviews }\end{array}$ & $\begin{array}{c}\text { Client-Provider } \\
\text { Observations }\end{array}$ & $\begin{array}{c}\text { Family } \\
\text { Planning } \\
\text { Client Exit } \\
\text { Interviews }\end{array}$ \\
\hline Botswana & 184 & 456 & 406 & 386 \\
\hline Côte d'Ivoire & 337 & 685 & 509 & 509 \\
\hline Ghana & 13 & 51 & 163 & 355 \\
\hline Kenya & 313 & 570 & 819 & 811 \\
\hline Madagascar & 216 & 448 & 741 & 741 \\
\hline Nigeria & 159 & 316 & 1163 & 1163 \\
\hline Senegal & 178 & 289 & 393 & 390 \\
\hline Tanzania & 180 & 361 & 1123 & 1123 \\
\hline Zambia & 348 & 598 & 451 & 451 \\
\hline Zanzibar & 254 & 358 & 396 & 392 \\
\hline Zimbabwe & 101 & 191 & 144 & 144 \\
\hline
\end{tabular}


The Situation Analysis methodology calls for observation of all clients who come to the SDP on that day, but this is clearly not possible in major hospitals with many staff and clients. For most SDPs, then, no weight is necessary since the observed clients constitute a census, but for hospitals, no weight is possible because there is no information on the universe of clients on the day of the visit. The same argument holds for the family planning client exit interviews.

The SDP weight alone could also have been applied to the other three modules in the absence of other weights. This was not done because of a combination of time and logistical constraints, as well as a desire to keep the n's on the other modules close to their original values. (Since the numbers of staff and clients vary among SDPs, applying the SDP weight would significantly alter the n's on the other modules.)

Following are brief descriptions of the weighting procedure applied to the inventory of each study.

Botswana. The sample in Botswana is fairly straightforward and is stratified by type. It includes $72 \%$ of all hospitals, $59 \%$ of all clinics, and $15 \%$ of health posts. The data were weighted accordingly.

Burkina Faso. In Burkina Faso, the fieldwork began as a census of all SDPs, but after 8 of 30 provinces had been covered, it was switched to a sample stratified by province. Where a sample was drawn, the sampling fraction by province ranged from about .25 to .57 , and the remaining provinces had a sampling fraction of 1 . The weights correct for this skewed sample.

Côte d'Ivoire. The Situation Analysis in Côte d'Ivoire assessed 13 pilot SDPs in Abidjan, which represented a census of SDPs offering family planning at the time. No weighting is necessary.

Ghana. The Ghana sample is stratified by type: $50 \%$ of hospitals, $25 \%$ of maternities, $25 \%$ of $\mathrm{MOH}$ clinics, and $100 \%$ of Planned Parenthood Association of Ghana clinics. The actual achieved sampling fractions differed slightly from these, and the weights are adjusted accordingly.

Kenya. The Kenya sample cannot be weighted because of difficulties with identifying universes and sampling fractions. However, all Nairobi City
Council (NCC) clinics were included purposively, which is probably the most skewed element of the sample. For this reason, several of these clinics were removed from the data sets, resulting in a proportion similar to the overall sampling fraction. This mitigates the overrepresentation of NCC clinics in the sample.

Madagascar. This Situation Analysis is not nationally representative, but instead focuses on a census of SDPs in two major provinces: Antananarivo and Fianarantsoa. Because the data included scattered SDPs in other provinces, these SDPs were excluded from the analysis. The remaining data were not weighted since they consist of a census.

Nigeria. The Nigeria sample is complex. First, 6 of 30 provinces were selected based on target provinces for upcoming $\mathrm{MOH}$ and Department for International Development (DfID) projects. These projects had not begun at the time of the fieldwork, and the 6 provinces were judged by program managers to be representative of the country (Askew et al., 1994). Then a total sample size of 171 was calculated and divided evenly among the 6 provinces, for 30 SDPs each. In this way, the sampling fraction in each province differs because the universes differ. Furthermore, within each province, the 30 SDPs were stratified by type, with various levels of representation among hospitals, health centers, clinics, and Planned Parenthood Federation of Nigeria (PPFN) clinics. The weights correct for these differences in representation at the type and province levels.

Senegal. This Situation Analysis is a census of all SDPs in the country, so no weights are needed.

Tanzania. The Tanzania sampling plan was based on the six zones of the country, each of which is made up of three to four regions. In each zone, one region was randomly selected, and a census of SDPs was taken. For this reason, the weights are based solely on the number of regions per zone. In addition, Dar Es Salaam was purposively included, so it is weighted accordingly.

Zambia. The Zambia sample was not drawn randomly. Instead, it consists mainly of SDPs that are targeted for program interventions by organizations such as USAID, CARE, the United Nations Fund for Population Activities (UNFPA), and the Family Planning Service Expansion and Technical 
Support (SEATS) Project. In only a handful of cases had the intervention already begun at the time of the fieldwork. No information is available on how these SDPs were selected, so the sample cannot be said to be representative. However, it does constitute a substantial proportion of all SDPs, it is clearly distributed by region and type, and it is judged by program managers to be reasonably representative. The data are not weighted.

Zanzibar. This Situation Analysis consisted of a census of SDPs, so no weights are necessary.

Zimbabwe. The sample for the 1996 Situation Analysis consists of the same SDPs that were visited in the 1991 study, with a few small corrections. In 1991, the sample was randomly drawn such that it was self-weighting and representative by province and type. The universe of SDPs changed insignificantly between the two studies, so small weights are applied in 1996 to adjust the sample accordingly.

\section{REFERENCES}

The Alan Guttmacher Institute. 1998. Into A New World: Young Women's Sexual and Reproductive Lives. The Alan Guttmacher Institute, New York.

Askew, I., B. Mensch, and A. Adewuji. 1994. "Indicators for measuring the quality of family planning services in Nigeria." Studies in Family Planning, 25,5:268-283.

Bertrand, J. 1991. "Recent lessons from Operations Research on service delivery mechanisms." In Seidman, M. and M. Horn, Operations Research: Helping Family Planning Programs Work Better. John Wiley \& Sons, New York.

Bruce, J. 1990. "Fundamental elements of the quality of care: A simple framework." Studies in Family Planning, 21,2:61-91.

Caldwell, J.C. and P. Caldwell. 1988. "Is the Asian family planning program model suited to Africa?" Studies in Family Planning, 19,1:19-28.

Center for Human Services. 1988. Primary Health Care Thesaurus: A List of Services and Support Indicators. Center for Human Services, Chevy Chase, Maryland.

Fisher, A., J. Laing, J. Stoeckel, and J. Townsend. 1991. Handbook for Family Planning Operations Research Design, Second Edition. Population Council, New York.

Fisher, A. 1993. "Family planning in Africa: A summary of recent results from Operations Research studies."
In Africa Operations Research and Technical Assistance Project: End-of-Project Conference, Nairobi, 4-7 October. Population Council, New York.

Fisher, A. and K. Miller. 1996. “Conditions required at SDPs to deliver quality family planning services: Why so many do so little." Paper presented at the Annual Meeting of the American Public Health Association, New York.

Frerichs, R. 1989a. "Simple analytic procedures for rapid microcomputer-assisted cluster surveys in developing countries." Public Health Reports, 104,1:24-34.

Frerichs, R. and K. Tar Tar. 1989b. "Computer-assisted rapid surveys in developing countries." Public Health Reports, 104,1:14-23.

Huntington, D., K. Miller, and B. Mensch. 1996. “The reliability of the Situation Analysis observation guide." Studies in Family Planning, 27,5:277-282.

Kirk, D. and B. Pillet. 1998. "Fertility levels, trends, and differentials in sub-Saharan Africa in the 1980s and 1990s." Studies in Family Planning, 29,1:1-20.

Maggwa, N. and I. Askew. 1997. Integrating STI/HIV Management Strategies into Existing MCH/FP Programs: Lessons from Case Studies in East and Southern Africa. Population Council, Nairobi, Kenya.

Mensch, B., R. Miller, A. Fisher, J. Mwita, N. Keyonzo, F.M. Ali, and C. Ndeti. 1994. "A Situation Analysis of city commission clinics." International Family Planning Perspectives, 20,2:48-54.

Miller, K. and A. Rosenfield. 1996. "Population and women's reproductive health: An international perspective." Annual Review of Public Health, 17:359-382.

Miller, R., L. Ndhlovu, M. Gachara, and A. Fisher. 1992. "Situation Analysis study of Kenya's family planning program." In Jain, A., Ed., Managing Quality of Care in Population Programs. Kumarian Press, West Hartford.

Miller, R. and R. Frerichs. 1992-1993. “An integrated approach to Operations Research for strengthening family planning programs: A case example in Kenya." International Quarterly of Community Health Education, 13,3:183-199.

Miller, R., A. Fisher, K. Miller, L. Ndhlovu, N. Maggwa, I. Askew, D. Sanogo, and P. Tapsoba. 1997. The Situation Analysis Approach to Assessing Family Planning and Reproductive Health Services: A Handbook. Population Council, New York.

National Research Council. 1993. Factors Affecting Contraceptive Use in Sub-Saharan Africa. National Academy Press, Washington, D.C.

Ndhlovu, L. 1998. "Lessons learned from Situation Analysis studies in Africa." Paper presented at the Annual Meeting of the Population Association of America, Chicago.

Phillips, J. and W. Greene. 1993. Community Based Distribution of Family Planning in Africa: Lessons from 
Operations Research (Final Report). Population Council, New York.

Pritchett, L. 1994. "Desired fertility and the impact of population policies." Population and Development Review, 20,1:1-55.

Rosen, J. and S. Conly. 1998. Africa's Population Challenge: Accelerating Progress in Reproductive Health. Population Action International, Washington, D.C.

Ross, J., W.P. Mauldin, and V. Miller. 1993. Family Planning and Population: A Compendium of International Statistics. United Nations Population Fund and Population Council, New York.

Shane, B. and K. Chalkley. 1998. From Research to Action: How Operations Research Is Improving Reproductive Health Services. Population Reference Bureau, Washington, D.C.

UNAIDS and World Health Organization. 1998. Report on the Global HIV/AIDS Epidemic: June 1998. UNAIDS and WHO.

Van de Walle, E. and A. Foster. 1990. Fertility Decline in Africa: Assessment and Prospects. Technical Paper No. 125, African Technical Department Series, World Bank, Washington, D.C.

\section{NOTES}

1 Throughout this chapter, as elsewhere in the volume, clients are referred to in the feminine form, since virtually all clients of African integrated maternal and child health $(\mathrm{MCH})$ centers are women. At the same time, it is recognized that the clients of some programs are men.

2 Problem identification, strategy selection, strategy experimentation, dissemination of results, and utilization of results.

3 A more complete description of the Situation Analysis methodology is presented in the Situation Analysis Handbook (Miller et al., 1997).

4 See Annex 1-1 for details on sampling.

5 Data from the fifth instrument (exit interview with $\mathrm{MCH}$ clients) are not used in this volume, so sample sizes are not given.

\footnotetext{
12 Clinic-Based Family Planning and Reproductive Health Services in Africa
} 


\title{
Descriptions of the Family Planning Programs Studied
}

\author{
Kate Miller and Brian Pence
}

Situation Analysis results should be interpreted in light of the unique set of circumstances facing each family planning program at the time of the study. Political support, client characteristics, program maturity, and the state of the AIDS epidemic in each country all affect the ability of programs to deliver high-quality care, and they all vary among the 12 study sites included here. This chapter provides a brief description of each of the 12 study sites and the status of its family planning program at the time of the Situation Analysis fieldwork. The descriptions also include information on the population of family planning clients, such as age, marital status, and reproductive intentions, as gathered from the Situation Analysis exit interviews. Annex 2-1 contains the detailed results of these client characteristics.

Program maturity is categorized using an approach developed by Destler and colleagues (1990). This framework groups family planning programs into five levels based on the prevalence rate of modern contraceptives (CPR) in that country. The programs in a given category tend to share certain general characteristics:

- Emergent programs ( $0 \%$ to $7 \%$ CPR) have limited service delivery and low levels of family planning awareness among the population.

- Launch programs (8\% to 15\%) possess a broader institutional base as compared with the emergent level and offer increased access to a wider range of contraceptive methods.

- Growth programs (16\% to $34 \%$ ) have successfully reached a large portion of the more urban and better-educated populace, with demand growing for services among other segments of the population and for long-term and permanent methods among all segments.
- Consolidation programs (35\% to $49 \%)$ have a more heterogeneous, younger clientele; a high CPR among the urban and educated populations; and expanding services for the rural and poor. In addition, while the public sector remains the primary provider for clinical methods such as the IUD and sterilization, the private sector is beginning to assume responsibility for delivering temporary methods.

- Mature programs (50\% and over) are effectively reaching most segments of the population. The most popular methods are sterilization, the IUD, and oral contraceptives.

The majority of the sites have CPRs below 15\%:1 6 of the 12 programs studied fall into the launch category, and three others have emergent programs. The analysis also includes three of the region's most successful family planning programs: the programs of Botswana, Kenya, and Zimbabwe have all attained growth status (and yet Botswana and Zimbabwe are two of the countries hardest hit by the HIV / AIDS pandemic). All the sites show a substantial increase in program effort scores between 1982 and $1989,{ }^{2}$ and in most cases, contraceptive prevalence is on the rise. The sites exhibit high total fertility rates (TFRs), ranging from 4.5 to nearly $7.4^{3}$; TFRs are declining in all sites, however, in some more precipitously than in others. In all sites, the local Ministry of Health is by far the largest source of family planning services for contraceptive users.

Estimates of HIV seroprevalence among the general populations of the study sites range from $0.1 \%$ in Madagascar to $17-18 \%$ in Botswana, Zambia, and Zimbabwe. Two-thirds of the study sites have seroprevalence rates over 6\%. Among urban antenatal clients, HIV seroprevalence rates 
were measured at over $10 \%$ in two-thirds of the sites and at over 25\% in Botswana, Zambia, and Zimbabwe. ${ }^{4}$ Since heterosexual sex is by far the most common mode of HIV transmission in subSaharan Africa (World Bank, 1997), family planning programs in the region have an increased responsibility to inform and protect clients in this regard.

The following descriptions of program context are based on Situation Analysis reports, Demographic and Health Survey (DHS) data, United Nations and World Bank databases, and results from the 12 Situation Analysis studies themselves. The list of references at the end of this chapter includes citations of all the DHS and Situation Analysis reports used here.

\section{BOTSWANA}

\section{Population: 1.5 million}

The Botswana family planning program is unique in many ways. For one, family planning services in Botswana have historically been integrated with other health services, so no separate family planning program has been established. Between 1982 and 1989, the government sharply increased its efforts on family planning services, and perhaps as a result, Botswana has one of the highest CPRs in the region ( $29 \%$ of all women of reproductive age in 1988), and has experienced one of the steepest drops in TFR over the last few decades (from 6.4 in the late 1970s to 4.5 in the late 1990s). The method mix in Botswana consists mainly of pills $(61 \%)$, IUDs $(16 \%)$, and injectables $(11 \%)$, a mix that is fairly typical for the region. ${ }^{5}$ Botswana is also extremely wealthy relative to the other 11 study sites: in 1992 its per capita gross domestic product was over $\$ 3,000$, as compared with a range of $\$ 100$ to $\$ 1,000$ among the other countries. ${ }^{6}$

Botswana is a fairly small country, so the program also caters to a relatively small number of women of reproductive age. When the Situation Analysis was carried out in 1995, there were 364,000 women of reproductive age in the country, as compared with several million in the other study sites included here. ${ }^{7}$ However, Botswana is one of the countries hardest hit by the HIV epidemic: seroprevalence in late 1994 was estimated by the World Health Organization at fully $18 \%$ among the general adult population, and was measured at 34\% of urban antenatal clients in 1995.

The population of family planning clients in Botswana differs sharply from that in other countries. Only $35 \%$ of clients in Botswana are in a monogamous union, and fully $46 \%$ are not in a formal union, a much higher percentage than in any other study site. The clients are fairly young, with a mean age of 27; fully $13 \%$ are under 20 years old. The clients have a relatively small number of living children (mean 2.5), and 59\% want more children. Clients in Botswana are also particularly highly educated, and have an unusual pattern of religion: $37 \%$ are African Spiritual, 31\% are Protestant, and $22 \%$ practice no religion at all.

\section{Summary: Botswana}

At the time of the 1995 Situation Analysis study, Botswana had:

- A growth-level family planning program.

- High levels of political support for family planning and a strong basis for domestic program funding.

- Significant contraceptive use.

- A particularly educated client base.

$\square$ A young and largely unmarried client base.

口 Extremely high HIV seroprevalence.

\section{BURKINA FASO}

\section{Population: 10.2 million}

The Ministry of Health in Burkina Faso began offering family planning services in 1985 in the capital city of Ouagadougou, and services quickly expanded thereafter across the country. An ambitious population policy adopted in 1991 set the target CPR at $60 \%$. Although the government's increased attention to family planning is reflected in a substantial rise in its program effort scores between 1982 and 1989, the 1993 DHS found that the CPR among all women of reproductive age was only $4 \%$. About half of these users were taking oral 
contraceptives, $22 \%$ were using condoms, and $17 \%$ IUDs. The TFR in Burkina Faso was consistently high (6.5) until quite recently, when it began to drop slightly.

The first Burkina Faso Situation Analysis took place in 1992 and the second in 1995 (see Chapter 11 for a comparison of the results of these studies). The country suffered a sizable loss in donor support when the United States Agency for International Development (USAID) country mission was closed in 1994. Thus, although the family planning program had been in operation for 11 years by the time of the 1995 Situation Analysis, it still ranked in the emergent category and had not achieved a high level of sustainability or effectiveness.

Burkina Faso's client base is almost $60 \%$ Muslim and about 30\% Catholic. Although 60\% are in a monogamous union, fully $28 \%$ are in polygamous unions, a finding that has ramifications for contraceptive method mix because of the associated risk of sexually transmitted infection (STI). In 1994, HIV prevalence among the general adult population was estimated to be $6.7 \%$, and was measured at $12 \%$ of urban antenatal clients. Family planning clients are among the least educated across the 12 study sites: $55 \%$ have no formal education, and fewer than $10 \%$ can read easily in either French or a local language. Reflecting the country's fairly high fertility, clients have an average of 3.5 living children, and two-thirds want more chil-

\section{Summary: Burkina Faso}

At the time of the 1995 Situation Analysis study, Burkina Faso had:

$\checkmark$ A long-standing but still emergent family planning program.

- Increasing levels of domestic political support for family planning, but decreasing donor program support.

$\checkmark$ Very low contraceptive use.

- A fairly uneducated client population, largely Muslim.

$\checkmark$ A large proportion of clients in polygamous unions.

๑ Fairly high HIV prevalence. dren. The mean client age is 29 , and $6 \%$ are teenagers.

\section{CÔTE D'IVOIRE}

\section{Population: 12.7 million}

The government of Côte d'Ivoire vigorously opposed the adoption of a family planning policy long after many of its neighbors had initiated family planning services; it was not until 1991 that the government decided to offer family planning services through its maternal and child health $(\mathrm{MCH})$ program. In that year, family planning services were introduced in 13 clinics in the capital city of Abidjan, with the intention of eventually expanding services throughout the country. The Situation Analysis was undertaken in 1992 to help planners identify the strengths and weaknesses of the program in these clinics prior to the program's expansion. This Situation Analysis is therefore unique among the 12 in that it involves only these 13 clinics-not a representative sample, but a census of the pilot project SDPs. The Côte d'Ivoire program is also by far the youngest of the 12 family planning programs at the time of the Situation Analysis fieldwork. The results from Côte d'Ivoire must be interpreted with these points in mind.

Like Burkina Faso, Côte d'Ivoire has one of the most persistently high TFRs in the region (over 7 until quite recently). It also has a very low CPR of under $5 \%$ of currently married women, showing no great increase between 1980 and 1994. Much of this prevalence is accounted for by condom use (33\% of all users). HIV prevalence among the general adult public in Côte d'Ivoire was estimated at $6.8 \%$ in 1994, and measured at $12 \%$ in 1995-1996 among urban antenatal clients. However, the Situation Analysis study took place several years before these estimates, so HIV prevalence may have been lower at that time. Côte d'Ivoire is also relatively wealthy. In 1992, its per capita gross domestic product was over $\$ 1,000$, much larger than that of all other study sites except Botswana.

Family planning clients in Côte d'Ivoire have an average of 3.2 living children, but fully $85 \%$ express a desire for more children, reflecting the 
country's extremely high fertility. About half of clients are in a monogamous union, about $40 \%$ are single, and the remaining clients are in polygamous unions. About a third have no formal education. Religious affiliations are largely split among Muslim, Catholic, and Animist. The clients are relatively young: $11 \%$ are under 20 years old.

\section{Summary: Côte d'Ivoire}

At the time of the 1992 Situation Analysis study, Côte d'Ivoire had:

- An emergent pilot family planning program.

- Low but rising levels of domestic political support for family planning, with possibilities for domestic program funding.

- Very low contraceptive use.

- A large population of spacing clients.

- Situation Analysis results from 13 extremely new clinics.

๑ Fairly high HIV prevalence.

\section{GHANA}

\section{Population: 17.8 million}

Although a National Family Planning Secretariat had been established in Ghana in 1969, its activities did not at first succeed in significantly raising the CPR, which was about $10 \%$ of all women in 1979 , or decreasing the TFR, which remained above 6 until the late 1980s. However, government family planning efforts increased in the course of the 1980s, as shown by an increase in program effort scores, and in 1991 the Ghana Family Planning and Health Project was established to increase contraceptive prevalence and combat the spread of HIV and other STDs. This project placed renewed emphasis on family planning services, and since it was undertaken, the TFR has fallen to 5.5. The CPR has remained steady at $10 \%$. The contraceptive method mix as measured in 1993 was characterized by a fairly large reliance on condoms (28\%), pills $(30 \%)$, and injectables $(13 \%)$.

HIV seroprevalence in Ghana is low relative to other countries reviewed here, but still reached an estimated $2.3 \%$ of the general population in 1994 . The prevalence rate was measured at $2.2 \%$ of urban antenatal clients in 1995 . In general, the population of Ghana shows one of the smallest differences in HIV prevalence between populations at high and low risk of infection.

Most clients in Ghana are in a monogamous union $(72 \%)$, and about a fifth are in polygamous unions. Clients' mean age is fairly high (31), and only $2 \%$ are teenagers. Their religious affiliations are divided among Protestant, Catholic, other Christian, and Muslim. The mean number of children among clients is 3.6, and a relatively low percentage $(55 \%)$ express the desire for more children, reflecting the declining TFR. Clients are not very well educated: $37 \%$ have had no education at all, and only $22 \%$ can read a letter in English or a local language.

\section{Summary: Ghana}

At the time of the 1996 Situation Analysis study, G hana had:

- A launch-level family planning program.

$\checkmark$ A declining TFR and a steady CPR.

- Increasing political attention to family planning.

$\square$ Relatively low HIV prevalence.

- A relatively older, less-educated client base, with declining fertility desires.

\section{KENYA}

\section{Population: 30.5 million}

The government of Kenya began to offer family planning services in 1967, long before many of its neighbors. However, the program evolved extremely slowly, and was judged "weak" by several evaluation efforts over the ensuing 20 years (see Miller et al., 1992). Nonetheless, the TFR in Kenya did decline from about 8 in the late 1970s to about 7 by the late 1980s. In 1987, the government 
initiated a more serious commitment to family planning services, which is reflected in a large increase in program effort scores between 1982 and 1989. After 1987, the TFR continued to decline, and the CPR continued to rise. Although the TFR is still high (5.8), its decline over the last two decades has been one of the largest in the region. Thus, the 1995 Situation Analysis was carried out on a growth-level family planning program which was mature and largely effective, catering to a relatively large population of 6.25 million women of reproductive age.

The client population in Kenya is over 90\% Protestant, Catholic, or other Christian, and fully $78 \%$ of clients are in monogamous unions. Clients are also fairly well educated: only $11 \%$ have no formal education, and $62 \%$ can easily read in English. Their average age is 28 , and $5 \%$ are below age 20 . The mean number of living children per client is 3.2, but only $41 \%$ want more children (the lowest result of all 12 study sites), reflecting Kenya's declining TFR. The method mix among users in Kenya consists mainly of pills $(36 \%)$, injectables $(27 \%)$, and IUDs $(14 \%)$. Again consistent with the falling TFR, 19\% of users have had tubal ligations, a remarkably high percentage among these study sites. This method mix has consequences for STD transmission: the HIV seroprevalence was estimated in 1994 to be $8.3 \%$ of the general adult population, and in 1995 to be almost $14 \%$ of those attending urban antenatal clinics.

\section{Summary: Kenya}

At the time of the 1995 Situation Analysis study, Kenya had:

$\square$ A growth-level family planning program.

- High levels of domestic political support for family planning.

$\checkmark$ A rising CPR and falling TFR.

口 A fairly well-educated, largely Christian, and monogamous population of clients.

$\checkmark$ A relatively small population of spacing clients.

๑ Fairly high HIV prevalence.
MADAGASCAR (Antananarivo and Fianarantsoa)

\section{Population: 15.4 million}

Although family planning services were available through an International Planned Parenthood Federation (IPPF) affiliate in Madagascar starting in 1967, the Ministry of Health itself did not begin a serious effort to offer services until about 1991, after a population policy had been put in place in 1990. Since that date, there has been steady growth in the number and type of clinics offering family planning; in 1995, a population of 3.3 million women of reproductive age was served. However, the TFR in Madagascar remained persistently high (6.6) until recently, and the 1992 DHS found a CPR among all women of reproductive age of only $4 \%$, indicating that the program remains in the emergent category. Of these users, about a third used oral contraceptives, another third injectables, and $14 \%$ condoms. A relatively high $17 \%$ have had tubal ligations.

The 1996 Situation Analysis in Madagascar did not cover the entire country, only the main urban provinces of Antananarivo and Fianarantsoa. The data are therefore not nationally representative, although for simplicity this study is referred to as the Madagascar Situation Analysis.

HIV seroprevalence in Madagascar is extraordinarily low. It was estimated in 1994 to be $0.1 \%$ of the adult population, and the same prevalence was found among urban antenatal clients in 1995. Even among high-risk populations in major cities, HIV prevalence has been measured at only $0.3 \%$. For this reason, the family planning program in Madagascar has a unique opportunity to assist the country's health system in preventing the epidemic altogether.

About a quarter of clients in Madagascar are in a polygamous union, and $69 \%$ are in a monogamous union. The client population appears to be fairly well educated: only 5\% have had no formal education, and although almost none can read in French, $66 \%$ can read easily in their local language. The average age of clients is 30 , and only $3 \%$ are teenagers. The vast majority of clients are either Protestant or Catholic. Fewer than half express a 
desire for more children, a relatively low result that is probably related to the fairly high prevalence of tubal ligation.

\section{Summary: Madagascar}

At the time of the 1996 Situation Analysis study, Madagascar had:

- An emergent family planning program.

口 Increasing levels of domestic political support for family planning.

A very low CPR and high but falling TFR.

- A fairly well-educated, largely Christian population of clients.

- A relatively small population of spacing clients.

• Extraordinarily low HIV prevalence.

\section{NIGERIA}

\section{Population: 102.1 million}

Nigeria is by far the most populous country in Africa. At the time of the Situation Analysis study in 1992, the family planning program in Nigeria had to serve an overwhelming population of over 23 million women of reproductive age. Serious governmental attention to family planning services did not begin until 1983, when substantial donor support became available, and a national population policy was adopted in 1988. The effect of this attention can be seen in a steep rise in program effort scores between 1982 and 1989. However, the TFR in Nigeria has consistently remained at about 6.5, and the low CPR $(8 \%)$ showed no change between 1981 and 1990. In more recent years, the TFR has begun to fall.

HIV prevalence was estimated in 1994 to be $2.2 \%$ of the general adult population, and was measured at $3.8 \%$ among a population of urban antenatal clients in 1993-1994. The Situation Analysis took place a few years before these estimates, so prevalence may have been lower at that point.

Family planning clients in Nigeria have on average 4.3 living children, the largest number of any study site included here; $60 \%$ would like to have another child. The client population is moderately educated, and split fairly evenly among Protestants, Muslims, and Catholics. These clients are among the oldest across the study sites: the mean age is 31 , and only $1 \%$ are below age 20 . Twothirds are in a monogamous union, and another quarter are in polygamous unions.

\section{Summary: Nigeria}

At the time of the 1992 Situation Analysis study, Nigeria had:

- A family planning program that was on the border between emergent and launch level, despite being long-standing.

- Increasing levels of domestic political support for family planning.

- A persistently low CPR and a high TFR.

$\checkmark$ A moderately educated and relatively old population of clients, commonly in polygamous unions.

- Relatively low HIV prevalence.

\section{SENEGAL}

\section{Population: 8.1 million}

In 1981 and 1982, major projects by USAID and the United Nations Population Fund (UNFPA) enabled the first widespread provision of family planning services in Senegal through several nongovernmental organizations (NGOs). In 1991, the adoption of a national population policy by the government allowed the Ministry of Public Health to coordinate the NGO efforts through a national program of family planning. The increase in program effort scores between 1982 and 1989 reflects this growing interest on the part of the government.

The TFR in Senegal dropped steadily from 7 in the late 1970 s to 5.6 in the late 1990s. The CPR, although rising, remained under $10 \%$ throughout the 1980s. The method mix comprises mostly pills (45\%), IUDs (27\%), and condoms (16\%); injectable 
use is quite uncommon (2\%). HIV prevalence in Senegal is fairly low: in 1994, $1.4 \%$ of the general adult population were estimated to be infected, and $1.1 \%$ of urban antenatal clients were HIV-positive. Rates among high-risk populations are somewhat higher.

The vast majority of family planning clients in Senegal are Muslim. A relatively high proportion $(27 \%)$ are in polygamous unions, and $60 \%$ are in monogamous unions. The level of education is quite low: $41 \%$ have no formal education, $20 \%$ can read in French, and only $2 \%$ can read in a local language. Senegalese clients have an average of almost 4 living children, a relatively high number for these study sites, and $73 \%$ want more children, reflecting the higher TFR of 1994 . The mean age is 30 , and $4 \%$ are teenagers.

\section{Summary: Senegal}

At the time of the 1994 Situation Analysis study, Senegal had:

- A launch-level family planning program.

- High levels of political support for family planning.

- A low but rising CPR and high but falling TFR.

- A largely Muslim, uneducated client base with high fertility desires.

A relatively high proportion of clients in polygamous unions.

- Relatively low HIV prevalence.

\section{TANZANIA (See also Zanzibar)}

\section{Population: 27.3 million}

In 1974, the government of Tanzania officially permitted its health facilities to offer family planning, but the actual provision of services was slow to come about. By 1982, 37\% of clinics were offering family planning, and by 1987, 80\%. A national population policy was approved in 1992, and donor support began to increase thereafter. Of all the study sites included in this analysis, Tanzania has since the late 1980s had the lowest per capita gross domestic product (\$100 in 1992).

Tanzania is one of the most populous countries in sub-Saharan Africa; in 1992 the population of women of reproductive age was 6.27 million. HIV prevalence is fairly high, estimated at $6.4 \%$ of the general adult population in 1994, and measured at about $14 \%$ of clients of urban antenatal clinics in 1995-1996. The TFR in Tanzania has declined from 6.8 in the late 1970 s to a recent 5.5. The CPR among all women has been growing steadily, but remains at just above $10 \%$. Most users take oral contraceptives $(41 \%)$, but a large proportion use the injectable (32\%) and condoms (11\%); sterilization accounts for a relatively high $12 \%$ of the method mix.

Clients in Tanzania have an average of 3.4 living children, and fully three-quarters express the desire for more children. The population is fairly young, with a mean age of 27 , and $8 \%$ are under age 20. In light of these high fertility desires and this young age distribution, the relatively common use of tubal ligation is surprising. The majority of clients $(70 \%)$ are in monogamous unions, with the rest divided almost evenly between polygamous unions and single status. Clients are divided among the Muslim, Catholic, and Protestant faiths, and levels of education are moderate as compared with the other study sites $(18 \%$ have no formal education).

\section{Summary: Tanzania}

At the time of the 1992 Situation Analysis study, Tanzania had:

- A launch-level family planning program.

- Low but rising political support for family planning, and little basis for domestic financial program support.

- A low but rising CPR and declining TFR.

- A mixed-religion, moderately educated, relatively young client base with high fertility desires.

- A relatively high proportion of clients in polygamous unions.

$\square$ Fairly high HIV prevalence. 


\section{ZAMBIA}

\section{Population: 9.4 million}

Zambia's National Family Planning Program was launched in 1992 in response to a population policy adopted in 1989. The program is focused on service delivery; information, education, and communication (IEC); commodity and equipment logistics; and training. In 1993, USAID launched a Zambia Family Planning Services project to assist in strengthening family planning services. Although this project did not get fully under way until the early 1990s, the TFR in Zambia has been falling since the late 1970s, from 7.2 to about 5.5 in 1997. Program effort scores rose dramatically between 1982 and 1989, but the CPR, although increasing, was still only about $10 \%$ of all women in 1996. The method mix is typically heavy on the pill $(41 \%)$ and injectables (32\%), but sterilization is also relatively common $(12 \%)$.

As part of the "AIDS belt" in Southern Africa, Zambia has an extremely high HIV prevalence rate. It was estimated in 1994 that $17 \%$ of the general adult population were infected, and prevalence was measured at $27.9 \%$ of urban antenatal clients in the same year.

Family planning clients in Zambia are relatively well educated ( $7 \%$ have no formal education). A majority $(71 \%)$ are in monogamous unions, and the rest are split about evenly between polygamous and single. The vast majority of clients are either Protestant or Catholic, and the mean age is 28. Clients in Zambia have an average of 3.4 children, and $62 \%$ express the desire for more children.

\section{Summary: Zambia}

At the time of the 1997 Situation Analysis study, Zambia had:

- A launch-level family planning program.

- Increasing domestic political support for family planning.

$\checkmark$ A steadily declining TFR but low CPR.

$\checkmark$ Extremely high HIV prevalence.

- Relatively well-educated clients, most in monogamous unions.

\section{ZANZIBAR (see also Tanzania)}

\section{Population: 800,000}

Zanzibar consists of several islands lying off the coast of Tanzania, and although it shares certain government structures with Tanzania (home and foreign affairs, defense, communications, currency, and higher education), it is otherwise separate and independent (see Mapunda, 1996). Since the two regions have separate health systems, Zanzibar is included in this report as a separate study.

In contrast to Tanzania, Zanzibar has a very small client base: in 1995 the islands had an estimated 180,000 women of reproductive age. Family planning services first became available through the $\mathrm{MCH}$ system in 1985 under the Zanzibar Family Planning Project, funded by UNFPA. These services were available at 6 clinics initially, and at all 104 SDPs by 1994. The DHS studies for Tanzania included Zanzibar, so the results can be used as a proxy for Zanzibar as well. Again, fertility was stubbornly high (6.8) until recently, when it began to decline, and the CPR is increasing, although it is still just above $10 \%$.

Family planning clients in Zanzibar have an average of 4.2 children, almost the highest across the study sites. Fully 75\% express a desire for more children, reflecting the high fertility of the region. The clients are also almost exclusively Muslim (95\%), and as in Tanzania, $71 \%$ are in a monogamous union, with the rest divided fairly evenly between polygamous and single. The mean age is 29 , and $10 \%$ have no formal education.

\section{Summary: Zanzibar}

At the time of the 1995 Situation Analysis study, Zanzibar had:

- A launch-level family planning program.

- Low but rising political support for family planning, and little basis for domestic financial program support.

$\checkmark$ A low but rising CPR and persistently high TFR.

- A Muslim client base with high fertility desires. 


\section{ZIMBABWE}

\section{Population: 11.4 million}

The family planning program in Zimbabwe is clearly one of the most successful in sub-Saharan Africa. Although the first scattered family planning services were available in Zimbabwe in the 1950s, the government officially introduced family planning into its general health system in 1981. In 1985, the Zimbabwe National Family Planning Council was formed to coordinate all the country's family planning activities.

Zimbabwe's TFR fell from 6.6 in the late 1970s to 4.5 about 20 years later. Moreover, the CPR among all women of reproductive age is the highest across the countries included here: 31\% in 1994 (a rate rivaled only by Botswana). This high level of contraceptive use is credited mainly to the Zimbabwe National Family Planning Council's emphasis on community-based distribution (CBD), which serves up to $30 \%$ of family planning clients. Because the CBD program has focused on pills, the method mix in Zimbabwe is strongly dominated by that method ( $77 \%$ of users).

HIV prevalence in Zimbabwe is among the highest in Africa. It was estimated at $17.4 \%$ of the general adult population in 1994, and measured at fully $35 \%$ among urban antenatal clients in
1995-1996. Among urban, high-risk populations, the prevalence is $86 \%$.

Fully $86 \%$ of clients in Zimbabwe are in a monogamous union, by far the highest proportion of all the study sites; only $2 \%$ are in a polygamous union, and the remaining $12 \%$ are single. Clients have an average of 3 living children, and 55\% express a desire for more; both these figures are relatively low and reflect the plunging TFR in Zimbabwe. The great majority (85\%) of clients are Protestant, Catholic, or other Christian. Clients are fairly well educated: $47 \%$ can read in English, 71\% can read in a local language, and only $8 \%$ have had no formal education. The mean age is 28 .

\section{Summary: Zimbabwe}

At the time of the 1997 Situation Analysis study, Zimbabwe had:

$\checkmark$ A growth-level family planning program.

- Extraordinarily high levels of domestic political support for family planning.

$\square$ A strong CBD program that serves a third of contraceptive users.

a Extremely high HIV prevalence.

口 A fairly well-educated client population with declining fertility desires. 


\section{Annex 2-1: Family Planning Client Characteristics}

Annex Table 2-1 presents descriptive information on the population of family planning clients, drawn from exit interviews in the Situation Analysis studies. In this table, blank cells mean the question was not asked or the data are not usable. For percentages, the sample sizes (n's) listed are the denominators; likewise for means, the n's are the total valid n's. The n's vary according to missing data.
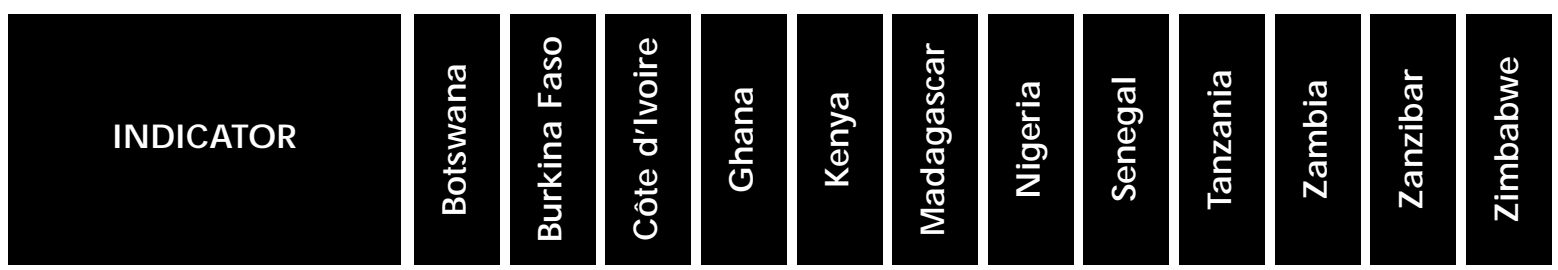

ANNEX TABLE 2-1. POPULATION OF FAMILY PLANNING CLIENTS, 12 STUDY SITES

\begin{tabular}{|c|c|c|c|c|c|c|c|c|c|c|c|c|}
\hline Mean age of clients & \begin{tabular}{|c|}
27.3 \\
$n=372$ \\
\end{tabular} & \begin{tabular}{|c|}
28.6 \\
$n=453$ \\
\end{tabular} & \begin{tabular}{|c|}
27.2 \\
$n=341$ \\
\end{tabular} & \begin{tabular}{c|}
30.6 \\
$n=728$
\end{tabular} & $\begin{array}{c}28.6 \\
n=695\end{array}$ & $\begin{array}{c}30.4 \\
n=1149 \\
\end{array}$ & $\begin{array}{c}31.1 \\
n=385\end{array}$ & \begin{tabular}{|c|}
29.8 \\
$n=1108$ \\
\end{tabular} & \begin{tabular}{c|}
26.8 \\
$n=414$ \\
\end{tabular} & $\begin{array}{c}27.9 \\
n=382 \\
\end{array}$ & $\begin{array}{c}28.7 \\
n=134\end{array}$ & $\begin{array}{c}27.9 \\
n=717 \\
\end{array}$ \\
\hline$\%$ of clients under age 20 & $\begin{array}{l}2.9 \\
=372\end{array}$ & $\begin{array}{c}5.5 \\
n=453\end{array}$ & $\begin{array}{c}10.6 \\
n=34\end{array}$ & $\begin{array}{c}1.5 \\
n=728\end{array}$ & $\begin{array}{c}3.9 \\
n=695\end{array}$ & $\begin{array}{c}3.0 \\
n=1149\end{array}$ & $\begin{array}{c}1.0 \\
n=385\end{array}$ & $\begin{array}{c}4.4 \\
n=1108\end{array}$ & $\begin{array}{c}7.5 \\
n=414\end{array}$ & $\begin{array}{c}6.0 \\
n=382\end{array}$ & $\begin{array}{c}2.2 \\
n=134\end{array}$ & $\begin{array}{c}7.1 \\
n=717\end{array}$ \\
\hline $\begin{array}{l}\text { Marital status: } \\
\% \text { in monogamous union } \\
\% \text { in polygamous union } \\
\% \text { not in formal union }\end{array}$ & $\begin{array}{c}38.0 \\
15.6 \\
46.4 \\
n=384\end{array}$ & \begin{tabular}{|c|}
59.9 \\
27.8 \\
12.3 \\
$n=489$
\end{tabular} & $\begin{array}{c}49.2 \\
11.9 \\
39.0 \\
n=354\end{array}$ & $\begin{array}{c}71.6 \\
20.8 \\
7.7 \\
n=784\end{array}$ & $\begin{array}{c}75.4 \\
11.0 \\
13.6 \\
n=715\end{array}$ & $\begin{array}{c}68.7 \\
24.9 \\
6.4 \\
n=1154\end{array}$ & $\begin{array}{c}67.4 \\
26.5 \\
6.2 \\
n=389\end{array}$ & $\begin{array}{c}60.2 \\
26.9 \\
12.8 \\
n=1121\end{array}$ & \begin{tabular}{|c|}
70.0 \\
13.5 \\
16.5 \\
$n=436$
\end{tabular} & $\begin{array}{c}81.8 \\
7.2 \\
11.0 \\
n=391\end{array}$ & $\begin{array}{c}70.7 \\
13.6 \\
15.7 \\
n=140\end{array}$ & $\begin{array}{c}85.9 \\
2.3 \\
11.8 \\
n=736\end{array}$ \\
\hline $\begin{array}{l}\text { Religion: } \\
\text { \% Protestant } \\
\text { \% Catholic } \\
\text { \% Other Christian } \\
\text { \% Muslim } \\
\text { \% Traditional } \\
\text { \% Animist } \\
\text { \% African Spiritual } \\
\text { \% Hindu } \\
\text { \% None } \\
\text { \% Other }\end{array}$ & $\begin{array}{r}31.3 \\
6.7 \\
.0 \\
.5 \\
.0 \\
.0 \\
37.3 \\
.3 \\
22.3 \\
1.6 \\
\mathrm{n}=386 \\
\end{array}$ & $\begin{array}{r}9.2 \\
29.3 \\
.0 \\
58.8 \\
.0 \\
2.7 \\
.0 \\
.0 \\
.0 \\
.0 \\
\mathrm{n}=488 \\
\end{array}$ & $\begin{array}{r}8.3 \\
32.6 \\
.0 \\
36.2 \\
.0 \\
14.2 \\
.0 \\
.0 \\
.0 \\
8.6 \\
\mathrm{n}=337 \\
\end{array}$ & $\begin{array}{r}23.8 \\
16.3 \\
41.2 \\
11.2 \\
1.9 \\
.0 \\
.0 \\
.0 \\
5.3 \\
.4 \\
\mathrm{n}=787 \\
\end{array}$ & $\begin{array}{r}51.0 \\
30.7 \\
10.7 \\
6.1 \\
.6 \\
.0 \\
.0 \\
.0 \\
.0 \\
1.0 \\
\mathrm{n}=710 \\
\end{array}$ & \begin{tabular}{|r|}
48.6 \\
43.6 \\
.0 \\
.1 \\
.0 \\
.0 \\
.0 \\
.0 \\
.0 \\
7.8 \\
$\mathrm{n}=1148$ \\
\end{tabular} & $\begin{array}{r}46.1 \\
21.6 \\
.0 \\
30.7 \\
.5 \\
.0 \\
.0 \\
.0 \\
.0 \\
1.0 \\
\mathrm{n}=388 \\
\end{array}$ & $\begin{array}{r}.0 \\
5.4 \\
.1 \\
94.5 \\
.0 \\
.0 \\
.0 \\
.0 \\
.0 \\
.0 \\
\mathrm{n}=1120\end{array}$ & $\begin{array}{r}20.4 \\
35.1 \\
.0 \\
40.4 \\
.0 \\
.2 \\
.0 \\
.0 \\
.0 \\
3.9 \\
\mathrm{n}=436 \\
\end{array}$ & $\begin{array}{r}68.6 \\
26.0 \\
.0 \\
.0 \\
.3 \\
.0 \\
.0 \\
1.0 \\
.3 \\
3.8 \\
\mathrm{n}=392 \\
\end{array}$ & $\begin{array}{r}.0 \\
3.6 \\
1.4 \\
95.0 \\
.0 \\
.0 \\
.0 \\
.0 \\
.0 \\
.0 \\
\mathrm{n}=140 \\
\end{array}$ & $\begin{array}{r}31.8 \\
16.9 \\
36.4 \\
.3 \\
.7 \\
.0 \\
.0 \\
3.0 \\
.0 \\
11.0 \\
\mathrm{n}=745 \\
\end{array}$ \\
\hline $\begin{array}{l}\% \text { of clients with no } \\
\text { formal education }\end{array}$ & $\begin{array}{c}13.2 \\
n=386\end{array}$ & $\begin{array}{c}55.0 \\
n=489\end{array}$ & \begin{tabular}{c|}
28.7 \\
$n=352$
\end{tabular} & $\begin{array}{c}37.1 \\
n=785\end{array}$ & $\begin{array}{c}12.4 \\
n=710\end{array}$ & \begin{tabular}{|c|}
5.0 \\
$n=1152$
\end{tabular} & $\begin{array}{c}23.1 \\
n=386\end{array}$ & $\begin{array}{c}41.0 \\
n=1120\end{array}$ & $\begin{array}{c}18.3 \\
n=437\end{array}$ & $\begin{array}{c}7.1 \\
n=392\end{array}$ & $\begin{array}{c}10.0 \\
n=140\end{array}$ & $\begin{array}{c}7.8 \\
n=732\end{array}$ \\
\hline $\begin{array}{l}\% \text { of clients who can read } \\
\text { easily in English/French }\end{array}$ & $\begin{array}{c}53.1 \\
n=386\end{array}$ & $\begin{array}{c}9.5 \\
n=379\end{array}$ & & $\begin{array}{c}22.0 \\
n=786\end{array}$ & $\begin{array}{c}59.0 \\
n=709\end{array}$ & $\begin{array}{c}3.7 \\
n=511\end{array}$ & & $\begin{array}{c}20.1 \\
n=819\end{array}$ & & & & $\begin{array}{c}47.4 \\
n=734\end{array}$ \\
\hline
\end{tabular}



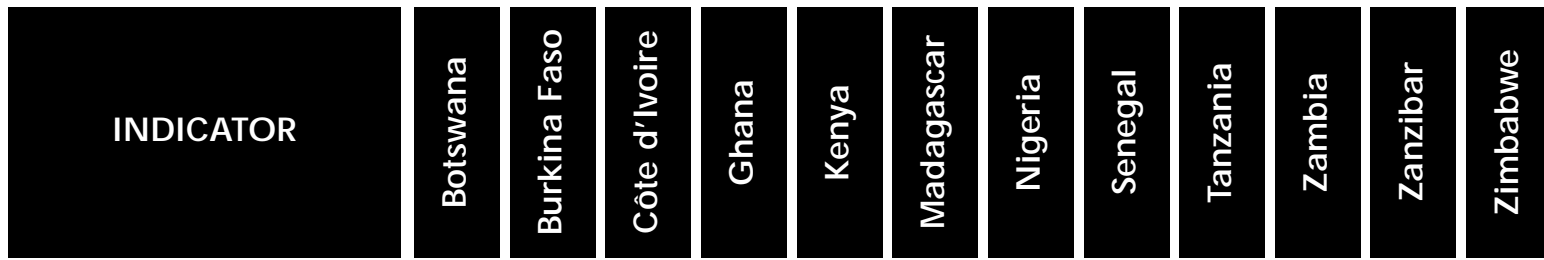

AN NEX TABLE 2-1. PO PULATION OF FAMILY PLAN NING CLIENTS, 12 STUDY SITES (concluded)

\begin{tabular}{|l|c|c|c|c|c|c|c|c|c|c|c|c}
\hline $\begin{array}{l}\text { \% of clients who can read } \\
\text { easily in a local language }\end{array}$ & $\begin{array}{c}79.0 \\
\mathrm{n}=386\end{array}$ & $\begin{array}{c}3.7 \\
\mathrm{n}=379\end{array}$ & & $\begin{array}{c}22.1 \\
\mathrm{n}=787\end{array}$ & & $\begin{array}{c}65.8 \\
\mathrm{n}=518\end{array}$ & & $\begin{array}{c}2.2 \\
\mathrm{n}=819\end{array}$ & & & $\begin{array}{c}71.4 \\
\mathrm{n}=746\end{array}$ \\
\hline $\begin{array}{l}\text { Mean number of living } \\
\text { children among clients }\end{array}$ & $\begin{array}{c}2.5 \\
\mathrm{n}=386\end{array}$ & $\begin{array}{c}3.5 \\
\mathrm{n}=460\end{array}$ & $\begin{array}{c}3.2 \\
\mathrm{n}=353\end{array}$ & $\begin{array}{c}3.6 \\
\mathrm{n}=785\end{array}$ & $\begin{array}{c}3.4 \\
\mathrm{n}=710\end{array}$ & $\begin{array}{c}3.6 \\
\mathrm{n}=1144\end{array}$ & $\begin{array}{c}4.3 \\
\mathrm{n}=389\end{array}$ & $\begin{array}{c}3.9 \\
\mathrm{n}=1112\end{array}$ & $\begin{array}{c}3.4 \\
\mathrm{n}=437\end{array}$ & $\begin{array}{c}3.4 \\
\mathrm{n}=391\end{array}$ & $\begin{array}{c}4.2 \\
\mathrm{n}=140\end{array}$ & $\begin{array}{c}2.9 \\
\mathrm{n}=733\end{array}$ \\
\hline $\begin{array}{l}\text { \% of clients who want } \\
\text { more childrenc }\end{array}$ & $\begin{array}{c}58.6 \\
\mathrm{n}=353\end{array}$ & $\begin{array}{c}67.4 \\
\mathrm{n}=475\end{array}$ & $\begin{array}{c}84.5 \\
\mathrm{n}=336\end{array}$ & $\begin{array}{c}57.0 \\
\mathrm{n}=739\end{array}$ & $\begin{array}{c}39.0 \\
\mathrm{n}=672\end{array}$ & $\begin{array}{c}42.8 \\
\mathrm{n}=1081\end{array}$ & $\begin{array}{c}59.9 \\
\mathrm{n}=354\end{array}$ & $\begin{array}{c}73.3 \\
\mathrm{n}=1094\end{array}$ & $\begin{array}{c}75.4 \\
\mathrm{n}=427\end{array}$ & $\begin{array}{c}62.3 \\
\mathrm{n}=374\end{array}$ & $\begin{array}{c}76.5 \\
\mathrm{n}=136\end{array}$ & $\begin{array}{c}55.4 \\
\mathrm{n}=677\end{array}$ \\
\hline
\end{tabular}

a "Monogamous union" includes monogamous marriage and cohabiting. "Polygamous union" includes polygamous marriage and visiting. "No formal union" includes single, divorced, separated, and widowed.

b French applies in Madagascar, Burkina Faso, and Senegal, whereas English applies in Ghana, Zimbabwe, Botswana, and Kenya.

" Excludes the $2 \%$ of clients whose fertility decisions "depend on God" or "depend on husband."

\section{REFERENCES}

\section{Demographic Health Surveys}

Botswana, Central Statistical Office. 1988. Botswana Demographic and Health Survey, 1988. Macro International, Calverton, MD.

Burkina Faso, Institut National de la Statistique et de la Démographie. 1994. Enquête Démographique et de Santé, Burkina Faso, 1993. Macro International, Calverton, MD.

Côte d'Ivoire, Institut National de la Statistique. 1995. Enquête Démographique et de Santé, Côte d'Ivoire, 1994. Macro International, Calverton, MD.

Ghana, Ghana Statistical Service. 1994. Ghana Demographic and Health Survey, 1993. Macro International, Calverton, MD.

Kenya, Central Bureau of Statistics. 1994. Kenya Demographic and Health Survey, 1993. Macro International, Calverton, MD.

Madagascar, Centre National de Recherches sur l'Environnement. 1994. Enquête Nationale Démographique et Sanitaire, 1992. Macro International, Calverton, MD.

Nigeria, Federal Office of Statistics. 1992. Nigeria Demographic and Health Survey, 1990. Macro International, Calverton, MD.

Senegal, Ministère de l'Economie, des Finances et du Plan. 1997. Enquête Démographique et de Santé au Sénégal, 1994. Macro International, Calverton, MD.
Tanzania, Bureau of Statistics. 1997. Tanzania Demographic and Health Survey, 1996. Macro International, Calverton, MD.

Zambia, Central Statistical Office. 1993. Zambia Demographic and Health Survey, 1992. Macro International, Calverton, MD.

Zanzibar. See Tanzania.

Zimbabwe, Central Statistical Office. 1995. Zimbabwe Demographic and Health Survey, 1994. Macro International, Calverton, MD.

\section{Situation Analysis Studies}

\section{Botswana}

Baakile, B., N. Maggwa, L. Maribe, and R. Miller. 1996. A Situation Analysis of the Maternal and Child Health/Family Planning (MCH/FP) Program in Botswana. Ministry of Health MCH/FP Unit and Population Council, Botswana.

\section{Burkina Faso}

Bakouan, D., P. Sebgo, I. Askew, Y. Ouedraogo, P. Tapsoba, C. Viadro, and S. Kanon. 1992. Analyse Situationnelle du Programme de Planification Familiale au Burkina Faso. Ministère de la Santé, de l'Action Sociale et de la Famille, Direction de la Santé de la Famille, and Population Council, Burkina Faso.

Bamba, A., B. Millogo, J. Nougtara, Y. Ouedraogo, P. Tapsoba, and I. Kabore. 1996. Rapport Final: Deuxième 
Analyse Situationnelle du Programme de Planification Familiale au Burkina Faso. Ministère de la Santé, Direction de la Santé de la Famille, and Population Council, Burkina Faso.

\section{Côte d'Ivoire}

Kouakou, K., K. Kouame, and D. Huntington. 1992. Analyse Situationnelle du Programme d'Extension des Services de Planification Familiale en Côte d'Ivoire. Association Ivoirienne pour le Bien-Être Familial and Population Council, New York.

\section{Ghana}

Twum-Baah, K., P. Wolf, P. Nyarko, and H. Odai. 1994. A Situation Analysis Study of Family Planning Service Delivery Points in Ghana. Ghana Statistical Service, USAID, and Population Council, Accra, Ghana.

Twum-Baah, K., E. Ameka, E. Okrah, and A. OheneOkai. 1997. Second Round Situation Analysis Study of Family Planning Service Delivery Points in Ghana. Ghana Statistical Service, Accra, Ghana.

\section{Kenya}

Miller, R., L. Ndhlovu, and M. Gachara. 1989. A Situation Analysis of the Family Planning Program of Kenya: The Availability, Functioning, and Quality of $\mathrm{MOH}$ Services. Population Council, New York.

Ndhlovu, L., J. Solo, R. Miller, K. Miller., and A. Omunde. 1997. An Assessment of Clinic-Based Family Planning Services in Kenya: Results from the 1995 Situation Analysis. Ministry of Health, Division of Family Health, Nairobi, Kenya, and the Population Council, New York.

\section{Madagascar}

Ministère de la Santé, République du Madagascar. 1996. Analyse Situationnelle du Système de Prestation de Services de Planification Familiale dans les Provinces d'Antananarive et de Fianarantsoa: Rapport de Synthèse. Population Council, New York.

\section{Nigeria}

Federal Ministry of Health, Nigeria. 1992. Nigeria: The Family Planning Situation Analysis Study. Population Council, New York.

\section{Senegal}

Ministère de la Santé et de l'Action Sociale du Sénégal and Population Council. 1995. Analyse Situationnelle du Système de Prestation de Services de Planification Familiale au Sénégal: Rapport Final. Population Council, Dakar, Senegal.

\section{Tanzania}

Ministry of Health, Tanzania. 1992. Tanzania: The Family Planning Situation Analysis Study. Population Council, New York.

\section{Zambia}

Central Statistical Office, Zambia. 1998. Zambia Situation Analysis, 1997. Central Statistical Office and Population Council, Zambia.

\section{Zanzibar}

Mapunda, P. 1996. The Zanzibar Family Planning Situation Analysis Study. Ministry of Health and Population Council, Tanzania.

\section{Zimbabwe}

Zimbabwe National Family Planning Council, Population Council, and SEATS. 1992. Zimbabwe: A Situation Analysis of the Family Planning Programme. Population Council, Harare, Zimbabwe.

Dube, H., C. Marangwanda, and L. Ndhlovu. 1998. An Assessment of the Zimbabwe Family Planning Programme: Results from the 1996 Situation Analysis Study. Evaluation and Research Unit, Zimbabwe National Family Planning Council, and the Population Council, Harare, Zimbabwe and Nairobi, Kenya.

\section{Other References}

Destler, H., D. Liberi, J. Smith, and J. Stover. 1990. Family Planning: Preparing for the 21st Century. United States Agency for International Development, Washington, D.C.

Lapham, R.J. and W.P. Mauldin. 1985. "Contraceptive prevalence: The influence of organized family planning programs." Studies in Family Planning, 16,3:117-137.

Mapunda, P.S. 1996. The Zanzibar Family Planning Situation Analysis Study. Zanzibar Family Planning Program, Zanzibar, Tanzania.

Miller, R., L. Ndhlovu, M. Gachara, and A. Fisher. 1992. "Situation Analysis Study of Kenya's Family Planning Program." In Jain, A., Ed., Managing Quality of Care in Population Programs. Kumarian Press, Connecticut.

Ross, J., W.P. Mauldin, and V. Miller. 1993. Family Planning and Population: A Compendium of International Statistics. United Nations Population Fund and Population Council, New York.

United Nations. 1997. Demographic Yearbook 1995. United Nations, Department for Economic and Social Information and Policy Analysis, Statistics Division, New York.

United Nations. 1996a. Demographic Yearbook 1994. United Nations, Department for Economic and Social Information and Policy Analysis, Statistics Division, New York.

United Nations. 1996b. Statistical Yearbook 1994. United Nations, Department for Economic and Social 
Information and Policy Analysis, Statistics Division, New York.

World Bank. 1997. Confronting AIDS: Public Priorities in a Global Epidemic. World Bank, Washington, D.C.

\section{NOTES}

1 All CPRs are from the relevant Demographic and Health Surveys (DHS) (see the list of references at the end of this chapter). Unless otherwise noted, $\mathrm{CPRs}$ are reported for all women of reproductive age and include only modern methods.

2 Program effort scores are from two rounds of studies (in 1982 and 1989) by Lapham, Mauldin, and Ross that measure a government's commitment to its family planning program. Information is collected on official national policies, service-related activities, national recordkeeping and evaluation programs, and availability and accessibility of contraceptive supplies and services. This information is then standardized to a score ranging from 0 to 100 that represents the percentage of the maximum effort that is being applied. For this chapter, all program effort scores are from Ross et al. (1993).

3 All TFRs are from United Nations (1997).

4 All HIV seroprevalence levels are from World Bank (1997). The 1994 prevalence rates among general adult populations are World Bank estimates, and prevalence rates among urban antenatal clients are from studies contained in the U.S. Census HIV / AIDS Surveillance Database. All rates are for HIV-1.

5 All information on method mixes is from the relevant DHS studies, and is for all women of reproductive age using modern methods.

6 All gross domestic products are from United Nations (1996b).

7 All populations of women of reproductive age are calculated from United Nations (1996a), with supplemental information on women of reproductive age as a percent of total population from United Nations (1997). 



\section{BASIC STUDY FINDINGS $\&$ THEIR UTILIZATION}

Indicators of
Readiness
and Quality:
Basic Findings
presents the overall findings of
approximately 100 indicators
of the readiness and quality of
family planning services in the
12 study sites.

\begin{tabular}{l}
\hline \\
Using Situation \\
Analysis to Improve \\
Reproductive \\
Health Programs \\
examines how Situation \\
Analysis is being used not only \\
to assess program strengths \\
and weaknesses, but also, for \\
example, to evaluate changes \\
in programs over time and \\
measure the impact of \\
interventions. \\
\end{tabular}





\section{KEY POINTS AND CONCLUSIONS Indicators of Readiness and Quality: Basic Findings}

One objective of Situation Analysis studies is to describe the readiness and quality of family planning services offered at service delivery points (SDPs). A general description of services in subSaharan Africa can be constructed from over 100 indicators generated by each of the 12 Situation Analysis studies included in this volume. That description indicates various strengths and weaknesses of the 12 programs and, most important, identifies those aspects of services most critically in need of attention. To some extent, the quality of a country's services is related to the maturity of its family planning program, but certainly not all the results reported here follow this pattern.

Many of the consistent program strengths revolve around readiness to provide services:

- The infrastructure of most SDPs, especially in urban areas, is quite serviceable, and many have the equipment required for basic family planning services.

- The pattern of methods offered is in some ways quite appropriate, the one serious problem being the lack of full availability of condoms in some countries.

- Stockout rates for most methods appear generally to be under control, with the exception of injectables.

Several strengths are also evident in the quality of services given to clients:

- The physical assessment of new family planning clients with weight, blood pressure, last menstrual period, and medical history, for example, is carried out fairly consistently.
- The quality of injections is quite high in most countries, especially with regard to aseptic procedures-an important finding given the increasing use of injectables in many contexts.

- Across all the study sites, the vast majority of clients are given revisit dates, thereby encouraging continuity of method use.

However, three overall weaknesses are clearly evident and call for direct attention:

- Counseling on family planning is broadly lacking across all study sites, in terms of both information taken from the client and information given to the client about her method. These activities are particularly important because they are directly related to client satisfaction, appropriateness of method selected, continuity of use, and sexually transmitted disease (STD) / HIV risk.

- Aseptic procedures are not always followed, which is extremely serious given the high STD/HIV prevalence in many countries. In particular, findings related to availability of clean water, handwashing and glove use during pelvic exams, and use of sterile instruments during IUD insertion raise this concern.

Clients' STD/HIV risk is generally not adequately addressed. Availability of condoms is quite low in some contexts, and where available, they are often not discussed with clients. Moreover, clients are rarely told how their method relates to STD/HIV transmission or screened for STD symptoms. 



\section{Indicators of Readiness and Quality: Basic Findings}

Kate Miller, Heidi Jones, and Marjorie C. Horn

This chapter presents the basic findings from the most recent Situation Analysis studies in the 12 subSaharan African countries described in Chapter 2. It includes over 100 indicators calculated in as many studies as the data will allow, resulting in an overall picture of the quality of family planning services in the region. These indicators reflect the readiness of programs to provide services and the quality of services delivered, as defined by the Bruce-Jain quality-of-care framework (see Chapter 1 for a discussion of this framework). ${ }^{1}$

The indicators are grouped under the various elements of readiness, such as infrastructure and commodity management, and of quality of care delivered, such as technical competence and information exchange. The indicators within each element are discussed individually, and a summary of each element is then presented under the heading "General Assessment." For ease of tracking and cross-referencing, the elements and indicators are numbered consecutively according to the following scheme: elements under readiness and quality are numbered, respectively, R.1, R.2, ... and Q.1, Q.2, .... Indicators are then numbered R.1-1, R.1-2, and so on. For example, R.1-1 is the first indicator discussed under the first element of readiness, while Q.3-2 is the second indicator discussed under the third element of quality. Readers who are interested in an overview of the results may want to read only the general assessment sections and the concluding section of the chapter. Those who want more detail on a particular element may wish to read the individual indicator results and also refer to Annex 3-1, which contains all the results and sample sizes in tabular form, in the order in which they are discussed in the text. Annex 3-1 also provides a more detailed breakdown of the indicators discussed in the main text. References for all of the Situation Analysis studies and Demographic and Health Surveys (DHS) used here are given at the end of Chapter 2.

\section{READINESS TO PROVIDE SERVICES}

\section{R.1 Infrastructure and Facilities}

\section{R.1-1 Do SDPs have adequate waiting areas?}

Family planning clients should have an appropriate place to wait for services. For this indicator, an "adequate" waiting area is defined as an area protected from both rain and sun and with enough seats to accommodate the average daily client load. The results range from about $50 \%$ of service delivery points (SDPs) in Tanzania and Madagascar to 100\% in Côte d'Ivoire. In 7 of the 12 study sites, $86 \%$ or more of the SDPs have an adequate waiting area.

\section{R.1-2 Do SDPs have electricity and piped running water?}

Electricity and clean water are both essential to the provision of certain types of family planning services. Although they may not be necessary for the dispensing of pills, they are needed for the insertion of IUDs, which requires aseptic procedures and sterile instruments. In most cases, injectables are given with disposable needles, but in some clinics, sterilization of needles is still necessary.

The availability of electricity and water varies considerably across the study sites. ${ }^{2}$ In Senegal and Ghana, almost all SDPs have electricity, but in Zanzibar, the proportion is just 36\%. Similarly, $91 \%$ of SDPs have piped running water in Botswana, 
Figure 3-1. Percent of SDPs with adequate water in exam room

"Adequate": A sufficient quantity of dean water for wasting hands and equipment

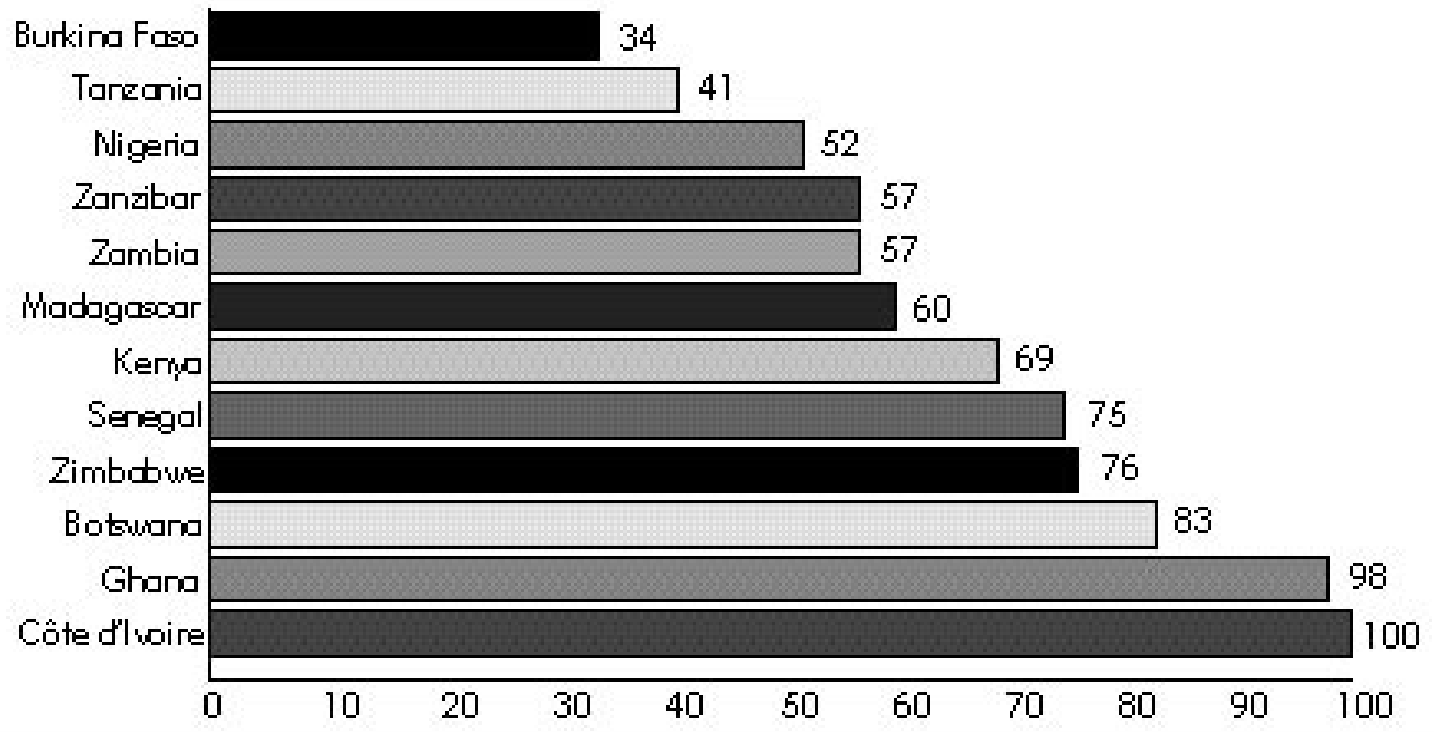

but only $44 \%$ in Zambia. The availability of electricity and water is strongly determined by the location of the SDP. For each study site, both electricity and water are significantly more available in urban and semi-urban SDPs than in rural SDPs $(\mathrm{p}<.01) .^{3}$ However, rural SDPs may obtain both water and energy from alternative sources. The Kenya Situation Analysis report in particular notes that some rural SDPs collect rainwater and burn gas or firewood for heating.

\section{R.1-3 Do SDPs have working toilets for clients?}

In many cases, clients wait for several hours for family planning services, so SDPs should have working toilets available to clients. In 10 of the 12 study sites, $74 \%$ or more of the SDPs have working toilets for clients. The proportion is somewhat lower in Madagascar (56\%) and Zanzibar (61\%), but in general, toilets are available to clients.

\section{R.1-4 Do SDPs have adequate examination areas?} An examination area is considered "adequate" if it is clean, has sufficient light, and has a supply of clean water. All of these characteristics are desirable for a physical exam and necessary for a pelvic exam. In all the study sites, $79 \%$ or more of SDPs have sufficient light in their exam rooms. In half the study sites, the vast majority of SDPs have clean examination areas $(89 \%$ to $100 \%)$, whereas in the other half the proportion is about two-thirds (63\% to $73 \%$ ).

As Figure 3-1 shows, more variation is apparent in the results for the availability of adequate water, which is defined as a sufficient quantity of clean water for washing hands and equipment. The water supply does not have to include piped running water, only a source of clean water that can be refreshed in the examination room. In 5 study sites, three-quarters or more of SDPs have adequate water in their exam rooms, but in 7 other sites, the availability ranges from $69 \%$ (Kenya) to only $34 \%$ (Burkina Faso). For the latter countries, this indicator is significantly affected by location: the vast majority of urban SDPs have water in exam rooms, but in Burkina Faso and Madagascar, for example, as few as $29 \%$ of rural SDPs have adequate water.

\section{R.2 Accessibility}

\section{R.2-1 Do SDPs open on time?}

Failure of an SDP to open at its stated opening time can represent a restriction on the accessibility of services to clients. Eight of the study sites show great variation in the proportion of SDPs open on time. About $80 \%$ of SDPs in Tanzania and Zanzibar 


\section{General Assessment of Infrastructure and Facilities}

With a few exceptions, adequate waiting areas (R.1-1) and toilets for clients (R.1-3) are generally available in SDPs at the study sites. Not surprisingly, the other indicators are heavily determined by location: in general, more urban SDPs have electricity and running water (R.1-2) and adequate examination rooms (R.1-4) than do rural SDPs. (See Chapter 7 for more discussion of this point.)

The infrastructural item of greatest concern is the availability of clean water in examination rooms. This water is used for washing hands between exams and for washing equipment, thereby reducing the risk of cross-infection of clients. In areas of high unrecognized STD/HIV prevalence, this is a matter of critical importance.

were open on time the day of the study visit, whereas in Kenya the proportion was only $5 \%$. Other sites range between these extremes.

Some final Situation Analysis reports state that SDPs do not open on time because staff are busy preparing for the day's services by sterilizing instruments and cleaning examination areas. However, from the client's point of view, access is still restricted if the clinic is not giving services at the stated hour. If service preparation is causing the delay in opening, the schedule for preparation might be rearranged or the stated opening hour delayed.

\section{R.2-2 Do SDPs have signs announcing family planning services?}

Signs announcing an SDP's family planning services raise clients' awareness of those services. Across the 12 study sites, $68 \%$ or fewer of the SDPs have such a sign either inside or outside the facility. This indicator shows a good deal of variation, ranging from $68 \%$ in Ghana to $10 \%$ in Tanzania. Generally, signs announcing family planning service availability are not common.

The appropriateness of signs announcing family planning services is debated in the Senegal and Nigeria Situation Analysis reports. Both family planning programs question the utility of such signs when many clients would like to protect their anonymity; thus signs might actually be a deterrent to obtaining contraceptive services. However, in Zimbabwe and Kenya, signs are not seen as playing an important role in influencing clients, and thus are not emphasized.

\section{General Assessment of Accessibility}

The two indicators included under this element-opening on time and having signs announcing family planning services-are illustrative only, and are not intended to present a full picture of accessibility. Accessibility also includes factors such as travel time, cost, and psychosocial accessibility, all of which are outside the scope of the Situation Analysis studies. (However, see Q.7, "Appropriateness and Acceptability of Services," for discussion of related issues.)

However, these two indicators do reveal a good degree of variation in accessibility of services. The variation does not seem to be determined by the overall maturity of the program, since some of the most mature programs score poorly and vice versa. Although the countrylevel performance is difficult to explain, within countries these indicators seem to vary with the type of SDP. For both opening on time (R.2-1) and signs announcing family planning services (R.2-2), the larger SDPs, such as hospitals and clinics with maternities, do significantly better than health posts and smaller clinics. This difference is statistically significant for opening times in Ghana, Nigeria, Tanzania, and Zambia, and for family planning signs in Zimbabwe, Zanzibar, Zambia, Kenya, G hana, Burkina Faso, and Botswana $(p<.05)$. Furthermore, the availability of signs appears to be related to the location of the SDP: in several study sites, urban SDPs are more likely than rural SDPs to have signs announcing family planning services. This difference is statistically significant in Zambia, Tanzania, Nigeria, Kenya, G hana, and Burkina Faso $(p<.05)$. 


\section{R.3 Methods Offered}

This section addresses the methods that are usually offered at SDPs, not whether those methods were actually in stock on the day of the study visit, or whether the SDP has the appropriate equipment in working order for each method. Rather, the discussion focuses on whether the SDPs report that each method is usually available.

\section{R.3-1 How available are hormonal methods?}

Combined oral contraceptives (COCs) generally dominate the method mix of each study site, and are by far the most commonly offered method as well. Across all 12 study sites, fully $91 \%$ or more of SDPs normally offer COCs. Similarly, injectables are often widely available; as Figure 3-2 shows, however, 4 of the study sites-Tanzania, Zambia, Burkina Faso, and Senegal-had not integrated injectables fully into their programs at the time of the study. ${ }^{4}$ In fact, at the time of the Situation Analysis in Zambia, injectables had not been legally registered, so the fact that half of the SDPs there reported they offer injectables is surprising. Progestin-only pills (POPs) are widely available in some countries, but in other cases are quite uncommon: only $23 \%$ of SDPs in Nigeria, for example, offer POPs. At the time of many of the studies,
NORPLANT ${ }^{\oplus}$ was only available experimentally in a handful of clinics.

\section{R.3-2 How available are condoms and dual methods?*}

In 9 of the study sites, $89 \%$ or more of SDPs normally offer condoms, so in these cases the method is largely available. In Zanzibar and Tanzania, about three-quarters of SDPs offer condoms, and in Burkina Faso only about half.

Dual-method counseling involves encouraging the client to use condoms in addition to her normal family planning method for increased STD/HIV protection. Data on the availability of dual-method counseling exists in only three studies: $50 \%$ of the SDPs in Zambia reportedly offer dual methods, $67 \%$ in Ghana, and $71 \%$ in Zimbabwe.

\section{R.3-3 How available are IUDs?}

The availability of IUDs varies widely by study site (see Figure 3-3). In Senegal, Nigeria, and Côte

* The term "dual methods" (using a condom in addition to the regular contraceptive method to provide STD protection) is slightly less encompassing than "dual protection," which additionally includes choosing a condom to serve both as contraception and as STD protection. While acknowledging that it is not as broad a term, we use "dual methods" here to follow the phrasing of the Situation Analysis instruments.

Figure 3-2. Percent of SDPs that off er iniectables

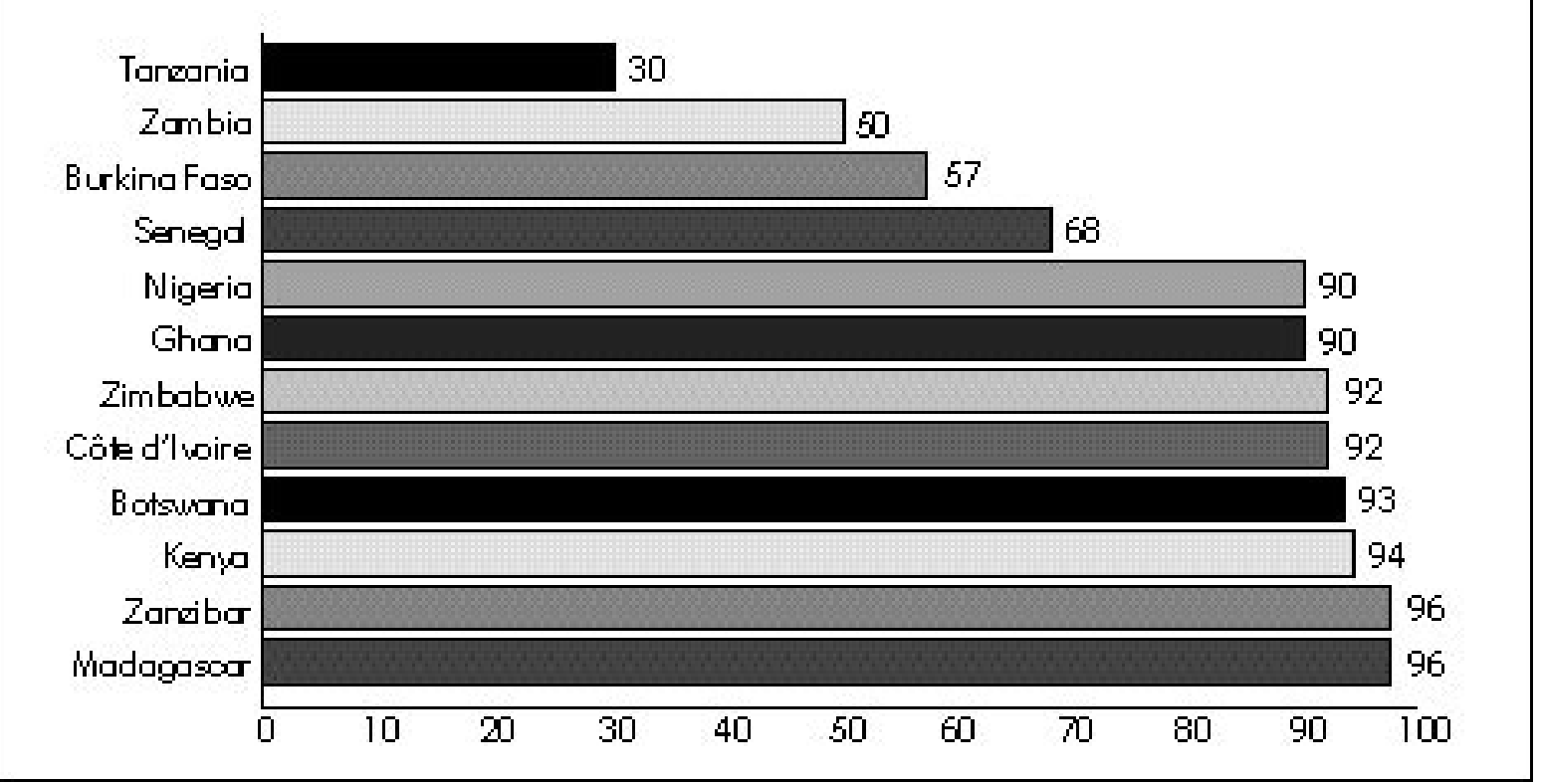


d'Ivoire, over $80 \%$ of SDPs offer IUDs, whereas in Zimbabwe, Tanzania, Zambia, and Burkina Faso, the proportion is $25 \%$ or less. ${ }^{5}$ In all cases, hospitals and clinics with maternity services offer IUDs more frequently than do health posts, often because of equipment needs or national service policy.

\section{R.3-4 How available are tubal ligation and vasectomy?}

These methods are the most unavailable across all study sites because they require substantial infrastructure and specially trained staff. For this reason, most SDPs that offer surgical sterilization in the study sites are hospitals or high-level clinics.

Ghana has by far the greatest availability of both methods: $23 \%$ of SDPs offer vasectomy and $50 \%$ tubal ligation. These methods are also prevalent in Kenya, where $29 \%$ offer tubal ligation and $14 \%$ vasectomy. In other countries, $10 \%$ or fewer offer tubal ligation and $5 \%$ or fewer vasectomy.

Overall, these methods are not widely available, and vasectomy is less available than tubal ligation. A simple comparison of those SDPs that offer these methods shows that if an SDP offers vasectomy, it probably offers tubal ligation as well, but the reverse is not true; thus it is extremely rare to find SDPs that offer vasectomy and not tubal ligation.
R.3-5 How available are natural family planning and lactational amenorrhea methods?

These two methods require staff trained to counsel properly, but do not require any equipment or infrastructure. The availability of natural family planning counseling ranges widely, from 3\% in Tanzania to fully $74 \%$ in Ghana. Information on the availability of lactational amenorrhea method (LAM) is presented in only 4 studies, but it too shows great variability: $10 \%$ of SDPs offer LAM in Burkina Faso, 39\% in Zimbabwe, 63\% in Zambia, and $83 \%$ in Ghana.

\section{R.4 Contraceptive Supplies and Logistics}

\section{R.4-1 How often do SDPs experience stockouts of} contraceptive supplies?

If SDPs run out of stocks of methods, clients' choice of methods and continuation of method use are necessarily constrained. For this reason, contraceptive stockouts affect the quality of services that can be delivered. Stockout rates of contraceptive supplies vary greatly by method and by country. ${ }^{6}$ Stockout rates are the highest for injectables. Over 6 of the study sites, an average of $22 \%$ of SDPs offering injectables had experienced a stockout.

Figure 3-3. Per cent of SDPs that off er IUDs

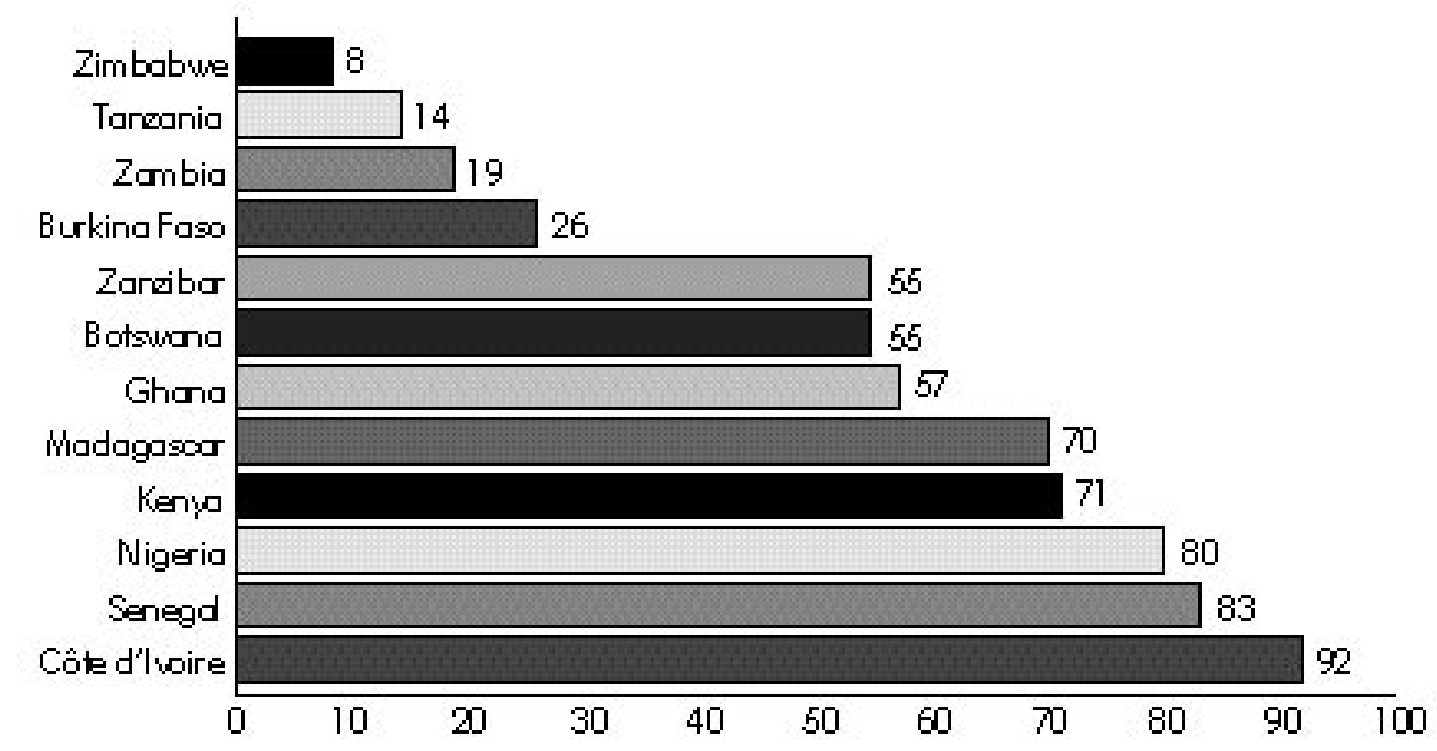




\section{General Assessment of Methods Offered}

Three broad characteristics of sub-Saharan African populations should influence the appropriate range of method availability: reproductive intentions, STD risk, and breastfeeding status. ${ }^{7}$ In many of the study sites, a majority of clients express the desire for spacing rather than limiting their births, and thus the high availability of COCs and injectables (R.3-1) is appropriate in terms of meeting the needs of spacers. Similarly, because the expressed demand for limiting is not currently very high, the relatively low availability of tubal ligation and vasectomy (R.3-4) may be appropriate. Demand for limiting may well rise in coming decades, however (see Westoff, 1991), and availability of these methods could be expected to rise accordingly. At the same time, because these methods require intensive investment in staff training and infrastructure, broadening their availability is more difficult than for many other methods. (See indicators R.6-3 through R.6-9 for more information on the availability of trained staff for the various methods.)

Clients may also be at serious risk of STD/HIV, and pills, injectables, and surgical methods offer no protection against STD/HIV transmission. For this reason, the availability of condoms and use of dual methods (R.3-2) are particularly crucial. Although the majority of facilities in most countries do offer condoms, some do not. Moreover, as will be seen later, condoms and dual methods are often not discussed with clients even when available (see Q .3-5). Dual-method counseling is important since clients may not be aware that condoms can be used for STD/HIV prevention in conjunction with other methods. The 3 studies that collected this information do not show full availability of dual-method counseling. Again, because of the high risk of STD/HIV transmission in many of these sites, both condoms alone and counseling for dual methods should be available at every SDP.

The high prevalence of STD/HIV also critically affects IUD users, since the insertion procedure may not be aseptic; clients may therefore be placed at risk of iatrogenic infections, and clients with undiagnosed STDs placed at risk of pelvic inflammatory disease (PID). Availability of IUD insertion (R.3-3) does vary with the size of the SDP, which is appropriate since insertion involves a certain amount of equipment and infrastructure. However, as will be discussed later (see Q.5-3), the providers of this method have a particular responsibility to protect clients against STD transmission, and in many cases clients are not being adequately protected. In short, the sheer a vailability of IUD services seems appropriate, but the implementation of these services may require attention.

Lastly, the breastfeeding status of clients affects method choice because breastfeeding is a contraindication for COC use. Instead, breastfeeding clients who would otherwise use COCs should use PO Ps. Given that a large proportion of clients are breastfeeding, and in most study sites large proportions are potential COC users, the availability of PO Ps (R.3-1) should be more widespread than the results here reveal. Upcoming sections will address whether COCs and PO Ps are actually mentioned to clients (see Q.3-2 and Q.3-3), and reveal that in many cases COCs are being used where PO Ps would be more appropriate.

Because of high breastfeeding rates, LAM would probably be an appropriate method for many clients, and indeed most women in Africa know about suppressed fertility during breastfeeding. However, LAM has not been emphasized by many programs because of misunderstanding of the difference between breastfeeding and LAM. If LAM is to work reliably, appropriate counseling and support should be available to clients. This method shows varying degrees of availability in the study sites (R.3-5). Yet because LAM counseling requires no infrastructure or equipment, only staff training is necessary to add it to the list of methods offered at an SDP.

36 Clinic-Based Family Planning and Reproductive Health Services in Africa 
Fully 50\% of SDPs in Nigeria had experienced an injectable stockout in the previous 6 months. The relatively high injectable stockout rates may reflect the increasing popularity of injectables in subSaharan Africa and the relative novelty of the method. (See R.4-2 for more discussion of injectable stockouts.)

Condoms have the lowest overall stockout rates. In all the study sites, about one-quarter or fewer SDPs had experienced a stockout of condoms in the 6 months previous to the Situation Analysis study. COCs and POPs have slightly higher stockout rates: in all the study sites about one-third or fewer SDPs had experienced these stockouts. IUDs have a stockout rate of $22 \%$ or less in most of the study sites, but in Kenya, $36 \%$ of SDPs offering IUDs had run out of them in a 6-month period.

NORPLANT $^{\circledast}$ was being introduced experimentally at the time of many of the Situation Analysis studies, so it was offered by only a handful of SDPs. In Senegal, Nigeria, Madagascar, Burkina Faso, and Botswana, none of the SDPs offering NORPLANT ${ }^{\circledast}$ had experienced a stockout in the previous 6 months. However, the stockout information for NORPLANT ${ }^{\circledast}$ is not very representative given the unavailability of this method at the time of the studies.
Stockout rates also vary by country. Figure 3-4 shows the percentage of SDPs in each country that had experienced at least one stockout of at least one offered method in the 6 months preceding the study. At $65 \%$, Nigeria had experienced the most stockouts, and about half of SDPs in Kenya, Zambia, and Zimbabwe had experienced at least one stockout. Senegal, Madagascar, and Zanzibar had the lowest overall stockout rates.

\section{R.4-2 Do SDPs have commodity inventory lists,} and are they related to stockout rates?

One way an SDP can control its stocks of contraceptives is to keep a running commodity inventory to monitor stock levels. The percentage of SDPs with commodity inventories varies widely across the study sites, ranging from 29\% in Burkina Faso to $100 \%$ in Côte d'Ivoire. The presence of a written commodity inventory might be associated with fewer stockouts, since it helps clinic managers identify low supplies and reorder before a method is fully stocked out. However, a simple comparison of SDPs with and without commodity inventories shows no clear relationship between such inventories and stockouts. In most countries and for most methods, no statistically significant relationship holds. However, certain methods in some countries are significantly less likely to be stocked out if

\section{Figure 34. Per cent of SDPs experiencing a sto dko ut of at least one offer ed method in last 6 months}

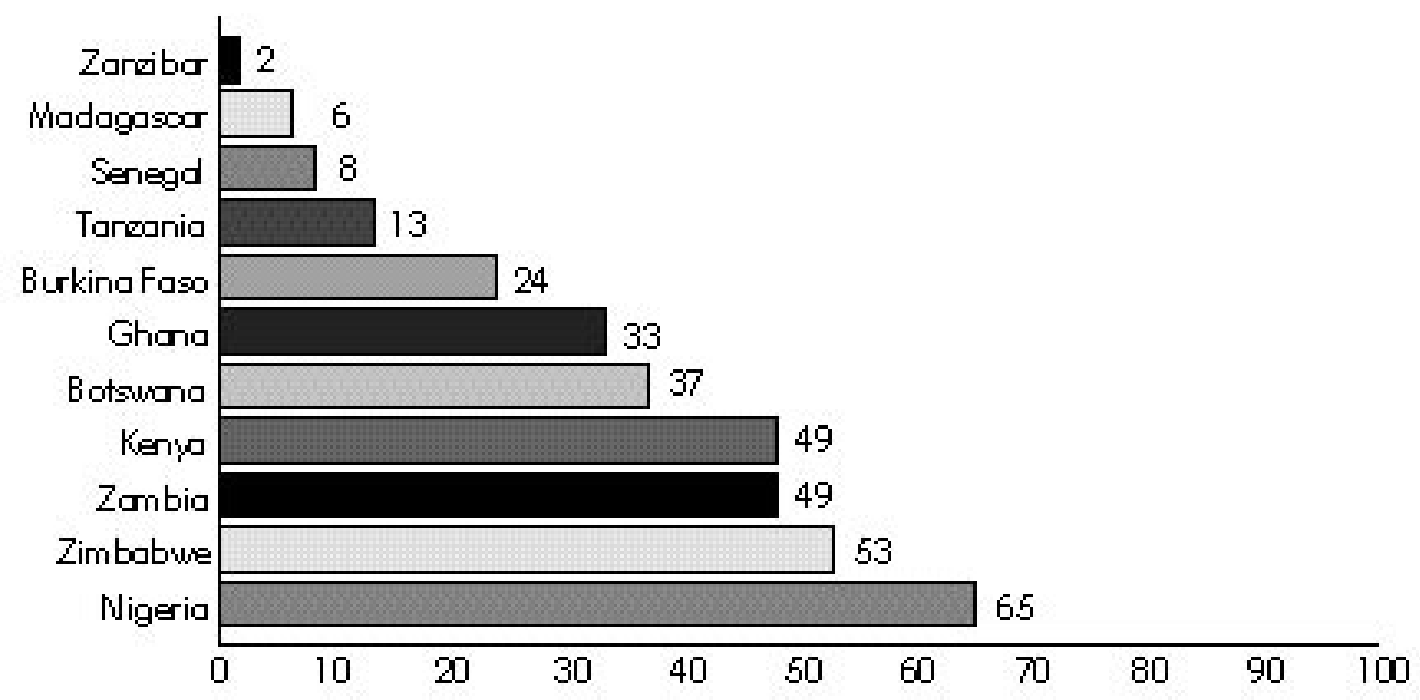


a commodity register is present: POPs in Burkina Faso, COCs and IUDs in Ghana, and IUDs in Zanzibar. These results do not fall into a simple pattern.

This observed lack of a clear relationship between commodity inventories and stockout rates may be due to the fact that stockouts often result from systemwide distribution problems rather than clinics neglecting to order stocks. For example, 19\% of SDPs in Ghana had experienced a stockout of injectables in the 6 months prior to the study, even though $98 \%$ of the SDPs had a commodity register. However, in that period the national supplier of injectables changed from the United States Agency for International Development (USAID) to the United Nations Development Programme (UNDP), and difficulties in keeping distribution constant during the switchover resulted in a nationwide 3-month stockout. Local commodity registers would of course have no effect on this type of problem.

\section{R.4-3 Do SDPs have adequate storage for contraceptive stocks?}

For this indicator, "adequate" is defined as protected from rain, sun, adverse temperatures, ${ }^{8}$ and pests. In almost all the study sites, three-quarters or more of SDPs have adequate contraceptive storage. The exception is Côte d'Ivoire, in which only 1 of 13 SDPs reported adequate contraceptive storage. In the Situation Analysis report for Côte d'Ivoire, the reasons for inadequate storage are cited as poor ventilation, storage of commodities on the floor, and storage of commodities in the manager's office.

\section{R.5 Equipment}

Equipment needs vary depending on the type of SDP. For the present analysis, SDPs have been divided into three general categories: hospitals, large clinics with maternities, and small clinics or health posts. Clearly, differences in client loads and services in these types of SDPs affect their equipment needs. For this analysis, an SDP is considered to have the minimum equipment needed if it meets the requirements shown in Table 3-1, which were drawn up for the purposes of this analysis. Equipment that is necessary only for specific methods,

\section{General Assessment of Contraceptive Supplies and Logistics}

In general, storage areas for contraceptive stocks are appropriate (R.4-3), but stockouts can pose a larger problem (R.4-1). The general rate of stockouts, as shown in Figure 3-4, varies greatly by country and seems to be related to overall contraceptive prevalence. That is, the more users a program serves, the more commodities are consumed, and the greater are the chances for a stockout. Zimbabwe, Botswana, and Kenya have some of the highest contraceptive prevalence rates and also among the highest stockout rates for at least one offered method. Conversely, Madagascar, Senegal, and Tanzania have low contraceptive prevalence and lower stockout rates. This correlation is also reflected in the consistently higher stockout rates of injectables, a rapidly growing method in the region. Since this method is new relative to pills and condoms, the supply mechanisms may not yet be as firmly established as those for other methods. The one counterexample is Nigeria, which has the highest overall stockout rate by far, but quite low contraceptive prevalence. However, the Nigerian family planning program serves about 23 million women, four to ten times as many as any of the other study sites. The contraceptive prevalence rate is low, but the sheer number of users is dauntingly large and may pose extraordinary distribution problems.

Lastly, stockout rates do not seem to be related to the presence of a commodity inventory (R.4-2). This is probably because stockouts often result from central distribution problems, such as incorrect amounts supplied or transportation difficulties, rather than on individual SDPs' neglecting to order stocks on time.

such as uterine sounds for IUDs, is reported only for SDPs that offer those methods. 
Table 3-1. Minimum equipment thresholds used in this analysis

\begin{tabular}{lccc} 
Equipment Type & Hospitals & $\begin{array}{c}\text { Clinics with } \\
\text { Maternity }\end{array}$ & $\begin{array}{c}\text { Health } \\
\text { Posts }\end{array}$ \\
Blood pressure machines & 3 & 1 & 1 \\
\hline Stethoscopes & 3 & 1 & 1 \\
\hline Access to sterilizer & 5 & 1 & 1 \\
\hline Adult scales & 1 & 1 & 1 \\
\hline Examination couches & 3 & 1 & 1 \\
\hline Angle poise lamps/flashlights & 3 & 1 & 1 \\
\hline Specula & 15 & 5 & 1 \\
\hline Gloves* & 100 & 30 & 5 \\
\hline Sterilization lotion (liters) & 5 & 1 & 1 \\
\hline Tenacula & 3 & 1 & 1 \\
\hline Uterine sounds $^{*}$ & 3 & 1 & 1 \\
\hline Needles and syringes & 15 & 5 & 1 \\
\hline No RPLAN ${ }^{\circledR}$ kits & 3 & 1 & 1 \\
\hline
\end{tabular}

* This item refers to the availability of sterile gloves. It includes the number of disposable pairs, plus the number of reusable pairs if a functioning sterilizer is present.

\section{R.5-1 Do SDPs have basic equipment?}

Although the basic equipment necessary for family planning provision differs widely across countries, the present analysis focuses on four very basic items: stethoscope, blood pressure machine (sphygmomanometer), adult weighing scale, and access to a means of sterilizing equipment.

Of these four items, adult weighing scales are the most available across all the study sites. Over $73 \%$ of all SDPs across all sites have such scales. Blood pressure machines and stethoscopes show a wider range of availability. Across 10 of the study sites, between $55 \%$ and $90 \%$ of SDPs have blood pressure machines, and stethoscope availability ranges between $51 \%$ and $94 \%$ of SDPs. Access to sterilizing equipment also varies greatly across all the sites (see Figure 3-5).

One additional issue is the clustering of equipment. That is, if an SDP has one item of basic equipment, does it tend also to have the other three? For all study sites with available data, correlation coefficients among these four items consistently show that blood pressure machines and stethoscopes are highly correlated. That is, if an SDP has a blood pressure machine, it probably also has a stethoscope and vice versa. Correlation coefficients for the other pairs of equipment are much lower.

\section{R.5-2 Do SDPs have equipment for pelvic exams?}

Whether to give pelvic exams to new clients as a matter of course is a controversial issue in subSaharan Africa. On the one hand, some providers argue that pelvic exams are part of a normal STD screening program and should be performed for the client's safety before a method is provided. Moreover, in many contexts, women have very few contacts with the healthcare system; thus if they come for family planning care, the argument is that these services should be integrated with overall health care. On the other hand, pelvic exams may be seen by women as overly intrusive and may present a barrier to obtaining family planning services. Also, for purely practical reasons, if an SDP does not have the capability to do pelvic exams, it 
Figure 3-5. Per cent of 5DPs with access to sterilir ing equipment

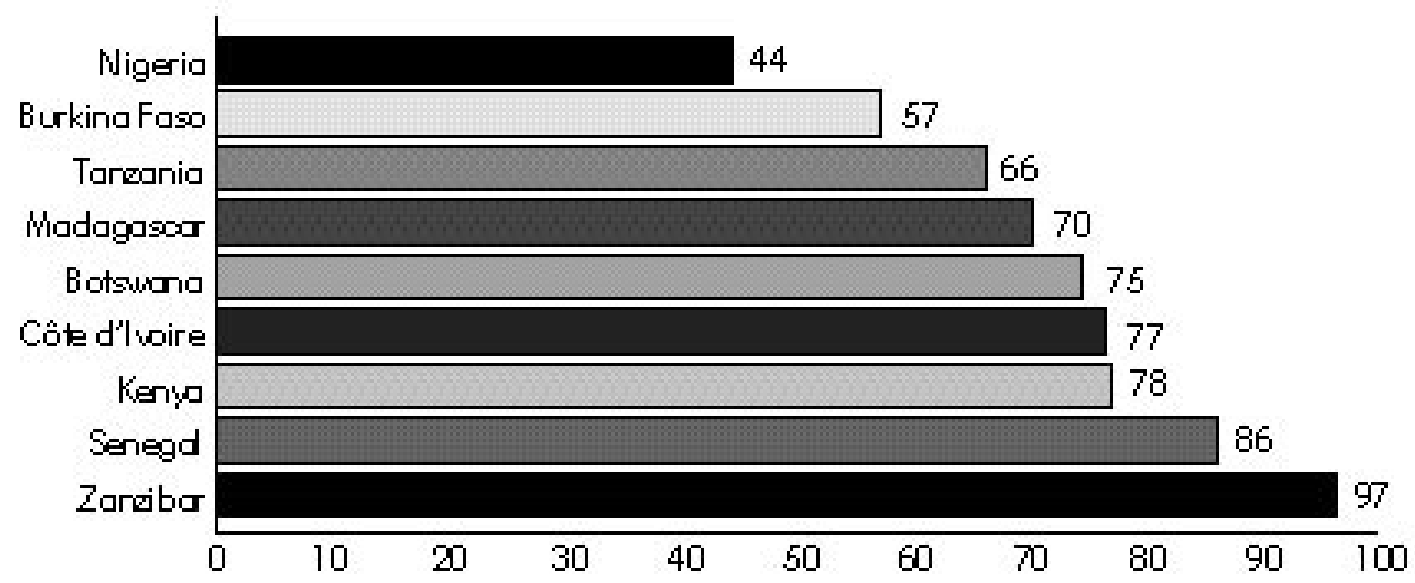

should not be prevented from dispensing certain methods, such as pills or injectables. (See Chapter 9 for further discussion of this point.)

Nonetheless, in at least some cases a pelvic exam will be indicated for particular clients. The Situation Analysis offers information on the availability of five basic items needed for providing pelvic exams: examination couch, angle poise lamp or working flashlight, specula, gloves, and sterilizing lotion. All of these items show considerable variation across the study sites. Across 9 of the sites, from about 50\% of SDPs (Tanzania) to $95 \%$ (Botswana) have examination couches, and from $18 \%$ of SDPs (Tanzania) to $85 \%$ (Côte d'Ivoire) have angle poise lamps or flashlights.

The availability of gloves is critically important. Figure 3-6 shows that in 4 of the study sites, about $45 \%$ of SDPs have sufficient gloves, and in an additional 7 sites, the proportion is $60 \%$ or higher. In Burkina Faso, glove supplies are seriously inadequate. Specula are also necessary for a pelvic exam, and availability of this equipment ranges from $38 \%$

\section{Figure 3-6. Percent of SDPs with disposdble or nondisposdble gloves}

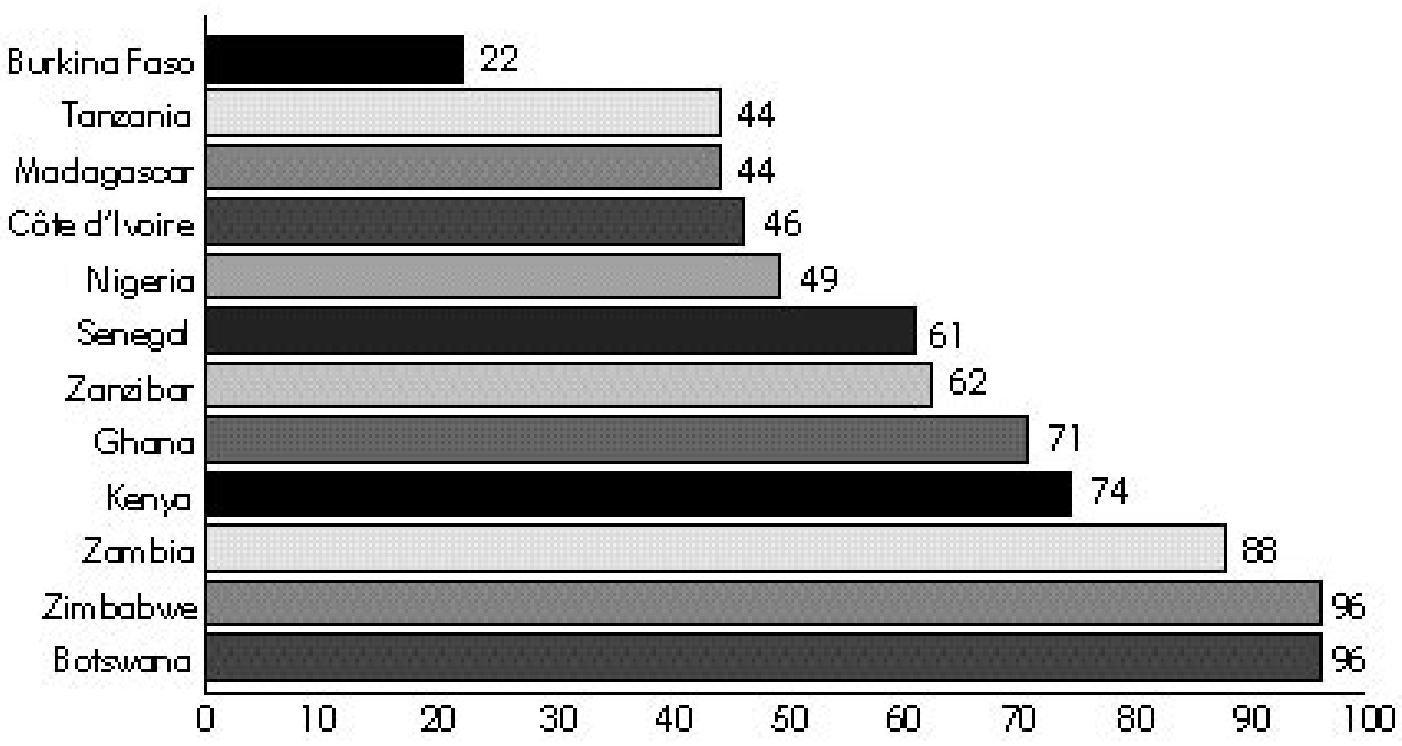

40 Clinic-Based Family Planning and Reproductive Health Services in Africa 
Figure 3-7. Average results on equipment avdilability by courtry

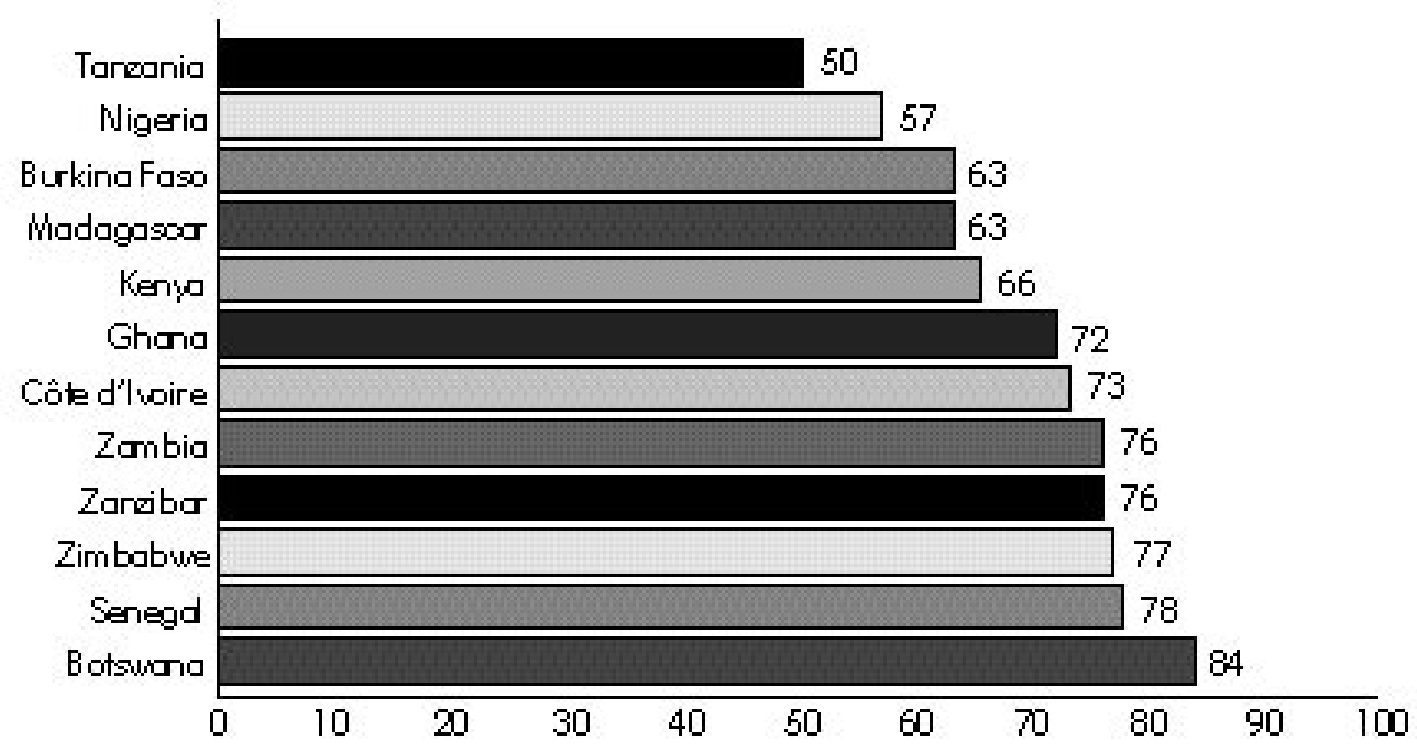

of SDPs in Kenya to 94\% in Senegal. Similarly, the availability of sterilizing lotion ranges from only $3 \%$ of SDPs (Tanzania) to $96 \%$ (Botswana).

It may be argued that if an SDP is going to perform pelvic exams, it needs these five items together. However, the presence of these items is not highly correlated. In most of the study sites, the correlation coefficients between any two items range from .10 to .30. In Côte d'Ivoire, Madagascar, and Nigeria, the correlation coefficients range up to .45 . Overall, however, the findings indicate that these items of equipment do not usually appear together. In fact, for 7 of 8 studies with complete information, fewer than $25 \%$ of all SDPs have all five items; the availability of all five items together is somewhat better in Botswana $(41 \%)$. (See Chapter 10 for further assessment of readiness for pelvic exams.)

\section{R.5-3 Do SDPs have equipment for IUD insertion?}

A tenaculum, uterine sound, gloves, and speculum are all necessary for IUD insertion. Although gloves and specula are covered above under equipment for pelvic exams (R.5-2), the discussion in this section is restricted to those SDPs that offer IUD insertion. Generally, the availability of gloves and specula among this subset of SDPs is slightly better, especially among those middling sites identified under the preceding indicator in which $40 \%$ to $70 \%$ of SDPs have the item.

Uterine sounds and tenacula are generally available at SDPs that offer IUDs. For 8 of the study sites, from $41 \%$ of SDPs (Zimbabwe) to $91 \%$ (Ghana) have tenacula, and across all 12 sites, from $49 \%$ (Zimbabwe) to $97 \%$ (Senegal) have uterine sounds.

As with pelvic exams, if an SDP offers IUD insertion, it should have all four of these items. The individual correlation coefficients show that uterine sounds and tenacula are highly correlated across most of the study sites; the correlation coefficients between these two items range around .4 and in some cases are substantially higher. In Zambia, Tanzania, and Zanzibar, all four items are highly correlated.

\section{R.5-4 Do SDPs have equipment for injections?}

Depo Provera injections require sterile needles and syringes. The availability of needles/syringes ranges from $90 \%$ in Botswana to only $22 \%$ in Burkina Faso, with a wide variety of availability at other sites.

\section{R.5-5 Do SDPs have equipment for NORPLANT ${ }^{\circledR}$} insertion?

The equipment necessary for NORPLANT ${ }^{\circledR}$ insertion is normally distributed as a kit containing the 
NORPLANT $^{\circledR}$ rods, compresses, sterile gloves, antiseptic, local anesthetic, and a scalpel. NORPLANT $^{\circledast}$ services also require the presence of a

\section{General Assessment of Equipment Availability}

In general, stethoscopes, adult weighing scales, and blood pressure machines are available at most SDPs (R.5-1). Access to sterilizing equipment is more restricted in some of the study sites, which raises concern over possible use of unsterilized instruments. Equipment for pelvic exams (R.5-2) shows a range of availability, with a lack of gloves in particular being observed in some contexts. Those SDPs that offer IUD insertion show a general availability of the necessary equipment (R.5-3), but some study sites show serious deficiencies in this regard. Moreover, the items needed for basic family planning, pelvic exams, and IUDs generally do not appear together in one SDP, although some clustering of blood pressure machines and stethoscopes and of uterine sounds and tenacula does occur.

Assessing equipment availability by country is difficult because results on all indicators are not available for all the study sites. Instead of counting pieces of equipment, one can examine the average percentage results on all equipment availability indicators to get an idea of the equipment availability by country (see Figure 3-7). ${ }^{10}$ These results, although blunt, show that the more mature launch and growth family planning programs are on average better equipped than the emergent programs. (See Chapter 2 for discussion of this terminology.) The one counterexample is Côte d'Ivoire, which had a very young program at the time of the Situation Analysis, but this study included only 13 SDPs in the capital city. These results suggest that as programs mature, their ability to distribute and maintain equipment increases, rather than their being subject to increasing loss and breakage of equipment. specifically trained provider, but this section is concerned with equipment only. In all study sites included here, NORPLANT ${ }^{\oplus}$ was being offered on a trial basis, and staff had been trained as part of a special pilot program. In many of the study sites, NORPLANT $^{\oplus}$ was available on a trial basis at only a handful of SDPs, and some studies did not ask about the availability of NORPLANT ${ }^{\circledast}$ kits at all. However, in Nigeria (1 clinic), Senegal (4 clinics), and Burkina Faso (6 clinics), all the SDPs for which data are available had NORPLANT ${ }^{\oplus}$ kits. In Kenya, 20 of 21 clinics had them, and in Madagascar 6 of 8 . In Botswana, only 2 clinics were offering NORPLANT $^{\oplus}$ and had data on kit availability: 1 had the method, and 1 did not. In general, these levels of availability are fairly high, but they cannot be taken as indicative of the availability of NORPLANT $^{\circledast}$ kits once the method has been fully integrated into more SDPs in each country.

\section{R.6 Staffing}

R.6-1 What types of staff provide family planning services?

The majority of family planning services in subSaharan Africa are provided by nurses. Across all the study sites, about three-quarters or more of SDPs had a nurse on duty on the day of the study visit, except Zanzibar, in which 38\% of SDPs had a nurse on duty. Doctors are less common in family planning settings, since most methods do not require physician training. In many countries $8 \%$ or fewer of the SDPs had a physician on duty. In 4 of the study sites-Côte d'Ivoire, Madagascar, Nigeria, and Zambia-a third or more of SDPs were found to have a physician on duty. ${ }^{11}$

\section{R.6-2 How experienced are family planning staff?}

Levels of staff experience in delivering family planning services vary among the study sites. In 7 sites, staff were asked how long they had been providing family planning services to clients. Their responses range from a mean of 3.4 years in Burkina Faso to 6.3 years in Tanzania. In 2 additional sites (Kenya and Botswana), staff were asked how long they had been providing family planning services at this particular SDP. Their responses are not quite 


\section{Figure 3-8. Percent of provider $s$ whose basic training induded family planning by whether basic th cining o ccur red before or after stat of fanily planning prog an}

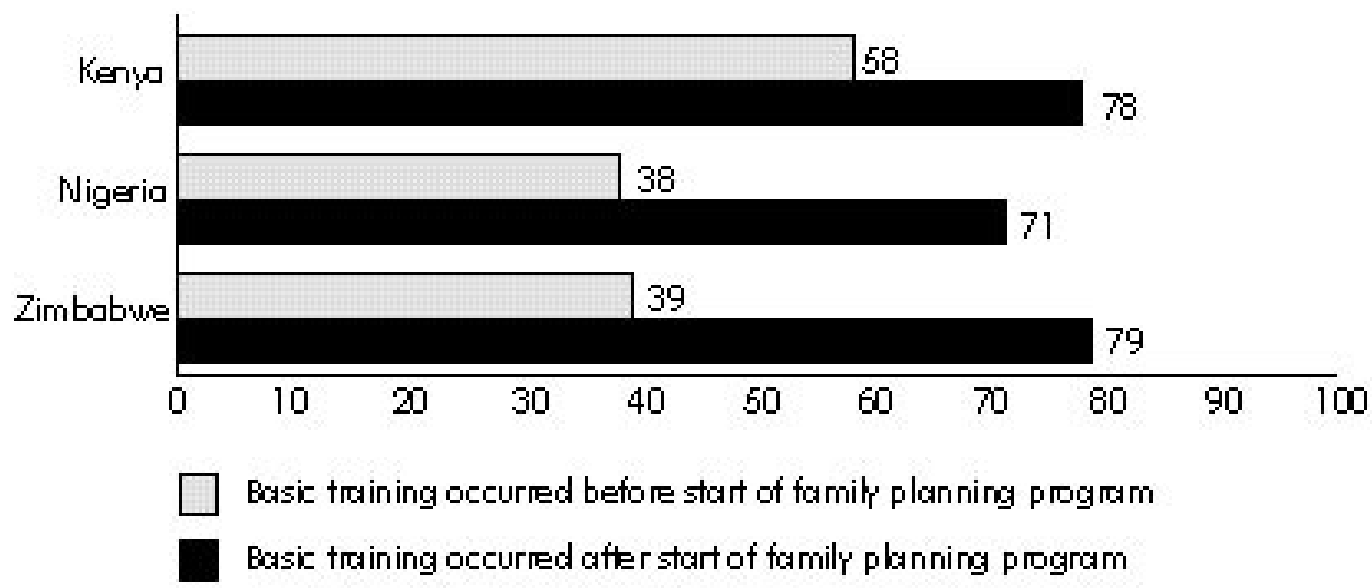

comparable with the others, since they do not include years spent providing family planning services at other SDPs. Nonetheless, these responses are fairly similar to the others: 4.4 years in Kenya and 6.6 years in Botswana.

The results on this indicator are directly related to the age of the family planning program. In Burkina Faso, Nigeria, Senegal, and Zanzibar, very few providers reported offering family planning services for longer than the family planning program itself had been in place. However, $27 \%$ of providers in Madagascar and 15\% in Kenya reported offering family planning services before the major emphasis had been placed on such services. These results indicate the prevalence of family planning activities in those countries-for example, through International Planned Parenthood Federation (IPPF) affiliates-before major attention was paid by the respective governments. This correlation with age is probably the reason providers in Botswana reported the longest period of providing family planning services; the family planning program there is the oldest among the 12 study sites, so providers have had the greatest opportunity to offer these services for the longest period of time.

R.6-3 Did providers' basic training include family planning, or have they ever had training in clinical family planning?

Every family planning provider should have been trained at least once in basic clinical family planning skills. In 9 of the study sites, providers were asked whether their basic training had included family planning, and in the remaining 3 sites, providers were asked whether they had ever had general clinical family planning training. Because these questions were asked somewhat differently, the two groups are not directly comparable, so they are addressed separately here.

In the first group (9 sites), the proportion of providers whose basic training had included family planning ranged from over $90 \%$ in Ghana and Botswana to only $41 \%$ in Côte d'Ivoire. These results are probably strongly affected by the start date of family planning services in each country. Figure 3-8 presents for 3 of the 9 countries with appropriate data the percentage of providers whose basic training had included family planning, by whether the training took place before or after the start of the family planning program. This graph shows that once family planning services had started in earnest, the vast majority of providers were being trained. Yet the problem remains of the many providers whose basic training did not include family planning, but who are offering these services, although they may have been trained in family planning outside of basic training. For 6 of these 9 countries, the next indicator (R.6-4) addresses whether these providers had received post-basic or refresher training in family planning. 
In the remaining 3 study sites (Madagascar, Senegal, and Burkina Faso), providers were asked whether they had ever had general clinical family planning training, either basic, post-basic, or refresher training. These results are in the same range as those for the other 9 sites: $40 \%$ in Madagascar, 62\% in Senegal, and 68\% in Burkina Faso. Once again, the results relate to the date of the start of family planning services. In all 3 of these countries, over $85 \%$ of the providers who had had this training took the course after the family planning program had officially begun. The large proportion of providers who have not been trained in family planning in any courses yet are providing these services reveals significant training needs in these 3 countries.

\section{R.6-4 Have providers had refresher training in family planning?}

In 6 of the study sites, providers were further asked whether they had had refresher training in family planning. In 5 countries, the results range from $74 \%$ (Ghana) to $54 \%$ (Zambia). In Côte d'Ivoire, only $6 \%$ of providers had had refresher training, which is understandable given that the family planning program was only a year old at the time of the study.

The previous indicator (R.6-3) gave the percentage of providers who had been trained in family planning during their basic training or had received general clinical family planning training. In many cases in which providers' basic training had occurred before the start of the family planning program, that training did not cover family planning. However, the information from the present indicator can be added to those results to determine the percentage of providers who had covered family planning in either basic or refresher training. ${ }^{12}$ The results show that the vast majority of providers had been trained at some point in family planning in Zambia (92\%), Botswana (98\%), Kenya (87\%), Zimbabwe (94\%), and Ghana (98\%). Again, the result in Côte d'Ivoire is much lower (41\%) because of the very recent start of family planning activities.

\section{R.6-5 Do family planning staff report that they also provide STD/HIV services?}

One measure of integration of family planning and STD/HIV services is whether staff provide both services themselves. In 5 of the study sites, staff providing family planning were asked whether they also provide STD or HIV management at this SDP. In Zambia and Zimbabwe, over $90 \%$ of providers reported that they do provide some form of HIV counseling or testing or other STD counseling, diagnosis, or treatment. In Ghana and Botswana, about two-thirds of providers offer similar services, and in Kenya only $18 \%$ reported doing so. However, observations with clients show that most providers do not discuss these issues during family planning counseling (see Q.2-7, Q.2-8, Q.4-3, and Q.4-4). (See Chapter 10 for more information on integration of STD/HIV and family planning services.)

\section{R.6-6 Have providers had basic or refresher training in STD/HIV, and how recently?}

Given the prevalence of STDs and particularly HIV in sub-Saharan Africa, staff training in these areas is particularly critical. Unfortunately, information on STD/HIV training among staff is available for only 5 of the study sites, ${ }^{13}$ and these are mainly countries that are placing extra emphasis on their STD/HIV programs. For this reason, these results are probably not representative of the area, but are higher than average.

STD or HIV management had been covered in the basic training of $49 \%$ of providers in Kenya, $66 \%$ in Botswana, and $70 \%$ in Zambia. When these figures are revised to reflect those providers who had had either basic or refresher training in STDs or HIV, the results rise to $54 \%$ in Kenya, $77 \%$ in Zambia, and fully $84 \%$ in Botswana.

The Ghana and Zimbabwe studies asked only whether a provider had had refresher training in STDs or HIV, but not basic training. Affirmative responses were given by $34 \%$ of providers in Ghana and $48 \%$ in Zimbabwe. However, it is difficult to assess the true level of staff training in these countries because of the lack of information about basic training.

\section{R.6-7 Have providers had training in family planning counseling/information, education, and communication (IEC), and how recently?}

This indicator includes training in "family planning counseling" or "IEC skills" as refresher or post-basic training. The questionnaires differ on 


\section{Figure 3-9. Per cent of pr ovider $s$ who had IEC skils tr cining}

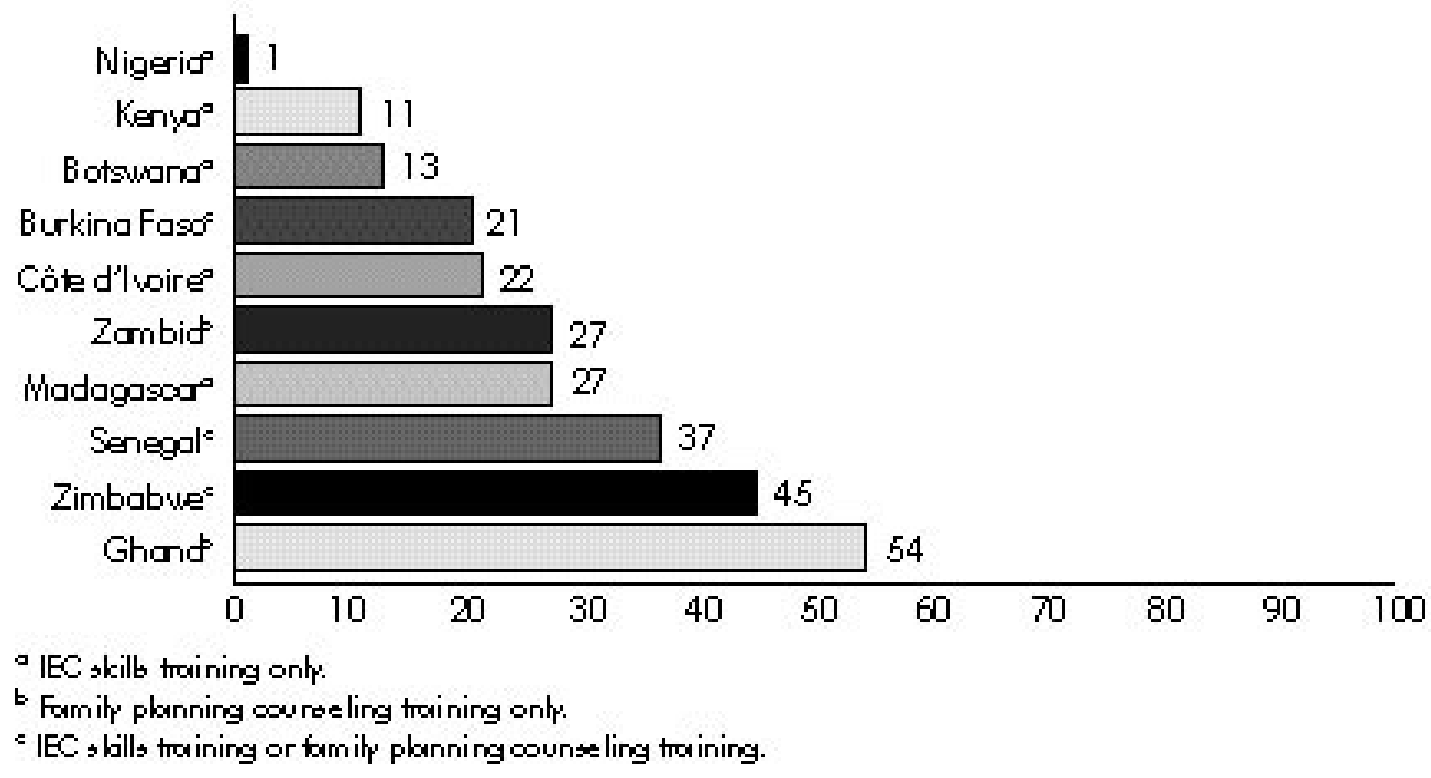

this training question. Some ask about IEC skills training alone, some about family planning counseling training alone, and some about both. The results presented here combine all the available information for each country, and they are not encouraging (see Figure 3-9). In 7 of 10 study sites, about $25 \%$ or less of staff had received any such training. In Senegal, Zimbabwe, and Ghana, the proportions are somewhat higher, but $50 \%$ or more of providers still had had no IEC training.

In 7 of the study sites, providers reported the date of their most recent training in family planning counseling/IEC. The dates reported are generally quite recent, ranging from 2.4 years ago in Zambia and Madagascar to 5.3 years ago in Kenya. In Zambia, among providers who had had this training, 65\% had received it less than 1 year prior to the study. As with clinical family planning training, in most cases the recency of counseling/IEC training is related to the age of the family planning program itself. However, in Senegal and Zimbabwe, the family planning programs are quite mature, but counseling/IEC training has occurred more recently. In Burkina Faso, fully $90 \%$ of the staff who had had such training received it within 3 years prior to the study, although the family planning program had been in place for 11 years. Thus counseling/IEC training is a more recent addition to clinical family planning training in some programs.

\section{R.6-8 Have providers had training in IUD insertion/removal, and how recently?}

The proportion of all providers who had had this type of training varies greatly-from only $7 \%$ in Madagascar to over $80 \%$ in Zanzibar. The recency of IUD training ranges from 2.4 years in Zambia to 7 years in Kenya. In fact, in Zambia the training was extremely close in time to the Situation Analysis fieldwork: over 60\% of IUD-trained staff reported that they had received their training within the previous year.

Because IUD services are not offered at all SDPs, these results may be more informative if calculated only for providers at those SDPs that offer such services. ${ }^{14}$ Interestingly, the results for this subset of providers are not very different. In most cases, the staff trained in IUD insertion/removal are not concentrated in SDPs that actually offer IUD services. The one exception is Zimbabwe, where 18\% of all staff had been trained in IUD insertion/ removal, but fully $50 \%$ of staff at those SDPs that offer IUDs had been so trained. Thus staff are concentrated in SDPs where their skills can be put to use. However, 
according to the Demographic and Health Survey (DHS), IUDs account for only $2 \%$ of the method mix in Zimbabwe, and this efficient distribution of staff would be more useful in places such as Botswana, Burkina Faso, and Senegal where IUDs comprise a much higher proportion of the method mix.

The results on mean years since IUD training among staff working at SDPs that offer IUDs do not differ greatly from the results among the general population of staff. Again, the one exception is Zimbabwe: mean years since IUD training among all staff is 5.7, whereas the figure among staff at SDPs that offer IUDs is 8.2 years. Thus the staff that have been trained more recently are not necessarily being placed at SDPs that can utilize their skills; rather, the staff trained less recently are those concentrated in the SDPs that offer IUD services.

\section{R.6-9 Have providers had surgical training in tubal ligation, and how recently?}

In some of the study countries, only certain types of staff are permitted to have sterilization training; in several countries, however, community health nurses and even nurses' aides have been trained in tubal ligation. For this reason, results for this indicator are not restricted to a particular type of staff, such as physicians or high-level nurses.

The results show that very few providers have had this type of training: $10 \%$ or fewer in 7 of 9 study sites. The percentages are a bit higher in Ghana (14\%) and Zimbabwe (26\%). However, as discussed above, only a very small percentage of SDPs offer tubal ligation, with the exception of Kenya, Nigeria, and Ghana, where the procedure is more available. It is worth noting that tubal ligation comprises over 10\% of the mix in Tanzania and Zambia and almost $20 \%$ of the mix in Kenya and Madagascar according to DHS data.

This indicator may be more informative if restricted to providers working at those SDPs that actually offer sterilization. The results for Botswana, Senegal, Burkina Faso, and Zambia are not encouraging. In Botswana, the sample included only 2 providers who had been so trained, in Senegal 8, and in Burkina Faso 35. In all 3 of these sites, all the trained providers were interviewed at SDPs that do not offer tubal ligation. In Zambia, only 1 of 22 providers thus trained worked at an
SDP that offers sterilization. ${ }^{15}$ In short, those SDPs that do offer tubal ligation were without staff trained in performing the procedure. Before concluding that the staff are poorly distributed, however, it is important to note that the prevalence of training in tubal ligation is quite low, and the Situation Analysis sample in these countries may not represent this subpopulation well. In other words, these results may be due to small sample sizes. In addition, the fact that no providers trained in tubal ligation were interviewed at SDPs offering the method in Botswana, Senegal, and Burkina Faso does not necessarily imply that untrained providers are performing sterilizations at these SDPs. Providers trained in tubal ligation who work at these SDPs may simply not have been present on the day of the Situation Analysis data collection.

The other 5 countries have complete data, the n's are larger, and the results are more credible. In Kenya and Ghana, the percentage of trained staff among those working at SDPs offering tubal ligation does not differ much from the percentage among all staff. As with the previous indicator on IUDs (R.6-8), this finding may point to trained staff not being concentrated at SDPs that can use their skills.

On the other hand, the proportion of trained staff at SDPs that offer tubal ligation is $27 \%$ in Madagascar and $64 \%$ in Zimbabwe. In both cases, these figures are much higher than the proportions among all staff, indicating that staff trained in tubal ligation are concentrated in those SDPs that offer this service.

The mean years since training in tubal ligation among all staff ranges from 1.7 in Zambia to 5.7 in Kenya. As with the IUD training results, training in tubal ligation in Zambia seems to have taken place quite recently. Again, over $60 \%$ of trained providers in Zambia reported that they had been trained within the year. For all studies, these means are not significantly different among staff working at SDPs that offer sterilization.

\section{R.7 IEC Materials and Activities}

\section{R.7-1 Do SDPs have IEC materials on family planning?}

IEC materials are excellent aids to communication with clients, and many family planning programs 


\section{General Assessment of Staffing}

The indicators under this element generate an overall picture of staff training that is in some ways quite appropriate. N urses provide most of the services (R.6-1), and have a suitable level of training for most family planning methods. Training in clinical family planning has generally been integrated into basic training since the start of family planning services (R.6-3), and IEC training has been on the rise in recent years in most of the study sites (R.6-7). In a few countries, the majority of family planning providers have also had training in STD/HIV services (R.6-6).

$\mathrm{O} n$ the other hand, the presence of even one provider with no training in clinical family planning is of concern to any program. Many of the providers who have not had family planning training appear to have been through basic training prior to the launch of or reemphasis on family planning services, so their lack of training is only a historical accident. However, this does not mitigate the fact that they are providing services without formal training. Moreover, the relatively low prevalence of training in IEC/family planning counseling (R.6-7) may have a detrimental effect on services: counseling indicators covered below under "information exchange" (Q .2 and Q.4) show that providers with IEC training may perform better on those elements.

The distribution of specially trained staff may also pose a problem in some contexts. In most of the study sites, staff that have been trained in IUD insertion and removal (R.6-8) are not placed at SDPs that offer this method. Similarly, staff trained in tubal ligation (R.6-9) do not seem to be clustered at SDPs where this method is provided, although this result may be due to an extremely small number of cases in the Situation Analysis data.

Most Situation Analysis studies do not ask about STD/HIV training (R.6-6), although family planning providers do offer these services in some contexts. In the 5 study sites with information on STD/HIV training, the training levels are fairly high in Zambia and Botswana, but lower in Kenya, Ghana, and Zimbabwe. Again, the prevalence of STDs and HIV in most of the study sites calls for giving STD/HIV training to all family planning providers.

include the procurement and distribution of these materials. The present analysis includes three types of IEC materials on family planning: posters, brochures, and flip charts. Posters can be used for reference during counseling, brochures are often given to clients to read at home, and flip charts can be used interactively with clients to describe how various methods work or to address other reproductive health issues. The indicators under this element measure whether these materials are available at SDPs.

In general, IEC materials on family planning are not widely available. Posters are by far the most prevalent, appearing in over half of SDPs in 10 of the 12 study sites. Brochures on family planning are largely available in Nigeria and Côte d'Ivoire, but are available in $60 \%$ or fewer of SDPs in 9 other study sites. In Zambia and Tanzania, they appear in fewer than $10 \%$ of clinics. Flip charts are the least available: in 8 of the 10 study sites they are available in fewer than half of the SDPs.

Moreover, these items are not usually clustered at one SDP. In all the studies, the correlation coefficient for each pair of items is about .3 or well below. The only exception is a strong correlation between the presence of flip charts and brochures in Madagascar and Nigeria.

\section{R.7-2 Do SDPs have IEC materials on STDs or HIV?}

Unfortunately, IEC materials on STDs or HIV are even less common than family planning materials. Again, the most available items are posters. Among 5 of the study sites, a range of $46 \%$ (Zambia) to $66 \%$ (Botswana) of SDPs have posters about HIV on the wall, while only 19\% (Ghana) to $43 \%$ (Botswana) have posters on STDs. 


\section{Figure 3-10. Per cent of health talks that induded information on family planring HWv, or other STDs (countries with data on dl three types)}

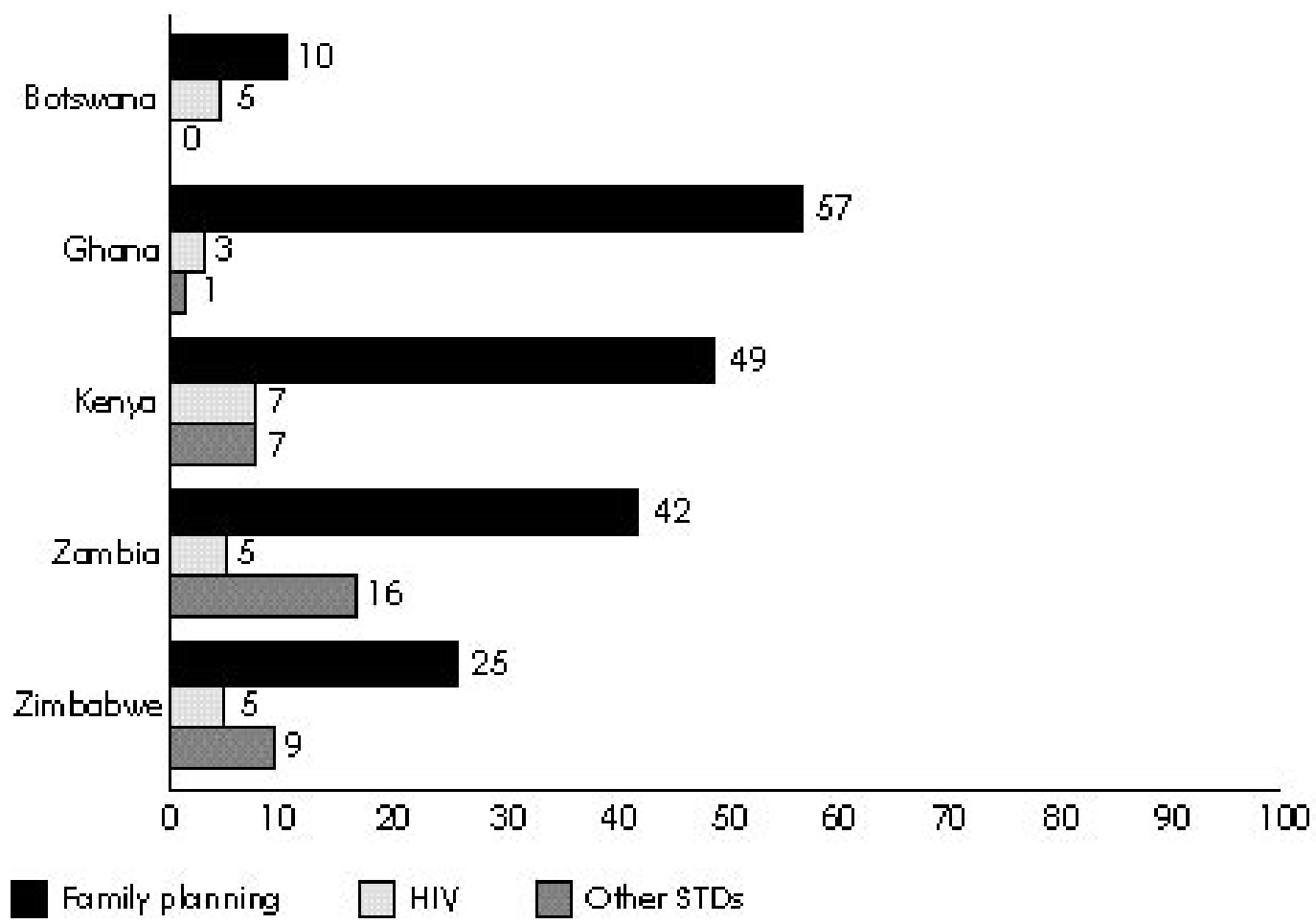

In Botswana, $45 \%$ of SDPs have a brochure on STDs and $63 \%$ a brochure on HIV, but the results in other countries are not as encouraging. Fewer than $25 \%$ of SDPs in 4 other study sites have a brochure on STDs or HIV. And no study found more than $18 \%$ of SDPs in possession of a flip chart on STDs or HIV.
In each country, three-quarters or more of the SDPs that have any of these six IEC items on STDs or HIV have only one or two; thus in large part the items do not cluster. This effect is somewhat reduced in Botswana, where a greater proportion of SDPs tend to have more than one or two items.

\section{General Assessment of IEC Materials and Activities}

IEC materials on family planning (R.7-1) are widely available in a few of the study sites, but are rather uncommon in most; IEC materials on STDs or HIV (R.7-2) are extremely uncommon in almost all study sites. Botswana has markedly higher availability of all six IEC items, reflecting a particular emphasis on IEC and STDs/HIV in that program. Health talks generally cover family planning more often than they cover STDs or HIV (R. 7-3), but these results are still not encouraging in most study sites.

The lack of IEC materials at the SDP level can have several causes. It may be that the family planning program simply has not generated these materials, or it may be that all the consumable IEC materials, such as brochures, have been used up. Commonly, however, these materials have been produced centrally, but the distribution system is faulty. In these cases, the IEC materials may be centrally located, simply waiting for distribution. The Botswana Situation Analysis report describes exactly this situation. 
R.7-3 Do group health talks at SDPs address family planning, STDs, or HIV?

This indicator is calculated for those SDPs that gave a group health talk on the day of the study visit. For family planning, the results range enormously. In Senegal, $100 \%$ of the health talks witnessed by the study teams included information on family planning, but in Botswana only $10 \%$ did so. Figure 3-10 shows that inclusion of information on STDs or HIV is extremely uncommon: $16 \%$ or fewer of health talks included information on STDs, and $7 \%$ or fewer addressed HIV.

\section{R.8 Recordkeeping and Supervision}

\section{R.8-1 Do SDPs have client record cards in good condition?}

A usable client record card system is essential to a family planning program, since it allows a client's medical history, clinical information, and family planning experience to be available to providers at every visit. This in turn enables better continuity of care.

In most of the study sites, client records are kept in good condition. "Good condition" is defined as well-ordered enough to be usable, ${ }^{16}$ and is restricted to those SDPs that keep client records on site rather than leaving them with clients. In 9 of the 11 sites for which information on this indicator is available, two-thirds or more of the SDPs keep their cards in such condition. In Zimbabwe, the figure is 55\% of SDPs and in Tanzania only $29 \%$. With a few exceptions, client record cards appear to be well maintained. However, this finding does not indicate whether the information on the cards is accurate or complete, but only whether the recordkeeping system is working.

\section{R.8-2 How often are SDPs supervised?}

Supervision is critical to any family planning program, but it is difficult to measure meaningfully. Various Situation Analysis studies approach the question differently; the simplest and most comparable measure that can be included here is whether each SDP had had at least one visit from a supervisor, for the purpose of supervision, in the 6 months preceding the study. Even using this blunt level of measurement, there are some visible difficulties with supervision.

The results fall into roughly three groups. In Botswana, Tanzania, Zanzibar, and Zimbabwe, over $93 \%$ of SDPs had received a supervisory visit in the previous 6 months, so the amount of supervision in these sites may meet some minimum level. ${ }^{17}$ In Zambia, Kenya, and Ghana, 78\% to $79 \%$ of SDPs had received a visit. This is still a relatively high percentage, but it means that about $20 \%$ of SDPs had not been supervised over a 6month period, which could have serious consequences for service quality. Lastly, only $48 \%$ of SDPs had received a supervisory visit in Senegal and only $22 \%$ in Burkina Faso. In both of these countries, supervision systems require serious attention.

\section{QUALITY OF SERVICES}

\section{Q.1 Interpersonal Relations}

Interpersonal relations is one of the most difficult elements to measure because it is so subjective. The two sources of information for this element are (1) observation of the client-provider interaction, in which an observer notes the provider's interpersonal treatment of the client, and (2) the exit interview, in which the client herself reports on how she was treated. Both of these approaches have methodological difficulties.

During the interaction, observers may be asked to judge subjective events, such as whether a greeting is "friendly" or not. In the training of observers, a clear definition of "friendly greeting" is agreed upon, using local customs and norms as guides. But this concept still may be measured differently by different providers, making this sort of data collection less reliable than might be desired.

On the other hand, reports from clients themselves are subject to a strong "courtesy bias," whereby clients understandably do not want to appear rude or ungrateful for the services they have just received. For this reason, reports of satisfaction from clients normally are universally high 
and show little variation. This effect is eliminated to some extent if the questions are very specific, or if they do not relate to the provider herself but to other aspects of the experience, such as waiting time or cost.

For the above reasons, the Situation Analysis is not particularly strong on measuring this element, which might be measured more satisfactorily with qualitative methods removed from the clinical environment. Nonetheless, four indicators of interpersonal relations are presented here, although they should be interpreted in light of the methodological difficulties involved.

\section{Q.1-1 Are clients greeted in a friendly manner?}

A friendly greeting helps put clients at ease. Information on this indicator was collected during the observation of client-provider interactions, using a definition of "friendly greeting" relative to each context, as discussed above. The results are almost universally high: in 9 of 10 sites, the percentage of positive findings on this indicator is $80 \%$ or higher; in Côte d'Ivoire, the percentage is lower $(66 \%)$.

\section{Q.1-2 Do clients ask questions and get} satisfactory responses?

An important part of interpersonal relations is making clients feel comfortable enough to ask questions. Once asked, the questions should be treated respectfully and answered in full. In the exit interview, clients were asked whether they had posed a question and if so, whether it had been answered satisfactorily. However, not all clients may have a question, so this indicator is not calculated for all clients. Instead, it is assumed that all new clients, returning clients with a problem, returning clients who wanted to switch methods, and returning clients who wanted to stop using could all reasonably be expected to have at least one question. These groups of clients form the denominator for this indicator.

The results for this indicator fall roughly into two groups. In Madagascar, Senegal, Botswana, and Kenya, $70 \%$ or more of clients had asked a question. In 5 other sites, only $42 \%$ to $49 \%$ of clients had done so. These results suggest there is room for improvement in making clients comfortable enough to ask questions and encouraging them to do so.

\section{Figure 3-11. Inf or ming clients dur ing pelvic exams}

Percent of pelyic exams in which the client yose informed about the prooedure beforehand.

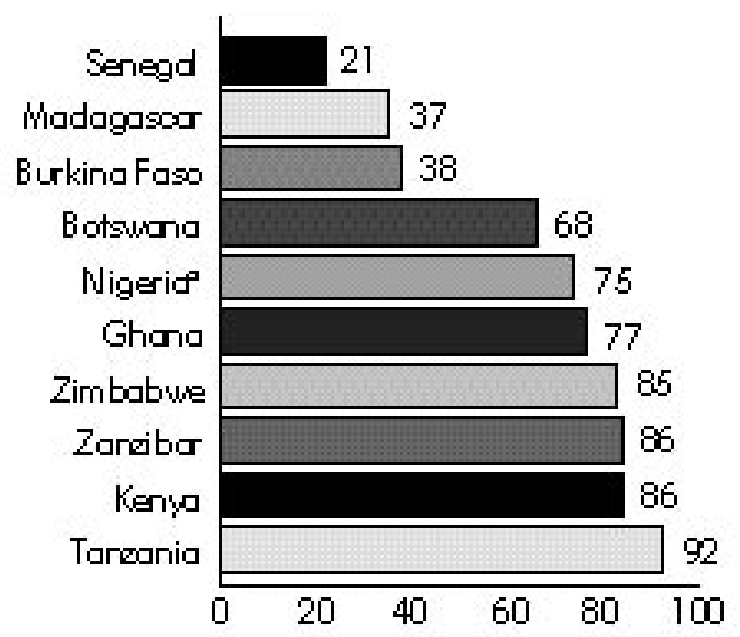

- Include petyic exams on new' clients ontp.
Perent of pelvic exams in which the client uncs told the results afteryand.

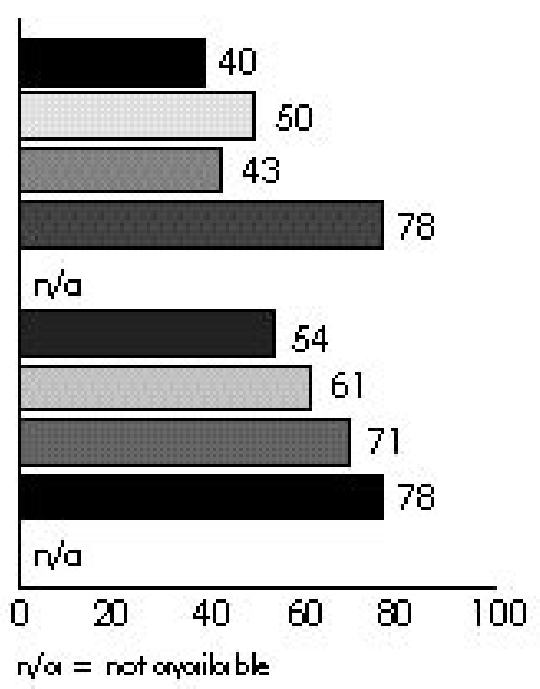


Figure 3-12. Percent of new Family planring dients asked their reproductive intentions

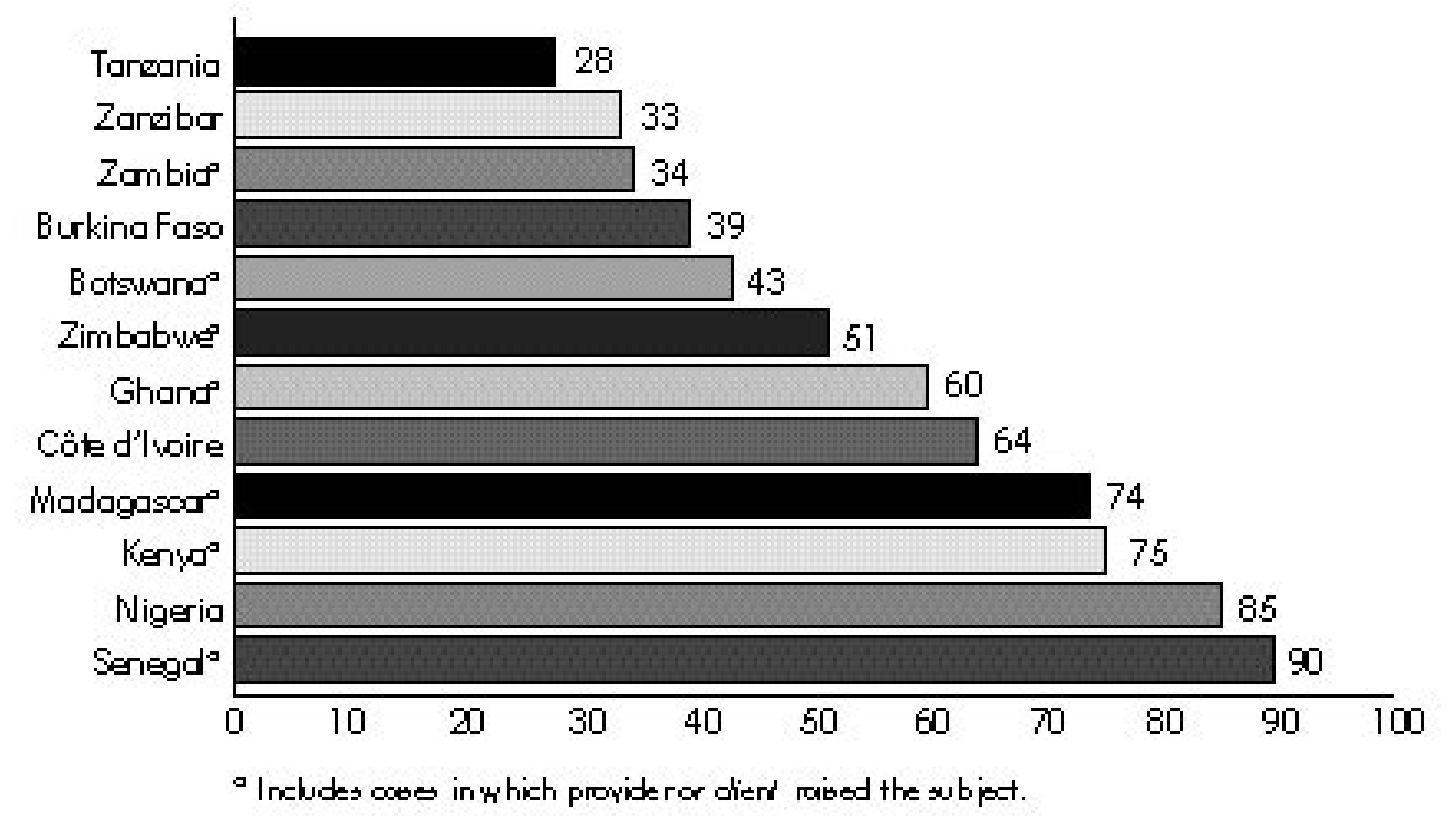

Among the clients who had asked questions, over $90 \%$ were satisfied with the responses across all study sites. However, the fact that this result is so consistently high is probably due to the courtesy bias discussed above.

\section{Q.1-3 Do clients find providers easy to understand?}

If providers use language that is too complicated for clients, the clients will not feel at ease and may even decide against adopting or continuing family planning. In exit interviews, clients were asked whether they had found the provider easy to understand. In 11 of the study sites, $96 \%$ or more of clients answered this question positively. This finding does not necessarily reflect clients' actual understanding of providers, however, because this question is also subject to the courtesy bias discussed above.

\section{Q.1-4 During pelvic exams, are clients informed} about the procedure beforehand and told the results afterward?

In many contexts, pelvic exams may be frightening, invasive, or simply uncomfortable for clients. For this reason, if a pelvic exam is carried out on a client, it is particularly critical that the provider ease her concerns by explaining the procedure beforehand and telling her of the outcome afterward.

The results on this indicator show enormous variation (see Figure 3-11). Positive responses on informing clients beforehand range from $21 \%$ (Senegal) to $92 \%$ (Tanzania) and on giving clients results afterward from $40 \%$ (Senegal) to $78 \%$ (Kenya and Botswana). A smaller proportion of clients had been both informed about the exam beforehand and told about the outcome: in Zanzibar and Kenya about 75\%; in Botswana, Ghana, and Zimbabwe about 50\%; and in Burkina Faso, Madagascar, and Senegal 25\% or fewer. (See also the discussion of pelvic exam quality under Q.5-2.)

\section{Q.1-5 During IUD insertion, are clients given moral support?}

As with pelvic exams, IUD insertion may be psychologically troubling for clients who may not be used to invasive procedures. For this reason, moral support should be offered during the course of the procedure to reassure clients. Again, 


\section{General Assessment of Interpersonal Relations}

Measurement of interpersonal relations is hampered by methodological difficulties. For example, the results on whether providers are easy to understand are not entirely informative since they do not vary much and are subject to a strong courtesy bias. $O f$ the indicators included under this element, those on pelvic exams and IUD insertion (Q.1-4 and Q .1-5) appear to be the most informative, although the IUD information suffers from very small sample sizes on the individual country level. These indicators are examined more closely here.

The results on informing clients during pelvic exams do not seem to vary by the maturity of the family planning program, but they do seem to differ between anglophone and francophone countries. The 3 francophone countries for which data on this indicator are available-Senegal, Madagascar, and Burkina Faso-do appear to have consistently lower results than those for the other countries, which are all anglophone. Since interpersonal relations are plausibly influenced by cultural factors, this finding may point to cultural differences between the two groups of countries, such as the degree of hierarchical relations between professional staff and lower-status clients. This finding generally holds for giving moral support during IUD insertion as well, although not as clearly. The countries do fall into francophone and anglophone groups, but the difference between the groups is not as large, and Ghana has the lowest percentage of all.

Interestingly, provider training (R.6) does not appear to be strongly related to these two indicators. In 5 of 6 countries for which the data can be linked, provider training in neither clinical family planning nor IEC/counseling had any effect on whether the provider informed the client before and after pelvic exams. The only counterexample is Kenya, where providers with training in family planning counseling are significantly more likely to inform clients about pelvic exams $(p<.05)$. O verall, however, the connection is not strong. (See Q .5-2 for information on training and the technical quality of pelvic exams.)

Findings on the relationship between training and giving moral support to clients during IUD insertion are similar. In several of the study countries, IUD insertions had been performed by providers who reported in the staff interview that they had never received training in IUD insertion and removal- $a$ finding that is in itself of great concern. Comparison of this untrained group with those trained in the procedure shows that, as with the pelvic exam results, providers trained in IUD insertion are not more likely to give moral support. The only counterexample is Ghana, in which IUD training is positively correlated with giving moral support $(p<.05)$. Similarly, training in IEC/counseling is not related to giving moral support during IUD insertion. (See Q.5-3 for information on IUD training and the technical quality of IUD insertions.)

Although the evidence for this element is scant and may be affected by methodological difficulties, collectively it suggests that interpersonal relations are influenced by cultural factors. Traditional training interventions may not substantially improve this element of quality, possibly because they do not directly address these issues. Further research is needed to develop more valid indicators of this element of service quality.

the proportion of positive results on this indicator varies widely, from only $17 \%$ in Madagascar to $100 \%$ in Botswana and Zimbabwe. However, the sample sizes on these insertions can be quite low, so these results may not be representative of all IUD insertions. (See also the discussion of IUD insertion quality under Q.5-3.)

\section{Q.2 Information Exchange: Information Taken from Clients}

\section{Q.2-1 Are new clients asked their reproductive intentions?}

A client's reproductive intentions are among the most important information that must be 
ascertained before a method can be provided. If a client does not want any more children, she should receive more information about long-term methods, whereas if she does want more children, she should receive information about a method that will allow her to space births appropriately. Results on this indicator vary greatly, from $28 \%$ in Tanzania to $90 \%$ in Senegal (see Figure 3-12).

\section{Q.2-2 Are new clients asked their breastfeeding status?}

Breastfeeding status is also among the most important information to be gathered from a client. COCs are not appropriate for breastfeeding women because estrogen can affect the quality and quantity of breastmilk (Hatcher et al., 1994). Instead, breastfeeding clients who want to use orals should be provided with a progestin-only method, such as POPs or injectables. Breastfeeding rates among clients in the study sites are probably fairly high, given the relatively high fertility and the traditionally prolonged period of breastfeeding (18 to 23 months) in many sub-Saharan African contexts (Jolly and Gribble, 1993).

Results on this indicator vary from $28 \%$ in Ghana to $79 \%$ in Senegal..$^{18}$ It may be argued that providers may not be inquiring in many cases because the client is an injectable or POP user, and thus her breastfeeding status is not as important. However, this indicator was also calculated among only those clients who had accepted COCs by the end of their consultations. The results are similar, ranging from $30 \%$ in Botswana to $94 \%$ in Nigeria. Thus with a few exceptions, a substantial proportion of COC clients are not being asked their breastfeeding status.

\section{Q.2-3 Are new clients asked their method preference?}

Client method preference is important because clients have been found to continue longer on a preferred method (Pariani et al., 1987). However, a client should not automatically receive her preferred method because it may not address her spacing/limiting needs properly, or may be contraindicated. Rather, asking a new client's preference is a starting point for a family planning coun- seling session. The positive results on this indicator are moderate, ranging from $42 \%$ in Burkina Faso to $81 \%$ in Senegal.

\section{Q.2-4 Are new clients asked about previous method use?}

A client who has previously used a method probably already knows what she likes and dislikes about that method. This existing knowledge is extremely useful to the provider in prescribing an appropriate method. Again, positive results on this indicator vary widely, from only 19\% in Zanzibar to over $80 \%$ in Zimbabwe.

\section{Q.2-5 Are revisit clients asked whether they had problems with their method?}

Most indicators of information exchange refer to new clients only, because these items should clearly be addressed in initial counseling sessions. On the other hand, problems with their method is one of the few issues that should clearly be addressed with all revisit clients. As with the other indicators under this element, results vary widely, from $37 \%$ in Zanzibar to $84 \%$ in Kenya.

\section{Q.2-6 Are new clients asked whether they discuss family planning with their partner?}

A client's relationship with her sexual partner has ramifications for the method she should adopt. If her partner is unaware or unsupportive of her use of family planning, she may want to select a method that is not visible, such as the injectable rather than the pill. The positive results on this indicator are generally lower than for the other indicators under this element: 8 of 9 studies found that about half or fewer clients had been asked whether they discuss family planning with their partners. The exception is Ghana, in which $69 \%$ of clients had been asked.

\section{Q.2-7 Are clients asked about their sexual relations?}

The nature, frequency, and timing of a client's sexual relations should also affect her method choice. In addition, this information gives providers an idea of exposure to STD risk, which may affect the method provided as well. 
Figure 3-13. Percent of family planring dients asked about unvsud bleeding

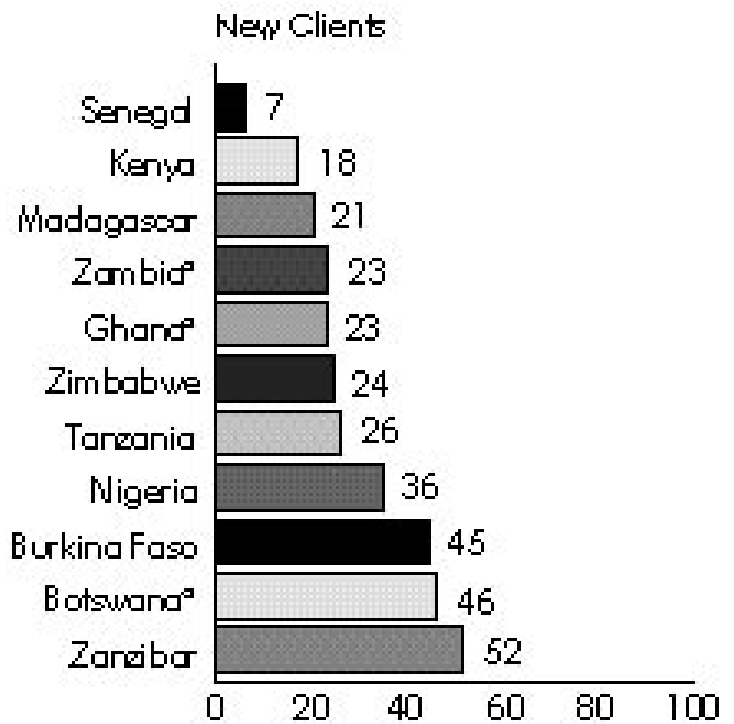

- Inelude coeses in which proyide ror dient ruised the subject.

Reyisit Clients

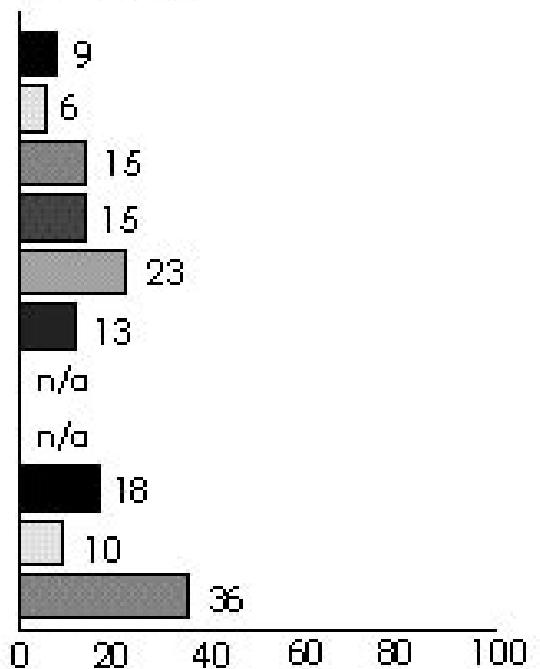

ror $=$ not oncribable
This indicator addresses both new and revisit family planning clients. Among new clients, the results are quite low, ranging from $33 \%$ (Kenya) to only $5 \%$ (Zambia) for 8 of 9 study sites. In Zanzibar,
$59 \%$ of new clients had been asked about this issue. For revisit clients the results are even lower: fewer than $10 \%$ of revisit clients had been asked this question in 6 of 7 countries, and only 13\% in the

\section{Figure 3-14. Percent of family planring dients asked about unvsud discharge}
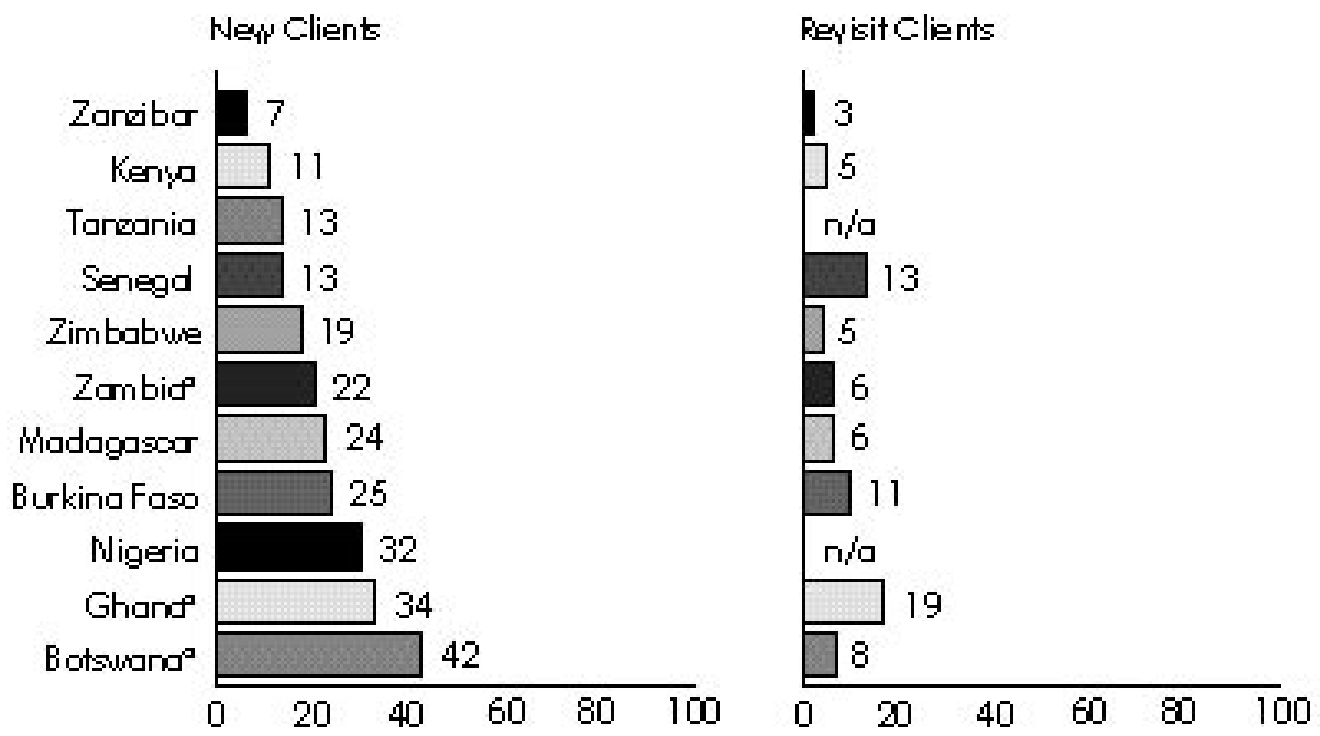

anclude coeses in which proyide ror dient rubed the subject.

$r a=$ not ancilable 
seventh (Kenya). (See Chapter 6 for more information on this point.)

\section{Q.2-8 Are clients asked about STD symptoms?}

Since family planning clients are sexually active, they are at risk for STDs and should be screened. The exact screening questions that should be used are a subject of controversy, since not all symptoms are indicative of STD infection in particular, rather than other conditions. Moreover, a client's judgment about what constitutes unusual symptoms or even pain can vary greatly, so reporting of physical symptoms may not be fully informative.

The available Situation Analysis data reveal a fairly blunt measure of screening activities: whether the provider asks clients about any unusual bleeding or discharge. The proportion of positive results on this measure, which are available for both new and revisit clients, is generally quite low.

Figure 3-13 shows that in 11 of the study sites, half or fewer of new clients had been asked about unusual bleeding. In Senegal, only $7 \%$ had been

\section{General Assessment of Information Taken from Clients}

Given the large range of results on the indicators under this element, it is difficult to form a clear overall picture of how information is being solicited from clients. O ne way to clarify the picture is to calculate the mean number of items asked of each client per country. Figure 3-15 presents the mean number of questions asked of new and revisit clients under each of the above indicators in each country. In each case, some studies are excluded because they did not include all the questions.

Figure 3-15 shows that for new clients, the more mature family planning programs identified in the country descriptions generally do take more information from clients during counseling. However, even in the highest-scoring country, Senegal, only about 4 of the 8 specific questions shown are being asked of clients. As for revisit clients, the same observation applies about the maturity of the program, but findings for Botswana are surprisingly low, while $\mathrm{N}$ igeria fares quite well. Results for Kenya and Nigeria are the highest, with 1.1 of 4 specific questions being asked of revisit clients on average.

Can the results be further explained by provider training? For 8 of the study sites, information is available on all the questions asked of new clients, and among these, the data from 5-Burkina Faso, Kenya, Senegal, Zambia, and Zimbabwe-can link staff information with observation information. This allows for a comparison of counseling behavior between staff that have and have not received different types of training. In Burkina Faso, Kenya, and Zambia, those providers that had received training in IEC/counseling (R.6-7) had asked clients on average a significantly $(p<.05)$ higher number of the questions shown in Figure 3-15. ${ }^{21}$ The same relationship holds for Zimbabwe, but the results are not significant. Interestingly, in all 5 countries, no significant difference was found between providers that had been trained in clinical family planning (R.6-3) and those that had not.

Do the above findings also hold for treatment of revisit clients? In this case, data are available from 7 countries about questions asked of revisit clients, and for 4 of these (G hana, Kenya, Zimbabwe, and Zambia), staff information and observation information can be linked. These results show no significant difference in questions asked with either IEC/counseling training or clinical training. The exception is Zimbabwe, in which providers trained in counseling had asked a significantly higher number of questions of revisit clients than had those not so trained; those trained in clinical skills had also asked significantly more questions than those without that training.

O verall, the results on this element reveal serious shortcomings in obtaining critical information from clients, even among the best family planning programs in the region. The lack of screening for STD symptoms is especially troubling. Moreover, information gathering from new clients may improve with training in IEC/ counseling skills, but does not appear to be related to training in clinical family planning. 
Figure 3-15. Mean number of items asked of new and revisit dients

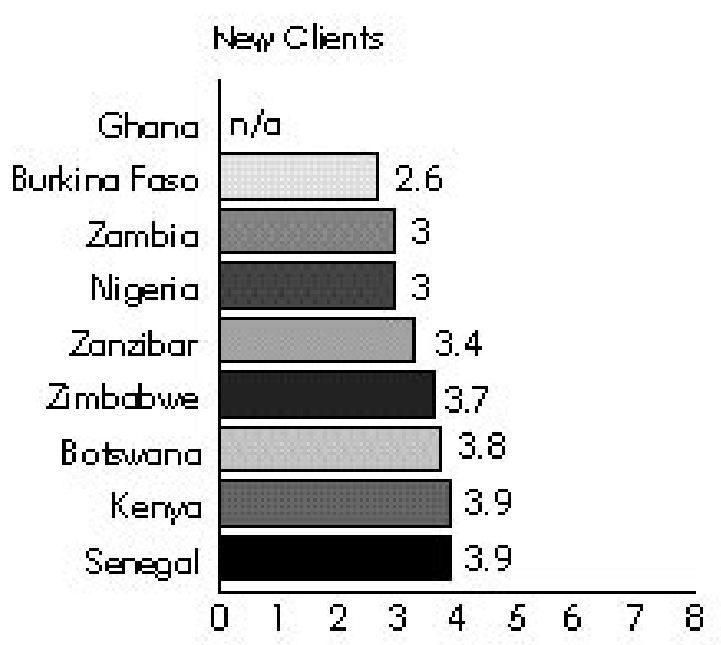

Eight possible items caled of new client.

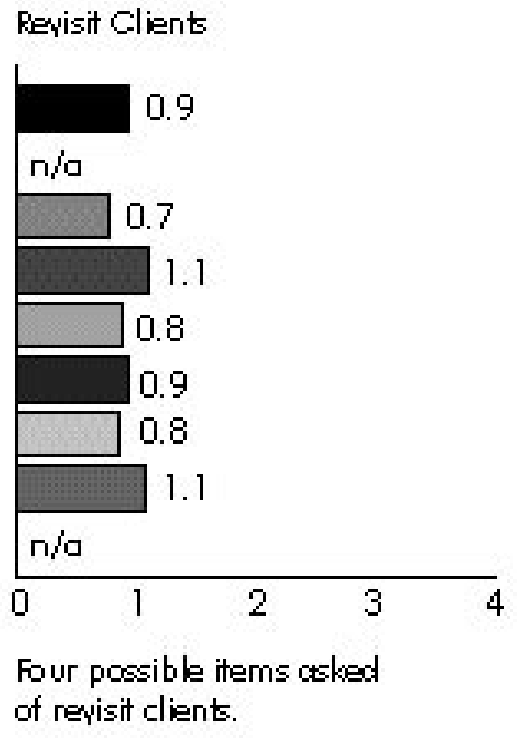

asked about this issue. The results for revisit clients are even lower, ranging from $6 \%$ in Kenya to $36 \%$ in Zanzibar. Similar proportions of clients had been asked about unusual discharge (see Figure 3-14). From $7 \%$ (Zanzibar) to $42 \%$ (Botswana) of new clients had been asked about unusual discharge, and from only 3\% (Zanzibar) to 19\% (Ghana) of revisit clients. These results indicate that STD screening, at least through introductory questions on possible symptoms, is generally not taking place. ${ }^{19}$ (See Chapter 10 for more information on this point.)

On the other hand, IUD clients are arguably the most in need of STD screening because of the risk of PID with IUD insertion, and they are in fact asked about unusual bleeding and unusual discharge more often than other clients. In 6 of 7 study sites, the results for bleeding range from $29 \%$ (Kenya) to 50\% (Burkina Faso), and the results for discharge range from 25\% (Burkina Faso) to 60\% (Zimbabwe). These results are all significantly higher than those for non-IUD clients. ${ }^{20}$ In the seventh country, Senegal, only 3\% of IUD clients had been asked about unusual bleeding and only $17 \%$ about unusual discharge. However, the overall result remains that in most contexts, IUD clients are screened more often for STDs than other clients, but many IUD clients are not screened.

\section{Q.3 Choice of Methods}

Most indicators under this element of quality address whether various methods are mentioned to clients during a family planning consultation. ${ }^{22}$ Many factors can affect whether a method is mentioned: whether the method is usually offered at that SDP, whether it is currently in stock, whether the provider is trained in that method, and whether the client prefers or is contraindicated from the method. For these reasons, not all methods are expected to be mentioned to all clients. However, a client can reasonably be expected to be told about at least two methods (Q.3-1).

For the following indicators, the proportion of clients that are told about a method is related only to whether that method is usually offered by that SDP. Although actual supplies of contraceptives are crucial, temporary stockouts should not prevent the discussion of a method with a client, who may be willing to use a temporary method until 
stocks of the preferred method become available. Moreover, given the available data, analysis of the other factors affecting whether a method is mentioned proved to be too cumbersome.

\section{Q.3-1 Are new clients told about at least two methods?}

In order to make even the simplest of choices, a client must be told about more than one method. The appropriateness of each method varies with the client's characteristics, and the optimum number of methods to present to clients is debated (see Chapter 6 for further discussion). The present indicator is a crude measure of choice, particularly since the client may have knowledge of methods from other sources and may present a preference to the provider. Nonetheless, this indicator gives a very rough measure of choice of methods offered to the client.

The results on the indicator vary, but in all 12 study sites, about $50 \%$ or more of clients have this extremely basic choice. In Kenya and Ghana, the figures are $85 \%$ and $87 \%$ respectively. The results for the indicator do not appear to vary with the maturity of the program. The results for Zimbabwe are among the lowest, even though it has a very strong family planning program. The relationship between the number of methods offered and the number mentioned is addressed in the "General Assessment" section at the end of this element.

\section{Q.3-2 How often do providers mention COCs to new clients?}

COCs are by far the most common method used by women in almost all the study sites; in Zimbabwe they comprise over three-quarters of the method mix. COCs can be safely prescribed to most women, so it is not surprising that they are easily the method most frequently mentioned to clients. However, COCs are not appropriate for breastfeeding women (see Q.2-2), so clients should be asked about their breastfeeding status when this method is mentioned. As noted under Q.2-2, in most contexts a large proportion of COC clients are not asked about their breastfeeding status, a finding that casts doubt on the quality of screening for this method.
Since COCs are also offered by almost all SDPs in all the study sites, most clients are attending SDPs where this method is usually available. This means that in almost all cases in which a client is not told about COCs, the method is in fact available at the SDP. The most extreme example is Zimbabwe, where fully $66 \%$ of new clients attend SDPs that offer COCs, but the method was not mentioned during counseling. However, in recognition of the breastfeeding issue, the Zimbabwe family planning program has been emphasizing POP use (see Q.3-3 below), which explains the low proportion of clients hearing about COCs in that country.

\section{Q.3-3 How often do providers mention POPs to new clients?}

POPs are designed for use by breastfeeding women, but they are mentioned to a relatively small proportion of clients. In 9 of 11 study sites, POPs were mentioned to well below $50 \%$ of clients. Again, the relative lack of questioning about breastfeeding status suggests that POPs may not be being presented to the proper audience. Two counterexamples are Zimbabwe and Kenya, in which about two-thirds of clients were told about POPs. In Zimbabwe in particular, this finding reflects a new emphasis on POP use at the time of the fieldwork (see Q.3-2 above).

POPs are fairly commonly available method. In 6 of 11 study sites, nearly half or more of clients attend SDPs that offer POPs, but were not told about them. In Senegal, fully $71 \%$ of clients were not told about POPs, although the method is offered at their SDP. Again, providers may avoid mentioning this method because the client is not breastfeeding, but the relative lack of discussion of breastfeeding, along with the high breastfeeding rates in the area, undermines this supposition.

In Zanzibar, $12 \%$ of clients were told about POPs although the method is not available at their SDP. This finding suggests the beginnings of a referral system in which methods are mentioned to a client regardless of their source, and the client is referred to another SDP for the method if necessary. In no other study site was such a large proportion of clients told about POPs at SDPs that do not offer the method. 


\section{Figure 3-16. Percent of new Family planring dients who we re told cbout condoms}

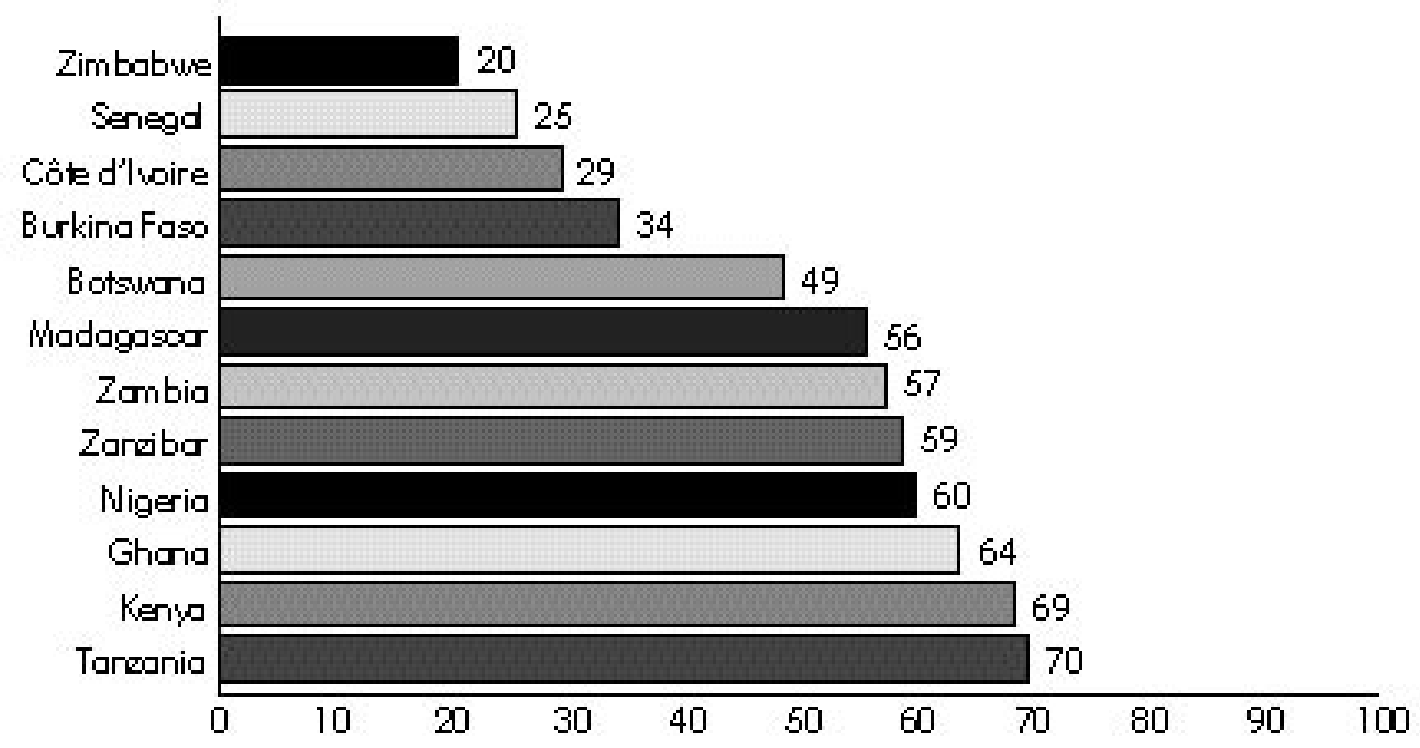

Q.3-4 How often do providers mention injectables to new clients?

Injectables were introduced into sub-Saharan African programs in the last decade or so, and although some controversy surrounded their safety in the 1980s, they have grown to comprise a major portion of the method mix in most countries. This method has very few contraindications, can easily be used by breastfeeding women, and can be used as a spacing or limiting method. The results on this indicator vary enormously. In 4 of the 12 study sites, $75 \%$ or more of clients were told about the injectable. In an additional 6 sites, 50\% to $60 \%$ were told about the method, whereas the figure was less than $50 \%$ in Botswana and Senegal. These results correspond roughly to the injectable's share of the method mix in each country: the higher the prevalence of injectables, the more new clients are told about them during family planning counseling.

Once again, however, a large number of clients are not being told about injectables even though the method is offered at their SDP. The largest proportion of such cases is in Botswana, where fully $65 \%$ of clients were not told about injectables even though the method is offered. Findings for other sites range from $8 \%$ of clients (Tanzania) to $47 \%$ (Zimbabwe). This may reflect the relatively high frequency of injectable stockouts, since providers may not mention a method they know is out of stock.

On the other hand, in Tanzania 20\% of clients were told about injectables even though the method is not offered at their SDP, and in Zanzibar this figure is $8 \%$. As with POPs, these findings suggest that in these two countries, clients are referred to other SDPs if their method of choice is not available at their SDP. The proportion of such cases is smaller in all other study sites.

\section{Q.3-5 How often do providers mention condoms to new clients?}

Given the prevalence of HIV and other STDs in many of the study sites, condoms should be mentioned to all clients, either as a primary form of contraception or as part of dual protection (see R.32 ). However, the results for this indicator show that only from $20 \%$ (Zimbabwe) to $70 \%$ (Tanzania) of new clients are told about condoms during their interactions with providers (see Figure 3-16). In the majority of study sites, fewer than two-thirds of clients are told about condoms.

These findings cannot be compared with findings on whether condoms are offered at the SDP, since condoms are such a commonly offered method. In fact, in Zimbabwe, Senegal, and Côte d'Ivoire, $99 \%$ of clients attend SDPs that offer con- 
doms, but three-quarters of clients were not told about the method during consultation.

On the other hand, in Ghana and Tanzania, about $18 \%$ of clients were told about condoms although the method is not offered at their SDP. Again, this is a positive sign of the beginnings of a referral system, which in the case of condoms may simply involve informing clients about the nearest source of supply. In all other study sites, if the SDP does not offer condoms, this method is generally not mentioned to clients.

This distribution of mentioning condoms is not easy to analyze. It does not vary with the maturity of the family planning program, nor does it reflect the share of condoms in the method mix or even the HIV prevalence of the country. Zimbabwe and Botswana have the two most advanced family planning programs, and they both suffer extreme- ly high HIV prevalence, yet half or fewer of their new clients hear about condoms. Failure to mention condoms may reflect providers' discomfort with discussing sexual relations with clients, although this is conjectural. It may also be argued that where condoms are not mentioned in the context of family planning, they may be mentioned at another time in the context of disease prevention. See the discussion below of whether new family planning clients are told that condoms prevent STDs (Q.4-4) for results on this point.

\section{Q.3-6 How often do providers mention IUDs to} new clients?

Again, the results on this indicator vary greatly, from $22 \%$ in Zimbabwe to $78 \%$ in Nigeria. Interestingly, these results do not seem to reflect the IUD's share of the method mix (as measured by

\section{General Assessment of Choice of Methods}

Of the methods mentioned or not mentioned to clients, the one that warrants most concern is condoms (Q .3-5). Although in many cases the majority of clients are told about more than one method, those methods clearly do not always include condoms. Given the prevalence of HIV in many of these study sites, condoms should be mentioned to every new client without fail.

Hormonal methods are mentioned most frequently, but they are not necessarily targeted to the appropriate users. COCs (Q .3-2) are mentioned quite often, but frequently not in conjunction with the important screening question about breastfeeding status. PO Ps (Q .3-3) are mentioned less often, although they would be a better method for breastfeeding clients. Injectables (Q .3-4) would also be appropriate for breastfeeding clients, but the extent of their presentation to clients appears to reflect their existing share of the method mix in each country. In the best case, a client would be asked her breastfeeding status, and the answer to that question would determine whether she would hear about COCs or POPs. This does not seem to be the case currently in most of the study sites.

With regard to IUDs (Q.3-6) and tubal ligation (Q.3-7), their mention to clients appears to be roughly determined by their availability at the SDP. However, not all clients at SDPs that offer these methods are actually told about them. In general, about half the clients at SDPs that offer IUDs are told about them during consultation.

O verall, then, the methods presented to clients do not seem to be related to the methods usually offered at the SDP, except in the case of IUDs and tubal ligation. Providers' mention of condoms and hormonal methods is governed by factors other than availability, but does not appear to take screening questions into account. Moreover, for effective choice of methods, clients should be able to take advantage of a referral system, but such systems generally do not appear to be in place, except to some extent in Tanzania and Zanzibar, and more for tubal ligation than for other methods. In addition, in about $10 \%$ to $30 \%$ of cases, one method is emphasized to clients over others. In all study sites, client choice could be improved through proper screening and mention of all appropriate methods, whether offered at the SDP or available through referral. (See Chapter 6.) 
the DHS). For example, IUDs make up $27 \%$ of the method mix in Senegal, and half of clients hear about them, whereas IUDs account for only $8 \%$ of the mix in Ghana, and three-quarters of clients hear about them.

Instead, these results seem to vary with the availability of IUDs at SDPs in a country. Fully $80 \%$ of SDPs offer IUDs in Nigeria, and three-quarters of clients are told about them, whereas only $8 \%$ of SDPs offer IUDs in Zimbabwe (see endnote 5), and only $22 \%$ of clients are told about them. ${ }^{23}$ The other countries generally follow this pattern, except for Zambia, Tanzania, and Zanzibar, which seem to have healthy referral systems for IUDs. In Tanzania, for example, fully $33 \%$ of clients attend SDPs that do not offer IUDs, yet they are told about IUDs in consultation. In Zanzibar this figure is 31\% and in Zambia 22\%.

\section{Q.3-7 How often do providers mention tubal ligation to new clients?}

This indicator is unique because tubal ligation would presumably be mentioned only to clients who want no more children-a minority in many of the study sites included here. Therefore, this indicator is restricted to clients who in their exit interview expressed the desire to have no more children. Since the information on whether tubal ligation is mentioned was gathered from the observation, it is not clear whether providers actually knew the client's status as a limiter. However, because few clients are asked their reproductive intentions (see Q.2-1 above), it is fair to assume that many providers do not know a client actually wants to limit her births.

Few limiting clients are told about tubal ligation. The proportions range from only $3 \%$ in Botswana to $39 \%$ in Kenya, and may be related to the percentage of SDPs that actually offer this method. Ghana and Kenya provide the most opportunities for tubal ligation, and they fare better than most on this indicator.

Tubal ligation is one method that clearly calls for a referral system, since it is usually offered only at larger SDPs (see R.3-4). In fact, referral systems are more in evidence for this than for other methods: in 6 of the study sites, from 11\% (Ghana) to $30 \%$ (Tanzania) of limiting clients were told about tubal ligation even though the method is not offered at their SDP. Thus although tubal ligation should probably be discussed more frequently with limiting clients, the beginnings of a referral system are already in place in some contexts.

\section{Q.3-8 Do providers overemphasize particular methods to new clients?}

During the observation, the observer answered the question: "Did the provider emphasize one method in particular?" The answer to this question is admittedly subjective, but the definition of "overemphasize" was carefully reviewed in the observer training, and was always defined to exclude cases in which methods are emphasized because of a contraindication for that client or other medical reason. Instead, this question encompasses cases in which providers emphasize one method regardless of the client's condition or desires. It is designed to identify clients who may have been pressed into using a method as a result of provider bias.

The results on this indicator are remarkably consistent, ranging between $11 \%$ and $28 \%$ for 9 study sites. In 5 countries, observers recorded which method was overemphasized, and pills, IUDs, and injectables accounted for most or all of the cases. Sterilization is rarely overemphasized in these countries. It may also be noted that emphasizing one method over others during consultation does not appear to be related to whether the provider has been trained in IEC/ counseling (R.6-7).

\section{Q.4 Information Exchange: Information Given to Clients}

\section{Q.4-1 Are new clients told how to use their method?}

Once a client has decided on a method, she must be told how to use it, or failure rates will probably increase. This is an especially important point for users of pills and condoms, which are particularly user dependent. Even an IUD user, however, must know how to check that the strings are in place and that she must return for checkups. Likewise, injectable users must be told about the schedule of reinjection. If this sort of information is omitted, 


\section{General Assessment of Information Given to Clients}

The first four indicators under this element naturally fall into two pairs: how to use the method (Q.41 ) and its side effects (Q.4-2), and how the method relates to STD transmission (Q .4-3) and the fact that condoms prevent STDs (Q .4-4). The first pair shows a range of results from low to high and addresses purely contraceptive issues, whereas the second pair is quite universally low and addresses STD prevention. The two pairs are addressed separately here.

The percentage of new clients who were told both how to use their method and its side effects is presented in Figure 3-17. As with information taken from clients $(Q .2)$, the younger programs do not generally fare as well as the more mature programs in this regard, although there are some exceptions, such as Zimbabwe, which has a fairly low result. Moreover, the results overall are not encouraging. Even in the best case, Botswana, only two-thirds of clients are receiving critical information about their method.

A look at the relationship between training and the provision of this information shows that providers who have had clinical family planning training (R.6-3) tend to give clients more of this information, but these results are not statistically significant in any of the studies for which the data on these indicators can be linked (Burkina Faso, G hana, Kenya, ${ }^{24}$ Senegal, Zambia, and Zimbabwe).

The same is true for training in IEC/counseling (R.6-7), but the results here are statistically significant in Zambia, where $77 \%$ of providers trained in IEC/counseling told clients both pieces of information, but only $44 \%$ of providers without this training did so $(p<.01)$. This suggests that training in $\mathrm{IEC} /$ counseling may indeed improve information given to clients.

Information on the pair of indicators related to STD transmission is available for only 4 of the study sites. The results for these 4 sites show that very few clients are receiving information on both their method's ability to prevent STDs and the fact that condoms prevent STDs. The percentages are $12 \%$ for Botswana, 7\% for Zambia, 2\% for Zimbabwe, and 2\% for G hana. Because these percentages are so small, no tests of provider training are statistically significant. However, the results do show a possible relationship to training in that only providers trained in clinical family planning or IEC/counseling actually provided this information to clients.

As for use of IEC materials, no summary measure of this indicator is necessary since use of these materials is universally low. In general, IEC materials are not being used with clients in any program, the only exception being clients in G hana and Zambia, who are sometimes counseled with flip charts. The use of IEC materials may be related to training in IEC/counseling, but the data on this issue do not yield many statistically significant results. Findings on this indicator were compared among providers who have had such training and those who have not; the results show only scattered statistical significance, but they are in the expected direction. For example, in Zambia $61 \%$ of clients who were counseled by providers trained in IEC were shown flip charts, compared to only $15 \%$ of clients counseled by untrained providers $(p<.01)$. This finding suggests that IEC training may be related to use of IEC materials.

In general, information given to clients is an extremely weak program element in all countries surveyed, both in terms of family planning information and even more so in terms of STD/HIV information. However, more mature family planning programs do seem to fare relatively better. Providers who are trained in IEC/counseling or basic clinical family planning may do a better job of giving information to clients, but the relationship is not extremely strong, and providers trained in IEC/counseling do not necessarily use IEC materials more often. (See Chapter 5 for more detail on Kenya.) 


\section{Figure 3-17. Percent of family planring acceptors who were told how to use their method and its side effeds}

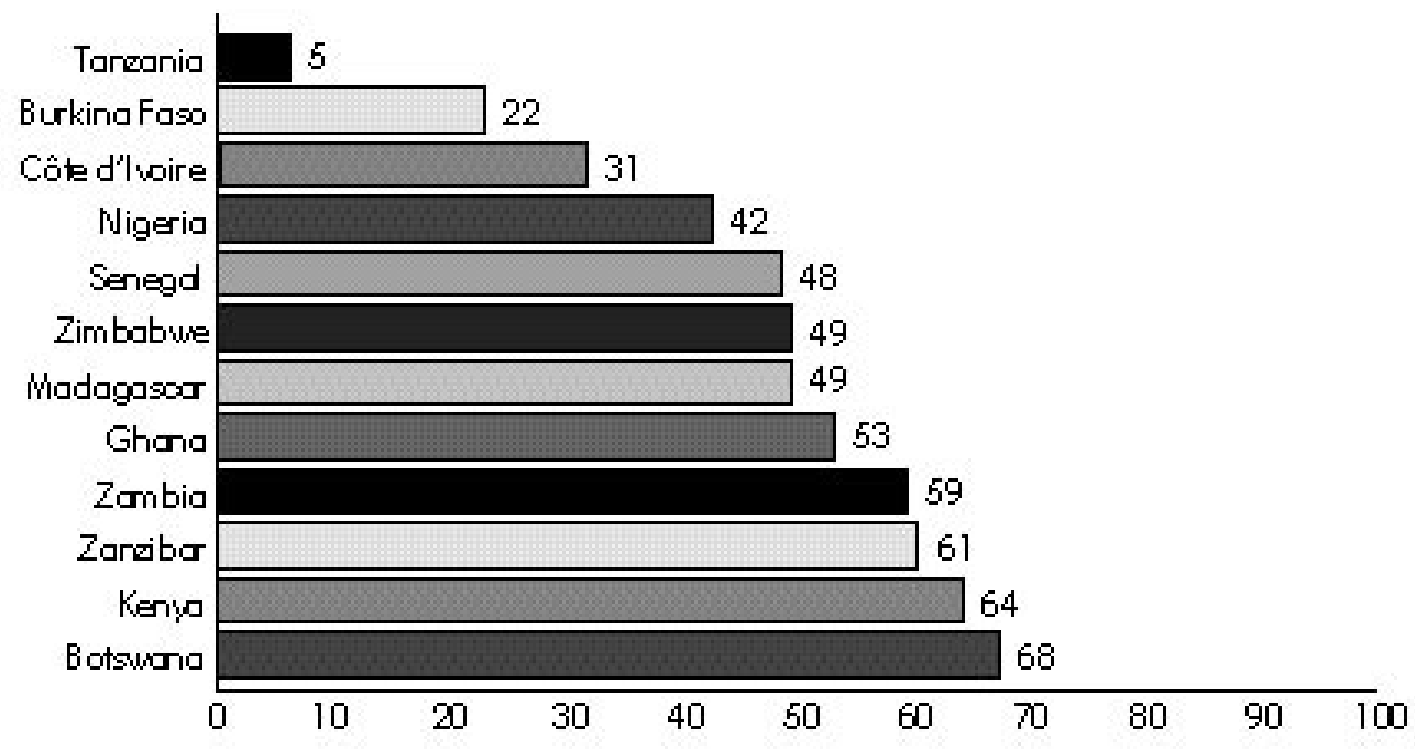

the client will probably not have a satisfactory long-term experience with the method. The results on this indicator are in general quite high: in 9 of 11 study sites, almost $80 \%$ or more of new clients were told how to use their method; $62 \%$ were told in Côte d'Ivoire, and only 14\% in Tanzania.

\section{Q.4-2 Are new clients told the side effects of their method?}

Awareness of side effects is particularly important, since they are a common reason for discontinuing many methods (Ross and Frankenberg, 1993). If clients are aware of side effects and their management beforehand, they may not discontinue the method as quickly should a side effect occur. Unfortunately, the results for this indicator are not particularly high, ranging from $24 \%$ in Burkina Faso to $68 \%$ in Botswana.

\section{Q.4-3 Are new clients told how their method relates to STD/HIV transmission?}

Given the high prevalence of HIV and other asymptomatic STDs in the region, family planning programs have a particular responsibility to inform clients as to whether their method can protect them from STD transmission. This question was included in only 4 of the Situation Analysis studies, and in all cases yielded very low results: 19\% of new clients in Botswana, 9\% in Zambia, 5\% in Zimbabwe, and only 3\% in Ghana. These findings are especially troubling because in all 4 countries, pills, IUDs, and injectables comprise a large portion of the method mix and do not provide protection against STDs.

\section{Q.4-4 Are new clients told that condoms prevent STDs/HIV?}

Aside from hearing about the ability of their chosen method to prevent STDs, clients should be told that condoms are the most effective method of STD prevention. Again, the high STD/HIV prevalence in the region makes this an issue of great importance. Unfortunately, the results on this indicator are not encouraging. Fewer than one-third of new clients generally and in some areas fewer than $10 \%$ of clients receive this information. (See also Q.3-5 above.)

\section{Q.4-5 Are IEC materials used with new clients?}

The content of information given to clients is critically important, but the form of communication also has an effect on whether the information is retained. Use of posters, flip charts, brochures, anatomical models, and other supports enables clients to understand and remember information 
more effectively. This indicator reveals whether new clients are being counseled using posters, brochures, and flip charts on family planning. In each case, the results include only clients at SDPs with a poster, brochure, or flip chart available. With a few exceptions, use of IEC materials is remarkably low. (See Chapter 6 for more discussion of this point; see also R.7-1 above.)

Where flip charts are available, they are used to counsel more than $50 \%$ of clients in Ghana and Zambia. In 9 other study sites, however, they are used with only about $25 \%$ or fewer clients, and in Zanzibar and Botswana the flip charts are simply never used. In all 12 study sites, $25 \%$ or fewer clients had been counseled using brochures, and in 10 study sites only $11 \%$ or fewer clients had been counseled using posters. In each case, the brochure or poster was available, but had not been not used with the vast majority of clients.

\section{Q.5 Technical Competence}

\section{Q.5-1 How are new clients assessed?}

Many of the countries included here have service protocols governing the assessment of new family planning clients. Because these protocols differ and can be quite complex, the present analysis focuses on five simple assessments: medical history, weight, last menstrual period (LMP), blood pressure, and physical exam..$^{25}$ Not all of these procedures are necessary for all new clients. For example, the Kenya and Zimbabwe programs have dropped their requirement for medical history and weight. International family planning guidelines rarely require these procedures (United States Agency for International Development, 1994). In some cases, these procedures may even constitute a barrier to services for clients who are uncomfortable with medical procedures. On the other hand, clients who come for family planning services deserve a broader concern for their general reproductive health (a theme of the International Conference on Population and Development [ICPD]), and they may appreciate the medical attention (Ndhlovu, 1995). Regardless of this debate, this indicator addresses only whether these five simple assessments were performed on new clients (see Chapter 9 for more information).

Weight, LMP, and blood pressure are almost universally taken. ${ }^{26}$ The results for weight range from $66 \%$ of clients in Ghana to $92 \%$ in Botswana, and the results for LMP range from $73 \%$ of clients in Zimbabwe to $97 \%$ in Burkina Faso. Similarly, blood pressure is taken with $69 \%$ (Ghana) to $93 \%$ (Senegal) of clients. Results for medical history

\section{Figure 3-1 8. Mean number of Five assessments carried out with new dients}

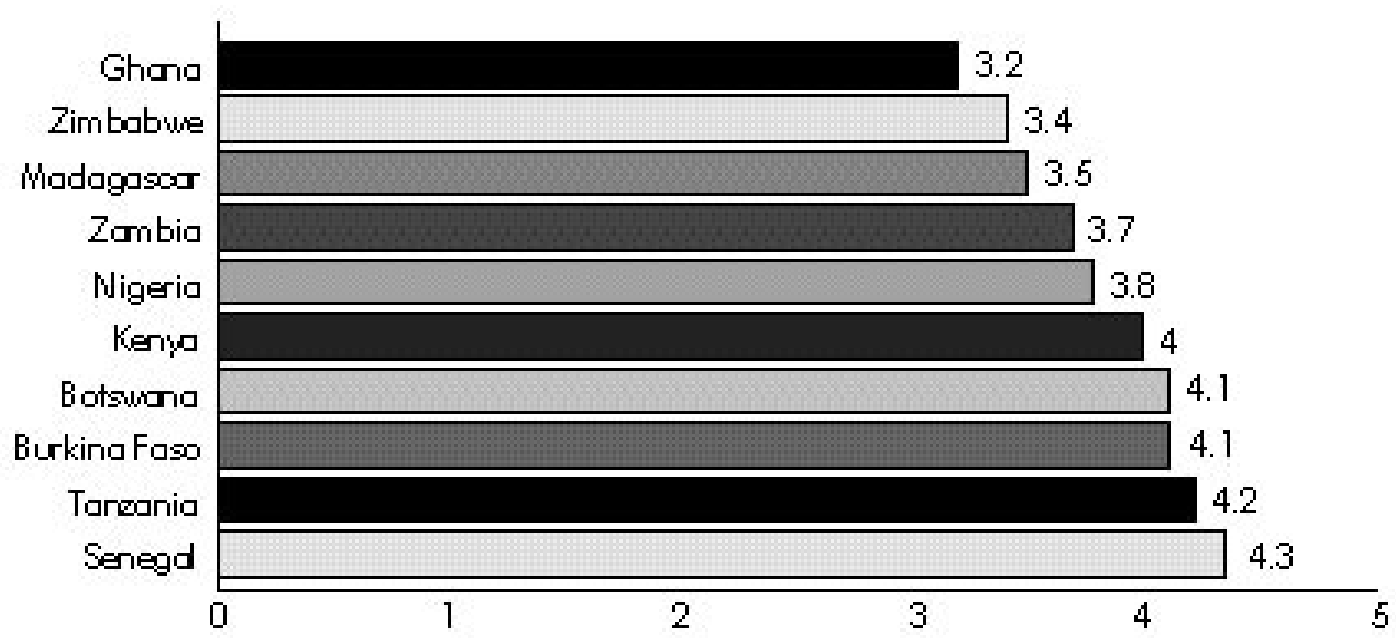

Fye assessment: medical histor, LWF, yeight, blood pressure, physioal exam. 


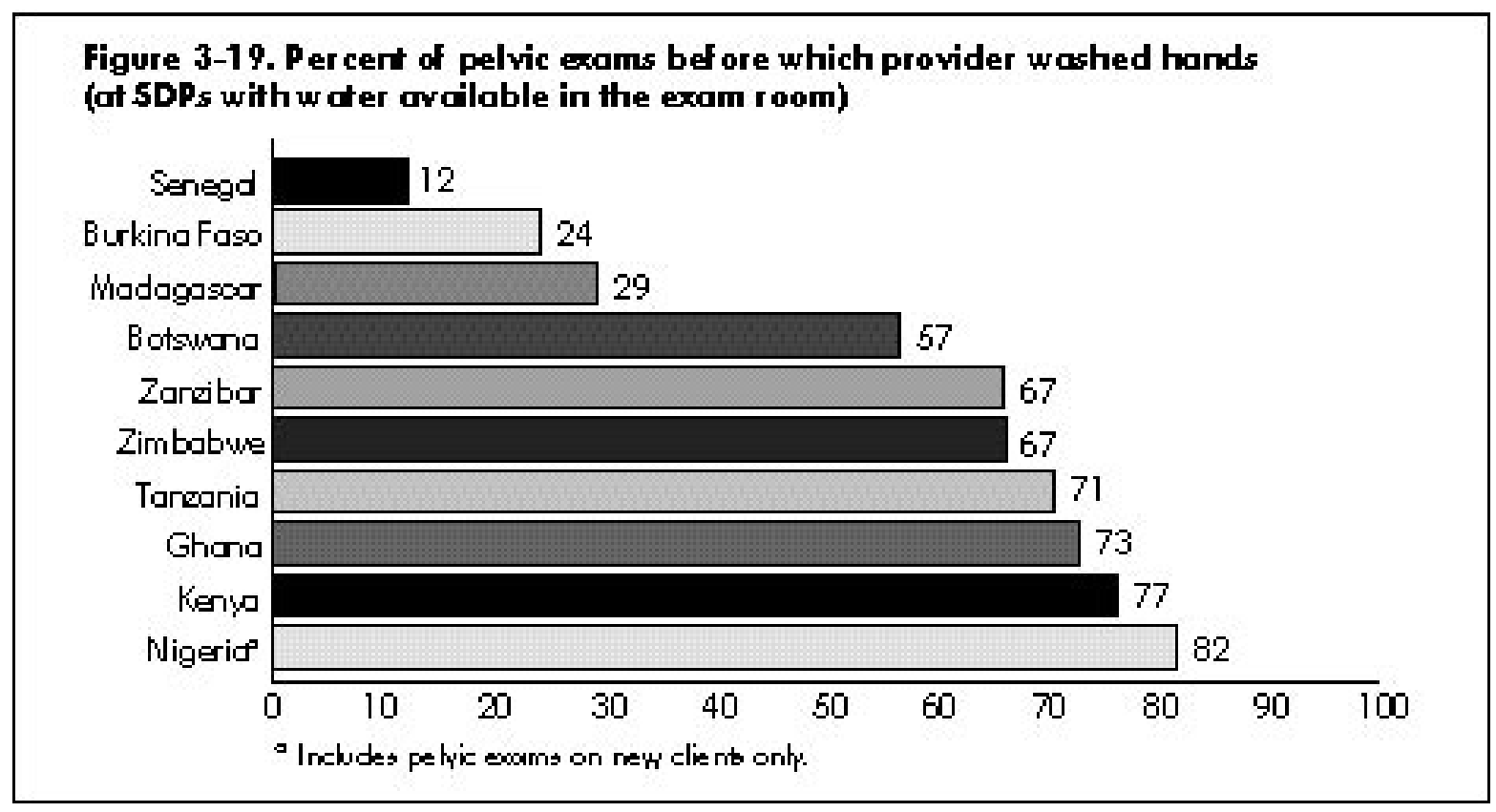

show a little more variation, fueled mainly by lower percentages for Zimbabwe (51\%) and Ghana $(60 \%)$. The others range from $69 \%$ (Zambia) to $86 \%$ (Côte d'Ivoire).

Physical exams are the least common item of the five. Although $85 \%$ of new clients in Burkina Faso receive a physical exam, this is the case in only $31 \%$ (Ghana) to 65\% (Senegal) of the remaining sites. The rates at which physical exams are performed do not appear to vary with the method accepted by clients.

In general, the results for this indicator are higher than those for information exchange (Q.2 and Q.4) and choice of methods (Q.3). One way to summarize the client assessment indicator is to calculate the mean number of the above five items performed with clients in each country. Figure 3-18 shows that these results exhibit a fairly narrow range, although the higher means are statistically different from the lower means. ${ }^{27}$ Interestingly, this result does not seem to vary with the maturity and effectiveness of the family planning program: Burkina Faso's mean is relatively high, while Zimbabwe's is relatively low.

A comparison of the clinical training of providers and their assessment of clients is possible for Burkina Faso and Senegal. (In all other countries, so few providers had not had clinical family planning training that a comparison of trained and untrained groups was not possible.) In both cases, providers trained in clinical family planning carried out a significantly higher number of assessment procedures with new clients than did untrained providers. In Senegal, providers trained in clinical family planning carried out 4.5 of the 5 assessments on average, versus 3.9 among untrained providers $(p<.01)$. In Burkina Faso, trained providers carried out an average of 4.3 assessments, versus 3.4 among untrained providers $(\mathrm{p}<.05)$.

\section{Q.5-2 What is the technical quality of} pelvic exams?

The quality of pelvic exams is usually measured according to six items in the Situation Analysis: informing clients about the procedure beforehand, washing hands beforehand, using a clean or sterile speculum, using clean or sterile gloves, washing hands afterward, and informing clients of the results. $^{28}$ Two of these items-informing clients beforehand and afterward-are covered above under Q.1-4. Three others-washing hands before, washing hands after, and using clean or sterile gloves-relate to protecting clients from crossinfections. Providers often disagree on whether all three of these steps are necessary with each pelvic exam, or whether one or two are sufficient for ensuring cleanliness. For the purposes of this analysis, performing all three items represents the 
Figure 3-20. IUD insertion qudity

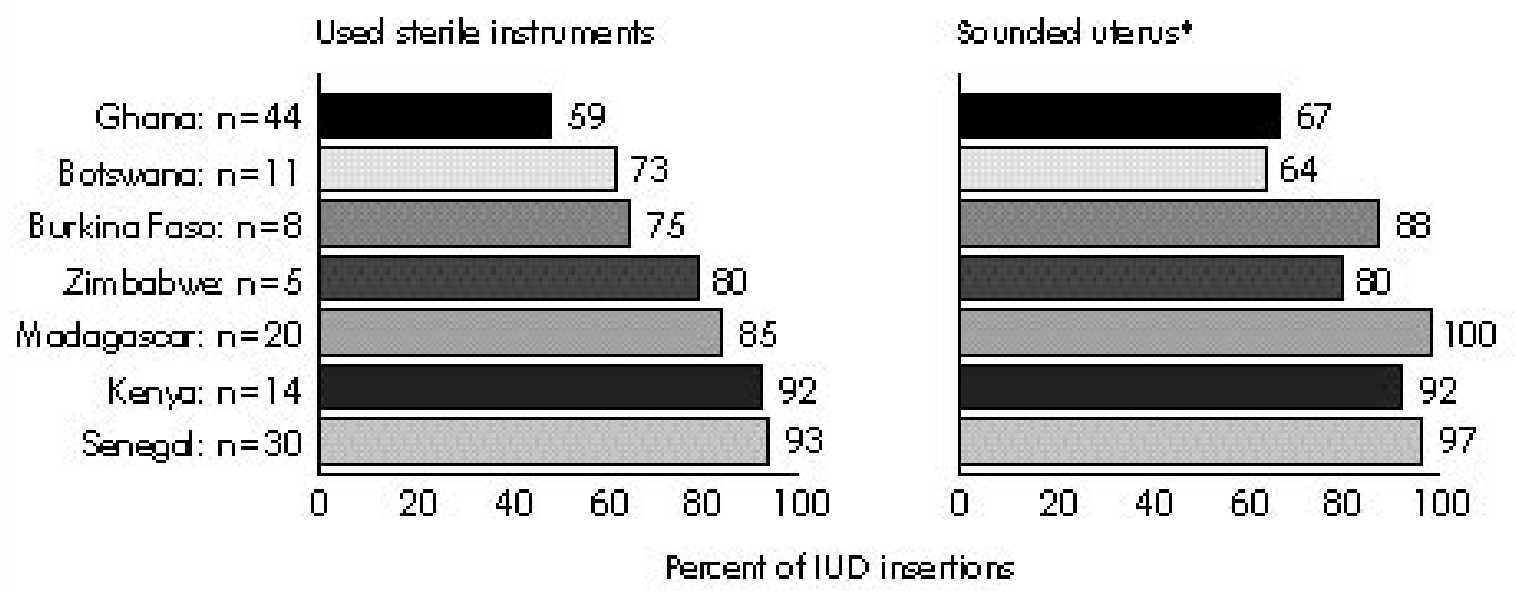

- At SDPs withuterine sound onvalable.

highest-quality pelvic exam, since it best protects the client from infection. Clearly, performing none of these actions puts clients at serious risk.

The results for handwashing before pelvic exams are given in Figure 3-19, and range from only $12 \%$ in Senegal to $82 \%$ in Nigeria. In 4 of the countries, information is also available on washing hands after the exam, and the results are higher: Botswana $(71 \%)$, Zimbabwe $(75 \%)$, Ghana $(81 \%)$, and Kenya $(87 \%)$. However, if the results on handwashing beforehand are compared with those on handwashing afterward in these 4 sites, one still finds that a moderate percentage of pelvic exams involve no handwashing at all. The percentages of pelvic exams during which the provider washed hands before or after are $76 \%$ for Botswana, 81\% for Zimbabwe, 89\% for Ghana, and 94\% for Kenya.

Use of gloves is also particularly important for averting infections, and the results on this item are somewhat higher, ranging from $72 \%$ in Botswana to $98 \%$ in Kenya. However, providers may be selecting between using gloves and washing hands before exams: in the 9 studies for which the data are available, $80 \%$ or more of pelvic exams involved either gloves or handwashing beforehand. This result is better than that for handwashing alone, but up to $20 \%$ of clients may be experiencing pelvic exams with no protection from crossinfection at all.
In Senegal and Burkina Faso, the data allow a comparison of training in clinical family planning with handwashing before and glove use. Disappointingly, in both countries those providers trained in clinical family planning show no statistically significant difference in handwashing from those not trained. In Senegal, trained providers do use gloves statistically more often $(\mathrm{p}<.05)$.

The final indicator relates to using clean or sterile specula. Speculum use is fairly high for 7 of 10 study sites, ranging from $83 \%$ in Senegal to $97 \%$ in Tanzania. In the other 3 sites, the percentage is considerably less: 65\% in Ghana, 58\% in Burkina Faso, and 52\% in Madagascar. Again, these results are only for SDPs that have at least two specula available.

In general, the quality of pelvic exams is variable. One of the most critical technical aspects of the exam is use of clean techniques, but many of the studies found this lacking, which places clients at risk of cross-infection.

\section{Q.5-3 What is the technical quality of IUD insertions?}

IUD insertions are fairly rare events in many of the study sites included here. In the 6 countries for which data on this indicator are available, the number of IUD insertions observed ranges from only 5 in Zimbabwe to 44 in Ghana. For this reason, these results must be interpreted with great 
Figur e 3-21. Percent of clients satiss ied with time of consultation

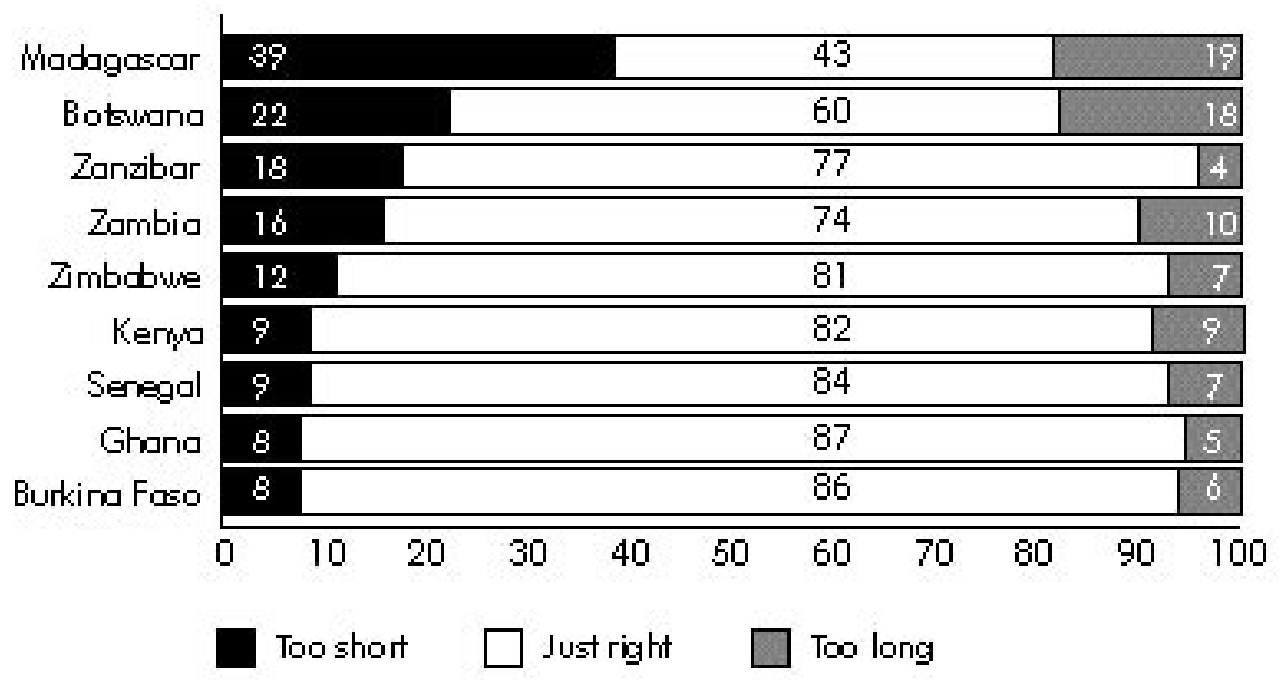

caution. Quality of IUD insertion is measured according to three items in Situation Analysis: sounding the uterus, ${ }^{29}$ using sterile instruments, and offering moral support. Findings on offering moral support are covered above under Q.1-5.

The results for using sterile instruments are not very encouraging (see Figure 3-20). Although in Senegal and Kenya over $90 \%$ of IUD insertions involve sterile instruments, results in the other 4 sites range from $59 \%$ in Ghana to $85 \%$ in Madagascar. In these contexts, a considerable proportion of clients are being exposed to cross-infection through the use of nonsterile instruments.

Sounding the uterus is important because if the IUD is not placed properly within the uterus, there is a risk of discomfort, expulsion, or perforation. In Madagascar, Senegal, and Kenya, almost all clients are sounded, but the results are lower for Burkina Faso $(88 \%)$, Zimbabwe $(80 \%)$, Ghana $(67 \%)$, and Botswana (64\%).

For 5 of the countries, it was possible to link information from the staff interview to the observation data, so a comparison between quality of IUD insertion and provider training in the procedure could be made. In all 5 countries, at least some of the observed IUD insertions were being performed by providers who reported in the staff interview that they had received no particular training in IUD insertion, which is itself a pressing issue (see R.6-
8). ${ }^{30}$ Among those who did report such training, the quality of IUD insertion was significantly higher on all three of the above items. For example, $89 \%$ of the providers trained in IUD insertion used sterile instruments, as compared with $59 \%$ of untrained providers. Similarly, $89 \%$ of trained providers used a sound, versus $70 \%$ of untrained providers. Lastly, $78 \%$ of trained providers offered moral support, as compared with $48 \%$ of untrained providers. ${ }^{31}$

\section{Q.5-4 What is the technical quality of injections?}

Injections are more common than IUD insertions in the study sites, so the sample sizes on this indicator are larger and more reliable. The quality of injections is measured according to four items: disinfecting the injection site, shaking the vial, using a sterile needle, and not massaging the injection site. In general, the results on these indicators are quite high, with the exception of not massaging the injection site, which shows more variation.

Across all study sites, $95 \%$ or more of observed injections involved sterile needles. This result is often attributable to the fact that Depo Provera, one of the most common injectables, is delivered prepackaged in a sterile, disposable needle and syringe. Because infectious material can reside on the outer skin, it is imperative to disinfect the injection site so that the needle itself does not act as an instrument of infection. In 6 of 9 sites, over $94 \%$ of 


\section{General Assessment of Technical Competence}

$O$ verall, most clients are assessed with weight, blood pressure, and LMP, with a little more variation in frequency of medical histories and physical exams. Findings on the quality of pelvic exams and IUD insertions are not encouraging. The main concern is clean or aseptic procedures, particularly handwashing and use of gloves during pelvic exams. Asepsis during injections is more under control generally, although most injections involve massaging the injection site, which is not appropriate.

The findings on these indicators suggest an overall relationship between training and technical competence. Although results for the effects of clinical training are available from only two countries (Senegal and Burkina Faso), they show that providers trained in clinical family planning perform more assessments of clients. However, the association between training in clinical family planning and observance of aseptic procedures is extremely sketchy, showing statistical significance only in Senegal, and only for using gloves. Clinical training does not seem to have an effect on handwashing. On the other hand, training in IUD insertion and removal does seem to improve the quality of IUD insertions significantly. Perhaps the more specific a training course is, the stronger is its effect on quality of care.

injections involved disinfecting the injection site. In Zimbabwe and Zanzibar, about $75 \%$ of the injection sites were disinfected, and in Ghana only $39 \%$.

In most cases, providers vigorously shook the injectable vial before injection. The results range from $89 \%$ in Senegal to $100 \%$ in Botswana. In Burkina Faso, however, this figure was just $63 \%$.

For most injections, such as antibiotics or vaccines, massaging the injection site after injection is standard practice, as it aids in uptake of the material. However, Depo Provera is carefully timed to release hormones at a specific rate into the bloodstream over several months' time. Massaging the injection site interferes with this process (United States Agency for International Development, 1994). In 7 of 9 study sites, massaging of injection sites appears to be common practice, since only about $25 \%$ of injections were not massaged. In Burkina Faso, 47\% were not massaged, and in Ghana $74 \%$.

\section{Q.6 Mechanisms to Encourage Continuity}

Q.6-1 Are clients told that they can switch methods?

Many clients who discontinue a method do so because of dissatisfaction with side effects, mode of action, or intrusiveness of the method itself. If clients are aware that they can switch methods should they encounter problems, they may be more likely to return to the SDP for a new method rather than discontinue altogether (Ross and Frankenberg, 1993). However, not many clients are told that they can switch. The results range from only $11 \%$ in Ghana to 54\% in Zanzibar, and none of these results are significantly related to staff training in IEC/ counseling (R.6-7).

Q.6-2 Are clients given a date for a return visit? If clients leave the SDP without a date for a return visit, they may feel it is unnecessary to return to the clinic for follow-up care or may not know when and where to obtain care. Conversely, having a return date encourages clients to continue their method. Giving clients a return visit date appears to be fairly standard practice across all study sites. The results range from $78 \%$ of clients in Zimbabwe to fully $100 \%$ in Senegal.

\section{Q.7 Appropriateness and Acceptability of Services}

\section{Q.7-1 Do clients receive the information} and services they desire?

This question was asked of clients as they left the SDP, and it is subject to a strong courtesy bias (see Q.1). The results are uniformly high, ranging from $87 \%$ in Botswana to $99 \%$ in Madagascar. Again, these results are not highly informative, but some 
of the following indicators are more specific and generate more variation in response.

\section{Q.7-2 Are clients satisfied with the duration of their consultation?}

Clients were asked whether the time of consultation was too short, just right, or too long. Figure 3-21 shows that where there was a complaint, clients tended to feel that their consultation was too short; this was particularly the case in Madagascar. Interestingly, the results differ for new and revisit clients. In all the study countries, substantially more revisit than new clients reported that their visit was too short. ${ }^{32}$ It may be assumed that a revisit client simply wants to refill her method and be on her way, but the results show that some of these clients would appreciate more time for discussion.

\section{Q.7-3 Do clients find waiting time reasonable?}

Actual waiting times vary greatly among clients and study sites, but the information is not collected through a patient-flow analysis. Instead, the Situation Analysis asks clients to estimate how

\section{General Assessment of Appropriateness and Acceptability of Services}

All family planning programs are aimed at satisfying clients in terms of both the method accepted and the acceptability of the visit itself. The indicators under this element attempt to get at client satisfaction with services, but many are not extremely informative since they are strongly affected by a courtesy bias and show little variation. In short, client satisfaction might better be measured using qualitative techniques rather than the Situation Analysis.

long they waited, and whether they feel this length of time was reasonable. The estimates of waiting time are not generally reliable, but for programmatic purposes, the clients' opinions on the acceptability of the waiting time can reveal whether they are waiting too long, however they define this. Although the results are subject to a courtesy bias, they do show some variation: from $67 \%$ (Côte d'Ivoire) to $92 \%$ (Nigeria) of clients felt the wait was reasonable. This indicator is calculated only for those clients who waited.

\section{Q.7-4 Do clients find the cost of services acceptable?}

In some family planning programs, services and commodities are free, and in others the charges vary by sector and method. In those study sites that charge for services, clients were asked whether they find the cost of services reasonable or affordable. Again, this question is subject to a courtesy bias, but it does show some variation, from $78 \%$ in Zimbabwe to $100 \%$ in Zanzibar.

Measurement of cost and affordability is a complex subject that cannot be addressed with one question. For proper measurement, one would need at least to add detailed information on the price paid and the financial resources of clients. However, these items currently lie outside the scope of the Situation Analysis methodology.

\section{CONCLUSION}

The myriad individual pieces of information on family planning service readiness and quality presented in this chapter can be used to construct an overall picture of service quality in sub-Saharan Africa. The Situation Analysis methodology in no way measures every aspect of quality, nor is it free from various biases. The results are informative nonetheless, and the general picture of service quality given here is presented in the spirit of recognizing the valuable information Situation Analysis can offer.

In general, the maturity of the family planning program alone predicts much of the quality of family planning services. Overall, Botswana and Zimbabwe, with two of the most mature and wellfunded programs in sub-Saharan Africa, fare relatively well on many indicators, while the opposite is true for some of the less developed programs. This result is not surprising, and in fact one need only read the country descriptions in Chapter 2 to 
anticipate many of the results. However, program maturity in no way determines all of the results. The sheer size of the population of women of reproductive age served, the method mix of each country, the quality and extent of training programs, and even the various cultures all affect service quality directly.

Many of the stronger points of family planning and reproductive health services in sub-Saharan Africa appear to revolve around readiness to provide services. The infrastructure of most SDPs, especially in urban areas, is quite adequate, and many have the equipment required for basic family planning services. Moreover, the pattern of methods offered is in some ways quite appropriate (the one serious problem being the lack of full availability of condoms in some countries), and stockout rates for most methods appear generally to be under control, excepting injectables.

Several strengths are also evident in the quality of services given to clients. The physical assessment of new family planning clients with weight, blood pressure, LMP, and medical history, for example, is carried out fairly consistently. The quality of injections is quite high in most countries, especially with regard to aseptic procedures-an important finding given the increasing use of injectables in many contexts. Also, across all the study sites, the vast majority of clients are given revisit dates, thereby encouraging continuity of method use.

The overall weaknesses of the programs revolve mainly around family planning counseling, asepsis, and STD/HIV integration. With regard to counseling, two aspects must be considered: information taken from clients about reproductive intentions, breastfeeding status, and other items that relate to family planning use, and information given to clients about the method accepted and STD risk. In both of these aspects, counseling is broadly lacking across all study sites. These activities are particularly important because they are directly related to client satisfaction, appropriateness of method selected, continuity of use, and STD/HIV risk. Moreover, IEC materials are extremely uncommon in most contexts, and even where they are present, are not usually used with clients. This nonuse of existing IEC materials rep- resents missed opportunities to communicate effectively with clients about their methods. (See Chapter 6 for more discussion of this point.)

The issue of asepsis also stands out as an area of concern. Many examination rooms do not have adequate water for washing hands or equipment, and handwashing and/or glove use are not practiced consistently during pelvic exams, even where water and gloves are available. In some cases, clients undergoing pelvic exams are given no protection whatsoever against cross-infection from other clients. Moreover, sterile instruments were not used for all observed IUD insertions. Because STD and HIV prevalence is so high in many of the study contexts, there can be no margin for error allowed on asepsis, so even the smallest lapses should be viewed as extremely serious.

Family planning clients are by definition sexually active, so they are at risk for STDs and HIV, but most programs do not adequately address this risk. Admittedly, most of the Situation Analysis studies included in this chapter were carried out before the ICPD in Cairo, at which the importance of STD/HIV integration with family planning services was stressed. Nonetheless, the results presented here reveal the great distance that many sub-Saharan African programs must travel to reach full integration. To begin with, the availability of condoms is in some places quite low, and even where they are available in most SDPs, they are often not discussed with clients with respect to either family planning or prevention of STD/HIV transmission. Moreover, clients are rarely told how their method relates to STD transmission or screened for STD symptoms. (See Chapter 10 for a more detailed discussion of STI/HIV integration.)

Many of the above observed strengths and weaknesses depend on the actions of staff, who are charged with the actual provision of family planning services. In turn, it is the responsibility of family planning programs to train providers adequately so their actions will result in high-quality care. The results of staff interviews show that family planning has in fact been integrated into many basic training courses, so most of the newer providers are trained. In addition, many programs appear recently to have been increasing their refresher training programs in IEC/counseling. 
However, a certain proportion of providers who attended basic training before the inclusion of family planning in the curriculum are still providing services. Of even more concern is the small number of providers who reported that they had had no training in IUD insertion and removal, yet were observed inserting IUDs. Thus although training programs may be improving in their preparation of new staff, older and untrained staff are still being used for service delivery. Moreover, the distribution of specially trained staff may be a problem in some contexts. That is, staff who are trained in IUD insertion or tubal ligation do not appear to be placed at SDPs that provide these services.

Some aspects of the relationship between provider training and activities with clients can also be seen from these data. Certain aspects appear to be clear and encouraging. An example is the increase in quality of counseling (information taken from and given to clients) among providers trained in IEC/counseling. Similarly, IEC training may be related to increased use of IEC materials. Interestingly, these activities do not appear related to training in clinical family planning. Clinical training may, however, increase the number of physical assessments of clients carried out. In addition, IUD training appears to be related to increased quality of IUD insertions. Yet not all of the relationships between training and quality of care are as positive. For example, training in IEC/counseling does not appear to be related to refraining from inappropriate overemphasis of a method. Also, in Senegal and Burkina Faso, clinical family planning training does not appear to be related to following aseptic procedures during pelvic exams. Perhaps not as surprisingly, training in IEC/counseling is not associated with improved interpersonal skills. In general, however, the data suggest that provider training can improve the quality of actions and interactions with clients, and the more specific the training, the stronger its effect on specific aspects of the quality of service provision. 


\section{Annex 3-1: Table of Results}

This table presents all the results for the indicators drawn from the Situation Analysis studies, grouped under the elements of readiness and quality, in the order in which they are discussed in the text. The numbering scheme used for the elements and indicators is explained in the introduction to the chapter. Note that the table often provides a further breakdown of the indicators presented in the text. In this table, blank cells mean that the question was not asked or the data are not usable. For percentages, the n's listed are the denominators; likewise for means, the n's are the total valid n's. The n's vary according to missing data and weighting.

The sources for the data are coded as follows: $\mathrm{I}=$ inventory, $\mathrm{O}=$ observation of client-provider interaction, $\mathrm{E}=$ exit interview of family planning clients, $\mathrm{S}=$ staff interview.
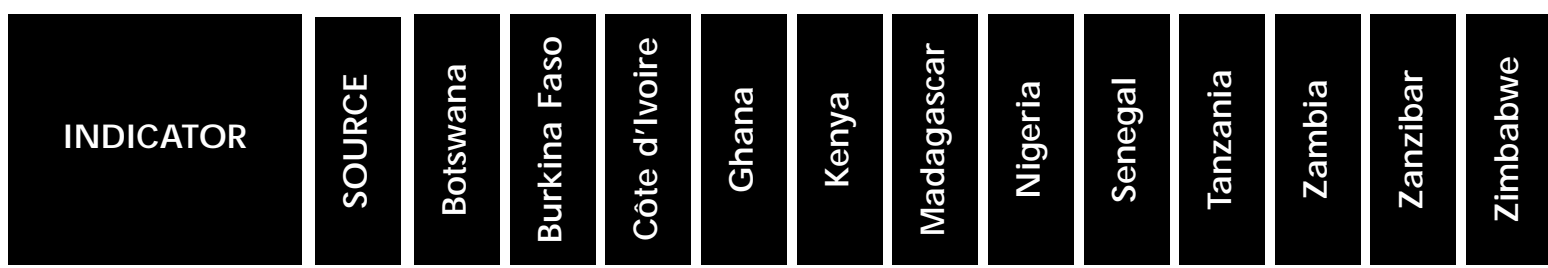

R. I READINESS TO PROVIDE SERVICES: INFRASTRUCTURE AND FACILITIES

\begin{tabular}{|c|c|c|c|c|c|c|c|c|c|c|c|c|c|}
\hline $\begin{array}{l}\text { R.1-1 \% of SDPs with } \\
\text { an adequate waiting } \\
\text { area }\end{array}$ & 1 & $\begin{array}{c}56 \\
n=184 \\
\end{array}$ & $\begin{array}{c}84 \\
n=340 \\
\end{array}$ & $\begin{array}{c}100 \\
n=12\end{array}$ & $\begin{array}{c}94 \\
n=269 \\
\end{array}$ & $\begin{array}{c}88 \\
n=211 \\
\end{array}$ & $\begin{array}{c}50 \\
n=159 \\
\end{array}$ & $\begin{array}{c}91 \\
n=165\end{array}$ & $\begin{array}{c}87 \\
n=180 \\
\end{array}$ & $\begin{array}{c}47 \\
n=345\end{array}$ & $\begin{array}{c}66 \\
n=253 \\
\end{array}$ & $\begin{array}{c}89 \\
n=99\end{array}$ & $\begin{array}{c}71 \\
n=190 \\
\end{array}$ \\
\hline $\begin{array}{l}\text { R.1-2 \% of SDPs with } \\
\text { working electricity }\end{array}$ & I & $\begin{array}{c}41 \\
n=184\end{array}$ & & & $\begin{array}{c}93 \\
n=194\end{array}$ & $\begin{array}{c}50 \\
n=216\end{array}$ & $\begin{array}{c}61 \\
n=158\end{array}$ & & $\begin{array}{c}90 \\
n=180\end{array}$ & & $\begin{array}{c}72 \\
n=253\end{array}$ & $\begin{array}{c}36 \\
n=99\end{array}$ & $\begin{array}{c}53 \\
n=192\end{array}$ \\
\hline $\begin{array}{l}\% \text { of SDPs with piped } \\
\text { running water }\end{array}$ & 1 & $\begin{array}{c}91 \\
n=179\end{array}$ & $\begin{array}{c}74 \\
n=341\end{array}$ & & $\begin{array}{c}84 \\
n=137\end{array}$ & $\begin{array}{c}58 \\
n=214\end{array}$ & $\begin{array}{c}61 \\
n=159\end{array}$ & & $\begin{array}{c}82 \\
n=180\end{array}$ & & $\begin{array}{c}44 \\
n=253\end{array}$ & $\begin{array}{c}46 \\
n=99\end{array}$ & $\begin{array}{c}70 \\
n=192\end{array}$ \\
\hline $\begin{array}{l}\text { R.1-3 \% of SDPs with } \\
\text { working toilet for } \\
\text { clients }\end{array}$ & 1 & $\begin{array}{c}88 \\
n=176 \\
\end{array}$ & $\begin{array}{c}77 \\
n=341 \\
\end{array}$ & $\begin{array}{c}77 \\
n=13\end{array}$ & $\begin{array}{c}93 \\
n=230 \\
\end{array}$ & $\begin{array}{c}85 \\
n=214 \\
\end{array}$ & $\begin{array}{c}56 \\
n=159 \\
\end{array}$ & $\begin{array}{c}88 \\
n=170 \\
\end{array}$ & $\begin{array}{c}79 \\
n=180 \\
\end{array}$ & $\begin{array}{c}74 \\
n=345 \\
\end{array}$ & $\begin{array}{c}90 \\
n=251 \\
\end{array}$ & $\begin{array}{c}61 \\
n=99\end{array}$ & $\begin{array}{c}94 \\
n=191 \\
\end{array}$ \\
\hline $\begin{array}{l}\text { R.1-4 \% of SDPs with } \\
\text { adequate light in exam } \\
\text { room }^{34}\end{array}$ & 1 & $\begin{array}{c}88 \\
n=183\end{array}$ & $\begin{array}{c}83 \\
n=341\end{array}$ & $\begin{array}{c}100 \\
n=13\end{array}$ & $\begin{array}{c}100 \\
n=283\end{array}$ & $\begin{array}{c}86 \\
n=215\end{array}$ & $\begin{array}{c}79 \\
n=159\end{array}$ & $\begin{array}{c}88 \\
n=164\end{array}$ & $\begin{array}{c}92 \\
\mathrm{n}=180\end{array}$ & $\begin{array}{c}84 \\
n=339\end{array}$ & $\begin{array}{c}85 \\
n=236\end{array}$ & $\begin{array}{c}83 \\
n=99\end{array}$ & $\begin{array}{c}92 \\
n=192\end{array}$ \\
\hline $\begin{array}{l}\% \text { of SDPs with clean } \\
\text { exam room } 35\end{array}$ & & $\begin{array}{c}97 \\
\mathrm{n}=181 \\
\end{array}$ & $\begin{array}{c}68 \\
n=341 \\
\end{array}$ & $\begin{array}{c}100 \\
n=13\end{array}$ & $\begin{array}{c}99 \\
n=285\end{array}$ & $\begin{array}{c}65 \\
n=215\end{array}$ & $\begin{array}{c}67 \\
n=159\end{array}$ & $\begin{array}{c}65 \\
n=161 \\
\end{array}$ & $\begin{array}{c}95 \\
\mathrm{n}=180\end{array}$ & $\begin{array}{c}71 \\
n=337\end{array}$ & $\begin{array}{c}73 \\
n=236\end{array}$ & $\begin{array}{c}89 \\
n=99\end{array}$ & $\begin{array}{c}90 \\
n=192\end{array}$ \\
\hline $\begin{array}{l}\% \text { of SDPs with } \\
\text { adequate water in } \\
\text { exam room }\end{array}$ & 1 & $\begin{array}{c}83 \\
n=181\end{array}$ & $\begin{array}{c}34 \\
n=341\end{array}$ & $\begin{array}{c}100 \\
n=13\end{array}$ & $\begin{array}{c}98 \\
n=235\end{array}$ & $\begin{array}{c}69 \\
n=213\end{array}$ & $\begin{array}{c}60 \\
n=159\end{array}$ & $\begin{array}{c}52 \\
n=165\end{array}$ & $\begin{array}{c}75 \\
n=180\end{array}$ & $\begin{array}{c}41 \\
n=342\end{array}$ & $\begin{array}{c}57 \\
n=235\end{array}$ & $\begin{array}{c}57 \\
n=98\end{array}$ & $\begin{array}{c}76 \\
n=192\end{array}$ \\
\hline
\end{tabular}



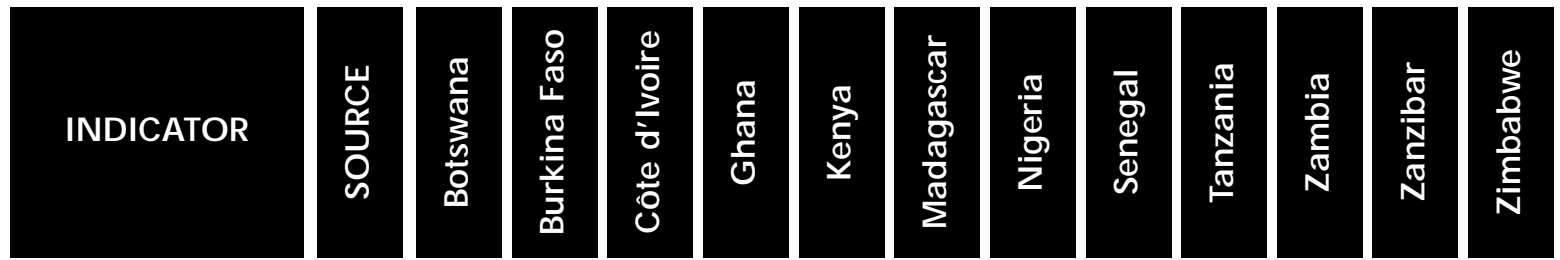

R. 2 READINESS TO PROVIDE SERVICES: ACCESSIBILITY

\begin{tabular}{|l|l|l|c|c|c|c|c|c|c|c|c|c|c|}
\hline $\begin{array}{l}\text { R.2-1 \% of SDPs open } \\
\text { on time }\end{array}$ & I & & & & $\begin{array}{c}61 \\
n=214\end{array}$ & $\begin{array}{c}5 \\
n=191\end{array}$ & $\begin{array}{c}27 \\
n=143\end{array}$ & $\begin{array}{c}64 \\
n=148\end{array}$ & $\begin{array}{c}22 \\
n=68\end{array}$ & $\begin{array}{c}81 \\
n=269\end{array}$ & $\begin{array}{c}43 \\
n=233\end{array}$ & $\begin{array}{c}80 \\
n=98\end{array}$ \\
\hline $\begin{array}{l}\text { R.2-2 \% of SDPs with } \\
\text { sign announcing } \\
\text { family planning } \\
\text { services inside or } \\
\text { outside SDP }\end{array}$ & & & & & & & & & & & & \\
\hline
\end{tabular}

R. 3 READINESS TO PROVIDE SERVICES: METHODS OFFERED

\begin{tabular}{|c|c|c|c|c|c|c|c|c|c|c|c|c|c|}
\hline $\begin{array}{l}\text { R.3-1 \% of SDPs that } \\
\text { offer COCs }\end{array}$ & I & $\begin{array}{c}97 \\
n=183\end{array}$ & $\begin{array}{c}98 \\
n=341\end{array}$ & $\begin{array}{c}100 \\
n=13\end{array}$ & $\begin{array}{c}91 \\
n=313\end{array}$ & $\begin{array}{c}98 \\
n=214\end{array}$ & $\begin{array}{c}97 \\
\mathrm{n}=159\end{array}$ & $\begin{array}{c}95 \\
n=168\end{array}$ & $\begin{array}{c}99 \\
\mathrm{n}=180\end{array}$ & $\begin{array}{c}96 \\
n=348\end{array}$ & \begin{tabular}{|c|}
95 \\
$n=250$
\end{tabular} & $\begin{array}{c}98 \\
n=100\end{array}$ & $\begin{array}{c}99 \\
n=191\end{array}$ \\
\hline $\begin{array}{l}\% \text { of SDPs that offer } \\
\text { injectables }\end{array}$ & 1 & $\begin{array}{c}93 \\
n=184\end{array}$ & $\begin{array}{c}57 \\
n=337\end{array}$ & $\begin{array}{c}92 \\
n=13\end{array}$ & $\begin{array}{c}90 \\
n=313\end{array}$ & $\begin{array}{c}94 \\
n=213\end{array}$ & $\begin{array}{c}96 \\
n=159\end{array}$ & $\begin{array}{c}90 \\
n=161\end{array}$ & $\begin{array}{c}68 \\
n=180\end{array}$ & $\begin{array}{c}30 \\
n=348\end{array}$ & $\begin{array}{c}50 \\
n=250\end{array}$ & $\begin{array}{c}96 \\
n=95\end{array}$ & $\begin{array}{c}92 \\
n=190\end{array}$ \\
\hline $\begin{array}{l}\% \text { of SDPs that offer } \\
\text { POPs }\end{array}$ & I & $\begin{array}{c}96 \\
n=183\end{array}$ & $\begin{array}{c}62 \\
n=341\end{array}$ & $\begin{array}{c}92 \\
n=13\end{array}$ & $\begin{array}{c}73 \\
n=313\end{array}$ & $\begin{array}{c}93 \\
n=213\end{array}$ & $\begin{array}{c}77 \\
n=159\end{array}$ & $\begin{array}{c}23 \\
n=165\end{array}$ & $\begin{array}{c}98 \\
n=180\end{array}$ & $\begin{array}{c}56 \\
n=348\end{array}$ & $\begin{array}{c}69 \\
n=249\end{array}$ & $\begin{array}{c}51 \\
n=73\end{array}$ & $\begin{array}{c}99 \\
n=191\end{array}$ \\
\hline $\begin{array}{l}\% \text { of SDPs that offer } \\
\text { N ORPLANT }^{\circledR 38}\end{array}$ & 1 & $\begin{array}{c}1 \\
n=184\end{array}$ & $\begin{array}{c}2 \\
n=337\end{array}$ & & $\begin{array}{c}7 \\
n=313\end{array}$ & $\begin{array}{c}14 \\
n=212\end{array}$ & $\begin{array}{c}5 \\
n=159\end{array}$ & $\begin{array}{c}1 \\
n=153\end{array}$ & $\begin{array}{c}3 \\
n=180\end{array}$ & $\begin{array}{c}0 \\
n=348\end{array}$ & $\begin{array}{c}0 \\
n=249\end{array}$ & & $\begin{array}{c}4 \\
n=188\end{array}$ \\
\hline $\begin{array}{l}\text { R.3-2 \% of SDPs } \\
\text { that offer condoms }\end{array}$ & I & $\begin{array}{c}99 \\
n=183\end{array}$ & $\begin{array}{c}53 \\
n=341\end{array}$ & $\begin{array}{c}92 \\
n=13\end{array}$ & $\begin{array}{c}89 \\
n=313\end{array}$ & $\begin{array}{c}98 \\
n=214\end{array}$ & $\begin{array}{c}94 \\
n=159\end{array}$ & $\begin{array}{c}94 \\
n=160\end{array}$ & $\begin{array}{c}97 \\
\mathrm{n}=180\end{array}$ & $\begin{array}{c}74 \\
n=348\end{array}$ & $\begin{array}{c}97 \\
n=251\end{array}$ & $\begin{array}{c}70 \\
n=79\end{array}$ & $\begin{array}{c}98 \\
n=190\end{array}$ \\
\hline $\begin{array}{l}\% \text { of SDPs that offer } \\
\text { dual-method } \\
\text { counseling }\end{array}$ & I & & & & $\begin{array}{c}67 \\
n=313\end{array}$ & & & & & & $\begin{array}{c}50 \\
n=251\end{array}$ & & $\begin{array}{c}71 \\
n=191\end{array}$ \\
\hline $\begin{array}{l}\text { R.3-3 } \% \text { of SDPs that } \\
\text { offer IUDs }\end{array}$ & I & $\begin{array}{c}56 \\
n=182\end{array}$ & $\begin{array}{c}26 \\
n=341\end{array}$ & $\begin{array}{c}92 \\
n=13\end{array}$ & $\begin{array}{c}57 \\
n=313\end{array}$ & $\begin{array}{c}72 \\
n=214\end{array}$ & $\begin{array}{c}70 \\
n=159\end{array}$ & $\begin{array}{c}80 \\
n=168\end{array}$ & $\begin{array}{c}83 \\
n=180\end{array}$ & $\begin{array}{c}14 \\
n=348\end{array}$ & $\begin{array}{c}19 \\
n=250\end{array}$ & $\begin{array}{c}55 \\
n=69\end{array}$ & $\begin{array}{c}8 \\
n=190\end{array}$ \\
\hline $\begin{array}{l}\text { R.3-4 \% of SDPs that } \\
\text { offer tubal ligation }\end{array}$ & 1 & $\begin{array}{c}5 \\
n=184\end{array}$ & $\begin{array}{c}1 \\
n=341\end{array}$ & $\begin{array}{c}0 \\
n=13\end{array}$ & $\begin{array}{c}50 \\
n=313\end{array}$ & $\begin{array}{c}29 \\
n=214\end{array}$ & $\begin{array}{c}6 \\
n=159\end{array}$ & $\begin{array}{c}10 \\
n=152\end{array}$ & $\begin{array}{c}5 \\
n=180\end{array}$ & $\begin{array}{c}2 \\
n=348\end{array}$ & $\begin{array}{c}9 \\
n=250\end{array}$ & & $\begin{array}{c}9 \\
n=191\end{array}$ \\
\hline $\begin{array}{l}\% \text { of SDPs that offer } \\
\text { vasectomy }\end{array}$ & 1 & $\begin{array}{c}3 \\
n=184 \\
\end{array}$ & $\begin{array}{c}0 \\
n=341\end{array}$ & $\begin{array}{c}0 \\
n=13\end{array}$ & $\begin{array}{c}23 \\
n=313\end{array}$ & $\begin{array}{c}14 \\
n=213\end{array}$ & $\begin{array}{c}3 \\
n=159\end{array}$ & $\begin{array}{c}1 \\
n=153\end{array}$ & $\begin{array}{c}1 \\
n=180\end{array}$ & $\begin{array}{c}0 \\
n=348\end{array}$ & $\begin{array}{c}5 \\
n=251\end{array}$ & & $\begin{array}{c}3 \\
n=191\end{array}$ \\
\hline $\begin{array}{l}\text { R.3-5 \% of SDPs that } \\
\text { offer natural family } \\
\text { planning }\end{array}$ & 1 & $\begin{array}{c}12 \\
n=183\end{array}$ & $\begin{array}{c}12 \\
n=341\end{array}$ & $\begin{array}{c}54 \\
n=13\end{array}$ & $\begin{array}{c}74 \\
n=313\end{array}$ & $\begin{array}{c}16 \\
n=214\end{array}$ & $\begin{array}{c}4 \\
n=159\end{array}$ & $\begin{array}{c}37 \\
n=156\end{array}$ & $\begin{array}{c}4 \\
n=180\end{array}$ & $\begin{array}{c}3 \\
n=348\end{array}$ & $\begin{array}{c}53 \\
n=249\end{array}$ & $\begin{array}{c}31 \\
n=100\end{array}$ & $\begin{array}{c}28 \\
n=190\end{array}$ \\
\hline $\begin{array}{l}\% \text { of SDPs that offer } \\
\text { LAM } \\
n=190\end{array}$ & I & & $\begin{array}{c}10 \\
n=341\end{array}$ & & $\begin{array}{c}83 \\
n=313\end{array}$ & & & & & & 63 & $n=251$ & 39 \\
\hline
\end{tabular}

72 Clinic-Based Family Planning and Reproductive Health Services in Africa 

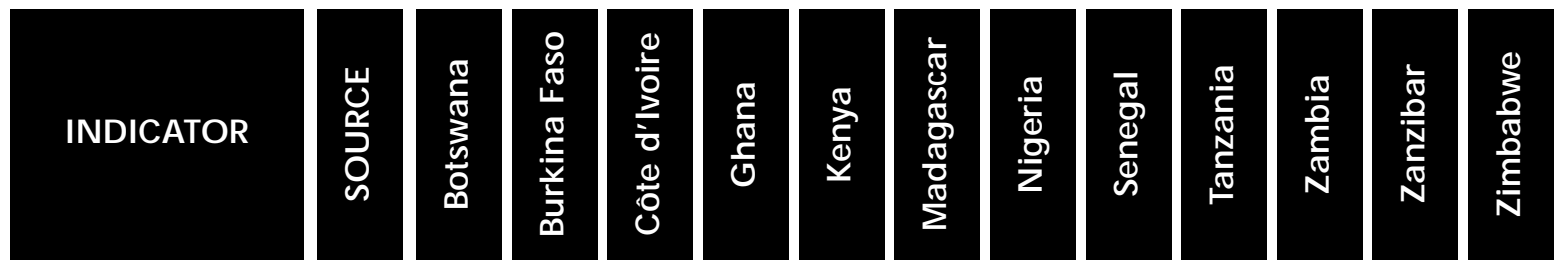

R.4 READINESS TO PROVIDE SERVICES: CONTRACEPTIVE SUPPLIES AND LOGISTICS ${ }^{39}$

\begin{tabular}{|c|c|c|c|c|c|c|c|c|c|c|c|c|c|}
\hline $\begin{array}{l}\text { R.4-1 \% of SDPs that } \\
\text { have experienced a } \\
\text { stockout of condoms } \\
\text { in the previous } \\
6 \text { months }^{40}\end{array}$ & 1 & $\begin{array}{c}22 \\
n=171\end{array}$ & $\begin{array}{c}16 \\
\mathrm{n}=175\end{array}$ & & $\begin{array}{c}7 \\
n=279\end{array}$ & $\begin{array}{c}14 \\
n=194\end{array}$ & $\begin{array}{c}6 \\
n=145\end{array}$ & $\begin{array}{c}16 \\
n=137\end{array}$ & $\begin{array}{c}6 \\
n=173\end{array}$ & $\begin{array}{c}5 \\
n=249\end{array}$ & $\begin{array}{c}12 \\
n=235\end{array}$ & $\begin{array}{c}0 \\
n=53\end{array}$ & $\begin{array}{c}29 \\
n=180\end{array}$ \\
\hline $\begin{array}{l}\% \text { of SDPs that have } \\
\text { experienced a } \\
\text { stockout of COCs in } \\
\text { the previous } \\
6 \text { months }\end{array}$ & 1 & $\begin{array}{c}10 \\
n=168\end{array}$ & & & $\begin{array}{c}14 \\
\mathrm{n}=285\end{array}$ & $\begin{array}{c}18 \\
n=195\end{array}$ & & $\begin{array}{c}35 \\
n=149\end{array}$ & & $\begin{array}{c}6 \\
n=323\end{array}$ & $\begin{array}{c}30 \\
n=236\end{array}$ & $\begin{array}{c}0 \\
n=97\end{array}$ & $\begin{array}{c}19 \\
n=185\end{array}$ \\
\hline $\begin{array}{l}\% \text { of SDPs that have } \\
\text { experienced a } \\
\text { stockout of POPs } \\
\text { in the previous } \\
6 \text { months }^{42}\end{array}$ & 1 & $\begin{array}{c}13 \\
n=167\end{array}$ & $\begin{array}{c}20 \\
n=200\end{array}$ & & $\begin{array}{c}17 \\
n=229\end{array}$ & $\begin{array}{c}18 \\
n=187\end{array}$ & & $\begin{array}{c}32 \\
n=23\end{array}$ & $\begin{array}{c}3 \\
n=175\end{array}$ & $\begin{array}{c}13 \\
n=186\end{array}$ & $\begin{array}{c}34 \\
n=170\end{array}$ & $\begin{array}{c}0 \\
n=37\end{array}$ & $\begin{array}{c}22 \\
n=186\end{array}$ \\
\hline $\begin{array}{l}\% \text { of SDPs that have } \\
\text { experienced a } \\
\text { stockout of IUDs in } \\
\text { the previous } \\
6 \text { months }{ }^{43}\end{array}$ & 1 & $\begin{array}{c}12 \\
n=82\end{array}$ & & & $\begin{array}{c}10 \\
\mathrm{n}=177\end{array}$ & $\begin{array}{c}36 \\
n=135\end{array}$ & & $\begin{array}{c}21 \\
n=126\end{array}$ & & $\begin{array}{c}3 \\
n=42\end{array}$ & $\begin{array}{c}16 \\
n=45\end{array}$ & $\begin{array}{c}5 \\
n=38\end{array}$ & $\begin{array}{c}22 \\
n=15\end{array}$ \\
\hline $\begin{array}{l}\% \text { of SDPs that have } \\
\text { experienced a } \\
\text { stockout of } \\
\text { injectables in the } \\
\text { previous } 6 \text { months }{ }^{44}\end{array}$ & 1 & & & & $\begin{array}{c}19 \\
n=281\end{array}$ & & & $\begin{array}{c}50 \\
n=135\end{array}$ & & $\begin{array}{c}5 \\
n=92\end{array}$ & $\begin{array}{c}28 \\
n=123\end{array}$ & $\begin{array}{c}1 \\
n=88\end{array}$ & $\begin{array}{c}29 \\
n=172\end{array}$ \\
\hline $\begin{array}{l}\% \text { of SDPs that have } \\
\text { experienced a } \\
\text { stockout of } \\
\text { NORPLANT }^{\circledast} \text { in the } \\
\text { previous } 6 \text { months }{ }^{45}\end{array}$ & 1 & $\begin{array}{c}0 \\
n=2\end{array}$ & $\begin{array}{c}0 \\
n=6\end{array}$ & & $\begin{array}{c}5 \\
n=22\end{array}$ & $\begin{array}{c}22 \\
n=23\end{array}$ & $\begin{array}{c}0 \\
n=4\end{array}$ & $\begin{array}{c}0 \\
n=1\end{array}$ & $\begin{array}{c}0 \\
n=5\end{array}$ & & $\begin{array}{l}100 \\
n=1\end{array}$ & & $\begin{array}{c}14 \\
n=7\end{array}$ \\
\hline $\begin{array}{l}\% \text { of SDPs that have } \\
\text { experienced at least } \\
\text { one stockout of an } \\
\text { offered method in } \\
\text { the last } 6 \text { months }\end{array}$ & 1 & $\begin{array}{c}37 \\
n=174\end{array}$ & $\begin{array}{c}24 \\
n=258\end{array}$ & & $\begin{array}{c}33 \\
n=293\end{array}$ & $\begin{array}{c}49 \\
n=199\end{array}$ & $\begin{array}{c}6 \\
n=147\end{array}$ & $\begin{array}{c}65 \\
n=152\end{array}$ & $\begin{array}{c}8 \\
n=179\end{array}$ & $\begin{array}{c}13 \\
n=336\end{array}$ & $\begin{array}{c}49 \\
n=247\end{array}$ & $\begin{array}{c}2 \\
n=98\end{array}$ & $\begin{array}{c}53 \\
n=188\end{array}$ \\
\hline $\begin{array}{l}\text { R. } 4-2 \% \text { of SDPs with } \\
\text { a commodity } \\
\text { inventory }\end{array}$ & 1 & $\begin{array}{c}77 \\
n=169\end{array}$ & $\begin{array}{c}29 \\
n=335\end{array}$ & $\begin{array}{c}100 \\
n=13\end{array}$ & $\begin{array}{c}98 \\
n=212\end{array}$ & $\begin{array}{c}56 \\
n=176\end{array}$ & $\begin{array}{c}85 \\
n=157\end{array}$ & $\begin{array}{c}85 \\
n=160\end{array}$ & $\begin{array}{c}52 \\
n=172\end{array}$ & $\begin{array}{c}38 \\
n=343\end{array}$ & $\begin{array}{c}71 \\
n=248\end{array}$ & $\begin{array}{c}82 \\
n=100\end{array}$ & $\begin{array}{c}93 \\
n=191\end{array}$ \\
\hline $\begin{array}{l}\text { R.4-3 } \% \text { of SDPs with } \\
\text { adequate storage } \\
\text { facilities for } \\
\text { commodities }^{46}\end{array}$ & 1 & $\begin{array}{c}77 \\
n=162\end{array}$ & $\begin{array}{c}76 \\
n=304\end{array}$ & $\begin{array}{c}8 \\
n=13\end{array}$ & $\begin{array}{c}100 \\
n=261\end{array}$ & $\begin{array}{c}86 \\
n=190\end{array}$ & $\begin{array}{c}84 \\
n=155\end{array}$ & $\begin{array}{c}84 \\
n=156\end{array}$ & $\begin{array}{c}79 \\
n=173\end{array}$ & $\begin{array}{c}83 \\
n=326\end{array}$ & $\begin{array}{c}89 \\
n=235\end{array}$ & $\begin{array}{c}81 \\
n=100\end{array}$ & $\begin{array}{c}99 \\
n=190\end{array}$ \\
\hline
\end{tabular}



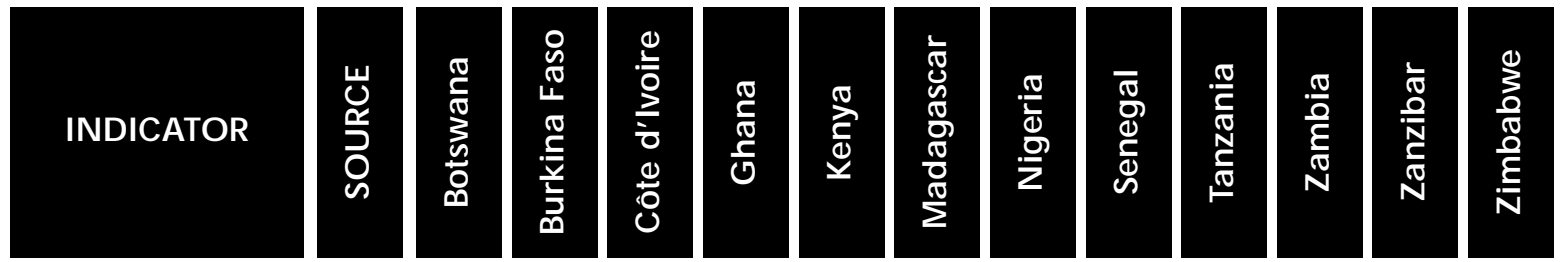

R.5 READINESS TO PROVIDE SERVICES: EQUIPMEN T ${ }^{47}$

\begin{tabular}{|c|c|c|c|c|c|c|c|c|c|c|c|c|c|}
\hline $\begin{array}{l}\text { R.5-1 \% of SDPs with } \\
\text { enough adult } \\
\text { weighing scales } \\
\end{array}$ & 1 & $\begin{array}{c}95 \\
n=184 \\
\end{array}$ & $\begin{array}{c}89 \\
n=341 \\
\end{array}$ & $\begin{array}{c}85 \\
n=13\end{array}$ & & $\begin{array}{c}89 \\
n=214 \\
\end{array}$ & $\begin{array}{c}84 \\
n=159 \\
\end{array}$ & $\begin{array}{c}74 \\
n=166 \\
\end{array}$ & $\begin{array}{c}91 \\
n=178 \\
\end{array}$ & $\begin{array}{c}75 \\
n=348 \\
\end{array}$ & $\begin{array}{c}96 \\
n=254 \\
\end{array}$ & $\begin{array}{c}93 \\
n=100 \\
\end{array}$ & $\begin{array}{c}98 \\
n=192 \\
\end{array}$ \\
\hline $\begin{array}{l}\% \text { of SDPs with } \\
\text { enough blood } \\
\text { pressure machines }\end{array}$ & 1 & $\begin{array}{c}90 \\
n=183\end{array}$ & $\begin{array}{c}83 \\
n=341\end{array}$ & $\begin{array}{c}69 \\
n=13\end{array}$ & & $\begin{array}{c}60 \\
n=214\end{array}$ & $\begin{array}{c}70 \\
n=159\end{array}$ & $\begin{array}{c}56 \\
n=159\end{array}$ & $\begin{array}{c}75 \\
n=178\end{array}$ & $\begin{array}{c}55 \\
n=348\end{array}$ & & $\begin{array}{c}87 \\
n=100\end{array}$ & $\begin{array}{c}85 \\
n=187\end{array}$ \\
\hline $\begin{array}{l}\% \text { of SDPs with } \\
\text { enough stethoscopes }\end{array}$ & I & $\begin{array}{c}94 \\
n=184\end{array}$ & $\begin{array}{c}87 \\
n=339 \\
\end{array}$ & $\begin{array}{c}77 \\
n=13\end{array}$ & & $\begin{array}{c}65 \\
n=214 \\
\end{array}$ & $\begin{array}{c}69 \\
n=159 \\
\end{array}$ & $\begin{array}{c}52 \\
n=160 \\
\end{array}$ & $\begin{array}{c}80 \\
n=178 \\
\end{array}$ & $\begin{array}{c}62 \\
n=348 \\
\end{array}$ & & $\begin{array}{c}85 \\
n=100 \\
\end{array}$ & $\begin{array}{c}90 \\
n=185\end{array}$ \\
\hline $\begin{array}{l}\% \text { of SDPs with } \\
\text { access to sterilizing } \\
\text { equipment }\end{array}$ & 1 & $\begin{array}{c}75 \\
n=184\end{array}$ & $\begin{array}{c}57 \\
n=341\end{array}$ & $\begin{array}{c}77 \\
n=13\end{array}$ & & $\begin{array}{c}78 \\
n=211\end{array}$ & $\begin{array}{c}70 \\
n=159\end{array}$ & $\begin{array}{c}44 \\
n=152 \\
\end{array}$ & $\begin{array}{c}86 \\
n=178\end{array}$ & $\begin{array}{c}66 \\
n=348 \\
\end{array}$ & & $\begin{array}{c}97 \\
n=100 \\
\end{array}$ & \\
\hline $\begin{array}{l}\text { R.5-2 \% of SDPs with } \\
\text { enough exam couches }\end{array}$ & I & $\begin{array}{c}95 \\
n=183\end{array}$ & $\begin{array}{c}66 \\
n=341\end{array}$ & $\begin{array}{c}85 \\
n=13\end{array}$ & & $\begin{array}{c}81 \\
n=214\end{array}$ & $\begin{array}{c}70 \\
n=159\end{array}$ & $\begin{array}{c}72 \\
n=167\end{array}$ & $\begin{array}{c}92 \\
n=178\end{array}$ & $\begin{array}{c}53 \\
n=348\end{array}$ & & $\begin{array}{c}86 \\
n=100\end{array}$ & \\
\hline $\begin{array}{l}\% \text { of SDPs with } \\
\text { enough angle poise } \\
\text { lamps or flashlights }\end{array}$ & 1 & $\begin{array}{c}48 \\
n=184\end{array}$ & $\begin{array}{c}64 \\
n=341\end{array}$ & $\begin{array}{c}85 \\
n=13\end{array}$ & & $\begin{array}{c}35 \\
n=210\end{array}$ & $\begin{array}{c}54 \\
n=159\end{array}$ & $\begin{array}{c}61 \\
n=160\end{array}$ & $\begin{array}{c}72 \\
n=178\end{array}$ & $\begin{array}{c}18 \\
n=348\end{array}$ & & $\begin{array}{c}54 \\
n=100 \\
\end{array}$ & \\
\hline $\begin{array}{l}\% \text { of SDPs with } \\
\text { enough specula }{ }^{48}\end{array}$ & 1 & $\begin{array}{c}80 \\
n=179\end{array}$ & $\begin{array}{c}80 \\
n=341\end{array}$ & $\begin{array}{c}85 \\
n=13\end{array}$ & $\begin{array}{c}47 \\
n=254\end{array}$ & $\begin{array}{c}40 \\
n=211 \\
\end{array}$ & $\begin{array}{c}41 \\
n=159\end{array}$ & $\begin{array}{c}52 \\
n=157\end{array}$ & $\begin{array}{c}92 \\
n=178 \\
\end{array}$ & $\begin{array}{c}38 \\
n=348\end{array}$ & $\begin{array}{c}60 \\
n=248\end{array}$ & $\begin{array}{c}86 \\
n=100 \\
\end{array}$ & $\begin{array}{c}81 \\
n=186\end{array}$ \\
\hline $\begin{array}{l}\% \text { of SDPs with } \\
\text { enough gloves }\end{array}$ & I & $\begin{array}{c}96 \\
n=184\end{array}$ & $\begin{array}{c}22 \\
n=341\end{array}$ & $\begin{array}{c}46 \\
n=13\end{array}$ & $\begin{array}{c}71 \\
n=200\end{array}$ & $\begin{array}{c}74 \\
n=208\end{array}$ & $\begin{array}{c}44 \\
n=159\end{array}$ & $\begin{array}{c}49 \\
n=154\end{array}$ & $\begin{array}{c}61 \\
n=178\end{array}$ & $\begin{array}{c}44 \\
n=348\end{array}$ & $\begin{array}{c}89 \\
n=243\end{array}$ & $\begin{array}{c}62 \\
n=100\end{array}$ & $\begin{array}{c}96 \\
n=186\end{array}$ \\
\hline $\begin{array}{l}\% \text { of SDPs with } \\
\text { enough sterilizing } \\
\text { lotion }^{49}\end{array}$ & 1 & $\begin{array}{c}96 \\
n=175\end{array}$ & $\begin{array}{c}43 \\
n=341\end{array}$ & $\begin{array}{c}39 \\
n=13\end{array}$ & & $\begin{array}{c}52 \\
n=200 \\
\end{array}$ & $\begin{array}{c}63 \\
n=159 \\
\end{array}$ & $\begin{array}{c}38 \\
n=147\end{array}$ & & $\begin{array}{c}3 \\
n=348\end{array}$ & & $\begin{array}{c}39 \\
n=100 \\
\end{array}$ & \\
\hline $\begin{array}{l}\text { R.5-3 } \% \text { of SDPs with } \\
\text { enough tenacula }\end{array}$ & 1 & $\begin{array}{c}64 \\
n=101\end{array}$ & & & $\begin{array}{c}91 \\
n=165\end{array}$ & $\begin{array}{c}73 \\
n=151 \\
\end{array}$ & & $\begin{array}{c}79 \\
n=123\end{array}$ & & $\begin{array}{c}57 \\
n=49\end{array}$ & $\begin{array}{c}70 \\
n=47\end{array}$ & $\begin{array}{c}84 \\
n=69 \\
\end{array}$ & $\begin{array}{c}41 \\
n=16\end{array}$ \\
\hline $\begin{array}{l}\% \text { of SDPs with } \\
\text { enough uterine } \\
\text { sounds }{ }^{51}\end{array}$ & 1 & $\begin{array}{c}73 \\
n=101\end{array}$ & $\begin{array}{c}91 \\
n=87\end{array}$ & $\begin{array}{c}83 \\
n=12\end{array}$ & $\begin{array}{c}92 \\
n=170\end{array}$ & $\begin{array}{c}79 \\
n=152\end{array}$ & $\begin{array}{c}79 \\
n=111\end{array}$ & $\begin{array}{c}84 \\
n=133\end{array}$ & $\begin{array}{c}97 \\
n=148\end{array}$ & $\begin{array}{c}66 \\
n=49\end{array}$ & $\begin{array}{c}72 \\
n=46\end{array}$ & $\begin{array}{c}83 \\
n=69 \\
\end{array}$ & $\begin{array}{c}49 \\
n=17\end{array}$ \\
\hline $\begin{array}{l}\% \text { of SDPs with } \\
\text { enough gloves } \\
\end{array}$ & 1 & $\begin{array}{c}94 \\
n=101\end{array}$ & $\begin{array}{c}23 \\
n=87\end{array}$ & $\begin{array}{c}50 \\
n=12\end{array}$ & $\begin{array}{c}72 \\
n=124\end{array}$ & $\begin{array}{c}76 \\
n=147\end{array}$ & $\begin{array}{c}52 \\
n=111 \\
\end{array}$ & $\begin{array}{c}55 \\
n=128\end{array}$ & \begin{tabular}{|c|}
62 \\
$n=148$ \\
\end{tabular} & $\begin{array}{c}50 \\
n=49\end{array}$ & $\begin{array}{c}82 \\
n=45\end{array}$ & \begin{tabular}{c|}
71 \\
$n=38$ \\
\end{tabular} & $\begin{array}{c}93 \\
n=16\end{array}$ \\
\hline $\begin{array}{l}\% \text { of SDPs with } \\
\text { enough specula }\end{array}$ & 1 & $\begin{array}{c}86 \\
n=98\end{array}$ & $\begin{array}{c}92 \\
n=87\end{array}$ & $\begin{array}{c}92 \\
n=12\end{array}$ & $\begin{array}{c}60 \\
n=172\end{array}$ & $\begin{array}{c}38 \\
n=152 \\
\end{array}$ & $\begin{array}{c}48 \\
n=111\end{array}$ & $\begin{array}{c}57 \\
n=131\end{array}$ & $\begin{array}{c}94 \\
n=148\end{array}$ & $\begin{array}{c}55 \\
n=49\end{array}$ & $\begin{array}{c}62 \\
n=45 \\
\end{array}$ & $\begin{array}{c}84 \\
n=38 \\
\end{array}$ & $\begin{array}{c}64 \\
n=15\end{array}$ \\
\hline $\begin{array}{l}\text { R.5-4 \% of SDPs with } \\
\text { enough needles and } \\
\text { syringes }\end{array}$ & 1 & $\begin{array}{c}90 \\
n=184\end{array}$ & $\begin{array}{c}22 \\
n=341\end{array}$ & & & $\begin{array}{c}82 \\
n=206\end{array}$ & $\begin{array}{c}74 \\
n=159 \\
\end{array}$ & $\begin{array}{c}27 \\
n=146\end{array}$ & $\begin{array}{c}33 \\
n=178\end{array}$ & $\begin{array}{c}58 \\
n=347\end{array}$ & & $\begin{array}{c}55 \\
n=100\end{array}$ & \\
\hline $\begin{array}{l}\text { R.5-5 } \% \text { of SDPs with } \\
\text { enough N ORPLANT } \\
\text { kits }^{54}\end{array}$ & I & $\begin{array}{c}50 \\
n=2\end{array}$ & $\begin{array}{l}100 \\
n=6\end{array}$ & & & $\begin{array}{c}95 \\
n=21\end{array}$ & $\begin{array}{c}75 \\
n=8\end{array}$ & $\begin{array}{l}100 \\
n=1\end{array}$ & $\begin{array}{l}100 \\
n=4\end{array}$ & & & & \\
\hline
\end{tabular}

74 Clinic-Based Family Planning and Reproductive Health Services in Africa 

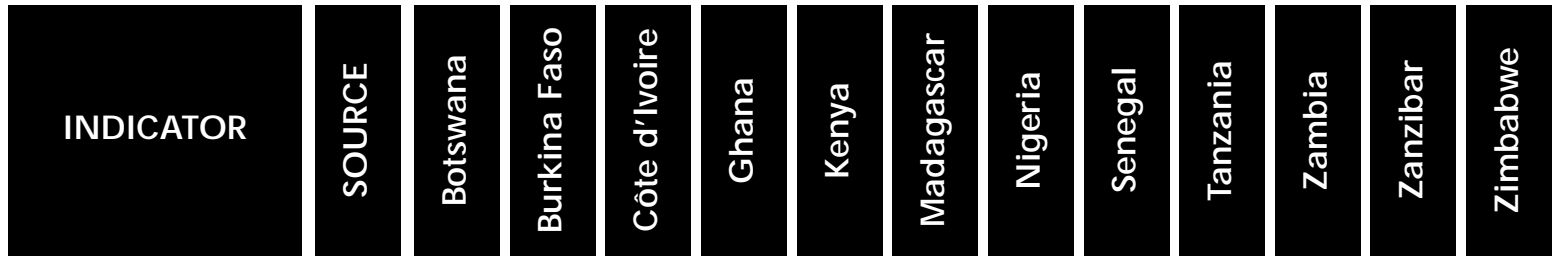

\begin{tabular}{|c|c|c|c|c|c|c|c|c|c|c|c|c|c|}
\hline $\begin{array}{l}\text { R.6-1 \% of SDPs with } \\
\text { at least one nurse on } \\
\text { duty on the day of } \\
\text { the study visit }\end{array}$ & 1 & $\begin{array}{c}72 \\
n=183 \\
\end{array}$ & $\begin{array}{c}87 \\
n=341\end{array}$ & $\begin{array}{c}100 \\
n=13\end{array}$ & $\begin{array}{c}94 \\
n=304\end{array}$ & $\begin{array}{c}91 \\
n=214\end{array}$ & $\begin{array}{c}72 \\
n=159\end{array}$ & $\begin{array}{c}95 \\
n=147\end{array}$ & $\begin{array}{c}94 \\
\mathrm{n}=180\end{array}$ & & $\begin{array}{c}75 \\
n=248\end{array}$ & $\begin{array}{c}38 \\
n=100 \\
\end{array}$ & $\begin{array}{c}92 \\
n=190\end{array}$ \\
\hline $\begin{array}{l}\% \text { of SDPs with at } \\
\text { least one doctor on } \\
\text { duty on the day of } \\
\text { the study visit }\end{array}$ & 1 & $\begin{array}{c}2 \\
n=183 \\
\end{array}$ & $\begin{array}{c}2 \\
n=341\end{array}$ & $\begin{array}{c}85 \\
n=13\end{array}$ & $\begin{array}{c}5 \\
n=309\end{array}$ & $\begin{array}{c}6 \\
n=202\end{array}$ & $\begin{array}{c}62 \\
n=159 \\
\end{array}$ & $\begin{array}{c}31 \\
n=31\end{array}$ & $\begin{array}{c}2 \\
n=180\end{array}$ & & $\begin{array}{c}31 \\
n=233\end{array}$ & $\begin{array}{c}0 \\
n=100\end{array}$ & $\begin{array}{c}8 \\
n=173\end{array}$ \\
\hline $\begin{array}{l}\text { R.6-2 Mean years staff } \\
\text { have been offering } \\
\text { family planning } \\
\text { services (at all) }\end{array}$ & $\mathrm{S}$ & & $\begin{array}{c}3.4 \\
n=680\end{array}$ & $\begin{array}{c}3.8 \\
n=51\end{array}$ & & & $\begin{array}{c}4.3 \\
n=316\end{array}$ & $\begin{array}{c}4.1 \\
n=283\end{array}$ & $\begin{array}{c}5.1 \\
n=360\end{array}$ & $\begin{array}{c}6.3 \\
n=590 \\
\end{array}$ & & $\begin{array}{c}4.1 \\
n=189 \\
\end{array}$ & \\
\hline $\begin{array}{l}\text { Mean years staff have } \\
\text { been offering family } \\
\text { planning services } \\
\text { (at this SDP) }\end{array}$ & $S$ & $\begin{array}{c}6.6 \\
n=394 \\
\end{array}$ & & & & $\begin{array}{c}4.4 \\
n=382 \\
\end{array}$ & & & & & & & \\
\hline $\begin{array}{l}\text { R. } 6-3 \% \text { of staff whose } \\
\text { basic training } \\
\text { included family } \\
\text { planning }\end{array}$ & $\mathrm{S}$ & $\begin{array}{c}92 \\
n=455 \\
\end{array}$ & & $\begin{array}{c}41 \\
n=51\end{array}$ & $\begin{array}{c}92 \\
n=549\end{array}$ & $\begin{array}{c}62 \\
n=448 \\
\end{array}$ & & $\begin{array}{c}53 \\
n=289\end{array}$ & & $\begin{array}{c}89 \\
n=598 \\
\end{array}$ & $\begin{array}{c}77 \\
n=342 \\
\end{array}$ & $\begin{array}{c}67 \\
n=189 \\
\end{array}$ & $\begin{array}{c}66 \\
n=280\end{array}$ \\
\hline $\begin{array}{l}\text { Mean years ago basic } \\
\text { training took place }\end{array}$ & $\mathrm{S}$ & $\begin{array}{c}11.6 \\
n=446\end{array}$ & & & $\begin{array}{c}10.8 \\
n=549\end{array}$ & $\begin{array}{c}12.8 \\
n=429\end{array}$ & & $\begin{array}{c}11.3 \\
n=283\end{array}$ & & $\begin{array}{c}9.7 \\
n=584\end{array}$ & $\begin{array}{c}14.2 \\
n=341\end{array}$ & & $\begin{array}{c}13.3 \\
n=280\end{array}$ \\
\hline $\begin{array}{l}\% \text { of staff who have } \\
\text { had training in clinical } \\
\text { family planning }\end{array}$ & $S$ & & $\begin{array}{c}68 \\
n=685\end{array}$ & & & & $\begin{array}{c}40 \\
n=316\end{array}$ & & $\begin{array}{c}62 \\
n=361 \\
\end{array}$ & & & & \\
\hline $\begin{array}{l}\text { Mean years ago } \\
\text { clinical training } \\
\text { took place }\end{array}$ & $\mathrm{S}$ & & $\begin{array}{c}3.3 \\
n=440 \\
\end{array}$ & & & & $\begin{array}{c}2.7 \\
n=125 \\
\end{array}$ & & $\begin{array}{c}5.7 \\
n=220 \\
\end{array}$ & & & & \\
\hline $\begin{array}{l}\text { R.6- } 4 \% \text { of staff who } \\
\text { have had refresher } \\
\text { training in family } \\
\text { planning }\end{array}$ & $S$ & $\begin{array}{c}60 \\
n=445\end{array}$ & & $\begin{array}{c}6 \\
n=51\end{array}$ & $\begin{array}{c}74 \\
n=570\end{array}$ & $\begin{array}{c}63 \\
n=442\end{array}$ & & & & & $\begin{array}{c}54 \\
n=357\end{array}$ & & $\begin{array}{c}62 \\
n=376\end{array}$ \\
\hline $\begin{array}{l}\% \text { of staff who have } \\
\text { had basic or refresher } \\
\text { training in family } \\
\text { planning }\end{array}$ & $\mathrm{S}$ & $\begin{array}{c}98 \\
n=450 \\
\end{array}$ & & $\begin{array}{c}41 \\
n=51\end{array}$ & $\begin{array}{c}98 \\
n=551 \\
\end{array}$ & $\begin{array}{c}87 \\
n=444 \\
\end{array}$ & & & & & $\begin{array}{c}92 \\
n=345 \\
\end{array}$ & & $\begin{array}{c}94 \\
n=291 \\
\end{array}$ \\
\hline $\begin{array}{l}\text { R.6-5 \% of staff who } \\
\text { provide STD/HIV } \\
\text { services at this SDP }\end{array}$ & $\mathrm{S}$ & $\begin{array}{c}69 \\
n=455\end{array}$ & & & $\begin{array}{c}67 \\
n=570\end{array}$ & $\begin{array}{c}18 \\
n=441\end{array}$ & & & & & $\begin{array}{c}91 \\
n=358\end{array}$ & & $\begin{array}{c}93 \\
n=376\end{array}$ \\
\hline $\begin{array}{l}\text { R.6- } 6 \% \text { of staff } \\
\text { whose basic training } \\
\text { included STD/HIV }\end{array}$ & $S$ & $\begin{array}{c}66 \\
n=455 \\
\end{array}$ & & & & $\begin{array}{c}54 \\
n=448\end{array}$ & & & & & $\begin{array}{c}71 \\
n=342\end{array}$ & & \\
\hline $\begin{array}{l}\% \text { of staff who have } \\
\text { had refresher training } \\
\text { in STD/HIV }\end{array}$ & $S$ & $\begin{array}{c}63 \\
n=445\end{array}$ & & & $\begin{array}{c}34 \\
n=570\end{array}$ & $\begin{array}{c}12 \\
n=442\end{array}$ & & & & & $\begin{array}{c}29 \\
n=358\end{array}$ & & $\begin{array}{c}48 \\
n=376\end{array}$ \\
\hline
\end{tabular}



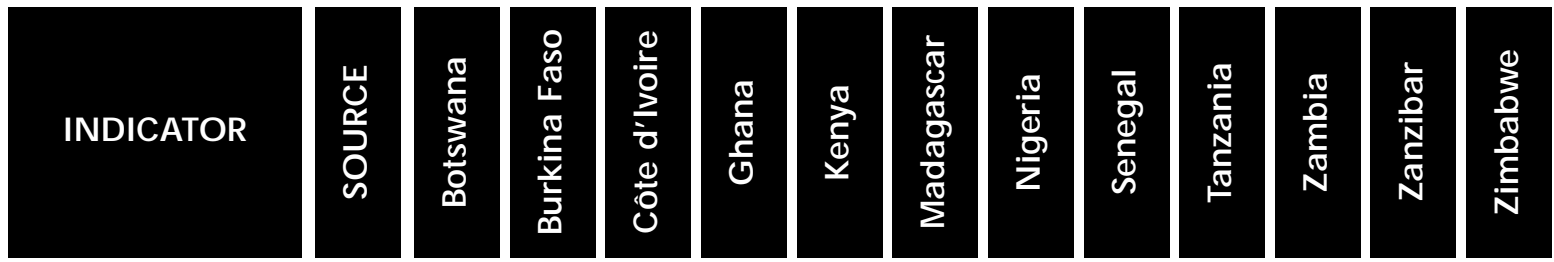

R. 6 READINESS TO PROVIDE SERVICES: STAFFING (contd.)

\begin{tabular}{|c|c|c|c|c|c|c|c|c|c|c|c|c|}
\hline $\begin{array}{l}\text { Mean years ago } \\
\text { refresher STD/HIV } \\
\text { training took place }\end{array}$ & $\mathrm{S}$ & & & & $\begin{array}{c}2.7 \\
n=166\end{array}$ & $\begin{array}{c}3.0 \\
n=51\end{array}$ & & & & $\begin{array}{c}1.5 \\
n=101\end{array}$ & & $\begin{array}{c}2.2 \\
n=179 \\
\end{array}$ \\
\hline $\begin{array}{l}\% \text { of staff who have } \\
\text { had basic or refresher } \\
\text { training in STD/HIV }\end{array}$ & $\mathrm{S}$ & $\begin{array}{c}84 \\
n=448\end{array}$ & & & & $\begin{array}{c}59 \\
n=443\end{array}$ & & & & $\begin{array}{c}77 \\
n=344\end{array}$ & & \\
\hline $\begin{array}{l}\text { R.6-7 \% of staff who } \\
\text { have had training in } \\
\text { family planning } \\
\text { counseling or IEC }\end{array}$ & $\mathrm{S}$ & $\begin{array}{c}13 \\
n=153 \\
\end{array}$ & $\begin{array}{c}21 \\
n=685\end{array}$ & $\begin{array}{c}22 \\
n=51\end{array}$ & $\begin{array}{c}54 \\
n=570 \\
\end{array}$ & $\begin{array}{c}11 \\
n=439\end{array}$ & $\begin{array}{c}27 \\
n=316\end{array}$ & $\begin{array}{c}1 \\
n=289\end{array}$ & $\begin{array}{c}37 \\
n=361\end{array}$ & $\begin{array}{c}27 \\
n=357 \\
\end{array}$ & $\begin{array}{c}80 \\
n=191 \\
\end{array}$ & $\begin{array}{c}45 \\
n=37\end{array}$ \\
\hline $\begin{array}{l}\text { Mean years ago } \\
\text { training in family } \\
\text { planning counseling } \\
\text { or IEC took place } \\
\end{array}$ & $\mathrm{S}$ & & $\begin{array}{c}1.9 \\
n=136\end{array}$ & & $\begin{array}{c}3.3 \\
n=268 \\
\end{array}$ & $\begin{array}{c}5.3 \\
n=48 \\
\end{array}$ & $\begin{array}{c}2.4 \\
n=85\end{array}$ & & $\begin{array}{c}3.5 \\
n=133\end{array}$ & $\begin{array}{c}2.4 \\
n=94\end{array}$ & & $\begin{array}{c}3.7 \\
n=169 \\
\end{array}$ \\
\hline $\begin{array}{l}\text { R.6-8 \% of staff who } \\
\text { have had training in } \\
\text { IUD insertion and } \\
\text { removal }\end{array}$ & $\mathrm{S}$ & $\begin{array}{c}25 \\
n=154 \\
\end{array}$ & $\begin{array}{c}42 \\
n=685\end{array}$ & & $\begin{array}{c}29 \\
n=570\end{array}$ & $\begin{array}{c}53 \\
n=440 \\
\end{array}$ & $\begin{array}{c}7 \\
n=316\end{array}$ & & $\begin{array}{c}59 \\
n=361\end{array}$ & $\begin{array}{c}17 \\
n=357\end{array}$ & $\begin{array}{c}82 \\
n=191 \\
\end{array}$ & $\begin{array}{c}18 \\
n=376\end{array}$ \\
\hline $\begin{array}{l}\text { Mean years ago IUD } \\
\text { training took place }\end{array}$ & $\mathrm{S}$ & & & & $\begin{array}{c}3.3 \\
n=149\end{array}$ & $\begin{array}{c}7.0 \\
n=227\end{array}$ & $\begin{array}{c}5.0 \\
n=22\end{array}$ & & & $\begin{array}{c}2.4 \\
n=60\end{array}$ & & $\begin{array}{c}5.7 \\
n=69\end{array}$ \\
\hline $\begin{array}{l}\text { R.6-9 \% of staff who } \\
\text { have had training in } \\
\text { tubal ligation } \\
\end{array}$ & $\mathrm{S}$ & $\begin{array}{c}1 \\
n=153\end{array}$ & $\begin{array}{c}5 \\
n=685\end{array}$ & & $\begin{array}{c}14 \\
n=570\end{array}$ & $\begin{array}{c}6 \\
n=440\end{array}$ & $\begin{array}{c}3 \\
n=316\end{array}$ & $\begin{array}{c}2 \\
n=289\end{array}$ & $\begin{array}{c}2 \\
n=361\end{array}$ & $\begin{array}{c}10 \\
n=357\end{array}$ & & $\begin{array}{c}26 \\
n=376\end{array}$ \\
\hline $\begin{array}{l}\text { Mean years ago tubal } \\
\text { ligation training took } \\
\text { place }\end{array}$ & $\mathrm{S}$ & & & & $\begin{array}{c}2.8 \\
n=65\end{array}$ & $\begin{array}{c}5.7 \\
n=25\end{array}$ & $\begin{array}{c}2.8 \\
n=10\end{array}$ & & & $\begin{array}{c}1.7 \\
n=33\end{array}$ & & $\begin{array}{c}4.8 \\
n=95\end{array}$ \\
\hline
\end{tabular}

\section{R.7 READINESS TO PROVIDE SERVICES: IEC MATERIALS AND ACTIVITIES}

\begin{tabular}{|c|c|c|c|c|c|c|c|c|c|c|c|c|c|}
\hline $\begin{array}{l}\text { R.7-1 } \% \text { of SDPs with } \\
\text { a poster on family } \\
\text { planning on the } \\
\text { walls }{ }^{58}\end{array}$ & 1 & $\begin{array}{c}59 \\
n=183\end{array}$ & $\begin{array}{c}66 \\
n=341 \\
\end{array}$ & $\begin{array}{c}54 \\
n=13\end{array}$ & $\begin{array}{c}85 \\
n=313\end{array}$ & $\begin{array}{c}77 \\
n=215\end{array}$ & $\begin{array}{c}70 \\
n=159 \\
\end{array}$ & $\begin{array}{c}69 \\
n=170 \\
\end{array}$ & $\begin{array}{c}82 \\
n=180 \\
\end{array}$ & $\begin{array}{c}36 \\
n=348\end{array}$ & $\begin{array}{c}48 \\
n=244 \\
\end{array}$ & $\begin{array}{c}79 \\
n=100\end{array}$ & $\begin{array}{c}83 \\
n=188 \\
\end{array}$ \\
\hline $\begin{array}{l}\% \text { of SDPs with a } \\
\text { brochure on family } \\
\text { planning }\end{array}$ & 1 & $\begin{array}{c}60 \\
n=179\end{array}$ & $\begin{array}{c}15 \\
n=341 \\
\end{array}$ & $\begin{array}{c}85 \\
n=13\end{array}$ & $\begin{array}{c}44 \\
n=313 \\
\end{array}$ & $\begin{array}{c}37 \\
n=213 \\
\end{array}$ & $\begin{array}{c}58 \\
n=159 \\
\end{array}$ & $\begin{array}{c}84 \\
n=98 \\
\end{array}$ & $\begin{array}{c}17 \\
n=180\end{array}$ & $\begin{array}{c}3 \\
n=348\end{array}$ & $\begin{array}{c}8 \\
n=245\end{array}$ & & $\begin{array}{c}30 \\
n=191 \\
\end{array}$ \\
\hline $\begin{array}{l}\% \text { of SDPs with a flip } \\
\text { chart on family } \\
\text { planning }\end{array}$ & 1 & $\begin{array}{c}35 \\
n=181 \\
\end{array}$ & $\begin{array}{c}27 \\
n=341\end{array}$ & & $\begin{array}{c}66 \\
n=313\end{array}$ & $\begin{array}{c}35 \\
n=213\end{array}$ & $\begin{array}{c}46 \\
n=159\end{array}$ & $\begin{array}{c}65 \\
n=57 \\
\end{array}$ & $\begin{array}{c}19 \\
n=180\end{array}$ & $\begin{array}{c}5 \\
n=348\end{array}$ & $\begin{array}{c}23 \\
n=252\end{array}$ & & $\begin{array}{c}21 \\
n=192 \\
\end{array}$ \\
\hline $\begin{array}{l}\text { R. } 7-2 \% \text { of SDPs with } \\
\text { a poster on HIV on } \\
\text { the walls }{ }^{61}\end{array}$ & 1 & $\begin{array}{c}66 \\
n=183\end{array}$ & & & $\begin{array}{c}49 \\
n=313\end{array}$ & $\begin{array}{c}64 \\
n=216\end{array}$ & & & & & $\begin{array}{c}46 \\
n=250\end{array}$ & & $\begin{array}{c}64 \\
n=185\end{array}$ \\
\hline $\begin{array}{l}\% \text { of SDPs with a } \\
\text { poster on STDs on } \\
\text { the walls }{ }^{62}\end{array}$ & I & $\begin{array}{c}43 \\
n=183\end{array}$ & & & $\begin{array}{c}19 \\
n=313\end{array}$ & $\begin{array}{c}29 \\
n=215\end{array}$ & & & & & $\begin{array}{c}37 \\
n=249\end{array}$ & & $\begin{array}{c}37 \\
n=180\end{array}$ \\
\hline
\end{tabular}

76 Clinic-Based Family Planning and Reproductive Health Services in Africa 

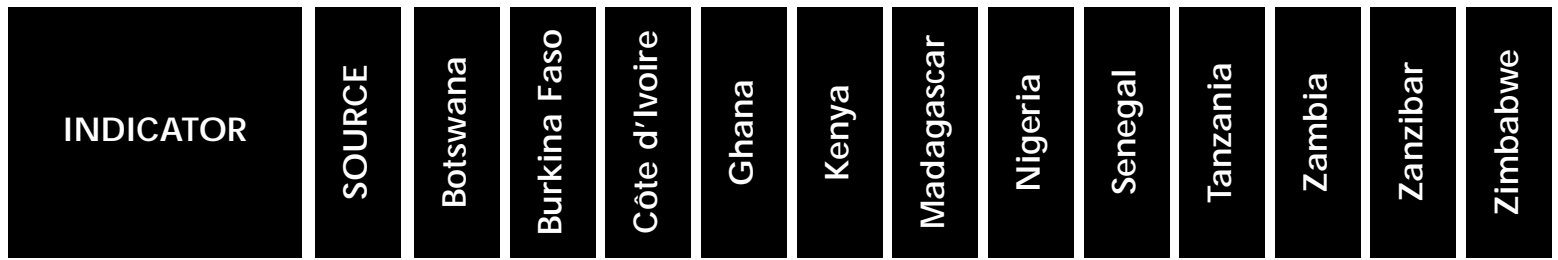

R. 7 READINESS TO PROVIDE SERVICES: IEC MATERIALS AND ACTIVITIES (contd.)

\begin{tabular}{|c|c|c|c|c|c|c|c|c|c|c|c|c|}
\hline $\begin{array}{l}\text { \% of SDPs with a } \\
\text { brochure on HIV }{ }^{63}\end{array}$ & I & $\begin{array}{c}63 \\
n=182 \\
\end{array}$ & & $\begin{array}{c}20 \\
n=313\end{array}$ & $\begin{array}{c}11 \\
n=216\end{array}$ & & & & & $\begin{array}{c}6 \\
n=246\end{array}$ & & $\begin{array}{c}18 \\
n=190 \\
\end{array}$ \\
\hline $\begin{array}{l}\% \text { of SDPs with a } \\
\text { brochure on STDs }\end{array}$ & I & $\begin{array}{c}45 \\
n=170\end{array}$ & & $\begin{array}{c}14 \\
n=313\end{array}$ & $\begin{array}{c}6 \\
n=216\end{array}$ & & & & & $\begin{array}{c}6 \\
n=245\end{array}$ & & $\begin{array}{c}16 \\
n=190\end{array}$ \\
\hline $\begin{array}{l}\text { \% of SDPs with a flip } \\
\text { chart on HIV }{ }^{65}\end{array}$ & I & $\begin{array}{c}18 \\
n=179\end{array}$ & & $\begin{array}{c}6 \\
n=313\end{array}$ & $\begin{array}{c}2 \\
n=215\end{array}$ & & & & & $\begin{array}{c}3 \\
n=250\end{array}$ & & $\begin{array}{c}14.9 \\
n=192 \\
\end{array}$ \\
\hline $\begin{array}{l}\% \text { of SDPs with a flip } \\
\text { chart on STDs }\end{array}$ & I & $\begin{array}{c}17 \\
\mathrm{n}=180\end{array}$ & & $\begin{array}{c}4 \\
n=313\end{array}$ & $\begin{array}{c}4 \\
n=216\end{array}$ & & & & & $\begin{array}{c}3 \\
n=249\end{array}$ & & $\begin{array}{c}3 \\
n=192\end{array}$ \\
\hline $\begin{array}{l}\text { R. } 7-3 \% \text { of health } \\
\text { talks that included } \\
\text { family planning }\end{array}$ & 1 & $\begin{array}{c}10 \\
n=22\end{array}$ & $\begin{array}{c}54 \\
n=48\end{array}$ & $\begin{array}{c}57 \\
n=72 \\
\end{array}$ & $\begin{array}{c}49 \\
n=41 \\
\end{array}$ & $\begin{array}{c}83 \\
n=53\end{array}$ & $\begin{array}{c}81 \\
n=50\end{array}$ & $\begin{array}{c}100 \\
n=10\end{array}$ & $\begin{array}{c}38 \\
n=119\end{array}$ & $\begin{array}{c}42 \\
n=95\end{array}$ & $\begin{array}{c}31 \\
n=36\end{array}$ & $\begin{array}{c}25 \\
n=48\end{array}$ \\
\hline $\begin{array}{l}\% \text { of health talks that } \\
\text { included STDs }\end{array}$ & 1 & $\begin{array}{c}0 \\
n=22\end{array}$ & & $\begin{array}{c}1 \\
n=72\end{array}$ & $\begin{array}{c}7 \\
n=41\end{array}$ & & & & & $\begin{array}{c}16 \\
n=95\end{array}$ & & $\begin{array}{c}10 \\
n=48\end{array}$ \\
\hline $\begin{array}{l}\% \text { of health talks that } \\
\text { included HIV }\end{array}$ & I & $\begin{array}{c}5 \\
n=21\end{array}$ & & $\begin{array}{c}3 \\
n=72\end{array}$ & $\begin{array}{c}7 \\
n=41\end{array}$ & & & & & $\begin{array}{c}5 \\
n=96\end{array}$ & & $\begin{array}{c}5 \\
n=48\end{array}$ \\
\hline
\end{tabular}

\section{R. 8 READINESS TO PROVIDE SERVICES: RECORDKEEPING AND SUPERVISION}

\begin{tabular}{|c|c|c|c|c|c|c|c|c|c|c|c|c|c|}
\hline $\begin{array}{l}\text { R.8-1 \% of SDPs with } \\
\text { client record cards in } \\
\text { good condition }{ }^{67}\end{array}$ & 1 & $\begin{array}{c}67 \\
n=40\end{array}$ & $\begin{array}{c}73 \\
n=294\end{array}$ & $\begin{array}{c}100 \\
n=13\end{array}$ & $\begin{array}{c}85 \\
n=277\end{array}$ & $\begin{array}{c}76 \\
n=167\end{array}$ & $\begin{array}{c}89 \\
n=131\end{array}$ & $\begin{array}{c}83 \\
n=152\end{array}$ & & $\begin{array}{c}29 \\
n=274\end{array}$ & $\begin{array}{c}79 \\
n=192\end{array}$ & $\begin{array}{c}76 \\
n=98\end{array}$ & $\begin{array}{c}55 \\
n=65\end{array}$ \\
\hline $\begin{array}{l}\text { R. } 8-2 \% \text { of SDPs that } \\
\text { have received a } \\
\text { supervisory visit in } \\
\text { the previous } 6 \text { months }\end{array}$ & 1 & $\begin{array}{c}93 \\
n=174\end{array}$ & $\begin{array}{c}22 \\
n=335\end{array}$ & & $\begin{array}{c}79 \\
n=277\end{array}$ & $\begin{array}{c}77 \\
n=186\end{array}$ & & & $\begin{array}{c}48 \\
n=177\end{array}$ & $\begin{array}{c}96 \\
n=326\end{array}$ & $\begin{array}{c}78 \\
n=242\end{array}$ & $\begin{array}{c}97 \\
n=97\end{array}$ & $\begin{array}{c}99 \\
n=171\end{array}$ \\
\hline
\end{tabular}

\section{Q.1 Q UALITY OF SERVICES: INTERPERSONAL RELATIONS}

\begin{tabular}{|c|c|c|c|c|c|c|c|c|c|c|c|c|c|}
\hline $\begin{array}{l}\text { Q.1-1 \% of all clients } \\
\text { given a friendly } \\
\text { greeting }^{68}\end{array}$ & 0 & $\begin{array}{c}90 \\
n=391\end{array}$ & $\begin{array}{c}96 \\
n=502\end{array}$ & $\begin{array}{c}66 \\
n=163\end{array}$ & & $\begin{array}{c}87 \\
n=737\end{array}$ & $\begin{array}{c}89 \\
n=1150\end{array}$ & $\begin{array}{c}98 \\
n=390\end{array}$ & $\begin{array}{c}80 \\
n=1085\end{array}$ & $\begin{array}{c}87 \\
n=438\end{array}$ & $\begin{array}{c}91 \\
n=396\end{array}$ & $\begin{array}{c}96 \\
n=139\end{array}$ & $\begin{array}{c}93 \\
n=758\end{array}$ \\
\hline $\begin{array}{l}\text { Q.1-2 \% of clients who } \\
\text { asked a question }{ }^{69}\end{array}$ & $E$ & $\begin{array}{c}78 \\
n=296\end{array}$ & $\begin{array}{c}34 \\
n=492\end{array}$ & & $\begin{array}{c}32 \\
n=785\end{array}$ & $\begin{array}{c}89 \\
n=471\end{array}$ & $\begin{array}{c}57 \\
n=1156\end{array}$ & & $\begin{array}{c}69 \\
n=1122\end{array}$ & & $\begin{array}{c}36 \\
n=392\end{array}$ & $\begin{array}{c}34 \\
n=140\end{array}$ & $\begin{array}{c}31 \\
n=736\end{array}$ \\
\hline $\begin{array}{l}\text { Among clients who } \\
\text { asked a question, \% } \\
\text { satisfied with the } \\
\text { response }\end{array}$ & $E$ & $\begin{array}{c}94 \\
n=290\end{array}$ & $\begin{array}{c}91 \\
n=507\end{array}$ & & $\begin{array}{c}99 \\
n=785\end{array}$ & $\begin{array}{c}99 \\
n=713\end{array}$ & $\begin{array}{c}99 \\
n=1153\end{array}$ & & $\begin{array}{c}98 \\
n=1121\end{array}$ & & $\begin{array}{c}96 \\
n=392\end{array}$ & $\begin{array}{c}94 \\
n=144\end{array}$ & $\begin{array}{c}96 \\
n=746\end{array}$ \\
\hline $\begin{array}{l}\text { Q.1-3 \% of clients } \\
\text { who found the } \\
\text { provider easy to } \\
\text { understand }\end{array}$ & $E$ & $\begin{array}{c}99 \\
n=374\end{array}$ & $\begin{array}{c}96 \\
n=492\end{array}$ & & $\begin{array}{c}99 \\
n=784\end{array}$ & $\begin{array}{c}98 \\
n=715\end{array}$ & $\begin{array}{c}98 \\
n=1143\end{array}$ & $\begin{array}{c}99 \\
n=388\end{array}$ & $\begin{array}{c}98 \\
n=1112\end{array}$ & $\begin{array}{c}97 \\
n=433\end{array}$ & $\begin{array}{c}98 \\
n=390\end{array}$ & $\begin{array}{c}97 \\
n=138\end{array}$ & $\begin{array}{c}98 \\
n=727\end{array}$ \\
\hline
\end{tabular}



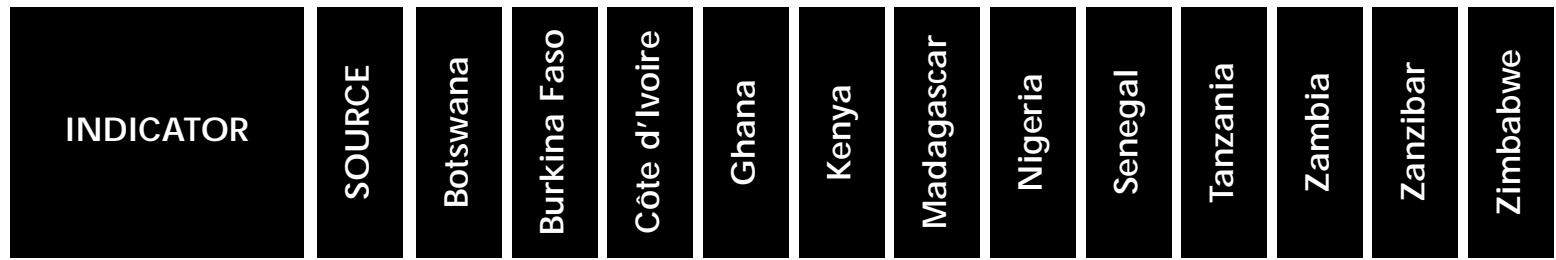

Q.1 QUALITY OF SERVICES: INTERPERSONAL RELATIONS (contd.)

\begin{tabular}{|c|c|c|c|c|c|c|c|c|c|c|c|c|}
\hline $\begin{array}{l}\text { Q.1-4 \% of pelvic } \\
\text { exams in which client } \\
\text { was informed about } \\
\text { the procedure } \\
\text { beforehand }\end{array}$ & 0 & $\begin{array}{c}68 \\
n=47\end{array}$ & $\begin{array}{c}38 \\
n=307\end{array}$ & $\begin{array}{c}77 \\
n=93\end{array}$ & $\begin{array}{c}86 \\
n=152 \\
\end{array}$ & $\begin{array}{c}37 \\
n=359 \\
\end{array}$ & $\begin{array}{c}75 \\
n=71 \\
\end{array}$ & $\begin{array}{c}21 \\
n=608\end{array}$ & $\begin{array}{c}92 \\
n=38\end{array}$ & $\begin{array}{l}100 \\
n=2\end{array}$ & $\begin{array}{c}86 \\
n=14 \\
\end{array}$ & $\begin{array}{c}85 \\
n=133 \\
\end{array}$ \\
\hline $\begin{array}{l}\% \text { of pelvic exams in } \\
\text { which client was } \\
\text { informed about the } \\
\text { results afterward }\end{array}$ & 0 & $\begin{array}{c}78 \\
n=45\end{array}$ & $\begin{array}{c}43 \\
n=307\end{array}$ & $\begin{array}{c}54 \\
n=93\end{array}$ & $\begin{array}{c}78 \\
n=152 \\
\end{array}$ & $\begin{array}{c}50 \\
n=359\end{array}$ & & $\begin{array}{c}40 \\
n=608\end{array}$ & & $\begin{array}{c}50 \\
n=2\end{array}$ & $\begin{array}{c}71 \\
n=14\end{array}$ & $\begin{array}{c}61 \\
n=133 \\
\end{array}$ \\
\hline $\begin{array}{l}\text { Q.1-5 \% of IUD } \\
\text { insertions in which } \\
\text { the client was given } \\
\text { moral support }\end{array}$ & 0 & $\begin{array}{c}100 \\
n=11\end{array}$ & $\begin{array}{c}75 \\
n=8\end{array}$ & $\begin{array}{c}52 \\
n=44\end{array}$ & $\begin{array}{c}86 \\
n=14\end{array}$ & $\begin{array}{c}85 \\
n=20\end{array}$ & & $\begin{array}{c}70 \\
n=30\end{array}$ & & & & $\begin{array}{l}100 \\
n=5\end{array}$ \\
\hline
\end{tabular}

\section{Q.2 QUALITY OF SERVICES: INFORMATION TAKEN FROM CLIENTS ${ }^{70}$}

\begin{tabular}{|c|c|c|c|c|c|c|c|c|c|c|c|c|c|}
\hline $\begin{array}{l}\text { Q.2-1 \% of new clients } \\
\text { asked about their } \\
\text { reproductive } \\
\text { intentions }\end{array}$ & 0 & $\begin{array}{c}44 \\
n=69 \\
\end{array}$ & $\begin{array}{c}39 \\
n=126 \\
\end{array}$ & $\begin{array}{c}64 \\
n=163 \\
\end{array}$ & $\begin{array}{c}60 \\
n=248\end{array}$ & $\begin{array}{c}75 \\
n=186 \\
\end{array}$ & $\begin{array}{c}74 \\
n=268 \\
\end{array}$ & $\begin{array}{c}85 \\
n=129 \\
\end{array}$ & $\begin{array}{c}90 \\
n=227 \\
\end{array}$ & $\begin{array}{c}28 \\
n=282 \\
\end{array}$ & $\begin{array}{c}34 \\
n=133 \\
\end{array}$ & $\begin{array}{c}33 \\
n=27 \\
\end{array}$ & $\begin{array}{c}51 \\
n=210 \\
\end{array}$ \\
\hline $\begin{array}{l}\text { Q.2-2 \% of new clients } \\
\text { asked their breast- } \\
\text { feeding status }\end{array}$ & 0 & $\begin{array}{c}62 \\
n=71 \\
\end{array}$ & $\begin{array}{c}58 \\
n=126\end{array}$ & $\begin{array}{c}66 \\
n=137\end{array}$ & $\begin{array}{c}28 \\
n=248\end{array}$ & $\begin{array}{c}55 \\
n=185\end{array}$ & $\begin{array}{c}44 \\
n=268\end{array}$ & $\begin{array}{c}54 \\
n=114\end{array}$ & $\begin{array}{c}79 \\
n=227\end{array}$ & $\begin{array}{c}52 \\
n=186\end{array}$ & $\begin{array}{c}49 \\
n=133\end{array}$ & $\begin{array}{c}78 \\
n=27\end{array}$ & $\begin{array}{c}65 \\
n=203\end{array}$ \\
\hline $\begin{array}{l}\% \text { of } \mathrm{COC} \text { acceptors } \\
\text { asked their breast- } \\
\text { feeding status }\end{array}$ & 0 & $\begin{array}{c}50 \\
n=38\end{array}$ & $\begin{array}{c}59 \\
n=80 \\
\end{array}$ & $\begin{array}{c}59 \\
n=66\end{array}$ & $\begin{array}{c}25 \\
n=49\end{array}$ & $\begin{array}{c}62 \\
n=39 \\
\end{array}$ & $\begin{array}{c}46 \\
n=65\end{array}$ & $\begin{array}{c}63 \\
n=16 \\
\end{array}$ & $\begin{array}{c}77 \\
n=92\end{array}$ & $\begin{array}{c}56 \\
n=93\end{array}$ & $\begin{array}{c}52 \\
n=82 \\
\end{array}$ & $\begin{array}{c}81 \\
n=16 \\
\end{array}$ & $\begin{array}{c}62 \\
n=26 \\
\end{array}$ \\
\hline $\begin{array}{l}\text { Q.2-3\% of new clients } \\
\text { asked their method } \\
\text { preference }\end{array}$ & 0 & $\begin{array}{c}79 \\
n=70 \\
\end{array}$ & $\begin{array}{c}42 \\
n=126\end{array}$ & $\begin{array}{c}62 \\
n=149 \\
\end{array}$ & $\begin{array}{c}79 \\
n=247\end{array}$ & $\begin{array}{c}72 \\
n=184 \\
\end{array}$ & $\begin{array}{c}58 \\
n=267 \\
\end{array}$ & $\begin{array}{c}65 \\
n=129 \\
\end{array}$ & $\begin{array}{c}82 \\
n=227 \\
\end{array}$ & $\begin{array}{c}68 \\
n=285 \\
\end{array}$ & $\begin{array}{c}66 \\
n=131 \\
\end{array}$ & $\begin{array}{c}48 \\
n=27\end{array}$ & $\begin{array}{c}57 \\
n=209 \\
\end{array}$ \\
\hline $\begin{array}{l}\text { Q.2-4 \% of new clients } \\
\text { asked about previous } \\
\text { method use }\end{array}$ & 0 & $\begin{array}{c}74 \\
n=68\end{array}$ & $\begin{array}{c}26 \\
n=126\end{array}$ & $\begin{array}{c}28 \\
n=134\end{array}$ & & $\begin{array}{c}75 \\
n=184 \\
\end{array}$ & $\begin{array}{c}49 \\
n=268\end{array}$ & & $\begin{array}{c}58 \\
n=227\end{array}$ & & $\begin{array}{c}63 \\
n=129\end{array}$ & $\begin{array}{c}19 \\
n=27\end{array}$ & $\begin{array}{c}81 \\
n=189 \\
\end{array}$ \\
\hline $\begin{array}{l}\text { Q.2-5\% of revisit } \\
\text { clients asked about } \\
\text { problems with their } \\
\text { method }\end{array}$ & 0 & $\begin{array}{c}59 \\
n=320\end{array}$ & $\begin{array}{c}60 \\
n=374\end{array}$ & & $\begin{array}{c}44 \\
n=569\end{array}$ & $\begin{array}{c}84 \\
n=551 \\
\end{array}$ & $\begin{array}{c}81 \\
n=882\end{array}$ & $\begin{array}{c}69 \\
n=247\end{array}$ & $\begin{array}{c}52 \\
n=866\end{array}$ & $\begin{array}{c}49 \\
n=152\end{array}$ & $\begin{array}{c}44 \\
n=259\end{array}$ & $\begin{array}{c}37 \\
n=111\end{array}$ & $\begin{array}{c}67 \\
n=538\end{array}$ \\
\hline $\begin{array}{l}\text { Q.2-6 \% of clients } \\
\text { asked if they discuss } \\
\text { family planning with } \\
\text { their partner }\end{array}$ & 0 & $\begin{array}{c}27 \\
n=71 \\
\end{array}$ & $\begin{array}{c}15 \\
n=126\end{array}$ & & $\begin{array}{c}69 \\
n=248\end{array}$ & $\begin{array}{c}50 \\
n=185\end{array}$ & $\begin{array}{c}25 \\
n=268\end{array}$ & & $\begin{array}{c}54 \\
n=227\end{array}$ & & $\begin{array}{c}41 \\
n=133\end{array}$ & $\begin{array}{c}48 \\
n=27\end{array}$ & $\begin{array}{c}46 \\
n=210\end{array}$ \\
\hline $\begin{array}{l}\text { Q.2-7 \% of new } \\
\text { clients asked the } \\
\text { nature of their } \\
\text { sexual relations }\end{array}$ & 0 & $\begin{array}{c}9 \\
n=69\end{array}$ & $\begin{array}{c}6 \\
n=126\end{array}$ & & $\begin{array}{c}22 \\
n=247\end{array}$ & $\begin{array}{c}32 \\
n=186\end{array}$ & $\begin{array}{c}11 \\
n=266\end{array}$ & & $\begin{array}{c}14 \\
n=227\end{array}$ & & $\begin{array}{c}5 \\
n=133\end{array}$ & $\begin{array}{c}59 \\
n=27\end{array}$ & $\begin{array}{c}16 \\
n=207\end{array}$ \\
\hline
\end{tabular}

78 Clinic-Based Family Planning and Reproductive Health Services in Africa 

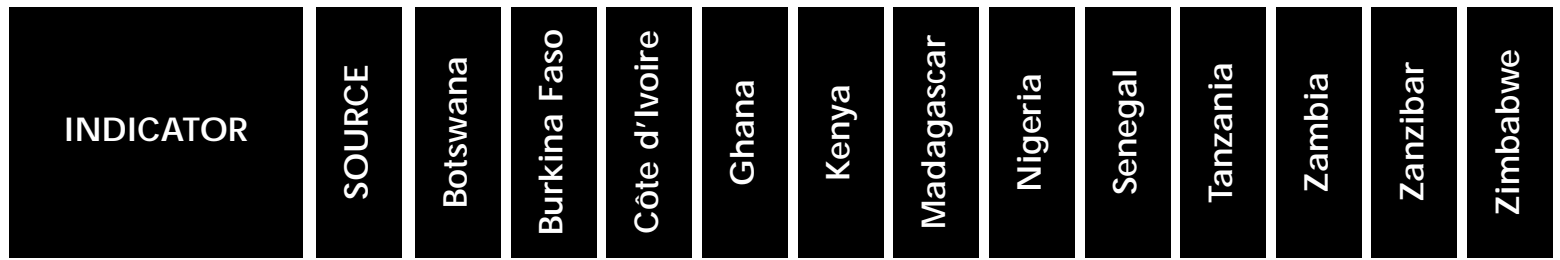

Q.2 QUALITY OF SERVICES: INFORMATION TAKEN FROM CLIENTS ${ }^{70}$ (contd.)

\begin{tabular}{|c|c|c|c|c|c|c|c|c|c|c|c|c|}
\hline $\begin{array}{l}\text { Q.2-8 \% of revisit } \\
\text { clients asked the } \\
\text { nature of their sexual } \\
\text { relations }^{72}\end{array}$ & 0 & $\begin{array}{c}3 \\
n=312\end{array}$ & & $\begin{array}{c}3 \\
n=569\end{array}$ & $\begin{array}{c}13 \\
n=551\end{array}$ & $\begin{array}{c}5 \\
n=882\end{array}$ & & & & $\begin{array}{c}1 \\
n=257\end{array}$ & $\begin{array}{c}8 \\
n=111\end{array}$ & $\begin{array}{c}9 \\
n=543\end{array}$ \\
\hline $\begin{array}{l}\text { Q.2-8 \% of new clients } \\
\text { asked about unusual } \\
\text { bleeding }\end{array}$ & 0 & $\begin{array}{c}46 \\
n=70 \\
\end{array}$ & $\begin{array}{c}45 \\
n=126\end{array}$ & $\begin{array}{c}23 \\
n=248\end{array}$ & $\begin{array}{c}18 \\
n=185\end{array}$ & $\begin{array}{c}21 \\
n=268\end{array}$ & $\begin{array}{c}36 \\
n=128\end{array}$ & $\begin{array}{c}8 \\
n=227\end{array}$ & $\begin{array}{c}26 \\
n=285\end{array}$ & $\begin{array}{c}23 \\
n=134\end{array}$ & $\begin{array}{c}52 \\
n=27\end{array}$ & $\begin{array}{c}24 \\
n=210\end{array}$ \\
\hline $\begin{array}{l}\% \text { of revisit clients } \\
\text { asked about unusual } \\
\text { bleeding }\end{array}$ & 0 & $\begin{array}{c}10 \\
n=322\end{array}$ & $\begin{array}{c}18 \\
n=376\end{array}$ & $\begin{array}{c}23 \\
n=571\end{array}$ & $\begin{array}{c}6 \\
n=550\end{array}$ & $\begin{array}{c}15 \\
n=882\end{array}$ & & $\begin{array}{c}9 \\
\mathrm{n}=866\end{array}$ & & $\begin{array}{c}15 \\
n=262\end{array}$ & $\begin{array}{c}36 \\
n=111\end{array}$ & $\begin{array}{c}13 \\
n=548\end{array}$ \\
\hline $\begin{array}{l}\% \text { of new clients } \\
\text { asked about unusual } \\
\text { discharge }\end{array}$ & 0 & $\begin{array}{c}42 \\
n=69 \\
\end{array}$ & $\begin{array}{c}25 \\
n=126\end{array}$ & $\begin{array}{c}34 \\
n=248\end{array}$ & $\begin{array}{c}11 \\
n=185\end{array}$ & $\begin{array}{c}24 \\
n=268\end{array}$ & $\begin{array}{c}32 \\
n=128\end{array}$ & $\begin{array}{c}13 \\
n=227\end{array}$ & $\begin{array}{c}13 \\
n=286\end{array}$ & $\begin{array}{c}22 \\
n=134\end{array}$ & $\begin{array}{c}7 \\
n=27\end{array}$ & $\begin{array}{c}19 \\
n=210\end{array}$ \\
\hline $\begin{array}{l}\% \text { of revisit clients } \\
\text { asked about unusual } \\
\text { discharge }\end{array}$ & 0 & $\begin{array}{c}8 \\
n=321 \\
\end{array}$ & $\begin{array}{c}11 \\
n=376\end{array}$ & $\begin{array}{c}19 \\
n=571\end{array}$ & $\begin{array}{c}5 \\
n=550\end{array}$ & $\begin{array}{c}7 \\
n=882 \\
\end{array}$ & & $\begin{array}{c}13 \\
n=866\end{array}$ & & $\begin{array}{c}6 \\
n=262 \\
\end{array}$ & $\begin{array}{c}3 \\
n=111\end{array}$ & $\begin{array}{c}5 \\
n=548\end{array}$ \\
\hline $\begin{array}{l}\% \text { of IUD clients } \\
\text { asked about unusual } \\
\text { bleeding }\end{array}$ & 0 & $\begin{array}{c}46 \\
n=11\end{array}$ & $\begin{array}{c}25 \\
n=8\end{array}$ & $\begin{array}{c}36 \\
n=44\end{array}$ & $\begin{array}{c}36 \\
n=14\end{array}$ & $\begin{array}{c}55 \\
n=20\end{array}$ & & $\begin{array}{c}17 \\
n=30\end{array}$ & & & & $\begin{array}{c}60 \\
n=5\end{array}$ \\
\hline $\begin{array}{l}\% \text { of IUD clients } \\
\text { asked about unusual } \\
\text { discharge }\end{array}$ & 0 & $\begin{array}{c}46 \\
n=11\end{array}$ & $\begin{array}{c}50 \\
n=8\end{array}$ & $\begin{array}{c}36 \\
n=44\end{array}$ & $\begin{array}{c}29 \\
n=14\end{array}$ & $\begin{array}{c}30 \\
n=20\end{array}$ & & $\begin{array}{c}3 \\
n=30\end{array}$ & & & & $\begin{array}{c}40 \\
n=5\end{array}$ \\
\hline
\end{tabular}

\section{Q.3 QUALITY OF SERVICES: CHOICE OF METHODS}

\begin{tabular}{|c|c|c|c|c|c|c|c|c|c|c|c|c|c|}
\hline $\begin{array}{l}\text { Q.3-1 \% of new clients } \\
\text { told about at least } \\
\text { two methods }\end{array}$ & 0 & $\begin{array}{c}61 \\
n=71\end{array}$ & $\begin{array}{c}64 \\
n=126\end{array}$ & $\begin{array}{c}49 \\
n=163\end{array}$ & $\begin{array}{c}87 \\
n=248\end{array}$ & $\begin{array}{c}85 \\
n=187 \\
\end{array}$ & $\begin{array}{c}74 \\
n=268\end{array}$ & $\begin{array}{c}61 \\
n=129\end{array}$ & $\begin{array}{c}61 \\
\mathrm{n}=227\end{array}$ & $\begin{array}{c}77 \\
n=286\end{array}$ & $\begin{array}{c}66 \\
n=134\end{array}$ & $\begin{array}{c}75 \\
n=28\end{array}$ & $\begin{array}{c}48 \\
n=210\end{array}$ \\
\hline $\begin{array}{l}\text { Q.3-2 \% of new clients } \\
\text { to whom COCs were } \\
\text { mentioned }\end{array}$ & 0 & $\begin{array}{c}77 \\
n=68\end{array}$ & $\begin{array}{c}77 \\
n=126\end{array}$ & $\begin{array}{c}76 \\
n=163\end{array}$ & $\begin{array}{c}81 \\
n=248\end{array}$ & $\begin{array}{c}83 \\
n=183 \\
\end{array}$ & $\begin{array}{c}74 \\
n=268\end{array}$ & $\begin{array}{c}72 \\
n=116\end{array}$ & $\begin{array}{c}75 \\
\mathrm{n}=227\end{array}$ & $\begin{array}{c}86 \\
n=286\end{array}$ & $\begin{array}{c}86 \\
n=134\end{array}$ & $\begin{array}{c}89 \\
n=27\end{array}$ & $\begin{array}{c}35 \\
n=21\end{array}$ \\
\hline $\begin{array}{l}\text { Q.3-3 \% of new clients } \\
\text { to whom POPs were } \\
\text { mentioned }\end{array}$ & 0 & $\begin{array}{c}36 \\
n=69\end{array}$ & $\begin{array}{c}32 \\
n=126 \\
\end{array}$ & & $\begin{array}{c}38 \\
n=248 \\
\end{array}$ & $\begin{array}{c}63 \\
n=183\end{array}$ & $\begin{array}{c}30 \\
n=268\end{array}$ & $\begin{array}{c}9 \\
n=115\end{array}$ & $\begin{array}{c}29 \\
\mathrm{n}=227\end{array}$ & $\begin{array}{c}30 \\
n=286\end{array}$ & $\begin{array}{c}40 \\
n=134\end{array}$ & $\begin{array}{c}26 \\
n=27\end{array}$ & $\begin{array}{c}66 \\
n=210\end{array}$ \\
\hline $\begin{array}{l}\text { Q.3-4 \% of new clients } \\
\text { to whom injectables } \\
\text { were mentioned }\end{array}$ & 0 & $\begin{array}{c}35 \\
n=68\end{array}$ & $\begin{array}{c}55 \\
n=126 \\
\end{array}$ & $\begin{array}{c}53 \\
n=163 \\
\end{array}$ & $\begin{array}{c}86 \\
n=248 \\
\end{array}$ & $\begin{array}{c}84 \\
n=183 \\
\end{array}$ & $\begin{array}{c}76 \\
n=268 \\
\end{array}$ & $\begin{array}{c}60 \\
n=115 \\
\end{array}$ & $\begin{array}{c}42 \\
n=227\end{array}$ & $\begin{array}{c}62 \\
n=286 \\
\end{array}$ & $\begin{array}{c}58 \\
n=134 \\
\end{array}$ & $\begin{array}{c}78 \\
n=27\end{array}$ & $\begin{array}{c}51 \\
n=210\end{array}$ \\
\hline $\begin{array}{l}\text { Q.3-5 \% of new clients } \\
\text { to whom condoms } \\
\text { were mentioned }\end{array}$ & 0 & $\begin{array}{c}49 \\
n=69\end{array}$ & $\begin{array}{c}34 \\
n=126\end{array}$ & $\begin{array}{c}29 \\
n=163\end{array}$ & $\begin{array}{c}64 \\
n=248\end{array}$ & $\begin{array}{c}69 \\
n=182\end{array}$ & $\begin{array}{c}56 \\
n=268\end{array}$ & $\begin{array}{c}60 \\
n=116\end{array}$ & $\begin{array}{c}25 \\
\mathrm{n}=227\end{array}$ & $\begin{array}{c}70 \\
n=286\end{array}$ & $\begin{array}{c}58 \\
n=134\end{array}$ & $\begin{array}{c}59 \\
n=27\end{array}$ & $\begin{array}{c}21 \\
n=210\end{array}$ \\
\hline $\begin{array}{l}\text { Q.3-6 \% of new clients } \\
\text { to whom IUDs were } \\
\text { mentioned }\end{array}$ & 0 & $\begin{array}{c}36 \\
n=69\end{array}$ & $\begin{array}{c}55 \\
n=126\end{array}$ & $\begin{array}{c}40 \\
n=163\end{array}$ & $\begin{array}{c}73 \\
n=248\end{array}$ & $\begin{array}{c}72 \\
n=182\end{array}$ & $\begin{array}{c}62 \\
n=268\end{array}$ & $\begin{array}{c}78 \\
n=116\end{array}$ & $\begin{array}{c}54 \\
n=227\end{array}$ & $\begin{array}{c}64 \\
n=286\end{array}$ & $\begin{array}{c}44 \\
n=134\end{array}$ & $\begin{array}{c}67 \\
n=27\end{array}$ & $\begin{array}{c}22 \\
n=210\end{array}$ \\
\hline
\end{tabular}



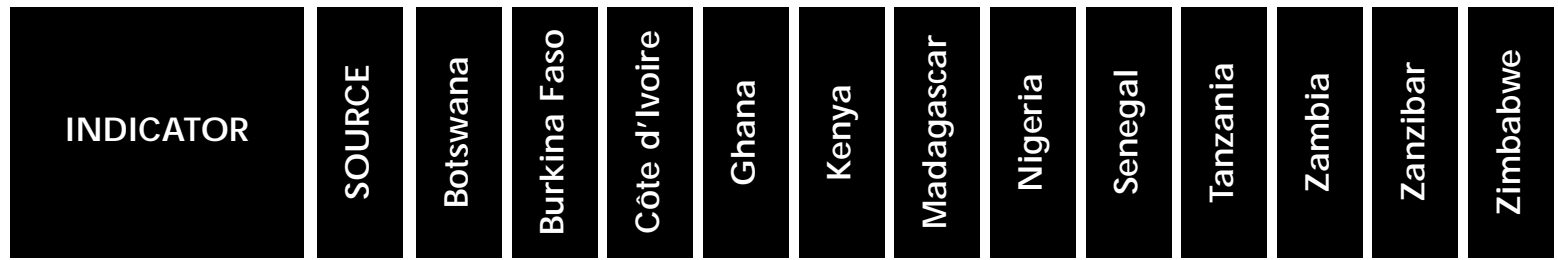

Q.3 QUALITY OF SERVICES: CHOICE OF METHODS (contd.)

\begin{tabular}{|c|c|c|c|c|c|c|c|c|c|c|c|c|c|}
\hline $\begin{array}{l}\text { Q.3-7 \% of new clients } \\
\text { to whom tubal } \\
\text { ligation was } \\
\text { mentioned }^{73}\end{array}$ & 0 & $\begin{array}{c}3 \\
n=69\end{array}$ & $\begin{array}{c}4 \\
n=126\end{array}$ & $\begin{array}{c}4 \\
n=163\end{array}$ & $\begin{array}{c}29 \\
n=248\end{array}$ & $\begin{array}{c}39 \\
n=183\end{array}$ & $\begin{array}{c}36 \\
n=268\end{array}$ & $\begin{array}{c}27 \\
n=115\end{array}$ & $\begin{array}{c}2 \\
n=227\end{array}$ & $\begin{array}{c}33 \\
n=286\end{array}$ & $\begin{array}{c}16 \\
n=134\end{array}$ & & $\begin{array}{c}10 \\
n=210\end{array}$ \\
\hline $\begin{array}{l}\text { Q.3-8 \% of new clients } \\
\text { to whom one method } \\
\text { was overemphasized }\end{array}$ & 0 & $\begin{array}{c}14 \\
n=70\end{array}$ & $\begin{array}{c}11 \\
n=126\end{array}$ & $\begin{array}{c}21 \\
n=140\end{array}$ & & $\begin{array}{c}16 \\
n=185\end{array}$ & $\begin{array}{c}15 \\
n=268\end{array}$ & & $\begin{array}{c}20 \\
n=227\end{array}$ & & $\begin{array}{c}28 \\
n=134\end{array}$ & $\begin{array}{c}15 \\
n=27\end{array}$ & $\begin{array}{c}14 \\
n=210\end{array}$ \\
\hline
\end{tabular}

\section{Q.4 QUALITY OF SERVICES: INFORMATION GIVEN TO CLIENTS}

\begin{tabular}{|c|c|c|c|c|c|c|c|c|c|c|c|c|c|}
\hline $\begin{array}{l}\text { Q.4-1 \% of new } \\
\text { acceptors told how } \\
\text { to use their method } \\
\end{array}$ & 0 & $\begin{array}{c}88 \\
n=64\end{array}$ & $\begin{array}{c}80 \\
n=111 \\
\end{array}$ & $\begin{array}{c}62 \\
n=151 \\
\end{array}$ & $\begin{array}{c}86 \\
n=225\end{array}$ & $\begin{array}{c}86 \\
n=172 \\
\end{array}$ & $\begin{array}{c}90 \\
\mathrm{n}=213\end{array}$ & $\begin{array}{c}57 \\
n=86\end{array}$ & $\begin{array}{c}86 \\
n=210 \\
\end{array}$ & $\begin{array}{c}14 \\
n=247\end{array}$ & $\begin{array}{c}87 \\
n=129\end{array}$ & $\begin{array}{c}79 \\
n=28\end{array}$ & $\begin{array}{c}81 \\
n=174 \\
\end{array}$ \\
\hline $\begin{array}{l}\text { Q.4-2 \% of new } \\
\text { acceptors told the } \\
\text { side effects of their } \\
\text { method }\end{array}$ & 0 & $\begin{array}{c}68 \\
n=63\end{array}$ & $\begin{array}{c}24 \\
n=111\end{array}$ & $\begin{array}{c}33 \\
n=151\end{array}$ & $\begin{array}{c}56 \\
n=225\end{array}$ & $\begin{array}{c}68 \\
n=171\end{array}$ & $\begin{array}{c}50 \\
n=213\end{array}$ & $\begin{array}{c}54 \\
n=82\end{array}$ & $\begin{array}{c}49 \\
n=210\end{array}$ & $\begin{array}{c}33 \\
n=247\end{array}$ & $\begin{array}{c}67 \\
n=129\end{array}$ & $\begin{array}{c}64 \\
n=28\end{array}$ & $\begin{array}{c}51 \\
n=174\end{array}$ \\
\hline $\begin{array}{l}\% \text { of new acceptors } \\
\text { told how to use their } \\
\text { method and its side } \\
\text { effects }\end{array}$ & 0 & $\begin{array}{c}68 \\
n=63\end{array}$ & $\begin{array}{c}22 \\
n=111\end{array}$ & $\begin{array}{c}31 \\
n=151\end{array}$ & $\begin{array}{c}53 \\
n=225\end{array}$ & $\begin{array}{c}64 \\
n=171\end{array}$ & $\begin{array}{c}49 \\
n=213\end{array}$ & $\begin{array}{c}42 \\
n=84\end{array}$ & $\begin{array}{c}48 \\
n=210\end{array}$ & $\begin{array}{c}5 \\
n=247\end{array}$ & $\begin{array}{c}59 \\
n=129\end{array}$ & $\begin{array}{c}61 \\
n=28\end{array}$ & $\begin{array}{c}49 \\
n=174\end{array}$ \\
\hline $\begin{array}{l}\text { Q.4-3\% of new } \\
\text { acceptors told about } \\
\text { their method's ability } \\
\text { to protect against } \\
\text { STD/HIV } \\
\end{array}$ & 0 & $\begin{array}{c}19 \\
n=63\end{array}$ & & & $\begin{array}{c}3 \\
n=225\end{array}$ & & & & & & $\begin{array}{c}9 \\
n=129\end{array}$ & & $\begin{array}{c}5 \\
n=174\end{array}$ \\
\hline $\begin{array}{l}\text { Q.4-4 \% of new clients } \\
\text { specifically told that } \\
\text { condoms protect } \\
\text { against STD/HIV }\end{array}$ & 0 & $\begin{array}{c}33 \\
n=63\end{array}$ & $\begin{array}{c}10 \\
n=126\end{array}$ & & $\begin{array}{c}25 \\
n=240\end{array}$ & $\begin{array}{c}14 \\
n=185\end{array}$ & $\begin{array}{c}17 \\
n=268\end{array}$ & & $\begin{array}{c}2 \\
n=227\end{array}$ & & $\begin{array}{c}36 \\
n=133\end{array}$ & $\begin{array}{c}31 \\
n=26\end{array}$ & $\begin{array}{c}7 \\
n=210\end{array}$ \\
\hline $\begin{array}{l}\text { Q.4-5 } \% \text { of new clients } \\
\text { with whom a flip } \\
\text { chart was used }^{74}\end{array}$ & 0 & $\begin{array}{c}0 \\
n=19\end{array}$ & $\begin{array}{c}11 \\
n=63\end{array}$ & & $\begin{array}{c}53 \\
n=191 \\
\end{array}$ & $\begin{array}{c}31 \\
n=85\end{array}$ & $\begin{array}{c}28 \\
n=183\end{array}$ & $\begin{array}{c}3 \\
n=33\end{array}$ & $\begin{array}{c}7 \\
n=89\end{array}$ & $\begin{array}{c}15 \\
n=20\end{array}$ & $\begin{array}{c}54 \\
n=52\end{array}$ & $\begin{array}{c}0 \\
n=7\end{array}$ & $\begin{array}{c}3 \\
n=58\end{array}$ \\
\hline $\begin{array}{l}\% \text { of new clients with } \\
\text { whom a brochure } \\
\text { was used }{ }^{75}\end{array}$ & 0 & $\begin{array}{c}8 \\
n=39\end{array}$ & $\begin{array}{c}0 \\
n=22\end{array}$ & $\begin{array}{c}7 \\
n=147\end{array}$ & $\begin{array}{c}13 \\
n=124\end{array}$ & $\begin{array}{c}4 \\
n=74 \\
\end{array}$ & $\begin{array}{c}25 \\
n=129\end{array}$ & $\begin{array}{c}0 \\
n=56\end{array}$ & $\begin{array}{c}12 \\
n=61\end{array}$ & $\begin{array}{c}19 \\
\mathrm{n}=16\end{array}$ & $\begin{array}{c}6 \\
n=16\end{array}$ & $\begin{array}{c}0 \\
n=26\end{array}$ & $\begin{array}{c}5 \\
n=84\end{array}$ \\
\hline $\begin{array}{l}\% \text { of new clients with } \\
\text { whom a poster was } \\
\text { used }^{76}\end{array}$ & 0 & $\begin{array}{c}11 \\
n=47\end{array}$ & $\begin{array}{c}3 \\
n=107\end{array}$ & $\begin{array}{c}6 \\
n=81\end{array}$ & $\begin{array}{c}11 \\
n=206\end{array}$ & $\begin{array}{c}4 \\
n=162\end{array}$ & $\begin{array}{c}4 \\
n=224\end{array}$ & & $\begin{array}{c}4 \\
\mathrm{n}=195\end{array}$ & & $\begin{array}{c}7 \\
n=86\end{array}$ & $\begin{array}{c}0 \\
n=22\end{array}$ & $\begin{array}{c}5 \\
n=176\end{array}$ \\
\hline
\end{tabular}

80 Clinic-Based Family Planning and Reproductive Health Services in Africa 

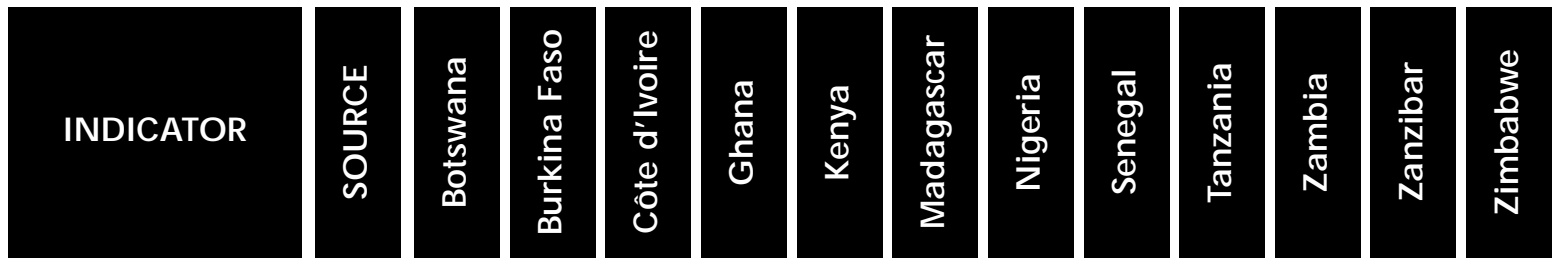

Q.5 QUALITY OF SERVICES: TECHNICAL COMPETENCE

\begin{tabular}{|c|c|c|c|c|c|c|c|c|c|c|c|c|c|}
\hline $\begin{array}{l}\text { Q.5-1 \% of new clients } \\
\text { who were weighed }{ }^{77}\end{array}$ & 0 & $\begin{array}{c}92 \\
n=65\end{array}$ & \begin{tabular}{|c|}
84 \\
$n=122$ \\
\end{tabular} & $\begin{array}{c}90 \\
n=123\end{array}$ & $\begin{array}{c}66 \\
n=212 \\
\end{array}$ & $\begin{array}{c}84 \\
n=160\end{array}$ & \begin{tabular}{|c|}
72 \\
$n=235$ \\
\end{tabular} & $\begin{array}{c}73 \\
n=113 \\
\end{array}$ & \begin{tabular}{|c|}
92 \\
$n=212$ \\
\end{tabular} & $\begin{array}{c}91 \\
n=234\end{array}$ & $\begin{array}{c}79 \\
n=130 \\
\end{array}$ & $\begin{array}{c}81 \\
n=26\end{array}$ & $\begin{array}{c}81 \\
n=202\end{array}$ \\
\hline $\begin{array}{l}\% \text { of new clients } \\
\text { whose LMP was taken }\end{array}$ & 0 & $\begin{array}{c}93 \\
n=70\end{array}$ & $\begin{array}{c}97 \\
n=126\end{array}$ & & $\begin{array}{c}86 \\
n=248\end{array}$ & $\begin{array}{c}95 \\
n=184\end{array}$ & $\begin{array}{c}88 \\
n=268\end{array}$ & $\begin{array}{c}94 \\
n=128\end{array}$ & $\begin{array}{c}94 \\
n=227\end{array}$ & $\begin{array}{c}93 \\
n=284\end{array}$ & $\begin{array}{c}90 \\
n=134\end{array}$ & $\begin{array}{c}85 \\
n=27\end{array}$ & $\begin{array}{c}73 \\
n=210\end{array}$ \\
\hline $\begin{array}{l}\% \text { of new clients } \\
\text { whose blood } \\
\text { pressure was taken }{ }^{78}\end{array}$ & 0 & $\begin{array}{c}88 \\
n=66 \\
\end{array}$ & $\begin{array}{c}76 \\
n=126\end{array}$ & $\begin{array}{c}81 \\
n=133 \\
\end{array}$ & $\begin{array}{c}69 \\
n=207\end{array}$ & $\begin{array}{c}85 \\
n=185 \\
\end{array}$ & $\begin{array}{c}76 \\
n=188 \\
\end{array}$ & $\begin{array}{c}73 \\
n=101 \\
\end{array}$ & $\begin{array}{c}93 \\
n=174 \\
\end{array}$ & $\begin{array}{c}89 \\
n=187\end{array}$ & $\begin{array}{c}86 \\
n=131\end{array}$ & $\begin{array}{c}78 \\
n=27\end{array}$ & $\begin{array}{c}87 \\
n=198\end{array}$ \\
\hline $\begin{array}{l}\% \text { of new clients } \\
\text { whose medical } \\
\text { history was taken }\end{array}$ & 0 & $\begin{array}{c}70 \\
n=70\end{array}$ & $\begin{array}{c}77 \\
n=126\end{array}$ & $\begin{array}{c}86 \\
n=138\end{array}$ & $\begin{array}{c}60 \\
n=248\end{array}$ & $\begin{array}{c}77 \\
n=184\end{array}$ & $\begin{array}{c}71 \\
n=268\end{array}$ & $\begin{array}{c}80 \\
n=128\end{array}$ & $\begin{array}{c}84 \\
n=227\end{array}$ & $\begin{array}{c}86 \\
n=285\end{array}$ & $\begin{array}{c}69 \\
n=134\end{array}$ & $\begin{array}{c}82 \\
n=27\end{array}$ & $\begin{array}{c}51 \\
n=210\end{array}$ \\
\hline $\begin{array}{l}\% \text { of new clients who } \\
\text { were given a general } \\
\text { physical exam }\end{array}$ & 0 & $\begin{array}{c}62 \\
n=71\end{array}$ & $\begin{array}{c}84 \\
n=126\end{array}$ & & $\begin{array}{c}31 \\
n=248\end{array}$ & $\begin{array}{c}64 \\
n=184\end{array}$ & $\begin{array}{c}45 \\
n=268\end{array}$ & $\begin{array}{c}56 \\
n=128\end{array}$ & $\begin{array}{c}65 \\
n=227\end{array}$ & $\begin{array}{c}62 \\
n=284\end{array}$ & $\begin{array}{c}46 \\
n=134\end{array}$ & & $\begin{array}{c}41 \\
n=210\end{array}$ \\
\hline $\begin{array}{l}\text { Q.5-2 \% of pelvic } \\
\text { exams before which } \\
\text { the provider washed } \\
\text { hands }^{79}\end{array}$ & 0 & $\begin{array}{c}57 \\
n=44\end{array}$ & $\begin{array}{c}24 \\
n=223\end{array}$ & & $\begin{array}{c}73 \\
n=70\end{array}$ & $\begin{array}{c}77 \\
n=116\end{array}$ & $\begin{array}{c}29 \\
n=263\end{array}$ & $\begin{array}{c}82 \\
n=50\end{array}$ & $\begin{array}{c}12 \\
n=608\end{array}$ & $\begin{array}{c}71 \\
n=24\end{array}$ & & $\begin{array}{c}67 \\
n=9\end{array}$ & $\begin{array}{c}67 \\
n=111\end{array}$ \\
\hline $\begin{array}{l}\% \text { of pelvic exams } \\
\text { after which the } \\
\text { provider washed } \\
\text { hands }^{80}\end{array}$ & 0 & $\begin{array}{c}71 \\
n=41\end{array}$ & & & $\begin{array}{c}81 \\
n=70\end{array}$ & $\begin{array}{c}87 \\
n=152\end{array}$ & & & & & & & $\begin{array}{c}75 \\
n=112\end{array}$ \\
\hline $\begin{array}{l}\% \text { of pelvic exams } \\
\text { during which clean/ } \\
\text { sterile speculum } \\
\text { was used }\end{array}$ & 0 & $\begin{array}{c}83 \\
n=47\end{array}$ & $\begin{array}{c}58 \\
n=279\end{array}$ & & $\begin{array}{c}65 \\
n=82 \\
\end{array}$ & $\begin{array}{c}94 \\
n=137\end{array}$ & $\begin{array}{c}52 \\
n=303\end{array}$ & $\begin{array}{c}94 \\
n=62\end{array}$ & $\begin{array}{c}83 \\
n=594\end{array}$ & $\begin{array}{c}97 \\
n=29\end{array}$ & $\begin{array}{l}100 \\
n=2\end{array}$ & $\begin{array}{c}86 \\
n=14\end{array}$ & $\begin{array}{c}87 \\
n=119\end{array}$ \\
\hline $\begin{array}{l}\% \text { of pelvic exams } \\
\text { during which clean/ } \\
\text { sterile gloves } \\
\text { were used }^{82}\end{array}$ & 0 & $\begin{array}{c}72 \\
n=32\end{array}$ & & & $\begin{array}{c}90 \\
n=72\end{array}$ & $\begin{array}{c}99 \\
n=132\end{array}$ & $\begin{array}{c}81 \\
n=330\end{array}$ & $\begin{array}{c}75 \\
n=55\end{array}$ & $\begin{array}{c}79 \\
n=283\end{array}$ & $\begin{array}{c}86 \\
n=29\end{array}$ & $\begin{array}{l}100 \\
n=2\end{array}$ & $\begin{array}{c}73 \\
n=11\end{array}$ & $\begin{array}{c}92 \\
n=116\end{array}$ \\
\hline $\begin{array}{l}\text { Q.5-3 \% of IUD } \\
\text { insertions during } \\
\text { which the uterus } \\
\text { was sounded } \\
\end{array}$ & 0 & $\begin{array}{c}64 \\
n=11 \\
\end{array}$ & $\begin{array}{c}88 \\
n=8\end{array}$ & & $\begin{array}{c}67 \\
n=30\end{array}$ & $\begin{array}{c}92 \\
n=12 \\
\end{array}$ & $\begin{array}{c}100 \\
n=20\end{array}$ & & $\begin{array}{c}97 \\
n=30\end{array}$ & & & & $\begin{array}{c}80 \\
n=5\end{array}$ \\
\hline $\begin{array}{l}\% \text { of IUD insertions } \\
\text { during which the } \\
\text { provider used sterile } \\
\text { instruments }\end{array}$ & 0 & $\begin{array}{c}73 \\
n=11\end{array}$ & $\begin{array}{c}75 \\
n=8\end{array}$ & & $\begin{array}{c}59 \\
n=44\end{array}$ & $\begin{array}{c}92 \\
n=13\end{array}$ & $\begin{array}{c}85 \\
n=20\end{array}$ & & $\begin{array}{c}93 \\
n=30\end{array}$ & & & & $\begin{array}{c}80 \\
n=5\end{array}$ \\
\hline $\begin{array}{l}\text { Q.5-4 \% of injections } \\
\text { during which a sterile } \\
\text { needle was used }\end{array}$ & 0 & $\begin{array}{c}100 \\
n=69\end{array}$ & $\begin{array}{c}100 \\
n=19\end{array}$ & & $\begin{array}{c}98 \\
\mathrm{n}=377\end{array}$ & $\begin{array}{c}95 \\
n=340\end{array}$ & $\begin{array}{c}100 \\
n=435\end{array}$ & & $\begin{array}{c}100 \\
n=55\end{array}$ & & $\begin{array}{l}100 \\
n=3\end{array}$ & $\begin{array}{c}98 \\
n=48\end{array}$ & $\begin{array}{c}100 \\
n=150\end{array}$ \\
\hline $\begin{array}{l}\% \text { of injections before } \\
\text { which the injection } \\
\text { site was disinfected }\end{array}$ & 0 & $\begin{array}{c}94 \\
n=70\end{array}$ & $\begin{array}{c}100 \\
n=19\end{array}$ & & $\begin{array}{c}39 \\
n=377\end{array}$ & $\begin{array}{c}98 \\
n=341\end{array}$ & $\begin{array}{c}100 \\
n=435\end{array}$ & & $\begin{array}{c}100 \\
n=55\end{array}$ & & $\begin{array}{l}100 \\
n=3\end{array}$ & $\begin{array}{c}77 \\
n=48\end{array}$ & $\begin{array}{c}75 \\
n=150\end{array}$ \\
\hline
\end{tabular}



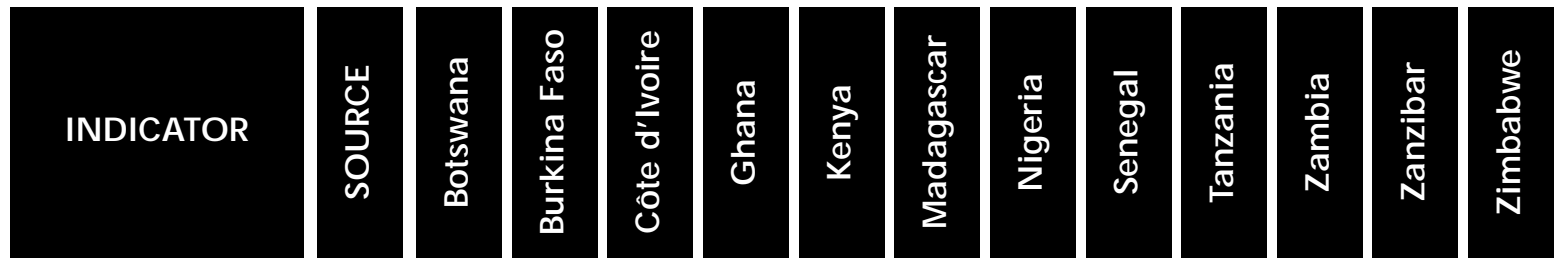

Q.5 QUALITY OF SERVICES: TECHNICAL COMPETENCE(contd.)

\begin{tabular}{|l|l|c|c|c|c|c|c|c|c|c|c|c|}
\hline $\begin{array}{l}\text { \% of injections before } \\
\text { which the vial was } \\
\text { vigorously shaken }\end{array}$ & 0 & $\begin{array}{c}100 \\
n=70\end{array}$ & $\begin{array}{c}63 \\
n=19\end{array}$ & & $\begin{array}{c}94 \\
n=329\end{array}$ & $\begin{array}{c}92 \\
n=435\end{array}$ & $\begin{array}{c}89 \\
n=55\end{array}$ & & $\begin{array}{c}98 \\
n=48\end{array}$ \\
\hline $\begin{array}{l}\text { \% of injections after } \\
\text { which the injection } \\
\text { site was not massaged }\end{array}$ & 0 & $\begin{array}{c}4 \\
n=70\end{array}$ & $\begin{array}{c}47 \\
n=19\end{array}$ & & $\begin{array}{c}74 \\
n=377\end{array}$ & $\begin{array}{c}13 \\
n=341\end{array}$ & $\begin{array}{c}27 \\
n=435\end{array}$ & $\begin{array}{c}16 \\
n=55\end{array}$ & $\begin{array}{c}0 \\
n=3\end{array}$ & $\begin{array}{c}19 \\
n=48\end{array}$ & $\begin{array}{c}19 \\
n=150\end{array}$ \\
\hline
\end{tabular}

Q.6 QUALITY OF SERVICES: MECHANISMS TO ENCOURAGE CONTINUITY

\begin{tabular}{|c|c|c|c|c|c|c|c|c|c|c|c|c|c|}
\hline $\begin{array}{l}\text { Q.6-1 \% of new } \\
\text { acceptors told that } \\
\text { they can switch } \\
\text { methods }\end{array}$ & 0 & $\begin{array}{c}25 \\
n=63\end{array}$ & $\begin{array}{c}19 \\
n=111\end{array}$ & $\begin{array}{c}16 \\
n=127\end{array}$ & $\begin{array}{c}11 \\
n=225\end{array}$ & $\begin{array}{c}37 \\
n=171\end{array}$ & $\begin{array}{c}32 \\
n=213\end{array}$ & & $\begin{array}{c}27 \\
n=210\end{array}$ & & $\begin{array}{c}31 \\
n=129\end{array}$ & $\begin{array}{c}54 \\
n=28\end{array}$ & $\begin{array}{c}12 \\
n=174\end{array}$ \\
\hline $\begin{array}{l}\text { Q.6- } 2 \% \text { of clients } \\
\text { given a date of } \\
\text { return }^{84}\end{array}$ & 0 & & $\begin{array}{c}93 \\
n=457\end{array}$ & $\begin{array}{c}78 \\
n=117\end{array}$ & $\begin{array}{c}96 \\
n=777\end{array}$ & $\begin{array}{c}99 \\
n=711\end{array}$ & $\begin{array}{c}95 \\
n=1001\end{array}$ & $\begin{array}{c}91 \\
n=390\end{array}$ & $\begin{array}{c}100 \\
n=1014\end{array}$ & $\begin{array}{c}90 \\
n=439\end{array}$ & $\begin{array}{c}96 \\
n=129\end{array}$ & $\begin{array}{c}97 \\
n=136\end{array}$ & $\begin{array}{c}78 \\
n=174\end{array}$ \\
\hline
\end{tabular}

\section{Q.7 QUALITY OF SERVICES: APPROPRIATENESS AND ACCEPTABILITY OF SERVICES}

\begin{tabular}{|c|c|c|c|c|c|c|c|c|c|c|c|c|c|}
\hline $\begin{array}{l}\text { Q.7-1 \% of clients } \\
\text { who received all the } \\
\text { information and } \\
\text { services they desired }\end{array}$ & $E$ & $\begin{array}{c}87 \\
n=381\end{array}$ & $\begin{array}{c}91 \\
n=492\end{array}$ & & $\begin{array}{c}95 \\
n=786\end{array}$ & $\begin{array}{c}94 \\
n=716\end{array}$ & $\begin{array}{c}99 \\
n=1156\end{array}$ & $\begin{array}{c}88 \\
n=388\end{array}$ & $\begin{array}{c}96 \\
n=1122\end{array}$ & $\begin{array}{c}92 \\
n=430\end{array}$ & $\begin{array}{c}93 \\
n=392\end{array}$ & & $\begin{array}{c}94 \\
n=737\end{array}$ \\
\hline $\begin{array}{l}\text { Q.7-2 Client } \\
\text { satisfaction with } \\
\text { time of consultation: } \\
\% \text { too long } \\
\% \text { just right } \\
\% \text { too short }\end{array}$ & $E$ & $\begin{array}{c}22 \\
60 \\
18 \\
n=384\end{array}$ & $\begin{array}{c}8 \\
86 \\
6 \\
n=491\end{array}$ & & $\begin{array}{c}8 \\
87 \\
5 \\
n=782\end{array}$ & $\begin{array}{c}9 \\
82 \\
9 \\
n=714\end{array}$ & $\begin{array}{c}39 \\
43 \\
19 \\
n=1151\end{array}$ & & $\begin{array}{c}9 \\
84 \\
7 \\
n=1120\end{array}$ & & $\begin{array}{c}16 \\
74 \\
10 \\
\mathrm{n}=392\end{array}$ & $\begin{array}{c}18 \\
77 \\
4 \\
n=141\end{array}$ & $\begin{array}{c}12 \\
81 \\
7 \\
n=736\end{array}$ \\
\hline $\begin{array}{l}\text { Q.7-3 \% of clients who } \\
\text { found the wait } \\
\text { reasonable }\end{array}$ & $\mathrm{E}$ & $\begin{array}{c}67 \\
n=378\end{array}$ & $\begin{array}{c}87 \\
n=489\end{array}$ & $\begin{array}{c}67 \\
n=353\end{array}$ & $\begin{array}{c}88 \\
n=768\end{array}$ & $\begin{array}{c}72 \\
n=714\end{array}$ & $\begin{array}{c}84 \\
n=1151\end{array}$ & $\begin{array}{c}92 \\
n=387\end{array}$ & $\begin{array}{c}68 \\
n=1112\end{array}$ & $\begin{array}{c}83 \\
n=435\end{array}$ & $\begin{array}{c}73 \\
n=386\end{array}$ & $\begin{array}{c}72 \\
n=140\end{array}$ & $\begin{array}{c}71 \\
n=739\end{array}$ \\
\hline $\begin{array}{l}\text { Q.7-4 \% of clients who } \\
\text { found the cost of } \\
\text { services acceptable }\end{array}$ & $\mathrm{E}$ & & $\begin{array}{c}97 \\
n=503\end{array}$ & $\begin{array}{c}98 \\
n=331\end{array}$ & $\begin{array}{c}94 \\
n=769\end{array}$ & $\begin{array}{c}84 \\
n=714\end{array}$ & $\begin{array}{c}95 \\
n=1144\end{array}$ & & $\begin{array}{c}92 \\
n=1107\end{array}$ & & $\begin{array}{c}86 \\
n=390\end{array}$ & $\begin{array}{c}100 \\
n=142\end{array}$ & $\begin{array}{c}78 \\
n=733\end{array}$ \\
\hline
\end{tabular}

82 Clinic-Based Family Planning and Reproductive Health Services in Africa 


\section{REFERENCES}

Askew, I., B. Mensch, and A. Adewuji. 1994. “Indicators for measuring the quality of family planning services in Nigeria." Studies in Family Planning, 25,5:268-283.

Hatcher, R., J. Trussell, F. Stewart, G. Steward, D. Kowal, F. Guest, W. Cates, Jr., and M. Policar. 1994. Contraceptive Technology, 16th Edition. Irvington Publishers, New York.

Jolly, L. and J. Gribble. 1993. “The proximate determinants of fertility." In Foote, K., K. Hill, and L. Martin, Eds., Demographic Change in Sub-Saharan Africa. National Academy Press, Washington, D.C.

Ndhlovu, L. 1995. Quality of Care in Family Planning Services in Kenya: Clients' and Providers' Perspectives. Ministry of Health, Kenya, and Population Council, New York.

Pariani, S., D. Heer, and M. Van Arsdol. 1987. "Continued contraceptive use in five family planning clinics in Surabaya, Indonesia." Studies in Family Planning, 22,6:384-390.

Ross, J. and E. Frankenberg. 1993. Findings from Two Decades of Family Planning Research. Population Council, New York.

Sinding, S., J. Ross, and A. Rosenfield. 1994. "Seeking common ground: Unmet need and demographic goals." International Family Planning Perspectives, 20,1:23-27.

United States Agency for International Development, Technical Guidance Working Group. 1994.

Recommendations for Updating Selected Practices in Contraceptive Use: Results of a Technical Meeting, Volume I and Volume II. INTRAH (University of North Carolina at Chapel Hill), Pathfinder International, USAID, and Family Health International, Washington, D.C.

Westoff, C. 1991. Reproductive Preferences. Comparative Studies 3, Demographic and Health Surveys, Macro Systems, Inc., Columbia, Maryland.

\section{NOTES}

1 Because Situation Analysis data should be interpreted in light of the maturity of the family planning program in each country, the reader is encouraged to refer to the brief descriptions of each country in the previous chapter.

2 The availability of water and electricity was measured on the day of the study visit. If an SDP had pipes but the water was not running that day, the facility was counted as not having water. In this way, the national estimates of water and electricity availability take water shortages and power outages into account.
3 In Botswana and Ghana, urban SDPs have a slightly higher availability of piped running water, but this result is not statistically significant.

4 The Tanzania study was done in 1992 and the Senegal study in 1994, when the safety of the method was being questioned, demand for injectables may not have been as high, and staff training programs for the method may not have been fully in place.

5 IUD-related results for Zimbabwe are strongly affected by weighting. All the clinics of the Zimbabwe National Family Planning Council were purposively included in the study, so they received a relatively small weight, decreasing their influence in the overall sample. However, their IUD services are generally of higher quality than those of other SDPs in the country, so when the influence of these clinics is weighted relatively lightly, the overall result is decreased substantially. Nevertheless, the weighted results paint a more representative picture of IUD services in the country.

6 All stockout rates are calculated only among those SDPs that offer each method.

7 This discussion emphasizes the appropriateness of methods based on meeting individual clients' and couples' reproductive intentions, rather than meeting demographic targets of contraceptive prevalence or fertility rates. Although some countries in subSaharan Africa do have stated demographic goals in their population policies, Sinding et al. (1994) found that meeting individual reproductive intentions will result in meeting or exceeding demographic targets. In this way, meeting individual reproductive intentions is actually consistent with meeting demographic targets.

8 Protection from adverse temperatures does not require that a storage space be air-conditioned.

9 See Askew et al. (1994) for more discussion of clustering of equipment.

$10 \mathrm{NORPLANT}^{\circledast}$ kits are not included in these averages because the number of SDPs offering NORPLANT ${ }^{\circledR}$ is so small.

11 The definition of "on duty" is not entirely clear in the data. It is taken to mean a physician physically present at the time of data collection, but it may refer to physicians on duty for an entire health facility, rather than just the maternal and child health $(\mathrm{MCH})$ / family planning unit. Therefore, the results from Côte d'Ivoire, Madagascar, Nigeria, and Zambia may be artificially high.

12 Unfortunately, data on the date of the refresher training are not available, so it is not possible to relate the refresher training to the start of the family planning program.

13 These are the 5 most recent studies included in this chapter.

14 In some countries, only a certain level of provider may attend IUD training. However, since these restrictions apply differently across all countries and the data vary, it was not possible to restrict the 
analysis to provider types who may attend IUD training.

15 Annex 3.1 lists 33 providers in Zambia trained in tubal ligation, rather than 22. The group of 22 is used here because of missing data on the crosstabulation.

16 This is one of the few areas in which field researchers are asked to make a qualitative judgment.

17 To truly evaluate the supervision in these countries, one would need to compare this visiting rate with the planned rate, and report information on what actions the supervisors took during the visits.

18 This indicator excludes the few clients who were breastfeeding in view of the provider at the time of the consultation, because in such cases the provider would not have to ask her status.

19 Another question in many of these studies asks directly whether the provider inquired about "current or previous STD symptoms." Because this question covers much the same ground as the indicators included under this element, and because the results are quite similar, this indicator has not been included here.

20 Most comparisons are significant at $\mathrm{p}<.05$.

21 The mean number of questions asked by providers trained in counseling vs. those not trained in counseling was 3.2 vs. 2.4 in Burkina Faso, 5.0 vs. 3.6 in Kenya, and 3.6 vs. 2.6 in Zambia.

22 Note that this includes methods mentioned only during the observed consultation, not during health talks.

23 Note that this discussion involves two units of analysis: the SDP and the client. For this reason, the two numbers are not strictly comparable, but in fact this finding holds even if the unit of analysis is switched to the client throughout. That is, the percentage of clients who hear about IUDs in consultation follows roughly the percentage of clients who attend SDPs that offer IUDs.

24 See Chapter 5, where significant results are found for Kenya using a different model for this issue.

25 The definition of "physical exam" varies from country to country.

26 The results for weight and blood pressure are reported only for clients attending SDPs that have adult scales and blood pressure machines available, respectively (see R.5-1).

27 In general, results for the higher-scoring countries (Nigeria, Kenya, Botswana, Burkina Faso, Tanzania, and Senegal) are significantly higher than those for the lower-scoring countries (Ghana, Zimbabwe, Madagascar, and Zambia) $(\mathrm{p}<.01)$. Within these two groups, means are not statistically different from each other.

28 Handwashing is calculated only for those SDPs that have adequate water in the exam room. Results for gloves are restricted to those SDPs that have gloves available, and results for specula are similarly restricted to those SDPs that have specula available. (See also R.5-1.)
29 Sounding the uterus is counted only for those IUD insertions at SDPs where uterine sounds are available.

30 In Botswana and Zimbabwe, this finding is explained by some staff having had on-the-job training in IUD insertion, which they did not report as formal IUD training in the staff interview.

31 Since the individual country sample sizes are so small, this relationship can be explored only in the aggregate. All results are significantly different at $\mathrm{p}$ $<.01$.

32 This result is statistically significant $(\mathrm{p}<.05)$ for all countries except Zimbabwe, Zanzibar, and Senegal.

33 "Adequate" is defined as protected from rain and sun, and with enough seats for clients. There are no data available for Botswana and Kenya on seating availability, and none for Zimbabwe on protection from rain and sun.

34 "Adequate" is defined as functioning electric light or sufficient natural light.

35 "Clean" is defined as floors swept and mopped at the start of the day, and no dust on tables or windowsills.

36 "Adequate" is defined as a sufficient quantity of clean water for washing hands and equipment.

37 In Côte d'Ivoire, this also includes signs in other units of the SDP.

38 All items related to NORPLANT ${ }^{\circledR}$ in the inventory are not weighted because so few SDPs offer this method.

39 For Burkina Faso, Madagascar, and Senegal, a number of stockout indicators are missing here although they were collected in the respective inventories. The reason is that in these studies, several brands were listed under each contraceptive, and each brand was marked as stocked out or not. This makes the overall stockout information very difficult to determine. If, for example, three brands of COCs are offered and one had a stockout, does that count as a stockout of the method if the others were available? Also, if all three were stocked out, one cannot know whether they were stocked out simultaneously or sequentially. Because these data are not comparable with those for the other countries, they are not included here.

40 Among SDPs that offer condoms.

41 Among SDPs that offer COCs.

42 Among SDPs that offer POPs.

43 Among SDPs that offer IUDs.

44 Among SDPs that offer injectables.

45 Among SDPs that offer NORPLANT ${ }^{\circledR}$. All items related to NORPLANT ${ }^{\circledR}$ in the inventory are not weighted because so few SDPs offer this method.

46 "Adequate" is defined as protected from rain, sun, adverse temperatures, and pests.

47 For the definitions of "enough" of each type of equipment, see Table 3-1 in the text. For Ghana, Zambia, and Zimbabwe, certain types of equipment are included in the inventory, but the data include only whether some of the equipment is available, not how many of each item. This makes it impossible to 
calculate these indicators in a manner comparable to that for the other countries.

48 In Burkina Faso and Madagascar, this combines small, medium, and large specula.

49 In Kenya, Tanzania, and Nigeria, this is "sterilizing lotion." In Côte d'Ivoire and Botswana it is "antiseptic lotion." In Burkina Faso it is "antiseptic/chemical lotion." In Madagascar it is "antiseptic lotion" or "formol comprimes." In Zanzibar, it is "Jik," "Dettol," or "other sterilizing lotion."

50 Among SDPs that offer IUDs.

51 Among SDPs that offer IUDs.

52 Among SDPs that offer IUDs.

53 Among SDPs that offer IUDs.

54 Among SDPs that offer NORPLANT ${ }^{\circledR}$. All items related to NORPLANT ${ }^{\circledast}$ in the inventory are not weighted because so few SDPs offer this method.

55 Not specified as part of basic, postbasic, or refresher training.

56 In Zambia and Ghana, this is family planning counseling training only. In Senegal, Burkina Faso, and Zimbabwe, this is training in IEC or family planning counseling. In all others, this is IEC training only.

57 In Senegal and Madagascar, this includes staff with formal minilap or formal laparoscopy training. In Burkina Faso, this is defined as "practical training in tubal ligation." In Zimbabwe, this includes staff trained in mini-laparotomy/local anesthetic (ML/LA) or mini-laparotomy/general anesthetic (ML/GA).

58 In Madagascar, Zimbabwe, and Ghana, this question does not specify "on the walls."

59 In Zambia, this includes only brochures "on display."

60 In Zambia, this includes only flip charts "on display."

61 In Zambia and Ghana, this question does not specify "on the walls."

62 In Zimbabwe and Ghana, this question does not specify "on the walls."

63 In Zambia, this includes only brochures "on display."

64 In Zambia, this includes only brochures "on display."

65 In Zambia, this includes only flip charts "on display."

66 In Zambia, this includes only flip charts "on display."

67 Among SDPs that keep client record cards at the clinic rather than giving them to clients.

68 In Côte d'Ivoire, this includes new clients only.

69 This is calculated among new clients, revisit clients who wanted to switch or stop, and revisit clients who were having a problem with their method. These clients can all reasonably be assumed to have at least one question to pose. For Kenya, Botswana, and Senegal, this is the percentage of clients whom the provider "let" ask a question, as opposed to other studies, which simply asked whether the client posed a question.
70 For Madagascar, Senegal, Kenya, Ghana, Zimbabwe, Botswana, and Zambia, the questions under this element address whether the discussion took place, regardless of whether the subject was brought up by the provider or the client. In the remaining studies, the observer noted only whether the provider raised the subject. This may seem a biasing factor, but the percentage of times subjects are brought up by the client is minuscule in each country.

71 In Zambia, Zimbabwe, and Ghana, new clients were asked "if more than one sexual partner in the last year." In Botswana, they were asked "the number of sexual partners in the last year," and in Kenya they were asked "the nature of the client's sexual relations" or "the number of sexual partners in the last year." In all other countries, this was phrased as "the nature of the client's sexual relations."

72 In Zambia, Zimbabwe, and Ghana, the client was asked "if more than one sexual partner in the past year," in Botswana, this is "the number of sexual partners since the last visit," and in Kenya it is "the nature of the client's sexual relations" or "the number of sexual partners since the last visit." In all other countries, this is "the nature of the client's sexual relations."

73 Among clients who want no more children, as identified through the exit interview.

74 Among clients at SDPs with at least one family planning flip chart available.

75 Among clients at SDPs with at least one family planning brochure available.

76 Among clients at SDPs with at least one family planning poster available.

77 Among clients at SDPs with adult weighing scales available.

78 Among clients at SDPs with blood pressure machines available.

79 Among pelvic exams at SDPs with water available in the exam room.

80 Among pelvic exams at SDPs with water available in the exam room.

81 Among pelvic exams at SDPs with at least two specula available.

82 Among pelvic exams at SDPs with sterile gloves available.

83 Among IUD insertions at SDPs with uterine sounds available.

84 In Zimbabwe, Côte d'Ivoire, and Zambia, this includes new clients only.

85 Among clients who waited at all.

86 Among clients who paid anything. In Senegal, Madagascar, and Zanzibar, clients were asked whether the cost is "reasonable," in Burkina Faso whether it is "affordable," and in Côte d'Ivoire whether it is "acceptable" or "not expensive." In all other sites, the word "acceptable" was used. 



\section{KEY POINTS AND CONCLUSIONS}

\section{Using Situation Analysis to Improve Reproductive Health Programs}

The Situation Analysis approach has been adopted in several countries as a way of providing managers with an assessment of the functioning of their program and the quality of services received by clients. Situation Analysis has clearly met an urgent need for such information as managers struggle with developing and refining what for many countries in Africa are still fairly new programs. Indeed, managers and donors still use the approach primarily for assessing the strengths and weaknesses of their programs.

With its evolution and refinement over time, however, Situation Analysis is now being used for several other purposes. In many countries, studies are now being undertaken at two or more points in time to evaluate whether a program has changed over time. The approach is also being adapted and used in operations research studies that test whether an intervention designed to improve the quality of services in clinics has had the intended effect. Research studies aimed at measuring whether different levels of service quality have an impact on the acceptance and continued use of family planning are using the approach to provide measures of service quality. As maternal and child health/family planning programs in Africa begin providing a more comprehensive and integrated package of reproductive health services, the Situation Analysis approach is also being broadened to collect information on other services, notably for sexually transmitted infections and HIV / AIDS, for child health, and for postabortion care. And as the number of data sets generated by Situation Analysis studies grows, so, too, does the opportunity to undertake comparative analyses of service-delivery issues across several programs and countries. This volume is the first systematic attempt to do so, but clearly it is not exhaustive, and further analyses are needed.

The purpose of a Situation Analysis study determines the way it is designed and implemented. Full participation by managers and a comprehensive strategy for disseminating the results have proven essential for ensuring that the information collected through any type of operations research is actually used. At two recently conducted workshops, program managers and others who had played an active role in implementing Situation Analysis studies and utilizing their results made the following suggestions:

- Ensure early and broad participation by service providers and managers from the regional and district levels so they can help identify the information to be collected and select the sample.

- Extend dissemination to all levels of the program, including clinics, so that all providers and managers are aware of the program's strengths and weaknesses and can take appropriate action themselves.

- Integrate data from Situation Analysis studies with data from other sources so that managers can have a broader picture of the relationships between the demand for and use of family planning and service-delivery activities.

- Although standardized instruments exist, ensure that each Situation Analysis study is tailored to meet the specific needs of the program, whether to plan future interventions, monitor and evaluate ongoing activities, or describe the overall program functioning.

These suggestions are relatively easy to implement, and to a large extent have been incorporated in the more recent Situation Analysis studies. 



\section{4}

\section{Using Situation Analysis to Improve Reproductive Health Programs}

\section{Ian Askew}

The publication of this volume marks the tenth anniversary of the development and initial application of the Situation Analysis approach. Those involved with the first study, conducted in Kenya in 1988-1989, will hardly recognize the approach as it is applied today. They certainly could not have envisioned the wide variety of applications to which that first simple study has led. As discussed in Chapter 1, the Kenya study originated with a simple request by the Kenya Ministry of Health $(\mathrm{MOH})$ for assistance in determining how well family planning services were being provided in their clinics. The $\mathrm{MOH}$ then used this information to make a number of improvements in clinic operations, most of which were accomplished with minimal or no additional resources.

Following the Kenya study, the Situation Analysis approach was rapidly adopted in several countries as a way of providing managers with a needs assessment of the functioning of their programs and the quality of services received by clients. There was a clear need for such information as managers struggled with developing and refining what for many countries in Africa were still fairly new family planning programs. Indeed, managers and donors still tend to use the approach primarily for this purpose.

As the Situation Analysis approach evolved and was refined over time and became more familiar to managers, donors, and researchers, Situation Analysis began to be used for several other purposes (see Box 1). For example, countries are conducting studies at two or more points in time to evaluate whether their program has changed over that time (see Chapter 11). The approach is also being adapted and used in operations research (OR) studies designed to test whether an intervention aimed at improving the quality of services in clinics has had the intended effect. Research studies that seek to measure whether different levels of service quality have an impact on the acceptance and continued use of family planning are using the approach to provide measures of service quality. As maternal and child health $(\mathrm{MCH})$ / family planning programs in Africa begin providing a more comprehensive and integrated package of reproductive health services, the Situation Analysis approach is being broadened to collect information on other services, notably for sexually transmitted infections (STIs) and HIV / AIDS (see Chapter 10), for child health, and for postabortion care. And finally, as the number of completed studies grows, so, too, does the opportunity to undertake comparative analyses of specific service-delivery issues across several programs. This volume is the first systematic attempt to do so, but it is clearly not exhaustive, and further analyses are certainly needed.

This chapter reviews and explores these different ways in which the Situation Analysis approach is being used. The purpose for which a Situation Analysis study is undertaken determines the way it is designed and implemented. Full participation by managers and a comprehensive strategy for disseminating the study results have proven essential for ensuring that the information collected through any type of OR is actually used (Solo et al., 1998). Accordingly, the chapter provides specific examples of how Situation Analysis studies have been implemented to illustrate how this can best be accomplished. The use of Situation Analysis for the following purposes is examined:

- Diagnosing and strengthening program readiness and service quality

- Analyzing and explaining quality of care 
- Evaluating national programs over time

- Developing and validating indicators of service delivery

- Evaluating specific interventions in $\mathrm{MCH} / \mathrm{fam}$ ily planning clinics

- Evaluating the impact of programs on family planning behavior and fertility

The chapter then reviews means by which the dissemination and utilization of Situation Analysis results can be maximized.

\section{Box 1}

Situation Analysis studies are being used to:

$\square$ Diagnose and strengthen program readiness and service quality.

- Analyze and explain quality of care.

- Evaluate national programs over time.

- Develop and validate indicators for service delivery.

- Evaluate specific interventions in maternal and child health $(\mathrm{MCH}) /$ family planning clinics.

- Evaluate the impact of programs on family planning behavior and fertility.

\section{DIAGNOSING AND STRENGTHENING PROGRAM READINESS AND SERVICE QUALITY}

As noted, the original purpose of Situation Analysis studies was to provide program managers with basic information on the readiness of their programs to offer a basic level of family planning services, and to give some indication of the quality of services received by family planning clients. This has continued to be the main purpose for carrying out such studies. For example, studies implemented in Mali, Mauritania, Cameroon, and Guinea $^{1}$ over the past 2 years were requested to give program managers (and the donor agencies supporting the programs) information that would help them strengthen their programs' subsystems. Basic Situation Analysis studies are most frequently requested when a donor agency, or the program itself, has acquired additional resources and needs information to guide the design of new programstrengthening activities.

Most commonly, the results from basic Situation Analysis studies are used first to identify areas of weakness in a program, and then to suggest ways in which those weaknesses can be reduced or even eliminated (see Box 2). The collection and analysis of data are designed so that the results can be presented in relation to the key subsystems of a family planning program (see Chapter 1). Thus data are collected on staff experience, training, and skills; information, education, and communication (IEC) materials; supervision procedures; recordkeeping and reporting; and infrastructure, equipment, and commodities and supplies logistics. In this way, managers find it relatively easy to identify specific administrative decisions that can improve their programs because there are usually separate units, departments, or individuals responsible for each of these subsystems. Moreover, donors and technical assistance agencies usually organize their support (and indeed sometimes their own operations ${ }^{2}$ ) according to these subsystems. Having study results arranged by subsystem therefore provides both managers and donors with an easily understandable set of data about how a program is functioning from which decisions about how to strengthen the program can be made.

Results from Situation Analysis studies are also used to guide the formulation of, as well as to evaluate, national or organizational policies and action plans (see Box 3). The study reports themselves have frequently been used as background resource materials during the planning process. All Situation Analysis study reports include a section presenting recommended actions toward the development of a stronger program offering higher-quality services. These recommendations have proven useful to those responsible for preparing policies and plans because they result from a focused analysis of the situation based on empirical data, rather than from anecdotal, impressionistic, or politically motivated evidence. Moreover, these recommendations usually have credibility with planners because they have been developed not solely by the researchers who collected the 


\section{Box 2 \\ Using Results from Situation Analysis Studies to Strengthen Program Subsystems}

\begin{tabular}{lll} 
Country & Date & Utilization of Results \\
Botswana & 1995 & Task Force established to review client record forms, service provider \\
guidelines, and IEC materials after weaknesses identified by study. \\
\hline
\end{tabular}

"Reference guide" on contraceptive methods developed to improve information provided.

Data analyzed by Gesellschaft für Technische Zussammenarbeit (GTZ) to strengthen services at those clinics it supports.

G uide for undertaking a training needs assessment developed by Program for International Training in Health (INTRAH).

IEC materials on contraceptives developed by Johns Hopkins University/Population Communication Services.

Findings used by Family Planning Service Expansion and Technical Support (SEATS) Project to advise the MOH on a program of integration.

$\begin{array}{lll}\text { Ghana } & 1993 & \text { Results of items on training used by the Johns Hopkins Program for } \\ \text { International Education in Reproductive Health (JHPIEG O) to } \\ \text { monitor implementation of revised curriculum. }\end{array}$

\begin{tabular}{lll} 
Kenya & 1990 & Results used by Nairobi City Commission (NCC) and Pathfinder to assess \\
and strengthen infection control, counseling, and IEC activities. \\
\hline Decision made by Pathfinder to support clinic strengthening in all \\
44 NCC clinics, rather than 10 as originally planned.
\end{tabular}

Results disseminated to six provinces and used by managers to plan improvements at subprovince level.

$\begin{array}{ll} & 1994 \\ & 1996\end{array}$

Decentralized dissemination led to rapid training sessions for improving providers' attitudes and beliefs about contraception.

Training manual developed for managing contraceptive supplies. 


\begin{tabular}{|c|c|c|}
\hline \multicolumn{3}{|c|}{$\begin{array}{l}\text { Box } 2 \text { (contd.) } \\
\text { Using Results from Situation Analysis Studies to } \\
\text { Strengthen Program Subsystems }\end{array}$} \\
\hline Country & Date & Utilization of Results \\
\hline \multirow[t]{2}{*}{ Zanzibar } & 1994 & Equipment found missing at specific facilities purchased by $\mathrm{MOH}$. \\
\hline & & Management and supervision courses added to program. \\
\hline \multirow[t]{13}{*}{ Zimbabwe } & 1991 & Basic family planning training curriculum revised by Zimbabwe \\
\hline & 1996 & $\begin{array}{l}\text { National Family Planning Council (ZNFPC) to encourage full } \\
\text { counseling on methods. }\end{array}$ \\
\hline & & $\begin{array}{l}\text { Counseling skills training module developed, with special emphasis } \\
\text { on long-term and permanent methods. }\end{array}$ \\
\hline & & Interpersonal communications training module developed. \\
\hline & & Checklist developed to improve follow-up visits to continuing clients. \\
\hline & & $\begin{array}{l}\text { Selection criteria for those being trained in family planning revised } \\
\text { to ensure that all clinics have at least one provider trained in family } \\
\text { planning. }\end{array}$ \\
\hline & & $\begin{array}{l}\text { Decentralized training initiated at district level to increase number of } \\
\text { staff trained in family planning. }\end{array}$ \\
\hline & & $\begin{array}{l}\text { Increased attention and resources allocated to training doctor-nurse } \\
\text { teams for sterilization and IUD services. }\end{array}$ \\
\hline & & $\begin{array}{l}\text { Frequency of refresher training increased through training of } \\
\text { provincial staff. }\end{array}$ \\
\hline & & System for distributing IEC materials to provinces improved. \\
\hline & & $\begin{array}{l}\text { Health Information Form improved, with training provided in } \\
\text { completion of the family planning component to improve service } \\
\text { statistics. }\end{array}$ \\
\hline & & $\begin{array}{l}\text { Proposal developed for study to train providers in reproductive tract } \\
\text { infection (RTI) management with IUD clients; proposal funded and } \\
\text { supported by JHPIEGO. }\end{array}$ \\
\hline & & $\begin{array}{l}\text { Pilot study to test depot-holder approach as alternative CBD strategy } \\
\text { developed and implemented. }\end{array}$ \\
\hline
\end{tabular}

data, but in conjunction with, and sometimes by, the managers and staff directly responsible for the day-to-day running of a program.

Immediately following the first Situation Analysis study in Kenya, a deliberate attempt was made to encourage program managers in that country to use the study results for identifying key service-delivery problems that could be addressed through OR studies. Three problems were identified, ${ }^{3}$ and a small-scale OR study that tested alternative solutions was completed for each (Miller and Frerichs, 1992-1993). This strategy of using Situation Analysis studies to diagnose problems and then identify and test potential solutions has rarely been used since. Although intuitively it may seem surprising that this strategy has not been more widely adopted, the realities facing program managers may help explain why. 


\section{Box 3 \\ Using Results from Situation Analysis Studies to Guide and Evaluate Policies and Programs}

\begin{tabular}{|c|c|c|}
\hline Country & Date & Utilization of Results \\
\hline \multirow[t]{4}{*}{ Botswana } & \multirow[t]{4}{*}{1995} & $\begin{array}{l}\text { Reproductive health section of G overnment of Botswana's 5-year } \\
\text { National Development Plan formulated. }\end{array}$ \\
\hline & & $\begin{array}{l}\text { Indicators for monitoring integrated reproductive health services } \\
\text { developed. }\end{array}$ \\
\hline & & $\begin{array}{l}\text { Data used by USAID as baseline measure for evaluating its } \\
\text { bilateral BOTSPA integrated services project. }\end{array}$ \\
\hline & & $\begin{array}{l}\text { United N ations Population Fund (UNFPA) 5-year Project } \\
\text { Review and Strategy Development Plan developed. }\end{array}$ \\
\hline \multirow{2}{*}{\multicolumn{2}{|c|}{$\begin{array}{r}\text { Burkina Faso } 1991 \\
1995\end{array}$}} & $\begin{array}{l}\text { Comparative analysis of results from } 1991 \text { and } 1995 \text { studies funded } \\
\text { by UNFPA, as was a regional-level analysis of the } 1995 \text { data, to } \\
\text { guide joint planning with the MOH. }\end{array}$ \\
\hline & & $\begin{array}{l}\text { Results used by } \mathrm{MOH} \text { to guide World Bank-funded decentralization } \\
\text { process. }\end{array}$ \\
\hline Ghana & $\begin{array}{l}1993 \\
1997\end{array}$ & $\begin{array}{l}\text { Results used by FHI to assess adherence by providers to new } \\
\text { standards and guidelines. }\end{array}$ \\
\hline \multirow[t]{4}{*}{ Kenya } & \multirow{4}{*}{$\begin{array}{l}1990 \\
1991 \\
1995\end{array}$} & $\begin{array}{l}\text { Study report used by program managers to identify specific resource } \\
\text { needs in discussions with donors. }\end{array}$ \\
\hline & & MOH Reproductive Health Strategy developed. \\
\hline & & $\begin{array}{l}\text { National Implementation Plan for Family Planning Program } \\
(1995-2000) \text { developed. }\end{array}$ \\
\hline & & $\begin{array}{l}\text { Data used by Marie Stopes International to develop proposals for } \\
\text { the World Bank. }\end{array}$ \\
\hline \multirow[t]{5}{*}{ Senegal } & 1994 & 1996 National Action Plan developed. \\
\hline & \multirow[t]{4}{*}{1996} & Fees policy reviewed and standardized nationwide. \\
\hline & & Standards and guidelines finalized. \\
\hline & & $\begin{array}{l}\text { Provision of injectables begun by International Planned Parenthood } \\
\text { Federation (IPPF) affiliate. }\end{array}$ \\
\hline & & $\begin{array}{l}\text { Data used as baseline for USAID bilateral child health/family planning } \\
\text { project implemented by Management Sciences for Health (MSH). }\end{array}$ \\
\hline Zambia & 1997 & $\begin{array}{l}\text { Data used as baseline for evaluating: } \\
\text { - USAID bilateral clinic-strengthening project implemented by John } \\
\text { Snow Incorporated (USI). } \\
\text { - USAID Child Health Project. } \\
\text { - UNFPA reproductive healthcare project in } 10 \text { districts. } \\
\text { - MOH Lusaka health program. }\end{array}$ \\
\hline Zanzibar & 1994 & Next 5-year program with UNFPA funding developed. \\
\hline \multirow[t]{2}{*}{ Zimbabwe } & 1991 & 1997-2001 national reproductive health program developed. \\
\hline & 1996 & Changes in program since 1991 assessed. \\
\hline
\end{tabular}


First, many of the identified problems lend themselves to immediate administrative action. If equipment is not available at clinics because it is locked up in a storeroom in the capital city, managers do not need a research study to find a solution. Many findings from Situation Analysis studies are of this nature, in which case the challenge is to optimize the procedures by which remedial action is taken. One potential drawback, however, is that because study results are normally presented in a clear-cut and precise fashion, managers usually draw from their own experience to identify a single solution to the problem that may or may not be the most appropriate approach. Thus having used research to identify a problem, they rely on intuition to solve it.

Second, most program managers rely heavily on external funds to support OR studies. Thus even if the need for an OR study is identified, a program manager is unlikely to be in a position to undertake such a study unless funds are available immediately for the purpose (as was the case in Kenya). The problem is in part the lack of resources allocated by service-delivery organizations to research generally. It is also due to the fact that, with the notable exception of the first Kenya study, Situation Analysis studies have not been designed or funded to allow for follow-on OR projects. To enhance the future utility of Situation Analysis studies, then, it is important for those who fund and support such studies to consider including additional resources to allow for possible follow-on work. (See Box 4.)

\section{ANALYZING AND EXPLAINING QUALITY OF CARE}

Despite 10 years of intensive attention by program managers, donor agencies, and technical assistance organizations to strengthening the quality of clinic services-and by researchers to defining and measuring quality-little is really known about why some clients receive higher-quality services than others, what determines these differences, and whether improving service quality actually makes a difference. As Shelton (in Miller et al., 1997:vi) points out, Situation Analysis "is the first systematic data collection tool which credibly documents

\section{Box 4 Importance of Follow-on Operations Research}

Ensuring that financial and technical support is available for follow-on OR studies is essential. For example, following the national Situation Analysis study in Botswana, the $\mathrm{MCH} /$ family planning unit realized that the client record forms it had been using in providing family planning services were inappropriate for recording the information needed to provide integrated services to clients. Although it was possible to simply redesign the form so that it included space for the additional information, the $\mathrm{MCH} /$ family planning unit wanted to test the new form on an experimental basis to determine how well providers were able to adapt to using it, and whether it improved their ability to offer integrated services. Because no funds were available for this study, either from within the $\mathrm{MOH}$ or from an external donor, it was not undertaken.

what goes on inside the formidable 'black box' of service delivery." To date, however, very little use has been made of the ever-increasing amount of data on quality of service delivery that has been collected through Situation Analysis studies. This volume marks the first systematic attempt to go beyond simply describing quality of care. It presents a number of analyses, none particularly refined methodologically, that seek to explain why clinics function the way they do and why providers often behave in ways that are less than optimal.

A few other attempts have been made to exploit these data sets more thoroughly. In particular, researchers have been seeking to establish whether generalizable patterns exist across programs in different countries. For example, Fisher (1993) used data from Zimbabwe, Nigeria, Ghana, and Tanzania to demonstrate that $20-30$ percent of all clinics deliver services to $70-80$ percent of all new family planning clients attending clinics, even after

94 Clinic-Based Family Planning and Reproductive Health Services in Africa 
controlling for clinic type. Mensch and colleagues examined the relationship of this finding to subsystem functioning. They found that "variation in the number of new acceptors is not adequately explained by subsystem functioning" (Mensch et al., 1996:26). Measham et al. (1996) looked at the quality of care provided to family planning clients who were breastfeeding at clinics in Nigeria and Senegal, and were able to develop a number of recommendations for improving the service offered to this important group of clients. Stein (1996) compared the perception of service quality by family planning and $\mathrm{MCH}$ clients in Botswana, Tanzania, and Kenya, and found that $\mathrm{MCH}$ clients were significantly less satisfied than family planning clients.

Clearly, there is tremendous scope for looking in greater detail at many aspects of quality of care and for identifying what determines the level of quality provided by a clinic. ${ }^{4}$ All Situation Analysis data sets have been standardized to the extent possible so that cross-study analyses can be undertaken, and all are in the public domain. ${ }^{5}$

\section{EVALUATING NATIONAL PROGRAMS OVER TIME}

Evaluation of family planning programs over time is acknowledged to be a difficult undertaking. Opinions differ about the level of effect or impact that should be measured, the most appropriate indicators to use, and the best means of collecting the data (Buckner et al., 1995; Samara et al., 1996). The Situation Analysis approach offers a new evaluation strategy that can be used to measure subsystem functioning and service quality at two or more points in time. The first country to undertake a second Situation Analysis study was Burkina Faso. Since then, Ghana, Kenya, Senegal, and Zimbabwe have completed second Situation Analysis studies, and Botswana and Zambia plan to do so in the near future. (See Chapter 11 for the key results from four of these comparisons over time.)

As experience in Burkina Faso and in Kenya (see Miller et al., 1996) has shown, however, unless the first and second Situation Analysis studies are deliberately planned for use in program evaluation, substantial differences in sampling and data collection may effectively render them noncomparable. In both Burkina Faso and Kenya, comparisons made over time could be only "accidental" rather than planned because methodological differences between the two studies (due to improvements in the methodology over time) meant that very few indicators could actually be measured in the same way. The first and second studies undertaken in Zimbabwe succeeded in having the same sampling plan, but substantial changes in the data collection instruments between 1991 and 1996 limited the number of indicators that were comparable for both studies. The two studies carried out in Ghana represented the first attempt at deliberately planning a series of Situation Analysis studies to measure changes in the national program over time (see Chapter 11); a third study is planned for 1999.

The use of the Situation Analysis approach for evaluation purposes has been most fully developed in Senegal. Whereas the studies in Ghana are comparing the national program at three points in time, effectively creating a time-series evaluation design (Fisher et al., 1991), the strategy in Senegal has gone two steps further in strengthening the use of the approach. ${ }^{6}$ First, the evaluation design includes an experimental group of clinics (all $\mathrm{MOH}$ clinics in four designated provinces) that have received a clinic-strengthening intervention and a comparison group of clinics that have not received this intervention (all clinics in the remaining six provinces). Following the first Situation Analysis study in 1994, which provided the baseline measures, ${ }^{7}$ the clinic-strengthening intervention was introduced by the Programme National pour la Planification Familiale (PNPF) with support from USAID's bilateral Child Survival and Family Planning Project. This evaluation design means that the clinics receiving the intervention can be compared in two ways: over time to see whether they themselves have changed, and with the clinics that did not receive the intervention to see whether changes in the two groups differ. Second, as discussed below, data collected from the first Situation Analysis study were used to develop a set of measurable indicators of program functioning and service quality. 


\section{DEVELOPING AND VALIDATING INDICATORS OF SERVICE DELIVERY}

To date, most Situation Analysis studies have been used as a reservoir of information for use by managers to serve particular purposes. As Situation Analysis studies are increasingly designed and used explicitly for evaluation purposes, however, the role of indicators that can be used and understood by program managers becomes more important.

The recently completed EVALUATION Project (implemented by the University of North Carolina, Tulane University, and others with funding from USAID) has made a tremendous contribution to program evaluation, particularly the identification of service-delivery indicators that can be used by program managers and donors (Bertrand et al., 1993). Drawing from the list of indicators, Askew et al. (1994) used data from the 1992 Situation Analysis study in Nigeria to validate those indicators proposed by the EVALUATION Project for measuring quality of care that could be measured using this data. A similar approach was taken by Brown and colleagues using data from a Situation Analysis study in Morocco (Brown et al., 1995).

These initial studies have paved the way for a more systematic consideration of indicator development and measurement during the adaptation of standard data collection instruments for national Situation Analysis studies (see Askew et al. [1995] for a review of how Situation Analysis data have been used for measuring indicators). Indeed, the Situation Analysis Handbook (Miller et al., 1997) includes a description of 138 programmatic indicators that can be measured using the standard data collection instruments.

The Situation Analysis Handbook also describes the indicators developed in Senegal, which provide managers with succinct empirical information about changes in program functioning and quality in the

\section{Box 5 \\ Example of Indicators Measured by USAID/Senegal Using Situation Analysis Data}

Indicators for Key Intermediate Result 1-Increase access to $\mathrm{MCH}$ /family planning and STI/AIDS services: ${ }^{\text {a }}$

$\checkmark$ Proportion of clinics offering family planning services to men and adolescents

- Proportion of clinics having trained staff and adequate equipment and supplies to offer family planning services

- Proportion of clinics having at least one person trained in managing diarrhea and an adequate stock of oral rehydration salts (ORS)

- Proportion of clinics having at least one person trained in malnutrition, as well as equipment needed to screen for malnutrition

a Proportion of clinics meeting the "baby-friendly hospital" criterion

Indicators for Key Intermediate Result 2-Improve the quality of $\mathrm{MCH}$ /family planning and ST/AIDS services: $\checkmark$ Proportion of clinics offering a complete range of contraceptive methods

$\checkmark$ Proportion of clinics at which clients receive counseling that conforms to senvice-delivery norms and standards

- Proportion of clinics receiving at least one supervisory visit every 3 months

- Continuation rate for family planning clients

Source: Touré et al. (1997a).

a Key intermediate results are those outcomes that must be accomplished to achieve a USAID Mission's strategic objective.

b This indicator refers to 10 questions regarding the existence and implementation of a breastfeeding policy, the existence of a support system for breastfeeding women, staff training, and staff attitudes and practices toward breastfeeding mothers and their children. 
experimental and control clinics. Most important, key decision makers from the PNPF and from the donors supporting the intervention directed the development of these indicators. Consequently, the indicators provide a credible means for evaluating the program. They were used for an interim evaluation of the situation in the experimental clinics in 1996; the first post-intervention study, in which all the clinics from the pre-intervention study were revisited, was completed in September 1998.

One of the major donors for the Senegal program (USAID) is also using the Situation Analysis data to measure its own indicators of progress with its bilateral support project. The USAID Mission in Senegal (with technical assistance from the Africa OR/TA Project II) has identified 10 indicators for evaluating progress on its strategic objective for service delivery, on which it is expected to report to USAID headquarters in Washington every 2 years (see Box 5). Data from the 1996 Situation Analysis study was used to measure these indicators (Touré et al., 1997a), as will the data from the 1998 study.

\section{EVALUATING SPECIFIC INTERVENTIONS IN MCH/FAMILY PLANNING CLINICS}

The Situation Analysis approach is essentially a means of collecting data that describe how clinic services are being provided. It can therefore be adapted fairly easily to evaluate interventions that are introduced at clinics. One of the first attempts to do so was undertaken by The Johns Hopkins University/Population Communication Services (JHU/PCS), which refined the observation and client interview instruments to collect more detailed data on the nature of information exchanged during client counseling (Kim and Lettenmaier, 1995). Data collected with these instruments have been used extensively to revise training curricula in Kenya, Tanzania, Zimbabwe, and Nigeria (Y. Kim, 1998, personal communication).

Since the increased focus on integrating STI/HIV / AIDS services at MCH/family planning clinics that resulted from the 1994 International Conference on Population and Development (ICPD) in Cairo (see Chapter 10), the Situation
Analysis approach has proved extremely useful in assessing the degree to which such integration is feasible (see Box 6). Maggwa and Askew (1997) report on a series of case studies undertaken in East and Southern Africa to examine projects that have sought to introduce interventions aimed at providing services in a more integrated fashion. These case studies used revised versions of the Situation Analysis instruments to collect data on whether the clinics had the necessary equipment, supplies, and drugs to offer both types of services; on the level of staff training and experience in providing STI/HIV/AIDS information and services; on how well the providers had implemented the revised procedures for integrated service delivery; and on clients' knowledge of the symptoms, modes of transmission, and means of protection for STIs. Versions of these case study instruments are currently being used by the Navrongo Health Research Centre in northern Ghana to evaluate the effect of an intervention (supported by JHPIEGO) to introduce STI management services in four district clinics.

Now that most national $\mathrm{MCH} /$ family planning programs in the region are integrating STI/ HIV / AIDS services within their standard servicedelivery procedures, many of the questions developed for the case study instruments are being included in the regular Situation Analysis instruments used for national studies. For example, Chapter 11 uses data from five national Situation Analysis studies that incorporated many of the questions on STI services developed for the case studies.

Botswana was the first country to include modules on STI management in its national Situation Analysis study (Baakile et al., 1996). The data collected gave the $\mathrm{MOH}$ a baseline for evaluating changes in the quality of care provided to clients after the introduction of an intervention that trained staff and equipped clinics to offer integrated services. Instead of repeating a complete national Situation Analysis study to measure the effect of this intervention, the $\mathrm{MOH}$ requested assistance from the Africa OR/TA Project II to develop and test a mechanism for quickly and accurately measuring the effect of the intervention on the way clinics and staff function. The resulting mechanism 


\section{Box 6}

\section{Lessons Learned About Integration of Services}

The following are key lessons learned about integrating $\mathrm{MCH}$ /family planning and STI/AIDS services from case studies using a modified Situation Analysis approach:

- Risk assessment and clinical history taking, which are essential for identifying potential STI cases among mainly asymptomatic $\mathrm{MCH} /$ family planning clients, are not performed consistently or according to guidelines.

$\checkmark$ A thorough general clinical examination and a pelvic examination, which are essential for detecting signs and symptoms associated with STIs, are not always undertaken.

- Little emphasis is placed on clinical examinations within STI management algorithms; client record forms do not record STI information; and basic equipment and supplies are commonly missing.

- Although staff have been trained in syndromic management and the associated algorithms are on display, many staff have problems completing the algorithms because of drug shortages or bureaucratic barriers that require referral to another provider or facility for treatment.

- Partner notification, implemented primarily by requesting clients to notify their partners verbally, has proven to be highly ineffective and remains a major problem if successful treatment of the woman is not to be undermined through reinfection by her untreated partner.

- HIV testing and counseling are seen as an essential component of any integrated approach in subSaharan Africa, but because of the resource-poor context, all programs refer clients elsewhere for these services.

- In line with national policy guidelines, programs are expected to offer syphilis screening for pregnant women. However, this service is not effectively implemented because clients must return later to obtain the results or go elsewhere for the test, and normally have to pay for the test as well. Little attention is paid to screening other STIs associated with adverse obstetric outcomes, such as gonorrhea and chlamydia.

- Information and education activities to prevent new infections and improve health-seeking behavior are seen as essential program components and could be improved greatly with minimal additional resources. Daily group health talks are mandated but are held infrequently, and information on STIS and HIV/AIDS is given only occasionally in both group talks and individual consultations.

- Condom promotion is undertaken to a differing extent by each program studied, but needs to be strengthened in all cases.

- Community-based workers raise awareness about STIs and facilitate access to information and services, but their efforts need to be integrated more thoroughly with the clinic-based services.

uses a combination of lot quality assurance sampling (LQAS) ${ }^{8}$ to identify a small but representative sample (approximately 25 clinics) and four short checklists (based on Situation Analysis instruments) for collecting data on a small number of indicators (approximately 50) that explicitly measure the changes expected as a result of the intervention (Maribe et al., 1997). The indicators were identified during a 1-week meeting of key program managers from all levels of the $\mathrm{MOH}$ who would use the results. The data were collected and analyzed by $\mathrm{MOH}$ staff themselves over a period of 3 months, and the findings were reported back to managers at a 2-day workshop. As a result of this rapid turnaround time, and the fact that those who would use the indicators had developed them, the findings have been used extensively by program managers. The expectation is that this mechanism will be used every 2 years to complement routine service statistics, thereby providing a more complete strategy for monitoring the integrated approach. 
Situation Analysis studies have also been used to explore providers' and clients' perceptions of unwanted pregnancies and the potential or actual role played by abortion in managing such situations (Huntington et al., 1993, 1996). Moreover, since the promotion of a more comprehensive approach to providing both reproductive health and $\mathrm{MCH}$ services, a number of modules have recently been developed and used to assess several other services being provided at $\mathrm{MCH} /$ family planning clinics. For example, in Zambia, Senegal, and Guinea, data are being collected on the quality of diarrhea management services for children through observations of client-provider interactions and interviews with the children's caregivers. In Guinea and Senegal, prenatal services are being observed and clients interviewed, and in Guinea, clients attending clinics for STI or HIV / AIDS services are being observed and interviewed. In addition, the Situation Analysis approach has been adapted by Sloan and colleagues to develop a rapid assessment tool for measuring the readiness and quality of emergency obstetric services at primary and secondary health facilities (Sloan et al., 1995).

With the promotion of a more comprehensive and integrated approach to providing reproductive health services, the role played by national service-delivery standards and guidelines is currently under close scrutiny. Originally developed to govern how clinic staff provide family planning services, these guidelines are now being revised by many countries to expand the number and types of services included. The results from Situation Analysis studies can contribute directly to this process (as has been the case in Ghana, Kenya, and Senegal) by demonstrating how services are currently being provided and offering suggestions for changes to the guidelines that would result in the provision of a better quality and wider range of services (Hardee et al., 1998). As discussed in Chapter 8, at present many providers do not always follow existing standards and guidelines, and thereby impose unnecessary barriers on the use of family planning by certain clients.

A number of programs are currently implementing more broad-based interventions to strengthen the overall ability of clinics and their staff to offer high-quality family planning services. The Situation Analysis approach has been adapted to evaluate the effectiveness of clinic-strengthening interventions in two West African countries. In Burkina Faso, the $\mathrm{MOH}$ has trained staff and reequipped 13 clinics in Bazèga Province as part of its experimental study to test alternative strategies for providing family planning services to rural populations. The national Situation Analysis study undertaken in 1995 provided baseline data for many of these clinics, ${ }^{9}$ as well as for the 8 clinics assigned to the comparison group. A miniSituation Analysis study was undertaken in 1997, 18 months after the intervention had been implemented, in all experimental and comparison clinics. A comparison is currently under way (using 15 summary indicators) to ascertain whether the intervention has in fact strengthened the clinics' readiness to offer quality services.

A slightly different approach is being taken in Senegal, where the PNPF has introduced a strategy for strengthening 14 selected clinics so that they will become "reference centers" or "model clinics." ${ }^{10}$ As part of a larger study to examine the impact on family planning behavior of improving service quality (see the next section), the quality of services provided at a sample of 5 model clinics is being measured and compared with that of 5 comparison clinics at three points in time.

\section{EVALUATING THE IMPACT OF PROGRAMS ON FAMILY PLANNING BEHAVIOR AND FERTILITY}

The idea that improving service quality will increase family planning acceptance and use has been broadly accepted since seminal papers by Jain (1989) and Bruce (1990) first proposed that such a relationship exists. To date, however, surprisingly little empirical evidence has been collected to either prove or disprove this relationship. In large part, this is because of the difficulty of designing research studies that can systematically link the quality of care offered in clinics with the family planning and ultimately the fertility behavior of clinic clients and the general population. Recently, Fisher et al. (1998) have proposed three ways in 
which Situation Analysis studies can be used to assess the impact of programs on family planning behavior and fertility.

The first, which they describe as an "output evaluation" study, seeks to evaluate how the readiness of clinics to offer high-quality services may have changed as a result of program improvements. Such an evaluation would require two Situation Analysis studies undertaken at least 3 years apart, using the same or similar samples of clinics. The comparisons undertaken in Burkina Faso and Ghana described above and in more detail in Chapter 11 are examples of this approach.

The second type of impact study would measure the effect of improving service quality on clients' contraceptive behavior. The ongoing studies at the model clinics in Senegal and in Bazèga, Burkina Faso (mentioned above) are examples of this approach. For the Senegal study, the objective is to determine whether clients attending clinics with better-quality services are likely to continue to use a family planning method more consistently and longer than clients attending clinics where service quality has not been strengthened. This impact is being measured by following cohorts of women attending both strengthened and nonstrengthened clinics over time. ${ }^{11}$ In Bazèga, impact is being measured at two levels: the study is examining whether clinics that have been strengthened attract and retain more new and revisit clients, and by adding a catchment area panel survey to the evaluation design, it is also assessing the impact on the use of family planning in the clinic catchment areas over time. A series of Situation Analysis studies conducted at the clinics over time describes changes in the quality of care offered.

The third type of impact study also seeks to relate use of services in the catchment area with the quality of care provided, but does so by linking a Situation Analysis study with a Demographic and Health Survey (DHS) in the same geographic area. Such a study was undertaken by Mensch and colleagues in Peru (Mensch et al., 1996). They found that there is a small but significant relationship between quality and use: if all women had access to clinics with the highest-quality care, contraceptive prevalence would be at least 16 percent higher. Unfortunately, the stringent and costly methodological requirements for this study, which sought to link data on contraceptive prevalence in DHS clusters with quality measures for clinics accessible to the cluster populations, have made it impossible to replicate the study elsewhere. Under the USAID-funded MEASURES Project, a number of studies have been proposed that would attempt to address this issue more efficiently by linking the collection of facility- and population-based data in a single study. The facility-based data would be collected using an adaptation of the Situation Analysis approach.

\section{MAXIMIZING THE DISSEMINATION AND UTILIZATION OF SITUATION ANALYSIS RESULTS}

As the Situation Analysis approach has evolved over time, so, too, has the process by which Situation Analysis studies are implemented. As demonstrated in this chapter, the data from Situation Analysis studies have been used for a variety of purposes, but the most important remains its use by managers to guide improvements in the functioning and quality of the services provided by their programs. Consequently, much attention has been paid to ensuring that Situation Analysis studies are implemented so that their results are widely disseminated and, wherever possible, they can be acted upon by program managers and donors.

A process for implementing Situation Analysis studies has emerged that focuses on ensuring direct participation by managers at all levels in each of the key phases of implementing a study. Use of a participatory approach has proven invaluable for increasing managers' understanding of the research process, and for ensuring that the study produces information the managers believe to be important and on which they can take action. First, a 2- to 3-day orientation and planning meeting is held among the senior managers, policy makers, and donors who will use the results and the research team that will undertake the study. At this meeting, several activities are completed:

- The methodology and data collection procedures are discussed in detail.

100 Clinic-Based Family Planning and Reproductive Health Services in Africa 
- The sampling plan is developed to ensure that everyone is satisfied that the results will be representative.

- The core data collection instruments (see Miller et al., 1997:Ch. 3) are adapted to the local needs and adjusted to meet any special circumstances.

- Participants agree to their roles during and after the fieldwork, with particular attention to facilitating the research teams' access to the sampled clinics.

The credibility of the results and recommendations from a Situation Analysis study rests heavily on the representativeness of the study's sampling plan. The sampling plans for the studies reported in this volume are described in Chapter 1 , where it can be seen that there is no universally applicable plan. The guiding principle followed for each sample was that it should be regarded as representative by those who would be using the data. Indeed, the 1994 and 1998 studies in Senegal and the study in Zanzibar did not draw a sample at all: the family planning program managers insisted that a census of every clinic that provides family planning services should be included.

More commonly, a sample is drawn that is national in scope, but is stratified in ways that are believed by program managers to best represent the program (e.g., by province, by type of clinic). In several studies, the sample has also deliberately included an oversampling of clinics representing a particular component of the program (e.g., in Nigeria and the first study in Ghana, all hospitals were sampled) or the clinics of another organization in addition to the MOH (e.g., in Ghana, samples were drawn of the IPPF-affiliate clinics and of the Ghana Registered Midwives Association [GRMA] program clinics, while in Kenya, all clinics of the Nairobi City Council were included).

The importance of having an acceptable and credible sample is emphasized here because quite often the study results may be contentious in that they frequently portray a program that is not functioning as well as was expected. When the results do not meet everyone's expectations, questions are usually raised about the sample because there is a feeling that "the wrong clinics" must have been visited. However well a sample was designed, it is always possible to find some fault with it, particularly if random selection was used. For example, the Tanzania study undertaken in 1992 used a random sampling plan for the whole country. Unfortunately, the random selection process drew a sample that did not include any clinics from one of the provinces in the north of the country. To compound the problem, this province has by far the highest contraceptive prevalence rate, so there had been great interest in finding out more about the family planning program there. Data collection proceeded at the clinics that had been randomly selected, and as a result the entire study suffered a lack of credibility because it did not include representation from every province. In hindsight, the sample should have been stratified by province to avoid this problem. This example highlights the importance of involving all those interested in the study results from the beginning of the process, including sample selection.

Once the data have been collected, rapid processing is essential for the production of frequency tabulations for all indicators, with some basic cross tabulations being done where necessary. A data interpretation workshop (usually lasting 1-2 days) is held for the same group of managers, policy makers, donors, and researchers to discuss these preliminary results. One of the basic purposes of this workshop is to allow managers to see the preliminary data before a final report has been written and before the data are disseminated more widely. In this way, managers are directly involved in giving meaning to the data, rather than having to rely on the interpretation of researchers as is the case with most research studies. The objective of the workshop is to obtain overall agreement on the programmatic implications of the data.

Following the data interpretation workshop, a draft report is written (usually by the researchers) to synthesize the key study results in a simple-toread format (see Miller et al., 1997:Ch. 4). This draft report serves as the background documentation for a national (or program-level) dissemination seminar at which the study results are presented to and discussed with a wider range of interested parties. Attention is paid to ensuring that the results are presented in an easily understandable form; to this end, bar charts are commonly used to present the data graphically. ${ }^{12}$ Programmatic recommenda- 
tions and commitments to ameliorative action are sometimes made prior to the dissemination seminar and presented for approval. Often, however, the drafting of such recommendations forms an integral part of the seminar itself. A final report is then produced, which describes both the study results and the programmatic recommendations.

Because many Situation Analysis studies are undertaken for a national program, a decentralized dissemination strategy has emerged that explicitly includes the presentation of results to subnational levels of the program. This strategy has been followed in Burkina Faso, Ghana, Kenya, Nigeria, and Zimbabwe, and has been most fully exploited in Senegal. Diop et al. (1996) describe how this decentralized strategy was developed for the first Situation Analysis study in Senegal. In summary, it consisted of four phases:

1. Analyzing the data by administrative region

2. Planning and organizing the regional dissemination seminars

3. Facilitating the 1-day seminars

4. Synthesizing the regional-level results and programmatic recommendations into a regional report

The objective of disseminating the results in this manner was twofold: to maximize the number of people at different levels of the program who would hear of the results, and to enable regional and district-level managers to develop recommendations and action plans that would be based on the findings from their clinics. In the implementation of this decentralized strategy, remarkable differences emerged between national- and regional-level managers with regard to the perceived strengths and weaknesses of the programs (see Diop et al. [1996:173] for examples). This outcome reinforced the resolve of the PNPF to strengthen its decentralized approach to program management. It has also resulted in subsequent Situation Analysis studies being planned and disseminated at both the national and regional levels (as was the case with the second Senegal study in 1996 [Touré et al., 1997b]).

To learn more from managers themselves about how best to maximize the dissemination and utilization of Situation Analysis study results, two regional workshops were convened-one at Nairobi, Kenya, in May 1996 (Leonard, 1997) and another in Dakar, Senegal, in June 1997. Program managers and others who had played an active role in implementing Situation Analysis studies and utilizing their results participated in these workshops. The major suggestions made were as follows:

- Ensure early and broad participation by service providers and managers from the regional and district levels so they can help identify the information to be collected and select the sample.

- Extend dissemination to all levels of the program, including clinics, so that all providers and managers are aware of the program's strengths and weaknesses and can take appropriate action themselves.

- Integrate data from Situation Analysis studies with data from other sources so that managers can have a broader picture of the relationships between the demand for and use of family planning and service-delivery activities.

- Although standardized instruments exist, ensure that each Situation Analysis study is tailored to meet the specific needs of the program, whether to plan future interventions, monitor and evaluate ongoing activities, or describe the overall program functioning.

These suggestions are relatively easy to implement, and to a large extent have been incorporated in the more recent Situation Analysis studies. ${ }^{13}$

\section{CONCLUSION}

The ways in which the Situation Analysis approach can be used have increased over time. From a tool used to describe the functioning and quality of family planning services provided at health facilities at a particular point in time, the approach has broadened in its application to serve more evaluative purposes. In addition to assessments of entire family planning programs over time, the approach has been adopted for evaluations of specific servicedelivery interventions. As this volume demonstrates, data from several studies can be used as well for secondary analyses that compare different programs and allow generalizations about patterns of service delivery. The utilization of the approach has also mirrored changes in the field of family planning and reproductive health generally, 
through the development of quality-of-care indicators that can be measured empirically, and through the development of modules that allow reproductive health services other than family planning to be described and assessed. Although tremendous progress has been made in a short period of time, there is scope for wider utilization of the approach, particularly for research studies aimed at exploring the impact of service quality on family planning and fertility behavior by linking facility- and population-based data.

\section{REFERENCES}

Askew, I., B. Mensch, and A. Adewuji. 1994. "Indicators for measuring the quality of family planning services in Nigeria." Studies in Family Planning, 25,5:268-283.

Askew, I., K. Miller, and B. Mensch. 1995. "Key indicators for measurement of quality of family planning services." Paper presented at The EVALUATION Project's Service Delivery Working Group Meeting, Washington, D.C., October.

Baakile, B., N. Maggwa, L. Maribe, and R. Miller. 1996. A Situation Analysis of the Maternal and Child Health/Family Planning (MCH/FP) Program in Botswana. Prepared by the Africa OR/TA Project II in cooperation with the Ministry of Health $\mathrm{MCH} / \mathrm{FP}$ Unit, Family Health Division. Gaborone, Botswana.

Bertrand, J., R. Magnani, and J. Knowles. 1993. Handbook of Indicators for Family Planning Program Evaluation. The EVALUATION Project, Carolina Population Center, Chapel Hill, North Carolina.

Brown, L., M. Tyane, J. Bertrand, D. Lauro, M. Abououakil, and L. deMaria. 1995. "Quality of care in family planning services in Morocco." Studies in Family Planning, 26,3:154-168.

Bruce, J. 1990. "Fundamental elements of the quality of care: A simple framework." Studies in Family Planning, 21,2:61-91.

Buckner, B., A. Tsui, A. Hermalin, and C. McKaig, Eds. 1995. A Guide to Methods of Family Planning Program Evaluation, 1965-1990. The EVALUATION Project, Carolina Population Center, Chapel Hill, North Carolina.

Diop, N., A. Cerulli, and D. Sanogo. 1996. "Regional dissemination of Senegal's Situation Analysis study results: A promising way to maximize Operations Research utilization." African Journal of Fertility, Sexuality and Reproductive Health, 1,2:169-173.

Fisher, A. 1993. "Family planning in Africa: A summary of recent results from Operations Research studies." In Africa Operations Research and Technical Assistance Project: End-of-Project Conference, Nairobi, 4-7 October. Population Council, New York.
Fisher, A., J. Laing, J. Stoekel, and J. Townsend. 1991. Handbook for Family Planning Operations Research Design. Population Council, New York.

Fisher, A., K. Miller, and R. Miller. 1998. "Situation Analysis: Assessing the functioning and quality of the service delivery environment." In Tsui, A. and J. Phillips, Eds., Methods for Evaluating Family Planning Program Impact. Oxford University Press, Oxford, United Kingdom.

Hardee, K., B. Janowitz, J. Stanback, and M. Villinski. 1998. "What have we learned from studying changes in service guidelines and practices?" International Family Planning Perspectives, 24,2:84-90.

Huntington, D., B. Mensch, and N. Toubia. 1993. “A new approach to eliciting information about induced abortion." Studies in Family Planning, 24,2:120-124.

Huntington, D., B. Mensch, and V. Miller. 1996. "Survey questions for the measurement of induced abortion." Studies in Family Planning, 27,3:155-161.

Jain, A. 1989. "Fertility reduction and the quality of family planning services." Studies in Family Planning, 20,1:1-16.

Kim, Y. and C. Lettenmaier. 1995. Tools to Assess Family Planning Counseling: Observation and Interview. John Hopkins School of Public Health Center for Communication Programs, April.

Leonard, A., Ed. 1997. Situation Analysis: How Can We Use It Better? Report of a workshop on strengthening the utilization of Situation Analysis for planning, managing, and evaluating reproductive health services, Nairobi, Kenya, May 29-31, 1996. Population Council, Nairobi, Kenya.

Maggwa, N. and I. Askew. 1997. Integrating STI/HIV Management Strategies Into Existing MCH/FP Programs: Lessons From Case Studies in East and Southern Africa. Population Council, Nairobi, Kenya.

Maribe, L., N. Maggwa, I. Askew, and K. Miller. 1997. Using a Rapid Assessment Approach to Evaluate the Quality of Care in an Integrated Program: The Experience of the Family Health Division, Ministry of Health, Botswana. Population Council, Nairobi, Kenya.

Measham, D., K. Stein, and B. Winikoff. 1996. The Unmet Family Planning Needs of Breastfeeding Women: Lessons from Nigeria and Senegal. Report of Technical Assistance provided by the Africa Operations Research and Technical Assistance Project II to Wellstart International and the Population Council's Ebert Program. Population Council, New York.

Mensch, B., M. Arends-Kuenning, and A. Jain. 1996. "The impact of the quality of family planning services on contraceptive use in Peru." Studies in Family Planning, 27,2:59-75.

Miller, R. and R. Frerichs. 1992-1993. “An integrated approach to Operations Research for strengthening family planning programs: A case example in Kenya." International Quarterly of Community Health Education, 13,3:183-199. 
Miller, R., K. Miller, L. Ndhlovu, J. Solo, and O. Achola. 1996. "A comparison of the 1995 and 1989 Kenya Situation Analysis study findings." African Journal of Fertility, Sexuality, and Reproductive Health, 1,2:162-168.

Miller, R., A. Fisher, K. Miller, L. Ndhlovu, N. Maggwa, I. Askew, D. Sanogo, and P. Tapsoba. 1997. The Situation Analysis Approach to Assessing Family Planning and Reproductive Health Services: A Handbook. Population Council, New York.

Samara, R., B. Buckner, and A. Tsui. 1996. Understanding How Family Planning Programs Work: Findings from Five Years of Evaluation Research. The EVALUATION Project, Carolina Population Center, Chapel Hill, North Carolina.

Sloan, N., C. Quimby, B. Winikoff, and N. Schwalbe. 1995. Guidelines and Instruments for a Situation Analysis of Obstetric Services. Population Council, New York.

Solo, J., A. Cerulli, R. Miller, I. Askew, and E. Pearlman. 1998. Strengthening the Utilization of Family Planning Operations Research: Findings from Case Studies in Africa. Africa OR/TA Project II, Nairobi, Kenya.

Stein, K. 1996. "Service quality among women receiving $\mathrm{MCH}$ and family planning services." African Journal of Fertility, Sexuality, and Reproductive Health, 1,2:146-152.

Touré, I., N. Diop, A. Diallo, and D. Sanogo. 1997a. Evaluation des programmes de santé de la reproduction. Prepared by the Africa OR/TA Project II in cooperation with the Ministère de la Santé et de l'Action Sociale, and Programme National de Planification Familiale, Dakar, Senegal.

Touré, I., N. Diop, and D. Sanogo. 1997b. Indicateurs de l'objectif strategique. Report of Technical Assistance provided by the Africa OR/TA Project II to the Ministère de la Santé. Dakar, Senegal.

Valadez, J. 1991. Assessing Child Survival Programs in Developing Countries: Testing Lot Quality Assurance Sampling. Department of Population and International Health, Harvard School of Public Health, Boston, Massachusetts.

Valadez, J., R. Transgrud, M. Mbugua, and T. Smith. 1997. "Assessing family planning service-delivery skills in Kenya." Studies in Family Planning, 28,2:143-150.

\section{NOTES}

1 The study in Mali was requested and funded by the USAID-funded community-based distribution (CBD) program (implemented by the Population Council). The study in Mauritania was requested and funded by the United Nations Population Fund (UNFPA). Both studies were undertaken by the Centre d'Etudes et de Recherche sur la Population pour le
Développement (CERPOD). The studies in Cameroon and Guinea were funded and undertaken through the USAID-supported Africa Operations Research and Technical Assistance (OR/TA) Project II.

2 For example, within the USAID system there are projects devoted solely to supporting logistics, management, training, IEC, research, etc.

3 The three problems were missed opportunities to educate $\mathrm{MCH}$ clients about family planning, little information on tubal ligation given during family planning counseling, and long waiting times at clinics.

4 To give just one example, the time spent by providers interacting with clients is frequently felt to be too short. Situation Analysis studies collect data on the duration of client-provider interactions. Thus it would be relatively easy to ascertain the average time spent with clients, whether this varies with the client's characteristics, whether it affects the amount of information exchanged, and so on.

5 Data sets are available upon request from the Population Council, New York.

6 A similar approach is planned for Zambia, in that the sample includes both experimental and comparison clinics; the baseline study was completed in 1996. To date, however, no attempt has been made to develop a set of indicators that will be used to make comparisons in the future.

7 This evaluation strategy is known by researchers as a "pre-intervention post-intervention nonequivalent comparison group" design, which is a form of quasiexperiment (Fisher et al., 1991).

8 The LQAS approach has its origins in industrial quality assurance and was adapted for evaluating primary healthcare interventions in developing countries (e.g., Valadez, 1991). More recently, the LQAS approach has been used to evaluate family planning training interventions in Kenya (Valadez et al., 1997).

9 Unfortunately, it was not possible to collect data from all experimental and comparison clinics during the national study.

10 This intervention consists of three broad activities: training staff; equipping clinics with medical supplies, laboratory tests, and all contraceptive methods; and establishing a mechanism to facilitate client follow-up.

11 This study is part of an ongoing program of research being undertaken by the Population Council (with funding from the Rockefeller Foundation) to address the issue of measuring the impact of improving service quality.

12 In Ghana, The Futures Group International assisted the $\mathrm{MOH}$ in presenting the data via user-friendly software, similar to the EASEVAL software used to present DHS data.

13 Solo et al. (1998) drew similar conclusions in their review of lessons learned on how to increase the utilization of results from OR studies generally. 


\section{FACTORS AFFECTING QUALITY}
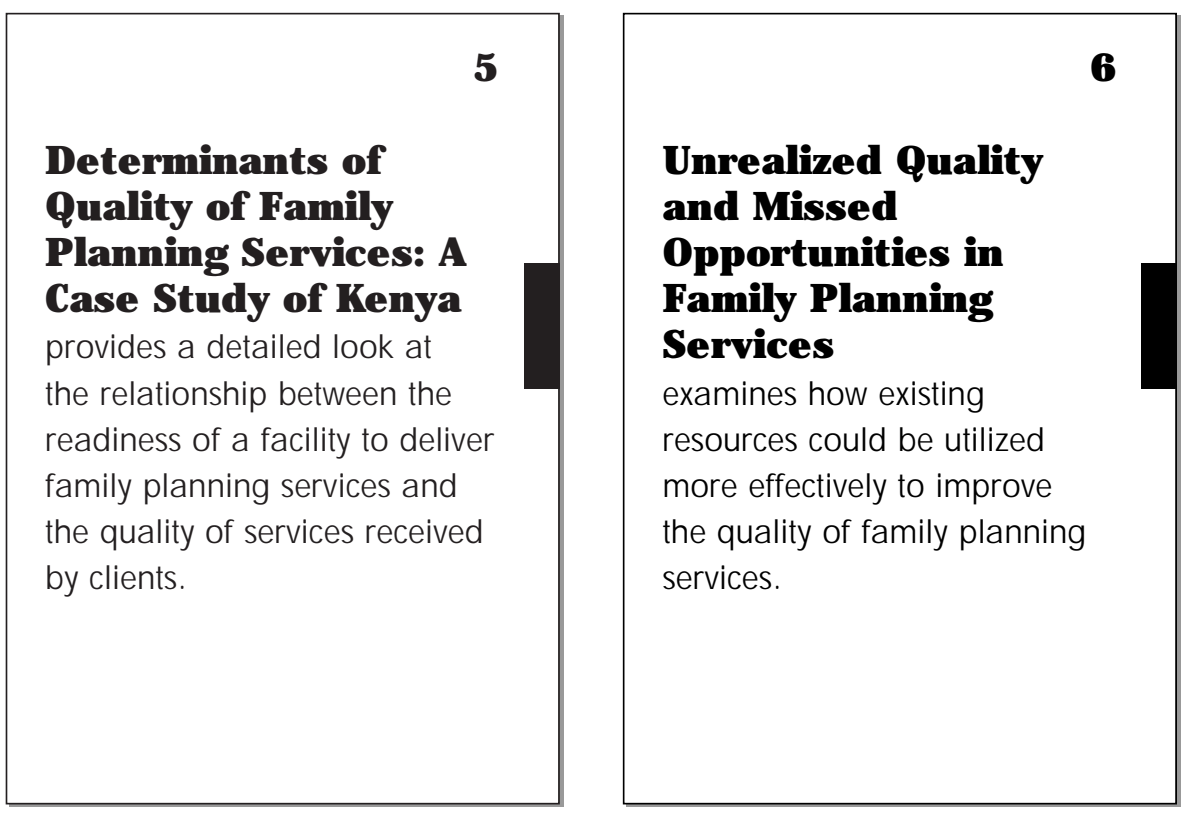

Urban and Rural
Family Planning
Services: Does Service
Quality Really Differ?
presents findings that
challenge the common
expectation that urban family
planning services are superior
to rural services.





\section{KEY POINTS AND CONCLUSIONS}

\section{Determinants of Quality of Family Planning Services: A Case Study of Kenya}

Using data from the 1995 Situation Analysis conducted in Kenya, this chapter analyzes the readiness and quality of family planning programs and the role of clients' personal characteristics to assess whether there is a relationship between how well prepared a facility is-in terms of infrastructure, equipment and supplies, and staff training - to deliver family planning services, and the quality of services received by clients. The analysis leads to the following conclusions:

- Only provider training in long-term or permanent contraceptive methods, such as NORPLANT $^{\circledast}$ and female sterilization, is related to the provider's mentioning those methods to clients. No similar relationship exists for shortterm methods, such as combined oral contraceptives (COCs) and condoms.

- Discussions with clients on IUDs, NORPLANT', and spermicides are related to the facility's preparedness to provide those methods, whereas discussions with clients on injectables, condoms, and COCs are not.

- The availability of medical equipment, such as blood pressure machines, at a facility is believed to motivate providers to use that equipment for assessing medical contraindications to certain contraceptive methods.

- There is an association among the presence of certain information, education, and communication (IEC) materials (specifically contraceptive samples and anatomical models) at a facility, their use in counseling, and the amount of information given to clients about their method of choice.
- Client characteristics play a mediating role in the way services are provided. Providers use client characteristics not only to identify the most suitable method, but also to restrict access to contraception. Specifically, marital status and number of children have the greatest influence on which methods providers mention to clients. Married women are more likely to hear about progestin-only pills (POPs), condoms, and IUDs, and women with more children are less likely to hear about any method other than female sterilization. On the other hand, client characteristics have no significant impact on whether a client is told about the advantages and side effects of a method or about the ability to switch methods if she so desires.

- There is an association between the client's educational level and the information she receives on how to use a method, its disadvantages, and what to do in case of a problem. Clients with secondary education are much more likely than clients with primary education to be told about problem management.

The overall conclusion of the analysis is that readiness indicators are not always directly related to quality indicators. For example, specific training on family planning methods does improve quality of care in terms of both counseling and the use of IEC materials with clients. However, general family planning training does not have the same impact on counseling and is not associated with our indicators of technical competence. 



\section{Determinants of Quality of Family Planning Services: A Case Study of Kenya}

\section{Lewis Ndhlovu}

\section{BACKGROUND}

Several Situation Analysis studies conducted in sub-Saharan Africa since 1989 have identified weaknesses in the region's family planning programs in terms of availability, functioning, and quality of family planning services (see Chapter 3 ). As discussed in Chapter 1, a major contribution of Situation Analysis has been the ability to identify program weaknesses and suggest interventions to improve program planning and operations. Interventions usually aim at improving the health facility infrastructure (see Chapter 11), under the assumption that improvements in infrastructure components lead to better service quality for clients. This chapter presents an analysis of the relationship between health facility infrastructure and service quality.

The analysis of service quality in this chapter is based on the Bruce-Jain conceptual framework (Bruce, 1990), an adapted version of which is depicted in Figure 5-1. The health facility infrastructure, or readiness-box A, Facility Preparedness, in the figure 1 - consists of five elements: (1) contraceptives and supplies; (2) physical facilities and equipment; (3) staff training and attitudes; (4) supervision and management; and (5) information, education, and communication (IEC) materials and activities. It is postulated that having fully prepared health facilities leads to improved service quality (box C), broadly defined as (1) method choice, (2) information given to clients, and (3) technical competence.

Clients' personal characteristics (box B) are also hypothesized to affect service quality. The services that providers offer to clients are mediated by consideration of the client's age, marital status, number of children, and educational level. In turn, improved service quality is hypothesized to have a positive impact on contraceptive behavior and demographic outcomes (box D), namely clients' knowledge, satisfaction, health, and contraceptive use; the total fertility rate; and contraceptive prevalence.

Since the mid-1980s, there have been a number of quality-of-care studies based on the Bruce-Jain framework, yet there has been limited research on the relationships among the components of the framework shown in Figure 5-1. This despite the fact that researchers have been keenly interested in determining the effects of service quality on women's behavior and demographic outcomes (Bertrand et al., 1995; Veney et al., 1993; Beegle, 1995; Feyisetan and Ainsworth, 1994; Oliver, 1995). This issue is of interest to both researchers and program managers for a number of reasons. Historically, population researchers were concerned mainly with population growth. Quality of care did not become a central concern until elaborated by Bruce and Jain in 1990 . Hence family planning programs were assessed largely for their potential impact on demographic outcomes, such as contraceptive prevalence, adoption, continuation, and total fertility rate. More recently, recommendations from and responses to the 1994 International Conference on Population and Development (ICPD) have added impetus to concerns about clients' health and satisfaction.

In examining the impact of health facility infrastructure on service quality, it is important to note that extensive evidence from Situation Analysis 


\section{Figure 5-1. Conceptual relationship between facility prepa edness and qudity of services provided to dients}

A=Facility Preparedness

+ Controceptives and supplies

+ Phrsical facilities and equipment

+ Staff training and attitudes

+ Superyision and mancsgement

+ IEC materiak and activities studies in the region and other studies shows a wide variability in the infrastructure of health facilities in sub-Saharan countries (see Chapter 3). This variability suggests that the quality of services clients receive is likely to vary as well. It is therefore important to investigate the factors that determine quality. It would be unrealistic to expect uniform standards of quality for all programs (Jain et al., 1992), but it is important to identify elements of the infrastructure that are correlates of quality.

Clients' views about service quality indicate that, in addition to good interpersonal relations with providers, they consider facility infrastructure to be important. For example, they perceive facilities to be of good quality if they have adequate supplies and equipment for medical examinations (Ndhlovu, 1995; Collins et al., 1996; United Nations
$\mathrm{D}=$ Import of High-Qualit' Services

+ Client knowledge

+ Client satistaction

+ Client health

+ Contraceptive use

- Aoceptance

- Continuation

+ Total fertility rate

+ Contraceptive preyalence

al Characteristics

of Client:

$$
\begin{aligned}
& \text { + Age } \\
& \text { + Marital status } \\
& \text { + Number of children } \\
& \text { + Educational bevel }
\end{aligned}
$$

Development Programme, World Bank, and WHO 1994). Haddad and Fournier (1995) describe a study in Zaire in which villagers stated that if the nearest health facility lost its equipment (for example, a microscope), they would seek services from another facility, even if it were farther away. A study by Tsui (1980) in rural Mexico corroborates the importance clients attach to the availability of medical supplies and equipment for examinations. Therefore, an analysis of the relationship between infrastructure and quality can assist in understanding clients' views of service quality and incorporating those views into the services provided (Veney et al., 1993).

Situation Analysis studies reveal that access to and quality of services are restricted by service providers based on certain background characteristics 
of clients (see Chapter 8). For example, service providers report that they consider the client's age, number of children, and marital status, as well as spousal consent, to be important factors in what contraceptives they recommend to clients. Data on the personal characteristics shown in box B in Figure 5-1 (age, marital status, number of children, and education) is analyzed below with respect to the elements of quality shown in box C. The hypothesis is that providers base their decisions on policy guidelines recommended by the Ministry of Health.

\section{DATA AND METHODS}

This chapter uses Kenya as an illustrative case study to analyze the relationships among facility preparedness, client characteristics, and the quality of services offered at health facilities. Though the Bruce-Jain framework has been used extensively by researchers, it does not provide means of linking the large array of indicators of facility infrastructure and service quality. Therefore, the first task of this analysis was to identify salient indicators in terms of logical connection, importance, and relevance. The process of identifying these indicators and the possible links among them involved consultations with program managers, researchers, and service providers.

This analysis is based on a 1995 Situation Analysis study of 254 health facilities in Kenya, including 473 interviews with providers and 241 observations of new family planning clients. ${ }^{2}$ Of the sampled service delivery points (SDPs), 58\% are in the governmental sector, which provides the majority of health services in Kenya. However, these facilities have been facing a gradual deterioration in infrastructure since the late 1980s. Nairobi City Commission (NCC) health facilities, which constitute $17 \%$ of the sample, represent a municipal authority that serves densely populated urban areas, and these facilities tend to have better resources than government centers. The rest of the sample consists of nongovernmental organization (NGO)-sector SDPs, which are supported by the Family Planning Association of Kenya, Marie Stopes International, and the Christian Association of Kenya.
In addition, facilities can be divided into hospitals, health centers, and dispensaries. Hospitals ( $24 \%$ of the sample) are generally best endowed in terms of staff, equipment, and range of services offered. Health centers ( $48 \%$ of the sample) and dispensaries $(28 \%)$ have fewer resources. Dispensaries are the smallest units of service delivery in Kenya, and they are nearest physically to the majority of the population.

Table 5-1 shows the hypothesized relationships between several elements of readiness that are investigated in this chapter and various indicators of quality. (Annex 5-1 lists the specific indicators of readiness, quality of care, and client characteristics used for this analysis.) The indicators under the elements of readiness shown in the center column of Table 5-1 are measured mainly at the SDP level, and the indicators of quality in the righthand column are measured at the client level. However, in order to relate readiness to quality, the two units of analysis must be the same. For this analysis, the comparison is carried out on the SDP level, so results from the client level are averaged to give overall results for each SDP.

The readiness indicators are fairly straightforward, having either a "yes" or "no" response for each SDP that is taken directly from the inventory and staff provider responses. For example, there is a yes / no response at the SDP level for the presence of a flip chart at an SDP. The only exception is indicators of staff training, which are taken at the staff level. An SDP may have two or more providers, each with different levels of training. For this analysis, if an SDP has at least one provider with the training of interest, it receives a "yes" on indicators measuring the presence of trained providers.

Assigning yes/no scores to the quality indicators is a bit more complex since they are usually based on several client observations. For example, suppose five clients were observed at an SDP that has a flip chart, and the flip chart was used with three of the five. In this analysis, if an action is taken with half or more of observed clients at an SDP, that SDP receives a "yes" on that quality indicator. Otherwise the SDP receives a "no." In this way, SDP-level indicators of readiness and quality can be correlated. 


\section{Table 5-1. Hypothesized relationships between readiness and quality indicators}

\begin{tabular}{|c|c|c|}
\hline & These readiness elements . . & $\begin{array}{l}\text {... may affect } \\
\text { these quality indicators }\end{array}$ \\
\hline \multirow{4}{*}{$\begin{array}{l}\text { Readiness elements } \\
\text { affecting method choice }\end{array}$} & \multirow[t]{2}{*}{ Staff training } & Individual methods mentioned \\
\hline & & Total methods mentioned ${ }^{a}$ \\
\hline & \multirow{2}{*}{$\begin{array}{l}\text { Equipment and supplies for } \\
\text { each method }{ }^{\mathrm{b}}\end{array}$} & Individual methods mentioned \\
\hline & & Total methods mentioned ${ }^{\mathrm{a}}$ \\
\hline \multirow{2}{*}{$\begin{array}{l}\text { Readiness elements } \\
\text { affecting information } \\
\text { exchange }\end{array}$} & Staff training ${ }^{c}$ & $\begin{array}{l}\text { Information given on accepted } \\
\text { method }^{d}\end{array}$ \\
\hline & Use of IEC materials ${ }^{\mathrm{e}}$ & $\begin{array}{l}\text { Information given on accepted } \\
\text { method }^{d}\end{array}$ \\
\hline \multirow{2}{*}{$\begin{array}{l}\text { Readiness elements } \\
\text { affecting technical } \\
\text { competence }\end{array}$} & $\begin{array}{l}\text { Equipment for client } \\
\text { assessment }^{f}\end{array}$ & Client assessment $^{9}$ \\
\hline & $\underline{S t a f f}_{\text {training }}{ }^{c}$ & Client assessment $^{9}$ \\
\hline
\end{tabular}

a Methods include combined oral contraceptives (COCs), progestin-only pills (PO Ps), condom, IUD, injectable, NO RPLANT ${ }^{\circledR}$, lactational amenorrhea method (LAM), vasectomy, and female sterilization.

b Equipment for COCs, condoms, and spermicide: blood pressure machine and scale; equipment for IUDs: gloves, cotton wool, swabs, speculum, tenaculum, and light source; equipment for injectables: cotton wool, antiseptic lotion, needles, and syringes; equipment for voluntary surgical contraception (VSC): minilap kit, gloves, local anesthesia, needles/syringes, sutures/needles, and antiseptic lotion.

c Training includes clinical family planning skills; IUD insertion/removal; mini-laparotomy/local anesthetic (ML/LA); NO RPLANT ${ }^{\circledast}$ insertion/removal; vasectomy; management/planning; supervision/evaluation; information, education, and communication (IEC) skills; LAM; and natural family planning (NFP) counseling.

d Information given includes how to use method, advantages, disadvantages, side effects, management of side effects, and possibility of switching methods.

e IEC materials include flip charts, contraceptive samples, and anatomical models.

f Equipment includes blood pressure machine and scales.

${ }^{9}$ Client assessment includes medical history, last menstrual period (LMP), weight, blood pressure, and physical exam.

It is also important to note certain limitations of the data. First, the quality indicators are based on a varying number of observations of client-provider interactions per facility. Of the 254 health facilities visited during the study, just over half $(53 \%)$ had at least one new family planning client observed. Of the latter facilities, $53 \%$ had only one client observed, $27 \%$ had two, $11 \%$ had three, and $9 \%$ had more than three. The remaining facilities, at which revisit clients but no new family planning clients were observed, were excluded from this analysis. Revisit clients were excluded from the analysis because the Ministry of Health policy guidelines do not require the performance of extensive procedures for these clients, and this limited the indica- tors of quality that could be observed. Thus for these facilities, it is not possible to describe the quality of services offered.

Second, the use of summary scores to characterize client-provider interactions needs cautious interpretation. A typical maternal and child health $(\mathrm{MCH})$ / family planning facility in Kenya has an average of one to two nurse midwives providing services, and the care providers gave during their interaction with clients on the day of the study visit is assumed to be representative of the quality of services delivered at that facility. This assumption has some validity since clients usually do not receive services from a single provider, but are counseled and examined by a number of different 
nurses before they finally obtain their contraceptives. For the client, the process of receiving care is an experience more with a health facility than with individual providers.

Although the Bruce-Jain framework proposes six elements of quality, only three of these are discussed in this chapter. Measurements of interpersonal relations from quantitative surveys have not yielded satisfactory findings, and hence this indicator is excluded. Clients' responses about interpersonal relations are subject to a courtesy bias and lack variation (see the discussion under Q.1 in Chapter 3). Another element of the framework that is subject to the same problem is mechanisms to ensure continuity of care. Similarly, only a few indicators of infrastructure that have logical and direct links with service quality indicators are examined; other infrastructure indicators, including supervision, management, and recordkeeping, could not be analyzed because relevant information for these indicators is not available.

The analysis of the relationship between client characteristics and quality of family planning services is carried out a bit differently. Information on clients' personal characteristics was gathered in exit interviews with the clients. As shown in Table $5-2$, this information is hypothesized to affect methods mentioned and information given to the client about the accepted method during the consultation. Because both the exit interview and the observation were conducted at the client level, no combining of scores is necessary. The unit of analysis is the client.

Most of the analysis presented here relies on simple statistical techniques: frequency distributions, cross-tabulations, and measures of association based on the chi square test of independence. Multivariate analysis using logistic regression is applied to the data on clients' personal characteristics.

The next section presents the results of the analysis with regard to readiness indicators affecting methods discussed with clients, readiness indicators affecting information given to clients about their selected method, readiness indicators affecting client assessment, client characteristics affecting methods discussed with clients, and finally client characteristics affecting information given to clients about their selected method. The final

\section{Table 5-2. Hypothesized relationship between dient characteristics and quality indicators}

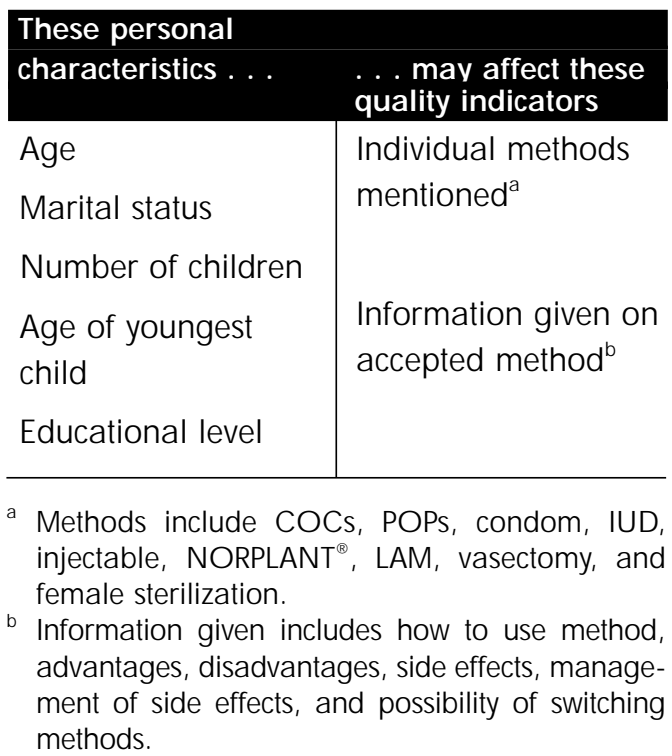

section of the chapter presents conclusions drawn from these results.

\section{RESULTS}

\section{Readiness Indicators Affecting Methods Discussed with Clients}

The availability of family planning methods at health facilities is hypothesized to influence the method choice actually offered to clients. Where service providers are able to counsel clients on a variety of methods, they may be more likely to meet client needs. In this section, provider training and equipment availability for individual methods (readiness) are related to the methods discussed with clients and the total number of methods mentioned to clients (quality).

\section{Staff Training}

Since the early 1980s, the Kenyan government has invested heavily in the training of staff for reproductive health. The 1995 Situation Analysis study showed that the majority of service providers in $\mathrm{MCH} /$ family planning units had attended at least 
one family planning course since their post-basic training. These courses covered IUD insertion and removal, clinical family planning skills, mini-laparotomy/local anesthetic (ML/LA), NORPLANT ${ }^{\oplus}$ insertion and removal, vasectomy, counseling for voluntary surgical contraception (VSC), management/planning, supervision and evaluation, IEC skills, lactational amenorrhea method (LAM), and natural family planning (NFP) counseling. A detailed analysis of this training shows that the courses varied in duration, depth, and breadth, and many of the topics covered were found to overlap.

Though it is widely assumed that training imparts skills to service providers and leads to improvements in service quality, this may not always be the case (Maggwa and Askew, 1997; see also Chapter 11). The present analysis shows that only specific training in long-term and permanent methods is related to higher rates of mentioning these methods to clients. For example, at $62 \%$ of facilities with at least one provider trained in female sterilization, the majority of clients were told about ML/LA. In contrast, the majority of clients were told about ML/LA at only $41 \%$ of SDPs without providers trained in this method. Generally, clients who attended health facilities with at least one provider trained in ML/LA, NORPLANT $^{\oplus}$, and vasectomy counseling were more likely to be told about these methods, as well as LAM. However, no type of refresher training appears to increase discussion of any other methods: no differences were observed with regard to individual methods discussed with clients at facilities with providers trained in clinical family planning, IUD insertion/removal, or IEC.

Staff training can also be related to the total number of methods discussed with clients, specifically with whether the majority of clients at an SDP were told about five or more methods. Only training in long-term methods, NFP, and LAM appears to be related to mentioning more methods to clients. For example, where there was at least one provider trained in ML/LA, five or more methods were discussed with the majority of clients at $52 \%$ of facilities, compared with $35 \%$ of facilities with providers not so trained. A similar pattern was observed for facilities with providers trained in NORPLANT ${ }^{\circledast}$, NFP, and LAM. As above, however, no difference in total methods mentioned to clients was observed at SDPs with at least one provider trained in clinical family planning, IUD insertion/ removal, or IEC skills.

The observed influence of training in long-term and permanent methods may be due to the fact that providers with these skills are more likely to be located at hospitals. However, providers at health centers and dispensaries are expected to refer clients for methods they do not provide. In fact, there is only a small difference in the mean number of methods providers discussed with clients at hospitals (six methods), health centers (six methods), and dispensaries (five methods). This finding leads to the conclusion that the number of methods mentioned is not a function of the size of the SDP. (See Chapter 6 for further discussion of providers not discussing available methods.)

\section{Commodity and Equipment Availability}

Referrals aside, the availability of contraceptive methods and the equipment needed to provide those methods are prerequisites for successful provision of any method. In this analysis, an SDP is considered fully prepared to deliver a method if it had all the equipment and supplies necessary, and if it had not experienced a stockout of the method in the 6 months prior to the study visit. Annex 5-1 lists the equipment deemed necessary for each method by an expert group of researchers and family planning managers. We now turn to the relationship between preparedness to deliver a method and whether that method is mentioned to the majority of clients. Note that male and female sterilization procedures were offered at only a limited number of facilities and thus could not be analyzed.

The results vary by method. For the IUD, NORPLANT $^{\oplus}$, and spermicides, the majority of clients were told about these methods at more SDPs that were prepared to deliver them, as compared with those that were not prepared. For Depo Provera, condoms, and oral contraceptives, which were generally available in most facilities, discussions during counseling sessions did not appear to be related to the availability of these methods. The lack of variation in the availability of these methods may explain the lack of correlation with their being mentioned to clients. 
Other indicators of method choice include the total number of methods discussed and whether clients were asked about their method preference. However, these indicators were not correlated with availability of equipment and contraceptive supplies at health facilities.

\section{Readiness Indicators Affecting Information Given to Clients About Their Selected Method}

Successful use of contraceptives is dependent upon proper and sufficient counseling for clients. In this section, information given to clients about their selected method includes how to use it, its advantages, its disadvantages, its side effects, management of side effects, and the possibility of switching methods. In general, it appears that providers discussed important information with clients: how to use the method ( $86 \%$ of clients), side effects $(65 \%)$, advantages $(66 \%)$, and disadvantages $(62 \%)$. Less than half of clients were told about the possibility of switching methods. Though the figures appear high for individual data items, providers did not offer a full range of information about each method (see Chapter 6 for further discussion of complete counseling).

\section{Staff Training}

Staff training, particularly in IEC skills, is hypothesized to increase the amount of information given to clients about their chosen method. Indeed, training in IEC skills, NFP, and VSC counseling appears to have an effect on the amount of information given to clients. The majority of clients received information about the advantages, disadvantages, and management of side effects of their chosen method at a larger proportion of facilities with at least one provider trained in IEC skills, NFP, and VSC counseling, as compared with facilities without these trained providers (see Chapter 3 for more information on the relationship between training in IEC skills and observed counseling).

The association with NFP and LAM training is particularly strong. For example, in $78 \%$ of facilities where providers were trained in NFP, the majority of clients were told about the disadvantages of their accepted method, compared with
$63 \%$ at SDPs where providers were not so trained. Likewise, the majority of clients were told about disadvantages at $90 \%$ of SDPs with at least one provider trained in LAM, compared with $62 \%$ of facilities where no providers had this training. Courses in these two user-dependent methods are known to pay strong attention to counseling and information exchange so clients can understand and properly use their method. The above findings suggest that intensive training in counseling for service providers can improve the quality of information given to clients about their accepted method.

Training in clinical family planning skills and other specific methods showed no relationship with information given to clients about their accepted method. This finding appears to be particularly notable for providers trained in IUD insertion/removal, who showed no difference in the information given clients about that method.

\section{IEC Use}

IEC materials normally found at health facilities include flip charts, brochures, pamphlets, samples of contraceptives, posters, anatomical models, charts, and calendars. The extent to which providers used IEC materials during counseling varied considerably. Most of the clients were counseled with contraceptive samples ( $80 \%$ of clients), followed by anatomical models (25\%) and flip charts (14\%). Other IEC materials were used in less than $5 \%$ of client-provider interactions. ${ }^{3}$

A provider can use IEC materials only if they are physically present at the SDP, which is a measure of facility readiness. In this section, the use of IEC materials is analyzed with respect to the information given to clients on their accepted method. This comparison relates an indicator associated with both quality and readiness (IEC use) to an indicator of quality (information given to clients).

Providers who used contraceptive samples and anatomical models during counseling sessions appeared to provide more contraceptive information to clients. Table 5-3 shows that at SDPs where providers generally used these IEC materials, the majority of clients were more likely to be told about important aspects of their accepted method. The relationship appears to hold as well for use of 


\section{Table 5-3. Percent of SDPs at which the majority of dients were given information on their accepted method, by use or nonuse of certain IEC materials}

\begin{tabular}{|c|c|c|c|c|c|c|}
\hline $\begin{array}{l}\text { Were Majority of } \\
\text { Clients Counseled } \\
\text { Using IEC Materials? }\end{array}$ & $\begin{array}{l}\text { How } \\
\text { to Use } \\
\text { Method }\end{array}$ & $\begin{array}{c}\% \text { OF SDPs A7 } \\
\text { Advantages } \\
\text { of } \\
\text { Method }\end{array}$ & $\begin{array}{l}\text { T WHICH Mc } \\
\text { Disadvan- } \\
\text { tages of } \\
\text { Method }\end{array}$ & $\begin{array}{l}\text { ST CLIENT } \\
\text { Side } \\
\text { Effects of } \\
\text { Method }\end{array}$ & $\begin{array}{l}\text { WERE TOL } \\
\text { Possible } \\
\text { to } \\
\text { Switch }\end{array}$ & $\begin{array}{l}\text { D: } \\
\text { Management } \\
\text { of } \\
\text { Problems }\end{array}$ \\
\hline $\begin{array}{l}\text { Flip Chart } \\
\text { Yes }(n=100) \\
\text { No }(n=75)\end{array}$ & $\begin{array}{r}100 \\
91\end{array}$ & $\begin{array}{l}60 \\
71 \\
\end{array}$ & $\begin{array}{l}70 \\
65\end{array}$ & $\begin{array}{l}70 \\
75\end{array}$ & $\begin{array}{l}60 \\
45\end{array}$ & $\begin{array}{l}67 \\
69 \\
\end{array}$ \\
\hline $\begin{array}{l}\text { Samples of } \\
\text { Contraceptives } \\
\text { Yes }(n=179) \\
\text { No }(n=43)\end{array}$ & $\begin{array}{l}92 \\
63^{\mathrm{a}} \\
\end{array}$ & $\begin{array}{l}73 \\
39^{a} \\
\end{array}$ & $\begin{array}{l}67 \\
42^{\mathrm{a}} \\
\end{array}$ & $\begin{array}{l}68 \\
52^{\mathrm{b}}\end{array}$ & $\begin{array}{l}45 \\
26^{\mathrm{b}}\end{array}$ & $\begin{array}{l}71 \\
50^{b}\end{array}$ \\
\hline $\begin{array}{l}\text { Anatomical Models } \\
\text { Yes }(n=46) \\
\text { No }(n=108)\end{array}$ & $\begin{array}{r}100 \\
82^{\mathrm{a}}\end{array}$ & $\begin{array}{l}83 \\
69^{a}\end{array}$ & $\begin{array}{c}78 \\
57^{\mathrm{a}}\end{array}$ & $\begin{array}{l}85 \\
63^{a}\end{array}$ & $\begin{array}{l}54 \\
36^{\mathrm{b}}\end{array}$ & $\begin{array}{l}84 \\
60^{\mathrm{a}}\end{array}$ \\
\hline
\end{tabular}

Note: Posters were not observed to be used for counseling, and pamphlets or brochures were used in only 10 interactions.

flip charts, although the results are not significant. In general, these results indicate an association among the presence of certain IEC materials, their use in counseling, and the amount of information given to clients about their selected method.

\section{Readiness Indicators Affecting Client Assessment}

There has been extensive debate among researchers and program managers about the importance and relevance of medical procedures such as taking clients' weight or blood pressure for family planning services (see Chapters 8 and 9). One view is that medical examinations constitute a barrier to services for women who need contraceptives, in part because of the associated cost, and in part because of the inconvenience, discomfort, or embarrassment experienced by many clients. Accordingly, the Kenyan program and many others in Africa no longer require clients to have medical examinations before most contraceptives can be prescribed (see Chapter 9). Another view, supporting sentiments expressed at the ICPD, encourages a broader approach to family planning services, whereby medical examinations are viewed as an important component of women's overall health. Evidence from qualitative studies conducted in Kenya shows that clients place great value on medical examinations, and this finding may indicate that clients view medical examinations as an important dimension of service quality (Solo et al., 1998; Ndhlovu, 1995).

In the present analysis, assessment of clients for medical contraindications based on LMP, medical history, blood pressure, and weight was found to be nearly universal in Kenyan health facilities. Providers generally asked clients about their LMP $(94 \%)$ and medical history $(81 \%)$. They also took the client's blood pressure (87\%) and weight $(82 \%)$. (See Chapter 9 for more discussion).

\section{Equipment Availability}

Availability of equipment at $\mathrm{MCH} /$ family planning units can motivate providers to use it for assessing clients' medical conditions. This is an important point since data collectors found that in 
some facilities, providers borrowed equipment from other departments in order to conduct medical examinations, a situation that discourages providers from taking blood pressure or weight. The point is supported by the data on availability and use of blood pressure machines and scales. At facilities where a blood pressure machine was available, $97 \%$ of providers took blood pressure, compared with $53 \%$ who had to borrow. Staff with a scale located at the $\mathrm{MCH} /$ family planning unit were nearly twice as likely to take weight as compared with those who had to borrow.

\section{Staff Training}

Table 5-4 shows the percentage of facilities at which most clients were assessed with various procedures, by whether the facility had at least one provider trained in various methods and skills. For most combinations of assessment and training, there appears to be a very weak relationship. On the other hand, among SDPs with a provider trained in IUD insertion/removal or in clinical family planning, the majority of clients were more likely to be assessed with medical history, LMP, and blood pressure. Among SDPs with a provider trained in IEC skills, the majority of clients were more likely to be assessed with medical history, blood pressure, and physical exam. These results indicate that, at least for courses in clinical family planning, IEC skills, and IUD insertion/removal, provider training may be associated with more frequent performance of certain client assessments.

\section{Client Characteristics Affecting Methods Discussed with Clients}

This section investigates the relationship between client characteristics and the methods they are told about, irrespective of the readiness of the facility. Client characteristics included in this analysis are age, marital status, parity, age of youngest child, reproductive intentions, and educational level. Providers can use these characteristics to identify and recommend the contraceptives most suitable to each client. On the other hand, findings from Situation Analysis studies strongly suggest that providers use the same characteristics to limit clients' access to contraceptives (see Chapter 8).
The various client characteristics were investigated with respect to factors providers mentioned to clients concerning their accepted method (Table 5-5). Results that are indicated as statistically significant in Table 5-5 reveal the client characteristics considered most important by providers during client counseling.

Of the five client characteristics included in Table 5-5, marital status and number of children had the greatest influence on which methods were mentioned. In general, married women were far more likely to hear about POPs, condoms, and IUDs than were unmarried clients. And women with more children were decreasingly likely to hear about any method except female sterilization. Interestingly, women with secondary education were almost six times as likely to hear about POPs as women with less education, but this relationship does not hold for the other methods. The ages of clients and their children do not figure significantly in the picture. Note also that Depo Provera is not included in Table $5-5$ because no client characteristics appeared to influence discussions about this method.

That providers offer different levels of counseling based on clients' education and marital status can likely be attributed to the providers' social values. The findings suggest that providers consider sex and use of contraceptives to be a preserve of marital relationships.

\section{Client Characteristics Affecting Information Given}

As noted earlier, important information that should be provided to clients about their accepted method includes how it should be used, its advantages, its disadvantages, possible side effects, management of side effects, and the possibility of switching to other methods. In this section, these six items of information are examined relative to clients' background characteristics.

Interestingly, client characteristics had no significant effect on whether a client was told about the advantages of the selected method, its possible side effects, or the ability to switch methods. This finding indicates that for some of the most important information, providers do not discriminate among clients based on their characteristics. 
Table 5-4. Percentage of health facilities where most clients were assessed for medical conditions, by whether at least one provider had attended different training courses

\begin{tabular}{|c|c|c|c|c|c|}
\hline $\begin{array}{l}\text { Did Facility Have At Least } \\
\text { One Provider Trained in: }\end{array}$ & $\begin{array}{l}\text { Medical } \\
\text { History }\end{array}$ & $\begin{array}{r}\text { FACIL } \\
\text { RE ASSE } \\
\text { LMP }\end{array}$ & $\begin{array}{l}\text { WHICH M } \\
\text { ITH TEST/ } \\
\text { Weight }\end{array}$ & $\begin{array}{l}\text { CLIENTS } \\
\text { CEDURE } \\
\text { Blood } \\
\text { Pressure }\end{array}$ & $\begin{array}{l}\text { Physical } \\
\text { Exam }\end{array}$ \\
\hline $\begin{array}{l}\text { IUD Insertion/Removal } \\
\text { Yes }(n=183) \\
\text { No }(n=19)\end{array}$ & $\begin{array}{l}91 \\
67^{\mathrm{a}} \\
\end{array}$ & $\begin{array}{l}98 \\
83^{\mathrm{a}} \\
\end{array}$ & $\begin{array}{l}86 \\
75 \\
\end{array}$ & $\begin{array}{l}92 \\
58^{a}\end{array}$ & $\begin{array}{l}74 \\
50\end{array}$ \\
\hline $\begin{array}{l}\text { Clinical Family Planning } \\
\text { Yes }(n=166) \\
\text { No }(n=36)\end{array}$ & $\begin{array}{l}91 \\
75^{\mathrm{a}} \\
\end{array}$ & $\begin{array}{l}99 \\
85^{\mathrm{a}}\end{array}$ & $\begin{array}{l}85 \\
85\end{array}$ & $\begin{array}{l}91 \\
75^{\text {a }} \\
\end{array}$ & $\begin{array}{l}71 \\
70 \\
\end{array}$ \\
\hline $\begin{array}{l}\text { ML/LA } \\
\text { Yes }(n=31) \\
\text { No }(n=171)\end{array}$ & $\begin{array}{l}91 \\
82\end{array}$ & $\begin{array}{l}43 \\
36\end{array}$ & $\begin{array}{l}30 \\
17\end{array}$ & $\begin{array}{l}96 \\
76^{\mathrm{a}}\end{array}$ & $\begin{array}{l}65 \\
57\end{array}$ \\
\hline $\begin{array}{l}\text { NO RPLANT }{ }^{\circledast} \text { Insertion/ } \\
\text { Removal } \\
\text { Yes }(n=38) \\
\text { No }(n=163)\end{array}$ & $\begin{array}{l}92 \\
87\end{array}$ & $\begin{array}{l}96 \\
97 \\
\end{array}$ & $\begin{array}{l}80 \\
86\end{array}$ & $\begin{array}{l}96 \\
86\end{array}$ & $\begin{array}{l}72 \\
71\end{array}$ \\
\hline $\begin{array}{l}\text { Vasectomy } \\
\text { Yes }(n=39) \\
\text { No }(n=163)\end{array}$ & $\begin{array}{r}100 \\
85\end{array}$ & $\begin{array}{r}100 \\
95 \\
\end{array}$ & $\begin{array}{l}96 \\
82\end{array}$ & $\begin{array}{l}96 \\
86\end{array}$ & $\begin{array}{l}91 \\
66^{\mathrm{a}}\end{array}$ \\
\hline $\begin{array}{l}\text { VSC Counseling } \\
\text { Yes }(n=81) \\
\text { No }(n=121)\end{array}$ & $\begin{array}{l}92 \\
85\end{array}$ & $\begin{array}{r}93 \\
100^{\mathrm{a}} \\
\end{array}$ & $\begin{array}{l}86 \\
84\end{array}$ & $\begin{array}{l}94 \\
84^{\mathrm{a}} \\
\end{array}$ & $\begin{array}{l}78 \\
66 \\
\end{array}$ \\
\hline $\begin{array}{l}\text { Supervision } \\
\text { Yes }(n=69) \\
\text { No }(n=133)\end{array}$ & $\begin{array}{l}90 \\
87\end{array}$ & $\begin{array}{r}100 \\
94\end{array}$ & $\begin{array}{l}85 \\
84\end{array}$ & $\begin{array}{l}93 \\
86\end{array}$ & $\begin{array}{l}76 \\
69\end{array}$ \\
\hline $\begin{array}{l}\text { IEC Skills } \\
\text { Yes }(n=62) \\
\text { No }(n=140)\end{array}$ & $\begin{array}{l}97 \\
84^{\mathrm{a}}\end{array}$ & $\begin{array}{r}100 \\
95\end{array}$ & $\begin{array}{l}92 \\
81\end{array}$ & $\begin{array}{l}97 \\
84^{\mathrm{a}}\end{array}$ & $\begin{array}{l}86 \\
64^{\mathrm{a}}\end{array}$ \\
\hline $\begin{array}{l}\text { NFP } \\
\text { Yes }(n=74) \\
\text { No }(n=128)\end{array}$ & $\begin{array}{l}94 \\
84\end{array}$ & $\begin{array}{l}98 \\
95\end{array}$ & $\begin{array}{l}85 \\
85\end{array}$ & $\begin{array}{l}90 \\
87\end{array}$ & $\begin{array}{l}67 \\
75\end{array}$ \\
\hline $\begin{array}{l}\text { LAM } \\
\text { Yes }(n=44) \\
\text { No }(n=158)\end{array}$ & $\begin{array}{l}93 \\
87\end{array}$ & $\begin{array}{r}100 \\
95\end{array}$ & $\begin{array}{l}85 \\
85\end{array}$ & $\begin{array}{l}96 \\
86\end{array}$ & $\begin{array}{l}70 \\
71\end{array}$ \\
\hline
\end{tabular}

Results for the other information are shown in Table 5-6. Clients' level of education had a significant effect on whether they were told about how to use a method, its disadvantages, and how to manage side effects. As compared with women having no education, those with secondary education 


\section{Table 5-5. Effects of clients' background characteristics on odds of being told about various contraceptives ${ }^{\mathrm{a}}$}

\begin{tabular}{|c|c|c|c|c|c|c|}
\hline & & & ODDS & RATIOS & & \\
\hline $\begin{array}{l}\text { Client } \\
\text { Characteristic }\end{array}$ & $\mathrm{COCs}$ & POPs & Condom & IUD & LAM & $\begin{array}{l}\text { Fem. } \\
\text { Steril. }\end{array}$ \\
\hline Age & & & & & & \\
\hline $15-19$ & 1.00 & 1.00 & 1.00 & $\mathrm{n} / \mathrm{a}$ & 1.37 & 0.47 \\
\hline $20-29$ & 1.20 & 0.92 & 0.58 & & 0.98 & 0.34 \\
\hline $30-46$ & 0.80 & 0.60 & 0.35 & & 1.00 & 1.00 \\
\hline Marital Status & & & & & & \\
\hline Married & 1.00 & 1.00 & 1.00 & 1.00 & 1.00 & 0.85 \\
\hline Not married & 0.34 & $0.19^{c}$ & $0.41^{d}$ & $0.35^{d}$ & 0.51 & 0.62 \\
\hline No. of Children & & & & & & \\
\hline $0-1$ & 1.00 & 1.00 & 1.00 & 1.00 & 1.00 & 1.00 \\
\hline $2-4$ & 0.77 & 0.75 & 0.65 & $0.38^{d}$ & 0.48 & 0.63 \\
\hline $5+$ & $0.24^{d}$ & $0.27^{\mathrm{d}}$ & $0.26^{d}$ & $0.15^{c}$ & $0.12^{d}$ & 1.36 \\
\hline Age of Youngest Child & & & & & & \\
\hline Up to 1 year & 1.00 & 1.00 & $\mathrm{n} / \mathrm{a}$ & $\mathrm{n} / \mathrm{a}$ & $\mathrm{n} / \mathrm{a}$ & 1.00 \\
\hline 1-2 years & 1.44 & 1.39 & & & & 0.40 \\
\hline$>2$ years & 2.03 & 0.94 & & & & 0.84 \\
\hline $\begin{array}{l}\text { Highest Educational } \\
\text { Level }\end{array}$ & & & & & & \\
\hline No school & 1.00 & 1.00 & 1.00 & 1.00 & 1.00 & 1.00 \\
\hline Primary & 1.89 & 2.32 & 1.54 & 2.07 & 1.33 & 1.94 \\
\hline Secondary & 2.67 & $5.91^{d}$ & 1.73 & 2.27 & 2.73 & 1.32 \\
\hline Significance of Model & 0.006 & 0.000 & 0.002 & 0.000 & 0.009 & 0.014 \\
\hline No. of Observations & 213 & 213 & 225 & 226 & 210 & 209 \\
\hline
\end{tabular}

a A multivariate logistic regression was carried out for each method.

b The odds ratio indicates the likelihood of the provider mentioning a particular contraceptive to a client given the client's background characteristics. For example, the top left box shows that a client in the age group 20-29 is 1.20 times as likely to hear about CO Cs than a client who is in the 15-19 year age group, whereas a client in the 30-46 year age group is only 0.80 times as likely to hear about CO Cs as one in the age group 15-19.

c $p<0.01$.

d $p<0.05$.

were 11 times more likely to be told about management of side effects, while those with primary education were 6 times more likely. Interestingly, since providers generally do not ask clients about their educational attainment, this judgment is likely to be based on visual appraisal of clients.

In addition, the client's age was shown to be an important factor in the information given by providers. Women over age 20 were nearly six times as likely to be counseled about management of contraceptive side effects than were adolescents.

Providers' decisions about counseling based on clients' age and number of children have their origins in the Kenyan family planning procedures manual of 1988, which was most probably followed by the majority of providers in 1995 (Cege et al., 1988). The manual states that women over age 35 are contraindicated for COCs and POPs. The 


\section{Table 5-6. Effects of clients' background characteristics on information given about accepted method ${ }^{\mathrm{a}}$}

\begin{tabular}{lccc} 
Background Characteristic & $\begin{array}{c}\text { How to Use } \\
\text { Method }\end{array}$ & $\begin{array}{c}\text { ODD RATIOS } \\
\text { Disadvantages }\end{array}$ & $\begin{array}{c}\text { Problem } \\
\text { Management }\end{array}$ \\
Age & 1.00 & 1.00 & 1.00 \\
$15-19$ & 2.30 & $3.25^{\mathrm{b}}$ & $4.09^{\mathrm{b}}$ \\
$20-29$ & 1.44 & 2.31 & $5.89^{\mathrm{b}}$ \\
$30-46$ & & & \\
\hline Marital Status & 1.00 & 1.00 & 1.00 \\
Married & 0.70 & 0.49 & 1.54 \\
Not married & & & \\
\hline No. of Children & $\mathrm{n} / \mathrm{a}$ & 1.00 & 1.00 \\
-1 & & 0.55 & 0.66 \\
$2-4$ & & 0.49 & 0.45 \\
5+ & & & \\
\hline Level of Education & 1.00 & 1.00 & 1.00 \\
No school & 5.05 & $4.13^{\mathrm{b}}$ & $6.18^{\mathrm{c}}$ \\
Primary & 5.50 & $4.54^{\mathrm{b}}$ & $11.59^{\mathrm{b}}$ \\
\hline Secondary & 0.045 & 0.005 & 0.001 \\
\hline Significance of Model & 214 & 213 & 211 \\
\hline No. of O bservations & & & \\
\hline
\end{tabular}

a A multivariate logistic regression was carried out for each piece of information.

b $p<0.01$.

c $p<0.05$.

manual also states that the remaining methods are suitable for women regardless of age. Although this guidance may have been misapplied, it is not surprising that providers used age as a basis for discussing most contraceptives. Providers' counseling criteria based on number of children were also misapplied. The manual advises providers to discuss vasectomy and female sterilization for couples with completed family sizes, but the number of children is not specified.

Tables 5-5 and 5-6 demonstrate the importance service providers place on clients' personal characteristics. New family planning clients seeking services at health facilities are a self-selected group with unmet need for family planning services. Regardless of their background characteristics, they visit health facilities to seek services, and the quali- ty of the services they receive can be affected by providers' judgments of personal circumstances.

\section{CONCLUSIONS}

The hypothesis that those health facilities that are better prepared to deliver services in terms of availability of equipment, contraceptives, trained providers, and IEC materials provide higher-quality services is partially supported by the findings presented here. The analysis demonstrates that facilities with staff who have attended certain training courses, with equipment and supplies for methods and client assessment, and with IEC materials available are more likely to carry out specific aspects of quality with most of their clients. 
However, many of the hypothesized relationships between readiness and quality are not borne out by these findings (see also Chapter 6).

The choice of methods offered to clients appears to be improved by the presence at an SDP of providers trained in long-term methods. In addition, training in NFP and LAM is associated with offering more methods to each client, possibly reflecting the strong counseling component of these methods. However, training in clinical family planning and IEC skills is not associated with offering an expanded choice of methods. The presence of commodities and equipment for a method is related to whether clients hear about the method, but only for IUDs, NORPLANT ${ }^{\varpi}$, and spermicides. For COCs, injectables, and condoms, no relationship was found, possibly because virtually all health facilities in Kenya offer these methods.

Detailed counseling about contraceptives was shown to be influenced by the type of IEC materials available at a health facility and used during counseling sessions. Use of available contraceptive samples and anatomical models was significantly associated with more detailed information given to clients about contraceptives, and the use of flip charts showed a similar, but not significant, association. It is not clear whether providers who used these materials were highly motivated to do so, or their availability at health facilities led to their greater use.

Moreover, the presence of providers trained in IEC skills, NFP, and LAM was found to be related to an increase in certain information given to the majority of clients on their accepted method. This finding is encouraging, although the presence of providers trained in clinical family planning and in IUD insertion/removal did not appear to have an effect on the information given to clients. The implication is that providers who attend courses with strong counseling components are likely to serve clients better. Therefore, it is recommended that family planning courses for service providers cover training in counseling skills, including the use of IEC materials.

Client assessment through weight and blood pressure is strongly affected by the presence of the necessary equipment in the $\mathrm{MCH} /$ family planning unit itself. In addition, the presence of at least one provider trained in clinical family planning, IEC skills, or IUD insertion/removal is associated with increased performance of certain client assessments. Interestingly, the presence of staff who had attended a range of other training courses appeared to have no significant effect on the client assessments carried out. Taking clients' weight appears to be a fairly universal practice. The usefulness of carrying out these assessments is debated, but the data show that the relationship between training and carrying out these assessments is not consistent.

Finally, providers' consideration of clients' personal characteristics in the provision of services poses a challenge to the Kenyan program. In some cases, such as the provision of injectables or telling clients about the advantages and side effects of their chosen method, providers do not appear to be affected by clients' personal characteristics. However, the methods presented to clients do appear to be affected by their marital status and parity. In addition, informing clients about how to use their method, its disadvantages, and how to manage side effects is clearly related to the clients' age and educational status. Many Situation Analysis studies in Africa have tended to concentrate on age, spousal consent, and number of children as barriers to clients' access to contraceptives (see Chapter 8). This analysis has shown that another factor, the client's educational level, plays a critical role in providers' decisions about the services offered to clients. This finding calls for more exploration of these provider biases in order to determine effective ways of overcoming them, thereby providing quality services in a more equitable manner.

\section{REFERENCES}

Beegle, K. 1995. "The quality and availability of family planning services and contraceptive use in Tanzania." Living Standards Measurement Study Working Paper No. 114, Policy Research Department, World Bank, Washington, D.C.

Bertrand J., K. Hardee, R. Magnani, and M. Angle. 1995. "Access, quality of care and medical barriers in family planning programs." International Family Planning Perspectives, 21:64-69. 
Bruce, J. 1990. "Fundamental elements of the quality of care: A simple framework." Studies in Family Planning, 21,2:61-91.

Cege, L.W., M.A. Odipo, et al. 1988. Family Planning Procedure Manual for Health Workers. Division of Family Health, Nairobi, Kenya.

Collins, D., J. Quick, S. Musau, and D. Kraushaar. 1996. Health Financing Reform in Kenya: The Fall and Rise of Cost Sharing, 1989-1994. Management Sciences for Health, Boston.

Feyisetan, B. and M. Ainsworth. 1994. "The impact of the availability, price and quality of services on the demand for contraception in Nigeria." Living Standards Measurement Study Working Paper No. 108, Policy Research Department, World Bank, Washington, D.C.

Haddad, S. and P. Fournier. 1995. "Quality, cost and utilization of health services in developing countries: A longitudinal study in Zaire." Social Science and Medicine, 40,6:743-753.

Jain, A., J. Bruce, and B. Mensch. 1992. "Setting standards of quality in family planning programs." Studies in Family Planning, 23,6:392-395.

Maggwa, N. and I. Askew. 1997. Integrating STI/HIV Management Strategies into Existing MCH/FP Programs: Lessons from Case Studies in East and Southern Africa. Population Council, Nairobi, Kenya.

Ndhlovu, L. 1995. Quality of Care in Family Planning Service Delivery in Kenya: Clients' and Providers' Perspectives. Population Council, Nairobi, Kenya.

Oliver, R. 1995. “Contraceptive use in Ghana: The role of service availability, quality and price." Living Standards Measurement Study Working Paper No. 111, Policy Research Department, World Bank, Washington, D.C.
Solo, J. et al. 1998. "Improving the management of sexually transmitted infections among $\mathrm{MCH} / \mathrm{FP}$ clients at the Nakuru Municipal Council health clinics." Unpublished draft. Population Council, New York.

Tsui, V.D. 1980. “Underutilization of health centres in rural Mexico: A qualitative approach to evaluation and planning." Studies in Family Planning, 11,4:145-153.

United Nations Development Programme, World Bank, and World Health Organization. 1994. "Quality of health care for women: Report of a workshop held in Budapest, Hungary, October 1994." Special Programme for Research and Training in Tropical Diseases; Division of Family Health; Special Programme of Research, Development and Research Training in Human Reproduction, WHO; and Ford Foundation.

Veney, J., R. Magnani, and P. Gorbach. 1993. "Measurement of the quality of family planning services." Population Research and Policy Review, $12: 243-259$

\section{NOTES}

1 The term "health facility infrastructure" is defined as the enabling environment for delivering services, which in turn is commonly referred to as "facility preparedness" or "program subsystems." These terms are often used interchangeably in this chapter.

2 These sample sizes differ slightly from those cited in Chapter 1 because of the different methods used to weight the data.

3 These results do not account for the presence or absence of the IEC materials at the SDP. 


\section{Annex 5-1: Indicators of Readiness, Quality, and Client Characteristics Used for This Analysis}

\section{Readiness Indicators}

\section{Equipment and Supplies}

Percentage of facilities with gloves, cotton wool, swabs, speculum, tenaculum, and light source Percentage of facilities with cotton wool, antiseptic lotion, needles, and syringes Percentage of facilities with blood pressure machines and scales

Availability of minilap kit, gloves, local anesthesia, needles/syringes, and antiseptic lotion

\section{Information, Education, and Communication}

Percentage of client-provider interactions using flip chart for counseling Percentage of client-provider interactions using contraceptive samples for counseling Percentage of client-provider interactions using anatomical models for counseling

\section{Staff Training}

Percentage of health facilities with staff trained in IUD insertion/removal Percentage of health facilities with staff trained in clinical family planning Percentage of health facilities with staff trained in mini-laparotomy/local anesthetic Percentage of health facilities with staff trained in NO RPLANT ${ }^{\oplus}$ insertion/removal Percentage of health facilities with staff trained in vasectomy counseling Percentage of health facilities with staff trained in supervision/evaluation Percentage of health facilities with staff trained in IEC skills Percentage of health facilities with staff trained in natural family planning Percentage of health facilities with staff trained in lactational amenorrhea method (LAM)

\section{Quality-of-Care Indicators}

\section{Methods Discussed}

Percentage of interactions in which clients were counseled about combined oral contraceptives (COCs) Percentage of interactions in which clients were counseled about progestin-only pills (PO Ps) Percentage of interactions in which clients were counseled about condoms Percentage of interactions in which clients were counseled about the IUD Percentage of interactions in which clients were counseled about NO RPLANT ${ }^{\oplus}$ Percentage of interactions in which clients were counseled about LAM Percentage of interactions in which clients were counseled about vasectomy Percentage of interactions in which clients were counseled about Depo Provera Percentage of interactions in which clients were counseled about female sterilization 


\section{Information Given About Method}

Percentage of clients counseled about how to use method accepted

Percentage of clients counseled about advantages of method accepted

Percentage of clients counseled about disadvantages of method accepted

Percentage of clients counseled about possible side effects of method accepted

Percentage of clients counseled about possibility of switching

Percentage of clients counseled about management of method problems

\section{Technical Competence: Assessment for Medical Conditions}

Percentage of health facilities where medical history was taken

Percentage of health facilities where clients were asked about last menstrual period

Percentage of health facilities where clients had their weight taken

Percentage of health facilities where clients had their blood pressure taken

Percentage of health facilities where physical examinations were conducted

\section{Clients' Personal Characteristics}

Age: percentage of clients in defined age groups (15-19 years, 20-29 years, 30-46 years)

Marital status: percentage of clients in defined marital status groups (married, not married)

Number of children: percentage of clients with number of children falling into defined categories $(0-1$, $2-4,>4)$

Age of youngest child: percentage of clients with children in defined age groups (up to 1 year, 1-2 years, above 2 years)

Highest level of education: percentage of clients falling into defined educational categories (no school, primary, secondary + ) 


\section{KEY POINTS AND CONCLUSIONS}

\section{Unrealized Quality and Missed Opportunities in Family Planning Services}

The 1994 International Conference on Population and Development in Cairo set forth the broad challenge of ensuring that clients receive an adequate quality of care. In light of this emphasis, the question naturally arises of whether the resources exist to meet this objective. While technological and financial inputs are often necessary to incorporate closely related reproductive health services into the scope of family planning programs, the analysis in this chapter suggests that substantial opportunities exist to increase the quality of care and its contribution to clients' reproductive health simply by using available resources more effectively. The following specific conclusions, based principally on data from Botswana, Burkina Faso, Kenya, Senegal, and Zambia, are drawn:

- The number of contraceptive options offered to new clients seeking to space pregnancies is consistently fewer than the number of spacing methods physically present in the clinic, restricting clients' ability to choose the most appropriate or desirable method.

- Providers generally do not make use of available information, education, and communication (IEC) materials, which could improve clients' understanding and assist them in making more fully informed decisions.

- The majority of providers see three or fewer clients (including both family planning and maternal and child health clients) per day, suggesting that a significant amount of staff time may be available to increase the length of client visits, improve the quality of information given, and expand the reproductive health services offered.

- Providers commonly make use of equipment such as blood pressure machines and uterine sounds when available; however, they often fail to follow asepsis procedures during pelvic exams, even when clean water and gloves are in the exam room, putting clients at unnecessary risk of infection.

- Providers frequently do not inquire about the client's sexual context (number and frequency of partners, whether the partner is included in the decision to seek contraception), and are thus unable to assess contraindications for a given method or recommend methods appropriate to the client's situation.

- The ability of a given method to protect against sexually transmitted infections, and in particular the dual-protection benefit of condoms, is rarely discussed.

Although these conclusions focus on only a few specific aspects of the quality of care received by clients, they imply that providers can substantially raise the level of quality of services through such practical measures as improving information exchange and observing proper asepsis procedures. In addition, in many clinics a significant amount of staff time appears to be untapped and could be harnessed to broaden the scope of services.

Taking advantage of these unused resources will arguably increase the cost-efficiency of resource use, but will not be cost-free. It will require establishing appropriate protocols that define the minimum information to be given to clients and the operational meaning of offering choice of methods, and that identify as a clear priority ensuring the safety of services. It will also be necessary to shift the emphasis of staff training to focus more on providing higher-quality services and being sensitive to clients' expressed needs, and to implement a supervisory program that regularly supports and evaluates providers in these areas. 



\section{Unrealized Quality and Missed Opportunities in Family Planning Services}

\section{Nicole Haberland, Kate Miller, Judith Bruce, and Goli Fassihian}

Much of the debate following the Cairo International Conference on Population and Development (ICPD) of 1994 has centered, directly or indirectly, on the feasibility of offering reproductive health services in a climate of diminishing foreign aid and limited national budgets (Potts, 1996). That is, do the resources needed to meet the challenges set forth in Cairo (and in the Beijing Fourth World Conference on Women of 1995) really exist? Indeed, some contend that implementation of the tenets of ICPD would be detrimental without additional funding: "...if broader, more comprehensive programs are attempted without additional funds either coverage will be reduced or quality will decline, the most likely prospect being that both elements will suffer" (Finkle and McIntosh, 1996:111-112).

There has been relatively little questioning of the efficiency of current resource utilization (see Zeitlin et al., 1994, for a critique of assumptions about and bias toward the supply-side approach in common resource requirements analysis). Thus in discussions about implementation of the principles of Cairo, questions such as the following remain largely unexamined: To what degree are existing inputs such as staff time, equipment, and supplies actually used within standard family planning services? Are service delivery points (SDPs) and staff already operating at full capacity? Will the changes called for by the ICPD strain a fragile and efficient system, or is there room within family planning programs to take on parts of the Cairo agendaright now, and with existing resources?

Traditional methods of data collection in the population field typically do not cast much light on questions of resource utilization. Two sets of data are often the focus: one defines the amount of money being spent, staff trained, clinics open, etc., as the inputs; the other (often assumed to be correlated with the first) may track the steady increase in immunization, or in this case numbers of contraceptive users or couple-years of protection (CYPs), as an indication that the program is working and that resources are being well allocated (Barberis and Harvey, 1997; Janowitz and Bratt, 1994). Unfortunately, comparison of these broad inputs against broad output models leaves much unknown, or, as some have pointed out, “...treat[s] the operations of the family planning program as a 'black box'..." (Bertrand et al., 1994:11). These analyses can tell us nothing about how equipment, supplies, and staff time are expended for clinic operations.

By using a sample of observations of what is actually happening in the clinic setting, along with information on supplies and resources available at a site, Situation Analysis can provide a far more accurate picture of what resources are being utilized in the process of client care, and whether in fact those resources are being used optimally. ${ }^{1}$ With this data, one can begin to understand how the available contraceptives, staff time, and clinical equipment are being mobilized, as well as whether critical discussions with clients are taking place.

The hypothesis explored in this chapter is that there is underutilization of existing resources, which diminishes quality and choice for clients. The chapter examines observation-based data from five sub-Saharan countries in order to begin gauging the gap between resource availability and the 
care clients actually receive. This gap, which we term "unrealized quality," represents the untapped capacity of SDPs to deliver care of adequate quality. Operationally, this means there are programmatic inputs, such as contraceptives or clinical instruments, within an SDP that are not used or offered in the course of client care. For instance, although a clinic may be ready to provide a broad range of methods in terms of its contraceptive stocks, clients may not be being told about the methods available.

We should caution that the discussion here is not based on an experimental analysis that began with a hypothesis, then developed methods, and collected and analyzed data accordingly. We do begin with a hypothesis, but use existing data sets that were not tailored to our inquiry. This analysis is thus intended to be preliminary and indicative, and to contribute to discussions on policy and program options and spur more structured investigation.

\section{METHODS}

Data from Situation Analysis studies conducted in five countries of sub-Saharan Africa were used for this analysis: Botswana (1995), Burkina Faso (1995), Kenya (1995), Senegal (1994), and Zambia (1996). (See Chapter 2 for descriptions of these countries' family planning programs and the relevant study references, and Chapter 1 for information on the sample sizes and sampling plans for these five studies.) These studies were chosen for the analysis because they collected the relevant information. For example, earlier Situation Analyses in Côte d'Ivoire and Tanzania did not include many of the salient indicators of client-provider interaction and information exchange. Even among the five data sets included here, some do not include all the relevant information for our selected indicators.

The question of the potential gap between what is available at the SDP and what clients receive (unrealized quality) was not included in the original Situation Analysis studies. Moreover, the degree to which sexuality, partnership, and social context are addressed in the client-provider exchange was not a central concern when the Situation Analysis instruments were initially developed. Thus the scope of this analysis, as well as the way different indicators are defined, is limited accordingly. Nonetheless, the available data allow us to examine the degree to which four components of existing resources and opportunities are currently employed by family planning programs:

- Contraceptive method choice

- Use of staff time (client load)

- Use of clinic equipment and water

- Consideration of social context and clients' sexual relationships

The results of the analysis for each of these components are given below. The discussion includes a description of the particular indicators used to explore the extent of unused quality in each case.

\section{RESULTS}

\section{Contraceptive Method Choice}

The importance of choice, outlined by women's health advocates and incorporated in the BruceJain quality-of-care framework developed in the decade before Cairo (Bruce, 1990), was underscored by the ICPD (Programme of Action, 1994). Expanding choice has been an active policy agenda for policy makers and program managers in many countries, as well as for nongovernmental organizations (NGOs) and international agencies. Some measures seek to improve choice-and reproductive rights-by removing barriers such as eligibility criteria (see Chapter 8 ), as well as targets and evaluation criteria that effectively promote one method over others or even pressure clients. Other measures seek to expand the range of contraceptive options available at the receiving point. We focus here on the latter, as well as the interpersonal exchanges that make the available contraceptive options "real" to clients.

Providing an expanded contraceptive method mix allows the client to choose the contraceptive that best meets her own reproductive needs, as well as being compatible with the sexual behavior of the client and her partner. For instance, a woman concerned about the risk of sexually transmitted infections (STIs) / HIV has the option of choosing a method that protects against disease transmission, 


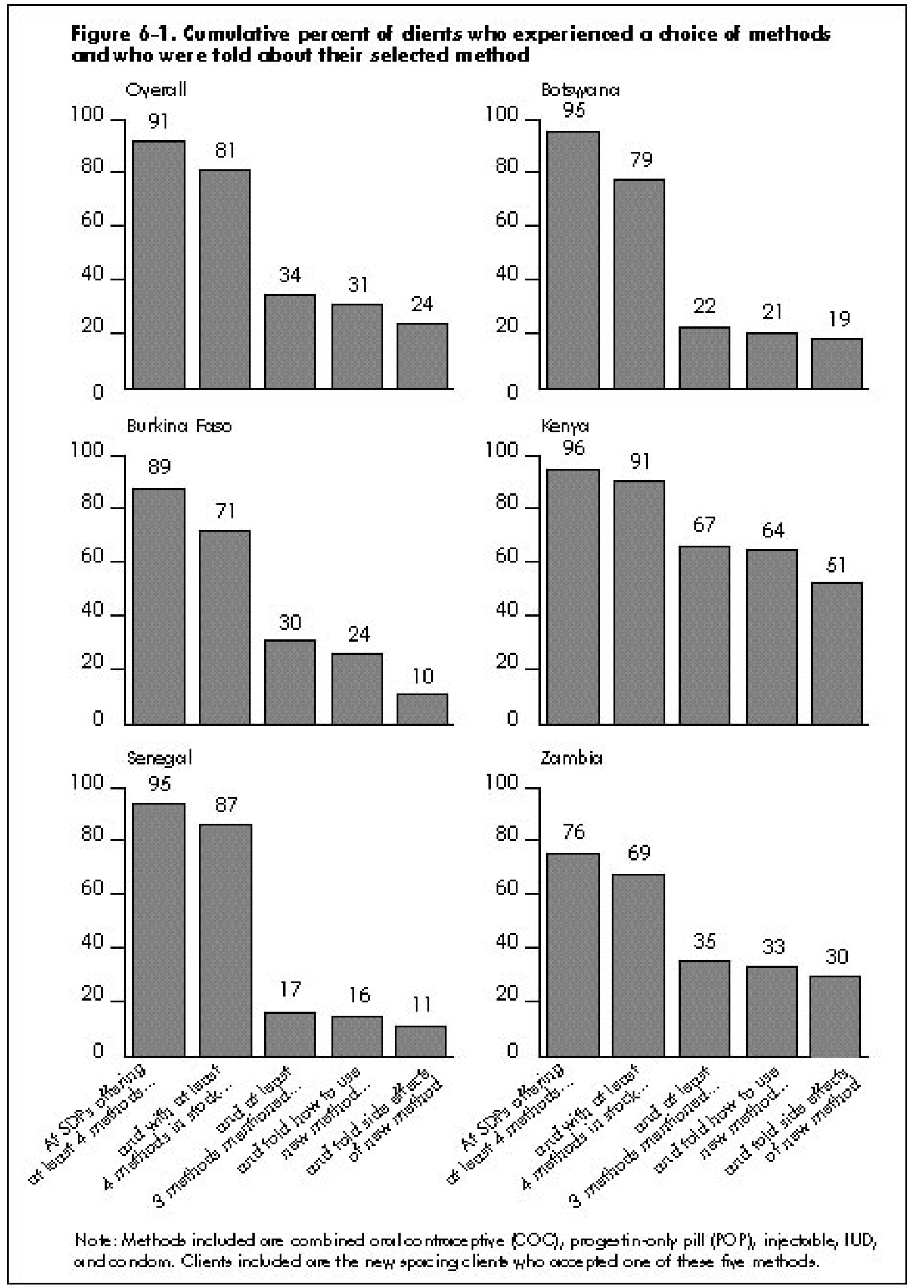


and a woman who does not want her partner(s) to know she is contracepting can choose a method that is surreptitious. Moreover, as all methods have disadvantages, offering a mix of methods also gives a woman the opportunity to select the method that is the most suitable for her.

One indicator of the breadth of method mix is the number of contraceptive methods mandated by a family planning program or the number of methods actually in stock at the SDPs. Although such cataloguing is informative about readiness to provide services, it tells us nothing about the level of quality clients actually receive (Bruce, 1990). That is, it does not provide us an accurate picture of whether clients are presented with a range of choices during their visit. For not only do various methods have to be in stock at the time of the visit, but clients need to be told which methods are on hand, or are available on referral, that would help them meet their reproductive intentions.

Figure 6-1 shows the proportion of clients who were offered a full range of methods in stock at their SDP. This analysis includes only new spacing clients ${ }^{2}$ because revisit clients may not need to hear about a range of methods, and limiting clients may require more permanent methods that are not widely available. Five spacing methods are included: combined oral contraceptive (COC), progestinonly pill (POP), injectable, IUD, and condom. Clients may not need to be told about all five methods; the number of available methods that should be mentioned depends on a client's preferences, breastfeeding status, and contraindications, but should include as many as possible given these considerations. $^{3}$ We therefore chose to identify those clients who were told about at least three of the five methods, allowing for methods that might not have been appropriate for individual clients. ${ }^{4}$

The overall results show that $81 \%$ of clients attended SDPs that, according to their own policy or procedures, included at least four of the five spacing methods in their repertoire and had at least four of these methods in stock on the day of the study visit. However, only $34 \%$ of clients were told about at least three of the five methods during counseling. This result reveals a significant gap in clients' method choice between the methods in stock at their SDP and those mentioned by providers. The size of the gap varies among countries, with Kenya having the smallest gap (91\% of clients attended an SDP with at least four methods in stock, and $67 \%$ of clients were told about at least three methods) and Senegal the largest (87\% of clients attended an SDP with at least four methods in stock, and $17 \%$ of clients were told about at least three methods).

Over the last decade, a good deal of effort has been directed to expanding method mix and reducing stockouts (Family Planning Logistics Management Project, 1997). Indeed, the finding that fully $81 \%$ of clients attended an SDP with at least four spacing methods in stock reflects a significant achievement. However, this achievement does not appear to be matched by an effort to ensure that clients are aware of this hard-won capacity (although in some contexts, the number of methods mentioned to clients appears to be increasing with time; see Chapter 11).

An adequate choice process, of course, does not consist only of being told what options are available, but also, for example, of being instructed on how to use the method selected and what side effects it may have. Figure 6-1 shows that the proportion of clients who received all of this basic information is quite low (although intersecting five variables, as in Figure 6-1, will inevitably lead to a decline in the proportion that falls in each subsequent category). Overall, only $24 \%$ of clients were told about at least three methods, told how to use their new method properly, and also told of the new method's potential side effects. Approximately half of clients in Kenya were given all this information, whereas the proportion was only $10 \%$ and $11 \%$ in Burkina Faso and Senegal respectively. ${ }^{5}$

Information, education, and communication (IEC) materials that might be used to facilitate such client-provider exchanges appear to be used infrequently. In all countries except Zambia, available IEC materials were used on average ${ }^{6}$ with $11 \%$ or fewer clients. In Zambia, these materials were used with $22 \%$ of clients. While these results may reflect the materials' being out of date or inappropriate, the figures are still strikingly low. IEC materials such as brochures, pamphlets, and models are generally not available at most SDPs (see Chapter 3), but even when they are, they are not used with clients. 
Figure 6-2. Distribution of family planning client load by quatile, rural SDPs

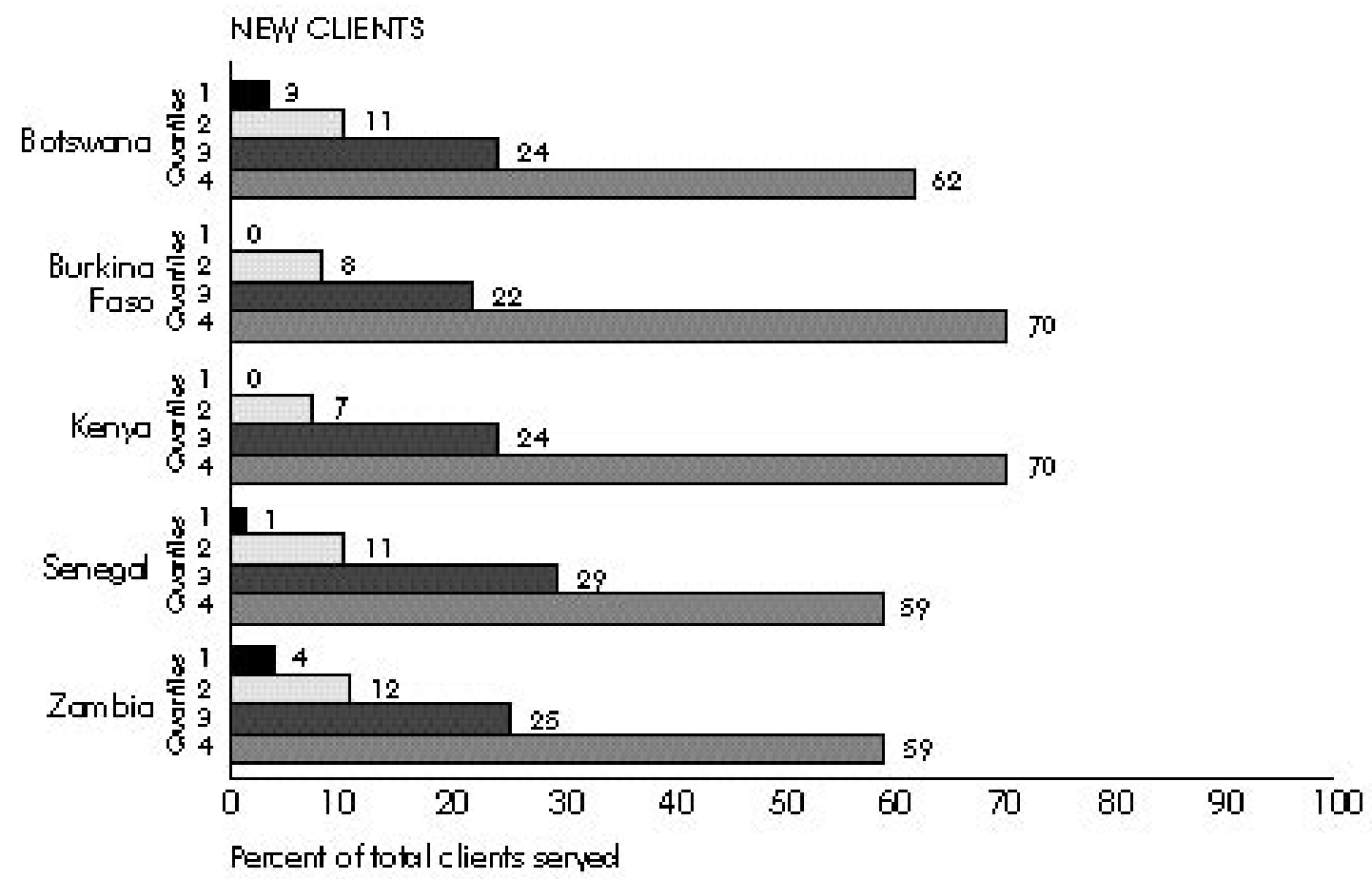

Percent of total clients seryed

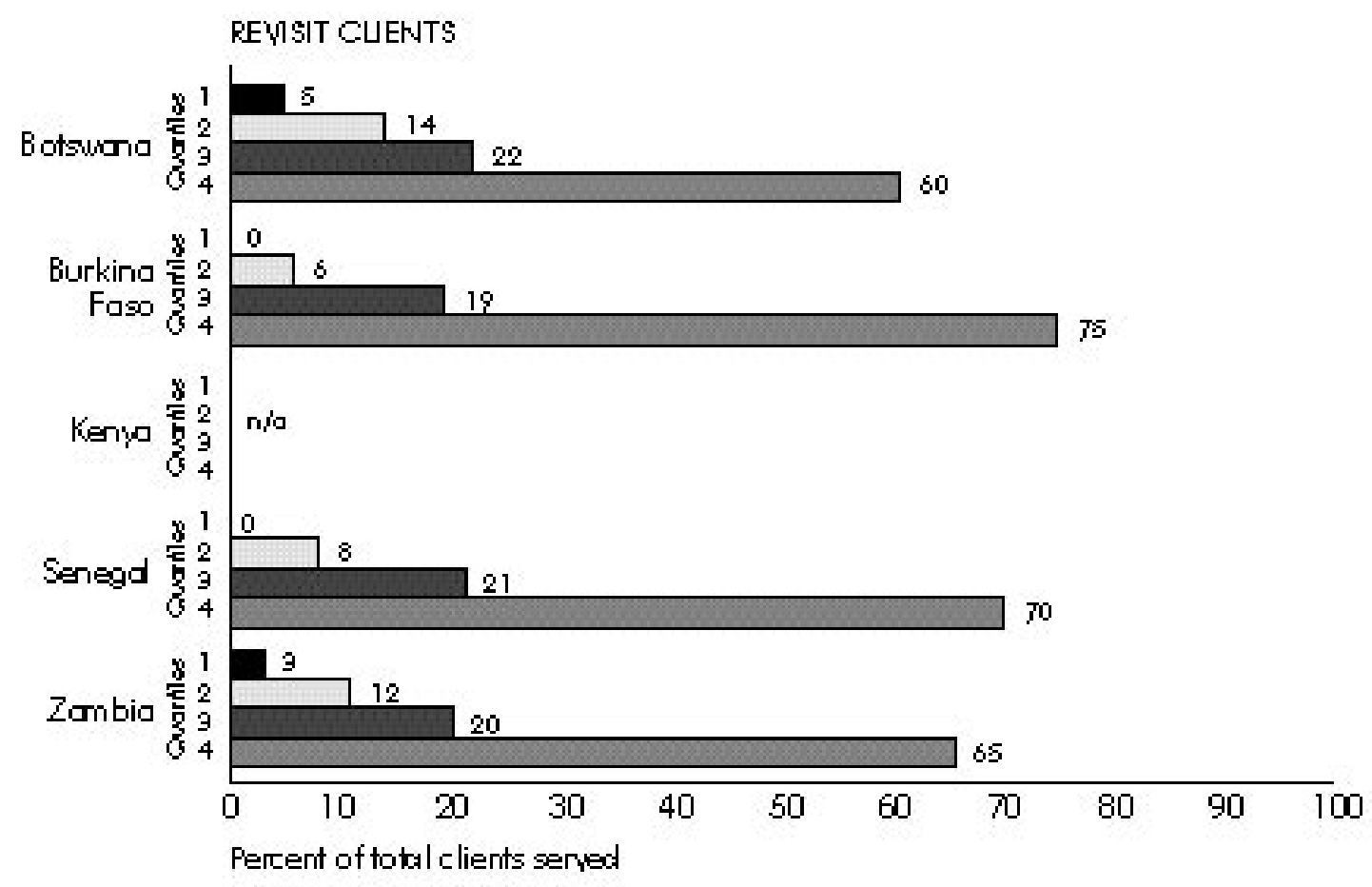




\section{Use of Staff Time (Client Load)}

One reason it may be difficult to improve quality of care in the context of existing resources is that clinics already carry a heavy client load, which places high demands on providers' time. The profile of client load across SDPs in the five countries included in this analysis, however, indicates that most clinics do not fall into this category. We grouped SDPs in the five countries into quartiles based on

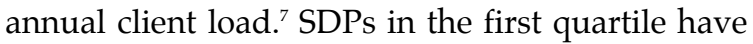
the lightest client loads, and those in the fourth quartile have the heaviest-with equal numbers of clinics placed in each quartile for a country. The results of this analysis (Figure 6-2) show that even in rural areas, $60 \%$ to $75 \%$ of clients are served by only a quarter of the SDPs (Fisher and Miller, 1996). In addition, the proportions do not change markedly among countries or between new and revisit clients. Very similar results are found among urban SDPs.

Figure 6-2 shows that some SDPs carry a disproportionate share of the family planning client load, which may relate to the number of staff working at that SDP. To examine this finding, we compared the annual number of all maternal and child health $(\mathrm{MCH})$ / family planning clients with the number of doctors, nurses, and midwives at each SDP. ${ }^{8}$ Figure 63 shows the number of $\mathrm{MCH} /$ family planning clients per day per provider served by SDPs. Overall, $27 \%$ of SDPs have one client or fewer per day per provider, and $58 \%$ of SDPs have three clients or fewer. Across countries, between 45\% (Burkina Faso) and $78 \%$ (Botswana) of SDPs have three or fewer clients per day per provider. These results indicate that most SDPs included in our analysis have a fairly light $\mathrm{MCH} /$ family planning client load, even allowing for daily administrative and preparatory tasks that may occupy providers' time. ${ }^{8}$

As these figures may reflect differences in population density, we stratified our results by rural and urban SDPs. Since the number of users may be lower in rural areas, the number of SDPs with few clients per provider might be expected to be higher than in urban areas. Surprisingly, 55\% of SDPs in rural areas have three or fewer clients per day per provider, a proportion only slightly lower than that among urban SDPs (63\%). As for individual coun- tries, only Senegal shows a higher proportion of rural than urban SDPs with three or fewer clients per day per provider; all other countries have the opposite pattern. This finding suggests that low population density does not fully explain the relatively low client-per-provider load.

Complete parity across sites cannot reasonably be expected; access, population density, and the scope of workers' responsibilities all shape the potential client/ provider ratio. Moreover, the way SDPs are configured can also affect the results. In the Situation Analyses included here, SDPs were defined as any facility offering family planning services, including hospitals, maternities, and health posts. The present analysis includes all $\mathrm{MCH}$ and family planning clients, but as some SDPs also serve other community health needs (such as curative services and emergency care), there may be other clients who make demands on providers' time. Presumably, such a diversity of needs is less likely in more specialized urban clinics. Yet the data show that the vast majority of urban SDPs also do not appear to have particularly high client loads.

The low client/provider ratio indicates, at the very least, that at most SDPs, overextended staff is probably not the reason clients are not receiving adequate care as defined here. It also suggests that there may be substantial staff resources that can be reoriented and better deployed. For example, family planning counseling is often neglected (see above and Chapter 3), whereas time for counseling may be available. Similarly, although broadening the range of reproductive health services offered to include, for instance, reproductive tract infection (RTI) diagnosis and treatment would require training and stocking of appropriate tests and medications, the staff time needed to provide these services may well be available.

\section{Use of Clinical Equipment and Water}

High-quality services require proper clinical procedures, including infection prevention. In many health and family planning clinics in low-resource settings, the ability of program managers and providers to ensure the use of such procedures is compromised by the context and realities of the environment. In some areas, for example, water 
Figure 6-3. Per cent of SDPs with 1 to $20+$ mater nal and child health/f anily plarming clients per day per provider
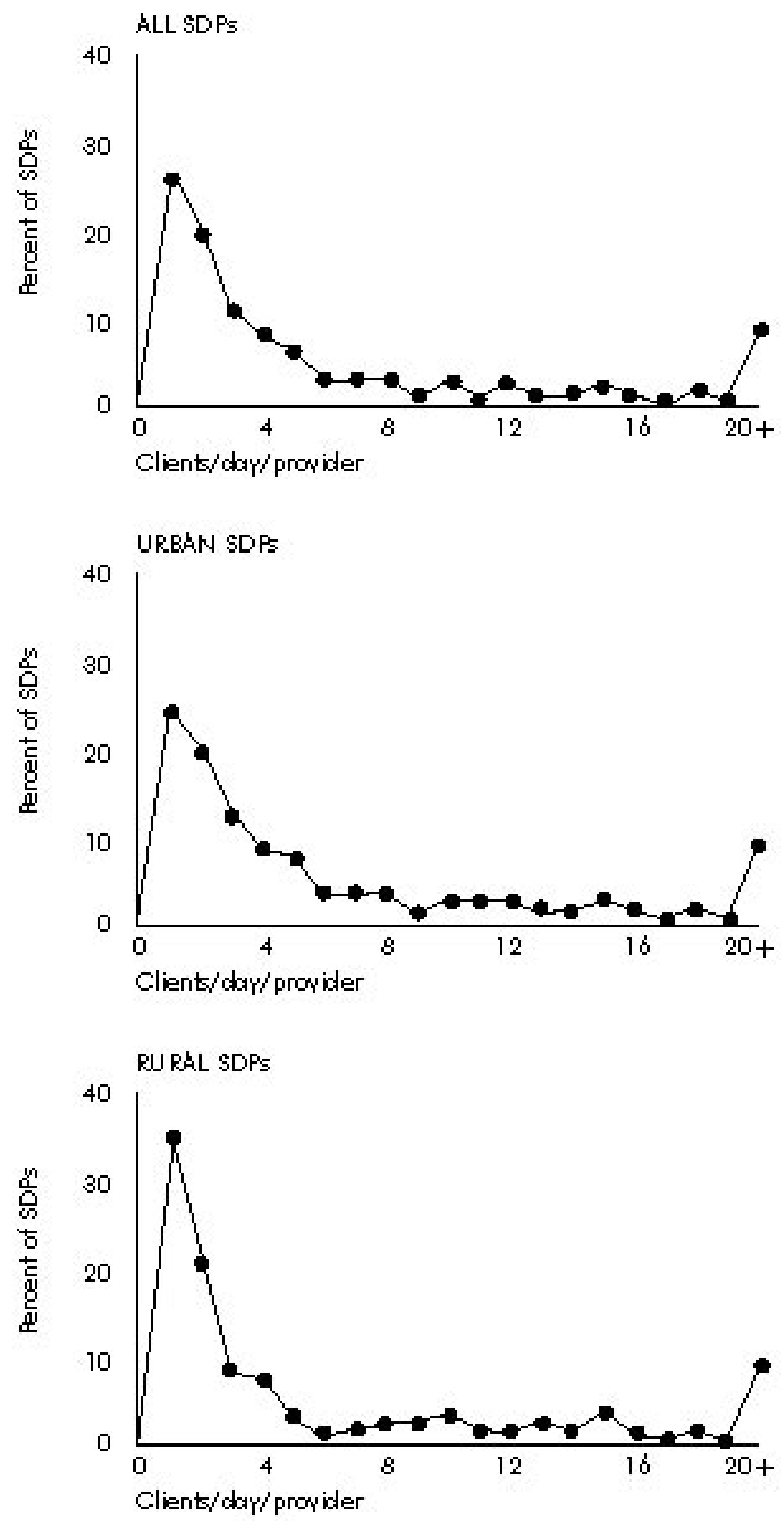
Figure 6-4. Per cent of clients with whom clinic resour ces w ere used

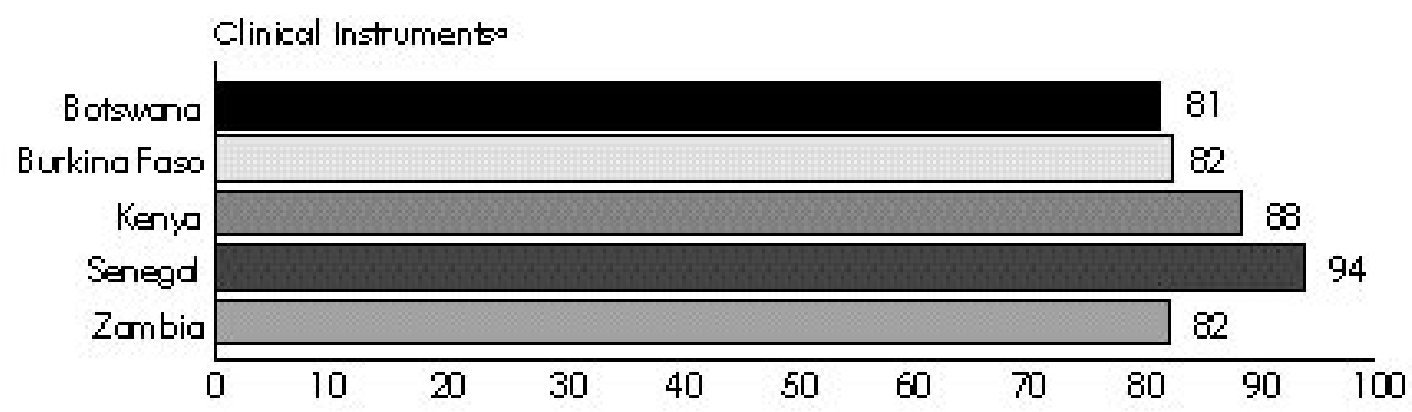

Woter (handyosthing before pelyic exams)

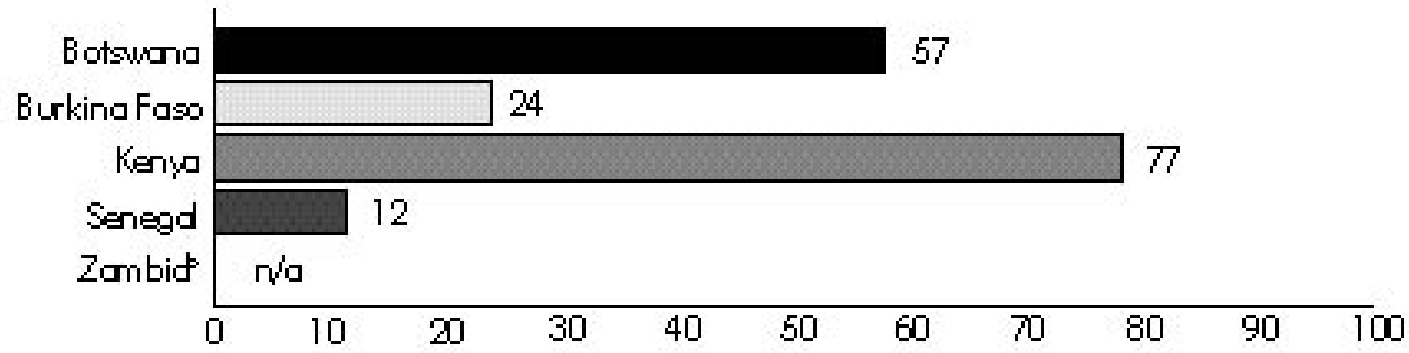

- Wean of \% of new client who were weighed (poaks), \% of new' client whoose bbodpresure wos taben (blood pressure mochinel, and \% of IUD elient whose ut nus kos sounded before IUD indertion (uterine soundl.

b Onty tho pelyiz exams 'were obseryed in Zambia.

may not be available regularly, if at all, or the program may not be able to afford sufficient equipment, such as blood pressure machines, for all its clinics. But how are such resources used in settings where they are available? To assess this, we looked at whether specific physical resources available and functioning in a clinic were used by providers during relevant clinical procedures. Figure 6-4 shows the mean proportion of new clients whose blood pressure was taken in clinics where blood pressure machines were available, who were weighed in clinics where a scale was available, and whose uterus was sounded for an IUD insertion in clinics where a uterine sound was available.

In all countries, the mean proportion of providers who used these available resources with new clients was quite high-ranging from $81 \%$ to $94 \%{ }^{10}$ Taken alone, the use of blood pressure machines in settings where they were available was also high, ranging from $76 \%$ (Burkina Faso) to 93\% (Senegal). This is a positive and heartening finding.
The appropriateness of carrying out each of these assessments is a local decision (see the discussion in Chapters 3 and 9), but the point here is that where instruments are available, they are being used.

Washing hands between pelvic exams is an important step in infection prevention. Figure 6-4 shows the proportion of providers at SDPs with water available in the exam room who washed their hands before pelvic exams. Between $23 \%$ and $88 \%$ of providers did not wash their hands before a pelvic exam despite the availability of water in the exam room. In four countries, 1024 pelvic exams were observed in settings where water was available in the exam room. Providers washed their hands before only $26 \%$ of these exams, though there is large variation by country.

This low proportion may be explained by the use of gloves instead of washing (see Chapter 3), or by the provider's having washed hands after the previous pelvic exam. Although the necessity of handwashing is debated, for our purposes the most 


\section{Table 6-1. Discussion of client context}

\begin{tabular}{|c|c|c|c|c|c|}
\hline & & Burkina & & & \\
\hline Indicator & Botswana & Faso & Kenya & Senegal & Zambia \\
\hline $\begin{array}{l}\% \text { of new clients who were } \\
\text { asked about the nature of } \\
\text { their sexual relations }\end{array}$ & 9 & 6 & 36 & 14 & 5 \\
\hline $\begin{array}{l}\% \text { of revisit clients who were } \\
\text { asked about the nature of } \\
\text { their sexual relations }\end{array}$ & 3 & & 22 & & 1 \\
\hline $\begin{array}{l}\% \text { of new clients who were } \\
\text { asked if they discuss family } \\
\text { planning with their partner }\end{array}$ & 27 & 15 & 54 & 54 & 41 \\
\hline $\begin{array}{l}\% \text { of new clients who were } \\
\text { told the protective effect of } \\
\text { their new method against } \\
\text { STIs/HIV }\end{array}$ & 19 & & & & 9 \\
\hline $\begin{array}{l}\% \text { of new clients who were } \\
\text { specifically told that condoms } \\
\text { protect against STIs/HIV }\end{array}$ & 33 & 10 & 12 & 2 & 36 \\
\hline
\end{tabular}

a This includes any accepted method, not just condoms.

important result is the percentage of pelvic exams during which the provider washed hands before or after or used gloves. These data are available for three countries: Botswana (80\%), Kenya (99\%), and Senegal $(84 \%)$. These results indicate that infection prevention during pelvic exams is practiced well in Kenya, but that in Botswana and Senegal, up to $20 \%$ of pelvic exams involve no observed infection control procedures at all. In these cases, a minimum level of hygiene was neglected in one of every five interactions, despite the availability of either water or gloves. Africa's high prevalence of STI/HIV adds urgency to these results.

\section{Social Context and Clients' Sexual Relationships}

There is increasing recognition that reproductive health services must take into account clients' social context, sexual behavior, and partner relations, including underlying gender issues such as the role of power in intimate relationships (Programme of
Action, 1994; Hardon, 1997; Spicehandler, 1997). While some innovative programs include addressing and discussing sexuality, power, and disease prevention with their clients (Diaz, 1996; Plata, 1996; Widyantoro, 1996), the vast majority do not (see Chapter 3). Yet discussion of certain basic issues not only is critical if clients are to make informed decisions, but also is imperative if providers are to assist clients in making appropriate contraceptive choices. For instance, whether a client has a steady or multiple sexual partners and whether a client's partner knows she is using a contraceptive are both important considerations in her selection of an appropriate method. Other examples of contextual factors that affect clients are domestic violence, ability to pay for commodities and services, and ability to insist on condom use.

Moreover, given the spread of HIV/AIDS, the role played by STIs in its transmission, and the reproductive morbidity that results from STIs, family planning providers should inform clients about the relationship of a contraceptive method to 
such infections, as well as emphasize the dual protection role of condoms (see Chapter 10). Clients' knowledge of this type of information improves the appropriateness of their method choice. In a recent study in Mexico, providers informed clients about the relationship of different contraceptive methods to STIs, including the dangers of IUD use when a woman has an infection, and the fact that a woman's, and her partner's, sexual behavior could place her at risk for STIs. Preliminary findings indicate that with this information, clients were much better than medical providers at screening themselves out of inappropriate IUD use (Langer et al., 1998).

To gauge the extent to which providers seize the opportunity to discuss such issues with clients, we identified variables that reflect conversations about sexual behavior or the client's context. The Situation Analysis methodology does not include many variables on this issue, but the more recent studies in Botswana and Zambia included relatively more questions on these topics.

Table 6-1 reveals limited consideration of women's sexual behavior, partner relations, and STI prevention among providers. Among new clients in all countries, only 5\% to $36 \%$ were asked the nature of their sexual relationships. ${ }^{11}$ In Botswana, Kenya, and Zambia, this same question was asked of only $1 \%$ to $22 \%$ of revisit clients (asking revisit clients about their partner relations and sexual behavior is not redundant, as sexual relations change with time). Providers more frequently-though only approximately half the time or less (15\% to 54\%) —asked new clients whether they discuss family planning with their partner.

Two studies looked at whether providers told new clients about the protection their chosen method offered against STIs. In Botswana, 19\% of providers did so, and in Zambia, only 9\%. All five studies examined whether providers told new clients about the ability of condoms to prevent STIs. Between $2 \%$ and $36 \%$ of clinicians shared this information with new clients.

The overall point is that providers do not systematically discuss with clients basic aspects of sexuality that bear on client safety and satisfaction. Yet findings from other settings, such as the International Planned Parenthood Federation/
Western Hemisphere Region affiliates in Brazil, Honduras, and Jamaica, indicate that providers, when properly supported and guided, can discuss intimate topics such as sexuality with their clients (Becker et al., 1997).

\section{DISCUSSION}

This analysis is not definitive, but rather indicative of the actual choice and quality of care given to clients in sub-Saharan Africa. The results presented above suggest that there is substantial unused quality within current resource and service configurations, and therefore missed opportunities to offer better care. This overall finding challenges the notion that services cannot be improved without significant additional resources; indeed, it appears that many critical resources needed to improve services are already at hand, but substantially underutilized. We do not imply that no new funding is needed to implement a broader array of reproductive health services. Rather, we suggest that the efforts expended on broadening family planning services might begin with using existing facilities and staff time more efficiently. Several more specific points can be made regarding each of the four areas discussed above.

Contraceptive Method Choice. Examination of the actual choice of methods given to clients demonstrates that it is necessary to look beyond inventories of contraceptives in the clinic storage room. Once one begins to observe the process of choice from beginning to end, it becomes evident that the contraceptive options presented to clients are consistently fewer than the methods physically available for distribution. Further, providers do not generally make use of available IEC materials. The client-provider interaction is a mediating variable that can significantly reduce the options available to the client.

Use of Staff Time. Although some staff are overburdened with client care, the data consistently show that most providers in the countries studied have a light case load. Thus, it appears that considerable staff capacity remains untapped. Perhaps training, supervisory support, protocols, and appropriate rewards would enable staff to pro- 
vide better-quality services within the existing narrow scope of services, and could also allow the expansion of services to include other reproductive and sexual health needs.

Use of Clinical Equipment and Water. Providers frequently use clinic equipment, such as blood pressure machines and uterine sounds, during consultations with clients. However, infection prevention procedures during pelvic exams appear to be lacking in many settings, even where water and gloves are available in the exam room.

Social Context and Clients' Sexual Relationships. The available data show that client context is not commonly discussed during family planning consultations. Few clinicians ask clients about the nature of their sexual relations, inform them of their method's ability to protect against STIs, or explain the role of condoms in preventing disease transmission. Addressing sexual behavior, partner relations, and power in intimate relationships is imperative from a public health perspective, particularly in the era of HIV / AIDS. Ignoring these aspects of the client context clearly decreases family planning service quality. In addition, given the importance of such discussions, the Situation Analysis methodology and other approaches used to measure the quality of client counseling should gather more information on these topics.

\section{CONCLUSION}

Situation Analysis data make it possible to examine the capacity of SDPs to offer high-quality services, as well as whether SDPs actually utilize this capacity. It appears that the deficits in quality of family planning services are often not traceable to overextension, but rather to underutilization of what is at hand. In short, technological and monetary inputs do not equal quality. The presence of resources is obviously essential in terms of having a mix of methods, clean water, and enough staff to serve clients adequately. However, observation of what is happening in the client-provider interaction reveals that these tangible resources alone are not sufficient.

The problem is not that providers are negligent, but that program emphasis, protocols, supervisory procedures, and the like must support providers in giving the best possible care with given resources. To maximize the degree of quality received by clients, it is necessary to (1) implement policies that make adequate service quality and sensitivity to clients' social context clear priorities, (2) generate protocols that guide providers in how to offer such service quality and conduct such discussions, (3) provide appropriate training, (4) support workers in providing a high level of care and addressing clients' social context and sexual behavior, and (5) improve the amount and quality of data collected on whether client context and sexuality are discussed with clients.

There are clearly resource and cost implications for such rethinking of priorities. For example, whereas staff time to take on additional services may exist, providers must be trained to offer those services, and whereas most health budgets allocate funds for training, curricula need revising. However, we suggest that achieving improved quality and a broadened scope of services requires less new funding and more will and creative thinking. These achievements will certainly not be free, but there are already resources on hand that can be better deployed or redirected..$^{12}$ Much of what we suggest to improve service quality and move family planning programs closer to the reproductive health approach put forth in Cairo is thus not an allocation of new resources, but an affirmation of client-centered principles of care and the mobilization of often underutilized human and material resources.

\section{REFERENCES}

Barberis, M. and P.D. Harvey. 1997. “Costs of family planning programmes in fourteen developing countries by method of service delivery." Journal of Biosocial Science, 29,2:219-233.

Becker, J., E. Leitman, and M.F. Fathalla. 1997. "Introducing sexuality within family planning: The experience of three HIV/STD prevention projects from Latin America and the Caribbean." Quality/Calidad/Qualité. No. 8, Population Council, New York.

Bertrand, J., R. Magnani, and J. Knowles. 1994. Handbook of Indicators for Family Planning Program Evaluation. The Evaluation Project, UNC-Chapel Hill, Chapel Hill, North Carolina. 
Bruce, J. 1990. "Fundamental elements of the quality of care: A simple framework." Studies in Family Planning, 21,2:61-91.

Diaz, M. 1996. "The evolution of a sexuality education program: From research to action." In Zeidenstein, S. and K. Moore, Eds., Learning About Sexuality: A Practical Beginning. Population Council, New York.

Family Planning Logistics Management Project. 1997. "Contraceptive fact sheets: A tool for logistics advisors." John Snow Inc., Arlington, Virginia.

Finkle, J. and A. McIntosh. 1996. "Cairo revisited: Some thoughts on the implications of the ICPD." Health Transition Review, 6,1:110-113.

Fisher, A. and K. Miller. 1996. "Conditions required at SDPs to deliver family planning services: Why so many do so little." Paper presented at the Annual Meeting of the American Public Health Association, New York.

Hardon, A. 1997. "Reproductive rights in practice: A comparative assessment of quality of care." In Hardon, A. and E. Hayes, Eds., Reproductive Rights in Practice: A Feminist Report on Quality of Care. Zed Books, Ltd., London and New York.

Janowitz, B. and J.H. Bratt. 1994. Methods for Costing Family Planning Services. United Nations Population Fund (UNFPA), New York.

Kim, Y. and C. Lettenmaier. 1995. Tools to Assess Family Planning Counseling: Observation and Interview. Johns Hopkins School of Public Health Center for Communication Program, Baltimore, Maryland.

Kim, Y., C. Marangwanda, and A. Kols. 1997. "Quality of counseling of young clients in Zimbabwe." East African Medical Journal, 74,8:4-5.

Kim, Y., A. Kols, and S. Mucheke. 1998. "Informed choice and decision making in family planning counseling in Kenya." International Family Planning Perspectives, 24,1:4-11.

Langer A., B. Winikoff, and C. Coggins. 1998. "Evaluation of information and self-selection for appropriate IUD provision in a family planning clinic." Unpublished results from a Population Council research study in collaboration with INSP and IMSS, Mexico.

Pariani, S., D. Heer, and D. Van Arsdol. 1991. “Does choice make a difference to contraceptive use? Evidence from East Java." Studies in Family Planning, 22,6:384-390.

Plata, M.I. 1996. "Bringing men and women together in family planning clinics." In Zeidenstein, $\mathrm{S}$. and $\mathrm{K}$. Moore, Eds., Learning About Sexuality: A Practical Beginning. Population Council, New York.

Potts, M. 1996. "The crisis in international family planning." Health Transition Review, 6,1:114-119.

Programme of Action of the International Conference on Population and Development. Cairo, 5-13 September 1994.

Spicehandler, J. 1997. "Issues to consider in operationalizing reproductive health." Advances in Contraception, 13,2-3:345-350.
Vernon, R. 1998. "Providing comprehensive sexual and reproductive health care." Paper presented at the Annual Meeting of the Population Association of America.

Widyantoro, N. 1996. "Learning about sexuality through family planning counseling sessions in Indonesia." In Zeidenstein, S. and K. Moore, Eds., Learning About Sexuality: A Practical Beginning. Population Council, New York.

Zeitlin, J., R. Govindaraj, and L. Chen. 1994. "Financing reproductive and sexual health services." In Sen, G., A. Germain, and L. Chen, Eds., Population Policies Reconsidered: Health, Empowerment and Rights. Harvard School of Public Health, Boston.

\section{NOTES}

1 Population Communication Services and other organizations have also developed methodologies that explore how providers use their time and what they do with clients (see Kim and Lettenmaier, 1995; Kim et al., 1997; and Kim et al., 1998).

2 Spacing clients are defined as those who wish to have another child, but not in the next 2 years. The small number of new clients who did not accept a family planning method were excluded from this analysis, since they arguably did not need to hear about all methods.

3 In another approach to this question, Pariani (1991) and colleagues argue that client preference is the most important of these issues and that providing methods other than those preferred by the client will lead to increased discontinuation.

4 See Kim et al. (1998) for a more detailed approach to this issue.

5 It is the case, however, that in 9 of the 11 study sites, nearly all of the new clients were told how to use their method-a crucial but single step in the choice process. (See chapter 3.)

6 Average of $\%$ of new clients with whom existing flip charts were used, $\%$ of new clients with whom existing brochures were used, and \% of new clients with whom existing posters were used.

7 Annual client loads are measured through clinic service statistics, where available.

8 We eliminated 104 SDPs in Zambia from this analysis because their client loads constituted extreme outliers, and the quality of the data was questionable. Similarly, data from Kenya was omitted because of concerns regarding data quality. Estimates of the number of clients per day are based on the average number of days per week SDPs are open for family planning in each country and a working year of 50 weeks per provider.

9 The fact that roughly $10 \%$ of SDPs in all settings have 20 or more clients per day per provider is another issue, possibly one of overextended resources. However, the great majority of SDPs with far fewer clients per provider are the focus here. 
10 The results for all constituent indicators were similarly high, so the mean of the indicators fairly represents the results.

11 Wording on these questions differed across countries, but most were posed as "the nature of the client's sexual relations," which was defined locally, or "the number of partners in the last year" (new clients) and "the number of partners since the last visit" (revisit clients).

12 For instance, operations research projects in Guatemala, Honduras, Mexico, and Peru have tested the use of job aids as a means of training providers to offer a broader spectrum of reproductive health services (Vernon, 1998). 



\section{KEY POINTS AND CONCLUSIONS}

\section{Urban and Rural Family Planning Services: Does Service Quality Really Differ?}

As urban growth rates quickly increase in subSaharan Africa, the infrastructure in cities, particularly that of urban health systems, becomes increasingly strained. However, it might still be assumed that the quality of urban health services is better than that of rural services for a variety of reasons, including a greater concentration of trained personnel, larger health facilities, and shorter supply routes. To help family planning program managers assess the readiness of service delivery points (SDPs) to provide quality services and design future reproductive health programs, this chapter compares the quality of services in urban and rural areas. The analysis is based on data from Situation Analysis studies conducted in Botswana, Burkina Faso, Ghana, Kenya, Zanzibar, and Zimbabwe.

Overall, there is less difference in services between urban and rural SDPs than might be expected. In fact, it appears that the quality of care in rural SDPs is in most cases as good as that in urban SDPs, and sometimes better. This finding may arise from the fact that quality of care is defined by indicators of counseling and information exchange, which do not rely heavily on infrastructure and readiness. Specifically, the data lead to the following conclusions:

- For indicators related to infrastructure, such as availability of electricity and water, urban SDPs show a clear advantage.
- Almost half or more of overall readiness indicators-including staffing; accessibility of services; infrastructure; logistics; equipment; supervision; information, education, and communication (IEC) materials; and contraceptive methods offered-show similar results for urban and rural SDPs.

- There is significantly less difference between the quality of care offered at urban and rural SDPs than there is between the readiness of urban and rural SDPs to provide services. The majority of quality-of-care indicators show no differences between urban and rural SDPs, and a significant number of indicators show a rural advantage.

- The quality and frequency of counseling and information exchange appear to be similar among urban and rural SDPs within each country, and the technical competence of providers appears to be roughly equal as well.

Are urban services deteriorating, are rural services improving, or has urban and rural service quality always been similar? Further research with time series data is required to answer these questions. Nevertheless, the results presented here suggest that there needs to be a reassessment of common assumptions about the quality of services in urban and rural areas and a potential refocusing of program design in order to prepare for the population changes expected in the coming century. 



\section{Urban and Rural Family Planning Services: Does Service Quality Really Differ?

\author{
Elizabeth Pearlman, Heidi Jones, Martin Gorosh, \\ Carolyn Gibb Vogel, and Melinda Ojermark
}

Africa currently has the highest rate of urban growth of any region in the world. The urban growth rate is $11 \%$ in Burkina Faso; $5-7 \%$ in Botswana, Kenya, and Zanzibar; and about $4 \%$ in Ghana and Senegal. It is estimated that by the year 2020, over half of the population in Africa will live in urban centers (United Nations, 1995). As African countries grapple with this population growth in their cities, the public- and private-sector infrastructure serving these populations is being heavily strained. Rapid urbanization has had enormous consequences for housing, water and electrical supplies, transportation, basic education, and economic development (Sadik, 1996), and in particular for urban health systems (Gorosh et al., 1995). A recent study states, "In sub-Saharan Africa, continuing massive out-migration from impoverished rural areas and high natural increase-despite years of sustained recession-fuels persistent rapid urban growth, at nearly twice the world average, putting increased pressure on already strained economies and management capacities of both large and small urban centers" (Brockerhoff and Brennan, 1997:14).

Nonetheless, family planning and other reproductive health services in urban areas might be assumed to be better than those provided in rural areas for a variety of reasons: there is generally a larger concentration of trained personnel living in urban areas, health facilities tend to be larger and to have better resources, supply routes are shorter, and supervision should be easier given the smaller distances between facilities. Furthermore, there is usually a stronger demand for and use of such services in urban areas, attributable to higher levels of education and greater desire for a smaller family size due to the social and economic characteristics of urban populations (Weinberger, 1991; Shah, 1994). On the other hand, many governments, with the support of external donors, promote a wide range of primary healthcare initiatives in rural areas because this is where the majority of the population currently lives and where access to services is most restricted (Gilson, 1995; Dean, 1991). Family planning and reproductive health programs are no exception to this trend.

Whether there is a difference between urban and rural areas in the readiness of clinics to offer high-quality services is an important issue for policy makers and healthcare planners. Reproductive health services in the largest African cities are often implemented and funded separately from the national program because these cities are administered as separate entities for all public-sector services. The allocation of resources to rural health services, combined with the increasing demands faced by urban services, may be causing a difference in rural and urban areas in terms of the quality of services offered. However, there is little empirical evidence describing the relative conditions of urban and rural services (for descriptions of urban services, see Muvandi et al., 1995; Maggwa et al., 1995a; and Maggwa et al., 1995b). Is there a difference between them? If so, which have the advantage, and what aspects of service delivery are different? Such information is becoming increasingly important to policy makers and donors as they face the realities of rapid urbanization. 
Situation Analysis data on the readiness and quality of family planning services in several subSaharan African countries can be used to illustrate the extent of the differences between services in urban and rural areas. This chapter provides descriptive and comparable information on the state of urban and rural services in six countries where such data were collected: Botswana, Burkina Faso, Ghana, Kenya, Senegal, and Zanzibar. ${ }^{1}$ This analysis can inform the discussion in these countries, and elsewhere in Africa, on the future allocation of resources for family planning and other reproductive health services.

\section{METHODOLOGY}

This analysis uses the Bruce-Jain framework (Bruce, 1990) for describing readiness and quality of care (see Chapter 1) to compare services at rural and urban service-delivery points (SDPs). The urban and rural sample sizes for the six Situation Analysis studies that provided the data for the analysis are shown in Table 7-1. ${ }^{2}$ Data from the inventory questionnaires in Botswana, Burkina Faso, Ghana, and Kenya are weighted. As part of the Situation Analysis approach, SDPs are normally identified as being located in urban, rural, or peri-urban areas, as defined by local program managers. For the purposes of this chapter, we have combined peri-urban and urban SDPs for the four country studies that included peri-urban locations. ${ }^{3}$
Not surprisingly, there is a correlation between type of SDP and urban or rural location: in all six countries, urban areas have more hospitals and larger SDPs, whereas rural areas have more health posts and small clinics. For this reason, differences between urban and rural areas may be due simply to their different types of SDPs, rather than the location itself. However, the present analysis is protected from this effect in two ways. First, most indicators are defined at a basic level that should be present in all SDPs. For example, having adequate storage for commodity stocks or clean water in the exam room should be required of all types of SDPs. All new clients should be asked their reproductive intentions, regardless of the type of SDP they are attending. An exception is the availability of long-term methods in urban and rural areas, which is in fact affected by the distribution of type of SDP, as discussed later in the chapter. Second, some indicators are calibrated differently based on the type of SDP or type of client. The indicators addressing the amount of equipment at SDPs are measured in terms of differing amounts of equipment required for different types of SDPs and their varying client loads. For example, a hospital must have more specula than a health post to be considered as having "enough" specula. (See Chapter 3 for more detailed discussion.) And the indicators of quality of IUD insertion are measured only among clients who have an IUD inserted, so the difference in availability of IUD services does not affect the indicator. For these reasons, the issue of the

Table 7-1. Rural, urban, and total sample sizes

\begin{tabular}{lc|cccccc} 
& & \multicolumn{4}{c}{} & \multicolumn{4}{c}{ Number of SDPs Visited } & \multicolumn{3}{c}{$\begin{array}{c}\text { Number of Family } \\
\text { Planning Clients } \\
\text { Observed } \\
\text { Country }\end{array}$} & Year & Rural & Urban & Total & Rural & Total \\
\hline Botswana & 1995 & 110 & 74 & 184 & 190 & 215 & 405 \\
\hline Burkina Faso & 1995 & 273 & 64 & 339 & 160 & 349 & 509 \\
\hline Ghana & 1996 & 170 & 143 & 313 & 378 & 441 & 819 \\
\hline Kenya & 1995 & 134 & 119 & 253 & 351 & 610 & 961 \\
\hline Senegal & 1994 & 48 & 132 & 180 & 88 & 1035 & 1123 \\
\hline Zanzibar & 1995 & 85 & 15 & 100 & 58 & 86 & 114 \\
\hline
\end{tabular}

144 Clinic-Based Family Planning and Reproductive Health Services in Africa 
distribution of types of SDPs does not affect the analysis greatly.

The indicators presented here were collected through an inventory of equipment and supplies, and observations of interactions between clients and providers. The unit of analysis for inventory indicators is the SDP, while for observations it is the client. It was not possible to address all elements of readiness and quality in this analysis, but the elements included are believed to be adequately representative of the services provided. All urban/rural comparisons are made on the basis of statistically significant differences, using a p-value of .05 .

The goal of this analysis is twofold: to compare patterns of quality of care between rural and urban SDPs within each of the six countries, and to compare overall patterns of quality of care between rural and urban SDPs within the region. If data from a majority of the six countries show similar results, we consider a regional pattern to be established. Comparisons are expressed in terms of "rural advantage" or "urban advantage" if there is a significant rural/urban difference in results for an indicator within a country; if there is no significant difference, this is expressed as "no difference."

\section{RESULTS}

Indicators of clinic readiness are discussed first. They include indicators under eight elements: staffing; accessibility; infrastructure; contraceptive supplies and logistics; equipment; supervision; information, education, and communication (IEC) materials; and methods available. The quality-ofcare indicators are then presented, and address four elements: choice of methods, information exchange, mechanisms to ensure continuity, and technical competence. ${ }^{4}$

\section{Readiness to Provide Services}

Table 7-2 shows rough results for 34 readiness indicators. To simplify the presentation, the indicators that showed no difference between urban and rural areas are left blank. Those with an urban advantage are shaded grey, and those with a rural advantage are shaded black. A glance at the table shows that overall there is more urban advantage than rural, but many indicators show no difference, and there is some rural advantage.

\section{Staffing}

The presence of a nurse on site is necessary for the majority of reproductive health services and many methods of contraception. In both rural and urban areas, a nurse was present in the vast majority of SDPs. The one exception was Zanzibar, in which the low percentage of SDPs with a nurse on site (60\% urban, 34\% rural) was attributed to sickness, training, or staff being on leave. Overall, urban clinics demonstrated a slight advantage over rural ones, although this difference was significant only in Botswana and Kenya.

The presence of a physician on site may not be required by the Ministry of Health and is not necessary for most contraceptive methods. Consequently, the proportion of family planning clinics in which doctors were reported to be on duty during study visits was under $10 \%$ in both rural and urban areas, and the Zanzibar study showed no presence of a doctor at any SDP. Contrary to what was expected, there were few significant differences between rural and urban clinics in this regard. In only two countries, Ghana and Kenya, was there an urban advantage. However, it is probable that when needed for emergencies or complex procedures, doctors are more available at urban sites because of the proximity of other medical facilities.

\section{Accessibility}

The first accessibility indicator is whether SDPs open on time. Although this result differed greatly among the six countries-with 3\% of SDPs in urban Kenya opening on time compared with $87 \%$ of SDPs in urban Zanzibar-the rates were similar between rural and urban sites within each country.

The second indicator, whether the SDP had a visible sign indicating the availability of family planning services, showed differences between rural and urban areas. In every country except Senegal, a much higher percentage of urban clinics had such a sign. However, as noted in Chapter 3 
Table 7-2. Significant urban and rural differences in readiness indicators

\begin{tabular}{|c|c|c|c|c|c|c|c|}
\hline 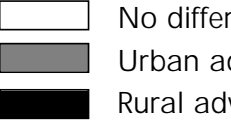 & $\begin{array}{l}\text { rence } \\
\text { dvantage } \\
\text { vantage }\end{array}$ & $\frac{\pi}{\frac{\pi}{0}}$ & 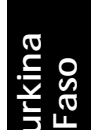 & $\underset{\frac{\sigma}{T}}{\frac{D}{C}}$ & $\stackrel{\mathbb{D}}{\overparen{C}}$ & $\overline{\mathbb{T}}$ & $\frac{\stackrel{c}{0}}{\stackrel{O}{N}}$ \\
\hline \multirow[t]{2}{*}{ Staffing } & $\%$ of SDPs with at least one nurse on duty & & & & & & \\
\hline & ... with at least one doctor on duty & & & & & & \\
\hline \multirow[t]{2}{*}{ Accessibility } & ... open on time & $\mathrm{n} / \mathrm{a}$ & $\mathrm{n} / \mathrm{a}$ & & & & \\
\hline & $\begin{array}{l}\text {... with a sign announcing family planning } \\
\text { services }\end{array}$ & & & & & & \\
\hline \multirow[t]{7}{*}{ Infrastructure } & .... with electricity & & $\mathrm{n} / \mathrm{a}$ & & & & \\
\hline & ... with a water source & & & & & & \\
\hline & ... with adequate water in exam room & & & & & & \\
\hline & ... with clean exam room & & & & & & \\
\hline & ... with adequate light in exam room & & & & & & \\
\hline & .... with a working toilet for clients & & & & & & \\
\hline & ... with an adequate waiting area & & & & & & \\
\hline \multicolumn{8}{|c|}{ Contraceptive ... with adequate storage for stocks } \\
\hline \multirow{5}{*}{$\begin{array}{l}\text { Supplies and } \\
\text { Logistics }\end{array}$} & $\ldots$ with a commodity inventory & & & & & & \\
\hline & $\begin{array}{l}\text {... that have experienced a stockout of } \\
\text { PO Ps* }\end{array}$ & & & & & & \\
\hline & $\begin{array}{l}\text {... that have experienced a stockout of } \\
\text { COCs* }\end{array}$ & & $\mathrm{n} / \mathrm{a}$ & & & $\mathrm{n} / \mathrm{a}$ & \\
\hline & $\begin{array}{l}\text {... that have experienced a stockout of } \\
\text { condoms* }\end{array}$ & & & & & & \\
\hline & ... that have experienced a stockout of IUDs* & & $\mathrm{n} / \mathrm{a}$ & & & $\mathrm{n} / \mathrm{a}$ & \\
\hline \multirow[t]{7}{*}{ Equipment } & $\ldots$ with enough blood pressure machines & & & $\mathrm{n} / \mathrm{a}$ & & & \\
\hline & ... with enough scales & & & & & & \\
\hline & ... with enough stethoscopes & & & $\mathrm{n} / \mathrm{a}$ & & & \\
\hline & ... with enough specula & & & & & & \\
\hline & $\ldots$ with enough gloves & & & & & & \\
\hline & ... with enough light sources & & & $\mathrm{n} / \mathrm{a}$ & & & \\
\hline & ... with enough sterilizers & & & $\mathrm{n} / \mathrm{a}$ & & & \\
\hline Supervision & $\begin{array}{l}\ldots \text {. that have received a supervisory visit in } \\
\text { last } 6 \text { months }\end{array}$ & & & & & & \\
\hline \multirow[t]{2}{*}{ IEC Materials } & $\ldots$ that have a brochure on family planning & & & & & & $\mathrm{n} / \mathrm{a}$ \\
\hline & ... that have a poster on family planning & & & & & & \\
\hline Methods & ... that usually offer PO Ps & & & & & & \\
\hline \multirow[t]{6}{*}{ Available } & ... that usually offer COCs & & & & & & \\
\hline & ... that usually offer condoms & & & & & & \\
\hline & $\ldots$ that usually offer injectables & & & & & & \\
\hline & ... that usually offer IUDs & & & & & & \\
\hline & . . . that usually offer NO RPLAN T ${ }^{\circledR}$ & & & & & & $\mathrm{n} / \mathrm{a}$ \\
\hline & ... that usually offer female sterilization & & & & & & $\mathrm{n} / \mathrm{a}$ \\
\hline
\end{tabular}

Note: $\mathrm{p}<0.05 . \quad \mathrm{n} / \mathrm{a}=$ not available. $\quad$ *For these indicators, an advantage means lower stockout rates. 
(indicator R.2-2) staff in some rural clinics feel that family planning signs compromise confidentiality, and therefore choose not to have a visible sign. Consequently, the appropriateness of a sign may depend on local context.

\section{Infrastructure}

Infrastructure comprises the basic facilities required for clinic functioning, such as water or electricity, and thus is an important facet of providing high-quality care to clients. While some infrastructure indicators do not demonstrate significant differences between rural and urban clinics, those that do show an urban advantage.

Not surprisingly, access to electricity and water was markedly higher in urban than in rural SDPs. The largest differences in electricity emerged in Botswana, Kenya, and Zanzibar, where fewer than $30 \%$ of rural clinics had electricity on the days on which data were collected, compared with over $82 \%$ of urban clinics. Availability of water at rural SDPs was greater than that of electricity, but was still as low as $41 \%$ (Zanzibar). The percentage of urban clinics with running water was never lower than $72 \%$ (Kenya). Botswana is the only country in which availability of water was similar in urban and rural areas.

One could debate the value of this measure, arguing that perhaps adequate water from wells, rainwater collection, or some other source of clean water is available. However, adequate water in exam rooms is essential for maintaining procedures that prevent infection. Water, either running or from other sources, proved to be more common in urban than in rural areas in Burkina Faso, Kenya, and Senegal, and differences between rural and urban areas were substantial. For example, in Burkina Faso only $29 \%$ of rural clinics had adequate water in the exam room, compared with $71 \%$ of urban clinics.

In three countries, a higher proportion of urban than rural SDPs had clean exam rooms. Results on this indicator were $80 \%$ or higher in all areas except rural Burkina Faso and Kenya generally, where the results ranged from $60 \%$ to $65 \%$. As for adequate light source in the exam room, an urban advantage was found only in Burkina Faso; however, this result did not fall below $80 \%$ in any region.
Working toilets for clients ${ }^{5}$ were significantly more common in urban than rural clinics in Botswana, Burkina Faso, and Zanzibar. In Kenya, however, toilets were more common in rural (90\%) than in urban $(66 \%)$ SDPs.

In the six countries studied, the data showed little difference between rural and urban areas in the percentage of SDPs with adequate waiting areas for clients. ${ }^{6}$ In fact, the data show only small differences among the countries. With the exception of rural Botswana, between $79 \%$ and $95 \%$ of SDPs had adequate waiting space. In Botswana, $44 \%$ of rural clinics had adequate waiting space for patients, compared with $83 \%$ of urban SDPs.

\section{Contraceptive Supplies and Logistics}

The element of contraceptive supplies and logistics includes not only each clinic's ability to provide contraceptives on site, but also its ability to manage its inventory through regulated systems. It could be argued that because rural clinics may have more difficulty acquiring materials, they may be less likely to keep systematized inventories. However, this was not borne out by the data.

No urban/ rural differences were found in any of the countries in terms of space for stocking equipment and goods. Moreover, no clear urban advantage was seen for use of systematized inventories. Although more urban than rural SDPs in Senegal and Burkina Faso had commodity inventory forms, the reverse was true in Botswana and Kenya, and there were no differences between rural and urban SDPs in Zanzibar and Ghana.

Similarly, for all methods of contraception, there was no difference between stockouts in rural and urban SDPs in the majority of countries.? Stockouts of progestin-only pills (POPs) were fairly equal between rural and urban SDPs, with the exception of SDPs in Kenya, where such stockouts were slightly more common in rural areas (i.e. there was an urban advantage). For combined oral contraceptives (COCs), stockouts were similarly found throughout each country in urban and rural SDPs. Condom stockouts varied, but in Burkina Faso and Botswana, urban SDPs showed an advantage on this indicator (meaning they had lower 
stockout rates). These results suggest that differences in commodity availability between rural and urban sites are not as great as might be expected. One possible explanation is that supplies are often distributed centrally, so stockouts may be beyond the control of individual clinics.

\section{Equipment}

One would expect access to equipment to be better in areas that are closer to central suppliers and have better transportation. Thus, urban SDPs might be expected to have more equipment on hand. However, as with contraceptives, this expectation rarely proved to be true. In most cases, the percentages of rural and urban SDPs with enough working equipment were fairly equal. When there was an advantage, it was more often rural over urban than the reverse.

The availability of an adequate number of blood pressure gauges, scales, stethoscopes, specula, gloves, light sources, and sterilizing facilities was generally similar in rural and urban SDPs. There were some exceptions: a rural advantage was found for blood pressure machines in Burkina Faso and Zanzibar, for sterilizers and stethoscopes in Kenya, and for lights and blood pressure machines in Zanzibar. An urban advantage was found for specula in Burkina Faso, gloves in Kenya, and lights in Botswana. However, these results show no overall trend in equipment availability across urban and rural contexts.

\section{Supervision}

As Figure 7-1 indicates, the percentage of SDPs receiving a supervisory visit in the 6 months prior to the study visit was comparable between rural and urban clinics within each country, with no significant differences. ${ }^{8}$ Thus although urban clinics are physically closer together, they are not necessarily supervised more frequently.

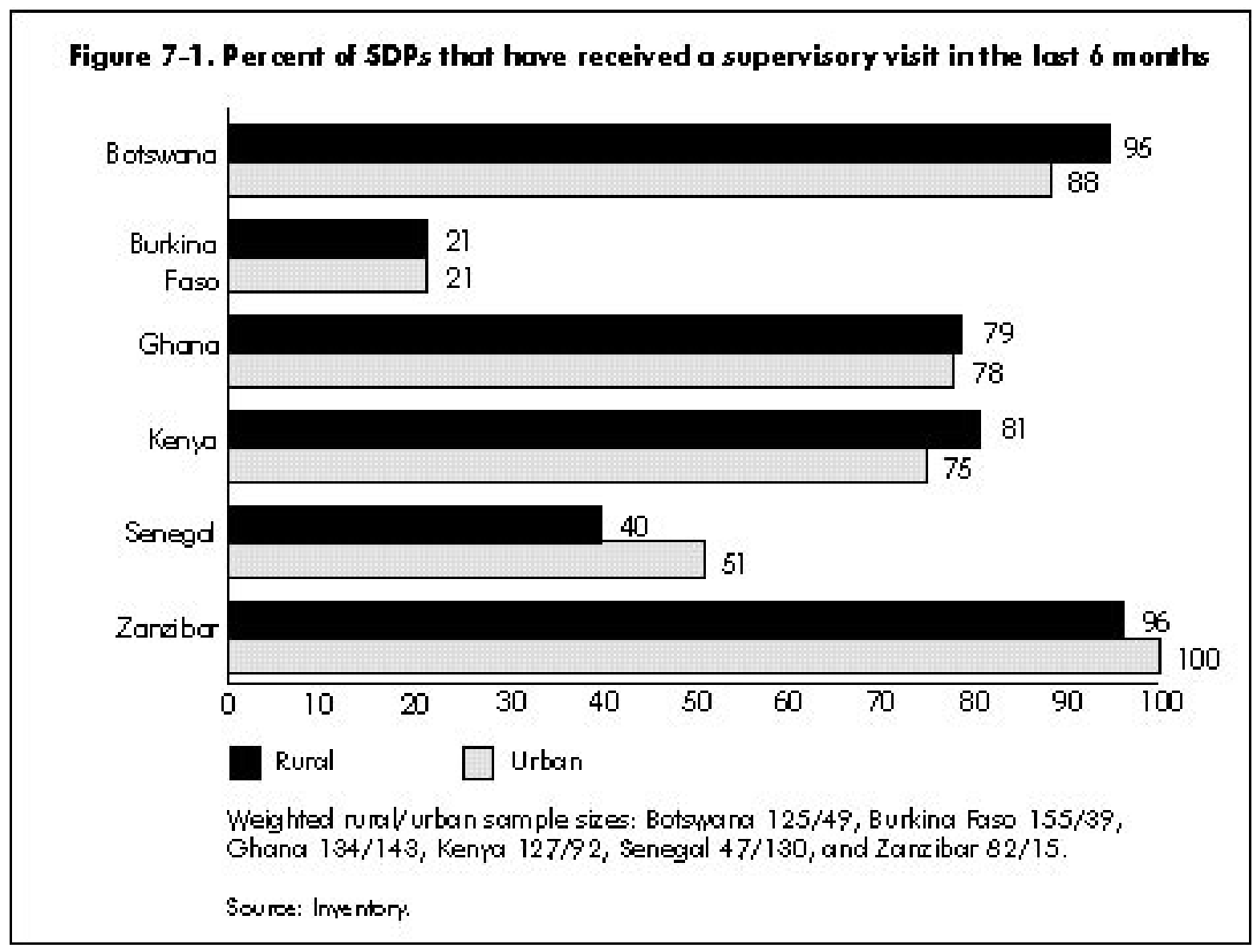

148 Clinic-Based Family Planning and Reproductive Health Services in Africa 


\section{Information, Education, and}

\section{Communication Materials}

The presence of brochures on family planning was fairly consistent across rural and urban areas within most countries, yet it varied considerably among countries (10\% in rural Senegal to $61 \%$ in rural Botswana). The presence of posters appeared to be more common in urban than rural areas in most countries, and showed a smaller range than that of brochures (55\% in rural Botswana to $90 \%$ in urban Ghana). Overall, more urban than rural SDPs had available stocks of IEC materials.

\section{Methods Available}

No distinguishable pattern was found for urban and rural SDPs that usually offer oral contraceptives, condoms, or injectables. Figure 7-2 shows that for POPs, which are used by women who are breastfeeding, there was no rural or urban advantage, with the exception of Botswana (rural advantage) and
Burkina Faso (urban advantage). Similarly, no difference was found for COCs, except in Botswana, which showed a rural advantage, and Ghana, which showed an urban advantage. Data on SDPs offering condoms revealed differences in Burkina Faso and Zanzibar, both showing an urban advantage. As for the injectable, Senegal and Burkina Faso showed an urban and Botswana a rural advantage. The fact that pills, condoms, and injectables are fairly easy to administer probably facilitates the equality commonly found between urban and rural areas on these indicators. Moreover, these methods are the most commonly available in general (see Chapter 3).

Not surprisingly, the differences become more striking among contraceptive methods that are relatively more complicated to administer: IUDs, NORPLANT $^{\circledast}$, and female sterilization. In almost all cases, the percentage of SDPs offering each method was higher in urban than rural areas. This would be expected because urban clinics are more likely to have the trained staff and infrastructure

\section{Figure 7-2. Per cent of SDPs that usually off er POPs}

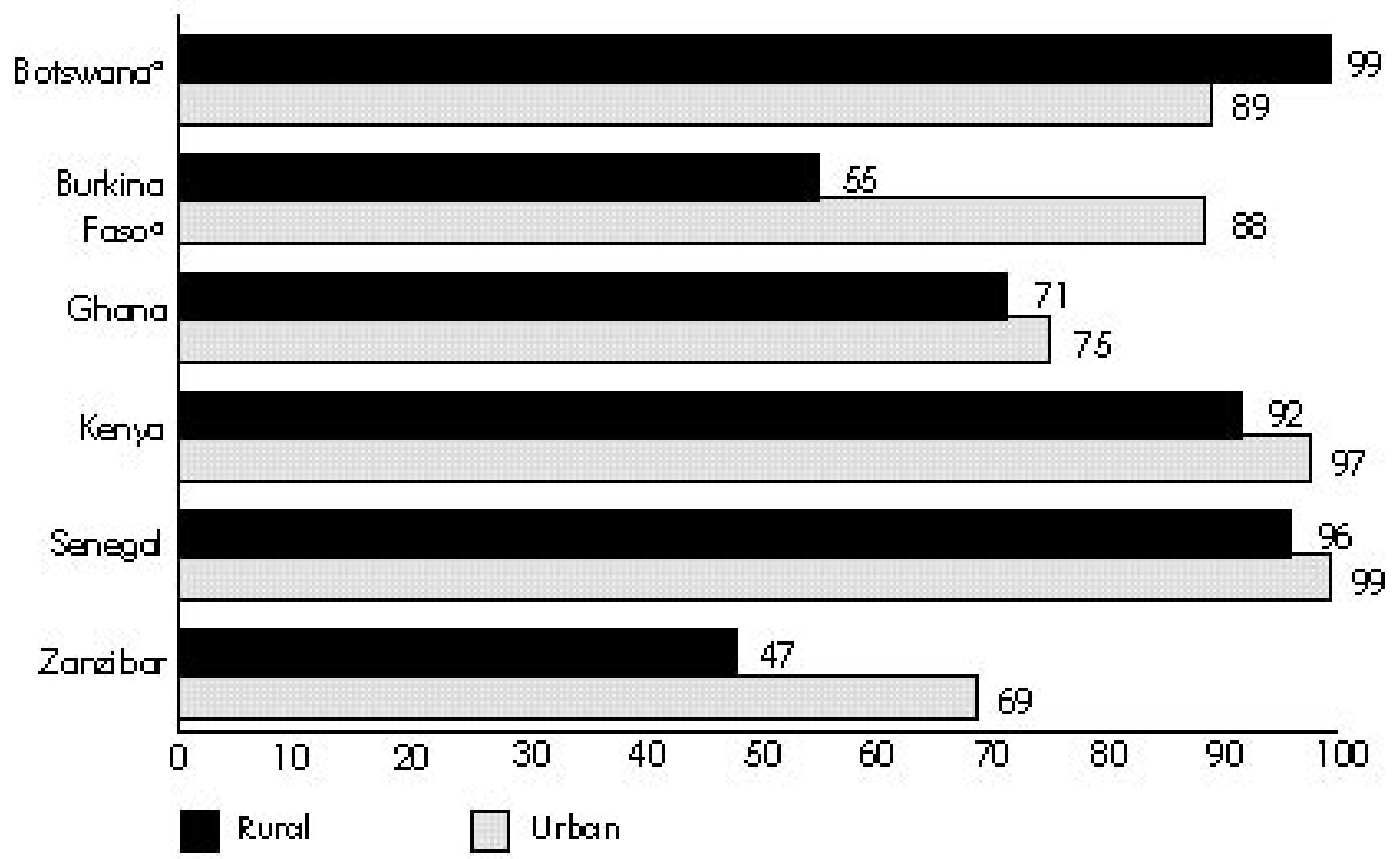

Weighted rumalurban sample sizes: Bot?inana 130/53, Burkina Faso 157/4 1, Ghana $153 / 161$, Kenra $132 / 119$, Senegal $48 / 132$, and Zarzibar $60 / 13$.

$a_{p}<0.05$. Source: Impentory. 
Figure 7-3. Percent of new clients told doout at least two metho ds

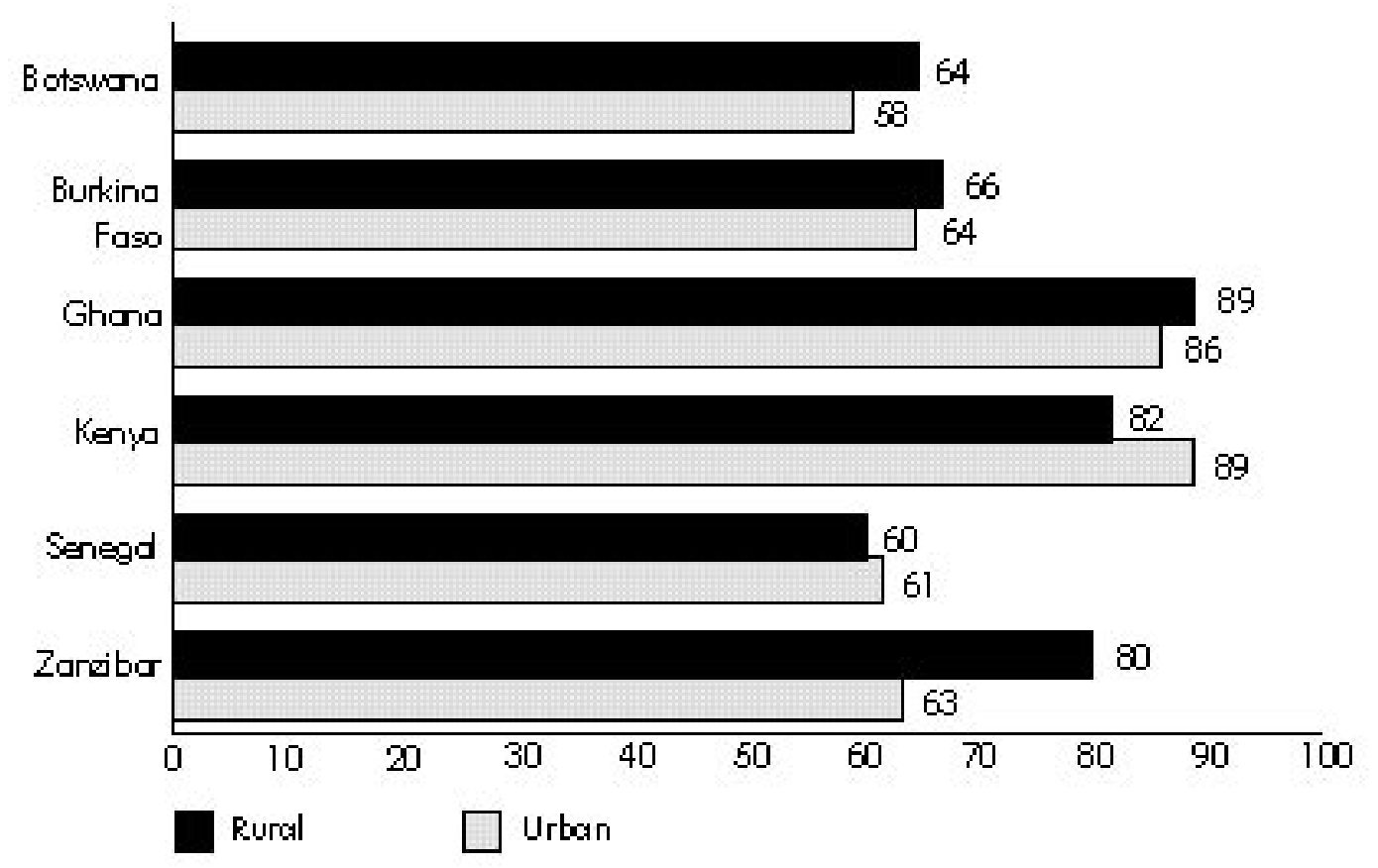

Weighted nura/urban sample sizes: Botzinana $28 / 43$, Burkina Faso $35 / 91$, Ghana $110 / 138$, Kenra $82 / 8$, Senegal $20 / 207$, and Zarzibar $20 / 8$.

Sourd: Imyentory.

required to administer these methods. The fact that more hospitals and larger clinics are clustered in urban areas affects these indicators strongly. The few cases that did not show a difference between urban and rural areas include Botswana for NORPLANT $^{\circledast}$ and female sterilization, and Burkina Faso for female sterilization. In all these cases, the overall availability of the method was extremely low, leading to the lack of a statistically significant difference between urban and rural regions.

\section{Quality of Care}

The results for 23 quality-of-care indicators are shown in Table 7-3. As in Table 7-2, cases with no significant difference are left blank, urban advantages are shaded gray, and rural advantages are shaded black. Immediately, this table appears quite different from the last, showing far fewer urban/rural differences overall.

\section{Choice of Methods}

As shown in Figure 7-3, none of the differences in the proportion of clients told about more than one method of contraception were significant. Similarly, the percentage of clients asked whether they had a contraceptive method preference was generally similar between rural and urban sites within each country, except in Ghana, which showed an urban advantage. This information demonstrates that although rural clinics may offer fewer methods because of constraints in readiness categories, they are as likely as urban SDPs to offer choices based on what is available on site (see Chapter 5).

\section{Information Exchange}

Information exchange is an important element for this analysis because it does not require equipment 
Table 7-3. Significant urban and rural differences in quality indicators

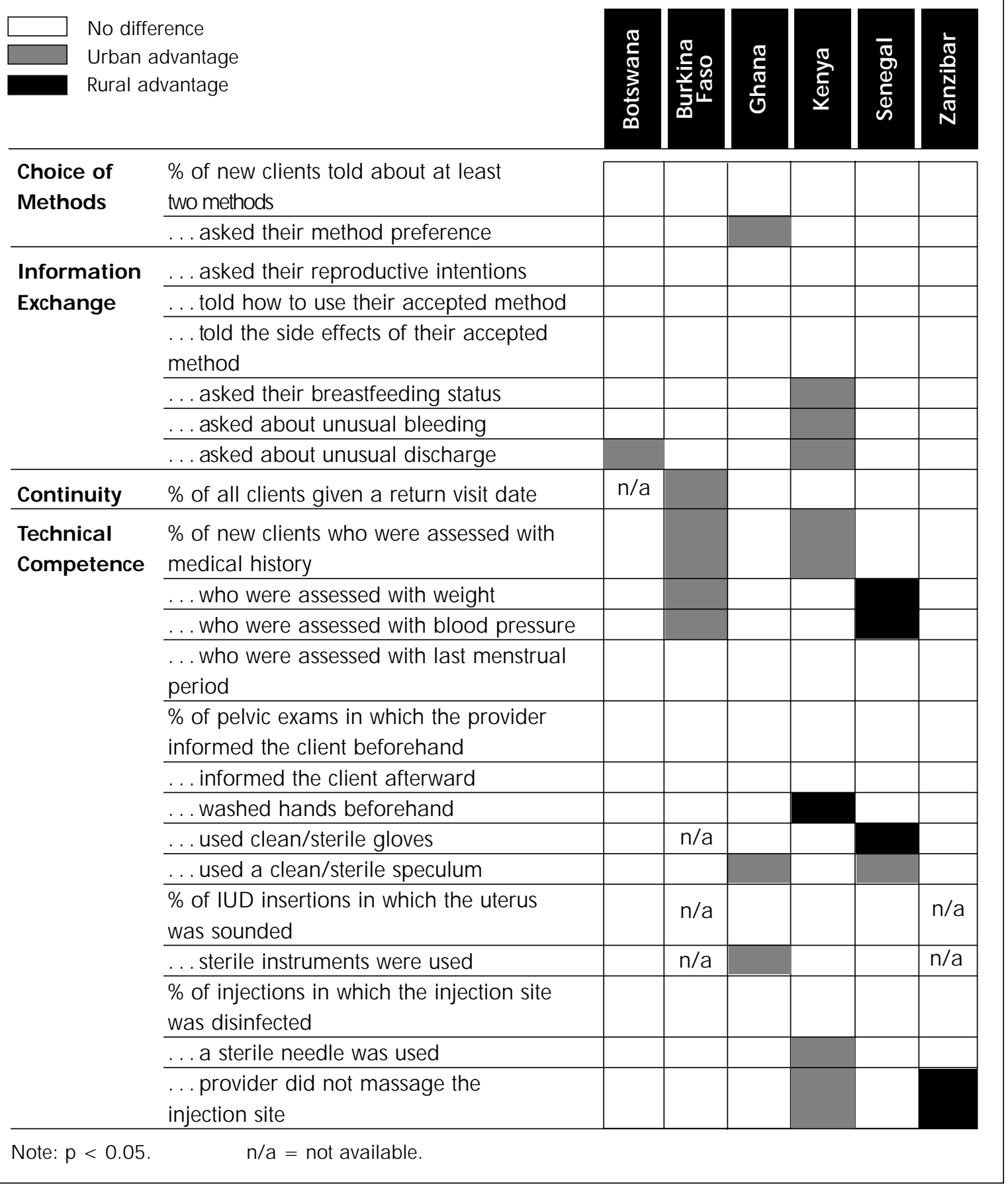

or infrastructure. In even the most remote or smallest SDP, trained staff can exchange information with clients. And in fact, rural and urban SDPs generally showed the same results for the information exchange indicators. In urban and rural SDPs, the same proportions of clients were asked their reproductive intentions, told how to use their method, and told about their method's side effects.

With some exceptions, no overall differences emerged between the percentages of rural and 


\section{Figure 74. Per cent of new clients asked dbout their br easfeeding status}

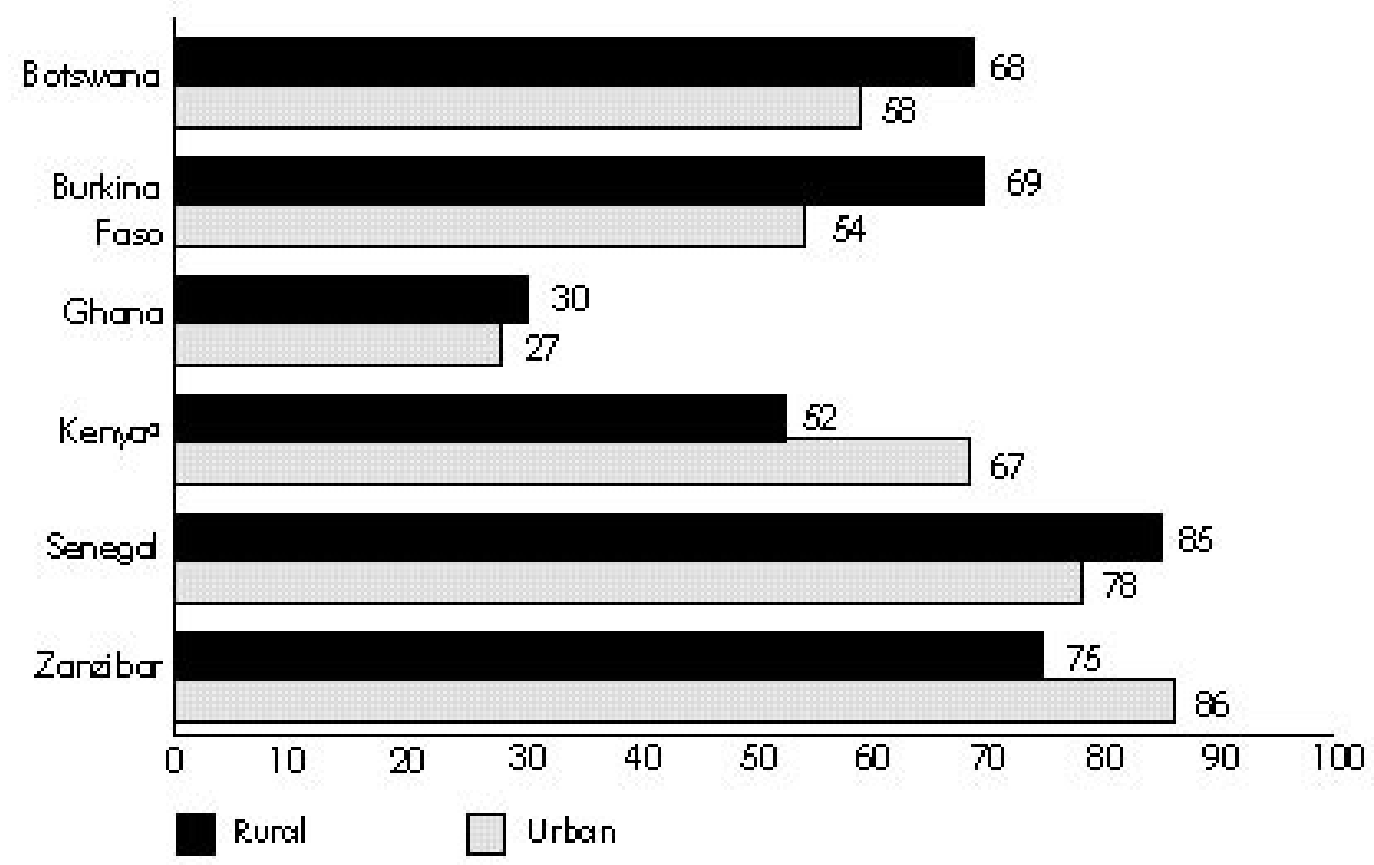

Weighted ruraVurban sample sires: Botsuana 28/43, Burkina Foso $35 / 91$, Ghana $110 / 138$, Kenra 87/153, Senegal 20/207, and Zarribar $20 / 7$.

$a_{p}<0.05$. Sourde: Imyentory.

urban clients who were asked whether they were breastfeeding (see Figure 7-4) or whether they had unusual bleeding or discharge. Kenya showed an urban advantage on these three indicators, and Botswana an urban advantage on clients asked about discharge. Overall, the results demonstrate that the frequency with which information is exchanged in counseling clients is similar among staff in rural and urban areas.

\section{Mechanisms to Encourage Continuity}

Clients should be encouraged to return to SDPs periodically to resupply their methods or check for problems with method use. The data show no difference in the percentage of clients given a date for a return visit in four of five countries. Only in Burkina Faso were significantly more urban $(96 \%)$ than rural $(87 \%)$ clients given a return visit date.

\section{Technical Competence}

The Situation Analysis approach gathers data on several aspects of the technical quality of family planning procedures (see Chapter 3). Technical competence is a major element of quality of care and encompasses a variety of indicators, from percentage of clients whose medical history was taken to percentage of injections for which a sterile needle was used.

The first set of indicators under technical competence addresses the physical assessment of clients through medical history, weight, blood pressure, and last menstrual period (LMP). As discussed elsewhere in this volume (see Chapters 3 and 9), the need to carry out each of these assessments with new clients is debated. For the purposes of this analysis, however, simple differences in rates of performing these procedures in urban and rural areas are presented. In the majority of cases, there were no differences between urban and rural 
Figure 7-5. Percent of readiness indicator $s$ with urban advantage, rurd advantage, and no difference

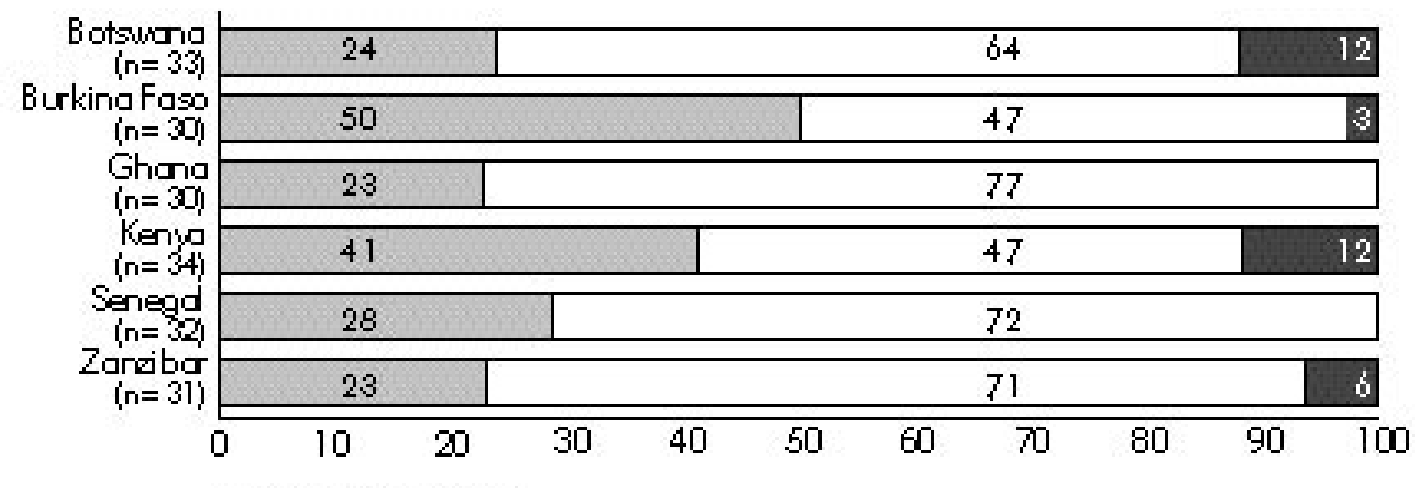

Perent of indicators

Urban advantage $\square$ No difference $\square$ Rumal adyantage

areas in rates of performing these assessments. Where differences did emerge, they tended to show an urban advantage, although weight and blood pressure showed a rural advantage in Senegal. Overall, no consistent differences were found.

For quality of pelvic exams, generally consistent services appear to be offered within each country. No urban/rural difference was found in any country between the percentages of clients who were informed about their exam before it started and after it was completed. On the indicators of handwashing before the exam and the use of gloves during the exam, there was one country with a rural advantage for each (Kenya for handwashing and Senegal for gloves). Conversely, an urban advantage was demonstrated for the use of sterile specula in Ghana and Senegal. It is important to note that the indicators of handwashing, sterile specula use, and glove use take into account whether clean water, specula, and gloves are available at the SDP. Again, however, no overall difference in quality of pelvic exams in urban and rural areas is apparent.

The next two indicators address whether proper procedures were followed during IUD insertions. These results indicate an urban advantage in one case, but do not show an overall pattern. The percentage of IUD insertions in which a uterine sound was used was the same for rural and urban
SDPs in each country (ranging from 50\% in rural Ghana to $100 \%$ in rural Kenya and Senegal). Moreover, the percentage of insertions during which sterile instruments were used was similar in rural and urban areas in three of the four countries surveyed on this indicator; Ghana was the exception, showing an urban advantage.

Injection quality shows similar results. There were no significant rural/urban differences in the percentage of injections for which the site was disinfected in any country. Sterile needles were used for roughly the same percentage of injections in rural and urban SDPs in five of the six countries, but showed an urban advantage in Kenya. Lastly, no differences emerged between rural and urban areas in terms of not massaging the injection site, except in Kenya (urban advantage) and Zanzibar (rural advantage). ${ }^{9}$

\section{DISCUSSION AND CONCLUSIONS}

Figures 7-5 and 7-6 display the percentage of indicators that show a rural advantage, urban advantage, or no difference for each country. Figure 7-5 addresses the 34 readiness indicators in Table 7-2, and Figure 7-6 the 23 quality indicators in Table 7-3.

Figure 7-5 shows that where differences exist in readiness indicators, urban areas tend to have the advantage in each country. This is most apparent 


\section{Figure $7-6$. Per cent of quality ind cators with urban advantage, rural advantage, and no dfffer ence}

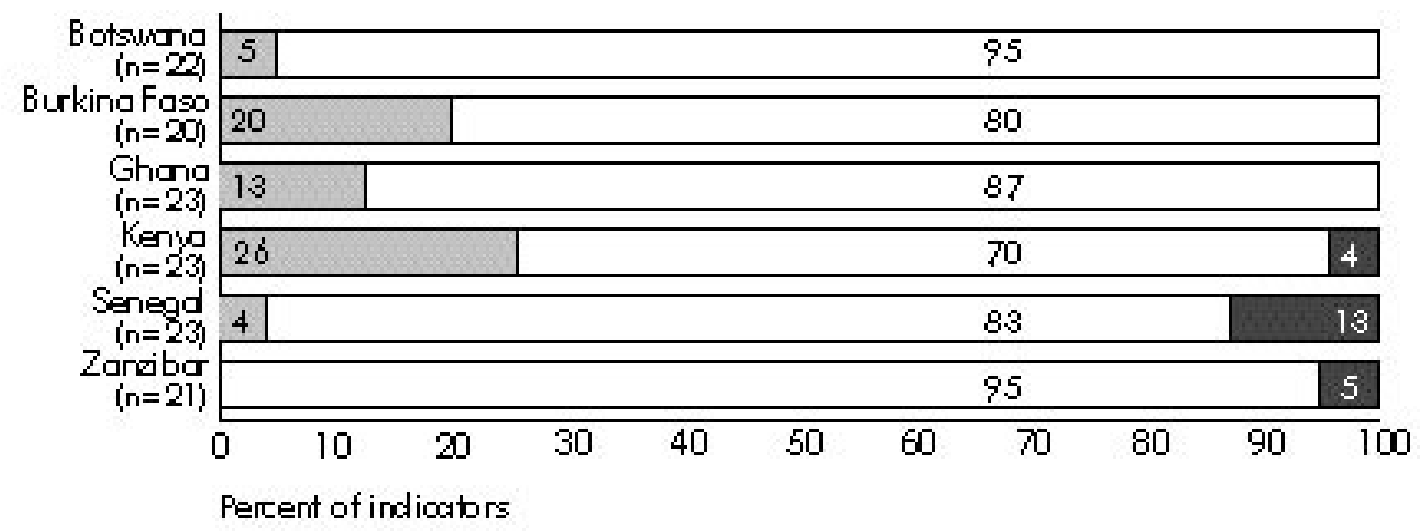

Urban advantoge $\square$ No difference $\square$ Rural adyantage

for indicators that relate to infrastructure, such as the availability of water or electricity, and of IUD, NORPLANT ${ }^{\circledast}$, and female sterilization services.

The remaining results are more unexpected, however. About half or more of the readiness indicators in each country show no significant urban/rural difference. In particular, there is no country in which an urban or rural advantage is shown for rates of supervision, stockouts of COCs, or storage areas for commodities. Moreover, up to $12 \%$ of the indicators for each country exhibit a rural advantage. These rural advantages are clustered around equipment availability, presence of commodity inventory, and, in Botswana, the availability of hormonal methods.

By country, Burkina Faso and Kenya appear to exhibit the most differences between urban and rural areas. In Burkina Faso, half of the indicators show an urban advantage. In Kenya, over a third of the indicators show an urban advantage, but fully $12 \%$ exhibit a rural advantage, as high as any country included here.

Comparison of these readiness results with the quality indicators shown in Figure 7-6 reveals a distinctly different pattern of urban and rural advantage. Although the quality of care in both urban and rural settings often demonstrates serious shortcomings (see Chapter 3), the majority of quality indicators show no difference between urban and rural SDPs. In Botswana and Zanzibar, fully $95 \%$ of the indicators show no difference between urban and rural contexts. Moreover, those urban and rural advantages that do exist do not cluster around any particular element of quality or show an easily generalizable pattern. Taken overall, the data show that quality of care across urban and rural areas in the six countries included here is roughly equal. This may be explained by the fact that these indicators of quality emphasize information exchange and counseling, which does not rely heavily on infrastructure or readiness. ${ }^{10}$ In addition, the Ministry of Health in each country is the main supplier of family planning services and runs standard training courses for its entire staff. As these staff are distributed among urban and rural SDPs, the consistency of their behavior may reflect their shared training.

How do the results presented here reflect on the assumption, presented at the beginning of the chapter, that urban services should be of higher quality than rural services? There are a number of considerations. First, because the majority of the population in Africa has historically lived in rural areas, government and nongovernmental organization (NGO) development efforts have focused primarily on rural areas (Gilson, 1995; Dean, 1991). Second, because urban standards of living have long shown a consistent advantage over rural areas, there has been an added emphasis on efforts to rectify the situation in rural areas. It may be that the attention 
given to rural services has improved them markedly, bringing them more in line with the higher-quality urban services. Alternatively, the increasing demands being faced by services in urban areas may be contributing to a decline in quality in those areas, bringing them closer to the levels of service provided in rural areas. A combination of these two factors could be at work. However, analysis of the situations in urban and rural areas over time would be needed to examine whether either or both of these trends are responsible (see Chapter 11). It is also possible, of course, that the assumed urban advantage never existed at all.

Furthermore, as noted earlier, this analysis is based on "quality" as defined by the Bruce-Jain framework. Although this framework is widely accepted, there may be differences between urban and rural services that are not measured using the approach applied here, and that would provide insights into the above conclusions. The Bruce-Jain framework emphasizes indicators of counseling, information exchange, and training. The use of other frameworks to compare rural and urban SDPs might result in different conclusions (United Nations, 1995).

Nevertheless, the results presented here suggest that policy makers and researchers need to examine common assumptions about the quality and readiness of reproductive health care in urban and rural settings. It is no longer safe to assume an urban advantage in clinic-based family planning services in sub-Saharan Africa. Moreover, with projections that sub-Saharan populations will be over $50 \%$ urban by the year 2020, refocusing and creating a new vision for urban family planning and reproductive health services is essential.

\section{REFERENCES}

Brockerhoff, M. and E. Brennan. 1997. "The poverty of cities in the developing world." Working Paper No. 96, Population Council, New York.

Bruce, J. 1990. "Fundamental elements of the quality of care: A simple framework." Studies in Family Planning, 21,2:61-91.

Dean, M. 1991. “Nairobi perspective: Health for how many by 2000?" Lancet, 330:239-240.
Gilson, L. 1995. "Management and health care reform in sub-Saharan Africa." Social Science \& Medicine, 40,5:695-710.

Gorosh, M., N. Maggwa, I. Muvandi, et al. 1995. Findings from the Sub-Saharan Africa Urban Family Planning Study: Overview of Studies Conducted in Blantyre, Malawi; Bulawayo, Zimbabwe; and Mombasa, Kenya. The Center for African Family Studies, Nairobi, Kenya; John Snow Inc., Arlington, VA; and the Center for Population and Family Health, New York.

Maggwa, N., I. Muvandi, M. Gorosh, et al. 1995a. "Findings from the sub-Saharan Africa urban family planning study: Mombasa city report." John Snow Inc., Arlington, VA.

Maggwa, N., I. Muvandi, M. Gorosh, et al. 1995b. "Findings from the sub-Saharan Africa urban family planning study: Blantyre city report." John Snow Inc., Arlington, VA.

Muvandi, I., N. Maggwa, M. Gorosh, et al. 1995. "Findings from the sub-Saharan Africa urban family planning study: Bulawayo city report." John Snow Inc., Arlington, VA.

Sadik, N. 1996. The State of World Population, 1996: Changing Places-Population, Development and the Urban Future. United Nations Population Fund, New York.

Shah, I.H. 1994. "Family planning in sub-Saharan Africa." World Health, 47,3:20-21.

United Nations. 1995. World Urbanization Prospects, the 1994 Revision. United Nations Department for Economic and Social Information and Policy Analysis, Population Division, New York.

Weinberger, M.B. 1991. "Recent trends in contraceptive behavior." In Demographic and Health Surveys World Conference: Proceedings, Vol. 1. IRD/Macro International, Inc., Columbia, Maryland.

\section{NOTES}

1 Although Zanzibar is part of Tanzania, it retains an independent health program and was therefore analyzed separately. For the purposes of this chapter, it is referred to as a country.

2 See Chapter 2 for information on these countries' family planning programs and Chapter 1 for study sampling information.

3 The following are the percentages of total SDPs used in this analysis that are peri-urban: Botswana, 17\%; Burkina Faso, 9\%; Kenya, 9\%; and Senegal, 25\%. The inclusion of these semi-urban SDPs in the urban category may have diluted urban/rural differences, particularly in Botswana and Senegal.

4 Note that for some of the indicators discussed in this section, data were not available for all six countries. 
In each case, the results presented are based on the available data.

5 Defined as flush toilets or any other type.

6 Adequate waiting areas are defined in a fairly consistent manner across all countries, and generally include seating for clients and a waiting space protected from rain.

7 Data on stockouts of injectables and NORPLANT ${ }^{\circledR}$ implants were not collected in all countries.
8 Although the differences were not significant, more rural than urban SDPs had been visited by a supervisor in four of the six countries.

9 Not massaging the injection site is believed to increase the efficacy of injectable contraception. See Chapter 3 for further detail.

10 It is not the purpose of this chapter to examine the relationship between quality of care and readiness in depth; this is done in Chapter 5. 


\section{STANDARDS AND GUIDELINES FOR SERVICES}

How Providers Restrict
Access to Family
Planning Methods:
Results from Five
African Countries
details the extent to which
providers impose various
restrictions-such as marital
status, parity, and age-on
women seeking family plan-
ning services, and compares
these restrictions against
national service protocols.

\begin{tabular}{l}
\hline \\
Tests and Procedures \\
Required of Clients in \\
Three Countries of \\
West Africa \\
reviews the requirements for \\
laboratory tests and other \\
medical procedures-such as \\
blood pressure, weight taking, \\
and pelvic exams-imposed \\
on clients and compares these \\
requirements against interna- \\
tional standards. \\
\end{tabular}





\section{KEY POINTS AND CONCLUSIONS}

\section{How Providers Restrict Access to Family Planning Methods: Results from Five African Countries}

Since 1992, Situation Analysis studies have gathered data on provider attitudes and behaviors to measure the extent to which providers impose various restrictions on the availability of contraceptive methods. A review of five recent and comparable studies on staff-imposed restrictions (in Botswana, Burkina Faso, Kenya, Senegal, and Zanzibar) and the analogous country service protocols leads to the following conclusions:

- Providers from Zanzibar are the most restrictive in providing contraceptive methods according to marital status. This is to be expected given the country's strong religious context and the Islamic norms of the service protocols.

- In all five countries, marital status restrictions are imposed most commonly in prescribing IUDs and female sterilization, and least commonly in prescribing condoms.

- An unmarried woman in Botswana, Burkina Faso, and Senegal might actually have an easier time obtaining contraception than a married woman who does not have her husband's consent. With the exception of female sterilization, however, none of the countries studied has consent restrictions on any method, implying that providers are imposing this barrier on their own initiative.

- A considerable proportion of providers impose parity requirements for the provision of IUDs and injectables. This is not surprising given that restrictions on IUDs are actually required by policy in Burkina Faso, Kenya, and Zanzibar. In Kenya, however, where the providers are the most restrictive of any of the five countries in terms of parity requirements, methods such as condoms are also significantly restricted on the basis of parity, even though service protocols do not require such restrictions.

- An examination of minimum age requirements suggests that providers generally consider COCs and condoms appropriate for younger women (average minimum age required: 1517); IUDs, injectables, and NORPLANT ${ }^{\circledast}$ appropriate for women in their middle reproductive years (minimum age: 17-27); and female sterilization appropriate for women who have completed their childbearing (minimum age: 35 or over). Apart from IUDs in Senegal and female sterilization, however, the service protocols have no such requirements, and international guidelines place no strict age requirements on any method.

- In each of the five countries, providers on average impose twice as many eligibility criteria as are required or encouraged by national guidelines; in Kenya, Botswana, and Zimbabwe, providers impose from one-quarter to twothirds of all eligibility criteria examined.

Overall, it can be concluded that providers' attitudes and behavior play a significant role in the way services are provided and in the restrictions imposed on particular methods. Interviews with providers in Ghana revealed that providers impose these restrictions with the intent of protecting both the client and society. Revised service protocols and training programs may remove the concerns about clients' wellbeing, but addressing broader societal and cultural concerns will require more careful attention. 



\section{How Providers Restrict Access to Family Planning Methods: Results from Five African Countries}

Kate Miller, Robert Miller, Goli Fassihian, and Heidi Jones

Access to family planning services and quality of care are both critical issues in the delivery of services. Access to services includes a client's ability to receive methods regardless of her age, parity, marital status, or spousal consent. This chapter addresses the ways in which providers restrict methods based on these "eligibility criteria," as measured through direct provider interviews.

Bertrand et al. (1995) provide an exhaustive classification of restrictions to family planning services, grouped as access barriers and medical barriers. To physically reach a service delivery point (SDP), a client must have geographic, economic, administrative, cognitive, and psychosocial access. Moreover, once at the SDP, a client should not be denied a family planning method because of medical barriers, outdated contraindications, eligibility criteria, process or scheduling hurdles, service provider qualifications, provider bias for or against methods, inappropriate management of side effects, or regulatory barriers. Shelton et al. (1992:1334) define these medical barriers "...as practices, derived at least partly from a medical rationale, that result in a scientifically unjustifiable impediment to, or denial of, contraception." Provider-imposed limitations on family planning services have been considered an underappreciated impediment to quality and have received substantial attention in the last 5 years (McDonald et al., 1995; Herndon, 1993; Jato et al., 1995).

Since 1992, Situation Analysis interviews with providers have documented the extent to which eligibility criteria are imposed by staff. In a followup to the 1994 Situation Analysis study in Ghana (Ghana Statistical Service, 1994), Twum-Baah and
Stanback (1995) used the Ghanaian data to identify SDPs at which providers impose the most eligibility criteria. They revisited these 46 highly restrictive SDPs to check the validity of the Situation Analysis data through a review of clinic records and reinterviewed providers on their views. The study concluded that service restrictions varied among and between different providers at each site, that providers had inadequate knowledge of contraindications and side effects of modern methods (especially injectables), and that providers imposed their personal moral standards on their clients.

Shelton et al. (1992) postulate that medical barriers appear at two levels of the provider-client interface-the macro level of policies, protocols, and regulations, and the micro level of individual provider attitudes and behaviors. This chapter addresses the macro level by examining family planning service protocols established by five Ministries of Health, and the micro level by reviewing provider reports about the eligibility criteria they impose. It explores how reports of provider restrictions relate to national program guidelines and considers other possible sources of restrictions. The objective is to describe possible regional patterns in these restrictions as a basis for further development of targeted interventions (Family Health International, 1994).

\section{METHODOLOGY}

\section{Situation Analysis Data}

Of the available Situation Analysis studies, five have recent and comparable data on staff-imposed 
restrictions on family planning access: ${ }^{1}$ Botswana (1995), Burkina Faso (1995), Kenya (1995), Senegal (1994), and Zanzibar² (1995). These five countries also constitute a broad geographical spread across the continent. ${ }^{3}$ The specific restrictions investigated are as follows:

- Marital status

- Spousal consent

- Parity requirements

- Minimum age requirements

- Maximum age requirements

Each restriction is examined with respect to six methods of contraception:

- Combined oral contraceptives (COCs)

- Condoms

- Intrauterine devices (IUDs)

- Injectables

- NORPLANT ${ }^{\circledast}$ implants

- Female sterilization Specifically, the interviews with providers included the following questions:

- Would you provide pill/condom/IUD/ injectable/NORPLANT ${ }^{\circledast}$ implants/female sterilization to an unmarried woman?

- Would you provide pill/condom/IUD/ injectable/NORPLANT ${ }^{\circledR}$ implants/female sterilization to a woman without her husband's consent?

- What is the minimum number of children a woman must have before you would provide or refer her for pill/condom/IUD/injectable/ NORPLANT $^{\circledR}$ implants / female sterilization?

- What is the age range outside of which you will not provide or refer a client for pill/condom/ IUD/injectable/NORPLANT ${ }^{\circledR}$ implants/female sterilization? (specify minimum and maximum)

These questions were asked similarly across the five studies, with some exceptions. NORPLANT ${ }^{\circledR}$ implants and female sterilization were not included in the list of methods for Botswana and Zanzibar. The interviews were conducted in French in Senegal and Burkina Faso, and in Swahili in Zanzibar. In Kenya, although the question on age restrictions was asked, the results cannot be reported because of difficulties with coding and interpreting the data.

All the results are presented later in the chapter in Figures 8-1 through 8-8. ${ }^{4}$ The results for marital status and spousal consent are expressed simply as the percentage of providers who reported that they impose these restrictions. The results for parity restrictions are expressed by two indicators: the percentage of providers who impose a restriction of at least one child, and among those providers, the mean number of children required. The age data are also presented as two indicators: the percentage of providers that impose the restriction, ${ }^{5}$ and among those providers, the mean age (both minimum and maximum) required. ${ }^{6}$

Situation Analysis studies collect information not only on the barriers a provider imposes, but also on the methods she has actually provided to clients in the last 3 months. All results were calculated among all staff, as well as among those that had provided in the last few months, by method. No marked differences emerged between these two groups, and the few differences that were found did not follow an easily interpreted pattern. For this reason, only results from among all staff are presented here.

\section{Family Planning Service Protocols}

To enable comparison of the eligibility criteria reported by providers with those required by national service protocols, service guidelines in effect at the time of the Situation Analysis fieldwork were collected (see the references at the end of the chapter for citations). Some of these guidelines have been updated since the Situation Analysis studies were conducted, but the comparison here is between providers' reports and the guidelines in effect at the time of the data collection.

\section{RESULTS}

\section{Family Planning Service Protocols}

The service protocols generally do not impose restrictions related to marital status or spousal consent. In four of the five countries, the protocols contain no statements that a client must be married before she can receive family planning services. The exception is Zanzibar, where protocols hint that marital status is required, stating, for example, 


\section{Table 8-1. Parity and age norms required by service protocols}

\begin{tabular}{|c|c|c|c|c|c|c|c|c|c|c|c|c|c|c|c|}
\hline \multirow[t]{2}{*}{ Country } & \multicolumn{4}{|c|}{ Parity } & \multicolumn{5}{|c|}{ Minimum Age } & \multicolumn{6}{|c|}{ Maximum Age } \\
\hline & COC Con IUD & Inj & Nor & $\mathrm{TL}$ & $\mathrm{COC}$ & Con IUD & Inj & Nor & $\mathrm{TL}$ & $\mathrm{COC}$ & Con & IUD & Inj & Nor & $\mathrm{TL}$ \\
\hline Botswana & & & & & & & & & & & & & & & \\
\hline Burkina Faso & 1 & & & 3 & & & & & 35 & $50^{a}$ & & & & & \\
\hline Kenya & $1^{\mathrm{b}}$ & $1^{\mathrm{c}}$ & $1^{c}$ & $1^{\mathrm{d}}$ & & & & & $\sigma^{\mathrm{e}}$ & 40 & & & & & \\
\hline Senegal & & & & & & $\checkmark^{f}$ & & & & & & & & & \\
\hline Zanzibar & 1 & & & $1^{9}$ & & & & & $30^{\mathrm{h}}$ & 40 & & & & & \\
\hline
\end{tabular}

a For the purposes of this analysis, 50 is not considered a restriction; 45 is the cut-off.

b Delivered 1 or more times; $2+$ is better.

c Proven fertility, assuming this means a completed pregnancy.

d Low parity requires especially careful counseling.

e Low reproductive age requires especially careful counseling.

$f$ Must have been menstruating for at least 2 years.

$\mathrm{g}$ Parity of 1 is not strictly required, but "those... with low parity must be counseled strongly about the permanence of the method."

$\mathrm{h}$ Recommended, but not strictly required: "those who are young (below 30 years) must be counseled strongly about the permanence of the method."

that condoms and foam tablets "can be supplied freely to any married person." The strong Islamic norms in Zanzibar lend force to these statements, and discussions with the Zanzibar Ministry of Health lead us to believe that a de facto marital status requirement is in place for all methods.

Similarly, spousal consent is not generally required across all five countries, except for sterilization. Interestingly, the countries whose protocols require spousal consent for sterilization do so for both male and female sterilization. In Botswana and Burkina Faso, spousal consent is required for both tubal ligations and vasectomies. In Senegal, spousal consent is desired for tubal ligations, with no mention of consent for vasectomy clients. Senegalese providers are encouraged to discuss the spouse's concerns with the tubal ligation client. In Kenya, protocols suggest that couples attend counseling together, and women or men whose partners oppose sterilization are to be assessed at the provider's discretion.

Parity, minimum age, and maximum age requirements are a bit more complex, and are summarized in Table 8-1. Burkina Faso, Kenya, and Zanzibar all impose some parity requirements on various methods, usually requiring only one child.
Burkina Faso has the most restrictive parity requirement found in these protocols: at least three children are required for tubal ligation clients.

Tubal ligation is also restricted by minimum age in Burkina Faso, Kenya, and Zanzibar. Injectables have a virtual minimum age restriction in Senegal, which requires that a client have been menstruating for at least 2 years before injectables are given. Maximum age requirements are imposed only on COCs-in Burkina Faso, Kenya, and Zanzibar.

Overall, service protocols in effect across all five countries infrequently impose eligibility criteria for family planning methods. Most combinations of country, barrier, and method have no official restriction at all. Female sterilization is the most restricted method, mainly by minimum age, parity, and spousal consent. IUDs are frequently restricted by parity, and COCs are restricted mainly by maximum age. As the notes to Table 8-1 indicate, many of these restrictions are not absolute, but constitute a recommendation or warning against use. Condoms are the least restricted method.

Across countries, the protocols of Botswana and Senegal are the least restrictive. Burkina Faso has some uncommonly stringent restrictions, such as 
three children and a minimum age of 35 required for sterilization. Kenya has several restrictions, but most are qualified in some way. Zanzibar presents a special case because of its strong religious context.

These restrictions should be assessed not only among countries, but also relative to accepted medical norms. The World Health Organization (WHO) (1996) guidelines for family planning service provision list internationally accepted norms for medical eligibility criteria for all contraceptive methods. A comparison of the WHO standards with the national protocols shows a variety of areas of both agreement and disagreement. ${ }^{7}$

The WHO guidelines include no parity restrictions on any method except the IUD. The parity requirements in Kenya, Burkina Faso, and Zanzibar on injections, NORPLANT ${ }^{\circledast}$ implants, and sterilization are therefore not deemed medically necessary by the international community. ${ }^{8}$ As for IUDs, the WHO guidelines state that nulliparous clients have an increased risk of expulsion; however, they conclude that the advantages of the method still outweigh the risks for nulliparous women (World Health Organization, 1996:70). For this reason, the parity-based restrictions on IUDs in Burkina Faso, Kenya, and Zanzibar can be seen as medically motivated, but stricter than necessary.

The WHO guidelines place no minimum age requirements on COCs, condoms, or IUDs, ${ }^{9}$ and this is reflected in all five national service protocols. The WHO guidelines do recognize some risk with progestin-only injections and NORPLANT ${ }^{\oplus}$ implants associated with young age: "for women under 16, there are concerns regarding hypo-oestrogenic effect due to POC [progestin-only contraceptive] use" (World Health Organization, 1996:54). Once again, however, the benefits of the method are seen to outweigh the risks, and young women are not medically barred from its use. In the national protocols, these methods are remarkably unrestricted on the basis of age. Only Senegal has a provision that injectable users must have been menstruating for 2 years, making the minimum age roughly 15 . With this small exception, the national norms for injectables and NORPLANT ${ }^{\circledast}$ implants are in accordance with the WHO guidelines in all countries. As for sterilization, although there are no medical reasons to deny tubal ligation to young women, the WHO guidelines do recommend thorough counseling. This is reflected in the protocols of Kenya and Zanzibar. Only Burkina Faso places an absolute minimum age of 35 on sterilization.

The WHO guidelines state there are no maximum ages beyond which it is medically required that condoms, IUDs, injectables, NORPLANT ${ }^{\circledast}$ implants, or sterilization not be provided. These guidelines are reflected in the protocols for all five countries, none of which imposes maximum ages on any of these methods. The maximum age limitations for COCs are a bit more complex. After age 40 , the risk of cardiovascular disease is increased and is exacerbated with COC use. However, the benefits of COC use still outweigh the risks, so WHO applies no blanket maximum age for COCs. Among women who smoke, however, WHO recommends against use of COCs after age 35, for both heavy and light smokers. For present purposes, this is the only case in which the WHO guidelines restrict a method based, at least partially, on the eligibility criteria presented here.

Table 8-1 shows that Burkina Faso, Kenya, and Zanzibar all apply maximum ages to COC use, regardless of smoking status. Because these limitations are independent of smoking status, they are more restrictive than the $\mathrm{WHO}$ guidelines. The country guidelines do reflect the smoking restriction as well, although not always related to age. The Botswana protocols place a "relative contraindication" on heavy smoking for COC users over 35, and the Burkina Faso guidelines call this an "absolute contraindication" for women who smoke 10 or more cigarettes per day. Kenya's guidelines prohibit COC use for women over 35 who "are smokers," and Zanzibar's guidelines call smoking 10 or more cigarettes per day a "relative contraindication" for COC use, regardless of age. These country guidelines do address the issue of smoking among older COC users, showing varying agreement with the WHO guidelines. However, since this combination of eligibility criteria was not addressed in the provider interviews, it cannot be evaluated here. The maximum ages reviewed in this chapter are independent of smoking status.

Marital status and spousal consent are not mentioned at all in the WHO guidelines and cannot be construed as medically necessary criteria. The

164 Clinic-Based Family Planning and Reproductive Health Services in Africa 
Figure 8-1. Marital status

Peroent of providers who report restricting mettods based on maritd status

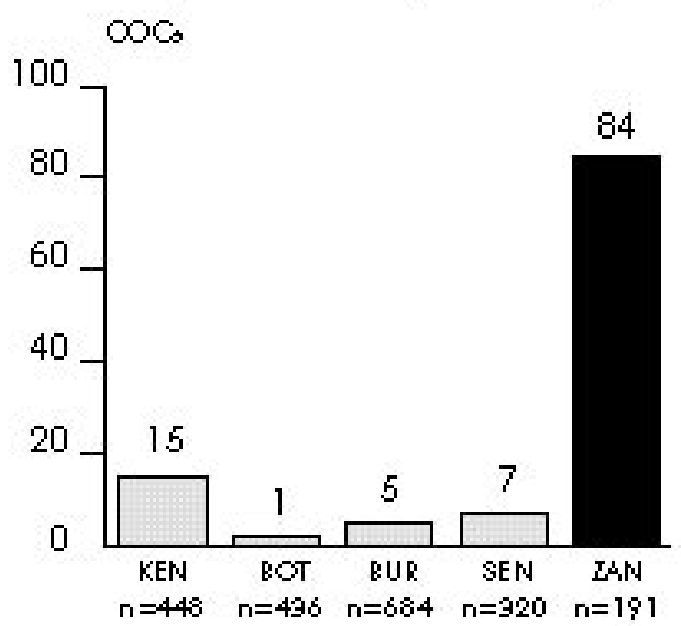

Condoms
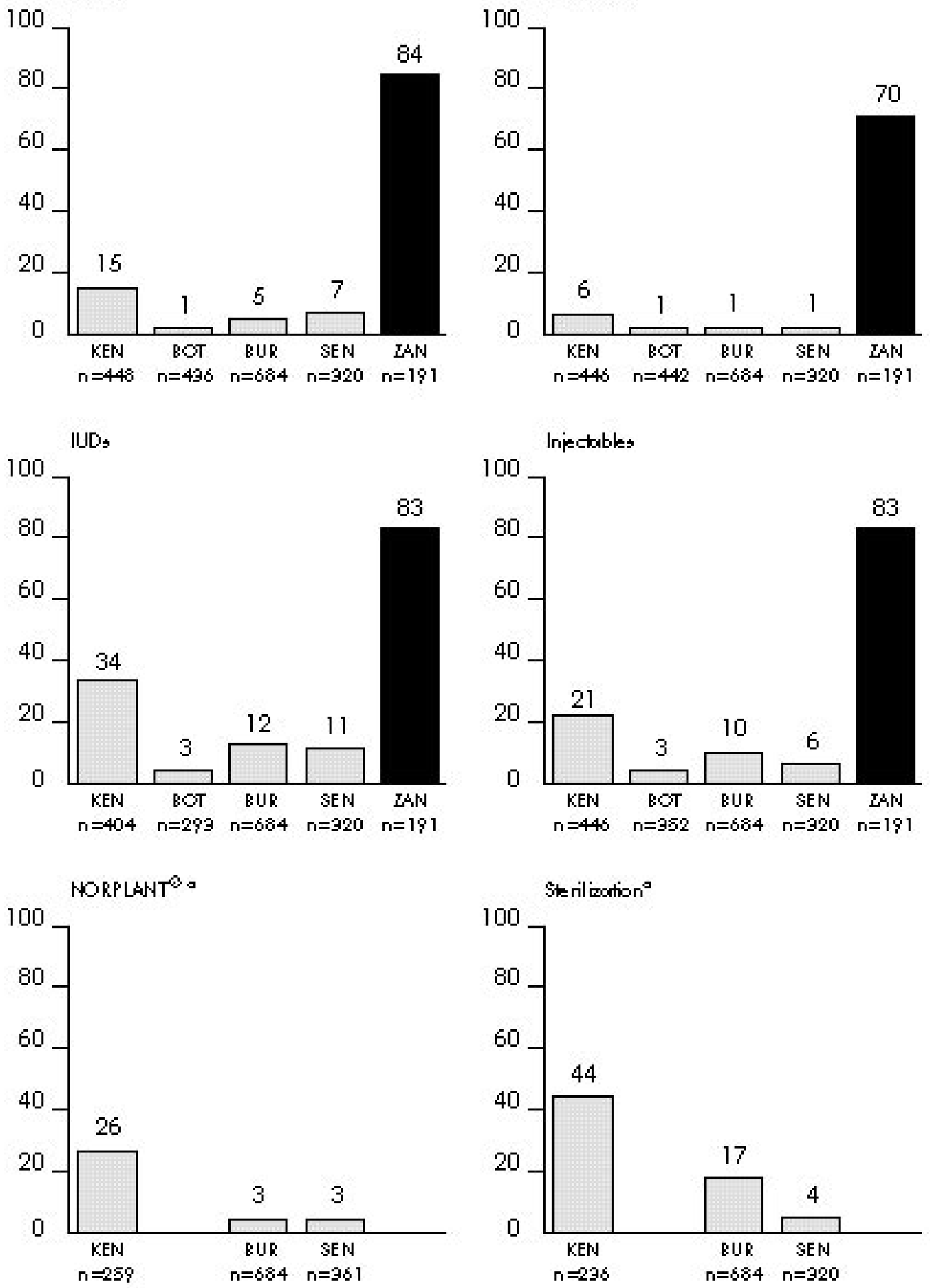

This refriction required or enoouraged br service protooob.

a Question not os ked in Bobrana or Zarzibar. 
requirements for spousal consent with sterilization and for marital status in Zanzibar are probably motivated more by social concerns than medical necessity.

Overall, the national protocols do reflect legitimate medical concerns in many cases. However, for all cases in which the WHO guidelines state medical cautions for a method, the benefits still outweigh the risks, and the method is not considered absolutely contraindicated. For this reason, any criteria in the national protocols that are absolute (such as one child required for an IUD in Burkina Faso) impose medical considerations more strictly than WHO recommends. These national service protocols appear to take local cultural factors into account as well, which may generate additional eligibility criteria motivated by social rather than medical concerns.

\section{PROVIDER REPORTS OF RESTRICTIONS}

Family planning service protocols set eligibility criteria for contraceptives, but providers interpret the protocols. During the Situation Analysis interviews, providers reported that they impose a considerably different and much more restrictive pattern of barriers on clients than that required by policy.

\section{Marital Status}

Figure 8-1 shows the percentage of providers that impose a marital status restriction on the various methods. Clearly, Zanzibar is the most restrictive context by far, as would be expected given its religious context. Over $70 \%$ of providers in Zanzibar require that a woman be married in order to receive COCs, IUDs, injectables, or even condoms. ${ }^{10}$

Kenya is the next most restrictive context. Although marital status is not required by policy, a third of Kenyan providers require that IUD clients be married, and almost half impose this requirement for sterilization. For the other methods, the percentage of providers requiring marriage ranges from $6 \%$ to $26 \%$.

Providers in Botswana, Burkina Faso, and Senegal are much less restrictive than those in
Zanzibar and Kenya for all methods, with the exception that $17 \%$ of Burkina Faso providers require that sterilization clients be married. Since the service protocols in these countries do not require married status for any method, the providers' actions are generally in concordance with the protocols.

\section{Spousal Consent}

Figure 8-2 presents data on spousal consent restrictions imposed by providers, by method. In Botswana, Burkina Faso, and Senegal, these results range from $6 \%$ to $32 \%$ and are all higher than those for marital status restrictions. An unmarried woman in these countries might actually have an easier time obtaining contraception than a married woman who does not have her husband's consent. With the exception of sterilization, none of these countries has spousal consent restrictions in its service protocols, so the providers are imposing this barrier of their own accord.

The pattern is markedly reversed in Zanzibar. Although Zanzibar's spousal consent restrictions are the highest of the five countries (32\% to 57\%), they are much lower than its marital status restrictions. Thus, a married woman without her husband's consent might have an easier time than a single woman obtaining contraceptives in Zanzibar. Again, however, protocols in Zanzibar do not require spousal consent, so providers that impose this barrier are motivated by other, possibly social or religious considerations.

In Kenya, the pattern is not as clear. As compared with the marital status results, fewer providers impose spousal consent requirements on COCs, IUDs, and injectables, but more providers impose these restrictions on condoms, NORPLANT $^{\circledast}$ implants, and sterilization. Once again, Kenya seems to be more restrictive than Botswana, Burkina Faso, and Senegal, but less restrictive than Zanzibar. Spousal consent is not required by Kenyan service protocols.

For all countries, the level of spousal consent requirements for condoms is discouraging, particularly since many of these regions have a high prevalence of HIV and other STDs. The Kenyan result is particularly troubling, since only $6 \%$ of 
Figure 8-2. 5pousd consent

Peroent of providers whiti report restricting methods based on spousal consent

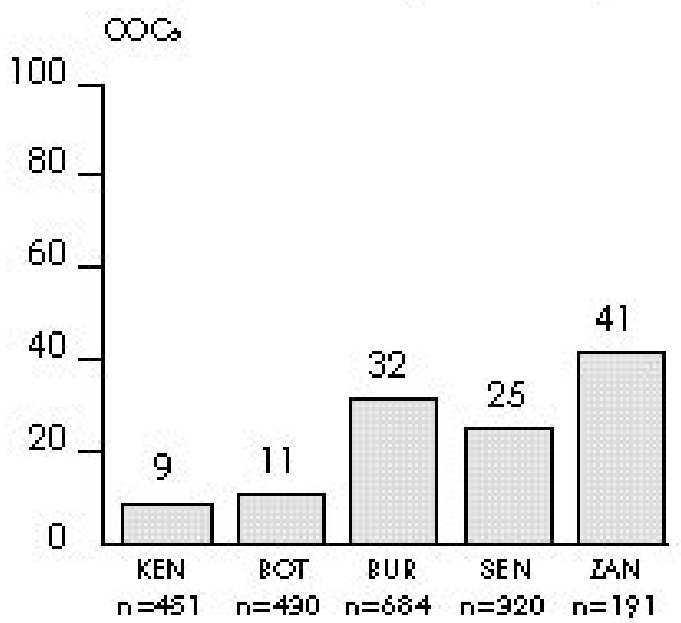

Condoms
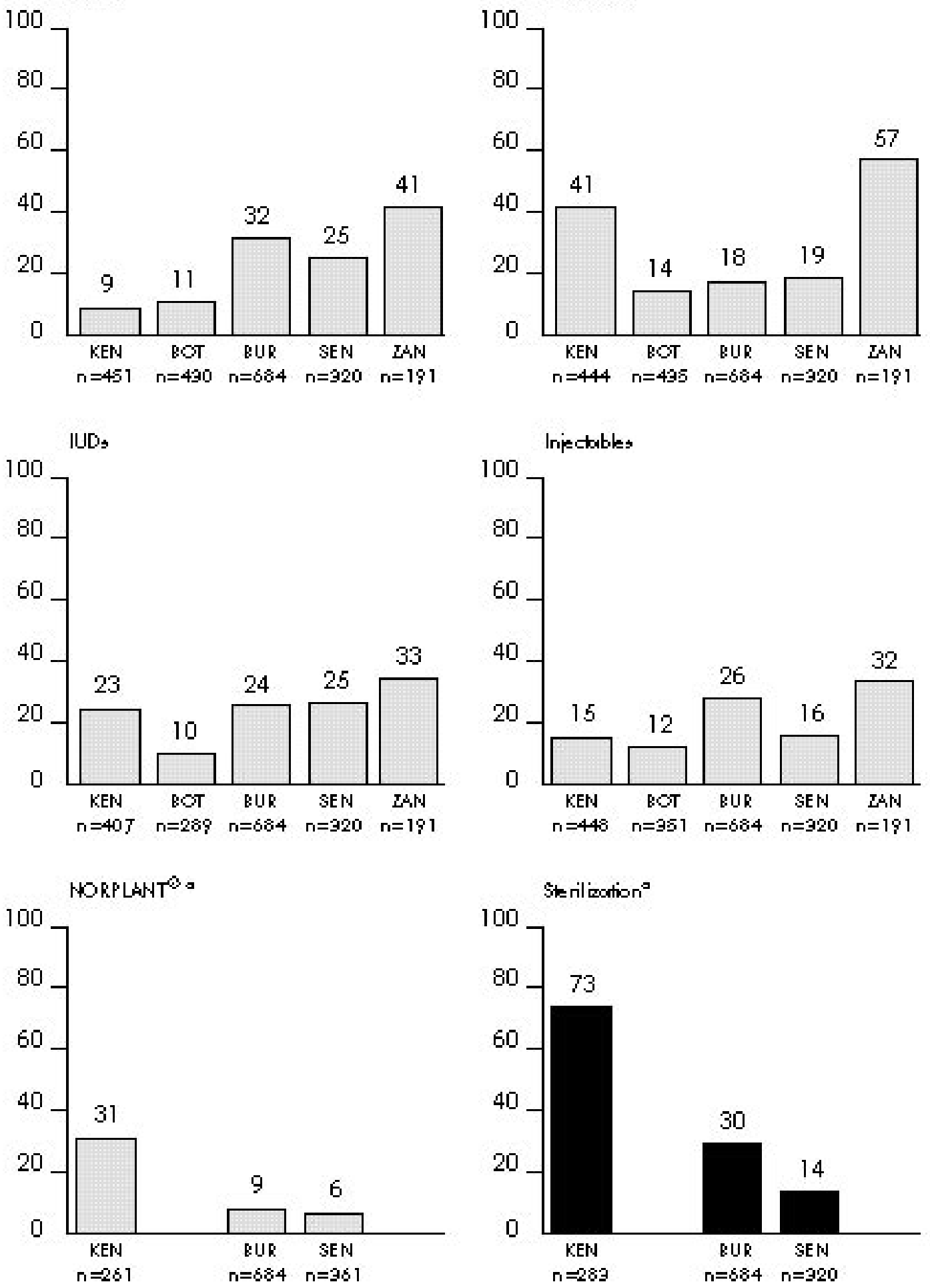

This restriction required or enoou roged by se ryice probools. "Quetion notasked in Botyka naor Zarzitar. 
Figure 8-3. Parity

Percent of providers who report restricting mettods based on parity

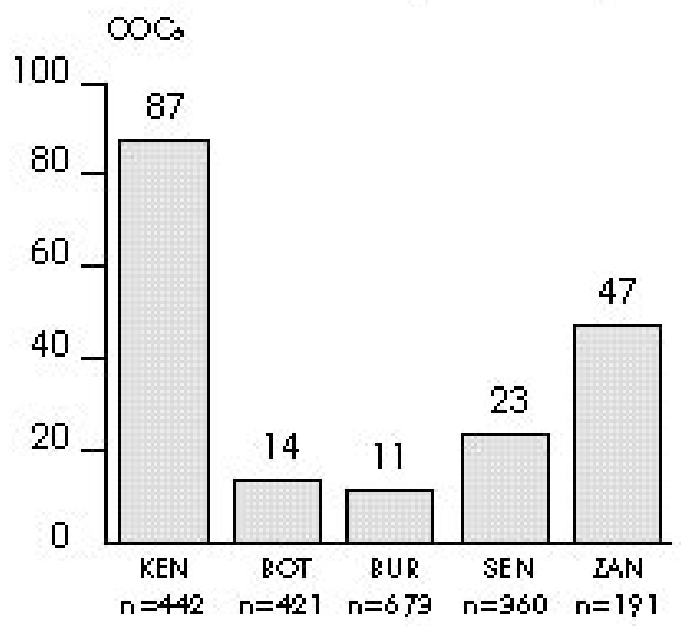

Condoms
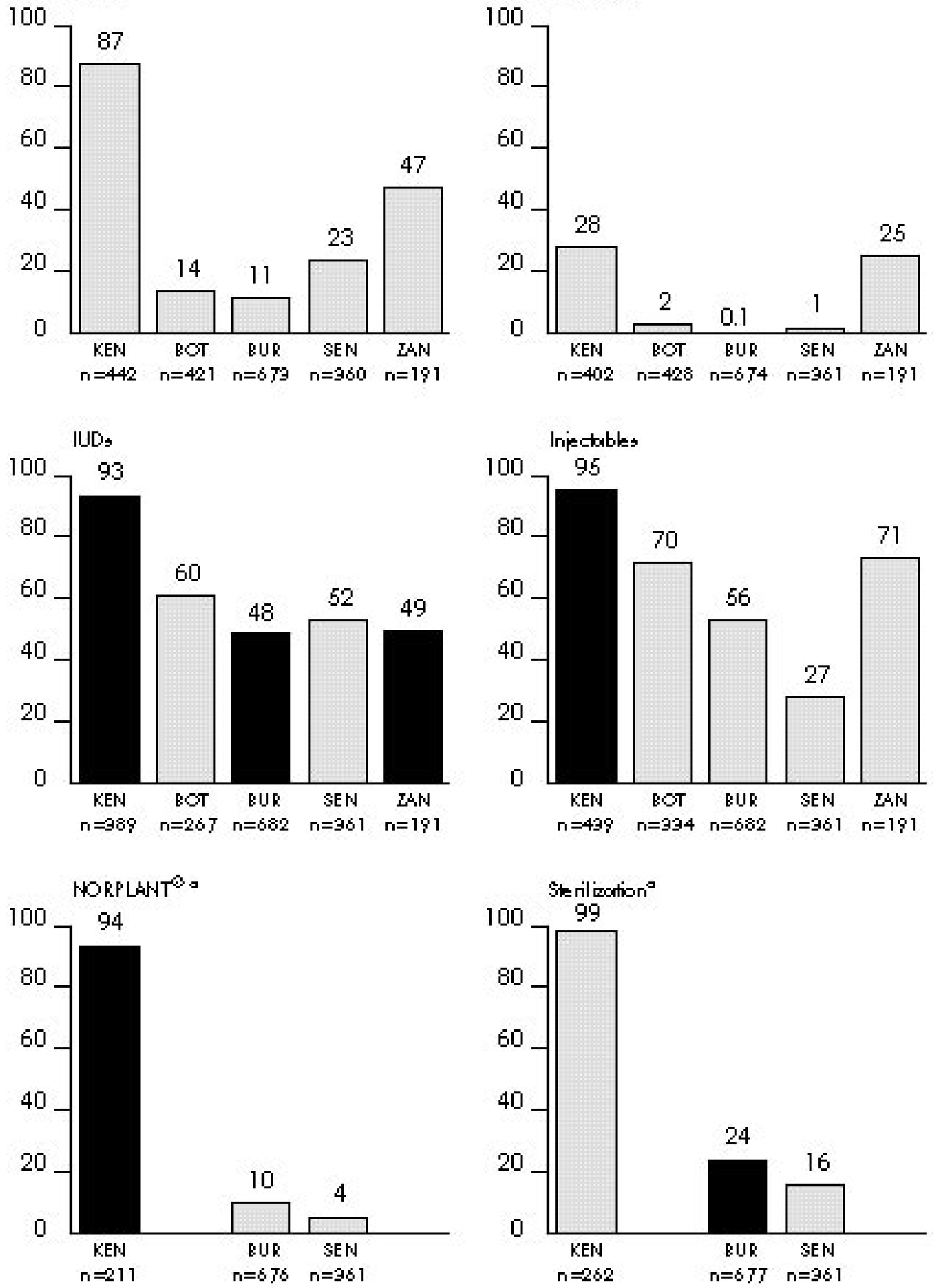

This refriction recuired or enocu raged by service protooob. "Question not os ked in Bobmarna or Zarzibar. 
Figure 8-4. Mean paity

Wean number of children required, among providers who meport restricting methods bosed on parity
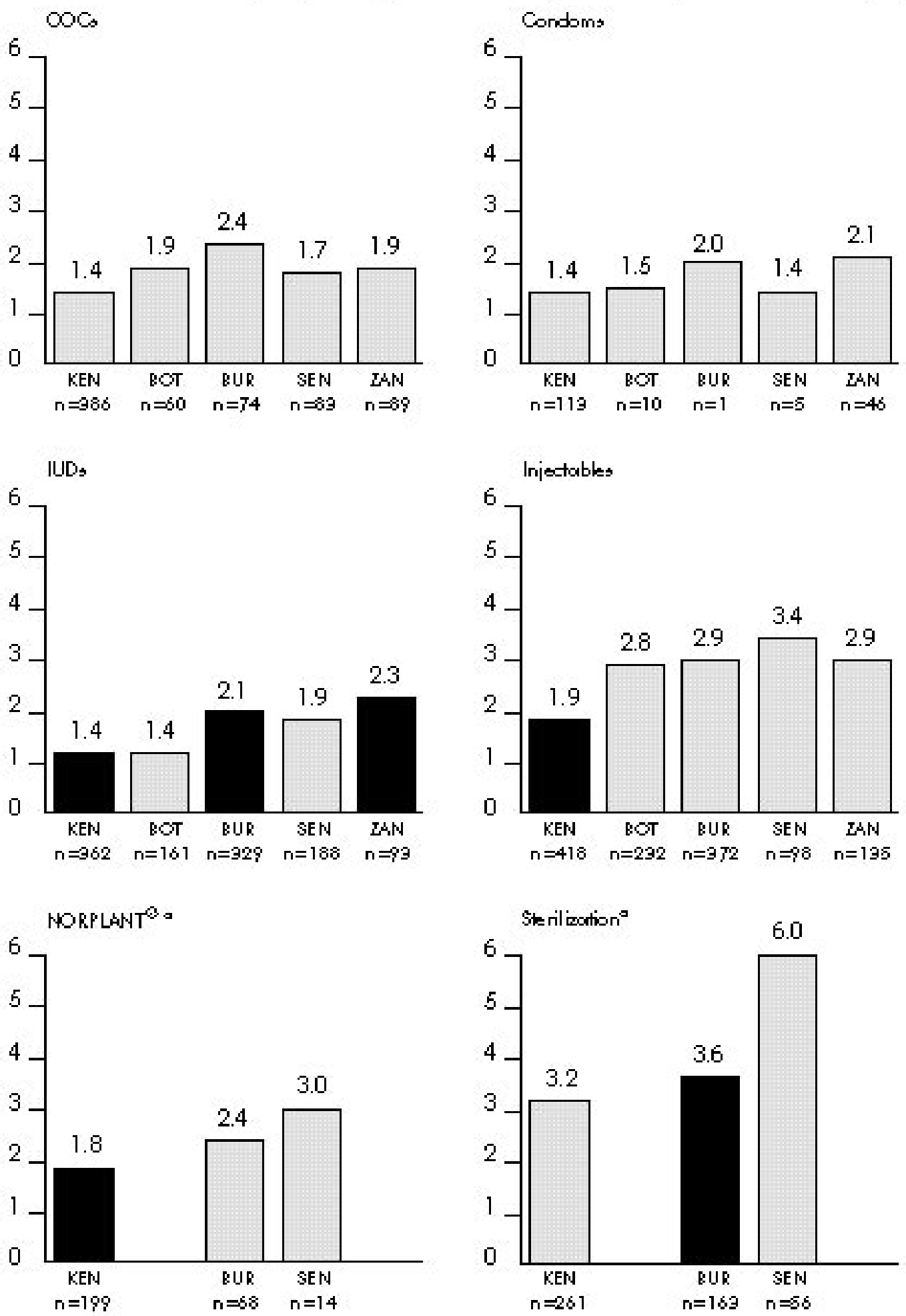

This retriction required or enoouraged by service protooob.

- Question not ouled in Bobyana or Zarzibar. 
providers require that a woman be married to obtain condoms, but fully $41 \%$ require that married women have spousal consent to do so. These findings may suggest an unwillingness to recognize the frequency of HIV transmission within married couples or a fear that the women would use the condoms in extramarital relationships.

The sterilization results vividly illustrate that the link between policy and practice is sometimes weak. Although spousal consent is required by policy in Burkina Faso and Senegal, only 30\% and 14\% of providers, respectively, actually impose this requirement. By contrast, almost two-thirds of providers in Kenya require spousal consent, although this restriction is not required by policy.

It may seem intuitive that there would be a correlation between imposing barriers of marital status and spousal consent. If a provider imposes a marital status barrier, is she more likely to impose a spousal consent barrier as well? An investigation with simple cross-tabulations by method did not show a strong relationship. In other words, often a provider does not require a client to be married in order to receive family planning, but if she is married, does require spousal consent. This finding may indicate providers' fear of reprisals from spouses who are unaware of their wives' contraceptive use.

\section{Parity}

In all five countries, a considerable proportion of providers impose parity requirements for the provision of IUDs and injectables (see Figure 8-3). As discussed above, the parity restriction for IUDs is actually required by policy in Burkina Faso, Kenya, and Zanzibar, and may be medically motivated. In fact, in Burkina Faso and Zanzibar, surprisingly few providers impose this restriction given that it is required.

The restriction on injectables may arise from a common misconception that they cause sub- or infertility. This concern may motivate providers to restrict use of the method, not only for the welfare of the client, but also to avoid blame or retribution from family members. The in-depth study of provider restrictions in Ghana showed that fully $94 \%$ of providers who restrict injectables based on parity do so because of concerns about infertility and blame from relatives (Twum-Baah and Stanback, 1995).

Kenya's providers are by far the most restrictive with regard to parity. These restrictions may arise from the service protocols that impose parity restrictions on IUDs, injectables, and NORPLANT ${ }^{\circledast}$ implants; within a generally restrictive context, providers may extend this criterion to COCs and female sterilization. Indeed, $28 \%$ of providers even require that a woman have children before prescribing condoms, which carry no risk to fertility whatsoever.

Figure 8-4 shows the mean number of children required among those providers that impose a parity requirement. Considerable variation is apparent among countries and methods. Moreover, where a large proportion of providers impose a parity requirement, it is not always for a large number of children. For example, whereas $87 \%$ of providers in Kenya impose a parity restriction for COCs, these providers require the least number of children (on average 1.4 children) before a client can receive COCs. Likewise, where a small proportion of providers impose a parity requirement, a large number of children may be required. In Senegal, for example, $27 \%$ of providers impose a parity requirement for injectables, and that subset of providers is particularly restrictive, requiring 3.4 children on average before a client can receive an injectable. In short, the percentage of providers who impose this requirement is not necessarily related to the number of children they require to meet it.

For sterilization, Burkina Faso's providers follow the protocols closely: 3 children are required by policy, and providers report requiring an average of 3.6 children in practice. The next-highest parity is required for injectables and NORPLANT ${ }^{\circledast}$ implants (1.8 to 3.4 children), whereas COCs, condoms, and IUDs require the lowest parity (1.4 to 2.4 children).

These parity requirements appear to fall into two groups: those that require demonstrated fertility and those that require completed fertility. For IUDs, for example, about 1 or 2 children are generally required. This may be a result of the restriction on this method for nulliparous women, so that 
Figure 8-5. Minimum age

Peroent of providers whio report restricting mettods based on a mirimum age of 13 or abover

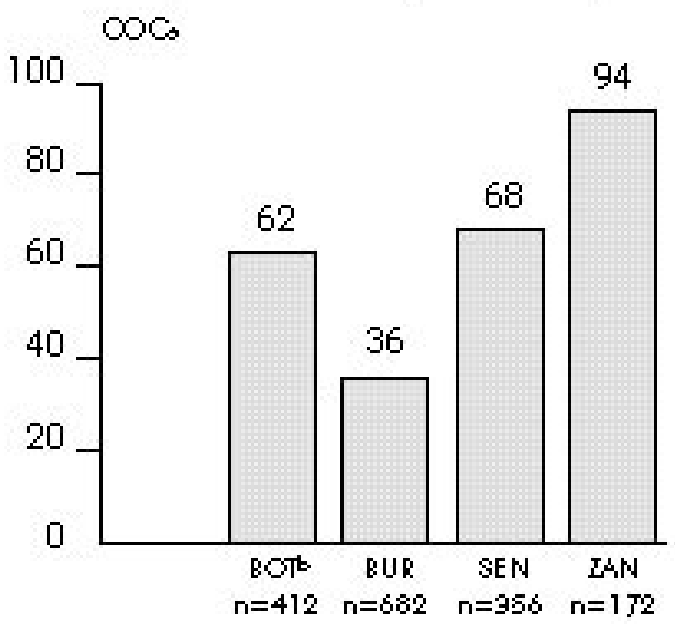
Condoms
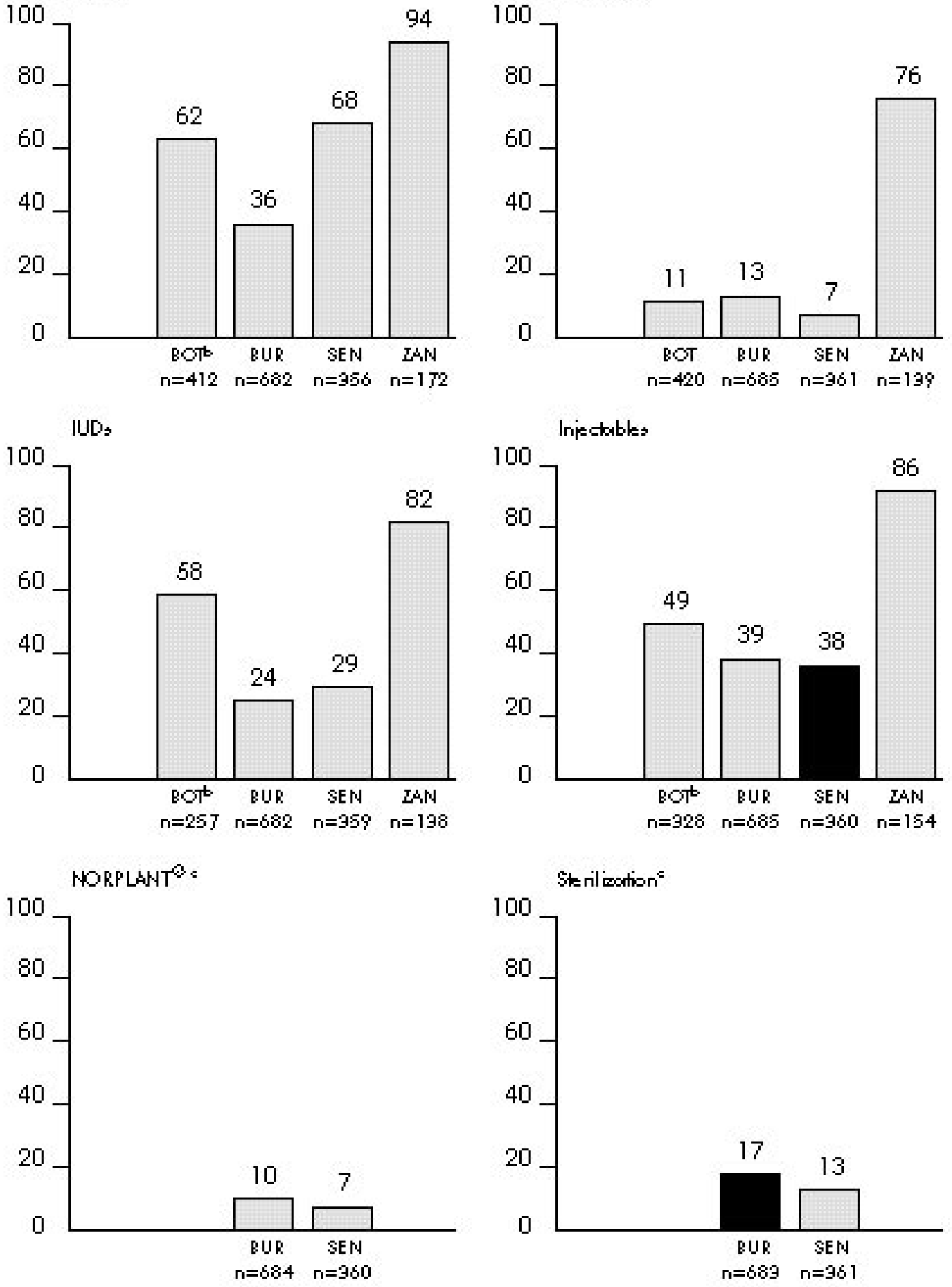

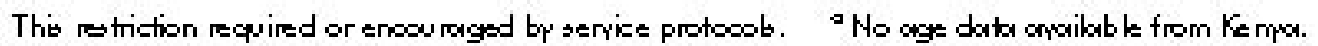

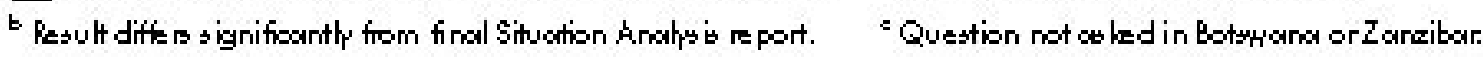


Figure 8-6. Mean minimum age

Mean minimum age required, among providers uto report resticing methods based on a mirimum age of 13 or above
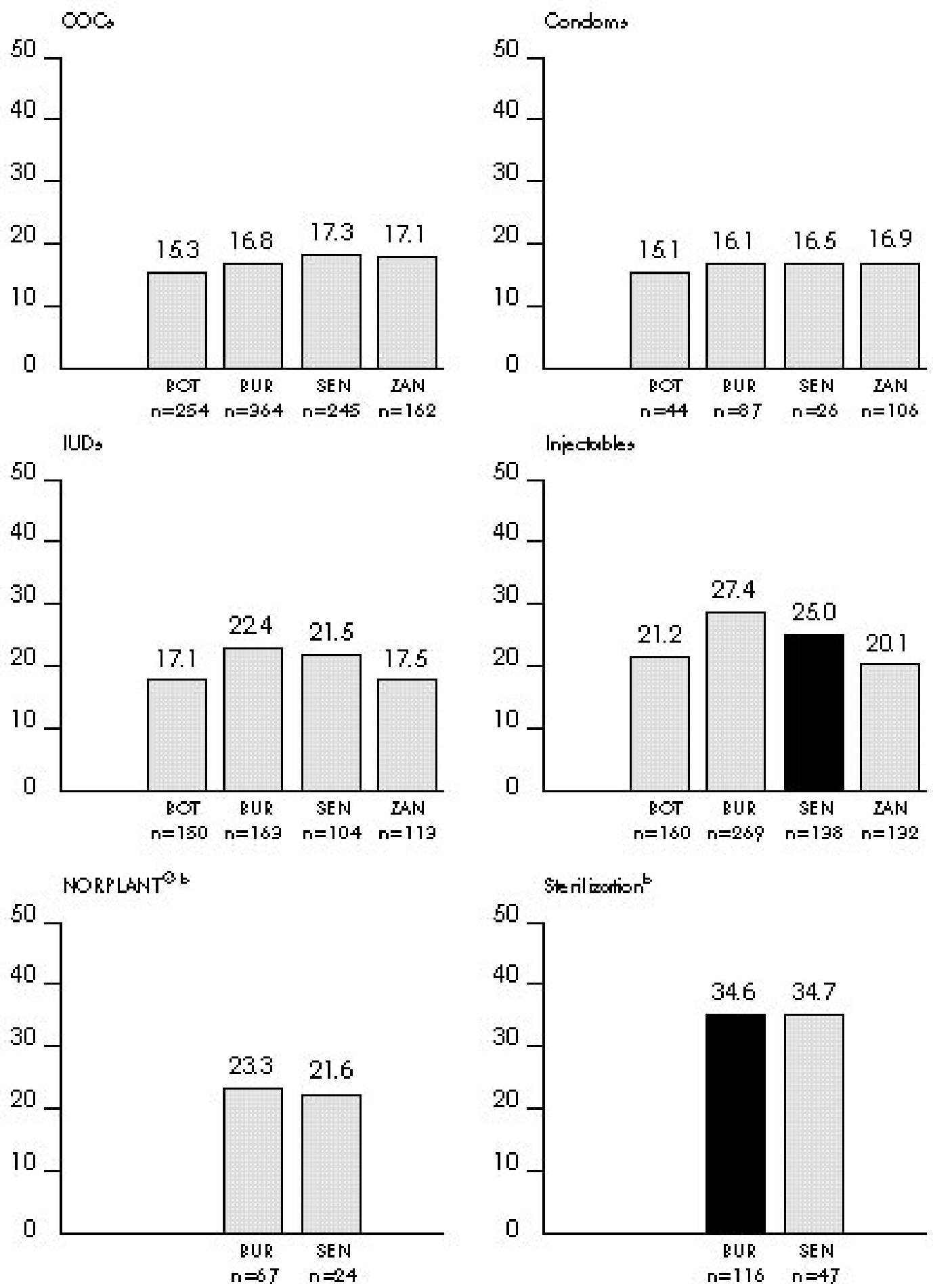

This retrition required or enoou roged by seryice protooob.

- Wo oge data onsilable from Kerpa.

EQuestion not as led in Botyrana or Zarzibar. 
Figure 8-7. Maximum age

Peroent of providers who report restriding mettods based on a masimum age of 45 or below?

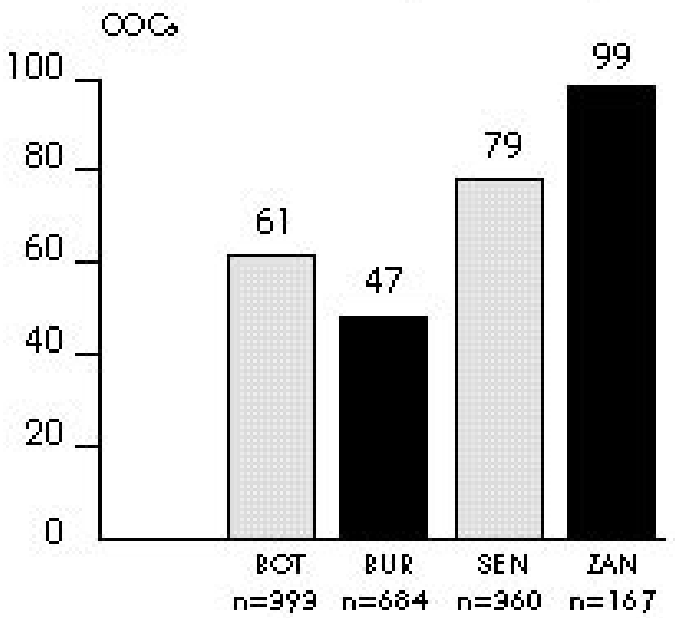

Condoms
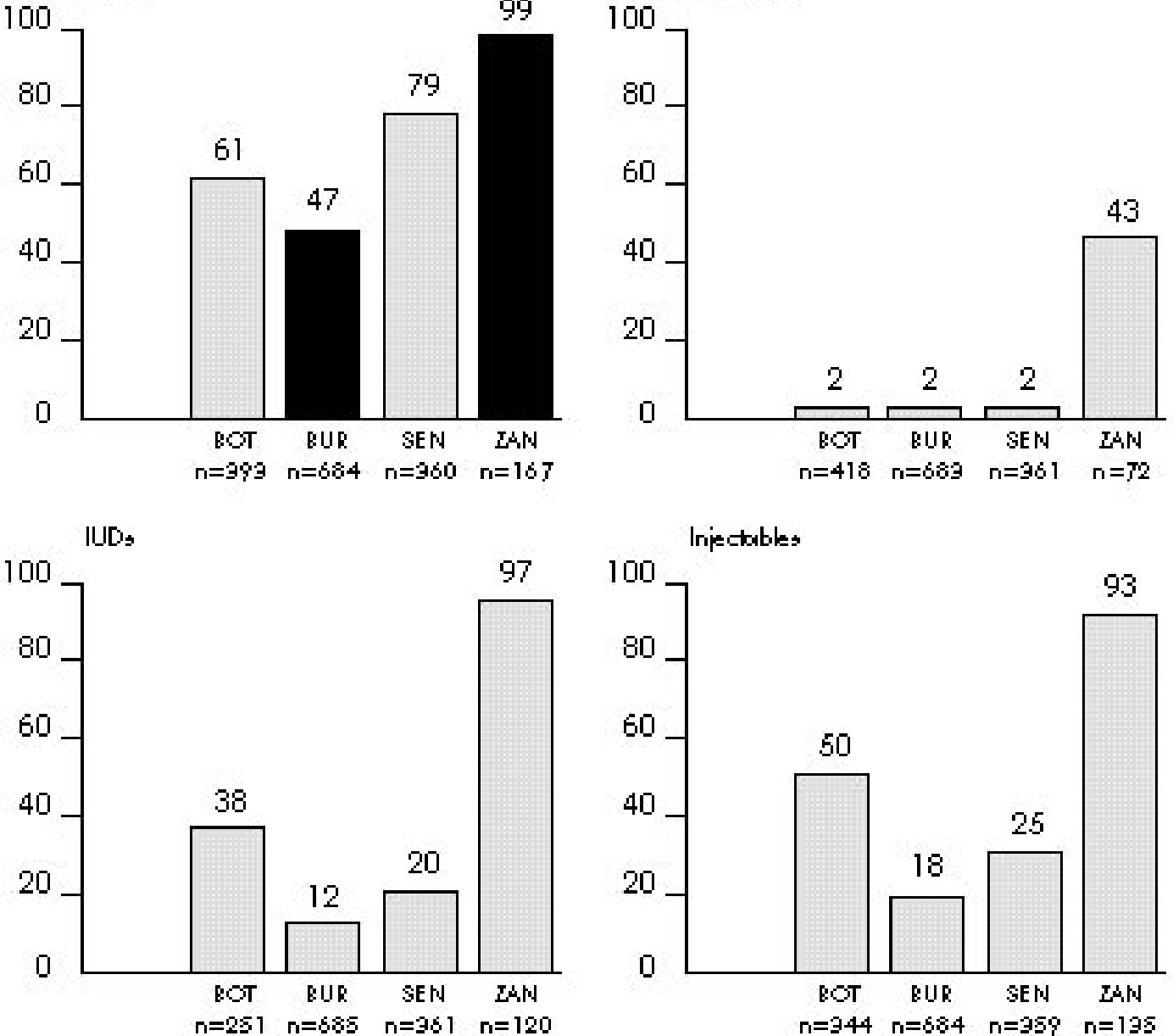

Inizctables,
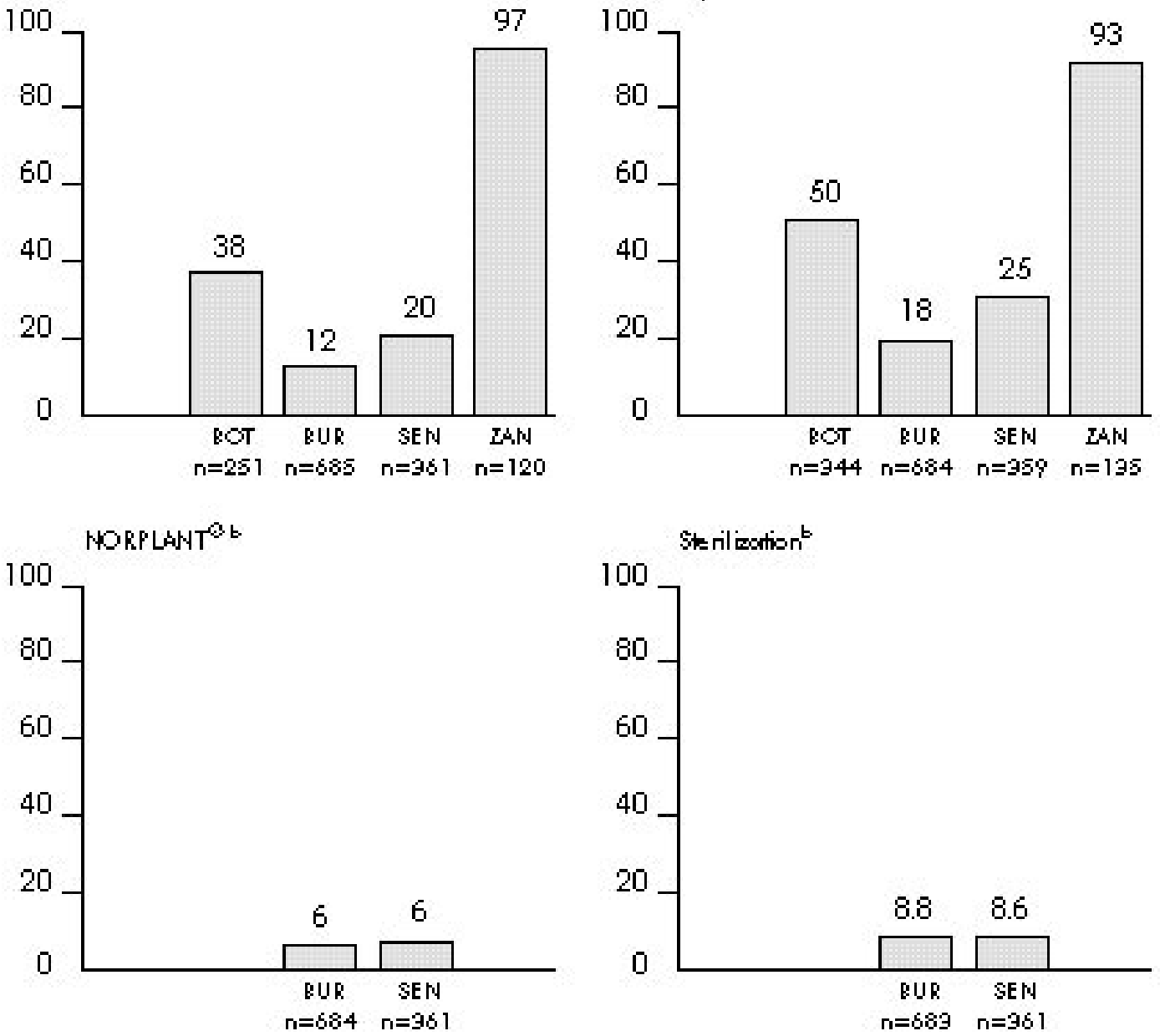

This retrition required or enocuraged by serpice protooob.

- No oge dora onorilable from Ke mpa.

- Question not celoed in Botynana or Zanzibar. 
demonstrated fertility is required. For sterilization, however, providers appear to require completed fertility (range from 3.2 to 6.0 children), for obvious reasons. In Kenya, the protocols state specifically that a woman must have proven fertility to receive an IUD, an injectable, or NORPLANT ${ }^{\circledR}$ implants; this requirement appears to be interpreted by providers as requiring 2 children. The mean number of children required for injectables suggests that providers are requiring completed fertility, particularly in Senegal (3.4 children), once again reflecting the notion that injectables cause infertility. The requirement for demonstrated fertility of up to 2 children for condoms is surprising, but relatively few providers $(0.1 \%$ to $28 \%)$ actually impose this restriction.

\section{Minimum Age}

Providers apply minimum age barriers quite commonly (see Figure 8-5). ${ }^{11}$ For COCs, IUDs, and injectables, $24 \%$ to fully $94 \%$ of providers require that a client be at least 13 years old. None of the protocols of the five countries requires a minimum age for these methods, so providers are imposing these barriers for other reasons. The only exception is Senegal, where injectable clients are required to be about age 15 (menstruating for at least 2 years). However, only $38 \%$ of the providers impose this barrier.

Condoms present an interesting case. In Botswana, Burkina Faso, and Senegal, few providers require that a client be at least 13 years old to receive a condom, which reflects the protocols in these countries. In Zanzibar, fully two-thirds of providers impose this minimum age requirement, probably reflecting once again the fact that Zanzibar is the most generally restrictive context of the five.

NORPLANT $^{\circledR}$ implants and sterilization appear to be surprisingly unrestricted by minimum age. Data are available only for Burkina Faso and Senegal, but in both cases, only $7 \%$ to $17 \%$ of providers require that a client be at least 13 to receive these methods. The protocols in Burkina Faso stipulate that a woman must be at least 35 to be sterilized, but only $17 \%$ of providers mentioned imposing this rule. One explanation for this finding could be that age limitations for these methods, particularly sterilization, seem so obvious to the providers that they did not think to mention them in the interview.

Figure 8-6 shows the mean minimum age requirement among those that do impose this barrier. Here, the variation among countries is not large, but the variation among methods is clear. COCs and condoms have the lowest age requirements, ranging from 15 to 17 . Clients are required to be a bit older for IUDs, injectables, and NORPLANT $^{\circledR}$ implants (17 to 27 ) and are generally required to be 35 before they can receive a tubal ligation. In Burkina Faso, the stipulated minimum age of 35 for sterilization appears to be upheld by the minority of providers that actually enforce this requirement.

Providers appear to consider different methods appropriate for the various stages of a woman's reproductive career. They consider COCs and condoms appropriate for younger women; IUDs, injectables, and NORPLANT ${ }^{\circledast}$ implants appropriate for women in their middle reproductive years; and sterilization appropriate for older women who have completed their childbearing. Although this pattern is arguably appropriate from a social or cultural standpoint, the WHO guidelines do not place any strict age restrictions on any of these methods. Based on age alone, then, clients are being restricted from various methods that are otherwise medically acceptable.

\section{Maximum Age}

The pattern of providers who impose a maximum age restriction (see Figure 8-7) is similar to that for minimum age barriers. COCs, IUDs, and injectables are the most restricted methods, and Zanzibar is the most restrictive country. Once again, condoms are restricted by very few providers in Botswana, Burkina Faso, and Senegal, but by almost half of providers in Zanzibar. Also, very small percentages of providers in Burkina Faso and Senegal $(6 \%$ to $9 \%)$ impose a maximum age on NORPLANT ${ }^{\circledR}$ implants or sterilization.

Maximum age limits are most commonly applied to COCs (47\% to 99\%). As mentioned above, some medical risk is associated with COC 
Figure 8-8. Mean maximum age

Mean maximum age required, anong providers uto reportresticing methods based on a maximum age of 45 or below"
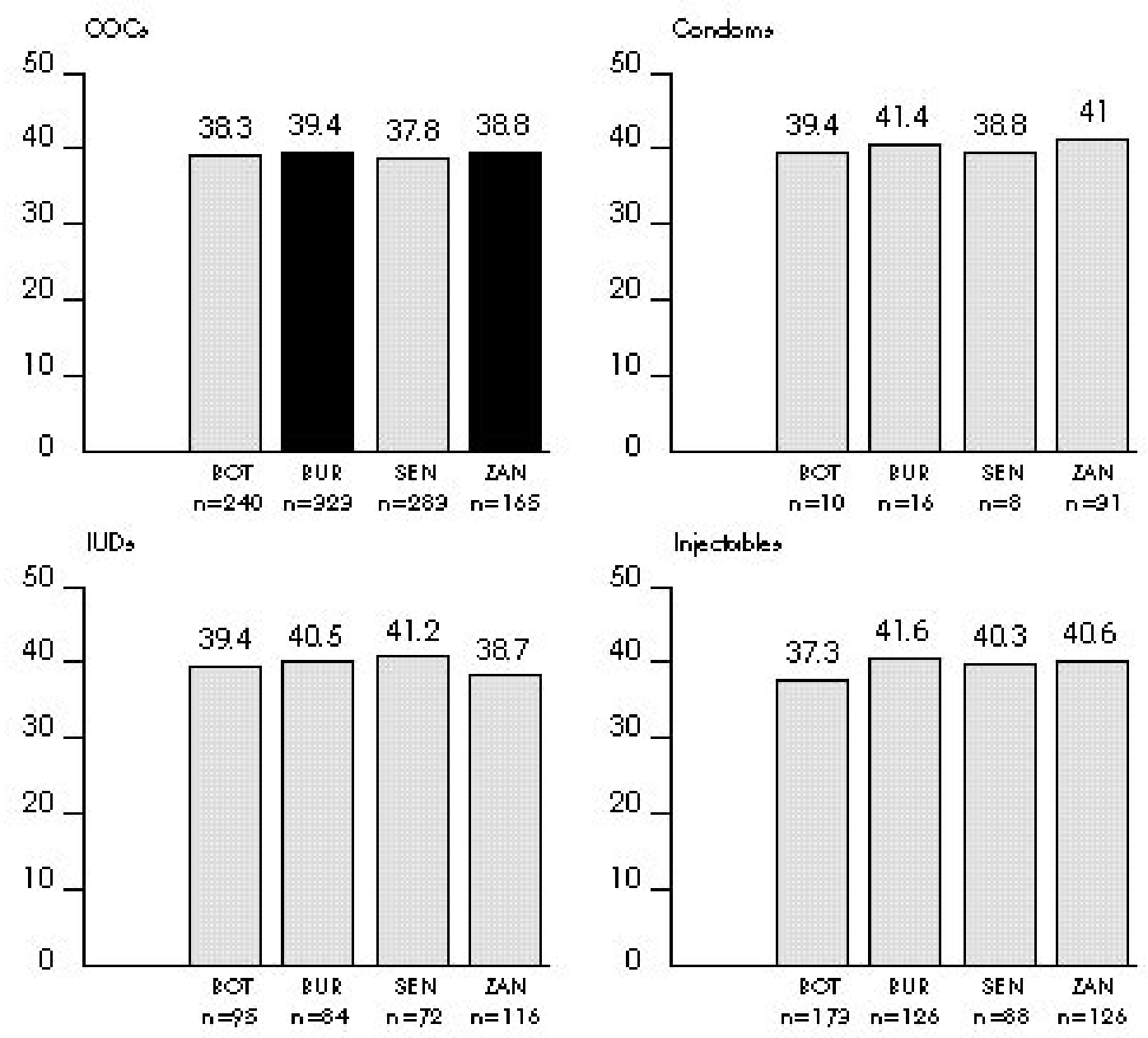

Inizctables
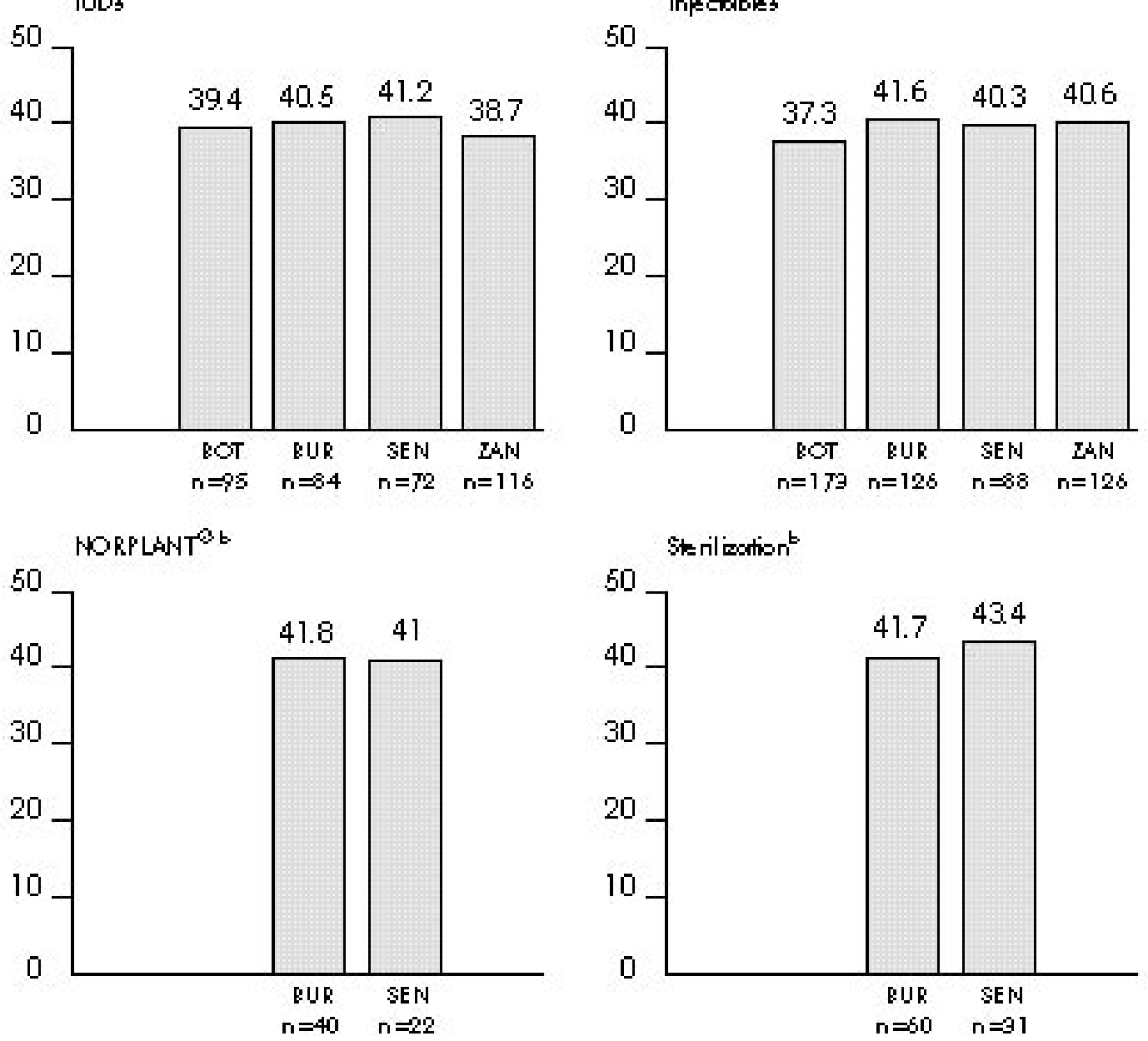

This restriation required or enoouraged by se ryise probools.

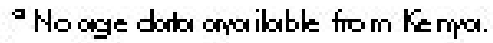

EQuestion not celed in Bobyara or Zarzibar. 
use after age 35 , which may motivate this restriction. Although the WHO guidelines gauge this risk to be small and still recommend COCs for women of any age, maximum ages for COC use are stipulated by service protocols in Zanzibar and Burkina Faso. In Zanzibar, this provision is implemented almost universally. However, almost all providers also impose this restriction on IUDs and injectables, which is not required by service protocols. The providers may therefore be imposing all maximum age requirements of their own accord rather than in response to the protocols. In Burkina Faso, a maximum age protocol of 50 does exist; $47 \%$ of providers apply a stricter requirement of 45 or below.

Figure 8-8 shows the mean maximum age required by those providers that impose this restriction. Very little variation among countries and methods is seen, with a range of 37 to 43 .

Taken together, these indicators of age across the five countries and six methods sketch an overall picture of how age restrictions are applied in sub-Saharan Africa. The following points broadly summarize the pattern of age restrictions found: ${ }^{12}$

- Age limits are often applied to COCs, and the range is roughly 16 minimum to 38 maximum.

- Age limits are rarely applied to condoms, but when they are, the range is about 16 to 40 .

- Age limits are sometimes applied to IUDs and injectables. When they are applied, the minimums are about 19 for IUDs and 23 for injectables, with maximums of 40 .

- Age limits are rarely applied to NORPLANT ${ }^{\circledast}$ implants or sterilization. When they are applied, the age range for NORPLANT ${ }^{\circledR}$ implants is about 22 to 41 , and that for sterilization is roughly 35 to 42 .

- Of the four countries for which data on provider age restrictions are available, Zanzibar is generally the most restrictive by age, followed by Botswana. Burkina Faso and Senegal are relatively less restrictive based on age requirements.

\section{DISCUSSION}

The Situation Analysis data on provider barriers is detailed and extensive. To summarize this information, we developed a composite measure of restrictiveness by provider. The providers were asked a series of yes/no questions about each eligibility criterion in combination with each method, generating several yes/no answers. To measure a provider's overall restrictiveness, we simply counted the number of yes answers given by each provider. The questions on NORPLANT ${ }^{\circledR}$ implants and sterilization were excluded, as data on these methods are available for only three countries. In addition, there is no information for Kenya on age barriers, so the total number of questions for Kenya is less than for the other countries. To make the scores comparable across countries, we calculated the percentage of the total eligibility criteria each provider applies:

\section{Number of eligibility criterion/method combinations for which the provider does impose a barrier \\ Number of eligibility criterion/method combinations for which we have data in that country}

Figure 8-9 presents the mean of this score among all providers in each country. The results confirm a repeated refrain in the above analysis: Zanzibar has by far the most restrictive providers, followed by Kenya, whereas providers as a whole are generally less restrictive in Botswana, Senegal, and Burkina Faso. Figure 8-9 also shows the percentage of eligibility criterion/method pairs required or encouraged by the protocols for each country. In Zanzibar, for example, 30\% of the eligibility criterion/method pairs are required, but providers reported that they apply on average $63 \%$ of the pairs. ${ }^{13}$

Two remarkable points are made clear in Figure 8-9. First, the most restrictive contexts, in terms of both policy and practice, are the anglophone East African countries, and the least restrictive are the francophone West African countries. This finding challenges the conventional assumption that West African providers are more restrictive than East African providers on eligibility criteria. As only five countries are included in this analysis, however, further investigation into regional differences is required.

Second, in all cases providers report that they vastly overapply restrictions to contraception above those required by protocol. In addition, in 


\section{Figure 8-9. Summary of eligbility criteria requir ed by protocol and those reported by providers}

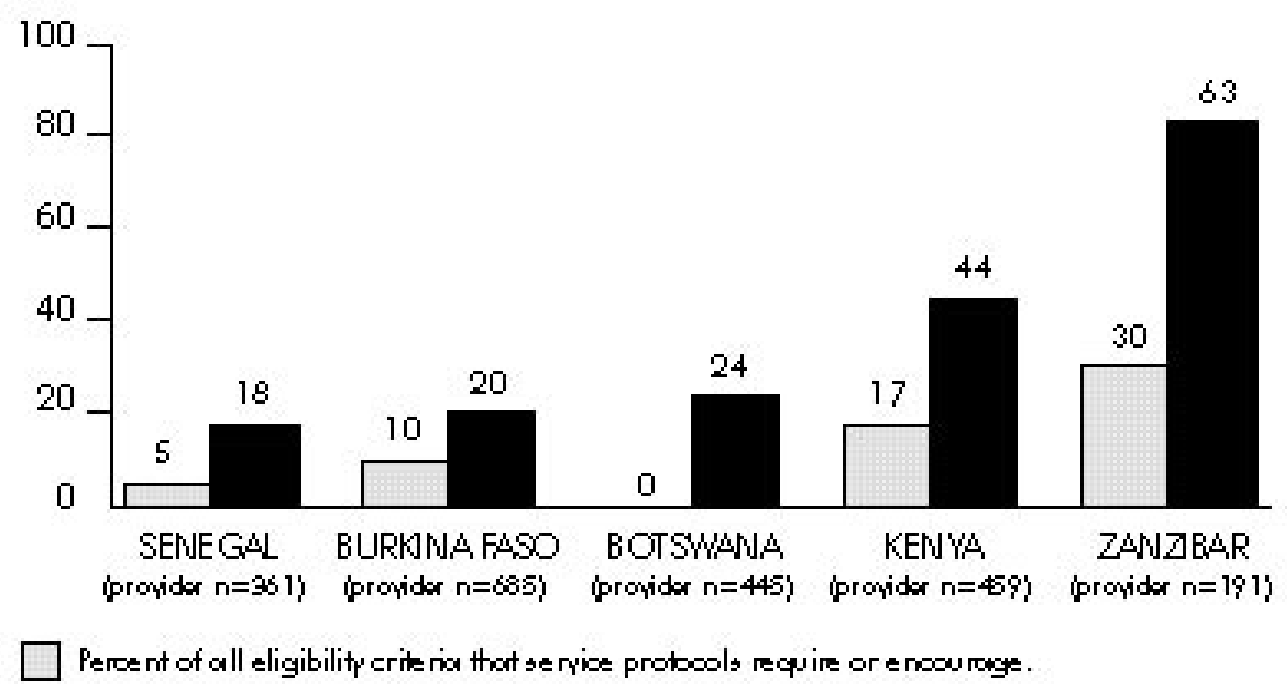

Wean perce nt of e ligibilitp crite ria that propide rs report they impose on elients.

many individual cases, providers seem unaffected by the protocols and may even underapply them. Moreover, no information is available for this study on how these reported eligibility criteria are actually applied to clients. This relationship among protocols, provider attitudes, and provider behavior is complex, but providers do report that they apply more eligibility criteria than the service guidelines recommend.

In-depth interviews about barriers with providers in Ghana revealed that "providers impose these restrictions with the best of intentions. Most feel that by doing so, they are protecting both the client and their society" (Twum-Baah and Stanback, 1995). If providers are motivated by protecting the health of their clients, revised service protocols and training programs should ameliorate the situation. And indeed, medical aspects of these concerns are being dealt with by such organizations as the United States Agency for International Development with training programs on contraceptive updates, in which the epidemiological evidence for restrictions on various methods is reviewed.

A provider's motivation for imposing limitations to protect her society, culture, or values is, however, an equally serious issue, and perhaps one less easily addressed. In this case, the resistance to change is probably stronger, and determining the appropriate means of changing provider behavior is more difficult. The cultural and societal concerns expressed by providers suggest that service protocols, although often medically based, are not applied in a thoroughly medical fashion. The cultural attitudes of providers have an enormous effect on how services are delivered and must be thoroughly addressed if training is to be successful in lifting these restrictions. Further, although access is an issue of great concern, quality of care must be addressed simultaneously if reproductive health services are to improve.

\section{REFERENCES}

\section{Service Protocols in Effect at Time of Situation Analysis Studies}

Botswana: Botswana Family Planning General Policy Guidelines and Service Standards. 1994. Maternal and Child Health/Family Planning Unit, Department of Primary Health Care, Ministry of Health, Botswana. 
Burkina Faso: Politique et Standards des Services SMI/PF au Burkina Faso. 1992. Ministèré de la Santé de l'Action Sociale et de la Famille, Direction de la Santé de la Famille, INTRAH, United States Agency for International Development, Burkina Faso.

Kenya: Family Planning Policy Guidelines and Standards for Service Providers. 1991. Family Planning Programme, Division of Family Health, Ministry of Health, Kenya.

Senegal: Protocoles des Services de Planification Familiale au Sénégal. 1996. Ministère de la Santé et de l'Action Sociale, Programme National de Planification Familiale, République du Sénégal.

Zanzibar: MCH/FP/EPI Service Guidelines. 1993. Ministry of Health, MCH/FP Unit, Zanzibar/Tanzania.

\section{Other References}

Baakile, B., N. Maggwa, L. Maribe, and R. Miller. 1996. A Situation Analysis of the Maternal and Child Health/Family Planning (MCH/FP) Program in Botswana. Ministry of Health MCH/FP Unit and Population Council, Botswana.

Bertrand, J., K. Hardee, R. Magnani, and M. Angle. 1995. "Access, quality of care, and medical barriers in family planning programs." International Family Planning Perspectives, 21, 2:64-69.

Direction de la Santé de la Famille and Population Council. 1996. Deuxième Analyse Situationnelle du Programme de Planification Familiale au Burkina Faso. Ministère de la Santé, Burkina Faso.

Family Health International. 1994. Improving Provider Practices: Highlights of the Workshop "Reduction of Medical Barriers to Contraception." FHI, Research Triangle Park, North Carolina.

Ghana Statistical Service. 1994. A Situation Analysis Study of Family Planning Service Delivery Points in Ghana. GSS, Accra, Ghana.

Herndon, N. 1993. "Next step for Egypt-access to more methods." NETWORK, 13,3:18-21.

Jato, M.N., C.A. Vondrasek, and D.N. Awasum. 1995. "Communication strategies to maximize access and improve the quality of family planning and reproductive health care services." Unpublished paper presented at the Francophone Regional Conference on Maximizing Access and Improving the Quality of Family Planning and Reproductive Health Services in Francophone Sub-Saharan Africa, Burkina Faso.

Mapunda, P.S. 1996. The Zanzibar Family Planning Situation Analysis Study. Zanzibar Family Planning Program, Zanzibar, Tanzania.

McDonald, O.P., K. Hardee, W. Bailey, M. Clyde, and M.T. Villinski. 1995. “Quality of care among Jamaican private physicians offering family planning services." Advances in Contraception, 11,3:245-254.
Omunde, A., L. Ndhlovu, J. Solo, R. Miller, and K. Miller. 1997. An Assessment of Clinic-Based Family Planning Services in Kenya: Results from the 1995 Situation Analysis. Ministry of Health, Division of Family Health, Kenya, and the Population Council, New York.

Programme National de Planification Familiale and Population Council. 1995. Analyse Situationnelle du Système de Prestation de Services de Planification Familiale au Sénégal: Rapport Final. Population Council, New York.

Shelton, J.D., M.A. Angle, and R.A. Jacobstein. 1992. "Medical barriers to access to family planning." The Lancet, 340,8831:1334-1335.

Twum-Baah, K.A. and J. Stanback. 1995. Provider Rationales for Restrictive Family Planning Service Practices in Ghana. Ghana Statistical Services and Family Health International, Ghana.

World Health Organization. 1996. Improving Access to Quality Care in Family Planning: Medical Eligibility Criteria for Contraceptive Use. WHO, Geneva, Switzerland.

\section{NOTES}

1 See Baakile et al. (1996), Direction de la Santé de la Famille (1996), Omunde et al. (1997), Programme National de Planification Familiale (1995), and Mapunda (1996).

2 While Zanzibar is politically a part of the United Republic of Tanzania, it has separate government structures in all areas except foreign affairs, defense, communications, currency, and higher education (Mapunda, 1996). For simplicity, Zanzibar is referred to as a country in this analysis.

3 See Chapter 2 for basic information about these five study contexts.

4 Each graph was tested for the significance of differences among countries using a one-way analysis of variation (ANOVA) and a post hoc Tukey's Honestly Significant Difference (HSD) test. The overall F tests for most of the graphs were significant at $p<.01$, and the Tukey's test showed a range of significant differences among countries. One test, mean parity imposed on condoms, was significant at $p<.05$. For NORPLANT ${ }^{\circledast}$ implants and sterilization, none of the indicators regarding age minimums and maximums showed a significant difference between any two countries. For the mean number of children required, minimum age imposed, and maximum age imposed, the results among countries presented here are significantly different from each other. However, since the analysis is not concerned with making fine distinctions among countries that differ only slightly, the details of statistical significance are not presented in the graphs. 
5 In some cases, a provider may report unusually extreme age limits. These indicators therefore impose a minimum threshold of 13 and a maximum threshold of 45 . Thus if a provider reports that she has a minimum age requirement of less than 13 for a method, she is counted as not having a minimum age requirement. Likewise, if a provider reports that she has a maximum age requirement of more than 45 for a method, she is counted as not having a maximum age requirement.

6 For parity, minimum age, and maximum age requirements, means are presented rather than medians. The medians were calculated, but they did not substantially differ from the means for any of the results. This is to be expected for the age data since all outliers were deliberately removed by the $13 / 45$ thresholds.

7 The WHO guidelines classify eligibility criteria into four categories: no restriction necessary, advantages generally outweigh risks, risks usually outweigh advantages, and health risk is unacceptable. The last two categories require that the provider suggest alternative contraceptive methods. Many of the national protocols studied use the same classification scheme.

8 The parity requirements for injectables and NORPLANT $^{\circledast}$ implants have been removed in Kenya's current, revised service protocols (June 1997).

9 In fact, the WHO guidelines do identify a relative contraindication for IUDs for women under 20 because "there is concern about the risk of expulsion in younger age groups due to nulliparity." In effect, this is a parity restriction that is correlated with younger age. However, the present analysis attempts to tease out each barrier individually. Thus, because WHO has not specified a medical reason to restrict IUDs based on age alone, age is not considered a medically necessary criterion for present purposes.

10 In fact, since the marriage requirement for contraceptive use is so strong in Zanzibar, it is remarkable that so many providers are willing to contradict that standard. For example, $30 \%$ of providers are willing to give condoms to unmarried women, an extraordinary finding in that context.

11 Kenya has age restriction protocols, but data on actual restrictions imposed by providers are not available from the Situation Analysis study, so that country is omitted from the discussion here and in the next subsection.

12 A study done by McDonald et al. (1995) in Jamaica shows remarkably similar results for provider restrictions based on age. All of the age ranges they report are fully comparable to those found here, with the exception that the mean minimum age for sterilization clients in Jamaica is about 27, much younger than our findings. In general, however, the similarity of the results suggests that the normative structure of these restrictions may not be limited to Africa alone.

13 This comparison must be interpreted with caution. In this graph, we do not know whether the barriers required by policy are the same as the ones being applied by staff. The comparison between the $30 \%$ and $63 \%$ in Zanzibar, for example, gives only a rough idea of the overapplication of barriers. 



\section{KEY POINTS AND CONCLUSIONS Tests and Procedures Required of Clients in Three Countries of West Africa}

While there is general agreement among the international medical community that laboratory tests are not necessary for the safe provision of family planning services, there is less agreement about other medical procedures, such as pelvic exams. While some argue that such procedures are helpful to women who may never otherwise have access to health care, others consider such procedures as barriers to contraceptive use. Historically, West African family planning programs were known for requiring numerous tests and procedures before providing a woman with a contraceptive method. However, many countries in the region have modified their service-delivery guidelines in response to recent studies demonstrating that laboratory tests, as well as other procedures, are in fact not necessary for safe contraceptive use.

A comparison of results from the Situation Analysis studies conducted in Burkina Faso, Senegal, and Ghana against international standards reveals the overapplication of several tests and procedures by family planning providers. Moreover, while most providers require and perform those tests and procedures considered essential for providing pills, the IUD, and injectables, there is some evidence of underapplication of screening for sexually transmitted infections by medical history in providing the IUD. Laboratory tests are required and performed far less frequently than other procedures for the methods examined in this chapter.

The overapplication of several procedures is quite common, particularly pelvic exam for the pill and injectable, blood pressure reading for the IUD and injectable, and breast exam and weight taking for all methods. The data indicates that blood pressure and weight are taken for most clients, regardless of method or the reason for the visit to the service delivery point. As these are relatively simple and noninvasive procedures, they may not in fact pose barriers to the use of family planning methods. However, it is not clear from the data whether performing these procedures may cause unnecessary delays in the provision of services.

In contrast, the high frequency of pelvic and breast exams may in fact discourage some women from using family planning. At the very least, these procedures are likely to take a substantial amount of the providers' time. In addition, breast exams and Pap smears, if included in a pelvic exam, are of quite limited usefulness in many West African contexts because treatment for breast and cervical cancer is generally not available. It is not possible to ascertain from the data whether women perceive these procedures as barriers to the use of family planning services. Women may welcome the procedures as they rarely have access to medical services (although this argument does not support the performance of these procedures for revisit clients).

The results presented in this chapter have implications for improving service delivery at both national and clinic levels. At the national level, the overapplication of several procedures suggests that service delivery guidelines may need to be reviewed in light of the international standards and the needs of the community. At the clinic level, the discrepancy between the tests that providers say they require and the ones that they are observed to require suggests that they may not always agree with the guidelines currently in place. In revising guidelines, national-level program managers should consult with supervisors, providers, and clients; this inclusion may help motivate providers to follow the guidelines more closely. In addition, provider training, both formal and on-the-job, may help ensure that providers implement national guidelines more accurately and effectively. 



\section{Tests and Procedures Required of Clients in Three Countries of West Africa}

\section{Lisanne F. Brown and Gwendolyn T. Morgan}

Historically, West African family planning programs were known for requiring numerous tests and procedures before providing a woman with a contraceptive method. In response to several recent studies demonstrating that laboratory testing and other procedures are in fact not necessary for safe contraceptive use (World Health Organization, 1996), several West African countries have modified their service-delivery guidelines (Diadhiou et al., 1990). However, there is some evidence to suggest that many providers still require clients to undergo a series of tests and procedures (TwumBaah and Stanback, 1995; Stanback et al., 1994). This chapter examines the tests and procedures women in West Africa must undergo prior to receiving a contraceptive method.

While policies requiring laboratory tests and other medical procedures are generally motivated by concern for women's health and the safe use of contraceptives, they can actually pose barriers to use (see Chapter 8). In addition, many of these tests and procedures are unnecessary. Laboratory tests, for example, are conducted to screen for medical contraindications such as diabetes, anemia, and high cholesterol. However, several studies have shown that the prevalence of medical contraindications to use of contraception is very low, thus rendering the universal laboratory testing of all clients unnecessary (Stanback et al., 1994; Buekens et al., 1990).

More important, requiring women to undergo numerous tests and procedures may discourage them from adopting or continuing to use contraception, especially if they must have the tests performed at their own expense outside the family planning clinic (Stanback et al., 1994; Diadhiou et al., 1993a). A study in Senegal, for example, showed that women seeking to use the pill were required to undergo numerous tests at a private laboratory at their own expense prior to receiving a prescription (Stanback et al., 1994); in some cases, women had to make up to nine visits (clinic and laboratory). In addition to discouraging patients, such practices may affect the functioning of the clinic by limiting the number of patients that can be seen or the methods that can be provided.

Unnecessary laboratory tests and other procedures, such as pelvic exams and numerous revisits, fall into the category of "process and scheduling hurdles," one of seven types of medical barriers to contraceptive use identified by Bertrand et al. (1995). ${ }^{1}$ More generally, medical barriers have been defined by Shelton et al. (1992:334) as "practices, derived at least partly from a medical rationale, that result in a scientifically unjustifiable impediment to, or denial of, contraception."

While there is general agreement among the international medical community that laboratory tests are not necessary for the safe provision of family planning services, there is less agreement about other medical procedures, such as pelvic exams. Some argue that such procedures are helpful to women who may never otherwise have access to health care (Kenya Ministry of Health and Population Council, 1995). Providers interviewed in Ghana, for example, stated that they conduct full physical and pelvic exams not only to identify contraindications, but also because Ghanaian women have limited opportunities for such medical care (Twum-Baah and Stanback, 1995). ${ }^{2}$ Others, however, regard such procedures as barriers to contraceptive 
use (Bertrand et al., 1995). As with laboratory tests, some women may not use family planning services if they know they must undergo a physical and/or pelvic exam.

Additional support for offering (not requiring) at least some basic tests and procedures came out of the International Conference on Population and Development (ICPD), which emphasized a broader view of family planning as a component of reproductive health. In this context, offering a variety of tests and procedures may respond to the health needs of women in developing countries.

\section{METHODOLOGY}

This chapter examines data on tests and procedures from Situation Analysis studies conducted in three countries of West Africa: Burkina Faso (1995), Senegal (1994), and Ghana (1993). ${ }^{3}$ Data from the provider interview and observation instruments (see Chapter 1) were examined to determine the frequency with which providers reported they require tests and procedures for hypothetical clients, and the actual tests and procedures clients underwent in order to receive a method. As part of the provider interview instrument, providers were asked which procedures and tests they consider necessary before providing contraceptive methods (responses are method specific). ${ }^{4}$ The actual tests and procedures clients underwent during clinic visits were assessed through the observation instrument.

The reported and actual tests and procedures thus determined were then compared with international guidelines that classify each test and procedure according to whether it is essential or unnecessary for safe use of each contraceptive method (as described below). Tests and procedures required for the pill, ${ }^{5}$ injectable, and IUD are included in the present analysis. ${ }^{6}$ NORPLANT $^{\circledR 7}$ and sterilization are excluded because of an insufficient number of clients and providers for these methods in the three countries at the time the Situation Analysis studies were conducted. Tests and procedures are analyzed separately for new/switching clients and revisit clients, as one would expect a difference in the tests and procedures required for these groups. The following tests and procedures were examined:
- Laboratory tests

- Pap smear

- Urine test

- Blood test

- STI screening (by history or laboratory test)

- Procedures

- Medical history

- Blood pressure reading

- Weight taking

- Pelvic exam

- Breast exam

\section{Classification of Tests and Procedures}

A standard classification for all three countries was adopted in order to permit the comparative analysis, although we recognize that each country may have its own clinical guidelines. The classification used for this analysis is based on international standards developed by the Technical Guidance/ Competence Working Group (1997).

Table 9-1 shows the classification of the tests and procedures used in this analysis. The four classes are defined as follows:

- Class A-essential and mandatory or otherwise important in all circumstances for safe and effective use of the contraceptive method.

- Class B-medically/epidemiologically rational in some circumstances to optimize the safe and effective use of the contraceptive method, but may not be appropriate for all clients in all settings.

- Class C - may be appropriate for good preventive health care, but not materially related to safe and effective use of the contraceptive method.

- Class D-not materially related to either good routine preventive health care or safe and effective use of the contraceptive method.

Medical history taking is considered Class A (essential) for all methods, as this is the recommended means of identifying existing conditions that may contraindicate the use of a contraceptive method. It is the only procedure considered essential for safe use of the pill and injectable. For safe use of the IUD, a pelvic exam and STI screening are also considered essential. Class B tests and procedures include blood pressure reading and breast 
Table 9-1. Classification of tests and procedures

\begin{tabular}{lccc} 
Class & COC & Injectable & IUD \\
Class A & & \\
Medical history & $\checkmark$ & $\checkmark$ & $\checkmark$ \\
\hline Pelvic exam & & $\checkmark$ \\
\hline STI screening (by history) & & $\checkmark$ \\
\hline
\end{tabular}

\section{Class B}

Blood pressure reading

Breast exam $\checkmark$

\section{Class C}

Blood pressure reading

\begin{tabular}{lccc}
\hline Pelvic exam & $\checkmark$ & $\checkmark$ & $\checkmark$ \\
\hline Pap smear & $\checkmark$ & $\checkmark$ & $\checkmark$ \\
\hline Breast exam & & $\checkmark$ & $\checkmark$ \\
\hline STI screen (by history and/or laboratory test) & $\checkmark$ & $\checkmark$ & $\checkmark$ \\
\hline
\end{tabular}

\section{Class D}

\begin{tabular}{lccc} 
Weight taking & $\checkmark$ & $\checkmark$ & $\checkmark$ \\
\hline Blood test (hemoglobin, sickle cell anemia) & $\checkmark$ & $\checkmark$ & $\checkmark$ \\
\hline Urine test & $\checkmark$ & $\checkmark$ & $\checkmark$ \\
\hline
\end{tabular}

a It is important to make the distinction between STI screening by medical history versus laboratory tests. STI Screening by history involves asking the patient about any past or current STIs and any risks for infection. STI screening by laboratory tests involves taking samples of blood, urine, or culture to determine the existence of an STI.

exam for the pill. Class $\mathrm{C}$ tests and procedures include pap smear and STI screening history and / or laboratory test for all methods, and blood pressure reading for the injectable and IUD. Class $\mathrm{D}$ tests and procedures include weight taking and laboratory (blood and urine) tests for all methods; all tests using blood and urine samples, regardless of their intended purpose, are considered unnecessary for safe family planning.

It is important to note that the international standards defined above differ in some cases from the standards used in the countries included in this analysis. Procedures such as weight taking and blood pressure reading are a standard part of clinical services in sub-Saharan Africa, and are performed regardless of the services for which women come to a clinic. In addition, blood pressure reading is considered necessary in many West African countries, particularly for pill use, and is required under the national service-delivery guidelines of the three countries included in this analysis. ${ }^{8}$

\section{Analysis Procedure}

All questions related to tests and procedures from the provider interview and observation instruments were analyzed and compared. The analysis of the provider interviews was restricted to staff who had provided at least one of the three methods included in this analysis in the 3 months prior to the study in order to focus on staff actively providing family planning methods. ${ }^{9}$ It is important to note that providers' reports of tests and procedures were spontaneous in Burkina Faso and Senegal, whereas providers were prompted in Ghana. 
The observation instrument included two types of clients. "New and switching clients" comprise all clients accepting a new method, including firsttime users of the family planning services and clients who have decided to change methods. "Revisit clients" include those obtaining a resupply of their method, and those having problems with their method and choosing to either continue or terminate its use.

The unit of analysis for the comparison of the provider interview and observation instruments is the provider. Therefore, we compared the tests and procedures providers said they require with those the providers actually performed on new/switching clients. This comparison was carried out only for new / switching clients because the questions in the provider interview referred to procedures required before prescribing a method for the first time, i.e., for clients new to the method.

Since in some cases the same provider was observed with several different clients, it was necessary to aggregate the observations by provider. If a provider was observed to have ordered a procedure with half or more of his/her clients, he/she was counted as performing the procedure in general. Missing cases were excluded.

When examining the results of the provider interview / observation comparison, it is also important to note the differences in sample sizes. The sample sizes for the observation instrument are much smaller than those for the provider interviews, as only those providers who were observed with a new client for each method are included in the analysis. In contrast, all providers who had provided a contraceptive method in the last 3 months were asked about the tests and procedures they would require before providing a method to a new client.

Procedures performed on revisit clients were also examined. These data are presented for informational purposes only, and were not compared with data from the provider interview or international standards, as there are no universal standards for revisit clients.

\section{Analysis Limitations}

This analysis has several limitations that should be noted. First, the initial analysis plan called for a matched analysis of the provider responses and the observed staff-client interactions-comparing what an individual provider said he/she required with what he/she was observed doing for each client. Although we did attempt to match providers and clients, the severely reduced sample sizes rendered such an approach impractical.

Second, the data do not permit a determination of whether tests are offered at the clinics included in this analysis, or clients are referred to a laboratory or other SDP with laboratory facilities. The only exception is the availability of Pap smears in Senegal (at $2 \%$ of clinics) and Ghana (at $9 \%$ of clinics). Thus a low frequency of a given test may indicate a lack of equipment or referral protocol, rather than a test not being required. Therefore, the estimates obtained in this analysis may understate the tests actually required. Discussions with clinical staff in Burkina Faso indicated that few clinics, apart from some in urban areas, have the various tests available. Therefore, most women are referred outside the clinic (generally to laboratories in town) if tests are deemed necessary. An exception is albumine tests, which are available in most urban and rural clinics because they are instantaneous.

Third, when providers say they require STI screening, we do not know whether they require laboratory tests or verbal diagnostic screening. This is an important distinction since the two are classified very differently by the international guidelines: STI screening by history is considered essential for the IUD, whereas STI screening by laboratory test is unnecessary for all methods.

Finally, results of the clients' medical histories are not available. It is not possible to determine whether clients were required to undergo specific tests because their medical histories indicated a possible contraindication to a method, or the provider ordered the tests indiscriminately.

\section{RESULTS}

\section{Comparison of Tests and Procedures Required and Performed by Providers}

This section presents results of the comparison of provider-related requirements, observed interactions with new/switching clients, and international 
standards for the three methods included in this analysis (pill, IUD, and injectable). Trends in overapplication of tests and procedures according to the standards defined above are also examined.

\section{Pill}

Medical history, ${ }^{10}$ blood pressure reading, and weight taking are the most consistently required and performed procedures for safe pill use in all three countries (see Figure 9-1). This finding may reflect the fact that these three procedures are performed routinely on all clients and are considered a standard part of health care, rather than being required for the pill in particular. The only counterexample is Burkina Faso, where far fewer providers reported that they require medical history prior to providing the pill; however, most take the medical history of clients during consultation. Breast exams are also frequently required and performed, although they are recommended only if there is a medical or epidemiological rationale (Class B). Providers commonly require pelvic exams in Burkina Faso and Senegal and less so in Ghana, even though these exams are not materially related to safe and effective use of the pill. Urine tests, blood tests, and STI screening, which are not necessary for safe pill use, are rarely required in Burkina Faso and Senegal. Providers in Ghana appear to require these tests markedly more often, although the different method of posing the question in Ghana (prompted rather than spontaneous) may be partly responsible for this result. In no country are these three tests performed frequently. This finding may reflect the unavailability of these tests, particularly in Ghana, where more providers than in the other two countries report they require the tests but do not provide them.

\section{IUD}

Medical history, pelvic exam, blood pressure reading, breast exam, and weight taking are the procedures most commonly required and performed prior to providing the IUD (see Figure 9-2). While the first two of these are considered essential for safe IUD use, the latter three are unnecessary and are most likely required and performed as part of the routine care provided to all clients, regardless of the method or the reason for the visit. Although STI screening (by history) is considered an essential procedure for safe IUD use according to the international standards, providers in all three countries rarely reported requiring this procedure and were never observed performing it. However, it is important to remember that the data does not distinguish STI screening by history or laboratory test. Pap smear, urine test, and blood test, all considered unnecessary procedures according to the international standards, are rarely required or performed in all three countries. The only exception is Ghana, where almost half the providers reported they require urine and blood tests; however, this result may again be due to the prompting of the providers.

\section{Injectable}

Medical history, blood pressure reading, breast exam, pelvic exam, and weight taking are the procedures most commonly required and performed for injectable users (see Figure 9-3). The only exceptions are medical histories in Burkina Faso and pelvic exams in Ghana. In Burkina Faso, far fewer providers report requiring a medical history, although many were observed asking clients about their history during the consultation. In Ghana, providers are far less likely than providers in Burkina Faso and Senegal to require or perform pelvic exams before providing the injectable to new users. Pap smear, STI screening, urine test, and blood test, all considered unnecessary procedures according to the international standards, are rarely required or performed in all three countries. The only exception is Ghana, where between one-third and one-half of the providers reported requiring these tests; once again, however, this result may be due to the prompting of the providers.

\section{TESTS AND PROCEDURES PERFORMED ON REVISIT CLIENTS}

Although there are currently no universal procedures required for revisit clients, we believe it is informative to examine the frequency of tests and procedures performed on these clients as measured through the observation instrument. 


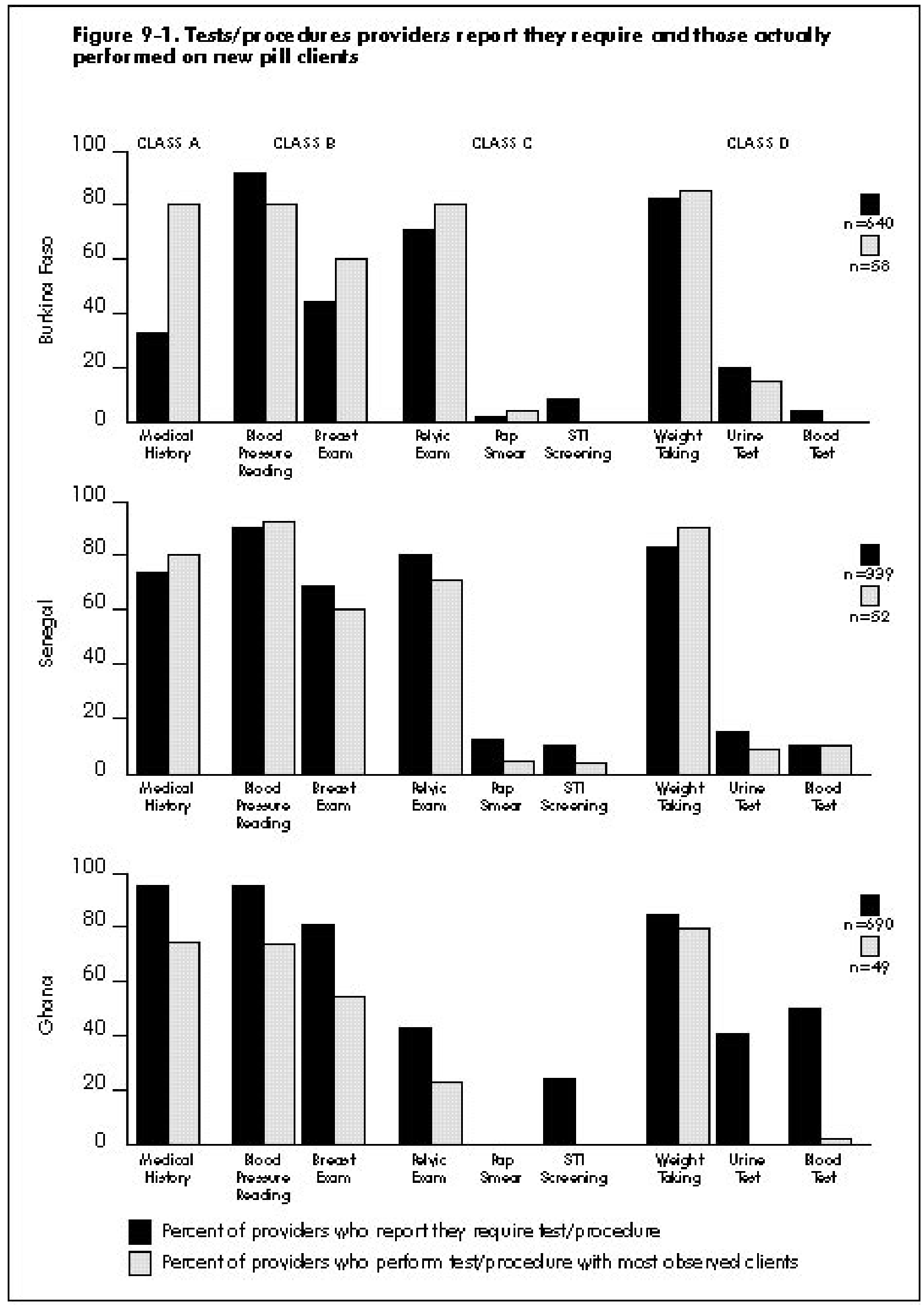

188 Clinic-Based Family Planning and Reproductive Health Services in Africa 
Figure 9-2. Tests/proce dures providers report they require and those actudly performed on new IUD clients
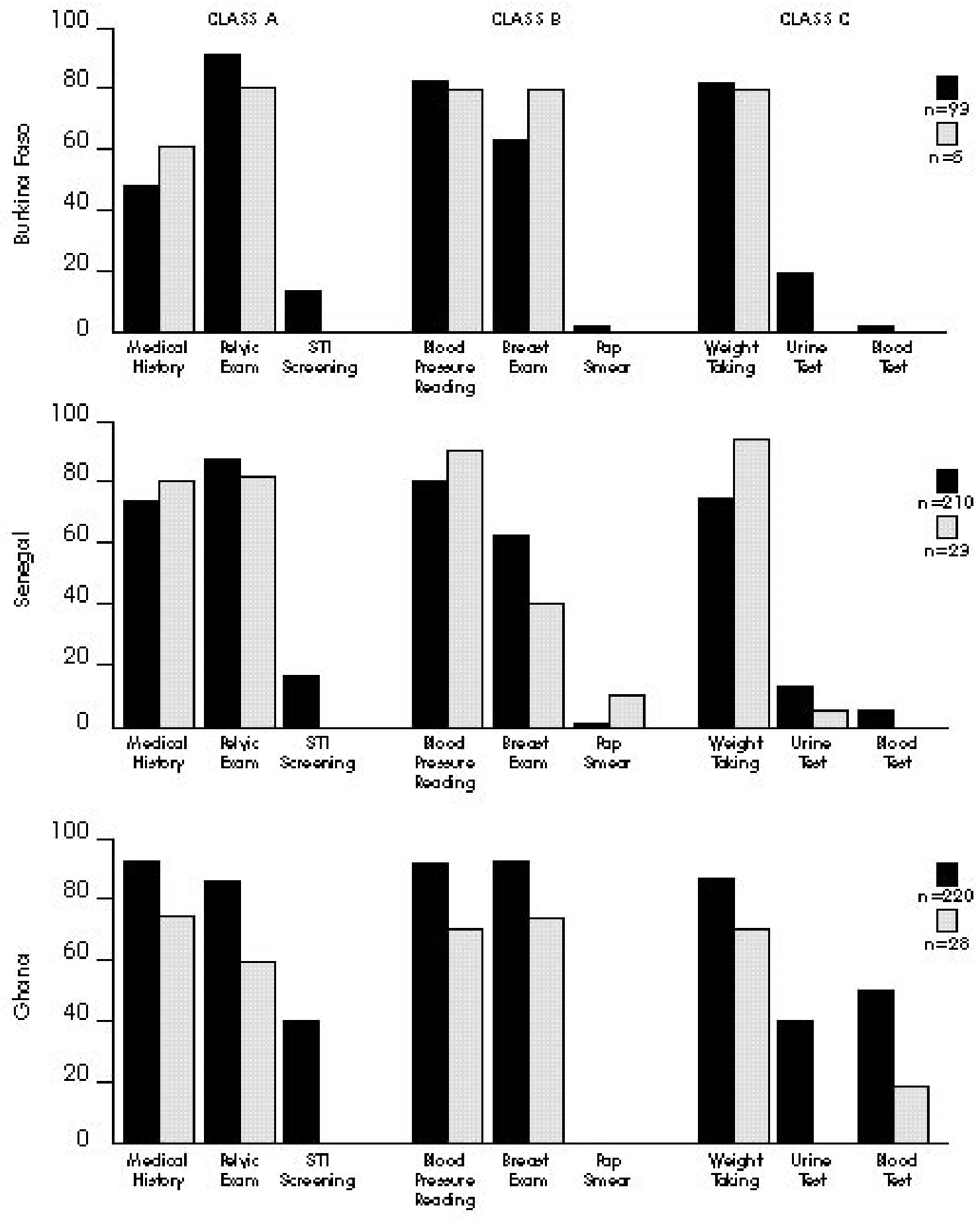

Percent of provickers who report ther' require testpocedure

Percent of provickers who perform test/procedure with most obeerved clients 
Figure 9-3. Tests/proce dures providers report they require and those actudly performed on new injedable clients
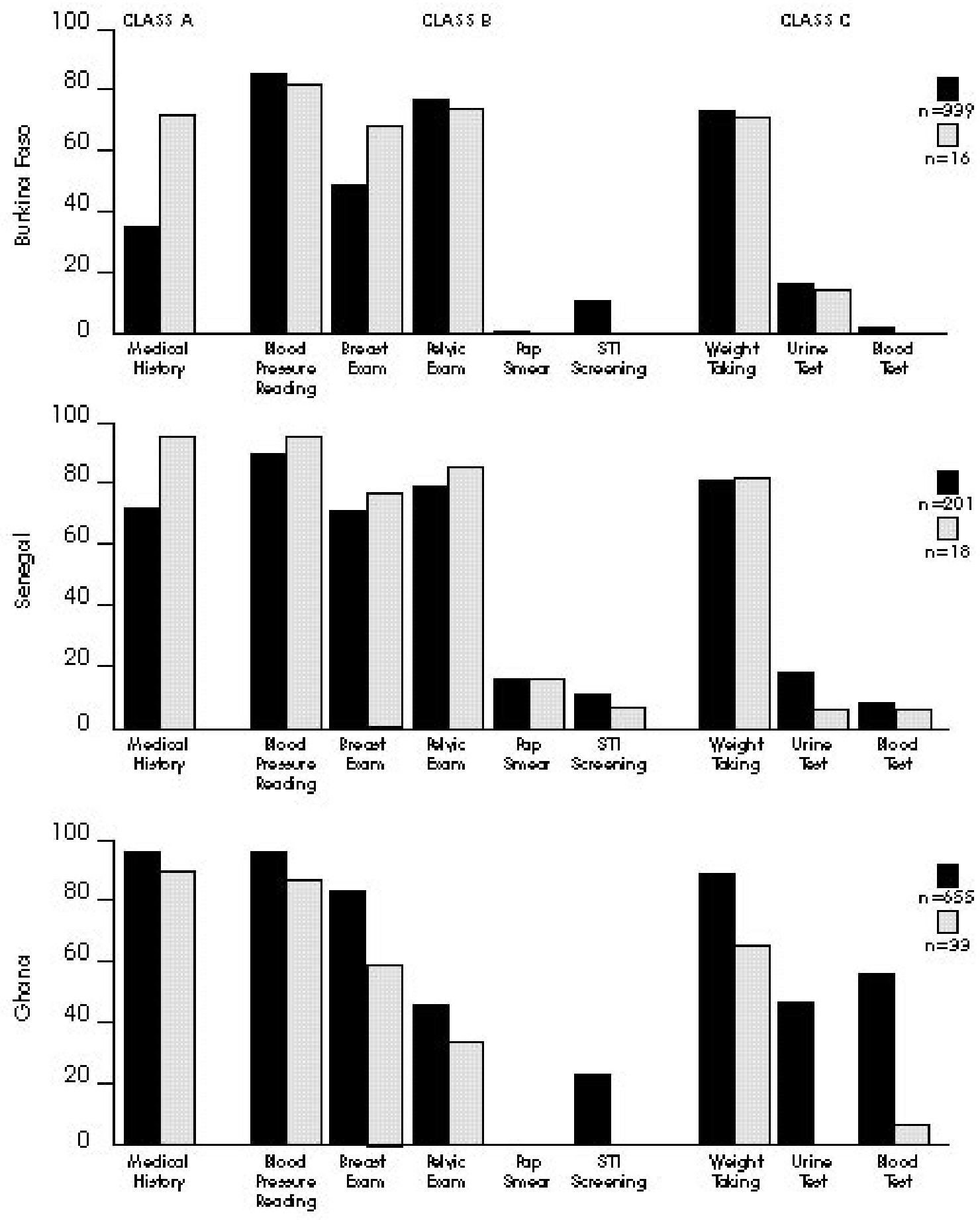

Percent of provickers who report ther' require testpocedure

Percent of provickers who perform test/procedure with most obeerved clients

190 Clinic-Based Family Planning and Reproductive Health Services in Africa 
However, we offer no conclusions about whether the tests and procedures performed were appropriate or not. More important, the data do not allow us to assess accurately why a client returned to the SDP; thus it is not possible to determine what percentage of clients returned because of problems with their method or their health.

\section{Pill}

Blood pressure reading and weight taking are the procedures most commonly performed on revisit pill users. Again, this finding may reflect the fact that these procedures are routinely performed on all clients. Pelvic and breast exams were performed on $5 \%$ to $40 \%$ of revisit pill users. Revisit pill users in Burkina Faso and Senegal underwent pelvic and breast exams most frequently (25-40\%), while only $5 \%$ of revisit users in Ghana had a pelvic exam and $25 \%$ a breast exam. Laboratory tests were required of fewer than $5 \%$ of revisit pill users.

\section{IUD}

A pelvic exam is the most common procedure performed on revisit IUD users in Burkina Faso and Senegal; only half of revisit IUD users in Ghana underwent a pelvic exam. Blood pressure reading and weight taking were also commonly observed among revisit IUD users, particularly in Senegal and Ghana; just one-third of revisit IUD users in Burkina Faso underwent these procedures. Laboratory tests and STI screening were required of very few revisit IUD users, with the exception of Pap smears in Senegal (observed for 10\% of revisit clients).

\section{Injectable}

As with the other methods, blood pressure reading and weight taking are the most commonly performed procedures for revisit injectable users, particularly in Senegal. Pelvic and breast exams were performed on between $10 \%$ and $45 \%$ of revisit injectable users across the three countries, again most frequently in Senegal. As with the pill and the IUD, very few revisit clients were required to undergo laboratory tests or STI screening.

\section{CONCLUSIONS}

A comparison of the results from the Situation Analysis studies in Burkina Faso, Senegal, and Ghana with the international standards reveals the overapplication of several tests and procedures. On the other hand, while most providers require and perform those tests and procedures considered essential (Class A) for providing the pill, IUD, and injectable, there is some evidence of underapplication of STI screening by history in providing the IUD.

The overapplication of several procedures is quite common, particularly pelvic exam for the pill and injectable, blood pressure reading for the IUD and injectable, and breast exam and weight taking for all methods. The data suggest that blood pressure and weight are taken for most clients regardless of the method or the reason for visiting the SDP. As these are relatively simple and noninvasive procedures, they may not in fact pose barriers to the use of family planning methods. However, it is not clear from the data whether performing these procedures may cause unnecessary delays in the provision of services.

In contrast, the high frequency of pelvic and breast exams revealed by the data may in fact discourage some women from using family planning. At the very least, these procedures are likely to take a substantial amount of the providers' time. In addition, the usefulness of breast exams and Pap smears, if included in a pelvic exam, is quite limited in many West African contexts because treatment for breast and cervical cancer is not generally available (Anim, 1993; Jain and Barone, 1996).

Interestingly, laboratory tests (urine and blood tests and STI screening) are required and performed far less frequently than other procedures for the three methods examined in this chapter. While 10\% and 20\% of providers in Burkina Faso and Senegal, respectively, and up to $60 \%$ of providers in Ghana reported that they require laboratory tests for the three methods examined in this analysis, far fewer clients were observed to have undergone these tests (fewer than $20 \%$ and generally $1-5 \%$ ). One possible explanation for this discrepancy is that clients are in fact required to undergo the tests, but must go outside the SDP for the purpose, and this is not recorded in the observation 
instrument. Otherwise it appears that women are in fact rarely required to undergo laboratory testing in order to receive the pill, IUD, or injectable. Therefore, laboratory tests may not be a barrier to family planning use in the three West African countries included in this analysis.

While laboratory testing does not appear to pose barriers to use of family planning methods, certain procedures-pelvic and breast examsmay do so. However, it is not possible to ascertain from the data whether women perceive these procedures as barriers. It is possible that they welcome these procedures since they recognize that they rarely have access to medical services (Kenya Ministry of Health and Population Council, 1995). However, this argument does not support the performance of these procedures for revisit clients.

The results presented in this chapter have implications for improving service delivery at both national and clinic levels. At the national level, the overapplication of several procedures suggests that service delivery guidelines may need to be reviewed in light of the international standards and the needs of the community. At the clinic level, the discrepancy between the tests that providers say they require and the ones that they are observed to require suggests that they may not always agree with the guidelines currently in place. In revising guidelines, national-level program managers should consult with supervisors, providers, and clients; this inclusion may help motivate providers to follow the guidelines more closely. In addition, provider training, both formal and on-the-job, may help ensure that providers implement national guidelines more accurately and effectively.

\section{REFERENCES}

Anim, J.T. 1993. "Breast cancer in sub-Saharan African women." African Journal of Medicine and Medical Sciences, 22,1:5-10.

Bertrand, J.T., K. Hardee, R.J. Magnani, and M.A. Angle. 1995. "Access, quality of care and medical barriers in family planning programs." International Family Planning Perspectives, 21,2:64-69 and 74.

Buekens, P., M. Vekemans, B. Dujardin, M. Boutsen, and E. Wollast. 1990. "Is blood testing necessary before OC prescription in Africa?" Studies in Family Planning, 213:178-180.
Diadhiou, F., M. Sangare, J. Stanback, T. Petrick, R. de Buysscher, and B. Gentile. 1990. Rapport Final, Symposium International sur le Bilan Paraclinique Préliminaire à la Contraception. Université Cheikh Anta Diop, Dakar, Senegal.

Diadhiou, F., C. Nare, E.O. Faye, C.T. Cisse, M. Ba Gueye, B. Jones, P.L. Bolton, D. Huntington, P. Tapsoba, and K. White. 1993a. Facteurs affectant la décision d'une femme de subir une ligature de trompes ou d'utiliser le NORPLANT ${ }^{\circledR}$. Rapport Final. Hôpital Aristide le Dantec Clinique Gynéco-Obstétrique and AVSC International

Diadhiou, F., C. Nare, E.O. Faye, M. Sangare, M. Ba Gueye, K. Katz, and C. Morrison. 1993b. Etude de la perspective de la cliente sur l'introduction du NORPLANT ${ }^{\circledR}$ au Sénégal. Hôpital Aristide le Dantec Clinique Gynéco-Obstétrique and Family Health International.

Jain, A. and M. Barone. 1996. "Preventing cervical cancer." AVSC News 34,3:2.

Janowitz, B., D. Hubacher, T. Petrick, and Nootan Dighe. 1994. "Should the recommended number of IUD revisits be reduced?" Studies in Family Planning, 25,6:362-367.

Kenya Ministry of Health, Division of Family Health and The Population Council. 1995. Quality of Care in Family Planning Service Delivery in Kenya: Clients' and Providers' Perspectives: Final Report.

Shelton, J.D., M.A. Angle, and R.A. Jacobstein. 1992. "Medical barriers to access to family planning." The Lancet, 340:1334-1335.

Stanback, J., J.B. Smith, B. Janowitz, and F. Diadhiou. 1994. "Safe provision of oral contraceptives: The effectiveness of systematic laboratory testing in Senegal." International Family Planning Perspectives, 20,4:147-149.

Technical Guidance/Competence Working Group and World Health Organization/Family Planning and Population Unit. 1996. "Family planning methods: New guidance." Population Reports Series J, 44:34.

Technical Guidance/Competence Working Group. 1997. Recommendations for Updating Selected Practices in Contraceptive Use. Volume II.

Twum-Baah, K.A. and J. Stanback. 1995. Provider Rationales for Restrictive Family Planning Service Practices in Ghana. Final Report. Ghana Statistical Service and Family Health International.

World Health Organization. 1996. Improving Access to Quality Care in Family Planning: Medical Eligibility Criteria for Contraceptive Use.

\section{NOTES}

1 The other six categories are: outdated contraindications; eligibility criteria (e.g., age or parity); service provider qualifications (i.e., restrictions on the type 
of healthcare provider that can offer family planning services); provider bias in recommending or presenting various methods to clients; government regulations, which may restrict or impede the development, approval, or promotion of certain contraceptives; and inappropriate management of side effects.

2 Unfortunately, this study does not include information from clients regarding their opinion of these procedures.

3 See Chapter 2 for the references for these studies. While more recent studies were conducted in Senegal and Ghana, they did not collect information on blood and urine tests, although they did collect information on the percent of SDPs offering screening and testing for a variety of sexually transmitted infections (STIs).

4 The exact question asked of providers was as follows (translated from the Burkina Faso and Senegal questionnaires): "As a professional, which procedures [clinical exams and complementary information] and tests do you consider necessary before you can offer the following methods?" In Ghana the question asked was: "For the following contraceptive methods, what, if any, examinations or tests do you conduct before you can offer the method?"

5 Combined oral contraceptives (COCs) and progestin-only pills (POPs) are both included in the analysis of the pill as they are not distinguished in the staff interviews of any of the Situation Analysis studies; in addition, there is an insufficient number of POP users for a separate analysis.

6 Hormonal methods are by far the most widely available across the three countries, whereas IUDs are less available. However, method availability varies with the type of service delivery point (SDP) (see Chapter 3 for more details).

7 NORPLANT ${ }^{\circledast}$ had been introduced only recently in these countries and was thus still experimental in most places at the time of the studies.

8 Blood pressure reading is considered a Class B procedure for the pill and a Class $\mathrm{C}$ procedure for most other methods.

9 In addition, the provider interview instrument for each study specified to interviewers that only providers who had provided a particular method in the last 3 months should answer the questions on required tests and procedures for that method. In practice, however, all staff who had provided any method during the period responded to these questions.

10 Medical history is taken only for new clients; thus this variable is not included for clients who change methods. 



\section{CURRENT AND FUTURE PROGRAM DIRECTIONS}

Integrating STI and
HIV/AIDS Services
at MCH/Family
Planning Clinics
reviews the extent to which
sexually transmitted infection
(STI) and HIV/AIDS services
have been integrated with
maternal and child health/
family planning services, and
examines the feasibility of
expanding integration of
services.

Changes in Quality
of Services Over Time
compares the findings of
successive studies conducted
in four of the study countries
to determine how these family
planning and reproductive
health programs have
changed in the interim.





\section{KEY POINTS AND CONCLUSIONS}

\section{Integrating STI and HIV/AIDS Services at MCH/Family Planning Clinics}

Since the Cairo International Conference on Population and Development in 1994, and based on the high prevalence of sexually transmitted infections (STIs) (especially HIV) found in sub-Saharan Africa, many countries in the region have begun integrating STI/HIV prevention and management services into their clinic-based maternal and child health $(\mathrm{MCH}) /$ family planning programs. Integration is seen as a potentially cost-effective means of reaching a large proportion of sexually active women, and indirectly their partners, with information and services that can help prevent infection and lead to the diagnosis and management of existing infections. Analysis of data from Situation Analysis studies completed in five countries (Botswana, Ghana, Kenya, Zambia, and Zimbabwe) shortly after service integration was initiated yields the following conclusions:

- At the time of the studies, less than one-third of staff had received refresher or post-basic training in STIs and/or HIV/AIDS, although over half claimed to be providing STI or HIV / AIDS information and services.

- Observations of client-provider interactions indicate that about one-quarter of clients are receiving some information about STIs and/or HIV / AIDS during their MCH or family planning consultation.

- Clients' ability to name STI symptoms is generally poor, their knowledge of means of HIV / AIDS transmission other than sex is limited primarily to needles and sharp implements, and personal fidelity is the most well-known means of transmission prevention.

- Apart from posters, other means of communicating information about STIs or HIV/AIDS (e.g., flip charts, brochures, group health talks) are lacking in most clinics.

- Condoms are available at virtually all clinics, and stockouts are rare. With marked variations among countries, however, condoms appear to be promoted primarily for family planning purposes, with less than one-fifth of clients hearing a dual-protection message.

- Virtually no family planning clients undergo any STI case detection procedures or HIV / AIDS management services.

- Evidence from at least one country (Zimbabwe) suggests that a well-supported and -organized STI drug supply program can ensure the availability of basic medication needed to treat STIs at most clinics; however, this situation appears to be the exception rather than the rule in most sub-Saharan African countries.

In general, the analysis shows that the communication of information to family planning clients about recognition of STI symptoms, modes of transmission, and protective behavior is feasible, and could be strengthened within clinics fairly easily. Moreover, in theory at least, such communication is more likely to reduce levels of infection by preventing transmission within the general population. The impact of such behavior-change communication strategies in transmission prevention, however, is not well understood.

STI case finding and management is clearly an essential component of a woman-centered approach that seeks to reduce the immediate suffering caused by such infections. This analysis reveals, however, that it has proved difficult for clinic-based programs to ensure that these procedures are effectively implemented. A more com- 
prehensive approach to introducing and supporting these activities is essential if they are to be successful. At the same time, however, it is questionable whether STI case finding and management among family planning clients in sub-Saharan
Africa has much effect on reducing the transmission of infection within the general population, as these clients are primarily monogamous women who are more likely to receive an infection from their regular partner than to transmit it to others.

198 Clinic-Based Family Planning and Reproductive Health Services in Africa 


\title{
Integrating STI and HIV/AIDS Services at MCH/Family Planning Clinics
}

\author{
Ian Askew, Goli Fassihian, and Ndugga Maggwa
}

\section{INTRODUCTION}

In recent years, considerable attention has been paid to the feasibility and effectiveness of integrating information and services for the management of sexually transmitted infections (STIs), ${ }^{1}$ including HIV / AIDS, into the existing services provided for women at maternal and child health $(\mathrm{MCH}) /$ family planning clinics. These efforts have complemented the broader range of STI and HIV/AIDS prevention and management activities that are being developed and tested, such as strategies for communicating behavior change among both the general and specific populations, promotion and social marketing of condoms, and targeting of men and commercial sex workers.

Within the overall strategy for preventing and managing STIs and HIV/AIDS at $\mathrm{MCH} /$ family planning clinics, the greatest attention is being focused on integrating these services into consultations with women attending for family planning services. Antenatal clients, however, are also considered an important audience for these services, given the seriousness of perinatal transmission of certain STIs, especially HIV, syphilis, and gonorrhea (Tsui et al., 1997; Howson et al., 1996; Bryson, 1996).

The rationale for the integration of STI and HIV / AIDS services at MCH/ family planning clinics appears to revolve around three main assumptions (Askew et al., 1998):

- A substantial proportion of women attending $\mathrm{MCH} /$ family planning clinics in sub-Saharan Africa are likely to have an infection of the reproductive tract (including those that are non-sexually transmitted), and this condition is serious enough, and the proportion of women suffering is large enough, to justify finding, diagnosing, and managing such cases.

- Informing and educating women about the transmission of these infections and ways to reduce the likelihood of becoming infected will lead to a reduction in the spread of such infections, notably HIV, among the general population.

- Providing STI information and case management services in the context of family planning and antenatal services is a cost-effective approach to increasing access to STI services, and will not adversely affect the provision of $\mathrm{MCH} /$ family planning services.

The above rationale was developed during and promoted following the Cairo International Conference on Population and Development (ICPD) in 1994. Since then, a consensus appears to have developed that such integration of services is essential, particularly in sub-Saharan Africa, where STI levels are the highest in the world and HIV infection is of pandemic proportions. This consensus reflects the need to find strategies not only for addressing reproductive health problems of individual women, but also for reducing the transmission of STIs, including HIV, within the general population (see Tsui et al. [1997] for a discussion of these two perspectives). As a consequence, most national $\mathrm{MCH} /$ family planning programs in the region are now actively revising their policy guidelines and procedures to advocate an integrated approach to serving family planning clients (and, albeit to a lesser extent, antenatal clients) (Mayhew, 1996). They are also seeking technical assistance and financial resources from donors so they can upgrade the capabilities of facility staff and assemble the infrastructure, equipment, and supplies 
needed to offer STI information and services in an $\mathrm{MCH} /$ family planning setting.

Recent evidence from case studies of clinicbased programs that have actively implemented an integrated approach (Maggwa and Askew, 1997a) suggests that such programs include, or seek to include, four component interventions:

- STI case finding, diagnosis, and treatment among family planning and, to a lesser extent, antenatal clients attending $\mathrm{MCH} /$ family planning clinics

- Communication of messages to clients about prevention of STI and HIV / AIDS transmission

- Screening and treatment for syphilis among antenatal clients

- Voluntary counseling and testing for clients suspected to have HIV / AIDS

The findings from case studies demonstrate, however, that it is not easy for programs to integrate these services, even when sufficient human and technical resources are available (e.g., Maggwa and Askew, 1997a; Iskander et al., 1998). Moreover, the assumptions stated earlier are now being questioned. Those assumptions were formulated when little empirical evidence was available on the nature and extent of STIs among the population of $\mathrm{MCH} /$ family planning clinic attendees, and little was known about the effectiveness of the component interventions, including STI case finding and management among family planning and antenatal clients, and behavior change communication through facility-based interactions.

Efforts to integrate services within $\mathrm{MCH} /$ family planning clinics appear, therefore, to be at a crossroads at this point in time. Before future directions can be mapped out with confidence, far more research is needed to gain a better understanding of both the nature and extent of the problem of STIs among women attending $\mathrm{MCH} /$ family planning clinics, and the effectiveness (and cost-effectiveness) of alternative solutions. Investigation is also needed of the role integration of services can play within the broader range of strategies to reduce the incidence of STIs among broader populations.

The purpose of this chapter is to contribute to the needed empirical evidence through an analysis of the current and potential integration of services at facilities within five national $\mathrm{MCH} /$ family planning programs in sub-Saharan Africa: Botswana (data collected in 1995), Ghana (1996), Kenya (1995), Zambia (1996), and Zimbabwe (1996). (See the Chapter 2 references for the relevant Situation Analysis studies.) These programs are represented here because when the Situation Analysis studies in these countries were being planned, the program managers expressed a need for such information. Given the current regionwide interest in integration, this information is now collected routinely for all national Situation Analysis studies undertaken in Africa.

The information presented on these five programs represents their situation shortly after the ICPD and Beijing conferences and immediately before the programs had undertaken substantial efforts to integrate STI and HIV/AIDS management with $\mathrm{MCH} /$ family planning services. For example, in Zimbabwe, the Ministry of Health $(\mathrm{MOH})$ had just begun to reorient its primary healthcare programs to the provision of STI services using an integrated approach. With financial and technical support from external donors, this reorientation involved training all nurses in the primary healthcare facilities in STI management and establishing logistic supply systems for the procurement and distribution of drugs and condoms. In Kenya, some programs (implemented by nongovernmental organizations [NGOs], the Nairobi City Commission, and the Nakuru Municipal Council) had been receiving limited support from donors to facilitate the integration of STI and HIV/AIDS services into their primary healthcare facilities since 1992 . When further funding became available in 1996, the $\mathrm{MOH}$ embarked on a national program to integrate STI management into all its primary healthcare facilities, starting with 10 districts. This effort (which started after the Situation Analysis study) has trained nurses in STI management; established the procurement and distribution of STI drugs; developed a reproductive health strategy; and revised the national family planning guidelines to include other reproductive health issues, especially STI and HIV / AIDS management.

The objective of this chapter is to provide program managers, donors, and technical assistance organizations with information on the preparedness of facilities to implement an integrated service- 
delivery approach. The discussion also includes some indication of the type and magnitude of support and resources needed if an integrated approach is to become the standard operating procedure for $\mathrm{MCH} /$ family planning programs in subSaharan Africa. The results of this analysis are presented in five sections, addressing (1) the extent to which services are currently integrated at clinical facilities, (2) whether clinic staff are prepared to offer an integrated approach to family planning and STI services, (3) the extent to which STI/ HIV/AIDS messages are being communicated to family planning clients, (4) the feasibility of providing STI/HIV/AIDS services to MCH/family planning clients, and (5) the awareness of STIs and HIV/AIDS among clients attending facilities. ${ }^{2}$ The final section presents conclusions and recommendations as to future directions these programs could take and the role of facility-based surveys in evaluating integrated programs.

It should be emphasized that the data collection instruments used, although broadly comparable, evolved over time and were modified to meet the specific information needs of each country. Consequently, more data were collected than is presented, and only those data for which generalizations can be derived across all five countries are included. It is believed that these are likely to be the most important indicators and the ones most widely applicable to other countries in the region.

\section{ARE SERVICES CURRENTLY INTEGRATED?}

The person "in charge" at each MCH/family planning facility (usually a senior nurse) was asked whether any STI or HIV management services are usually offered. The question was deliberately phrased in general terms so an overview could be gained of whether those working in the facilities consider STI/HIV management to be part of the routine services they are expected to provide. Across all five programs, more than two-thirds of facilities claimed to offer STI management services, and over half said they are currently offering HIV management services. Table 10-1 shows that for STI management services, these proportions are

\section{Table 10-1. Percent of facilities at which STI and HIV management services are routinely offered}

\begin{tabular}{lcc} 
Facilities & $\begin{array}{c}\text { Offer STI } \\
\text { Management } \\
\text { Services }\end{array}$ & $\begin{array}{c}\text { Offer HIV } \\
\text { Management } \\
\text { Services }\end{array}$ \\
\hline Hospitals & 72 & 49 \\
\hline Health centers & 71 & 62 \\
\hline Health posts & 69 & 51 \\
\hline Botswana & 90 & 46 \\
\hline G hana & 73 & 65 \\
\hline Kenya & 55 & 68 \\
\hline Zambia & 44 & 21 \\
\hline Zimbabwe & 99 & 25 \\
\hline
\end{tabular}

fairly consistent across the type of facility. Given the higher level of training required, the proportions offering HIV management services decrease at lower facility levels. There is considerable variation by country, with facilities in the Kenya and Zambia programs less likely to offer these services.

Because the data collection instruments evolved over time, the specific types of STI and HIV / AIDS management activities were clarified for three countries only (Ghana, Zambia, and Zimbabwe). As might be expected (see Table 10-2), counseling for both STIs and HIV/AIDS is the main service offered.

Overall, there is no difference in the likelihood of a facility offering STI services between facilities in urban and rural locations, or among the public, private, and NGO/Mission sectors.

However, there are such differences for HIV/AIDS services: the proportion of facilities offering these services is higher in urban (54\%) than in rural areas $(45 \%)$, and facilities in the NGO/Mission sector $(62 \%)$ are more likely than those in the public and private sectors $(46 \%)$ to offer some form of HIV management services. These findings may suggest that resources for strengthening HIV/AIDS prevention and control services have been focused more on NGO programs and on 


\section{Table 10-2. Percent of facilities offering different types of STI and HIV/AIDS management services in Ghana, Zambia, and Zimbabwe}

\begin{tabular}{lccccc} 
Country & $\begin{array}{c}\text { STI IEC } \\
\text { Counseling }\end{array}$ & $\begin{array}{c}\text { STI } \\
\text { Diagnosis }\end{array}$ & $\begin{array}{c}\text { STI } \\
\text { Treatment }\end{array}$ & $\begin{array}{c}\text { HIV/AIDS } \\
\text { Counseling }\end{array}$ & $\begin{array}{c}\text { HIV/AIDS } \\
\text { Testing }\end{array}$ \\
\hline Ghana & 66 & 36 & 46 & 66 & 20 \\
\hline Zambia & 36 & 29 & 28 & 26 & 2 \\
\hline Zimbabwe & 70 & $*$ & 74 & 56 & 17 \\
\hline
\end{tabular}

* Q uestion asked in terms of both laboratory diagnosis (95\%) and syndromic diagnosis (20\%).

facilities in urban areas than on public-sector programs and facilities in rural areas. (See Chapter 7 for more discussion of urban/rural differences.)

The frequency with which these services are currently provided to $\mathrm{MCH} /$ family planning clients is cause for concern among advocates of an integrated approach. Although attempts were made during the Situation Analysis studies to abstract the service statistics for STI and $\mathrm{HIV} /$ AIDS services from the MCH/family planning unit records, the notoriously poor quality of recordkeeping in these facilities (e.g., Ndhlovu, 1998) made it impossible to measure accurately the proportion of clients receiving such services on an annual basis. Nevertheless, valid data on the number of STI clients seen over a 12-month period was collected from 281 clinics in Ghana, Zambia, and Zimbabwe. The median number of clients seen was 203 (i.e., less than 1 per working day), with a range of 1 to 5,189 . Valid data on the number of clients receiving HIV/ AIDS services were collected from 86 clinics in these three countries and revealed a median of 12 clients (i.e., 1 per month), with a range of 1 to 3,082. Furthermore, among the 3,297 family planning clients and 6,797 $\mathrm{MCH}$ clients interviewed in these five studies, fewer than $0.5 \%$ reported having received an STI or HIV service (broadly defined) during their visit. ${ }^{3}$ Thus although staff are claiming to provide these services, they are clearly doing so infrequently.

More seriously, there would appear to be an underutilized capacity if the services are available but are not being provided or used on a regular basis. (See Chapter 6 for further discussion of this point.) This may be because staff are not routinely offering integrated services, and/or because $\mathrm{MCH} /$ family planning clients needing STI and HIV/AIDS services do not know they are available. Alternatively, it may be that the need for such services within the $\mathrm{MCH} /$ family planning population is low because the prevalence of STIs and HIV is low. This is a controversial yet crucial issue as it underlies one of the main assumptions supporting the need for integration, and it is an area in which more research is clearly needed.

\section{ARE STAFF PREPARED TO OFFER INTEGRATED SERVICES?}

Whether staff are adequately prepared to offer integrated services at an acceptable level of quality is an essential issue for all program managers (see also indicator R.6-6 in Chapter 3). Clearly, the most basic requirement is that staff be adequately trained. Table 10-3 shows the proportion of staff who said they had received post-basic or refresher training in STIs and/or HIV/AIDS. Post-basic or refresher training is the appropriate indicator because program managers believe that nurses' preservice training in STIs was inadequate and was usually completed too long ago to qualify them to provide these services competently. The data suggest that country programs are at different stages in training their staff in these services, and that overall, more than two-thirds of facility staff throughout the five countries had not been trained in STIs or HIV / AIDS at the time of the Situation Analysis studies. 


\begin{tabular}{|c|c|c|c|}
\hline Country & $\begin{array}{l}\text { Training in } \\
\text { HIV/AIDS }\end{array}$ & $\begin{array}{l}\text { Training in } \\
\text { STls }\end{array}$ & $\begin{array}{c}\text { Training in STI } \\
\text { Syndromic Management }\end{array}$ \\
\hline Botswana & 67 & 54 & 31 \\
\hline Ghana & 27 & 29 & 7 \\
\hline Kenya & 7 & 8 & 8 \\
\hline Zambia & 17 & 28 & 15 \\
\hline Zimbabwe & 31 & 44 & 34 \\
\hline
\end{tabular}

Training in these services is necessary but not sufficient for staff to implement an integrated approach to serving family planning clients. Some of the studies also collected data on the preparedness of staff to think in terms of offering integrated services to family planning clients. For example, staff in Botswana and Kenya were asked what procedures and tests new clients choosing the pill, IUD, or injectable must have before they can be offered the method. STI screening was mentioned by very few staff-ranging from a high of $23 \%$ of staff for IUD clients in Botswana to a low of 3\% for injectable clients in Kenya. (See also Chapter 9.) Moreover, when staff were asked which methods they would not recommend for a client with an STI, only three-quarters (with a range from $57 \%$ in Zambia to $91 \%$ in Kenya) mentioned the IUD, despite the fact that the IUD is normally contraindicated for women presenting with an STI.
The preparedness of staff to offer an integrated approach is also dependent on their experience with providing these services. Staff were asked which STI and HIV/AIDS services they themselves had offered over the last 3 months. ${ }^{4}$ Table 10-4 shows that, with the exception of Kenya, well over half of the staff interviewed indicated that they had counseled $\mathrm{MCH} /$ family planning clients on STIs, and a significant proportion had provided counseling on HIV / AIDS. Diagnosis and treatment of STIs appear to be provided more frequently by staff in Zambia and Zimbabwe. Caution is needed, however, in interpreting the frequency with which staff have provided such services. Because the timeframe for the question was within the last 3 months, the results could include both those staff who have provided the service only once and those who provide it on a daily basis.

\section{Table 10-4. Percent of staff indicating that they had provided STI and HIV/AIDS services in previous 3 months}

\begin{tabular}{lccccc} 
Country & $\begin{array}{c}\text { STI } \\
\text { Counseling }\end{array}$ & $\begin{array}{c}\text { STI } \\
\text { Diagnosis }\end{array}$ & $\begin{array}{c}\text { STI } \\
\text { Treatment }\end{array}$ & $\begin{array}{c}\text { HIV/AIDS } \\
\text { Counseling }\end{array}$ & $\begin{array}{c}\text { HIV/AIDS } \\
\text { Testing }\end{array}$ \\
\hline Ghana & 57 & 13 & 17 & 52 & 3 \\
\hline Kenya & 43 & $24^{\mathrm{a}}$ & 40 & 14 & 2 \\
\hline Zambia & 71 & 57 & 82 & 35 & 4 \\
\hline Zimbabwe & 87 & $85^{\mathrm{b}}$ & 87 & 60 & 4 \\
\hline
\end{tabular}

${ }^{a}$ Kenya includes $19 \%$ syndromic diagnosis, 10\% laboratory diagnosis.

b Zimbabwe includes 84\% syndromic diagnosis, 15\% laboratory diagnosis. 


\section{Figure 10-1. Readiness of facilities to co mmuricate messages on $5 T s$ and HV/AIDS}

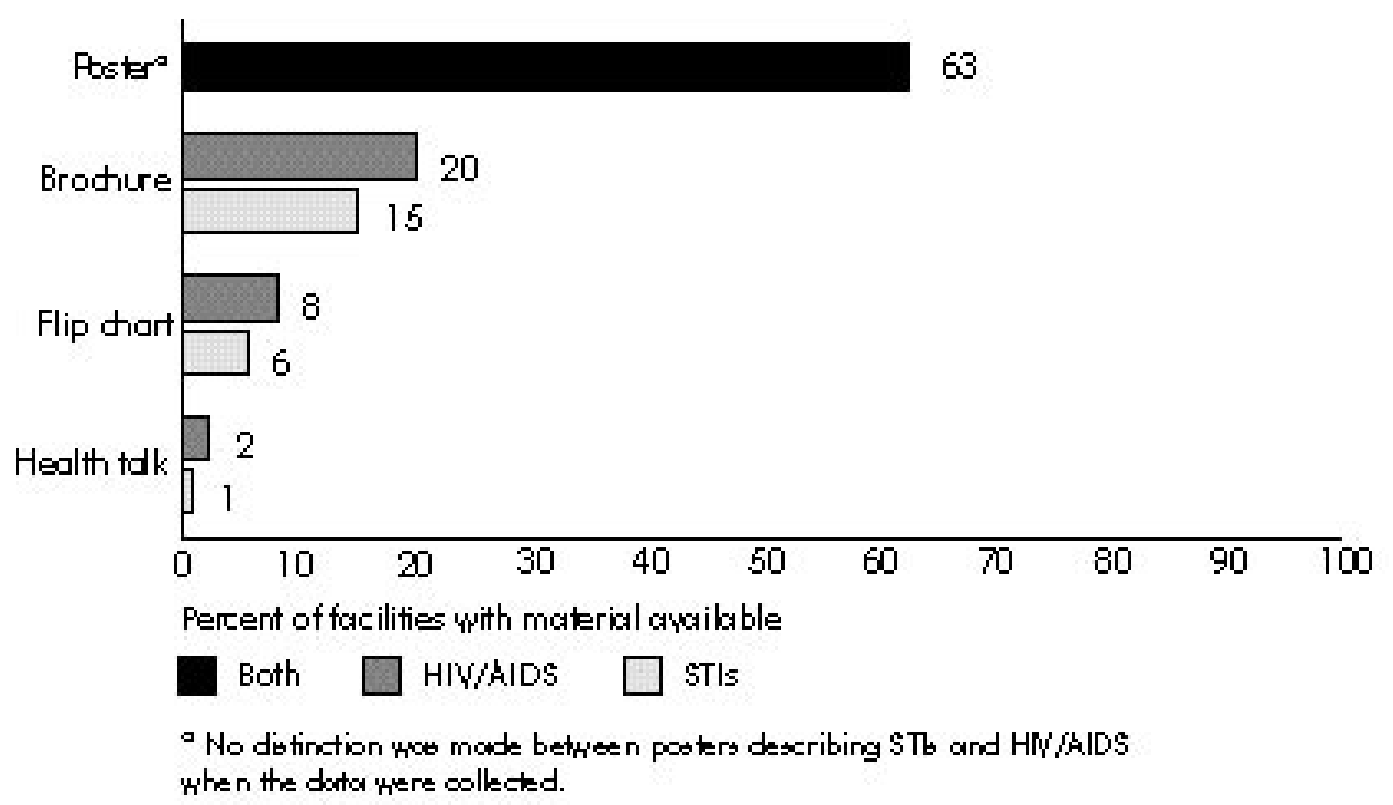

\section{ARE STI/HIV/AIDS MESSAGES BEING COMMUNICATED AT CLINICS?}

One of the main arguments for integrating STI information and services into $\mathrm{MCH} /$ family planning programs is that many women in the reproductive age group visit these facilities fairly frequently. Consequently, the $\mathrm{MCH} /$ family planning clinic is seen as an important venue at which messages on infection transmission, prevention, and treatment could be easily communicated to an important audience.

Figure 10-1 indicates the readiness of facilities to communicate these messages through different media (see also indicator R.7-2 in Chapter 3). Posters on the wall are clearly the most commonly available medium, in that almost two-thirds of facilities were found to have on the wall at least one poster with an STI and/or HIV/AIDS message. Although posters are useful for communicating simple messages to a broad audience, however, other information, education, and communication (IEC) materials are more appropriate for use during individual counseling. Brochures and flip charts are the most commonly used communication aides during individual meetings, but those covering STIs or HIV / AIDS appear not to be widely available at present.

For all five countries, group health talks are supposed to be held every morning. In only one-quarter of all facilities, however, was a talk observed on the day of data collection. Furthermore, STIs or HIV / AIDS were mentioned during only $8 \%$ and $5 \%$ of these health talks, respectively. Thus on the day of the research team's visit, only $2.5 \%$ of facilities could be said to have communicated messages to clients on either of these topics through group health talks. (See also indicator R.7-3 in Chapter 3.)

Clearly, there is a great deal of room for strengthening the capacity of facilities to exploit these communication media to better inform and educate $\mathrm{MCH} /$ family planning clients about STIs and HIV / AIDS. It would be prudent first, however, to undertake research to determine whether any or all of these media are effective in communicating messages that are likely to lead to a change in sexual behavior on the part of the client and/or, more important, her partner.

Overall, 22\% of new family planning clients were told something about STIs, HIV/AIDS, or both (the content of the discussion and whether the 


\section{Figure 10-2. Percent of new family planring dients with whom HIV/AIDS and STs were discussed}

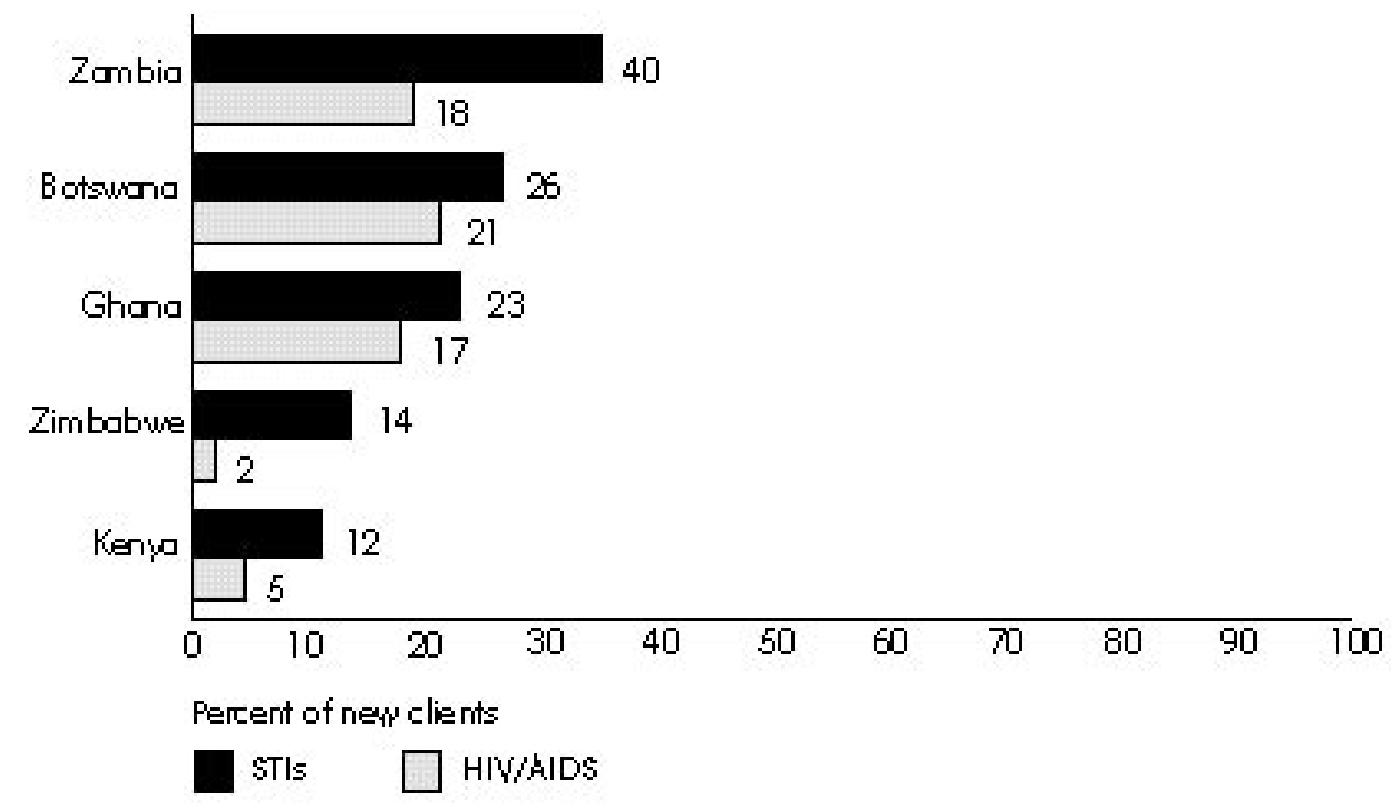

provider or client initiated it are not known). Virtually all of these women were told about STIs, and about half also were told about HIV / AIDS. Figure 10-2 shows the proportions by country. Although the proportions are not especially high, the findings (from Zambia and Botswana especially) suggest that it is possible for providers to incorporate such messages into their family planning consultations. Consequently, efforts could be made to increase the likelihood that a provider will discuss these issues.

The one message that would appear to be the simplest for $\mathrm{MCH} /$ family planning facilities to communicate to their clients is the role of condoms in protecting against both pregnancy and infection. Moreover, there is no problem in supplying condoms to clients. Virtually all $\mathrm{MCH} /$ family planning clinics offer condoms as a contraceptive method (over $95 \%$ of facilities in these five countries), and stockouts are not generally a problem, although about one-quarter of facilities in Botswana and Zimbabwe reported a condom stockout in the 6 months preceding the study (see also indicator R.4-1 in Chapter 3).

The willingness of providers to mention condoms to family planning clients appears to vary somewhat, as shown in Figure 10-3. For four of the countries, between half and three-quarters of new family planning clients were told about condoms during counseling about family planning, the one exception being Zimbabwe. ${ }^{5}$ Conversely, far fewer clients were explicitly told that condoms can be used to protect against contracting an STI. Thus although many staff appear to have little problem with mentioning condoms as a family planning method, the fact that condoms can also be used to protect against STIs is not always part of the message communicated to new family planning clients.

The data presented in Figure 10-3 describe the proportion of all new family planning clients who were told about the protection offered by condoms against either pregnancy or STIs. ${ }^{6}$ One component of an integrated approach that is currently receiving a great deal of attention is promotion of the male condom as the most effective contraceptive method, offering dual protection against both conception and infection. ${ }^{7}$ Figure $10-4$ shows the proportion of new family planning clients who received a "dual-protection" message, that is, who were told about the condom as part of family planning counseling and were also told about its ability to protect against STIs (see also indicator 


\section{Figure 10-3. Percent of new family planring dients hearing about condoms as protection against pregnancy or STLs}

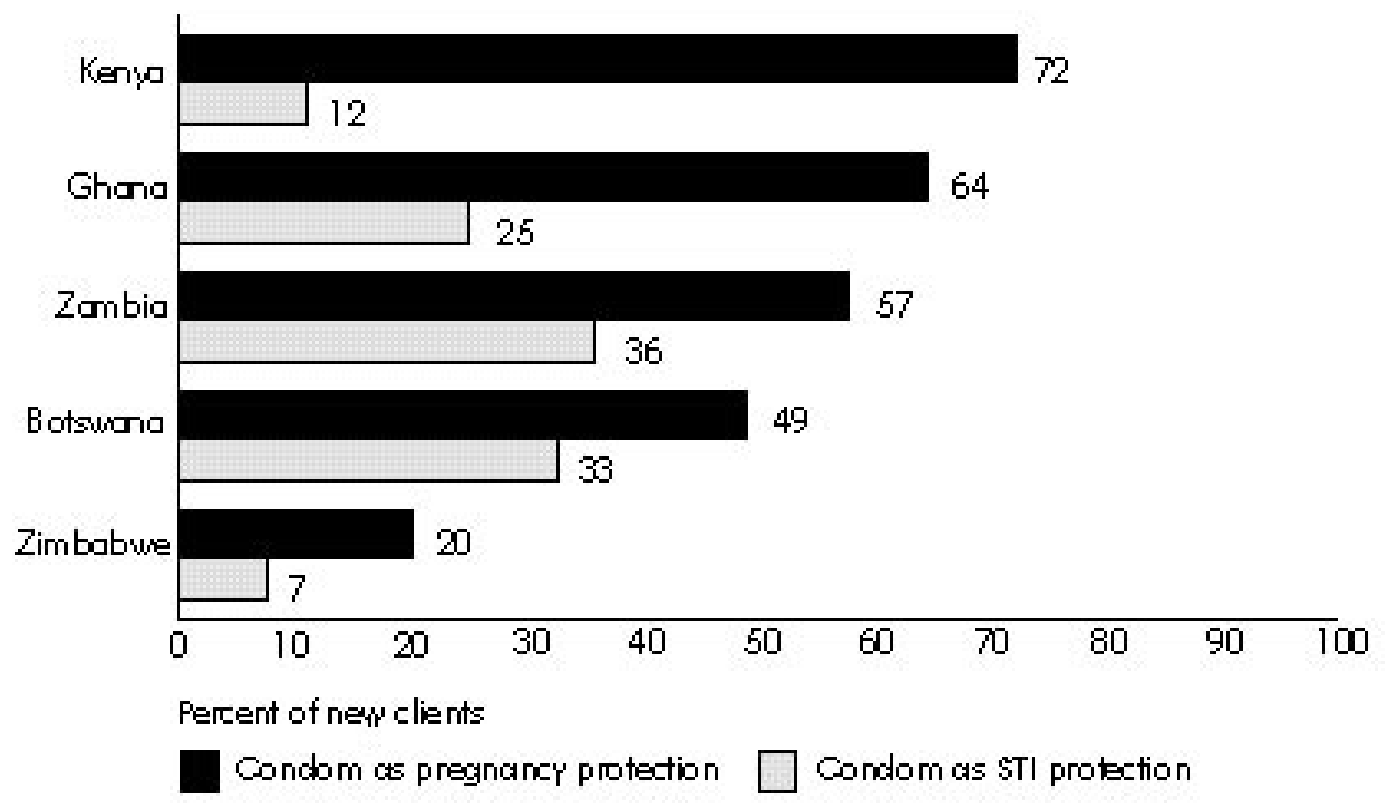

R.3-2 in Chapter 3). Overall, the proportion is low at $16 \%$ for all new clients observed, but it should be remembered that these studies generally preceded the current emphasis on dual-protection messages. There is clearly scope for strengthening this component of an integrated approach, as shown by the higher levels of communication of this message in Botswana, Zambia, and, to a lesser extent, Ghana.
Of all new family planning clients interviewed in the studies, 9\% had ever used condoms, and 7\% adopted the method during the observed visit. Given the low level of condom use among couples generally, and women specifically, in sub-Saharan Africa, this proportion is not surprising, and may even be seen as encouraging. It highlights again, however, the need to strengthen the role played by clinics as sources of information about condoms.

\section{Figure 10-4. Percent of new family planring dients hearing a dual-pr ofection messoge in relation to condoms}

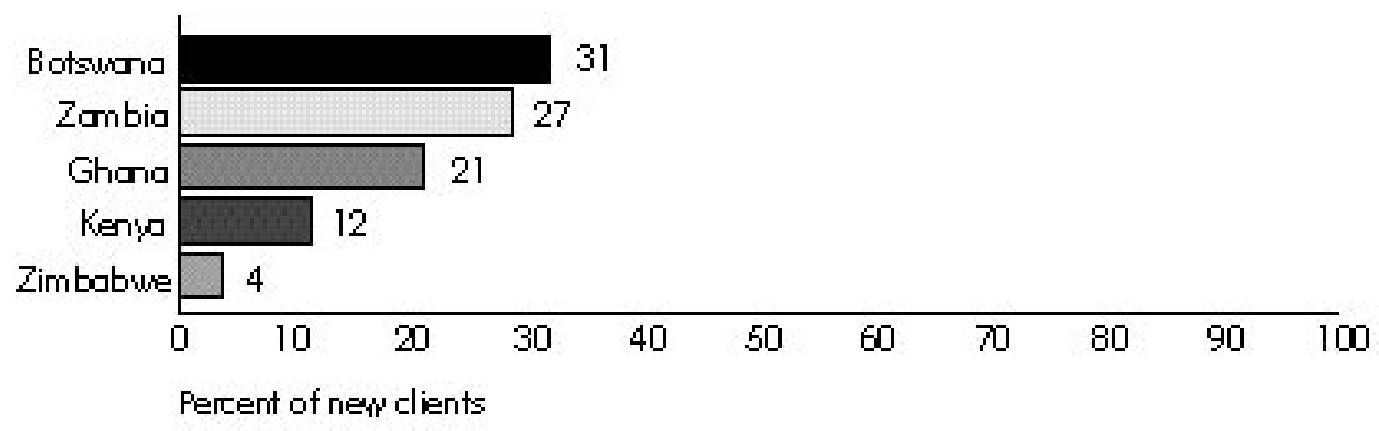




\section{CAN STI/HIV MANAGEMENT SERVICES BE PROVIDED TO MCH/FAMILY PLANNING CLIENTS?}

\section{STI Case Finding}

The most contentious issue associated with an integrated approach is whether $\mathrm{MCH} /$ family planning clinics are an appropriate venue for finding and managing STIs, given that the STI prevalence among their clients is likely to be relatively low. Finding cases of STI among women who are not presenting specifically for this problem is notoriously difficult, partly because many infected women are asymptomatic, and partly because many symptomatic women do not always recognize potential STI symptoms. At the time of these studies, however, STI case finding and management were being strongly promoted for women attending $\mathrm{MCH} /$ family planning clinics, and data were therefore collected on the preparedness of the facilities and their staff to undertake these services.

Case-finding procedures for STIs among $\mathrm{MCH} /$ family planning clients generally incorporate several activities to help the provider detect infections, including taking a medical history and performing a general physical examination; asking the woman risk assessment questions ${ }^{8}$; and, whenever possible, performing a pelvic examination. A pelvic exam with a speculum is believed to be useful in enabling the provider to distinguish between cervical and vaginal infections; this distinction can

\section{Table 10-5. Percent of new family planning clients having a medical history taken and receiving a general physical examination}

\begin{tabular}{lcc} 
Country & $\begin{array}{c}\text { Medical } \\
\text { History } \\
\text { Taken }\end{array}$ & $\begin{array}{c}\text { General } \\
\text { Physical } \\
\text { Examination }\end{array}$ \\
Botswana & 70 & 62 \\
\hline Ghana & 60 & 31 \\
\hline Kenya & 77 & 64 \\
\hline Zambia & 69 & 46 \\
\hline Zimbabwe & 51 & 41
\end{tabular}

be important as the former are generally more serious than the latter, and are more likely to be sexually transmitted than due to endogenous overgrowths. For these studies, certain items related to these procedures were observed during the clientprovider interactions, so it is possible to gain some insight into the frequency with which these procedures are currently followed.

Table 10-5 shows the proportion of new family planning clients whose medical history was taken and who had a general physical examination. The variation observed suggests that the expectations of staff about routinely performing these procedures for all new family planning clients differ across the five countries. Further analysis would

Figure 10-5. Per cent of new family planring dients asked bout comm on $5 T$ symptoms

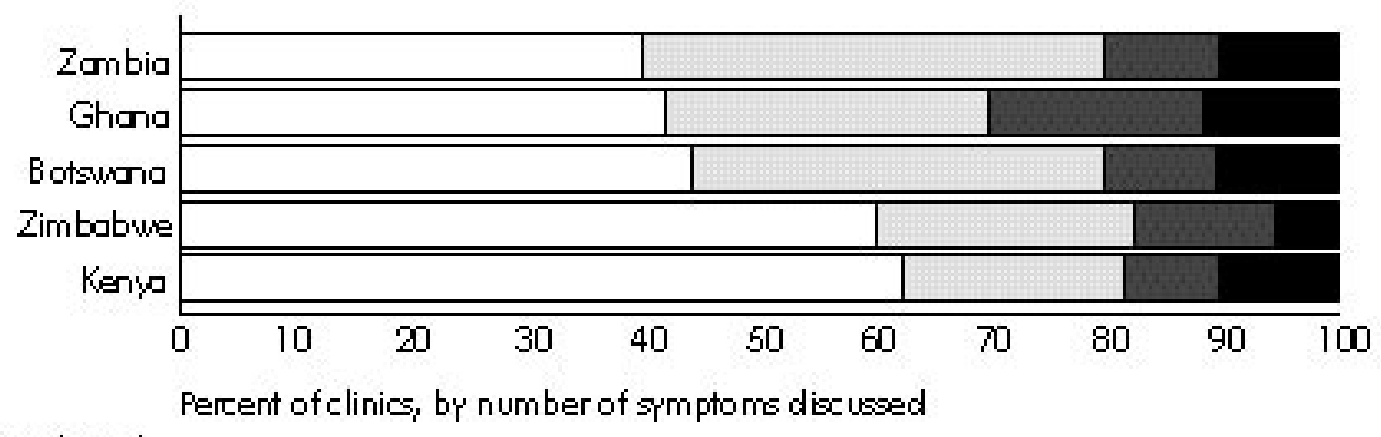

Number of

simptoms:

none $\square$ one

tyo

three/tour 
Table 10-6. Percent of new family planning clients asked risk assessment questions

\begin{tabular}{lcccccc} 
Country & $\begin{array}{c}\text { Marital } \\
\text { Status }\end{array}$ & $\begin{array}{c}\text { New Partners } \\
\text { in Last Year }\end{array}$ & $\begin{array}{c}\text { Concerns } \\
\text { About STls }\end{array}$ & $\begin{array}{c}\text { Sexual } \\
\text { Relations }\end{array}$ & $\begin{array}{c}\text { History of } \\
\text { STls }\end{array}$ \\
\hline Botswana & 52 & 9 & 18 & $\mathrm{n} / \mathrm{a}$ & 20 \\
\hline Ghana & 75 & 21 & 12 & 30 & 13 \\
\hline Kenya & $\mathrm{n} / \mathrm{a}$ & 14 & 6 & 36 & 12 \\
\hline Zambia & 74 & 5 & 19 & 34 & $\mathrm{n} / \mathrm{a}$ \\
\hline Zimbabwe & 68 & 16 & 13 & 5 & 15 \\
\hline
\end{tabular}

be needed to determine whether these differences are associated with specific characteristics of either the type of staff observed or the facilities visited. (See Chapter 9 on tests / procedures in West Africa.)

As part of an integrated approach, providers need to ascertain during history taking whether the client has any of the common STI symptoms; this information can then be used as a starting point for syndromic management. Figure 10-5 shows the proportion of new family planning clients who were observed to be asked by the provider about, or who themselves mentioned during the consultation, the presence of up to four common STI symptoms. ${ }^{9}$ Of the four symptoms considered (abnormal bleeding, abnormal discharge, abdominal pain, genital itching), genital itching was the symptom least discussed overall (in $11 \%$ of interactions), with the other three each being discussed in about $25 \%$ of interactions. The most commonly discussed symptom in Botswana, Kenya, and Zimbabwe was abnormal bleeding; in Ghana it was abnormal discharge; and in Zambia it was lower abdominal pain. Again, although there is room for improvement, the evidence (from Zambia in particular) suggests it is not necessarily problematic for providers to gather this information as part of routine counseling for new family planning clients. (Also see indicator Q.2-8 in Chapter 3.)

Risk assessment has been recommended as a means to increase the likelihood of finding potential STI cases among family planning clients and to improve the predictive value of syndromic

\section{Figure 10-6. Degree of comfort of stdf in discussing sexual behavio and $5 \mathrm{Th}$ with dient $s^{a}$}

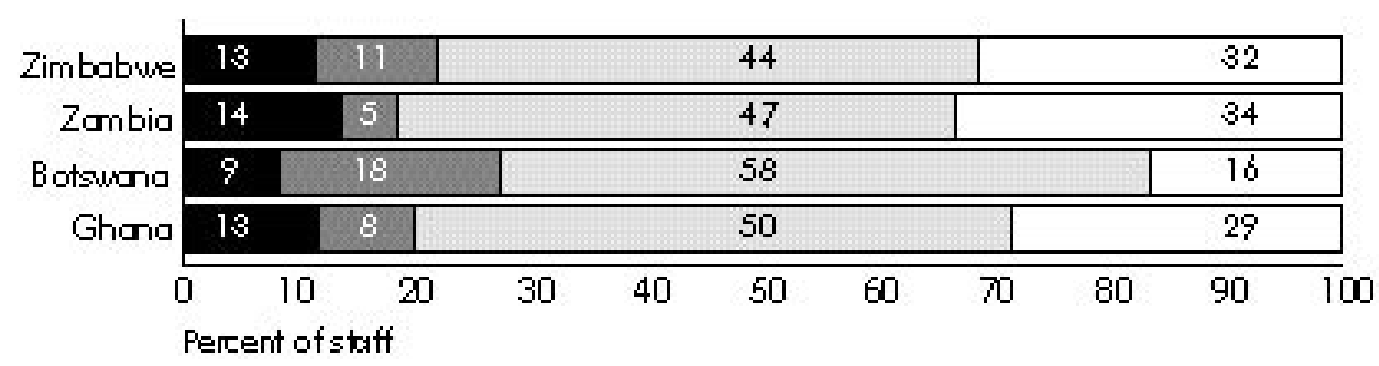

\footnotetext{
Q ver uncomfortable $\square$ someshiat uncomfortable

comfortable $\square$ ver comfortable

- Thie question woe not asked in Ke mpos.
} 


\section{Figure 10-7. Readiness of clinics to offer speculum pelvic examinations}

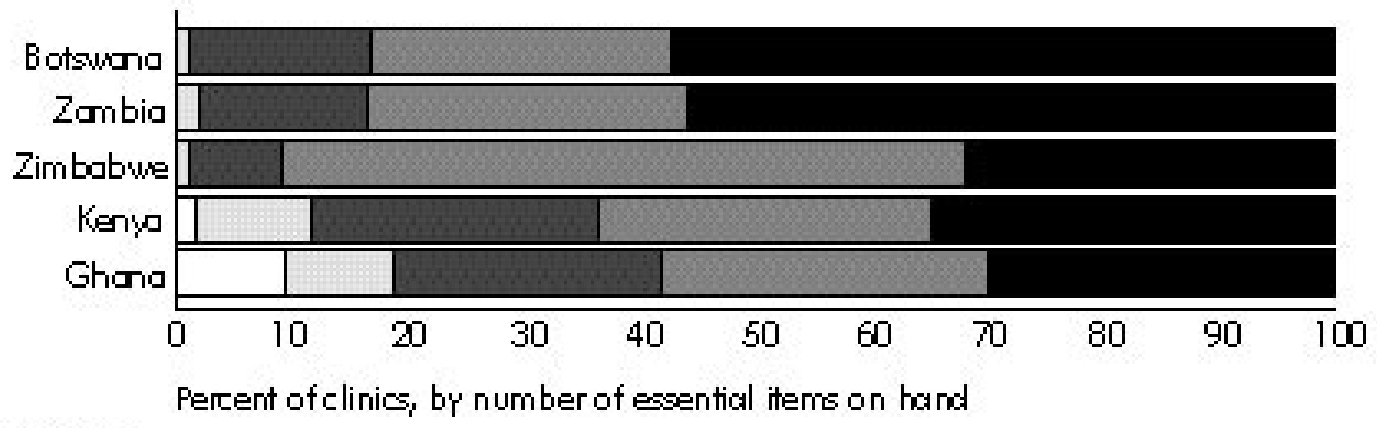

Number of

items: $\square$ none $\square$ one $\square$ tyo $\square$ three $\square$ four

management (see, e.g., Latif, 1996). Thorough counseling for a new family planning client should normally include such questions anyway, because knowing about a client's sexual behavior is important when deciding on the most appropriate type of contraceptive to use (see indicator Q.2-7 in Chapter 3). Table 10-6 shows the proportions of new family planning clients asked those questions that could also form part of an STI risk assessment procedure (programs vary in the information a provider is required to obtain from a new family planning client).

Marital status was the question most commonly asked. It is clearly the least difficult question to ask, as it does not require a discussion about sexual behavior or STIs. The other four questions (number of new partners in the last year, concerns about STIs, sexual relations, and history of STIs) were asked of smaller proportions of clients. This probably reflects either a perception by the provider that the client is not at risk and so need not be asked such questions, or the provider's embarrassment about discussing sexuality and STIs with family planning clients.

If staff are to effectively offer STI and HIV/AIDS information and services, they must feel comfortable discussing these subjects with their clients. Figure 10-6 indicates that on average, almost one-quarter of the staff interviewed felt very or somewhat uncomfortable when discussing these topics with their clients (see Maggwa et al., 1997b, for a discussion of this issue). This finding indicates the need to ensure that staff training includes how to discuss sexuality and STIs with clients.

To enable providers to undertake pelvic exams easily and in aseptic conditions, a facility must have available certain infrastructure, equipment, and supplies. A scale was created to measure the readiness of facilities to offer pelvic exams based on the availability of four essential items: a directable light source (either an angle poise lamp or flashlight), gloves (disposable or nondisposable), sterilizing lotion, and at least one speculum (see indicator R.5-2 in Chapter 3)..$^{10}$ The proportion of clinics that appear to be ready to offer a pelvic exam is shown in Figure 10-7. Overall, only $41 \%$ of clinics visited had all four essential items, and so could be judged fully ready to undertake a pelvic exam effectively and under safe conditions. A further $35 \%$ of clinics had three of the four items. The item most likely to be missing (in $40 \%$ of clinics) was the directable light source, and the item least likely to be missing (in $6 \%$ of clinics) was gloves; overall, only $17 \%$ of clinics were missing specula (although one-third of clinics in Ghana and onefifth of clinics in Zambia lacked this item).

Whether all new family planning clients should receive a pelvic exam, or only those accepting certain types of contraceptives (i.e., the IUD and sterilization), is a controversial issue. From the perspective of offering a client an integrated reproductive health consultation, a pelvic examination with a speculum would seem to be essential. Such an exam improves the likelihood of detecting an infection and, with rapid advances in simple 


\section{Figure 10-8. Per cent of new dients r eceiving a pelvic examination}

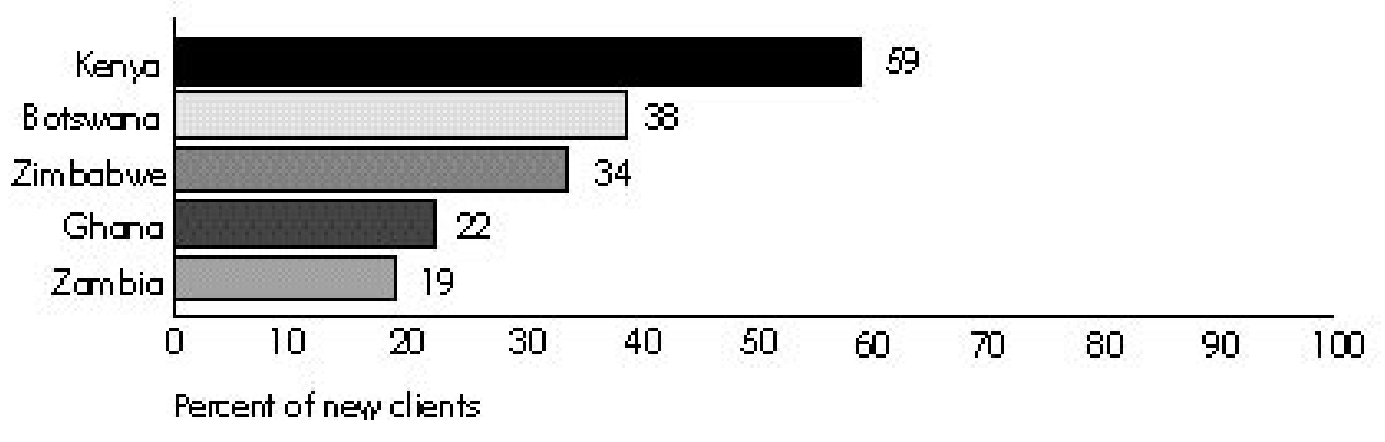

screening techniques, may increase the likelihood of early detection and management of cervical cancer (e.g., Chipato et al., 1995). However, unless infection prevention can be guaranteed, conduct of a pelvic exam for all but essential reasons is normally discouraged among family planning clients (Hatcher et al., 1997). (See also indicator Q.5-2 in Chapter 3.)

Figure 10-8 shows the proportion of clients who received a pelvic examination with a speculum. Explaining the variation across countries would require further analysis of factors such as national policies, clinic readiness to offer such exams, the methods accepted by clients, and staff training. The data do suggest, however, that all programs need to strengthen this procedure if it is to become a routine component of an integrated reproductive health service.

\section{Case Diagnosis and Treatment}

It is of little use to find potential STI cases among family planning and antenatal clients if these cases cannot then be correctly diagnosed and treated at the facility. ${ }^{11}$ Data given earlier in Tables 10-2, -3, and -4 show variability across the five countries in the readiness of their clinics and staff to offer STI diagnosis and treatment services. In recognition of the impossibility of equipping all health facilities to offer laboratory tests for specific STIs, syndromic management has been promoted as an alternative. Although the validity of this approach is currently being questioned (specifically for diagnosing and treating cervicitis through the vaginal discharge syndrome), all five countries had endorsed syndromic management at the time of these Situation Analysis studies. Staff training efforts were clearly

Table 10-7. Percent of facilities with on-site tests for specific infections

\begin{tabular}{lccccc}
\hline Facilities & HIV & Syphilis & Gonorrhea & Candidiasis & Chlamydia \\
All facilities & 9 & 21 & 18 & 15 & 9 \\
\hline Hospitals & 47 & 60 & 63 & 51 & 36 \\
\hline Health centers & 3 & 25 & 18 & 15 & 8 \\
\hline Health posts & 1 & 7 & 4 & 3 & 2 \\
\hline Botswana & 7 & 16 & 11 & 10 & 7 \\
\hline Ghana & 12 & 10 & 18 & 13 & 8 \\
\hline Kenya & 10 & 23 & 24 & 20 & 6 \\
\hline Zambia & 12 & 37 & 26 & 22 & 18 \\
\hline Zimbabwe & 0.5 & 22 & 7 & 7 & 16 \\
& & & & &
\end{tabular}


at an early stage, with a maximum of one-third of staff indicating that they had received post-basic or refresher training in syndromic management. However, a larger proportion of staff $(85 \%$ in Zimbabwe) claimed to have carried out STI diagnosis in the previous 3 months.

This is not to say that laboratory tests for STIs are not available at all. Table 10-7 indicates that almost one-fifth of facilities at all levels can offer syphilis and gonorrhea testing on site, although fewer than $10 \%$ offer HIV testing. As would be expected, the availability of tests varies by type and location of facility. For example, almost twothirds of hospitals offer on-site tests for syphilis and gonorrhea, about half offer HIV and candidiasis testing, and one-third offer chlamydia testing. On-site syphilis tests are available at only onequarter of the health centers and $7 \%$ of the health posts. As would be expected, private-sector facilities are the best equipped, and NGO facilities are better equipped than are public-sector facilities. Also, facilities in urban locations have more tests available than those in rural areas. The facilities in Zambia are the best equipped for all types of onsite tests, whereas those in Zimbabwe score very low for HIV tests (only $1 \%$ of all facilities visited), as well as for gonorrhea and candidiasis testing.

Treating a client who has a reproductive tract infection can be problematic in many African countries because on-site drug supplies are often not available. As a result, providers frequently must use alternative medications, often of a quality inferior to that of the recommended regimens, or send clients elsewhere to obtain the correct medication. Data from the Situation Analysis studies show that many health facilities often do not have available the first-line drugs recommended in their service provider guidelines for the management of vaginal discharge.

In Kenya, for example, a combination of Amoxycillin, Augmentin, and Erythromicin is recommended as the first-line treatment for cervicitis in pregnant women, yet Amoxycillin and Erythromicin were available at just $11 \%$ and $12 \%$ of health facilities, respectively. In Botswana, a country often cited as having few drug supply problems, Doxycycline and Ceftriaxime (both of which are recommended as first-line drugs for the treatment of cervicitis) were available at only $37 \%$ and $45 \%$ of facilities, respectively. The situation in Zimbabwe was better than that in the other countries, probably because of that country's ongoing STI program (supported by the World Bank and the Department for International Development), which provides drugs and supports the logistics of their distribution. The service provider guidelines in Zimbabwe (revised in 1997) recommend the use of Kanamycin, Cotrimoxazole, and Doxycycline as first-line drugs for the treatment of vaginal discharge, and these drugs were available at $92 \%, 87 \%$, and $64 \%$ of health facilities, respectively. These findings show, therefore, that the availability of STI drugs at health facilities varies from country to country, and is influenced by the availability of resources needed to purchase the drugs and the logistics required to ensure appropriate distribution.

\section{WHAT DO CLIENTS KNOW ABOUT STI AND HIV/AIDS SYMPTOMS, TRANSMISSION, AND PROTECTION? ${ }^{12}$}

Knowledge that diseases can be transmitted through sexual intercourse is high $(91 \%$ of all $\mathrm{MCH}$ clients and $93 \%$ of family planning clients). However, when asked whether they knew of any symptoms of diseases that can be transmitted sexually, 39\% of all $\mathrm{MCH}$ clients and $35 \%$ of family planning clients could not name any symptoms at all. The situation was particularly poor in Ghana, where only $41 \%$ of $\mathrm{MCH}$ clients and $50 \%$ of family planning clients could name at least one symptom. Of those clients who did know of any symptoms, most could cite only one or two. Figure 10-9 indicates that overall, the most common symptom cited as known was lesions/sores, followed by abnormal discharge and painful urination.

There are major differences among countries. For example, although the most cited symptom was lesions/sores in the three Southern African countries (Botswana, Zambia, and Zimbabwe), it was painful urination in Ghana and abnormal discharge in Kenya. Whether this variation reflects differences among countries in the prevalence of diseases and their symptoms or different perceptions 


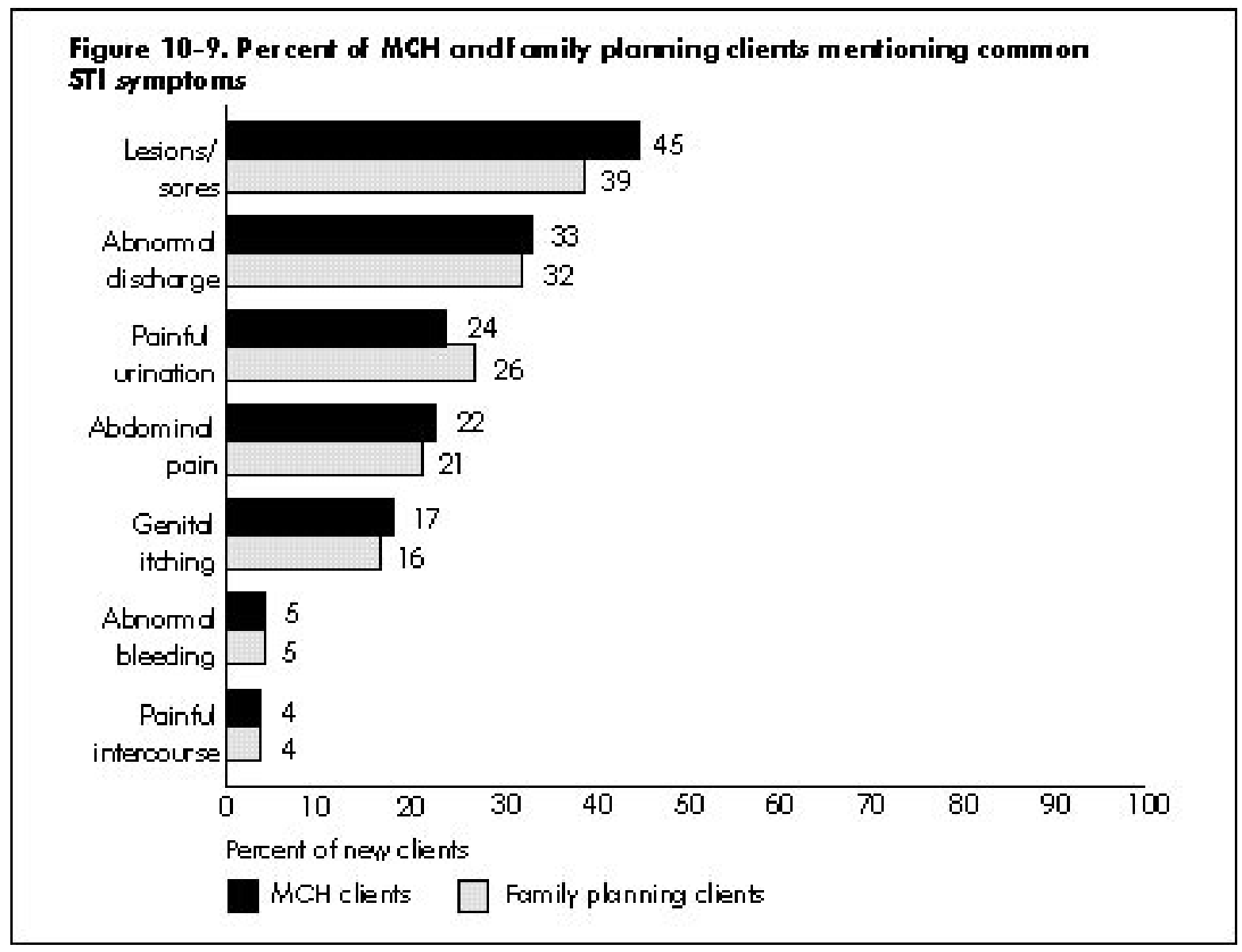

of what constitutes a symptom of an STI is not clear. This finding indicates the need for program managers to have greater knowledge of local perceptions, terminology, and experiences with STI symptoms so they can better train their staff in counseling and in case finding and diagnosis.

Knowledge of HIV / AIDS was found to be virtually universal among $\mathrm{MCH} /$ family planning clients. When asked whether they knew ways of contracting HIV/AIDS other than through sex, half of the $\mathrm{MCH} /$ family planning clients indicated that they knew of at least one other way (Figure 10-10). By far the most commonly mentioned mode of transmission was sharing needles, which probably reflects the messages being communicated by the national AIDS control programs in these countries. It is somewhat troubling, however, that so few women attending $\mathrm{MCH} /$ family planning clinics are aware of the possibility of perinatal HIV transmission.

Ways of protecting oneself against contracting an STI, including HIV/AIDS, were found to be well known: $86 \%$ of all MCH clients and $90 \%$ of family planning clients could mention at least one. Figure 10-11 shows the frequency with which the four main means were mentioned. There was some variation across countries in which means of protection were most frequently cited. In Ghana, Zambia, and Zimbabwe, $70 \%$ to $90 \%$ of clients mentioned being faithful to their partners. While this behavior is to be encouraged, given that for most $\mathrm{MCH} /$ family planning clients (who are almost exclusively women) it is their male partner who is the most likely source of infection, personal fidelity is probably less important than partner fidelity. Yet encouraging partner fidelity was mentioned by only one-quarter of respondents, perhaps reflecting women's perception that this is not an easy option to exercise in most relationships. Rather troubling is the fact that only one-half of respondents mentioned using condoms, although the proportion varied from over $90 \%$ in Botswana (where it was the most mentioned means) to about 


\section{Figure 10-10. Percent of MCH andfamily planring dients knowing ways of}

cont acting ST $s$, indu ding HIV/AIDS

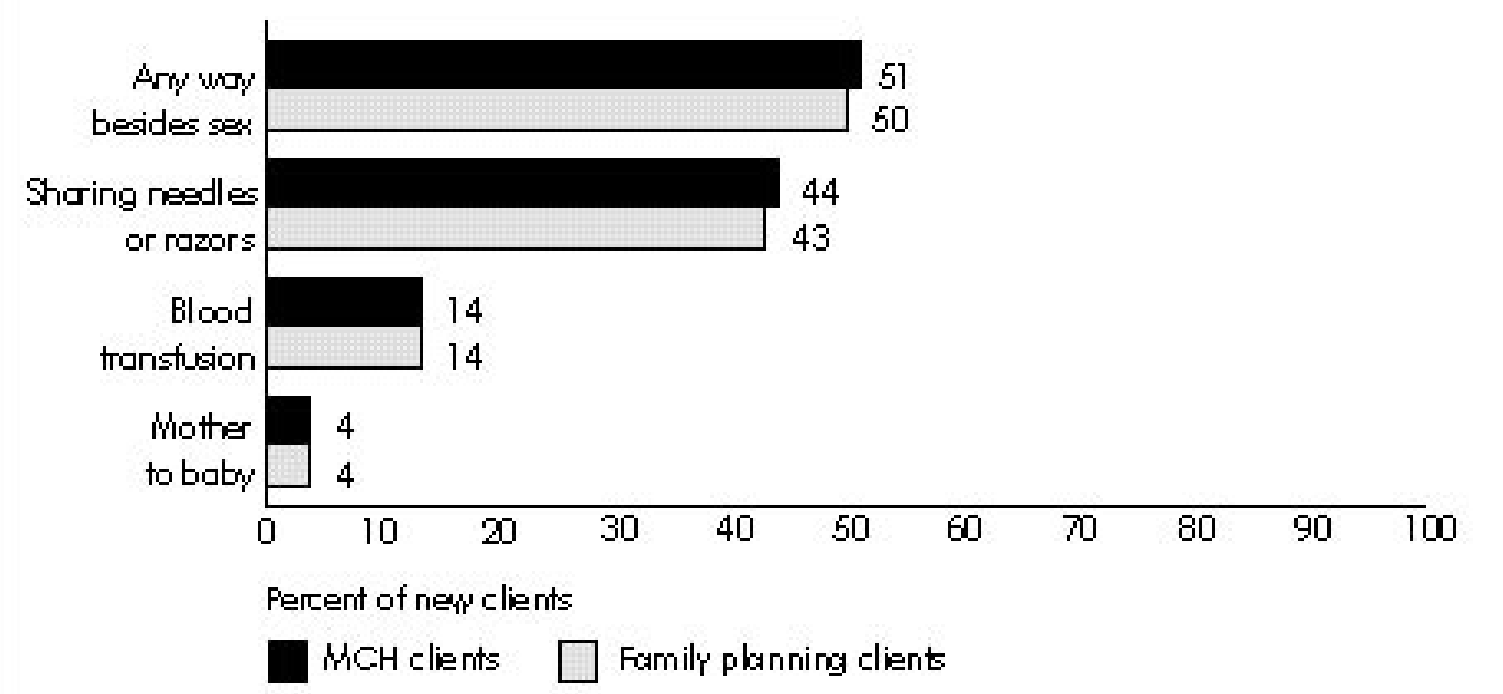

one-quarter in Ghana. Condom promotion was discussed earlier, and clearly programs are having different levels of success in reaching $\mathrm{MCH} /$ family planning clients with this message.

\section{CONCLUSIONS AND RECOMMENDATIONS}

The ICPD Program of Action promotes an expanded role for $\mathrm{MCH} /$ family planning facilities in providing a range of reproductive health services. This chapter has reviewed findings from representative samples of facilities within public-, NGO-, and private-sector programs in five countries of subSaharan Africa shortly after the ICPD to ascertain the feasibility of expanding their role to include information and services for STIs, including HIV / AIDS. Based on the results of the Situation Analysis studies, the following conclusions and recommendations can be offered:

- At the time of the surveys, less than one-third of staff had received refresher or post-basic training in STIs and/or HIV/AIDS, although over half claimed to be providing STI or HIV/AIDS information and services. Clearly there is a need to implement extensive programs of more comprehensive basic and regular refresher training for staff if these services are to be fully integrated with $\mathrm{MCH} / \mathrm{fam}$ ily planning services. Since these Situation Analysis studies were conducted, all five countries have initiated such training programs.

- Observations of client-provider interactions indicate that about one-quarter of clients are receiving some information about STIs and/or HIV/AIDS during their MCH or family planning consultation. Clearly consultations with $\mathrm{MCH}$ and family planning clients can be an appropriate and acceptable setting for communicating messages about STIs and HIV / AIDS. Efforts should be made to strengthen this aspect of the consultation without infringing on the quality of the $\mathrm{MCH}$ or family planning service being provided.

- Clients' ability to name STI symptoms is generally poor, their knowledge of means of HIV/AIDS transmission other than sex is limited primarily to needles and sharp implements, and personal fidelity is the most wellknown means of transmission prevention. These findings suggest that within a broader range of general communication strategies, those providing information and counseling within $\mathrm{MCH}$ / family planning clinics should (1) place more emphasis on educating women 


\section{Figure 10-11. Percent of MCH andfamily planning dients knowing ways to

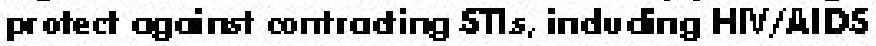

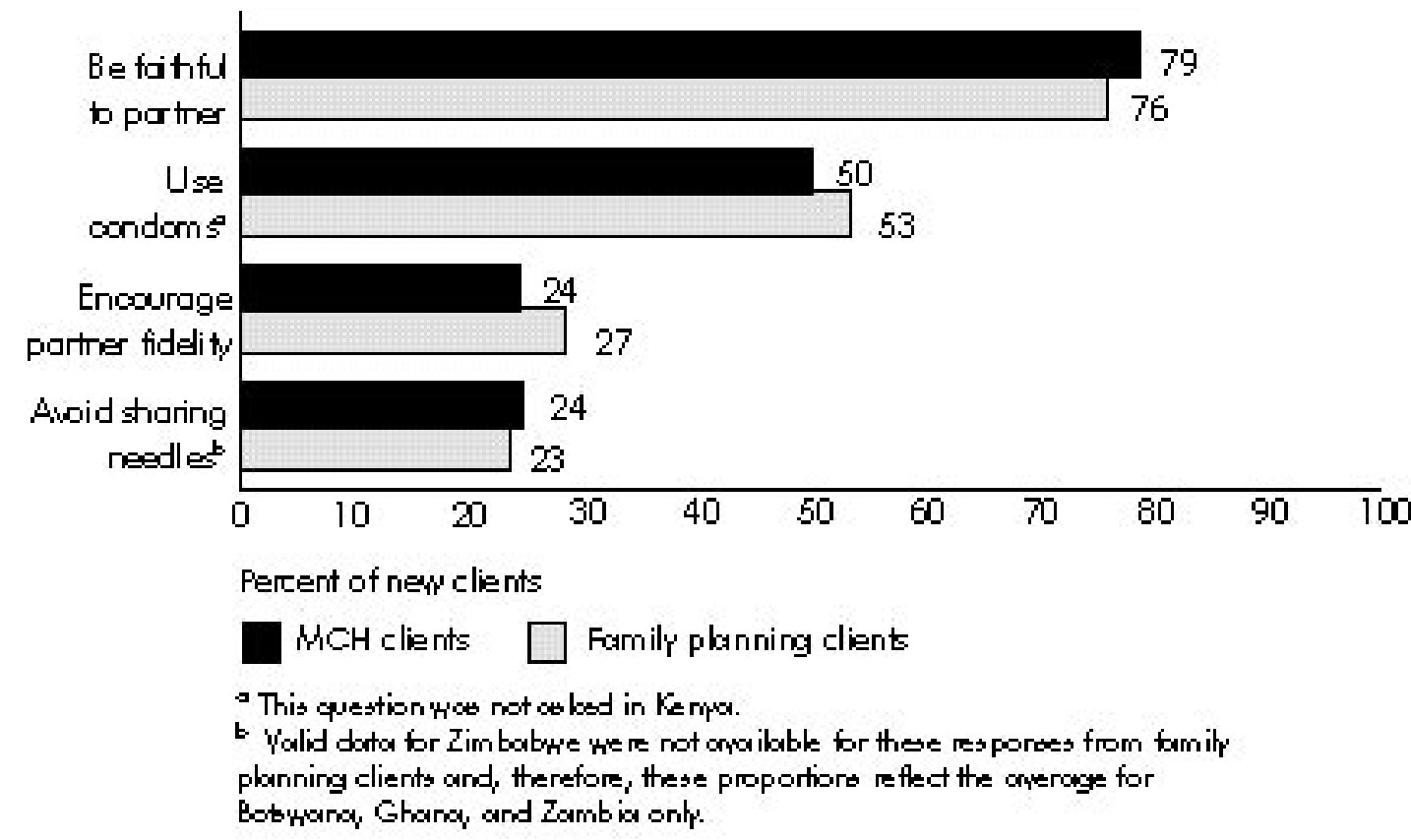

about potential STI symptoms (thus increasing the likelihood that clinic staff will be able to diagnose a symptomatic STI), (2) highlight the likelihood of perinatal transmission of HIV by infected women as an important mode of infection, and (3) stress the need for condom use and/or partner fidelity as the most important ways of preventing infection.

- Apart from posters, other means of communicating information about STIs or HIV/AIDS (e.g., flip charts, brochures, group health talks) are lacking in most clinics. Visual materials and verbal presentations have long been an integral part of communicating $\mathrm{MCH}$ and family planning messages, although a lack of print materials on these topics is also a longstanding problem. Attention needs to be paid to designing, producing, and distributing appropriate IEC materials, especially aides to guide verbal presentations on sensitive topics such as sexuality and reproductive tract infections.

- Condoms are available at virtually all clinics, and stockouts are rare. With marked variations among countries, however, condoms appear to be promoted primarily for family planning purposes, with fewer than one-fifth of clients hearing a dual-protection message. Encouraging and expecting staff to always mention the condom, its dual-protection role, and its availability during family planning consultations would appear to be a simple intervention that could have significant effects on use of this method by $\mathrm{MCH} /$ family planning clients.

- Virtually no family planning clients undergo any STI case detection procedures or HIV/AIDS management services. Although STI case finding, diagnosis, and treatment are rarely undertaken, there is scope for these procedures to be incorporated more systematically into a family planning consultation. For example, observations of client-provider interactions suggest that some degree of history taking, symptom identification, risk assessment, and physical examination is taking place, and could be strengthened to increase the likelihood of successful STI case detection. To do so would require thorough staff training and a reorientation of conventional consultation procedures for managing new family planning clients. 
Evidence from case studies that have tried this approach (Maggwa and Askew, 1997a), however, suggests that any such effort would need to be implemented very systematically and with substantial supervision.

- Evidence from at least one country (Zimbabwe) suggests that a well-supported and -organized STI drug supply program can ensure the availability of basic medications needed to treat STIs at most clinics; however, this situation appears to be the exception rather than the rule in most sub-Saharan African countries. Easy access to affordable and effective treatment for those found to be infected is crucial to ensure that complications are minimized, and the likelihood of further transmission is reduced. How such drug supply programs can be implemented and sustained is not well known, however.

These results need to be considered in the context of the current debates on the objectives and assumptions underlying the promotion of integration (as stated in the introduction). Currently, there appears to be agreement that communicating information to women about symptom recognition, modes of transmission, and protective behaviors is feasible within $\mathrm{MCH} /$ family planning clinics, and efforts to this end should be strengthened. Moreover, in theory at least, this strategy for changing behavior is expected to help prevent transmission of STIs/HIV, thereby reducing levels of STI/HIV infection in the general population, although such a behavior-change strategy urgently needs evaluation. The major question is whether STI case finding and management should always be a component of an integrated approach to service delivery at $\mathrm{MCH} /$ family planning clinics. Clearly, it is an essential component of a client-centered approach that seeks to alleviate immediate suffering and to prevent complications caused by such infections in individual women and their infants. However, the syndromic approach, which is currently used to detect and manage STI cases by most programs in sub-Saharan Africa, has been shown to have poor sensitivity and positive predictive values when used to manage vaginal discharge (a common syndrome in women), especially when the discharge is due to cervicitis. Therefore, for STI case finding and management to have the desired impact, there is need for improvements in the syndromic approach and/or development of more cost-effective alternative approaches. It is also questionable whether STI case finding and management in $\mathrm{MCH} /$ family planning clinics has much if any effect on reducing the transmission of infection in the general population. This is because most clients are primarily monogamous and are more likely to receive an infection from their regular partner than to transmit it to others. Moreover, unless the partner is also treated, the likelihood of the woman becoming reinfected is high.

In summary, operations research is needed to explore the effectiveness and cost-effectiveness of different approaches to integrating the communication of information on STIs and HIV/ AIDS as well as STI case finding and management into $\mathrm{MCH} /$ family planning clinic procedures. Moreover, research must be undertaken into the impact of such approaches on sexual behavior and on infection transmission and incidence, in both the population of women attending $\mathrm{MCH} /$ family planning clinics and the population resident in the clinic catchment areas. Without this information, policy makers do not have a sufficiently clear basis on which to promote clinic-based integration as a means of reducing STI and HIV transmission.

\section{REFERENCES}

Askew, I., N. Maggwa, and L. Kangas. 1998.

"Integrating STI and HIV / AIDS services into $\mathrm{MCH} / \mathrm{FP}$ programs in East and Southern Africa." Paper presented at the Annual Meeting of the Population Association of America, Chicago.

Bryson, Y. 1996. "Perinatal HIV-1 transmission: Recent advances and therapeutic interventions." AIDS, 10 (Supplement):S33-S42.

Chipato, T., M. Chirenje, P. Blumenthal, L. Gaffikin, and H. Sanghvi. 1995. "Cervical cancer screening pilot study in Zimbabwe." Unpublished report, JHPIEGO, Johns Hopkins University, Baltimore.

Hatcher, R., W. Rinehart, R. Blackburn, and J. Geller. 1997. The Essentials of Contraceptive Technology. Johns Hopkins School of Public Health, Population Information Program, Baltimore.

Howson, C., P. Harrison, D. Hotra, and M. Law, Eds. 1996. In Her Lifetime: Female Morbidity and Mortality 
in Sub-Saharan Africa. National Academy Press, Washington, D.C.

Iskander, M., J. Patten, S. Qomariyah, C. Vickers, and S. Molyneaux. 1998. Improved Reproductive Health and STI Services for Women Presenting to Family Planning Services in North Jakarta. Asia and Near East Operations Research and Technical Assistance Project and Indonesian Ministry of Health, Population Council, Jakarta, Indonesia.

Latif, M. 1996. A Report on a Study to Determine the Aetiology and Patterns of STDs amongst Men and Women Presenting to Health Centres in Harare, Zimbabwe and to Determine Risk Factors for Cervicitis among Symptomatic and Asymptomatic Women. University of Zimbabwe, Harare, Zimbabwe.

Maggwa, N. and I. Askew. 1997a. Integrating STI/HIV Management Strategies into Existing MCH/FP Programs: Lessons from Case Studies in East and Southern Africa. Africa Operations Research and Technical Assistance Project II, Population Council, Nairobi, Kenya.

Maggwa, N., N. Rutenberg, and I. Askew. 1997b. “A challenge to integrating STI/HIV management strategies into existing MCH/FP programs in East and Southern Africa: Talking about sex and sexually transmitted diseases." Paper presented at the Annual Meeting of the American Public Health Association, Indianapolis, Indiana.

Mayhew, S. 1996. "Integrating MCH/FP and STI/HIV services: Current debates and future directions." Health Policy and Planning, 11,4:339-353.

Ndhlovu, L. 1998. "Lessons learned from Situation Analysis studies in Africa." Paper presented at the Annual Meeting of the Population Association of America, Chicago.

Tsui, A., J. Wasserheit, and J. Haaga, Eds. 1997. Reproductive Health in Developing Countries: Expanding Dimensions, Building Solutions. National Academy Press, Washington, D.C.

\section{NOTES}

1 The term "sexually transmitted infection" is used here instead of "sexually transmitted disease" to reflect the need for healthcare programs not only to diagnose and treat the symptoms of sexually transmitted diseases, but also to prevent transmission of infection.

2 All results presented are for the times of data collection cited above.

3 It should be noted that this information was collected as part of a nonspecific question in which the family planning or $\mathrm{MCH}$ client was asked whether she had received any other services during her visit. It is also likely that some clients interpreted this to mean receiving treatment rather than information or counseling.

4 This question was not asked in Botswana.

5 There have been two Situation Analysis studies in Zimbabwe, in 1991 and 1996, and there has actually been a decrease in this proportion over time (the proportion in 1991 was 39\%). Paradoxically, Zimbabwe appears to have one of the highest per capita rates in the world for condom distribution through the public sector. Thus although $\mathrm{MCH} /$ family planning clinic staff do not appear to be discussing condoms with their clients, the clinic seems to be an acceptable and effective source for this method.

6 Only $3.5 \%$ of clients who were told that condoms protect against STIs were not told about them as a contraceptive method.

7 Cervical caps, female condoms, and, to a lesser extent, spermicides also provide dual protection, but are not widely available in African family planning programs.

8 Risk assessment involves asking the client about her sexual behavior and that of her partner(s).

9 The observation guide does not distinguish how the information was obtained, nor does it record whether the woman actually had the symptom; the intention is to measure whether these symptoms were discussed.

10 A slightly different approach to measuring readiness for undertaking a pelvic exam is presented in Chapter 3, which includes the presence of a couch as an essential item.

11 While referral of potential cases to other facilities for diagnosis and treatment is an important and necessary option when these services are not available on site, such an action is not within the spirit of offering women integrated services.

12 All data presented in this section are drawn from a series of open-ended questions to clients, and thus the responses were spontaneous. This approach probably led to some underreporting of knowledge.

The analysis in this chapter was jointly supported by the Population Council's HORIZONS Project and the Population Council's Africa Operations Research and Technical Assistance Project II. HORIZONS is funded by the United States Agency for International Development (USAID), Cooperative Agreement No. HRN-A00-97-00012-00. The Africa Operations Research and Technical Assistance Project II is also funded by USAID, Contract No. CCP-3030-C-00-3008-00. 


\section{KEY POINTS AND CONCLUSIONS Changes in Quality of Services Over Time}

In several sub-Saharan African countries, two or more Situation Analysis studies have been carried out several years apart. Comparison of the results of these studies reveals the changes in service quality that have occurred in the interval, and thus can be used to evaluate program interventions that have taken place. Such comparisons in Burkina Faso, Ghana, Kenya, and Zambia lead to several overall conclusions about how family planning and reproductive health services are changing:

- Choice of methods shows slight or partial improvements in all four countries. These improvements take the form of availability of an expanded range of methods, increased number of methods mentioned to clients, or larger numbers of clients being asked about method preference.

- All four countries are making some progress in increasing the availability of IUDs, NORPLANT ${ }^{\oplus}$, and/or permanent methods. At service delivery points that offer these methods, not all elements-training, equipment, technical competence, and counseling-required to offer the methods are fully in place in any of the countries, but efforts are driving in that direction.

- The quality of family planning counseling is generally low and is declining or stagnant in all four countries. This particular element of quality is suffering markedly more than technical competence or mechanisms to ensure continuity, and does not appear to be directly related to training interventions.

- The few indicators that address sexually transmitted disease (STD)/HIV integration issues either declined or remained constant over time. The prevalence of STDs/HIV in these contexts lends an extra urgency to these findings.

- These studies testify to the complexities involved in achieving real increases in quality of care. Improvements in the readiness of a program do not necessarily translate to improvements in the quality of care delivered, even if the changes are in the form of increased training or supervision.

These studies are limited in their ability to support evaluation of program interventions because they lack an experimental design in which the effect of one or more specific interventions is tested and the pathways between interventions and service quality improvements are clarified. As additional Situation Analysis studies are undertaken on a more regular basis in the future, the opportunity presents itself to design these evaluations more productively in this regard. 


\section{1}

\section{Changes in Quality of Services Over Time}

\section{Kate Miller ${ }^{1}$}

In several sub-Saharan African countries, two or more Situation Analysis studies have been carried out several years apart. Whereas each study stands on its own as a description of the readiness and quality of services at a particular point in time, comparison of the results can also reveal the changes in service quality that have occurred over the interval. This chapter summarizes the results of four such comparisons over time in Burkina Faso, Ghana, Kenya, and Zimbabwe. ${ }^{2}$

Each of these comparisons had certain specific objectives, but they all shared two common goals:

- To describe the changes in service quality over time

- To use that information to evaluate the activities that took place between the two studies

The first of these goals was achieved by all the studies included here. In each case, program managers selected a subset of indicators for the comparison. The selection of these indicators was affected in part by changes to the Situation Analysis methodology over the interval: in some cases, the study instruments had evolved significantly, and some indicators were not measured at both time periods. Also, the various program managers defined their indicators of interest based on their own service standards and research needs. For this reason, the indicator lists are different for each of the four countries.

The second goal represents a more powerful use of the comparative data, because it allows program managers to identify the most effective interventions and redesign those that are not effective. For example, if a large training program on family planning counseling had taken place between the two studies, the results would show whether the quality of counseling had improved, remained steady, or declined during the interval. All else being equal, if the quality of counseling among trained providers had improved, one could conclude that the training curriculum had probably had a positive effect. If the quality of counseling had remained steady or declined, however, one could conclude that the training curriculum might not have been effective and should be further investigated. Because resources are scarce for many programs, identifying the most effective interventions is particularly critical. (See Chapter 4 for more discussion on using this type of information.)

The study designs for these comparisons greatly affect their ability to assist in evaluating interventions. Under the best design, the first Situation Analysis would be carried out as a baseline study. An intervention to improve some aspect of services would then be carried out among a randomly selected group of service delivery points (SDPs) (the experimental group). All other SDPs (the control group) would carry out normal program activities. The second Situation Analysis would then measure the quality of service among the experimental and control groups, showing with some rigor whether the intervention had been effective in improving services.

This design is often difficult to carry out in the field, however, because of budgetary, methodological, and logistical difficulties. ${ }^{3}$ Most commonly, program managers would prefer to carry out interventions on predefined groups, such as large hospitals, clinics in particular geographic regions, or clinics targeted for improvements by donors, rather than on a randomly selected group of SDPs. In the former case, however, the observed changes in quality may be due to some factor other than the intervention, and it becomes much more difficult to achieve the goal of attributing increases or decreases in 
quality to the intervention alone. In all four cases described here, the Situation Analysis studies were undertaken as two separate activities, so their comparability rests largely on their sampling plans, which are described in Annex 11-1, and their instruments. Three of the four pairs of studies were used to evaluate interventions as responsibly as possible given the study design.

In this chapter, each of the four countries is addressed in turn. Background to the studies, program activities between the studies, results, and discussion are provided for each. The concluding section summarizes the results for the four countries.

\section{BURKINA FASO}

\section{Background}

Burkina Faso's family planning program was established in 1985, but by 1993 the contraceptive prevalence rate (CPR) for modern methods among all women was still only $4 \%$, and the total fertility rate (TFR) was above 6 , although declining. In 1991, an ambitious population policy was adopted, with the objectives of raising the CPR to $60 \%$ by the year 2000 and lowering fertility by $10 \%$ every 5 years after 2005, while respecting the fundamental rights of couples and individuals to decide the size of their families. The family planning strategy involved initiation of services at a small number of SDPs around the country, followed by expansion to other existing SDPs. In 1992, there were about 100 to 150 SDPs offering family planning services. The 1992 Situation Analysis study was carried out by the Ministry of Health $(\mathrm{MOH})$ to provide information about the actual functioning of the program before the expansion and program improvements took place.

Until 1993, by far the largest funding source for interventions and improvements to the Burkina

\section{Table 11-1. Changes in readiness indicators in Burkina Faso}

\begin{tabular}{|c|c|c|c|}
\hline Readiness Indicators & 1992 & 1995 & Significant? \\
\hline \multicolumn{4}{|l|}{ Infrastructure/ Facilities } \\
\hline B-R.1 \% of SDPs with a comfortable waiting room & 89 & 89 & \multirow[b]{3}{*}{$\Downarrow$} \\
\hline B-R. $2 \%$ of SDPs with a toilet for clients & 81 & 83 & \\
\hline B-R.3 \% of SDPs with a satisfactory examining area & 60 & 21 & \\
\hline \multicolumn{4}{|l|}{ Methods O ffered } \\
\hline B-R. $4 \%$ of SDPs that offer the complete range of methods & 24 & 21 & \\
\hline \multicolumn{4}{|l|}{ Contraceptive Supplies and Logistics } \\
\hline B-R.5 \% of SDPs with appropriate stocking areas & 83 & 79 & \\
\hline \multicolumn{4}{|l|}{ Equipment } \\
\hline $\begin{array}{l}\text { B-R. } 6 \% \text { of SDPs with the minimum necessary equipment } \\
\text { for family planning services }\end{array}$ & 9 & 19 & \\
\hline \multicolumn{4}{|l|}{ Staffing } \\
\hline $\begin{array}{l}\text { B-R. } 7 \% \text { of providers who have received training in } \\
\text { clinical family planning and IEC skills }\end{array}$ & 43 & 11 & $\Downarrow$ \\
\hline \multicolumn{4}{|l|}{ IEC Materials and Activities } \\
\hline $\begin{array}{l}\text { B-R. } 8 \% \text { of SDPs with at least one poster and one } \\
\text { brochure on } \mathrm{MCH} / \text { family planning }\end{array}$ & 28 & 18 & $\Downarrow$ \\
\hline \multicolumn{4}{|l|}{ Recordkeeping and Supervision } \\
\hline $\begin{array}{l}\text { B-R. } 9 \% \text { of SDPs that have received at least one } \\
\text { supervisory visit in the last } 6 \text { months }\end{array}$ & 57 & 25 & $\Downarrow$ \\
\hline
\end{tabular}




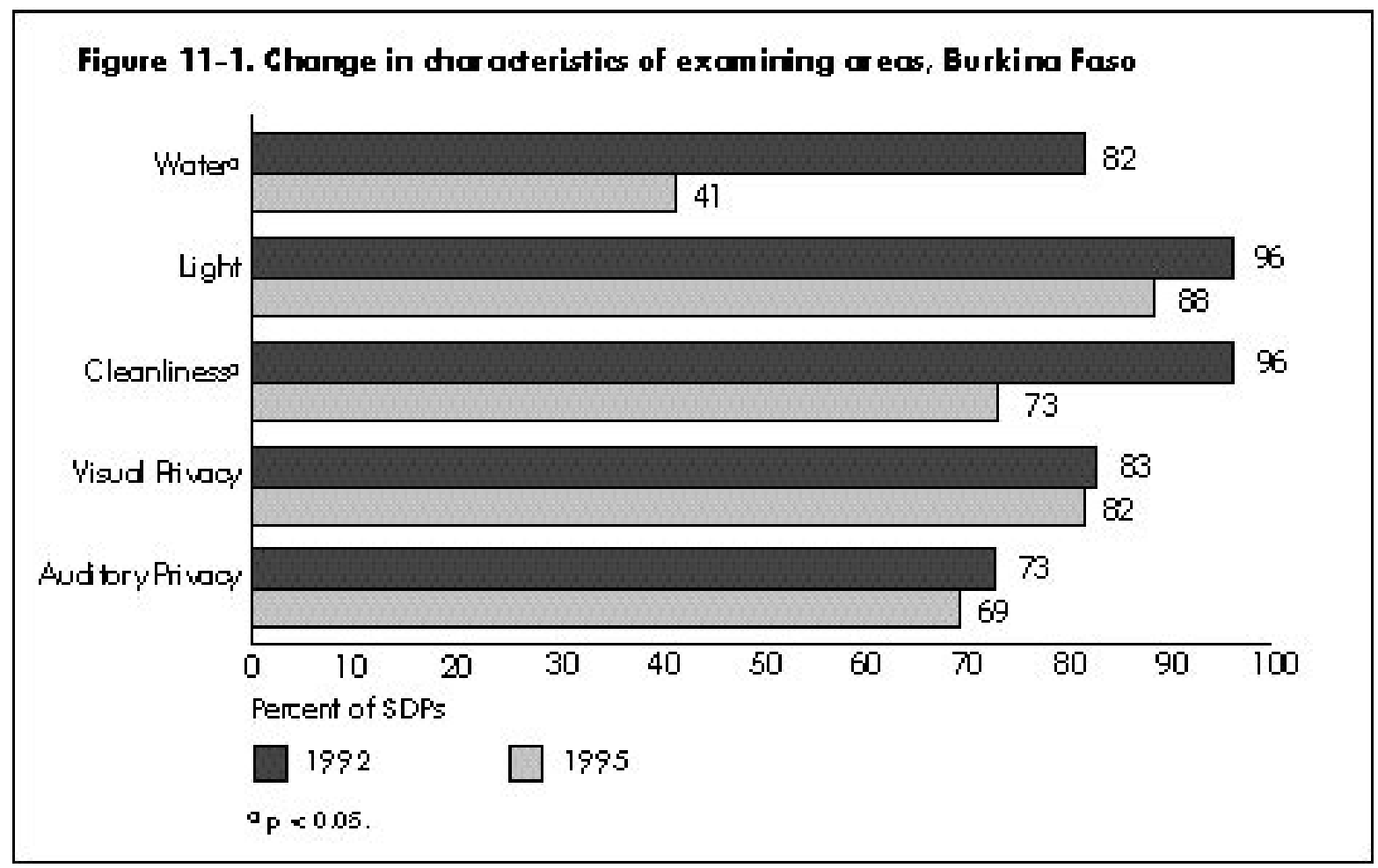

Faso family planning program was the United States Agency for International Development (USAID). However, USAID began phasing out its activities in Burkina Faso in 1993 and had ceased all activities by September 1995. In 1995, the United Nations Population Fund (UNFPA) began some activities, but on a much smaller scale than those of USAID. Despite this decrease in donor activity, however, by 1995 family planning services had been introduced into 585 SDPs throughout the 30 provinces of the country. This was an extraordinary increase in service availability in just 3 years, and the $\mathrm{MOH}$ undertook a second Situation Analysis study in 1995 to evaluate these new services.

Since the two Situation Analysis studies were not part of an experimental design, conclusions about the effects of interventions undertaken during the interval between the studies or about USAID's withdrawal cannot be drawn statistically, only anecdotally.

\section{Program Activities Between the Studies}

Information from the first Situation Analysis study was used to design several interventions, emphasizing primarily training. Since family plan- ning is covered in the basic training of midwives and nurses at the National School of Public Health, the interventions focused on post-basic training curricula in clinical family planning; information, education, and communication (IEC); management; and maternal and child health $(\mathrm{MCH})$ services. The length of these courses varied from 3 to 6 weeks according to the type of staff involved. Although the majority of post-basic training had previously taken place abroad, these courses were held in Burkina Faso. The Program for International Training in Health (INTRAH), the Johns Hopkins Program for International Education in Reproductive Health (JHPIEGO), and the Family Planning Service Expansion and Technical Support (SEATS) Project contributed significantly to these efforts.

In addition to the new training, the World Bank, UNFPA, and other partners in development assisted with distributing the minimum standard equipment to SDPs in certain provinces of the country. Also, the Bamako Initiative was implemented to decentralize health services in almost all provinces of the country. Health districts were established, directors were trained, and management committees were installed in individual SDPs, with 
Figure 11-2. Change in minimum equipment, Burkina Faso

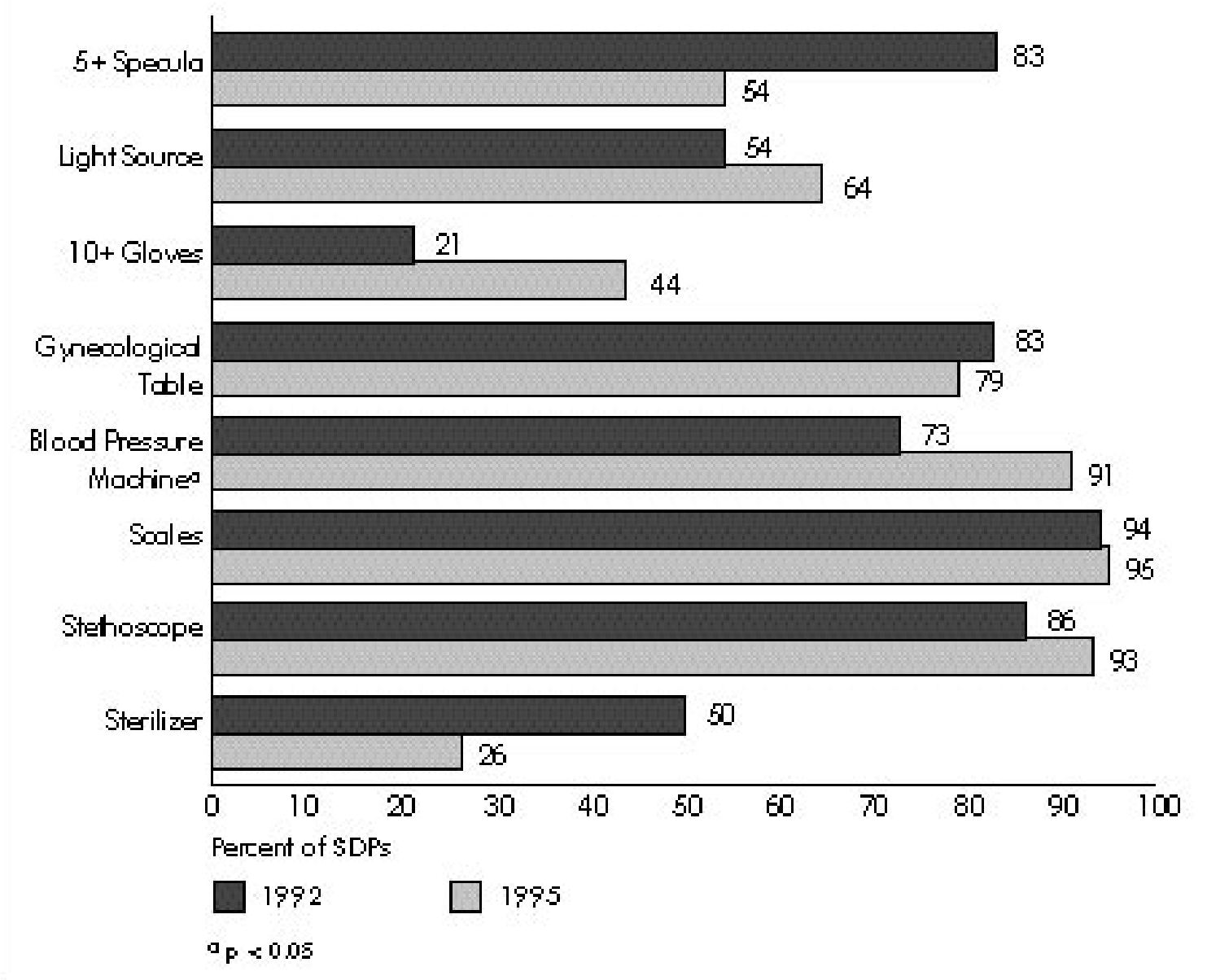

financial and technical assistance from SEATS. Moreover, to foster the promotion of family planning services, health talks and film screenings were organized at SDPs. In addition, SDPs were given posters on $\mathrm{MCH} /$ family planning, and television advertisements and radio bulletins were produced and aired. These activities benefited from the financial and technical assistance of the Department of Communication of The Johns Hopkins University, Population Communication Services.

\section{Results}

The results for all readiness indicators are presented in Table $11-1 .{ }^{4}$ Of the nine indicators, four showed a significant decline between 1992 and 1995, and the remaining five showed no significant change.
In both 1992 and 1995, the vast majority of SDPs had a comfortable waiting room and toilet for clients (B-R.1 and B-R.2). For indicator B-R.3, a "satisfactory" examining area is defined to include auditory privacy, visual privacy, cleanliness, sufficient light, and sufficient water. Figure 11-1 illustrates the change in these five components of examination rooms, and shows that cleanliness and water supply declined considerably, accounting for the drop in the overall indicator. ${ }^{5}$

The "complete range of methods" under B-R.4 is defined according to the type of facility. Smaller facilities are expected to offer at least combined oral contraceptives (COCs), injectables, condoms, and spermicides; midsize facilities are expected to offer these methods as well as IUDs; and hospitals should offer all of the above, as well as tubal ligation. ${ }^{6}$ The result shows no significant change in the 


\section{Table 11-2. Changes in quality indicators in Burkina Faso}

\begin{tabular}{|c|c|c|c|}
\hline Quality Indicators & 1992 & 1995 & Significant? \\
\hline $\begin{array}{l}\text { Interpersonal Relations } \\
\text { B-Q.1\% of new clients who received a friendly greeting }\end{array}$ & 71 & 100 & $\Uparrow$ \\
\hline $\begin{array}{l}\text { Information Taken from Clients } \\
\text { B-Q.2\% of new clients who were asked about their } \\
\text { reproductive intentions } \\
\text { B-Q.3\% of new clients who were asked about previous } \\
\text { experience with contraceptive methods }\end{array}$ & 53 & 25 & $\Downarrow$ \\
\hline $\begin{array}{l}\text { Choice of Methods } \\
\text { B-Q.4 \% of new clients informed about at least one } \\
\text { method other than the method chosen }\end{array}$ & 45 & 66 & $\Uparrow$ \\
\hline $\begin{array}{l}\text { Information G iven to Clients } \\
\text { B-Q .5\% of new clients properly informed about their } \\
\text { chosen method } \\
\text { B-Q.6\% of new clients with whom IEC materials were } \\
\text { used during the consultation, among SDPs with materials }\end{array}$ & 11 & 19 & $\Downarrow$ \\
\hline $\begin{array}{l}\text { Technical Competence } \\
\text { B-Q.7 \% of new clients who were assessed using standard } \\
\text { medical procedures } \\
\text { B-Q.8 \% of pelvic exams during which aseptic procedures } \\
\text { were followed }\end{array}$ & 56 & 68 & \\
\hline $\begin{array}{l}\text { Mechanisms to Encourage Continuity } \\
\text { B-Q.9\% of new clients informed about the possibility of } \\
\text { changing methods } \\
\text { B-Q.10\% of new clients who received a revisit date }\end{array}$ & $\begin{array}{l}43 \\
97\end{array}$ & $\begin{array}{l}18 \\
98\end{array}$ & $\Downarrow$ \\
\hline
\end{tabular}

percentage of SDPs offering the complete range of methods. However, this blunt result masks some large changes in the availability of individual methods. The availability of condoms and spermicides dropped precipitously, but injectables became far more widely available. COCs were consistently offered by virtually all SDPs. IUDs were consistently available at over $90 \%$ of the hospitals and midsize SDPs, but both studies found that IUD services were also being offered at some smaller SDPs. The change in availability of tubal ligation services cannot be documented with this data.

Under B-R.6, the "minimum necessary equipment" was defined as sterilizer, stethoscope, weighing scale, blood pressure machine, gynecological table, angle poise lamp/torch, gloves (10 or more pairs), and specula (5 or more). Although Table 11-1 shows an increase in the proportion of SDPs with all minimum equipment, this result must be interpreted with extreme care. First, the change is not statistically significant, so it may be attributable to sampling error. Second, counting the number of SDPs that have all the equipment conceals some details regarding individual items, as presented in Figure 11-2. Blood pressure machines and gloves became more common between the studies, but supplies of sterilizers and specula decreased. The availability of other items remained generally steady.

The percentage of providers reporting that they had been trained in both clinical family planning and IEC (B-R.7) declined sharply from $43 \%$ to $11 \%$ 
between the two studies. Most of this decrease is due to lower levels of IEC training: training in clinical family planning declined from $84 \%$ to $72 \%$, but training in IEC skills dropped from $47 \%$ to $14 \%$.

The result for indicator B-R. 8 shows a decline in SDPs with both posters and brochures on family planning, caused mainly by a decrease in supplies of brochures. Between 1992 and 1995, the proportion of SDPs with posters increased from $75 \%$ to $92 \%$, whereas the proportion with brochures declined from $36 \%$ to $20 \%$.

The proportion of SDPs having received at least one supervisory visit in the 6 months preceding the study (indicator B-R.9) declined sharply, from 57\% to only $25 \%$. This decline was also observed among the 45 individual SDPs visited for both situations.

The 10 indicators of service quality listed in Table 11-2 exhibit both increases and declines between 1992 and 1995. B-Q.1 measures interpersonal relations, and exhibits an increase between the studies. However, this indicator may not be fully valid because the arriving person is traditionally responsible for a greeting in Burkina Faso.

Indicators B-Q.2 and B-Q.3 address information taken from the client. About half of new clients were asked about their reproductive intentions (BQ.2), a percentage that did not change between 1992 and 1995. However, the proportion of new clients who were asked about previous experience with family planning (B-Q.3) fell precipitously from $72 \%$ to $25 \%$. B-Q.4 shows an increase in the percentage of clients informed about at least one method other than their selected method.

As for information given to clients, indicator BQ.5 shows a decrease in the proportion of clients who were properly informed about their chosen method, from $36 \%$ to $19 \%$. For this indicator, "properly informed" is defined as being told about how to use the method, as well as its side effects. The result for indicator B-Q.6 shows that very few clients were counseled with IEC materials, even among SDPs with IEC materials on hand, a result that did not change significantly between 1992 and 1995 .

Technical competence is measured by indicators B-Q.7 and B-Q.8, neither of which exhibited a significant change between 1992 and 1995. Indicator B-Q.7 addresses the physical assessment of new clients. For this indicator, "standard medical procedures" are defined as taking a medical history, weight, and blood pressure.

As for mechanisms to encourage continuity, over $95 \%$ of clients received revisit dates (B-Q.10), a figure that did not change between 1992 and 1995. However, the percentage of new clients informed about the possibility of changing methods (B-Q.9) dropped from $43 \%$ to $18 \%$.

\section{Discussion}

Overall, the readiness of the system to provide family planning services in Burkina Faso appears to have remained constant or deteriorated. The more permanent physical aspects of SDPs, such as waiting areas, toilets, and appropriate stocking areas, remained constant. But declines in readiness were associated with activities that can vary and supplies that need to be replenished, such as supervisory visits, IEC materials, and provider training. The decline in satisfactory examining areas is attributable not to the physical aspects of auditory and visual privacy, but to cleaning and ensuring water supplies.

The availability of basic equipment showed a range of improvements and declines in individual items, resulting in no significant change in the overall result. Moreover, the number of methods offered, which certainly depends on supplies and activities that can vary, did not change significantly overall. However, there was a range of changes in individual method availability, chiefly a drop for condoms and spermicide and a rise for injectables. These changes may simply reflect changes in method mix between the studies.

How do these changes reflect the interventions that took place between the studies? The decline in systems associated with activities that vary and supplies that need to be replenished is entirely consistent with the above-noted decline in donor activity. Most important, the loss of the SEATS Project on decentralization of services and management probably contributed to the decline in supervisory activities. Thus, the enormous increase in SDPs offering family planning services between 1992 and 1995 was not accompanied by an increase in resources for supervision, and supervisors may well have been overloaded. Moreover, training 
activities between the studies focused on refresher courses in clinical family planning and IEC, among others; the percentage of providers trained in family planning remained steady, and that of those trained in IEC dropped. Again, these results may reflect the cessation of USAID activity.

Other projects on very specific issues either increased readiness or perhaps halted a decline. For example, a large decline in the availability of equipment was probably avoided because of a World Bank project on supplying SDPs with basic equipment. The intervention on IEC materials dealt with items not measured by Situation Analysis, except posters on family planning, which did indeed increase. That supplies of brochures declined may reflect the fact that these particular items were not part of the intervention.

The quality of family planning services generally remained steady between the two studies, with a few improvements and declines. One improvement was found in interpersonal relations, but questions about the validity of indicator B-Q.1 prohibit any strong conclusions on this element of quality. The other improvement concerns the number of methods mentioned to clients (indicator BQ.4). However, other aspects of counseling appear to have declined. Indicators of information exchange (B-Q.2, B-Q.3, B-Q.5, and B-Q.6) either remained constant or declined, and the percentage of new clients who were asked about previous family planning experience (B-Q.3) declined by 47 percentage points, more than any other item in the analysis. The percentage of clients informed about changing methods (B-Q.9), which can be viewed as a counseling issue, also declined sharply. Moreover, by 1995 the levels of these indicators were quite low, many below $25 \%$.

It is fair to assume that the quality of counseling might improve with increased provider training and supervision. Although the training interventions focused on IEC, the actual proportion of providers trained in IEC fell (B-R.7 in Table 11-1), and supervision rates also fell precipitously (BR.9). These circumstances can reasonably be assumed to have contributed to the decline in counseling quality.

Technical competence, as measured by two indicators (B-Q.7 and B-Q.8), generally remained steady.
The intervention on clinical training might have been expected to affect this indicator, but as shown in Table 11-1, the proportion of providers trained in clinical family planning (B-R.7) did not change significantly, probably reflecting the USAID pullout. Moreover, the technical competence of providers probably also responds to supervision, which, as noted, declined sharply. In fact, given the circumstances, it is encouraging that these indicators did not show a significant decline. However, the fact that the overall levels of these indicators were still about $65 \%$ suggests that training could be strengthened to improve technical competence further.

\section{GHANA}

\section{Background}

The first population policy in Ghana was launched in 1969, but it did not at first succeed in significantly raising contraceptive prevalence or decreasing the TFR. In the early 1990s, however, the Ghana Family Planning and Health Program (GFPHP), funded by USAID, was established to combat sexually transmitted diseases (STDs) / HIV and improve access to family planning services. In 1993, a Situation Analysis study was conducted in conjunction with a Demographic and Health Survey (DHS), and information from these sources was used to revise the national population policy in 1994. The revised policy includes a goal of a TFR of 5.0 by 2000, down from 5.5 in 1993 . The GFPHP has since been revised to form the new USAID/Ghana Population and AIDS Project (GHANAPA), which will carry out activities through 2000. The second Situation Analysis study was undertaken in 1996 as part of this project. Its purpose was to evaluate the program changes made by the $\mathrm{MOH}$ and other agencies based on the 1993 Situation Analysis results. The instruments used in the two Situation Analysis studies in Ghana were quite similar, generating a wealth of comparable items.

\section{Program Activities Between the Studies}

Several program activities were carried out between 1993 and 1996 by the MOH, the Planned 
Parenthood Association of Ghana (PPAG), the Ghana Registered Midwives Association (GRMA), and USAID/GHANAPA. In particular, multisectoral, nationwide emphasis was placed on a postbasic course on IUD insertion, which also included basic family planning counseling skills and general reproductive health care. All organizations reported various numbers of staff who received this training. The $\mathrm{MOH}$ also instituted some separate training initiatives in general family planning clinical and counseling skills, as well as specific contraceptive technology updates. The PPAG and GRMA also ran various training programs for their own staffs.

AVSC International and USAID were involved in a vigorous program to increase the capacity of the system to provide long-term methods. Between 1992 and 1994, 35 doctor/nurse teams were trained in mini-laparotomy and vasectomy, and 15 hospitals were equipped with operating theaters. PPAG also established one center for mini-laparotomy/vasectomy, but found uptake quite low. Several NORPLANT ${ }^{\circledR}$ training courses were planned. The Catholic Church also carried out a campaign to increase the availability of natural family planning (NFP), particularly at mission hospitals. In addition, the popularity of injectables soared between the two studies.

Problems with contraceptive supplies were first addressed through policy. In 1992, oral contraceptives were no longer classified as a dangerous drug; they were later added to the essential drug list in an effort in improve the reliability of supplies. USAID took over this effort by trying to reduce the length of the national pipeline for contraceptive commodities. Injectable supplies were first provided at a national level by USAID, and subsequently by UNFPA. Because of logistical problems with this transition, there was a national stockout period of about 3 months.

Although an effort was made at broad-based IEC campaigns, these interventions were not very successful. The "We Care" campaign, launched by the $\mathrm{MOH}$ in 1990, had some success in the first few years, but was slowed in 1994 because of funding problems. A mass-market television campaign by the Ghana Social Marketing Foundation ran for 2 months, but was later canceled because the family planning message was seen by some groups as promoting promiscuity.

\section{Results}

The program managers in Ghana decided to present the results in detail, rather than combining indicators into a smaller number of summary indicators, as in the Burkina Faso analysis. Table 11-3 shows the results for readiness indicators included in the comparison report, ${ }^{7}$ which reveal a variety of improvements and declines. The infrastructure (GR.1 through G-R.4) has remained largely the same between 1993 and 1996, with no significant changes except an increase in light and water in examination rooms. Serious deficiencies in electricity and running water that persisted across the two studies were determined largely by urban/rural location.

Two of three indicators on the accessibility of SDPs declined significantly. The percentage of SDPs with signs announcing services (G-R.5) declined, probably because a major $\mathrm{MOH}$ project to supply signs to SDPs had taken place just before the first study, and by the second study many of those signs had fallen into disrepair. No special attention had been given the issue in the interim. About the same proportion of SDPs were open on time in the two studies (G-R.6), but significantly fewer SDPs were offering family planning services 5 days per week (G-R.7) in 1996 than in $1993 .{ }^{8}$

The results for methods offered (G-R.8) show a dramatic increase in the proportion of SDPs offering tubal ligation and vasectomy, as well as NFP. Although availability of COCs, condoms, and injectables declined slightly, these three methods continued to be offered by the vast majority of SDPs. IUD availability remained steady, and progestinonly pill (POP) availability increased somewhat.

The proportion of SDPs experiencing stockouts of COCs, IUDs, and condoms (G-R.9) did not change between 1993 and 1996, and these rates are fairly low. The large increase in injectable stockouts is expected because of the above-noted nationwide shortage during the switch from USAID to UNFPA as the major provider, coupled with the increasing popularity of the method. The decrease in POP stockouts is probably related to the rise in demand for injectables, which may have reduced the demand for POPs.

The two indicators of commodity management (G-R.10 and G-R.11) both dropped, although the 
Table 11-3. Changes in readiness indicators in Burkina Faso

\section{Readiness Indicators}

1993

1996 Significant?

Infrastructure/Facilities

G-R. $1 \%$ of SDPs with electricity

G-R.2 \% of SDPs with running water

$50 \quad 57$

G-R.3 \% of SDPs with clients' toilet

$44 \quad 37$

G-R $4 \%$ of SDPs with auditory privac

$72 \quad 69$

... visual privacy in exam room

... clean exam room

... adequate light in exam room

... adequate water in exam room

$89 \quad 91$

\section{Accessibility}

G-R.5\% of SDPs with a sign announcing family planning

services inside or outside

G-R. $6 \%$ of SDPs open on time

G-R. $7 \%$ of SDPs that offer family planning services

5 days a week or more

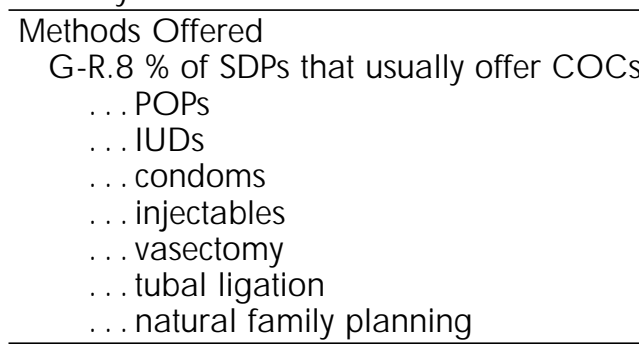

Supplies/ Logistics

G-R. $9 \%$ of SDPs that have experienced a stockout of

$\mathrm{COCs}$ in the previous 6 months

...PO Ps

...IUDs

.... condoms

... injectables

G-R.10\% of SDPs with a well-ordered inventory

G-R.11\% of SDPs with adequate storage facilities

Equipment

G-R.12 \% of SDPs with blood pressure apparatus

... stethoscope

$\ldots$. access to sterilizer ${ }^{a}$

... uterine sounds

... specula

... tenacula

$\begin{array}{lll}85 & 90 & \Uparrow \\ 69 & 74 & \Uparrow\end{array}$

$80 \quad 68 \quad \Downarrow$

$62 \quad 61$

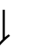

$62+2$

$\begin{array}{rll}97 & 91 & \Downarrow \\ 64 & 73 & \Uparrow \\ 50 & 57 & \\ 96 & 89 & \Downarrow \\ 95 & 90 & \Uparrow \\ 2 & 23 & \Uparrow \\ 4 & 50 & \Uparrow \\ 45 & 74 & \Uparrow\end{array}$

Staffing

G-R.13\% of providers imposing eligibility criteria

IEC Materials and Activities

G-R.14 \% of SDPs with posters on family planning

... flip charts on family planning

... brochures on family planning

... method samples

... anatomical models

\section{Supervision}

G-R.15\% of SDPs that received a supervisory visit in

previous 6 months

$\begin{array}{rrr}9 & 14 & \\ 36 & 17 & \Downarrow \\ 10 & 10 & \\ 10 & 7 & \\ 7 & 19 & \Uparrow \\ 75 & 68 & \Downarrow \\ 92 & 83 & \Downarrow \\ 92 & 91 & \\ 90 & 85 & \\ 47 & 80 & \Uparrow \\ 54 & 58 & \\ 67 & 71 & \\ 56 & 56 & \end{array}$

See Figure 11-3

In 1996, "sterilizer" was defined much more broadly in the instruments as compared with 1991. This observed increase in sterilizers may be due simply to the change in definition. 


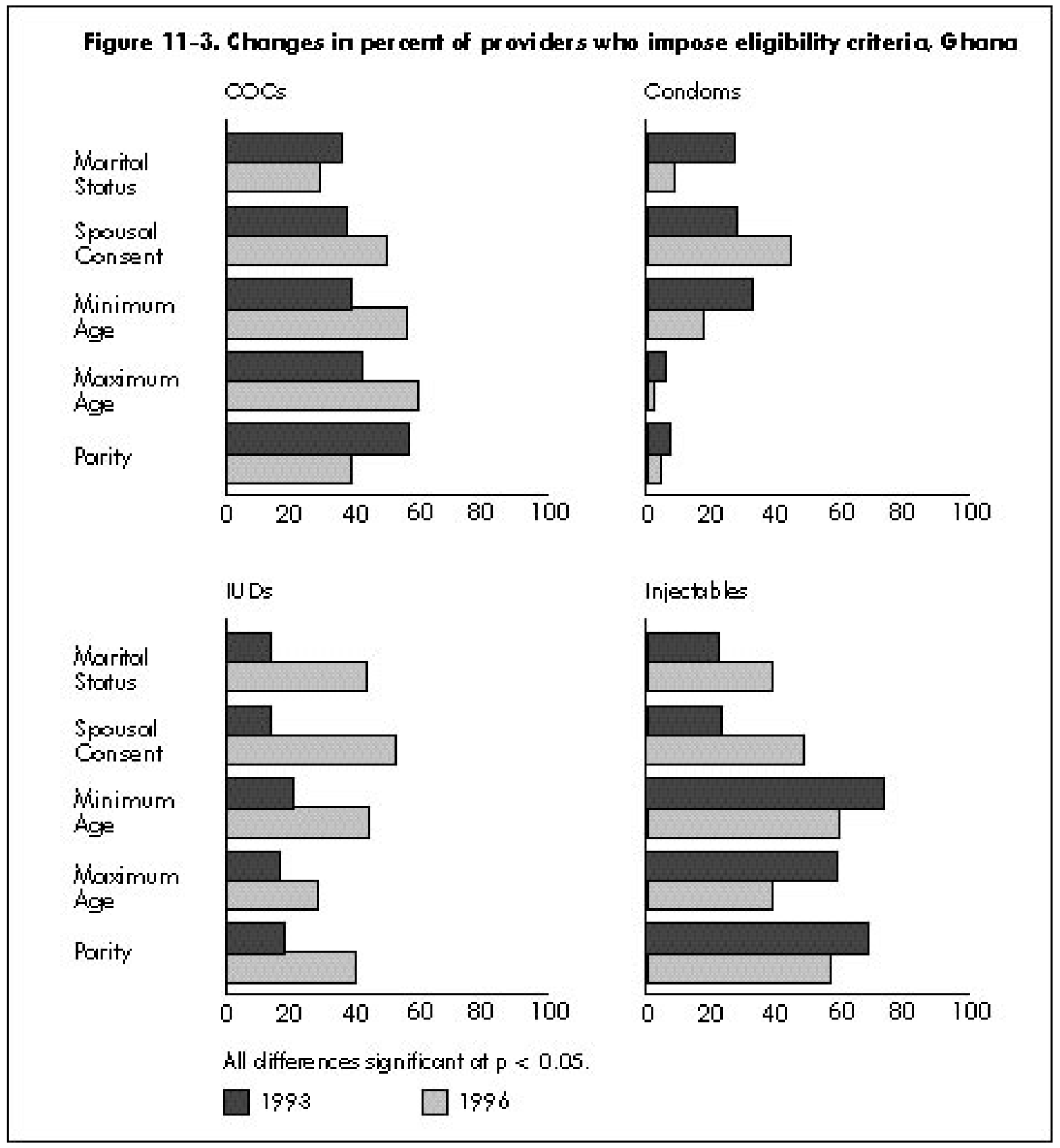

overall results remained fairly encouraging. The percentage of SDPs with a well-ordered inventory fell from $75 \%$ to $68 \%$, and the proportion of SDPs with adequate storage facilities dropped from $92 \%$ to $83 \%$.

The only item of equipment (G-R.12) that showed a change in availability between the two studies was access to a sterilizer, which increased dramatically. However, this increase is probably attributable to a change in the definition of "steril- izer" in the 1996 instrument to be much more inclusive than that in 1993.

Figure 11-3 shows the change in the percentage of providers who imposed eligibility criteria on four methods (G-R.13). The results show that in most cases, providers became considerably more restrictive during the interval. The increase in all eligibility criteria applied to IUDs is particularly notable. Program managers also note that the increase in spousal consent requirements for all 


\section{Table 11-4. Changes in quality indicators in Ghana}

\section{Quality Indicators $\quad 1993 \quad 1996$ Significant?}

Interpersonal Relations

G-Q.1\% of pelvic exams before which provider

informed the client

$74 \quad 77$

G-Q.2\% of pelvic exams after which provider informed

the client of result

$61 \quad 54$

G-Q.3\% of IUD insertions during which provider offered emotional support

$85 \quad 52 \quad \Downarrow$

Information Taken from Clients

G-Q.4\% of new clients asked about concerns about any method

G-Q.5\% of revisit clients asked if they would like to change methods

G-Q.6\% of revisit clients asked if they experienced problems with method

G-Q.7\% of new hormonal clients asked about unusual

bleeding

G-Q.8\% of new hormonal clients asked about unusual

discharge

\section{Choice of Methods}

G-Q.9\% of new clients asked a method preference

G-Q.10\% of new clients who are told about COCs

... about condoms

... about IUDs

... about injectables

... about tubal ligation

rmation $\mathrm{G}$ iven to Clients

G-Q.11\% of clients told how to use their

accepted method

$\begin{array}{lll}82 & 85 & \\ 66 & 69 & \\ 59 & 54 & \\ 57 & 53 & \\ 23 & 13 & \Downarrow \\ 29 & 11 & \Downarrow \\ 65 & 71 & \\ 33 & 23 & \end{array}$

... the advantages of method

$30 \quad 39$

$\ldots$. the side effects of method

G-Q .12\% of new clients with whom flip charts were used

... brochures were used

... posters were used

... method samples were used

$\begin{array}{ccc}19 & 11 & \Downarrow \\ 70 & 43 & \Downarrow \\ 46 & 27 & \Downarrow\end{array}$

$37 \quad 40$

... anatomical models were used

Technical Competence

G-Q.13\% of new hormonal clients who were weighed $\quad 69 \quad 72$

... whose LMP was taken

$88 \quad 90$

... whose medical history was taken

$79 \quad 69$

... whose blood pressure was taken

$74 \quad 72$

G-Q.14\% of pelvic exams before which provider washed hands 69

G-Q.15\% of IUD insertions during which provider used aseptic procedures

$83 \quad 59$

G-Q.16\% of IUD insertions before which provider sounded the uterus

$85 \quad 59$

Mechanisms to Encourage Continuity

G-Q.17\% of clients given a return date

92

92 
methods is probably attributable to providers' concern about possible reprisals from spouses unaware of their partner's use of family planning.

The availability of IEC materials (G-R.14) remained constant between the studies, except for a moderate increase in posters and a dramatic increase in the availability of anatomical models. The proportion of SDPs that received a supervisory visit in the 6 months preceding the study (GR.15) increased sharply, from $52 \%$ to $79 \%$, a major accomplishment for the program.

Table 11-4 shows that most indicators of service quality showed no significant change between 1993 and 1996, but most of those that did exhibited declines. The element of interpersonal relations was unchanged with regard to informing clients before and after pelvic exams (G-Q.1 and G-Q.2), but the proportion of clients given emotional support during IUD insertions (G-Q.3) dropped. This can also be seen as part of an overall decline in IUD insertion quality, discussed below.

Of the five indicators of information taken from clients (G-Q.4 through G-Q.8), three declined and two remained constant. By 1995, none of these results exceeded 50\%, indicating a serious difficulty with the quality of client counseling.

Under choice of methods, there was an encouraging increase in the percentage of new clients asked about their method preference (G-Q.9), which rose from $46 \%$ to $79 \%$. The percentage of clients hearing about various methods (G-Q.10) did not change significantly; the exception was injectables, which showed a moderate increase, probably reflecting the growing popularity of this method. Notably, the proportion of clients told about tubal ligation remained about $28 \%$.

As for information given to clients, most were told how to use their accepted method and its advantages (G-Q.11), but only just over half were told about its possible side effects. None of these proportions changed significantly between 1993 and 1996. Moreover, the proportion of clients with whom IEC materials were used (G-Q.12) either remained constant or dropped, as in the case of posters and brochures. ${ }^{9}$ With the exception of method samples, overall use of IEC materials was quite infrequent. Again, these results indicate serious deficiencies in client counseling.
The most significant finding on technical competence is the dramatic drop in the quality of IUD insertions, both for using aseptic procedures (GQ.14 and G-Q.15) and sounding the uterus (GQ.16). This is in addition to the decline in offering moral support during IUD insertions reported above (G-Q.3). The physical assessment of new clients (G-Q.13) did not change greatly between 1993 and 1996, except for a moderate drop in the proportion of new hormonal clients whose medical history was taken. Otherwise, the majority of new hormonal clients were assessed through weight, blood pressure, and last menstrual period. Moreover, the percentage of pelvic exams before which the provider washed his/her hands remained largely unchanged between the studies.

\section{Discussion}

In Ghana, there were some notable improvements in readiness indicators between the two studies. For example, the increase in availability of longterm methods is a strong accomplishment of the program, as are the dramatic increases in supervisory activities and in availability of certain IEC materials. Moreover, the effort expended on training in the interval between the studies leads to the reasonable conclusion that many staff were trained. In the 1996 study results, $69 \%$ of all staff report that they had attended family planning refresher training; $47 \%$ had attended a post-basic course in general clinical family planning skills; $54 \%$ in family planning counseling; and $29 \%$ in IUD insertion/removal. The training data from 1993 is not comparable, so we cannot measure the increase in training, but only recognize that the considerable effort put into training is reflected in these 1996 levels.

Many of the readiness indicators remained steady, suggesting that the program's efforts to improve readiness generally did not translate to higher-quality services. There were also declines in readiness, related to accessibility and commodity management. The effort to improve commodity supplies may have appreciably affected only POPs. As noted earlier, the increase in injectable stockouts probably resulted from a nationwide 3-month stockout due to a switch in suppliers. These are 
important program issues, but are not as central to service delivery as the above readiness indicators that improved considerably.

The quality of services, however, largely shows a decline. In particular, the quality of IUD services decreased sharply, in terms of both technical competence and the enforcement of eligibility criteria for the method. By 1996, 26\% of providers had been trained in IUD insertion/removal, but we do not know whether the particular providers observed to be inserting IUDs had been trained or not. ${ }^{10}$ Two possible conclusions can be drawn: that the observed providers had been trained and exhibited lower quality, or that IUDs were being inserted by untrained providers, in itself a compelling program issue. Either way, the emphasis on the IUD training between the studies did not have its intended effect of improving IUD services. In addition, the quality of information exchange appears to have remained steady or declined, even though the training also focused on counseling skills. However, counseling on choice of methods appears to have improved somewhat, which may reflect a success of the training.

Similarly, the impressive increase in SDPs offering sterilization services was not accompanied by an increase in clients being told about the method. Also, the improved availability of posters and anatomical models did not lead to increased use of these materials with clients. In fact, use of posters with clients dropped to only $11 \%$ at SDPs with posters available. In both cases, an increase in readiness to provide services did not appear to translate to the quality of services offered clients. And all of the declines in quality of care given clients took place in an environment of sharply increased supervision-a puzzling result.

\section{KENYA}

\section{Background}

In the 1970s and 1980s, Kenya's TFR was about 8, one of the highest in the world, but by 1993 it had fallen to 5.8. Although family planning services were first introduced in 1967, the program was not very effective until the late 1980s. The MOH's family planning program, with overall program and policy coordination by the National Council for Population and Development (NCPD), is credited with having contributed to the fertility decline.

The first Situation Analysis study carried out in Kenya in 1989 (see Chapter 1) was the first study of its kind, and was undertaken by the $\mathrm{MOH}$ and the Population Council to identify the strengths and weaknesses of the program. By the time the $\mathrm{MOH}$ decided to carry out its second Situation Analysis study in 1995, the methodology had evolved considerably. Although the new study gave a detailed picture of the state of services, there was not a large number of questions comparable with those of the first study.

Moreover, the activities undertaken between the studies took place over a long period of time, involved a variety of agencies, and affected various groups of SDPs. The resulting complexity of teasing out individual interventions reduces the ability to evaluate them. For these reasons, evaluating the effectiveness of program interventions is not possible with the Kenyan data. However, the data can still meet the goal of describing changes in program functioning.

\section{Program Activities Between the Studies}

The results of the first Situation Analysis study were used by a variety of organizations to improve Kenya's family planning program. For example, program managers were brought together to discuss the results and develop administrative plans for addressing some of the identified problems. The NCPD, the MOH, Nairobi University, and the Population Council collaborated on the development and implementation of operations research (OR) studies to experiment with possible solutions for some of the quality-of-care issues raised by the study. The Nairobi City Commission (NCC) immediately supplied clinical equipment stored in its warehouse to clinics where it was lacking, and also transferred personnel to better reflect the actual case loads of each of its clinics. The NCC also worked collaboratively to reorient the technical assistance program being planned with Pathfinder 


\section{Table 11-5. Changes in readiness indicators in Kenya}

\begin{tabular}{|c|c|c|c|}
\hline Readiness Indicators & 1989 & 1995 & Significant? \\
\hline \multicolumn{4}{|l|}{ Methods O ffered } \\
\hline K-R.1 \% of SDPs with COC stock on hand & 99 & 98 & \multirow{4}{*}{ II } \\
\hline ... condoms on hand & 85 & 91 & \\
\hline ... injectables on hand & 80 & 91 & \\
\hline$\ldots$...IUDs on hand & 60 & 51 & \\
\hline \multicolumn{4}{|l|}{$\begin{array}{l}\text { Staffing } \\
\text { See text }\end{array}$} \\
\hline \multicolumn{4}{|l|}{ IEC Materials and Activities } \\
\hline K-R.2 \% of SDPs with posters on family planning on the walls & 53 & 75 & $\Uparrow$ \\
\hline ... pamphlets or other IEC material available & 38 & 74 & $\Uparrow$ \\
\hline K-R.3 $\%$ of SDPs that gave a health talk & 32 & 21 & \\
\hline K-R.4 \% of health talks that included family planning & 17 & 9 & \\
\hline \multicolumn{4}{|l|}{ Recordkeeping and Supervision } \\
\hline $\begin{array}{l}\text { K-R.5 \% of SDPs that had at least one supervisory visit } \\
\text { in the previous } 6 \text { months }\end{array}$ & 78 & 78 & \\
\hline
\end{tabular}

International to better reflect the needs revealed by the study.

\section{Results}

The results of the comparison of the two Situation Analysis studies are shown in Tables 11-5 and 11-6. In Kenya, methods offered (K-R.1) refers to the methods physically in stock on the day of the study visit. ${ }^{11}$ The results show an increase in injectable stocks. The vast majority of SDPs had COCs and condoms in both 1989 and 1995, and about half had IUDs in both studies. NORPLANT ${ }^{\circledast}$ was offered in only one SDP in 1989, and in 6\% of SDPs in 1995. These results suggest a moderate increase in the range of methods available.

Methodological differences between the two studies make information on staff training difficult to compare. Nonetheless, a qualitative assessment of the data shows that there was probably an

\section{Table 11-6. Changes in quality indicators in Kenya}

\begin{tabular}{lccc} 
Quality Indicators & 1989 & 1995 & Significant? \\
Choice of Methods & & & \\
K-Q.1 \% of new clients who are told about COCs & 90 & 85 & \\
.. condoms & 77 & 72 & \\
... injectables & 90 & 83 & \\
... IUDs & 83 & 87 & \\
... tubal ligation & 17 & 34 & $\Uparrow$ \\
... vasectomy & 4 & 12 & $\Uparrow$ \\
\hline
\end{tabular}

Information Given to Clients

See Figure 11-4 
Figure 11-4. Change in irf or mation given to clients abo ut methods, Kenra
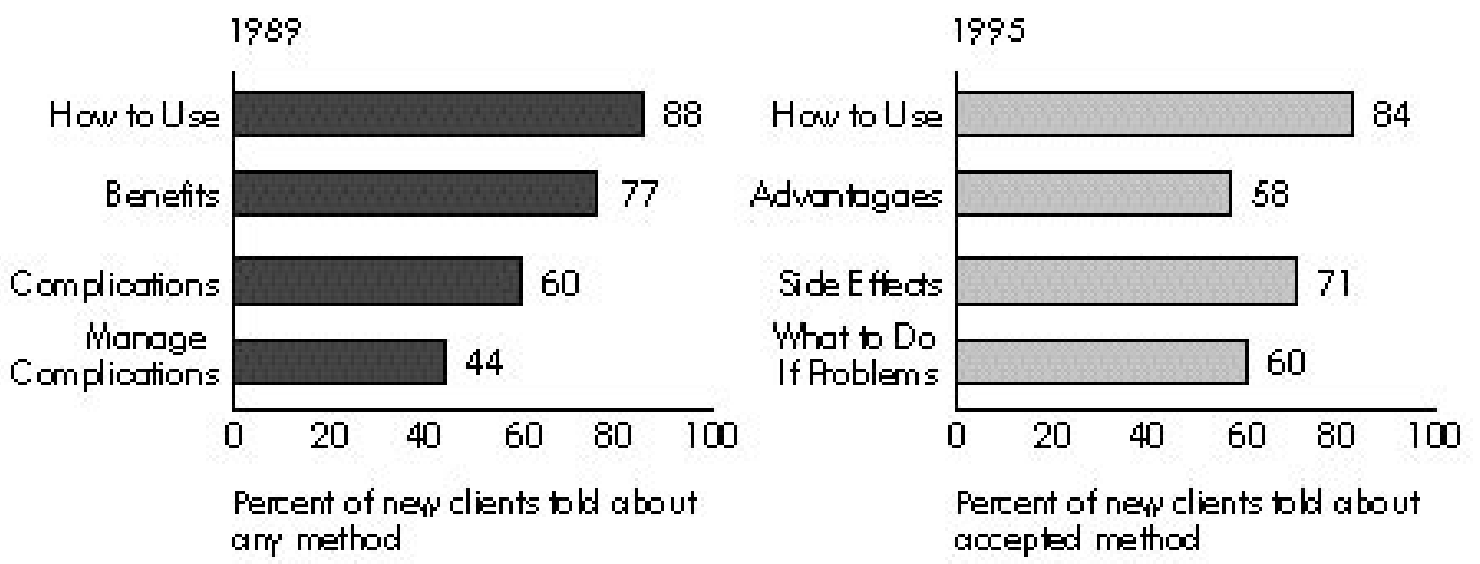

increase in the proportion of nurses who received in-service training, from about $32 \%$ in 1989 to about 60\% in 1995.

IEC materials in the form of posters and pamphlets or other materials (K-R.2) became increasingly available between the studies. However, no change was observed in health talks held (K-R.3) or in the proportion of health talks that included information on family planning (K-R.4). The frequency of supervision (K-R.5) was also not observed to change between the studies.

The only directly comparable indicator of service quality from the two studies is methods mentioned to clients (K-Q.1). Most clients were told about COCs, condoms, injectables, and IUDs, and these levels did not change significantly between 1989 and 1995. However, a significantly higher proportion of clients was told about long-term and permanent methods in 1995. NORPLANT ${ }^{\oplus}$ was introduced in Kenya in about 1989, but was not included in the first Situation Analysis study. By 1995, however, $35 \%$ of clients were being told about this method. Overall, there was an increase in the mean number of methods mentioned to clients from about 3.8 to $4.2 .^{12}$

The data on information given to clients cannot be directly compared between the two studies, but it can hint at some changes in the quality of counseling. In the 1995 study, observers noted whether clients were told various information about their chosen method, such as how it works and its side

effects, whereas in 1989, observers recorded what clients were told about any method, not the particular one they had chosen. Moreover, the categories of information changed between the studies. Nonetheless, a review of the training programs for observers suggests the comparability of four items (see Figure 11-4): how to use, benefits / advantages, complications/side effects, and how to manage complications/what to do if problems. Because the 1989 data relate to any method, percentages are probably inflated relative to the 1995 data. Given all these caveats, however, it may be fair to conclude that more clients were being told about side effects and their management in 1995 than in 1989, although conclusions about information on how to use the method and its benefits are more difficult to draw.

\section{Discussion}

Again, the circumstances of these Situation Analysis studies do not permit an evaluation of the program interventions. However, some service changes can be described. In general, most of the indicators remained constant between the studies, but all those that exhibited a change improved. Stocks of injectables and NORPLANT ${ }^{\circledast}$ rose, indicating a possible modest increase in method availability. IEC materials also showed an increase, although health talks declined in frequency and family planning content. Supervisory rates were 
unchanged. As for quality of services, the choice of methods appears to have improved, with increased discussion of long-term and permanent methods, and information given to clients on side effects and their management may have improved as well.

\section{ZIMBABWE}

\section{Background}

In 1985, the Zimbabwe National Family Planning Council (ZNFPC) was established to coordinate the family planning activities that had increasingly been taking place in the country since the 1950s. The resulting family planning program is generally regarded as one of the most successful in Africa. The TFR in 1997 was under 5, and the CPR for modern methods among all women of reproductive age was fully 31\% in 1994. Much of the credit for this CPR belongs to the active community-based distribution (CBD) program run by the ZNFPC, which serves up to $30 \%$ of users in the country. ${ }^{13}$ The majority of other users attend state clinics, most of which are run by the Ministry of Health and Child Welfare $(\mathrm{MOH} \& \mathrm{CW})$. Aside from the CBD program, the ZNFPC also runs some clinics, but it is mainly a policy-making and coordinating body.

The ZNFPC follows a 5-year planning cycle, and the first 5-year cycle came to an end in 1996. The first Situation Analysis study was undertaken in 1991 as a baseline study, and the second was carried out at the end of the planning cycle for comparison purposes.

\section{Program Activities Between the Studies}

In June 1992, as part of a project to increase the range of family planning methods available, the MOH\&CW lifted a ban on providing injectables. The ZNFPC subsequently conducted a number of workshops to refresh providers on all contraceptive methods, with particular attention to the injectable. Similar workshops were held throughout the country to disseminate new guidelines that removed eligibility criteria based on age, marital status, spousal consent, and parity, as well as other barriers to services.
In response to the 1991 Situation Analysis study finding that some facilities did not have the necessary equipment for family planning service delivery, the ZNFPC began a project to distribute equipment to SDPs. The establishment of a logistics unit within the ZNFPC in 1993 also helped facilitate the systematic procurement, storage, and distribution of contraceptive supplies and equipment.

Results from the 1991 Situation Analysis study were also used to revise the family planning training curriculum. The 1991 study showed that counseling skills were weak, as revealed by the limited information exchange that was observed. Unfortunately, the production of materials for use in training providers in counseling and interpersonal communication modules was delayed, and the result was a limited number of providers being trained between 1991 and 1996,

However, general training in family planning was enhanced by decentralizing training at $\mathrm{MCH} /$ family planning schools in selected districts and in-service training at city health departments, as well as including a family planning module in basic training for nurses. In particular, the ZNFPC responded to the finding that the provision of IUDs was limited and that providers hardly discussed or provided STD services by developing a module that integrated the diagnosis and management of genital and urinary tract infections. Furthermore, the ZNFPC piloted an on-the-job training program in IUDs and STD screening, as well as a privatesector program to train doctor/nurse teams in tubal ligation and NORPLANT ${ }^{\oplus}$.

IEC activities revolved around increasing the demand for long-term and permanent methods and training providers in selected sites on counseling skills for these methods. High-quality IEC materials were developed and produced for trainers, service providers, clients, and the general public.

\section{Results}

Table 11-7 shows that over half of the observed readiness indicators increased significantly, and the rest remained steady-an extraordinary achievement.

The infrastructure and facilities of the system did not change, as measured by availability of 


\section{Table 11-7. Changes in readiness indicators in Zimbabwe}

\begin{tabular}{|c|c|c|c|}
\hline Readiness Indicators & 1991 & 1996 & Significant? \\
\hline \multicolumn{4}{|l|}{ Infrastructure/Facilities } \\
\hline Z-R.1 \% of SDPs with adequate water available & 71 & 71 & \\
\hline Z-R.2 \% of SDPs with enough seating space & 74 & 71 & \\
\hline \multicolumn{4}{|l|}{$\begin{array}{l}\text { Methods O ffered } \\
\text { See text }\end{array}$} \\
\hline \multicolumn{4}{|l|}{ Contraceptive Supplies and Logistics } \\
\hline Z-R.3 \% of SDPs with proper stock keeping & 62 & 93 & $\Uparrow$ \\
\hline \multicolumn{4}{|l|}{ Equipment } \\
\hline Z-R.4 \% of SDPs with blood pressure machine & 93 & 94 & \\
\hline ... sterilizing equipment & 64 & 60 & \\
\hline ... examination couch & 72 & 94 & $\Uparrow$ \\
\hline ... adult weighing scale & 86 & 98 & $\Uparrow$ \\
\hline ... uterine sounds & 17 & 16 & \\
\hline ... specula & 78 & 94 & $\Uparrow$ \\
\hline ... tenacula & 16 & 15 & \\
\hline
\end{tabular}

\section{Staffing}

Z-R.5 \% of providers who attended a family planning refresher course Z-R. $6 \%$ of providers who attended IUD insertion/removal training

\begin{tabular}{lll}
6 & 60 & $\Uparrow$ \\
9 & 34 & $\Uparrow$ \\
\hline 15 & 25 & $\Uparrow$ \\
55 & 85 & $\Uparrow$
\end{tabular}

IEC Materials and Activities

Z-R.7 \% of SDPs that held a health talk Z-R.8 \% of SDPs with posters on family planning on the walls

Recordkeeping and Supervision

Z-R. $9 \%$ of SDPs with at least one supervisory visit in previous 6 months 58 99 $\Uparrow$

adequate water and seating space (Z-R.1 and ZR.2, respectively). As for methods offered, the data are largely not comparable on this point. However, information on supplies of IUDs, as well as other program information, leads to the conclusion that the availability of the IUD did not show a large increase between the studies.

There was a marked increase in the percentage of SDPs with proper stock keeping (Z-R.3), which is defined as keeping an up-to-date, legible inventory. Changes in equipment availability (Z-R.4) were all positive. By 1996, over $90 \%$ of SDPs had couches, scales, and specula, up from $86 \%$ or less. The percentage of SDPs with uterine sounds and tenacula did not change significantly, but these items are found only in SDPs that offer IUDs. Among these SDPs, 90\% or more had a uterine sound and speculum in 1996. Sterilizers remained available at about $60 \%$ of SDPs in both studies.

Increases in staff training were considerable. The percentage of providers who had been to a family planning refresher course (Z-R.5) rose from $6 \%$ to $60 \%$, and the percentage who had been to an IUD insertion/ removal course (Z-R.6) rose from $9 \%$ to $34 \%$. Similarly, the availability of IEC materials, in the form of health talks and posters (Z-R.7 and Z-R.8, respectively), rose significantly. The percentage of SDPs that had received at least one supervisory visit in the preceding 6 months (Z-R.9) increased from $58 \%$ to fully $99 \%$. 
The results for the quality indicators are more equivocal, as shown in Table 11-8. For example, both indicators of information taken from clients (Z-Q.1 and Z-Q.2) declined significantly, as did two of the three indicators of information given to clients (Z-Q.4). ${ }^{14}$ These results suggest a decline in the quality of counseling, although an increase was observed in the percentage of new clients told how their chosen method works.

The range of methods offered to clients (Z-Q.3) appears to have improved somewhat. Significantly more clients were told about POPs, injectables, and IUDs in 1996 than in 1991. The only decline in methods mentioned was in condoms, a finding that is of serious concern given the role of condoms in HIV prevention. Similar numbers of clients were told about COCs and permanent methods across the two studies. Also, in 1996 15\% of clients were told about NORPLANT ${ }^{\circledR}$, which was not available in 1991.

The only available indicators for technical competence relate to the assessment of clients (Z-Q.5). Although the vast majority of clients were assessed with weight and blood pressure in both studies, there was a drop in the proportion of clients whose medical history was taken. Similarly, the number of clients who received pelvic exams declined.

\section{Discussion}

The sizable increases in Zimbabwe's readiness indicators are unique among the four studies included here, and all seem to relate directly to program interventions. The training programs appear to have achieved considerable coverage, greatly increasing the percentage of staff with refresher family planning and IUD training. In addition, the efforts to equip SDPs properly were probably related to observed increases in several items of equipment, and the formation of a logistics unit appears to have led to increases in the percentage of SDPs with proper stock keeping. Although no intervention appears to have been directly focused on supervision, the rise in supervisory visits is another strong program achievement.

Efforts to increase the range of contraceptives available appear to have had more mixed results.
The number of SDPs offering IUD services did not increase between the studies, but the percentage of trained providers did. The lifting of the ban on injectables and the introduction of NORPLANT ${ }^{\circledR}$ certainly increased the range of methods available.

These changes in the readiness of the system were not strongly reflected in increases in quality of services, although some elements of quality did show improvement. With regard to the range of methods available, more clients were being told about several methods, including the injectable, which was mentioned to only 2\% of clients in 1991. On the other hand, fewer clients were being told about condoms, a particularly important method for combating HIV, and although there was some focus on increasing demand for permanent methods, there was no significant increase in the percentage of clients told about these methods. The quality of counseling appears to have declined, even though many providers had been trained in counseling, and supervision had increased dramatically. The only increase in counseling quality was in the percentage of clients told how their chosen method works.

As for technical competence, results for weight and blood pressure taking remained constant, whereas the percentage of clients whose medical history was taken declined. The decline in pelvic exams is seen by program managers as an achievement of the new protocols to reduce barriers to services.

\section{Conclusion}

The first goal of these comparisons was to describe changes in service quality, and the results of these studies do reveal some similar patterns. First, the quality of counseling is generally low (see Chapter 3 for more detail), and is declining or stagnant in all the countries included here. This particular element of quality is suffering markedly more than technical competence or mechanisms to encourage continuity, for example, and does not appear to be directly related to training interventions.

Second, choice of methods shows slight or partial improvements in all four countries. These improvements take the form of an expanded range of methods available, increased number of methods mentioned to clients, or larger numbers of clients being asked about method preference. 


\section{Table 11-8. Changes in quality indicators in Zimbabwe}

\begin{tabular}{|c|c|c|c|}
\hline Quality Indicators & 1991 & 1996 & Significant? \\
\hline $\begin{array}{l}\text { Information taken from clients } \\
\text { Z-Q.1\% of new clients asked their reproductive intentions } \\
\text { Z-Q.2\% of new clients asked their breastfeeding status }\end{array}$ & $\begin{array}{l}64 \\
83 \\
\end{array}$ & $\begin{array}{l}51 \\
62 \\
\end{array}$ & $\begin{array}{l}\Downarrow \\
\Downarrow\end{array}$ \\
\hline 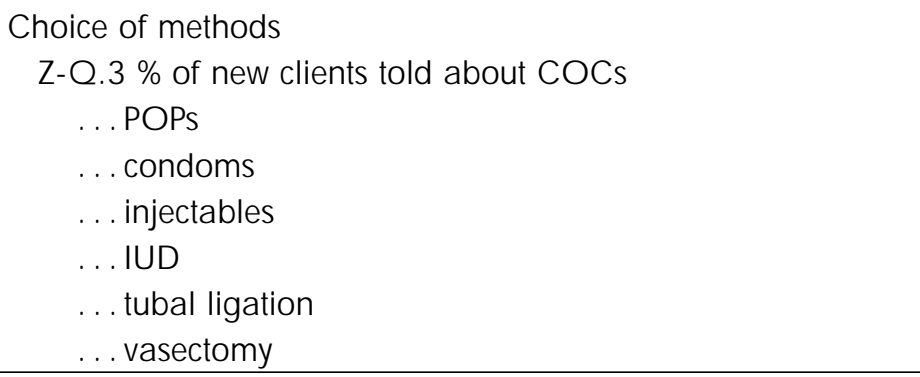 & $\begin{array}{r}32 \\
56 \\
39 \\
2 \\
14 \\
6 \\
1 \\
\end{array}$ & $\begin{array}{r}35 \\
66 \\
21 \\
51 \\
22 \\
10 \\
3 \\
\end{array}$ & $\begin{array}{l}\Uparrow \\
\Downarrow \\
\Uparrow \\
\Uparrow\end{array}$ \\
\hline $\begin{array}{l}\text { Information given to clients } \\
\text { Z-Q .4\% of new clients told how their chosen method works } \\
\text {... side effects of chosen method } \\
\quad \text {... management of side effects of chosen method }\end{array}$ & $\begin{array}{l}79 \\
72 \\
75 \\
\end{array}$ & $\begin{array}{l}93 \\
60 \\
51 \\
\end{array}$ & $\begin{array}{l}\Uparrow \\
\Downarrow \\
\Downarrow \\
\end{array}$ \\
\hline $\begin{array}{l}\text { Technical competence } \\
\text { Z-Q .5\% of new clients assessed through medical history } \\
\text {... weight } \\
\text {.. blood pressure } \\
\text {.. pelvic exam }\end{array}$ & $\begin{array}{l}71 \\
82 \\
91 \\
57\end{array}$ & $\begin{array}{l}62 \\
87 \\
91 \\
33\end{array}$ & $\Downarrow$ \\
\hline
\end{tabular}

Third, the prevalence of STIs/HIV places an extra urgency on condom promotion and infection control in family planning programs (see also Chapter 10). In all four countries, the few indicators that address these issues either declined or remained constant. The percentage of SDPs offering condoms fell in Burkina Faso and Ghana, and the percentage of clients told about condoms declined in Zimbabwe and remained constant in Kenya and Ghana. Pelvic exams in Burkina Faso exhibited no increase in aseptic procedures, and Ghana witnessed a decline in aseptic procedures during IUD insertions.

Finally, all four of these countries are making some progress in increasing availability for IUDs, NORPLANT $^{\oplus}$, and / or permanent methods. Ghana has markedly increased the availability of tubal ligation and vasectomy, and clients in Kenya are being told about permanent methods more often. In Zimbabwe, the percentage of providers trained in IUD insertion has risen dramatically. At SDPs that offer these methods, not all elements-training, equipment, technical competence, and counseling - needed to offer these methods are fully in place in any of the countries, but efforts are driving in that direction.

As for the goal of evaluating program interventions, these studies testify to the complexities involved in achieving real increases in quality of care. Improvements in the readiness of a program do not necessarily translate to improvements in the quality of care delivered, even if the changes are in the form of increased training or supervision (see Chapters 5 and 6). For example, in Burkina Faso, readiness indicators generally declined or remained constant, largely because of the USAID withdrawal. In particular, supervision declined significantly, and training somewhat as well. Quality of care also showed some declines, but in some ways remained constant. By comparison, in Zimbabwe readiness improved dramatically between the studies, including large increases in 
training and supervision. Yet the quality of care showed only modest increases, and some aspects of counseling declined considerably. In Ghana, the $\mathrm{MOH}$ emphasized a course on IUD insertion, and many providers were trained, but the quality of IUD insertions plummeted, and providers began imposing markedly more eligibility criteria on the method. The role of supervision, which is often seen as one of the most important program elements, is also questionable based on this data. Supervision declined drastically in Burkina Faso and increased dramatically in Zimbabwe and Ghana, but its effect on quality is not clear in any country. ${ }^{15}$

Based on these comparisons, then, the relationship between interventions and improvements in quality is tenuous. Nonetheless, two general lessons can be drawn from this exercise. First, improving service quality requires attention to several aspects of readiness at once. Increasing the availability of a method, for example, involves training providers both clinically and in counseling, ensuring effective logistical systems for commodities, establishing equipment and facilities at
SDPs, and generating relevant IEC materials. None of these elements alone will complete the task. For example, Ghana achieved a significant increase in the percentage of SDPs offering long-term methods, but providers were found not to be counseling clients about the methods any more than before the increase. In Zimbabwe, many providers were trained in IUD insertion, but the method was not being offered at a larger proportion of SDPs. Interventions that address all of the subsystems supporting a method may have more chance of actually improving the quality of services delivered to clients.

Second, these studies are limited in their ability to describe the effectiveness of program interventions. To meet this objective, the study designs need to be experimental, testing the effects of one or more specific interventions, as in the Senegal study design discussed earlier. In this way, the pathways between interventions and service quality improvements can become much clearer. As Situation Analysis studies are undertaken on a more regular basis in the future, the opportunity presents itself to design these evaluations more productively. 


\section{Annex 11-1: Comparability of Samples}

\section{Burkina Faso $(1992,1995)$}

In the 1992 Situation Analysis study, 10 of the 30 provinces were randomly selected for inclusion in the study, and all SDPs offering family planning services in those provinces were visited. The country's two main cities, Ouagadougou and Bobo-Diolasso, were also purposively included. The resulting total sample was 53 clinics. The observations of service delivery took place with new clients only.

By 1995, the universe of SDPs offering family planning services had increased dramatically, and a new sample was drawn. That sample included a census of 8 provinces and a stratified sample of the remaining 22 provinces. In addition, all hospitals and larger clinics were purposively included, for a total sample of 337. Because the chance of inclusion in the sample varies for each SDP, the 1995 data are weighted on a national level. Both new and revisit clients were observed with service providers.

For purposes of comparison, the 1995 data were reduced to the 12 geographical areas included in 1992 (10 provinces and 2 cities). Also, the 1995 observation data were reduced to new clients only. Thus, the 53 SDPs from 1992 are compared with 117 SDPs in the weighted sample from the same regions in 1995. Both of these samples include 45 individual SDPs that were visited in both studies. The Situation Analysis instruments changed between the studies, but not significantly.

\section{Ghana $(1993,1996)$}

The samples for both Situation Analysis studies in Ghana were stratified by type of SDP. In 1993, all hospitals and PPAG clinics were purposively included, as well as half of $\mathrm{MOH}$ clinics and maternities, resulting in a stratified random sample of 399 SDPs. By 1996, the universe of SDPs had increased considerably, so the same sampling plan would have resulted in a sample too large for the budget of the study. Instead, the 1996 sample was reduced to half of hospitals, a quarter of $\mathrm{MOH}$ clinics and maternities, and all PPAG clinics (44 SDPs). This new sample was randomly drawn, resulting in a total sample size of 313.

In the results for both Ghana studies, the data are weighted to account for the varying chance of inclusion for each SDP. Since each sample is representative of the country overall, the samples are comparable with each other on a national level. Although the samples were drawn separately, 109 SDPs were visited by chance in both studies. The Situation Analysis instruments did not change significantly between the studies.

\section{Kenya $(1989,1995)$}

The interval between the two Situation Analysis studies in Kenya was the longest among the comparisons included here, and the samples differ considerably. Moreover, as noted in the main text, the 1989 study was the very first Situation Analysis, and the methodology had evolved considerably by 1995 , greatly reducing the number of comparable questions. For these reasons, this comparison has less descriptive and evaluative power than the others.

The 1989 sample included a random sample of $\mathrm{MOH}$ clinics, stratified by province. Several districts were excluded because of logistical problems with the fieldwork and the sparse population of SDPs. The final sample included 99 SDPs and was self-weighting. Observations were carried out only on new family planning clients.

The 1995 random sample was stratified three ways: by type of SDP (hospital, health center, dispensary), by sector (government vs. nongovernmental organization [NGO]), and by province. 
This stratification scheme was self-weighting, but some additional facilities of certain NGOs were also purposively included. The final sample included 254 SDPs. ${ }^{16}$ Although the purposively included SDPs are not weighted, the sample is taken to be sufficiently nationally representative.

Thus, two sampling considerations must be kept in mind when interpreting the comparison of the Kenya studies. First, only $\mathrm{MOH}$ clinics were included in 1989, whereas $\mathrm{MOH}$ and NGO clinics were included in 1995. Second, the 1989 sample excluded five districts.

\section{Zimbabwe (1991, 1996)}

The 1991 sample of facilities in Zimbabwe was stratified by type among $\mathrm{MOH} /$ municipal clinics and private facilities, such as mission hospitals and industrial clinics. This stratification scheme was self-weighting. In addition, all ZNFPC clinics were purposively included, but difficulties with the data make weighting impossible. Although the total number of ZNFPC clinics is small, the sample is nonetheless considered to be nationally representative. To allow for the comparison, the same SDPs were visited for the 1996 study. The universe of SDPs had changed a bit during the interval, but not significantly. ${ }^{17}$ Neither data set is weighted in this comparison.

\section{REFERENCES}

\section{Burkina Faso}

Bakouan, D., P. Sebgo, I. Askew, Y. Ouedraogo, P. Tapsoba, C. Viadro, and S. Kanon. 1992. Analyse Situationnelle du Programme de Planification Familiale au Burkina Faso. Ministère de la Santé, de l'Action Sociale et de la Famille, Direction de la Santé de la Famille, and Population Council, Burkina Faso.

Bamba, A., B. Millogo, J. Nougtara, Y. Ouedraogo, P. Tapsoba, and I. Kabore. 1996. Rapport Final: Deuxième Analyse Situationnelle du Programme de Planification Familiale au Burkina Faso. Ministère de la Santé, Direction de la Santé de la Famille, and Population Council, Burkina Faso.

Miller, K., I. Kabore, N. Diop, P. Nebie, A. Bamba, Y. Ouedraogo, P. N’Diaye, and P. Tapsoba. 1997.
"Change in family planning service quality in Burkina Faso, 1992 to 1995." Unpublished.

\section{Ghana}

Ghana Statistical Service. Forthcoming. "Family planning service delivery in Ghana: Marching forward or marking time?" Ghana Statistical Service. Accra, Ghana.

Twum-Baah, K., P. Wolf, P. Nyarko, and H. Odai. 1994. A Situation Analysis Study of Family Planning Service Delivery Points in Ghana. Ghana Statistical Service, USAID, and Population Council, Accra, Ghana.

Twum-Baah, K., E. Ameka, E. Okrah, and A. OheneOkai. 1997. Second Round Situation Analysis Study of Family Planning Service Delivery Points in Ghana. Ghana Statistical Service, Accra, Ghana

\section{Kenya}

Miller, R., L. Ndhlovu, and M. Gachara. 1989. A Situation Analysis of the Family Planning Program of Kenya: The Availability, Functioning, and Quality of MOH Services. Population Council, New York.

Miller, R., L. Ndhlovu, M. Gachara, and A. Fisher. 1992. "Situation Analysis study of Kenya's family planning program." In Jain, A., Ed., Managing Quality of Care in Population Programs. Kumarian Press, Connecticut.

Ndhlovu, L., J. Solo, R. Miller, K. Miller, and A. Omunde. 1997. An Assessment of Clinic-Based Family Planning Services in Kenya: Results from the 1995 Situation Analysis. Ministry of Health, Division of Family Health, Nairobi, Kenya, and the Population Council, New York.

\section{Zimbabwe}

Dube, H., C. Marangwanda, and L. Ndhlovu. 1998. An Assessment of the Zimbabwe Family Planning Programme: Results from the 1996 Situation Analysis Study. Evaluation and Research Unit, ZNFPC, and the Population Council, Harare, Zimbabwe and Nairobi, Kenya.

Zimbabwe National Family Planning Council, Population Council, and SEATS. 1992. Zimbabwe: A Situation Analysis of the Family Planning Programme. Population Council, Harare, Zimbabwe.

\section{NOTES}

1 This chapter is based on work done by many researchers in four countries. In Burkina Faso: 
I. Kabore, N. Diop, P. Nebie, A. Bamba, Y.

Ouedraogo, P. N'Diaye, and P. Tapsoba. In Kenya: L. Ndhlovu, J. Solo, R. Miller, and O. Achola. In Ghana: K. Twum-Baah, E. Ameka, E. Okrah, and A. OheneOkai. In Zimbabwe: H. Dube, C. Marangwanda, and L. Ndhlovu.

2 The four reports are K. Miller et al., 1997 (Burkina Faso); Ghana Statistical Service, forthcoming (Ghana); Ndhlovu et al., 1997 (Kenya); and Dube et al., 1998 (Zimbabwe).

3 In Senegal, a quasi-experimental study with a strong evaluative function is currently under way, and certain other countries are planning similar studies. See Chapter 4 for further discussion.

4 For ease of reference within the text, the indicators in this chapter are numbered consecutively by country. Thus, for example, K-R.1 is the first readiness indicator discussed for Kenya, while G-Q.3 is the third quality indicator discussed for Ghana.

5 A similar decline in examining room quality was observed among the 45 individual SDPs that were visited in both studies.

6 These are not requirements of the $\mathrm{MOH}$, but levels of method availability that take into account the current state of each type of facility and what can reasonably be expected. Burkina Faso's national norms and standards establish more stringent requirements for method availability (Bakouan et al., 1992), but applying those standards yielded $0 \%$ for this indicator, which is not a useful or informative result.

7 Not all the indicators included in the Ghana report are reproduced here, mainly because of space constraints. The indicators shown in Tables 11-3 and 114 were chosen based on their rough comparability with indicators from the other three studies summarized here.

8 No decline in this statistic was recorded among the 109 revisited SDPs. Therefore, this result may be attributable to the inclusion in 1996 of more SDPs that did not offer family planning services 5 days per week, rather than some SDPs reducing the number of days they offered services.

9 These results were calculated among clients at SDPs with each type of IEC material available.

10 There were 47 IUD insertions observed in 1993 and 44 in 1996.

11 As compared with measuring the methods usually offered, regardless of whether stocks were available on the day of the visit, as in the other three studies included here.

12 The difference in these means cannot be tested for statistical significance because of difficulties with the data.

13 The comparison of the 1991 and 1996 Zimbabwe Situation Analysis studies includes a section on CBD quality, but these results are not presented in this chapter, whose focus is clinic-based services.

14 Changes in these three indicators of information given to clients must be interpreted with caution because they were collected with different methods in the two studies. In 1996, the data were taken from the observation, whereas in 1991, data were available only from the exit interview, in an unprompted question to clients.

15 This finding could be due to the crudeness of the Situation Analysis measurement of supervision. With more information on what supervisors actually do during visits, the effects of supervision might become clearer.

16 In Chapter 3, the total sample for Kenya is somewhat smaller because several NCC clinics were removed to increase the sample's national representativeness. These clinics were not removed for the comparison summarized here.

17 In Chapter 3, small weights are applied to the 1996 Zimbabwe data to account for the slight skewedness resulting from the change in universe. Thus, the 1996 results presented here will differ slightly from those presented in Chapter 3. 



\section{SUMMARY, CONCLUSIONS, FUTURE DIRECTIONS, AND RECOMMENDATIONS}

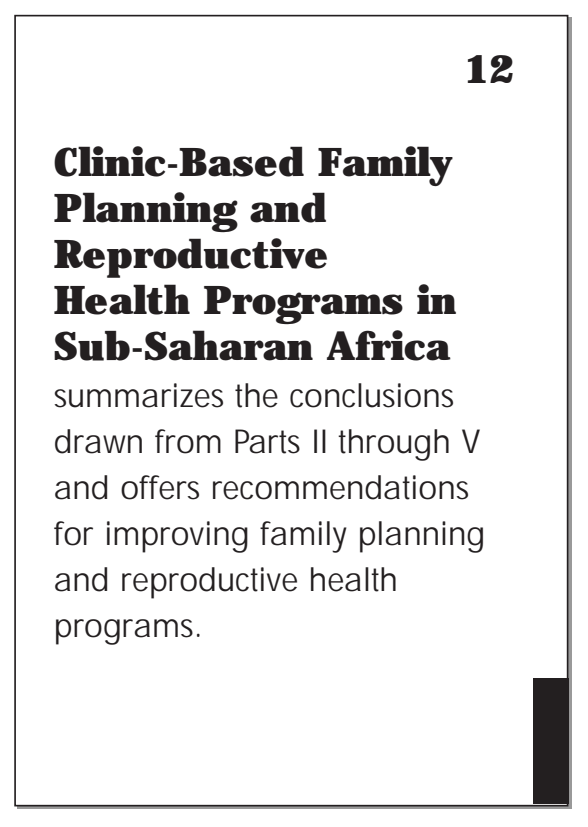





\title{
Clinic-Based Family Planning and Reproductive Health Programs in Sub-Saharan Africa
}

\author{
Robert Miller, Ian Askew, Marjorie C. Horn, and Kate Miller
}

\section{SUMMARY}

\section{Context}

Sub-Saharan Africa faces monumental reproductive health challenges, including the highest maternal mortality, population growth, and total fertility rates in the world; much unmet need for family planning; and substantial health problems resulting from unsafe abortions (see Chapter 1 in this volume and Rosen and Conly, 1998). The region also is the hardest hit by the world's HIV/AIDS pandemic, experiencing the highest rate of HIV/AIDS infection and $83 \%$ of global AIDS deaths (UNAIDS and World Health Organization, 1998). In an effort to address these extraordinary challenges, many national governments, with donor support, have established integrated family planning and maternal and child health programs that deliver services to women in thousands of public health facilities (as well as separate national AIDS programs that emphasize mass media-oriented information, education, and communication [IEC] and national surveillance). These public-sector clinic-based programs usually serve the majority of women using modern methods of contraception (up to $95 \%$ in Botswana).

This volume presents the results of a synthesis of basic findings from 12 Situation Analysis studies of these family planning and reproductive health programs. These studies were under taken over the last decade in Botswana, Burkina Faso, Côte d'Ivoire, Ghana, Kenya, Madagascar,
Nigeria, Senegal, Tanzania, Zambia, Zanzibar, ${ }^{1}$ and Zimbabwe.

When interpreting the findings presented in this volume, it is important to keep in mind the context for the programs examined, including the extent of the reproductive health problems these programs face and the economic conditions that characterize the region. Sub-Saharan Africa is the poorest region of the world, with economies that are deteriorating as the world experiences the global financial crisis of the late 1990s-a region where it is difficult to implement any public-sector program. Nevertheless, despite these caveats, the region's family planning and health programs are probably the best of its public-sector programs.

\section{The Situation Analysis Approach}

The Situation Analysis approach utilized for these studies is a process-oriented assessment methodology that for the first time provides a field-based, quantitative, large-scale framework for assessing national family planning programs. The methodology includes visits to a national, representative sample of family planning service delivery points (SDPs), where field researchers-most often nurses-conduct interviews with service providers and family planning and maternal and child health $(\mathrm{MCH})$ clients and observe the actual delivery of services to family planning clients (see Chapter 1 in this volume and Miller et al., 1997).

The underlying model guiding data collection emphasizes two elements: (1) the readiness of the 
service-delivery system to provide services, and (2) the actual quality of care delivered by providers and received by clients, following the Bruce-Jain quality framework (Bruce, 1990). Quality of care is considered important for its own sake. Although this assumption has not yet been convincingly demonstrated by research, quality of family planning services is considered a precursor to both important health-related individual outcomessuch as satisfaction with services, family planning use and continuation, and achievement of reproductive goals-and related societal demographic measures, such as population growth rates (see Figure 1-1 in Chapter 1).

Developed in 1988 in Kenya and rapidly disseminated around the world, the Situation Analysis methodology complements the older research tradition focused more on populationbased measures of family planning knowledge, attitudes, and practices than on those factors within the service-delivery system over which managers have more direct control (Fisher et al., 1991). Situation Analysis, for the first time, provides an appropriate framework for assessing national programs.

The 12 studies included in this volume were carried out in sub-Saharan countries where local interest was expressed and where the United States Agency for International Development (USAID) has been most active over the last 10 years. The national family planning programs studied include those considered the most effective in the region apart from South Africa, such as Botswana, Kenya, and Zimbabwe (National Research Council, 1993).

At the time of the studies, half the family planning programs were in the relatively early "launch" program phase; that is, the countries' contraceptive prevalence rates (CPRs) were between $8 \%$ and $15 \%$ (see Chapter 2). One-quarter of the programs were in the "emergent" phase, with CPRs below $8 \%$, and only one-quarter were in the more advanced "growth" phase, with CPRs of $16 \%$ to 34\% (Destler et al., 1990). Countries in East and Southern Africa were experiencing especially high rates of HIV / AIDS and other sexually transmitted infections (STIs) relative to other parts of the continent.
Smaller countries without USAID support and countries where political and factional fighting have been endemic are underrepresented in these studies. Thus, the volume is biased toward subSaharan countries with higher levels of resources and donor assistance and with functioning programs of higher quality relative to the region as a whole. Despite these limitations, however, the information presented here comprises the most comprehensive assessment of clinic-based servicedelivery practices yet undertaken in Africa, and most likely in any region of the world.

These studies represent a substantial collaboration among ministries of health, the Population Council's Africa Operations Research and Technical Assistance Projects, USAID, and numerous other agencies. Data for the basic findings were collected from approximately 2,500 SDPs; through observations of 7,000 client-provider interactions; and through interviews with 4,700 staff, 7,200 family planning clients, and 12,000 MCH clients. (An additional four studies are used for comparisons of programs over time in Chapter 11.) The volume describes the readiness of clinics to offer family planning and other reproductive health services and the quality of care received by clients. It also presents several comparative analyses that focus on different components of reproductive healthcare programs.

Situation Analysis studies are used primarily by program managers, policy makers, and donors to assess the strengths and weaknesses of reproductive health programs and to plan related activities designed to strengthen services. Many examples of how program managers have utilized Situation Analysis data are provided in this volume (see Chapter 4). These include modifying training curricula, redeploying personnel and equipment, changing management information systems, reorienting technical assistance plans, and planning follow-up research studies to test solutions to program problems.

In light of the vast amount of data examined here, what conclusions can be reached to aid the various groups involved in improving reproductive health policies and service delivery? The next section summarizes the key conclusions that emerge from the findings presented in Parts II 
through $\mathrm{V}$ of this volume with respect to availability of and access to services, SDP readiness, urban vs. rural services, quality of care, and changes in programs over time. This is followed by a discussion of future directions, as well as recommendations toward the development of improved quality of family planning and reproductive health services in the region.

\section{CONCLUSIONS}

\section{Availability of and Access to Services}

All countries represented in these studies have integrated family planning services within their broader primary healthcare structures, including the lowest level-the health post (see Chapter 1). Consequently, if a woman has access to a publicsector healthcare facility (which can itself be a limiting factor in many parts of Africa), she usually will also have access to family planning information and services. (Since the Situation Analysis approach is clinic-based, it cannot provide overall information on general access to services, which is instead found in population-based Demographic and Health Surveys.)

For the most part, clinic facilities are open daily Monday through Friday (and frequently on Saturdays). They usually open on or nearly on time. Providers generally stated that they offer family planning services at any time of the day (data not shown), although field researchers reported that providers were frequently found to encourage women to come in the mornings and to discourage afternoon attendance.

Providers were found to introduce, often of their own accord, a large number of eligibility criteria and other restrictions on access, such as minimum age, marital status, spousal consent, and minimum parity for different methods (see Chapter 8). Laboratory tests and procedures were also found to present barriers to access (see Chapter 9). These restrictive criteria based on client characteristics or laboratory testing, although not recommended by international standards, sometimes appear in national guidelines, which is itself a matter for concern. More important, the criteria are vastly overapplied by providers. In each of the five countries examined in Chapter 8, providers applied at least twice as many eligibility criteria as were required by national guidelines. This was especially the case in Kenya, Botswana, and Zanzibar, where from one-quarter to two-thirds of all eligibility criteria examined were applied by providers at their own discretion (see Figure 8-9 in Chapter 8).

\section{Service Delivery Point Readiness}

Readiness relates to the availability and functioning of infrastructure or subsystems that comprise the foundation for the service system, such as equipment, logistics and supplies, IEC activities, and management and supervision (see Chapter 1).

Overall, the majority of accessible public health facilities in the countries studied are ready to deliver a basic level of family planning information and services. They usually have trained nurses, selected basic equipment, and contraceptive supplies. A more complete review of the indicators summarized in Annex 3.1 to Chapter 3, however, reveals significant problems in a number of areas, including refresher training of staff and availability of IEC materials, running water, electricity, and various equipment. For example, among the 12 studies, the median percentage of SDPs with running water was 70\%. Gloves and systems for sterilizing instruments are also problematic in several countries, especially Burkina Faso, Nigeria, and Tanzania, where from one-third to one-half of SDPs reported that such equipment is not available in sufficient quantity. As a result, services are sometimes delivered without appropriate attention to the maintenance of cleanliness and/or aseptic conditions to prevent infections. This can be a problem when a pelvic exam is deemed necessary, and thus is a particular concern for staff expected to provide IUD and STI screening services. (When the indicators of washing hands before providing service, using gloves during service, and washing hands after providing service are examined together, under the assumption that any one of the three represents an effort to follow clean/aseptic procedures, this problem appears somewhat less severe.) 


\section{Staffing and Training}

SDPs are staffed mainly by nurses (and not by physicians, except in Côte d'Ivoire). In every study site except Zanzibar (where only 38\% of SDPs are staffed by nurses), more than $70 \%$ of the SDPs visited had at least one nurse on duty, and 6 of the 12 sites had more than $90 \%$ of SDPs staffed by nurses. In 5 of the 6 countries where data on basic or refresher training in family planning are available, at least $85 \%$ of the staff interviewed had received such training. (Training content is discussed further in the section below on the technical competence element of quality.)

\section{Equipment, Methods Offered, and Supplies}

One somewhat surprising but encouraging finding was that most SDPs do have the basic equipment, facilities, and commodities needed to deliver at least three or four types of family planning methods. In every country except Nigeria, scales, stethoscopes, equipment for sterilizing instruments, and blood pressure machines are generally available in more than two-thirds of SDPs. As would be expected, hospitals and some health clinics offer a more complete range of methods than do health posts, and are more likely to offer tubal ligation, vasectomy, or implants. Hospitals also have laboratories and are often capable of conducting various STI tests.

Contraceptive supplies are normally in stock at SDPs and are stored reasonably well. Among the 12 study sites, the median percentage of SDPs found to have experienced a stockout in the previous 6 months was $24 \%$ for injectables, $16 \%$ for combined oral contraceptives, $14 \%$ for condoms, and $14 \%$ for IUDs. Nigeria, Zimbabwe, Zambia, and Kenya had the highest stockout rates for offered methods in the previous 6 months, with about half or more of the SDPs reporting a stockout of at least one method. Zanzibar, Senegal, and Madagascar had fewer than $10 \%$ of SDPs reporting any stockouts in the previous 6 months.

\section{Urban vs. Rural Services}

The study findings reveal that SDPs in rural areas tend to be somewhat less ready to offer family planning services than those in urban areas, which are more advantaged with regard to the availability of water, electricity, staff, equipment and supplies, and many other factors (see Chapter 7). As can be seen in Figure 7-5, for each of 6 countries included in the analysis, an urban advantage was found for approximately one-quarter to one-half of the indicators examined, while a rural advantage appeared for only $4 \%$ to $16 \%$ of the indicators. Perhaps most surprising, little difference was found between urban and rural services on our measures of quality (see Figure 7-6).

\section{Quality of Care}

As noted earlier, the quality of care delivered to and received by clients is examined according to the Bruce-Jain model (Bruce, 1990) in Situation Analysis studies (see Chapter 1). Several elements of quality (choice of methods, mechanisms to encourage continuity, provider-client relations, and technical competence) show some strength in the 12 studies included here. However, our analyses reveal serious shortcomings in the information exchanged during counseling and in provision of the appropriate constellation of services (particularly the integration of STI/HIV / AIDS services).

\section{Choice of Methods}

On the positive side, more than $60 \%$ of clients are told about at least two family planning methods, except in Côte d'Ivoire and Zimbabwe. (It is possible that many clients who do not receive information on two or more methods come to the SDP with a choice already made. In such cases, providers may be discouraged from discussing other methods-a practice that has both supporters and detractors [see Pariani et al., 1987]). Many women do not, however, receive sufficient information about each method's possible side effects, its benefits and drawbacks, and its relationship to HIV / AIDS prevention (see Chapter 6 and the discussion of information exchange below).

\section{Mechanisms to Encourage Continuity of Care}

The most basic mechanisms to encourage continuity of use and follow-up are generally in place. Nearly all women are asked to come back for follow-up. Other data show that most are provided with written reminders, which is standard practice in all of 
the programs examined. However, the proportion of new clients told about possible side effects ranged from $68 \%$ to only $24 \%$ in our studies, with a median of $55 \%$. Problems with continuity are certainly exacerbated when women experience unexpected side effects with their method and therefore stop using it. Lack of discussion about the possibility of switching methods may also inhibit continuity. ${ }^{2}$

\section{Provider-Client Relations}

The vast majority of providers were observed to relate reasonably well with their clients, and few reports of poor social relations between providers and clients were documented. For example, more than $80 \%$ of clients were observed to receive a friendly greeting in all countries except Côte d'Ivoire. More than $95 \%$ of women interviewed in all 12 studies reported that the provider was easy to understand. It may be noted, however, that client satisfaction with services is notoriously difficult to measure through Situation Analysis or any survey approach because of "courtesy bias," whereby clients do not wish to appear rude or ungrateful in giving responses to such questions (Simmons and Elias, 1993).

Moreover, while provider-client relations score well on these direct measures, Situation Analysis indicators are somewhat superficial gauges of the nature of the provider-client relationship. Overall (as discussed under information exchange below), most providers appear reluctant to discuss intimate topics. It is unclear what factors underlie this reluctance. Possibilities include social norms against embarrassing or intimate discussions, social distance between a professionally trained nurse and a less-educated client, and lack of focused training to improve attitudes and skills in such matters.

\section{Technical Competence}

The technical competence of providers appears to be reasonably good with regard to certain specific tasks, such as using sterile needles for injections (observed in more than 95\% of the cases in all 9 countries where this indicator was studied). Similarly, in 9 of 11 countries, a clean or sterile speculum was used during pelvic exams in more than $80 \%$ of the observed cases.
One of the most obvious problems, however, is that some staff offer technical and invasive services, such as IUD insertion, pelvic examination, and STI/HIV screening and management, without having had appropriate or sufficient training. In the 3 countries where clinical training in family planning was specifically investigated, only $40 \%$ to $68 \%$ of staff reported having received such training. Nurses who were working prior to the establishment of the family planning program are less likely to have had family planning training. Similar gaps appear in STI/HIV/AIDS training. Lack of training in the proper use of less invasive methods, such as pills and condoms, though problematic, may not be quite as serious as is the case with more invasive methods since there is less chance of iatrogenic infections.

\section{Information Exchange}

In our view, the most serious limitation to the quality of care provided is the information exchanged during counseling. Providers usually do not ask for the background necessary to provide the full range of information, counseling, and contraceptive methods. For example, the median percentage of clients asked their reproductive intentions in the 12 studies was only $56 \%$, with a range of $28 \%$ in Tanzania to $90 \%$ in Senegal. The median proportion of provider-client interactions observed in which providers asked about the nature of the clients' sexual relations was $14 \%$. Information such as whether the woman's partner was included in the decision to seek family planning, the number of sexual partners over the past year, or whether the woman thinks she might be at risk of STIs as a result of her partner's behavior is rarely sought. Such information is central to the selection of an appropriate method and to the offering of other reproductive health information and services, such as STI/ HIV/AIDS prevention (see Chapters 6 and 10). Moreover, providers did not inquire about clients' breastfeeding status in $40 \%$ to $50 \%$ of the interactions observed, despite the fact that most programs have access to progestin-only contraceptives and in some countries many providers have been trained in lactational amenorrhea method (LAM).

Not only do providers not obtain full information on the client's individual situation, but they 
frequently do not provide all the information the client needs. While in 9 of 11 study sites almost $80 \%$ or more of new clients were told how to use their method, the results were less encouraging on such important dimensions as possible side effects of different methods (most notably the method accepted for use) and how to manage side effects, including the possibility of switching methods if desired.

In addition to a possible lack of skills, prevailing social norms, and/or social distance, failure to provide information may be due in part to the poor availability of printed and other materials to assist providers in communicating information. For example, brochures were available in less than half the SDPs in the majority of the 12 study sites. Additionally, those educational materials that are available are used very infrequently (with $11 \%$ of clients or fewer, except in Zambia, where available materials are used with $22 \%$ of clients) (see Chapter 6).

\section{Appropriate Constellation of Services}

The promotion of an integrated approach to reproductive health services, most notably by integrating STI/HIV/AIDS management and prevention into family planning services, has major implications for service delivery in sub-Saharan Africa (see Chapter 10). Unfortunately, use of the signs and symptoms of potential infection-the syndromic approach-is proving to be unreliable as an STI diagnostic and management tool for females. Moreover, inexpensive and easily administered laboratory testing is not now available in clinics and health posts in the region. Thus the view that STI detection and management could or should be routinely included as part of family planning consultation in African SDPs is being actively debated, with support waning in some circles as discussion of even the best examples of attempts at integration reveals severe problems with implementation (Maggwa and Askew, 1997).

In addition to the fact that laboratory testing for STI diagnosis and treatment is not now a viable option in most SDPs (except perhaps for syphilis testing), providers are not actively pursuing those options that are available to them for preventing or at least reducing the transmission of STI/HIV/
AIDS among their family planning clients, namely communicating messages about safer sexual practices, especially condom use. Because clients' sexual behavior is not usually discussed, an important opportunity to ascertain their risk of infection and to provide education on preventing infection is lost. Further, although condoms are mentioned to about half of clients (although there is significant variation across countries), this is almost always done in the context of contraception only; dual protection from both pregnancy and STI/HIV / AIDS is rarely mentioned to clients. The range of clients who were told specifically that condoms protect against STIs/HIV/AIDS varied from a low of $2 \%$ in Senegal to a high of only $36 \%$ in Zambia (see Annex 3.1 in Chapter 3).

Clients are also not told that methods other than condoms do not offer STI/HIV/AIDS protection. In 3 of the 4 countries in which this indicator was studied, fewer than $10 \%$ of clients were provided this information. Given the lack of availability of STI/HIV/AIDS testing and providers' limited attention to clients' sexual practices, partnership issues, and social context, it is difficult not to conclude that the existing reproductive health service system, with its emphasis on family planning alone, is making very little contribution to ameliorating the HIV / AIDS pandemic in the worst-affected region of the world.

In addition to this neglect of the STI/HIV/ AIDS issue, observations of client-provider interactions suggest that there is relatively little attention to health issues other than STIs/HIV / AIDS (such as nutrition, immunization, or baby care) when family planning services are being provided. Indeed, in several programs, family planning and other $\mathrm{MCH}$ services appear to be provided almost as vertical programs that happen to be offered under the same roof.

\section{Changes Over Time}

In several countries in the region-Burkina Faso, Ghana, Kenya, and Zimbabwe-two or more Situation Analysis studies were carried out a few years apart (see Chapter 11). Some similar patterns emerged. For example, the choice of methods offered to clients has improved, usually as a result 
of more reliable supplies of pills and condoms and the increased availability of injectables, IUDs, implants, and sterilization in the intervening years. At the same time, and requiring serious attention, the information offered to clients appears to be a significant and continuing problem in all four locations. This review, using Situation Analysis of service quality, draws attention to the complexities of achieving real increases in the quality of care, and yields two general lessons. First, improving service quality requires attention to several aspects of readiness at once. Secondly, while the situation analysis methodology can measure overall changes in various aspects of service quality, it cannot ascribe these changes to any particular intervention. The latter would require studies based on quasi-experimental design.

\section{FUTURE DIRECTIONS}

Considering the relatively short period of time during which many governments in Africa have been providing family planning and reproductive health services, the recency of the paradigm shift toward reproductive health and quality, and the large number of competing demands for healthcare resources, the achievements made in establishing the levels of program readiness and quality documented in this volume are substantial. In all cases, the development of these services has been assisted by external donor agencies. Both program managers and international donor agencies deserve considerable credit for introducing a major new health service rapidly and on a broad scale. Recall, however, that our 12 studies exclude many of the smaller countries with less-stable political systems and less external assistance, such as Liberia, Sierra Leone, and Somalia. It is quite likely, then, that the reproductive health programs of sub-Saharan Africa as a whole are less ready to provide services and have poorer-quality services than is suggested by the 12 studies included here. Thus the regional needs are probably far greater than is represented in this volume.

What should be done to improve the quality of family planning and reproductive health services in sub-Saharan Africa? Should efforts be made to increase the readiness of clinics, with the expectation that doing so will lead to improved quality? The analyses presented in Chapters 5 and 6 of this volume reveal that many of the components measured in Situation Analysis studies that contribute to clinic readiness to offer basic services do not appear to be associated directly with improved quality of care as defined by the Bruce-Jain model. An apparent exception to this general finding is that the provision of in-depth training programs specifically focused on family planning counseling and on the use of IEC materials with clients-such as those provided in courses on natural family planning (NFP) and LAM, methods that depend on quality counseling-does improve the quality of the provider-client information exchange (see Chapter 5). ${ }^{3}$ In contrast, general family planning training and technical updates alone have had little impact on counseling. Studies with more rigorous experimental or quasi-experimental research designs are required, however, to test efficient and effective means of delivering focused training on quality counseling.

In many African countries, considerable attention has recently been directed toward developing improved guidelines for policies and procedures and disseminating these widely and in creative ways-for example, through USAID's initiative to Maximize Access and Quality [MAQ] of family planning services. As noted earlier, the results from our Situation Analysis studies, which predate this USAID effort, show that program guidelines are frequently not followed by providers in imposing restrictions on and requiring tests and procedures for family planning methods. In deciding who is eligible for each method, providers appear to be guided more by social norms and personal beliefs about who should use which method than by existing national standards and guidelines (TwumBaah and Stanback, 1995). Thus USAID's MAQ initiative will require careful assessment to determine whether the increased attention to the development of new standards and guidelines and more aggressive dissemination activities improve actual provider behavior.

Although most infrastructure elements required for service delivery are in place, these elements are frequently not used by providers (see 
Chapter 6). One major challenge, then, is to bring the actual quality of care more in line with the potential that exists, given the current state of readiness. While it may be unlikely that all clinics will have running water and educational materials in the near future, providers must be encouraged to wash their hands before examining clients, and be trained to use IEC materials consistently, effectively, and with broader reproductive health objectives when such materials are available. Where national policy guidelines have been revised to give more complete and effective guidance, providers need to be encouraged to refer to those guidelines often and to use them, rather than devising their own rules and procedures.

In the future, new STI/HIV / AIDS testing technology can be expected to influence the ongoing debate on this subject. However, even with such improved technology, the situation can be expected to remain problematic. Frerichs (1994) has argued that there has been too much hesitation to use available public health technologies, thereby accepting nondetection of HIV as the norm. In Africa, as in other regions, there is considerable reluctance to perform HIV testing in the absence of careful AIDS counseling, which many providers have not been trained to offer (in 5 countries, the mean percentage of staff who had received any refresher training in STIs/HIV/AIDS was 48\%, with Botswana having the highest level). Also, as noted earlier, providers appear to avoid complex, intimate issues in discussions with clients.

Improving quality of services to take full advantage of current levels of readiness will clearly necessitate progress at two levels: the SDP and the national-level program manager. At the SDP level, AVSC International's Client Oriented and Provider Efficient (COPE) quality assurance approach (AVSC, 1995), which encourages the development of internal systems for identifying problems, causes, and solutions at an individual SDP, is one of the most effective approaches identified to date. AVSC is developing a guide for COPE that covers broad reproductive health issues and is expected to help providers understand integration and quality issues (AVSC, forthcoming). Several other tools have proven useful, including the "ABC" decision aide to guide providers through the counseling and decision-making process (Leon, 1995). Sloan and colleagues (1998) have recently developed a computer-based aid, somewhat analogous to a combination of a supervisory checklist and a limited Situation Analysis, to assist supervisors in improving quality of care in family planning programs. Overall, Shelton (1998) has recommended a synergy of components of MAQ promotion, which, at the SDP level, include problem solving; job aids; improved indicators and evaluation; and creation of a more supportive environment, particularly with regard to supervision.

In addition to quality assurance activities at the individual SDP level, the Situation Analysis approach is based on the assumption that there are a number of activities that can be undertaken only by national-level program managers, and that these national-level interventions may have broader impact than quality assurance efforts alone on the several thousand SDPs in the region that have not yet benefited from individual SDP efforts. Shelton's (1998) synergy of components of MAQ promotion also includes elements at this level: development of guidelines, improved program leadership, community promotion, and improvements to the organizational culture. Clearly, management decisions to give more attention to STI/HIV/AIDS prevention will need to begin and be supported at the national level.

Thus far, in the 4 countries in which two Situation Analysis studies have been conducted (see Chapter 11), it has not proved possible to measure the impact of the numerous managerial decisions and interventions adopted during the interval between the studies on the major quality problems noted earlier-counseling of clients and integration of STI/HIV / AIDS prevention. We suspect that this situation has several causes: (1) the managerial decisions and other interventions resulting from Situation Analysis studies were insufficiently focused on the specific quality problems found throughout the region; (2) the scope of various interventions was probably insufficient; (3) the relationship between interventions and improvements in quality is tenuous (see Chapter $11)$; and (4) the research designs of our time comparisons may not have been appropriate for assessing the impact of particular interventions. 


\section{RECOMMENDATIONS}

A large number of recommendations resulted in response to the myriad of problems revealed by each of the individual Situation Analysis studies included in this volume. Clearly there is substantial room for developing and evaluating new and creative approaches to improving national family planning and reproductive health programs in Africa. However, one of the benefits of reviewing the 12 studies together is to highlight certain widespread regional patterns, such as problems with counseling of clients and prevention of STI/HIV/ AIDS. Overall, then, we offer the following recommendations to program managers, providers, and donors who are interested in improving service quality in the region.

1. Use existing resources more efficiently. Some problems of service quality are not the result of a lack of resources, but can be alleviated through more efficient use of existing resources. Examples include using IEC materials that are present; using clean water that is available in the examination room; and taking the time to discuss clients' context, particularly at SDPs where few clients are seen per day by each provider. Using existing resources efficiently also means posting providers who are trained in specific methods at SDPs that offer those methods (see Chapters 3 and 6). Condoms are widely available, but they need to be recommended for protection against both unwanted pregnancy and the dangers of STIs/HIV / AIDS.

2. Recognize that improved readiness does not necessarily lead to improved quality of services. Interventions to improve services frequently focus on elements of readiness, such as staff training, equipment availability, or commodity management. However, these individual readiness improvements may not lead to changes in the quality of care delivered to clients. Thus interventions to improve readiness must also address how that improved readiness will be translated into improved quality (see Chapters 5, 7, and 11).

3. Train staff on specific issues, particularly on broader reproductive health counseling. All programs covered in our studies train their staff through basic, post-basic, and refresher training. The study results suggest, however, that training has a stronger impact on the quality of services if it is focused specifically on counseling; IEC; or family planning methods, such as LAM or NFP, for which the role of correct client counseling is crucial. Less-focused training is unlikely to affect the important dimensions of quality highlighted here (see Chapters 3 and 5).

4. Bridge the gap between protocols and provider behavior. The development of improved service protocols is critically important to improving services, but it may not lead immediately to changes in provider behavior. To be effective, protocols must be reinforced with training and supervision, and their introduction requires continued monitoring and assessment of their impact on provider behavior (see Chapters 8 and 9).

5. Strengthen supervisor training, and broaden the introduction of new supervisory tools. At present, Situation Analysis studies do not show a relationship between the quantity of supervision and the quality of services provided (see Chapter 11). This may be because the Situation Analysis data on supervision needs to better describe specific behaviors of supervisors, and/or because there may be no relationship between supervision as presently conducted and quality as we define it. Supervisors need guidance on what to observe, discuss, and evaluate on a consistent, ongoing basis. Improved supervision may support training in client counseling, STI/HIV/AIDS prevention through increased use of dual protection, and the provision of safe services.

6. Conduct Operations Research to test interventions on quality. To better understand how program goals can be reached, OR studies are needed to test whether interventions are effective, and how they can be scaled up to national and region-wide improvements in service delivery. Since the major problems identified are region wide, coordinated OR programs should be developed. Potential productive areas for OR include delivery of effective training to accomplish behavioral and attitudinal 
change, coordination of training and supervisory efforts to strengthen providers' counseling skills on family planning and the prevention of STI/HIV/AIDS, and means of making better use of existing resources. Experimental or quasi-experimental designs, including control groups, should be used to test interventions.

7. Conduct research to improve understanding of factors supporting the present pattern of service delivery, as well as client perspectives on quality. Further explanation may be required for providers' apparent reluctance to engage in certain types of intimate discussion with clients (see Chapters 3 and 5). While the Bruce-Jain conceptualization of quality has been enormously productive over the last decade, alternative perspectives on qualityperhaps ones that better integrate both the client and provider perspectives-need continuing investigation.

8. Strengthen the Situation Analysis methodology, and continue its use with a primary focus on assisting program managers. The Situation Analysis approach has become a standard tool, and the existing conceptual guidance for the methodology (see Chapter 1 ) has been relatively productive. Numerous interventions have resulted from Situation Analysis findings (see Chapter 4). Clearly, however, there is room for substantial improvements to this study methodology. The definition and selection of variables and the construction of indicators can be improved, and the number of variables included can probably be reduced. The logical links among readiness, quality, and impact need to be explored further with additional research, which can be used to strengthen the underlying model. The reliability and validity of the data require additional investigation as well. The primary objective of Situation Analysis studies to date has been to assist program managers in identifying and solving problems with readiness and quality; the secondary goal has been to advance research on these subjects. In our view, this order of priorities should continue.

The African Situation Analysis study results compiled over the last decade have made it possible to identify important program achievements in readiness and quality, as well as crucial region-wide shortcomings. The research findings presented in this volume also suggest potentially productive interventions to strengthen program quality. Taken together, the existing programs and the research findings represent both a solid foundation on which to build and a substantial challenge. It is our hope that program managers, providers, and donors will use these findings in their efforts to develop more comprehensive, higher-quality services that better meet the needs of African families.

\section{REFERENCES}

AVSC International. 1995. COPE: Client-Oriented, Provider-Efficient Services. AVSC International, New York.

AVSC International. Forthcoming. COPE Self-Assessment Guidelines for Reproductive Health Services. AVSC International, New York.

Bruce, J. 1990. "Fundamental elements of the quality of care: A simple framework." Studies in Family Planning, 21,2:61-91.

Destler, H., D. Liberi, J. Smith, and J. Stover. 1990. Family Planning: Preparing for the 21st Century. United States Agency for International Development, Washington, D.C.

Fisher, A., J. Laing, J. Stoeckel, and J. Townsend. 1991. Handbook for Family Planning Operations Research Design, Second Edition. Population Council, New York.

Frerichs, R. 1994. "Personal screening for HIV in developing countries." Lancet, 343,8903:960-962.

Leon, F. 1995. El ABC de la Atención Básica de Planificación Familiar, 4ta Edición. Population Council, Mexico.

Maggwa, N. and I. Askew. 1997. Integrating STI/HIV Management Strategies into Existing MCH/FP Programs: Lessons from Case Studies in East and Southern Africa. Population Council, Nairobi, Kenya.

Miller, R., A. Fisher, K. Miller, L. Ndhlovu, N. Maggwa, I. Askew, D. Sanogo, and P. Tapsoba. 1997. The Situation Analysis Approach to Assessing Family Planning and Reproductive Health Services: A Handbook. Population Council, New York.

National Research Council. 1993. Factors Affecting Contraceptive Use in Sub-Saharan Africa. National Academy Press, Washington, D.C. 
Pariani, S., D. Heer, and M. Van Arsdol. 1987. "Continued contraceptive use in five family planning clinics in Surabaya, Indonesia." Studies in Family Planning, 22,6:384-390.

Ross, J. and E. Frankenberg. 1993. Findings from Two Decades of Family Planning Research. Population Council, New York.

Rosen, J. and S. Conly. 1998. Africa's Population Challenge: Accelerating Progress in Reproductive Health. Population Action International, Washington, D.C.

Shelton, James. 1998. Keynote Presentation. In $M A Q$ : From Guidelines to Action; Report of a USAID Conference, May 12-13, 1998. PRIME, Chapel Hill, North Carolina.

Simmons, R. and C. Elias. 1993. The Study of ClientProvider Interactions: A Review of Methodological Issues. Programs Division Working Paper No. 7, Population Council, New York.

Sloan, N. 1998. "Computer-based assistance for supervisors to improve quality of care in family planning programs." Unpublished paper. Population Council, New York.

Twum-Baah, K.A. and J. Stanback. 1995. Provider Rationales for Restrictive Family Planning Service
Practices in Ghana. Ghana Statistical Services and Family Health International, Ghana.

UNAIDS and World Health Organization. 1998. Report on the Global HIV/AIDS Epidemic: June 1998. UNAIDS and $\mathrm{WHO}$.

\section{NOTES}

1 Zanzibar consists of several islands lying off the coast of Tanzania, and although it shares certain major government structures with Tanzania, it is separate and independent in several respects, including its health system. Zanzibar is therefore included in this report as a separate study.

2 The Population Council is currently undertaking a study in Senegal to test whether strengthening the quality of care offered does, in fact, reduce premature discontinuation (Ross and Frankenberg, 1993).

3 Similarly, the very specific and high-quality training programs that have been implemented on postabortion care have been shown to have a substantial impact on provider behavior. 